\title{
Regioselective Synthesis of Functionalized 3- or 5-Fluoroalkyl Isoxazoles and Pyrazoles from
}

\section{Fluoroalkyl Ynones and Binucleophiles}

Bohdan A. Chalyk, ${ }^{\mathrm{a}, \mathrm{d}}$ Andrii Khutorianskyi, ${ }^{\mathrm{a}, \mathrm{b}}$ Andrii Lysenko, ${ }^{\mathrm{a}, \mathrm{b}}$ Yulia Fil, ${ }^{\mathrm{a}, \mathrm{b}}$ Yuliya O. Kuchkovska, ${ }^{\mathrm{a}, \mathrm{b}}$ Konstantin S. Gavrilenko, ${ }^{\mathrm{a}, \mathrm{b}}$ Iulia Bakanovych, ${ }^{\mathrm{a}, \mathrm{b}}$ Yurii S. Moroz, ${ }^{\mathrm{b}, \mathrm{c}}$ Alina O. Gorlova ${ }^{\mathrm{a}, \mathrm{d}}$ and Oleksandr O. Grygorenko ${ }^{\mathrm{a}, \mathrm{b} *}$

${ }^{a}$ Enamine Ltd. (www.enamine.net), Alexandra Matrosova Street 23, Kyiv 01103, Ukraine

${ }^{b}$ National Taras Shevchenko University of Kyiv, Volodymyrska Street 64, Kyiv 01601, Ukraine

${ }^{c}$ Chemspace, Ilukstes iela 38-5, Riga, LV-1082, Latvia

${ }^{d}$ Institute of Organic Chemistry, National Academy of Sciences of Ukraine, Murmanska Street 5, Kyiv 02660, Ukraine

*Corresponding author. E-mail: gregor@univ.kiev.ua.

\section{Table of contents.}

1. Synthesis of functionalized derivatives.

2. Structure assignment.

3. Experimental section.

4. $\quad{ }^{1} \mathrm{H}$ NMR spectrum of the compound 13a.

5. ${ }^{13} \mathrm{C}\left\{{ }^{1} \mathrm{H}\right\}$ NMR spectrum of the compound 13a.

6. ${ }^{19}$ F NMR spectrum of the compound 13a.

7. ${ }^{1} \mathrm{H}$ NMR spectrum of the compound $\mathbf{1 3 b}$.

8. $\quad{ }^{13} \mathrm{C}\left\{{ }^{1} \mathrm{H}\right\}$ NMR spectrum of the compound $\mathbf{1 3 b}$.

9. ${ }^{19} \mathrm{~F}\left\{{ }^{1} \mathrm{H}\right\}$ NMR spectrum of the compound $\mathbf{1 3 b}$.

10. ${ }^{1} \mathrm{H}$ NMR spectrum of the compound 13c.

11. ${ }^{13} \mathrm{C}\left\{{ }^{1} \mathrm{H}\right\}$ NMR spectrum of the compound 13c.

12. ${ }^{19} \mathrm{~F}\left\{{ }^{1} \mathrm{H}\right\}$ NMR spectrum of the compound $\mathbf{1 3 c}$. 
13. $\quad{ }^{1} \mathrm{H}$ NMR spectrum of the compound 13d.

14. ${ }^{13} \mathrm{C}\left\{{ }^{1} \mathrm{H}\right\}$ NMR spectrum of the compound 13d.

15. ${ }^{19} \mathrm{~F}\left\{{ }^{1} \mathrm{H}\right\}$ NMR spectrum of the compound 13d.

16. ${ }^{1} \mathrm{H}$ NMR spectrum of the compound $13 \mathbf{e}$.

17. ${ }^{13} \mathrm{C}\left\{{ }^{1} \mathrm{H}\right\}$ NMR spectrum of the compound $\mathbf{1 3 e}$.

18. ${ }^{19} \mathrm{~F}\left\{{ }^{1} \mathrm{H}\right\}$ NMR spectrum of the compound $13 \mathbf{e}$.

19. ${ }^{1} \mathrm{H}$ NMR spectrum of the compound $\mathbf{1 3 f}$.

20. ${ }^{13} \mathrm{C}\left\{{ }^{1} \mathrm{H}\right\}$ NMR spectrum of the compound $\mathbf{1 3 f}$.

21. ${ }^{19} \mathrm{~F}\left\{{ }^{1} \mathrm{H}\right\}$ NMR spectrum of the compound $\mathbf{1 3 f}$.

22. ${ }^{1} \mathrm{H}$ NMR spectrum of the compound 13g.

23. ${ }^{13} \mathrm{C}\left\{{ }^{1} \mathrm{H}\right\}$ NMR spectrum of the compound 13g.

24. ${ }^{19}$ F NMR spectrum of the compound $13 g$.

25. ${ }^{1} \mathrm{H}$ NMR spectrum of the compound $\mathbf{1 3 h}$.

26. ${ }^{13} \mathrm{C}\left\{{ }^{1} \mathrm{H}\right\}$ NMR spectrum of the compound $\mathbf{1 3 h}$.

27. ${ }^{19} \mathrm{~F}\left\{{ }^{1} \mathrm{H}\right\}$ NMR spectrum of the compound $\mathbf{1 3 h}$.

28. $\quad{ }^{1}$ H NMR spectrum of the compound 16a.

29. ${ }^{13} \mathrm{C}\left\{{ }^{1} \mathrm{H}\right\}$ NMR spectrum of the compound $\mathbf{1 6 a}$.

30. ${ }^{19}$ F NMR spectrum of the compound 16a.

31. ${ }^{1} \mathrm{H}$ NMR spectrum of the compound $\mathbf{1 6 b}$.

32. ${ }^{13} \mathrm{C}\left\{{ }^{1} \mathrm{H}\right\}$ NMR spectrum of the compound $\mathbf{1 6 b}$.

33. ${ }^{19} \mathrm{~F}\left\{{ }^{1} \mathrm{H}\right\}$ NMR spectrum of the compound $\mathbf{1 6 b}$.

34. ${ }^{1} \mathrm{H}$ NMR spectrum of the compound 16c.

35. ${ }^{13} \mathrm{C}\left\{{ }^{1} \mathrm{H}\right\}$ NMR spectrum of the compound 16c. 
36. ${ }^{19} \mathrm{~F}\left\{{ }^{1} \mathrm{H}\right\}$ NMR spectrum of the compound 16c.

37. $\quad{ }^{1} \mathrm{H}$ NMR spectrum of the compound $\mathbf{1 6 d}$.

38. ${ }^{13} \mathrm{C}\left\{{ }^{1} \mathrm{H}\right\}$ NMR spectrum of the compound 16d.

39. ${ }^{19} \mathrm{~F}\left\{{ }^{1} \mathrm{H}\right\}$ NMR spectrum of the compound $\mathbf{1 6 d}$.

40. ${ }^{1} \mathrm{H}$ NMR spectrum of the compound 16e.

41. ${ }^{13} \mathrm{C}\left\{{ }^{1} \mathrm{H}\right\}$ NMR spectrum of the compound $\mathbf{1 6 e}$.

42. ${ }^{19} \mathrm{~F}\left\{{ }^{1} \mathrm{H}\right\}$ NMR spectrum of the compound 16e.

43. ${ }^{1} \mathrm{H}$ NMR spectrum of the compound $\mathbf{1 6 f}$.

44. ${ }^{13} \mathrm{C}\left\{{ }^{1} \mathrm{H}\right\}$ NMR spectrum of the compound $\mathbf{1 6 f}$.

45. ${ }^{19} \mathrm{~F}\left\{{ }^{1} \mathrm{H}\right\}$ NMR spectrum of the compound $\mathbf{1 6 f}$.

46. $\quad{ }^{1} \mathrm{H}$ NMR spectrum of the compound 16g.

47. ${ }^{13} \mathrm{C}\left\{{ }^{1} \mathrm{H}\right\}$ NMR spectrum of the compound $\mathbf{1 6 g}$.

48. $\quad{ }^{19}$ F NMR spectrum of the compound $\mathbf{1 6 g}$.

49. $\quad{ }^{1} \mathrm{H}$ NMR spectrum of the compound 18g.

50. ${ }^{13} \mathrm{C}\left\{{ }^{1} \mathrm{H}\right\}$ NMR spectrum of the compound $\mathbf{1 8 g}$.

51. ${ }^{19} \mathrm{~F}\left\{{ }^{1} \mathrm{H}\right\}$ NMR spectrum of the compound 18g.

52. $\quad{ }^{1}$ H NMR spectrum of the compound $\mathbf{1 6 h}$.

53. ${ }^{13} \mathrm{C}\left\{{ }^{1} \mathrm{H}\right\}$ NMR spectrum of the compound $\mathbf{1 6 h}$.

54. ${ }^{19} \mathrm{~F}\left\{{ }^{1} \mathrm{H}\right\}$ NMR spectrum of the compound $\mathbf{1 6 h}$.

55. $\quad{ }^{1} \mathrm{H}$ NMR spectrum of the compound 17a.

56. ${ }^{13} \mathrm{C}\left\{{ }^{1} \mathrm{H}\right\}$ NMR spectrum of the compound 17a.

57. ${ }^{19}$ F NMR spectrum of the compound 17a.

58. ${ }^{1} \mathrm{H}$ NMR spectrum of the compound $\mathbf{1 7 b}$. 
59. ${ }^{13} \mathrm{C}\left\{{ }^{1} \mathrm{H}\right\}$ NMR spectrum of the compound $\mathbf{1 7 b}$.

60. ${ }^{19} \mathrm{~F}\left\{{ }^{1} \mathrm{H}\right\}$ NMR spectrum of the compound $\mathbf{1 7 b}$.

61. ${ }^{1} \mathrm{H}$ NMR spectrum of the compound 17c.

62. ${ }^{19} \mathrm{~F}\left\{{ }^{1} \mathrm{H}\right\}$ NMR spectrum of the compound 17c.

63. $\quad{ }^{1} \mathrm{H}$ NMR spectrum of the compound 17d.

64. ${ }^{13} \mathrm{C}\left\{{ }^{1} \mathrm{H}\right\}$ NMR spectrum of the compound $\mathbf{1 7 d}$.

65. ${ }^{19} \mathrm{~F}\left\{{ }^{1} \mathrm{H}\right\}$ NMR spectrum of the compound 17d.

66. ${ }^{1} \mathrm{H}$ NMR spectrum of the compound 17e.

67. ${ }^{13} \mathrm{C}\left\{{ }^{1} \mathrm{H}\right\}$ NMR spectrum of the compound $\mathbf{1 7 e}$.

68. ${ }^{19} \mathrm{~F}\left\{{ }^{1} \mathrm{H}\right\}$ NMR spectrum of the compound $\mathbf{1 7}$.

69. ${ }^{1} \mathrm{H}$ NMR spectrum of the compound $\mathbf{1 7 f}$.

70. ${ }^{13} \mathrm{C}\left\{{ }^{1} \mathrm{H}\right\}$ NMR spectrum of the compound $\mathbf{1 7 f}$.

71. ${ }^{19} \mathrm{~F}\left\{{ }^{1} \mathrm{H}\right\}$ NMR spectrum of the compound $\mathbf{1 7 f}$.

72. $\quad{ }^{1} \mathrm{H}$ NMR spectrum of the compound $\mathbf{1 7 g}$.

73. ${ }^{13} \mathrm{C}\left\{{ }^{1} \mathrm{H}\right\}$ NMR spectrum of the compound $\mathbf{1 7 g}$.

74. ${ }^{19} \mathrm{~F}\left\{{ }^{1} \mathrm{H}\right\}$ NMR spectrum of the compound $\mathbf{1 7 g}$.

75. ${ }^{1} \mathrm{H}$ NMR spectrum of the compound $\mathbf{1 7 h}$.

76. ${ }^{13} \mathrm{C}\left\{{ }^{1} \mathrm{H}\right\}$ NMR spectrum of the compound $\mathbf{1 7 h}$.

77. ${ }^{19} \mathrm{~F}\left\{{ }^{1} \mathrm{H}\right\}$ NMR spectrum of the compound $\mathbf{1 7 h}$.

78. ${ }^{1} \mathrm{H}$ NMR spectrum of the compound 21a.

79. ${ }^{13} \mathrm{C}\left\{{ }^{1} \mathrm{H}\right\}$ NMR spectrum of the compound 21a.

80. ${ }^{19}$ F NMR spectrum of the compound 21a.

81. ${ }^{1} \mathrm{H}$ NMR spectrum of the compound 21b. 
82. ${ }^{13} \mathrm{C}\left\{{ }^{1} \mathrm{H}\right\}$ NMR spectrum of the compound 21b.

83. ${ }^{19} \mathrm{~F}\left\{{ }^{1} \mathrm{H}\right\}$ NMR spectrum of the compound 21b.

84. ${ }^{1} \mathrm{H}$ NMR spectrum of the compound 21c.

85. ${ }^{13} \mathrm{C}\left\{{ }^{1} \mathrm{H}\right\}$ NMR spectrum of the compound 21c.

86. ${ }^{19} \mathrm{~F}\left\{{ }^{1} \mathrm{H}\right\}$ NMR spectrum of the compound 21c.

87. ${ }^{1} \mathrm{H}$ NMR spectrum of the compound 21d.

88. ${ }^{13} \mathrm{C}\left\{{ }^{1} \mathrm{H}\right\}$ NMR spectrum of the compound 21d.

89. ${ }^{19} \mathrm{~F}\left\{{ }^{1} \mathrm{H}\right\}$ NMR spectrum of the compound 21d.

90. $\quad{ }^{1} \mathrm{H}$ NMR spectrum of the compound 21e.

91. ${ }^{13} \mathrm{C}\left\{{ }^{1} \mathrm{H}\right\}$ NMR spectrum of the compound 21e.

92. ${ }^{19} \mathrm{~F}\left\{{ }^{1} \mathrm{H}\right\}$ NMR spectrum of the compound 21e.

93. ${ }^{1} \mathrm{H}$ NMR spectrum of the compound 21g.

94. ${ }^{13} \mathrm{C}\left\{{ }^{1} \mathrm{H}\right\}$ NMR spectrum of the compound $\mathbf{2 1 g}$

95. ${ }^{19} \mathrm{~F}\left\{{ }^{1} \mathrm{H}\right\}$ NMR spectrum of the compound 21g.

96. ${ }^{1} \mathrm{H}$ NMR spectrum of the compound 21h.

97. $\quad{ }^{13} \mathrm{C}\left\{{ }^{1} \mathrm{H}\right\}$ NMR spectrum of the compound $\mathbf{2 1 h}$.

98. $\quad{ }^{19}$ F NMR spectrum of the compound $\mathbf{2 1 h}$.

99. ${ }^{1} \mathrm{H}$ NMR spectrum of the compound 20b.

100. ${ }^{13} \mathrm{C}\left\{{ }^{1} \mathrm{H}\right\}$ NMR spectrum of the compound $20 \mathbf{b}$.

101. ${ }^{19} \mathrm{~F}\left\{{ }^{1} \mathrm{H}\right\}$ NMR spectrum of the compound 20b.

102. ${ }^{1} \mathrm{H}$ NMR spectrum of the compound 20c.

103. ${ }^{13} \mathrm{C}\left\{{ }^{1} \mathrm{H}\right\}$ NMR spectrum of the compound 20c.

104. ${ }^{19} \mathrm{~F}\left\{{ }^{1} \mathrm{H}\right\}$ NMR spectrum of the compound 20c. 
105. ${ }^{1} \mathrm{H}$ NMR spectrum of the compound $20 f$.

106. ${ }^{13} \mathrm{C}\left\{{ }^{1} \mathrm{H}\right\}$ NMR spectrum of the compound $20 f$.

107. ${ }^{19} \mathrm{~F}\left\{{ }^{1} \mathrm{H}\right\}$ NMR spectrum of the compound $20 \mathrm{f}$.

108. ${ }^{1}$ H NMR spectrum of the compound 20g.

109. ${ }^{13} \mathrm{C}\left\{{ }^{1} \mathrm{H}\right\}$ NMR spectrum of the compound 20g.

110. ${ }^{19} \mathrm{~F}\left\{{ }^{1} \mathrm{H}\right\}$ NMR spectrum of the compound $\mathbf{2 0 g}$.

111. ${ }^{1} \mathrm{H}$ NMR spectrum of the compound 20h.

112. ${ }^{13} \mathrm{C}\left\{{ }^{1} \mathrm{H}\right\}$ NMR spectrum of the compound $\mathbf{2 0 h}$.

113. ${ }^{19} \mathrm{~F}\left\{{ }^{1} \mathrm{H}\right\}$ NMR spectrum of the compound $20 \mathbf{h}$.

114. ${ }^{1} \mathrm{H}$ NMR spectrum of the compound 22a.

115. ${ }^{13} \mathrm{C}\left\{{ }^{1} \mathrm{H}\right\}$ NMR spectrum of the compound 22a.

116. ${ }^{19}$ F NMR spectrum of the compound 22a.

117. ${ }^{1} \mathrm{H}$ NMR spectrum of the compound 28c.

118. ${ }^{13} \mathrm{C}\left\{{ }^{1} \mathrm{H}\right\}$ NMR spectrum of the compound 28c.

119. ${ }^{19} \mathrm{~F}\left\{{ }^{1} \mathrm{H}\right\}$ NMR spectrum of the compound 28c.

120. ${ }^{1} \mathrm{H}$ NMR spectrum of the compound 22c.

121. ${ }^{13} \mathrm{C}\left\{{ }^{1} \mathrm{H}\right\}$ NMR spectrum of the compound 22c.

122. ${ }^{19} \mathrm{~F}\left\{{ }^{1} \mathrm{H}\right\}$ NMR spectrum of the compound 22c.

123. ${ }^{1} \mathrm{H}$ NMR spectrum of the compound $\mathbf{2 2 h}$.

124. ${ }^{13} \mathrm{C}\left\{{ }^{1} \mathrm{H}\right\}$ NMR spectrum of the compound $\mathbf{2 2 h}$.

125. ${ }^{19}$ F NMR spectrum of the compound $\mathbf{2 2 h}$.

126. ${ }^{1} \mathrm{H}$ NMR spectrum of the compound 23a.

127. ${ }^{13} \mathrm{C}\left\{{ }^{1} \mathrm{H}\right\}$ NMR spectrum of the compound 23a. 
128. ${ }^{19}$ F NMR spectrum of the compound 23a.

129. ${ }^{1}$ H NOE NMR spectrum of the compound 23a.

130. ${ }^{1} \mathrm{H}$ NMR spectrum of the compound $\mathbf{2 3 b}$.

131. ${ }^{13} \mathrm{C}\left\{{ }^{1} \mathrm{H}\right\}$ NMR spectrum of the compound 23b.

132. ${ }^{19} \mathrm{~F}\left\{{ }^{1} \mathrm{H}\right\}$ NMR spectrum of the compound $23 \mathbf{b}$.

133. ${ }^{1} \mathrm{H}$ NMR spectrum of the compound $\mathbf{2 6 \mathbf { b }}$.

134. ${ }^{13} \mathrm{C}\left\{{ }^{1} \mathrm{H}\right\}$ NMR spectrum of the compound 26b.

135. ${ }^{19} \mathrm{~F}\left\{{ }^{1} \mathrm{H}\right\}$ NMR spectrum of the compound $\mathbf{2 6 \mathbf { b }}$.

136. ${ }^{1} \mathrm{H}$ NMR spectrum of the compound 23c.

137. ${ }^{13} \mathrm{C}\left\{{ }^{1} \mathrm{H}\right\}$ NMR spectrum of the compound 23c.

138. ${ }^{19} \mathrm{~F}\left\{{ }^{1} \mathrm{H}\right\}$ NMR spectrum of the compound 23c.

139. ${ }^{1} \mathrm{H}$ NMR spectrum of the compound 23d.

140. ${ }^{13} \mathrm{C}\left\{{ }^{1} \mathrm{H}\right\}$ NMR spectrum of the compound 23d.

141. ${ }^{19} \mathrm{~F}\left\{{ }^{1} \mathrm{H}\right\}$ NMR spectrum of the compound 23d.

142. ${ }^{1} \mathrm{H}$ NMR spectrum of the compound 23e.

143. ${ }^{13} \mathrm{C}\left\{{ }^{1} \mathrm{H}\right\}$ NMR spectrum of the compound 23e.

144. ${ }^{19} \mathrm{~F}\left\{{ }^{1} \mathrm{H}\right\}$ NMR spectrum of the compound $23 \mathbf{e}$.

145. ${ }^{1} \mathrm{H}$ NMR spectrum of the compound $\mathbf{2 3 h}$.

146. ${ }^{13} \mathrm{C}\left\{{ }^{1} \mathrm{H}\right\}$ NMR spectrum of the compound $\mathbf{2 3 h}$.

147. ${ }^{19}$ F NMR spectrum of the compound $\mathbf{2 3 h}$.

148. ${ }^{1} \mathrm{H}$ NMR spectrum of the compound 24a.

149. ${ }^{13} \mathrm{C}\left\{{ }^{1} \mathrm{H}\right\}$ NMR spectrum of the compound $24 a$.

150. ${ }^{19}$ F NMR spectrum of the compound 24a. 
151. ${ }^{1} \mathrm{H}$ NMR spectrum of the compound 24c.

152. ${ }^{13} \mathrm{C}\left\{{ }^{1} \mathrm{H}\right\}$ NMR spectrum of the compound 24c.

153. ${ }^{19} \mathrm{~F}\left\{{ }^{1} \mathrm{H}\right\}$ NMR spectrum of the compound 24c.

154. ${ }^{1} \mathrm{H}$ NMR spectrum of the compound $\mathbf{2 4 g}$.

155. ${ }^{13} \mathrm{C}\left\{{ }^{1} \mathrm{H}\right\}$ NMR spectrum of the compound 24g.

156. ${ }^{19}$ F NMR spectrum of the compound $\mathbf{2 4 g}$.

157. ${ }^{1} \mathrm{H}$ NMR spectrum of the compound $\mathbf{2 4 h}$.

158. ${ }^{13} \mathrm{C}\left\{{ }^{1} \mathrm{H}\right\}$ NMR spectrum of the compound $\mathbf{2 4 h}$.

159. ${ }^{19} \mathrm{~F}\left\{{ }^{1} \mathrm{H}\right\}$ NMR spectrum of the compound $\mathbf{2 4 h}$.

160. ${ }^{1} \mathrm{H}$ NMR spectrum of the compound 25a.

161. ${ }^{13} \mathrm{C}\left\{{ }^{1} \mathrm{H}\right\}$ NMR spectrum of the compound 25a.

162. ${ }^{19}$ F NMR spectrum of the compound 25a.

163. ${ }^{1} \mathrm{H}$ NMR spectrum of the compound 27a.

164. ${ }^{13} \mathrm{C}\left\{{ }^{1} \mathrm{H}\right\}$ NMR spectrum of the compound 27a.

165. ${ }^{19}$ F NMR spectrum of the compound 27a.

166. ${ }^{1} \mathrm{H}$ NMR spectrum of the compound $25 \mathbf{b}$.

167. ${ }^{13} \mathrm{C}\left\{{ }^{1} \mathrm{H}\right\}$ NMR spectrum of the compound 25b.

168. ${ }^{19} \mathrm{~F}\left\{{ }^{1} \mathrm{H}\right\}$ NMR spectrum of the compound $25 \mathbf{b}$.

169. ${ }^{1} \mathrm{H}$ NMR spectrum of the compound 25g.

170. ${ }^{13} \mathrm{C}\left\{{ }^{1} \mathrm{H}\right\}$ NMR spectrum of the compound 25g.

171. ${ }^{19}$ F NMR spectrum of the compound $25 \mathrm{~g}$.

172. ${ }^{1} \mathrm{H}$ NMR spectrum of the compound $\mathbf{2 5 h}$.

173. ${ }^{13} \mathrm{C}\left\{{ }^{1} \mathrm{H}\right\}$ NMR spectrum of the compound $\mathbf{2 5 h}$. 
174. ${ }^{19} \mathrm{~F}\left\{{ }^{1} \mathrm{H}\right\}$ NMR spectrum of the compound $\mathbf{2 5 h}$.

175. ${ }^{1} \mathrm{H}$ NMR spectrum of the compound 29a.

176. ${ }^{13} \mathrm{C}\left\{{ }^{1} \mathrm{H}\right\}$ NMR spectrum of the compound 29a.

177. ${ }^{19} \mathrm{~F}\left\{{ }^{1} \mathrm{H}\right\}$ NMR spectrum of the compound 29a.

178. ${ }^{1} \mathrm{H}$ NMR spectrum of the compound $29 \mathbf{b}$.

179. ${ }^{13} \mathrm{C}\left\{{ }^{1} \mathrm{H}\right\}$ NMR spectrum of the compound 29b.

180. ${ }^{19} \mathrm{~F}\left\{{ }^{1} \mathrm{H}\right\}$ NMR spectrum of the compound $\mathbf{2 9 b}$.

181. ${ }^{1} \mathrm{H}$ NMR spectrum of the compound 29d.

182. ${ }^{13} \mathrm{C}\left\{{ }^{1} \mathrm{H}\right\}$ NMR spectrum of the compound 29d.

183. ${ }^{19} \mathrm{~F}\left\{{ }^{1} \mathrm{H}\right\}$ NMR spectrum of the compound 29d.

184. ${ }^{1} \mathrm{H}$ NMR spectrum of the compound 30.

185. ${ }^{13} \mathrm{C}\left\{{ }^{1} \mathrm{H}\right\}$ NMR spectrum of the compound $\mathbf{3 0}$.

186. ${ }^{19} \mathrm{~F}\left\{{ }^{1} \mathrm{H}\right\}$ NMR spectrum of the compound $\mathbf{3 0}$.

187. ${ }^{1} \mathrm{H}$ NMR spectrum of the compound 33.

188. ${ }^{13} \mathrm{C}\left\{{ }^{1} \mathrm{H}\right\}$ NMR spectrum of the compound 33 .

189. ${ }^{19}$ F NMR spectrum of the compound 33.

190. ${ }^{1} \mathrm{H}$ NMR spectrum of the compound 37.

191. ${ }^{13} \mathrm{C}\left\{{ }^{1} \mathrm{H}\right\}$ NMR spectrum of the compound 37.

192. ${ }^{19} \mathrm{~F}\left\{{ }^{1} \mathrm{H}\right\}$ NMR spectrum of the compound 37.

193. ${ }^{1} \mathrm{H}$ NMR spectrum of the compound 34 .

194. ${ }^{13} \mathrm{C}\left\{{ }^{1} \mathrm{H}\right\}$ NMR spectrum of the compound 34 .

195. ${ }^{19} \mathrm{~F}\left\{{ }^{1} \mathrm{H}\right\}$ NMR spectrum of the compound 34 .

196. ${ }^{1} \mathrm{H}$ NMR spectrum of the compound 35b. 
197. ${ }^{13} \mathrm{C}\left\{{ }^{1} \mathrm{H}\right\}$ NMR spectrum of the compound 35b.

198. ${ }^{19} \mathrm{~F}\left\{{ }^{1} \mathrm{H}\right\}$ NMR spectrum of the compound 35b.

199. ${ }^{1} \mathrm{H}$ NMR spectrum of the compound 35c.

200. ${ }^{13} \mathrm{C}\left\{{ }^{1} \mathrm{H}\right\}$ NMR spectrum of the compound 35c.

201. ${ }^{19} \mathrm{~F}\left\{{ }^{1} \mathrm{H}\right\}$ NMR spectrum of the compound 35c.

202. ${ }^{1} \mathrm{H}$ NMR spectrum of the compound 35g.

203. ${ }^{13} \mathrm{C}\left\{{ }^{1} \mathrm{H}\right\}$ NMR spectrum of the compound 35g.

204. ${ }^{19}$ F NMR spectrum of the compound 35g.

205. ${ }^{1} \mathrm{H}$ NMR spectrum of the compound 40a.

206. ${ }^{13} \mathrm{C}\left\{{ }^{1} \mathrm{H}\right\}$ NMR spectrum of the compound 40a.

207. ${ }^{19} \mathrm{~F}\left\{{ }^{1} \mathrm{H}\right\}$ NMR spectrum of the compound 40a.

208. ${ }^{1} \mathrm{H}$ NMR spectrum of the compound 40b.

209. ${ }^{13} \mathrm{C}\left\{{ }^{1} \mathrm{H}\right\}$ NMR spectrum of the compound $40 \mathrm{~b}$.

210. ${ }^{19} \mathrm{~F}\left\{{ }^{1} \mathrm{H}\right\}$ NMR spectrum of the compound 40b.

211. ${ }^{1} \mathrm{H}$ NMR spectrum of the compound 40d.

212. ${ }^{13} \mathrm{C}\left\{{ }^{1} \mathrm{H}\right\}$ NMR spectrum of the compound 40d.

213. ${ }^{19} \mathrm{~F}\left\{{ }^{1} \mathrm{H}\right\}$ NMR spectrum of the compound 40d.

214. ${ }^{1} \mathrm{H}$ NMR spectrum of the compound 41a.

215. ${ }^{13} \mathrm{C}\left\{{ }^{1} \mathrm{H}\right\}$ NMR spectrum of the compound 41a.

216. ${ }^{19} \mathrm{~F}\left\{{ }^{1} \mathrm{H}\right\}$ NMR spectrum of the compound 41a.

217. ${ }^{1} \mathrm{H}$ NMR spectrum of the compound 42a.

218. ${ }^{13} \mathrm{C}\left\{{ }^{1} \mathrm{H}\right\}$ NMR spectrum of the compound 42a.

219. ${ }^{19} \mathrm{~F}\left\{{ }^{1} \mathrm{H}\right\}$ NMR spectrum of the compound 42a. 
220. ${ }^{1} \mathrm{H}$ NMR spectrum of the compound $\mathbf{4 2 b}$.

221. ${ }^{13} \mathrm{C}\left\{{ }^{1} \mathrm{H}\right\}$ NMR spectrum of the compound $\mathbf{4 2 b}$.

222. ${ }^{19} \mathrm{~F}\left\{{ }^{1} \mathrm{H}\right\}$ NMR spectrum of the compound $\mathbf{4 2 \mathbf { b }}$.

223. ${ }^{1} \mathrm{H}$ NMR spectrum of the compound 42d.

224. ${ }^{13} \mathrm{C}\left\{{ }^{1} \mathrm{H}\right\}$ NMR spectrum of the compound 42d.

225. ${ }^{19} \mathrm{~F}\left\{{ }^{1} \mathrm{H}\right\}$ NMR spectrum of the compound 42d.

226. ${ }^{1} \mathrm{H}$ NMR spectrum of the compound 38a.

227. ${ }^{13} \mathrm{C}\left\{{ }^{1} \mathrm{H}\right\}$ NMR spectrum of the compound 38a.

228. ${ }^{19} \mathrm{~F}\left\{{ }^{1} \mathrm{H}\right\}$ NMR spectrum of the compound 38a.

229. ${ }^{1} \mathrm{H}$ NMR spectrum of the compound 38b.

230. ${ }^{13} \mathrm{C}\left\{{ }^{1} \mathrm{H}\right\}$ NMR spectrum of the compound 38b.

231. ${ }^{19} \mathrm{~F}\left\{{ }^{1} \mathrm{H}\right\}$ NMR spectrum of the compound 38b.

232. ${ }^{1} \mathrm{H}$ NMR spectrum of the compound 38d.

233. ${ }^{13} \mathrm{C}\left\{{ }^{1} \mathrm{H}\right\}$ NMR spectrum of the compound 38d.

234. ${ }^{19} \mathrm{~F}\left\{{ }^{1} \mathrm{H}\right\}$ NMR spectrum of the compound 38d.

235. ${ }^{1} \mathrm{H}$ NMR spectrum of the compound 39a.

236. ${ }^{13} \mathrm{C}\left\{{ }^{1} \mathrm{H}\right\}$ NMR spectrum of the compound 39a.

S261

237. ${ }^{19} \mathrm{~F}\left\{{ }^{1} \mathrm{H}\right\}$ NMR spectrum of the compound 39a.

238. ${ }^{1} \mathrm{H}$ NMR spectrum of the compound 44a.

239. ${ }^{13} \mathrm{C}\left\{{ }^{1} \mathrm{H}\right\}$ NMR spectrum of the compound $44 \mathrm{a}$.

240. ${ }^{19}$ F NMR spectrum of the compound 44a.

241. ${ }^{1} \mathrm{H}$ NMR spectrum of the compound 45a.

242. ${ }^{13} \mathrm{C}\left\{{ }^{1} \mathrm{H}\right\}$ NMR spectrum of the compound 45a. 
243. ${ }^{19} \mathrm{~F}\left\{{ }^{1} \mathrm{H}\right\}$ NMR spectrum of the compound 45a.

244. ${ }^{1} \mathrm{H}$ NMR spectrum of the compound $\mathbf{4 4 h}$.

245. ${ }^{13} \mathrm{C}\left\{{ }^{1} \mathrm{H}\right\}$ NMR spectrum of the compound $\mathbf{4 4 h}$.

246. ${ }^{19} \mathrm{~F}\left\{{ }^{1} \mathrm{H}\right\}$ NMR spectrum of the compound $\mathbf{4 4 h}$.

S271

247. ${ }^{1} \mathrm{H}$ NMR spectrum of the compound 46a.

248. ${ }^{13} \mathrm{C}\left\{{ }^{1} \mathrm{H}\right\}$ NMR spectrum of the compound 46a.

249. ${ }^{19}$ F NMR spectrum of the compound $46 a$.

250. ${ }^{1} \mathrm{H}$ NMR spectrum of the compound 47a.

251. ${ }^{13} \mathrm{C}\left\{{ }^{1} \mathrm{H}\right\}$ NMR spectrum of the compound $47 \mathrm{a}$.

252. ${ }^{19} \mathrm{~F}\left\{{ }^{1} \mathrm{H}\right\}$ NMR spectrum of the compound $47 \mathbf{a}$.

253. $\quad{ }^{1} \mathrm{H}$ NMR spectrum of the compound 48.

254. ${ }^{13} \mathrm{C}\left\{{ }^{1} \mathrm{H}\right\}$ NMR spectrum of the compound 48 .

255. ${ }^{19} \mathrm{~F}\left\{{ }^{1} \mathrm{H}\right\}$ NMR spectrum of the compound 48.

256. $\quad{ }^{1} \mathrm{H}$ NMR spectrum of the compound $\mathbf{5 1 .}$

257. ${ }^{13} \mathrm{C}\left\{{ }^{1} \mathrm{H}\right\}$ NMR spectrum of the compound 51.

258. ${ }^{19} \mathrm{~F}\left\{{ }^{1} \mathrm{H}\right\}$ NMR spectrum of the compound 51.

259. ${ }^{1} \mathrm{H}$ NMR spectrum of the compound 49.

260. ${ }^{13} \mathrm{C}\left\{{ }^{1} \mathrm{H}\right\}$ NMR spectrum of the compound 49 .

261. ${ }^{19} \mathrm{~F}\left\{{ }^{1} \mathrm{H}\right\}$ NMR spectrum of the compound 49.

262. ${ }^{1}$ H NMR spectrum of the compound $\mathbf{5 0 .}$

263. ${ }^{13} \mathrm{C}\left\{{ }^{1} \mathrm{H}\right\}$ NMR spectrum of the compound $\mathbf{5 0 .}$

264. ${ }^{19} \mathrm{~F}\left\{{ }^{1} \mathrm{H}\right\}$ NMR spectrum of the compound $\mathbf{5 0 .}$ 
266. ${ }^{13} \mathrm{C}\left\{{ }^{1} \mathrm{H}\right\}$ NMR spectrum of the compound 52.

267. ${ }^{19} \mathrm{~F}\left\{{ }^{1} \mathrm{H}\right\}$ NMR spectrum of the compound 52.

268. ORTEP diagram for compound 29a.

S293

269. ORTEP diagram for compound 35g.

S294

270. ORTEP diagram for compound 24h.

S295 


\section{Synthesis of functionalized derivatives}

Jones oxidation of alcohols 21a,b,d and 20a-c,g gave carboxylic acids 29a,b,d and 35a-c,g, respectively, in 43-90\% yield (Scheme S1). Preparation of amines 38a,b,d and 39a was achieved by conversion of alcohols 21 and 20 to the corresponding chlorides $\mathbf{4 0}$ and $\mathbf{4 1}$, followed by subsequent nucleophilic substitution with azide anion, and reduction of $\mathbf{4 2}$ and $\mathbf{4 3}$ by Staudinger reaction (47-70\% overall yield for 3 steps). In addition, oxidation of alcohols 21a and 20a with pyridinium chlorochromate (PCC) resulted in aldehydes $44 a$ and $45 a$ with moderate yield ( $43 \%$ and $41 \%$, respectively), due to their high volatility. Attempts to run this reaction under the Parikh-Doering oxidation conditions (DMSO, py $\cdot \mathrm{SO}_{3}, \mathrm{NEt}_{3}$ ) was even less effective and resulted in no more than $21 \%$ yield of the target aldehydes. Still, the Parikh-Doering oxidation of $\mathbf{2 1 h}$ appeared to be more successful and gave ketone $44 \mathrm{~h}$ in $86 \%$ yield. Besides, oxidation of alcohol moiety to carboxylic acid group was also carried out for pyrazoles 24a and 25a,b, and led to the corresponding products $46 \mathbf{a}$ and $\mathbf{4 7 a} \mathbf{a}$ b in $81-85 \%$ yield.

In addition to the aforementioned reactions, products 42a and 43a were subjected to 'click' reaction with three different alkynes to give triazole-containing derivatives $\mathbf{4 8 - 5 0}$ (up to 79\% yield). Further hydrolysis of $\mathbf{4 8}$ resulted in acid 51 (83\% yield), whereas the reaction of 50 with $\mathrm{SO}_{2} \mathrm{Cl}_{2}$ led to the chlorinated derivative 52 ( $84 \%$ yield). 
$\mathrm{R}_{\mathrm{F}}$<smiles></smiles>

21a,b or d \begin{tabular}{|cc}
$\mathrm{SOCl}_{2}$ & $\mathbf{2 9 b}, 84 \%$ \\
$\mathrm{DMF}$ (cat) & $\mathbf{2 9 d}, 74 \%$ \\
$\mathrm{CH}_{2} \mathrm{Cl}_{2}$ &
\end{tabular}

$\mathrm{C}_{\mathrm{Cl}}^{\mathrm{R}}$ $\underset{\mathrm{DMF}}{\stackrel{\mathrm{NaN}_{3}}{\longrightarrow}} \underbrace{\stackrel{\mathrm{THF}_{\mathrm{F}}}{2) \mathrm{H}_{2} \mathrm{O}}}_{\mathrm{N}_{3}}$

40a

40b, $64 \%$

40d, $84 \%$

$\mathrm{F}_{2} \mathrm{HC}$<smiles>OCc1cc(Cl)on1</smiles>

42a, 75\% (2 steps)

42b, $79 \%$

42d, $88 \%$

$\mathrm{F}_{2} \mathrm{HC}$<smiles>O=Cc1cc(C=O)on1</smiles>

21a

(a) $\mathrm{R}_{\mathrm{F}}=\mathrm{CHF}_{2}$

(d) $\mathrm{R}_{\mathrm{F}}=\mathrm{C}_{2} \mathrm{~F}_{5}$

38a, $76 \%$

38b, $92 \%$ 38d, $84 \%$

$\mathrm{F}_{2} \mathrm{HC}$

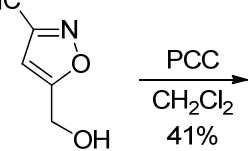

20a

$\mathrm{F}_{2} \mathrm{HC}$

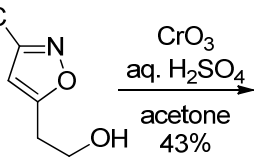

$20 \mathrm{~g}$
$\left.\mathrm{R}_{\mathrm{F}}\right\rangle \mathrm{N}$<smiles>O=C(O)c1ccno1</smiles>

(a) $\mathrm{R}_{\mathrm{F}}=\mathrm{CHF}$

(b) $\mathrm{R}_{\mathrm{F}}=\mathrm{CF}_{3}$

(c) $\mathrm{R}_{\mathrm{F}}=\mathrm{CF}_{2} \mathrm{CH}_{3}$

20a-c 35a, $89 \%$

$\mathrm{SOCl}_{2}, \mathrm{CH}_{2} \mathrm{Cl}_{2} \quad 35 \mathrm{~b}, 86 \%$

DMF (cat)

$60 \%$ (for 20a)
$\underset{\mathrm{Cl}}{\stackrel{\mathrm{DMF}}{\mathrm{NaN}}}$

41a

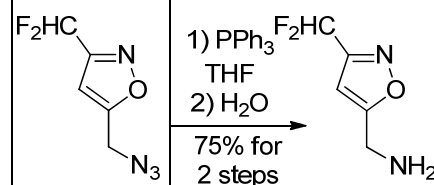

43a

21h $44 \mathrm{~h}$<smiles>CC(=O)c1cc(C)on1</smiles>

$4 h$

$\mathrm{F}_{2} \mathrm{HC}$<smiles>Cc1cc(C=O)on1</smiles>

$45 a$

$\mathrm{F}_{2} \mathrm{HC}$<smiles>CCC</smiles><smiles>Cc1cc(CC(=O)O)on1</smiles>

$35 \mathrm{~g}$

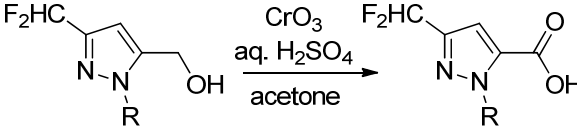

24a, $\mathrm{R}=\mathrm{H}$

25a, $\mathrm{R}=\mathrm{Me}$

$46 a, \mathrm{R}=\mathrm{H} \quad 82 \%$

47a, $\mathrm{R}=\mathrm{Me} \quad 85 \%$

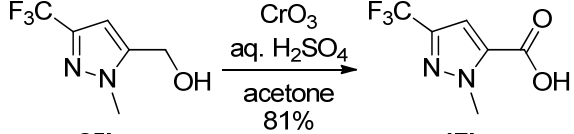

25b

47b

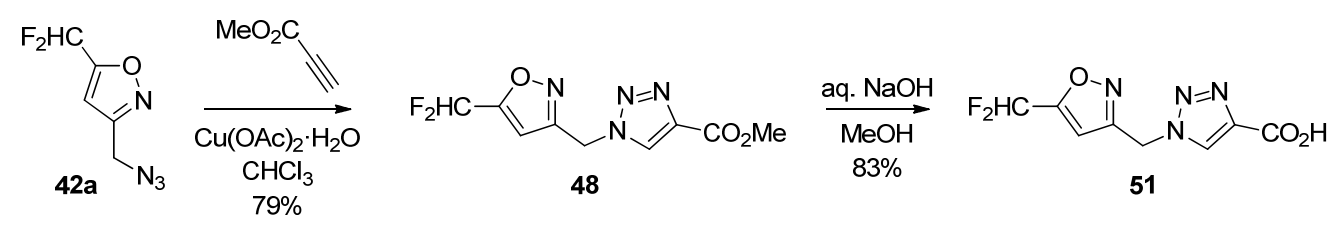<smiles>[R]Cc1cc(C(F)F)no1</smiles>

41a, $\mathrm{R}=\mathrm{Cl} \longrightarrow \mathrm{NaN}_{3} \quad \mathrm{CHCl}_{3}, 74 \%$ for 3 steps

50 52

Scheme S1. Synthesis of the derivatives 29, 35-52. 


\section{Structure assignment}

In order to confirm the structures of the synthesized 3- and 5-fluoroalkyl-substituted isoxazoles and pyrazoles, additional X-ray and NMR spectroscopy studies were carried out. In particular, X-ray structures of carboxylic acids 29a and 35g obtained by oxidation of 21a (the product of the 1,4-addition route) and $20 \mathrm{~g}$ (the product of the $\mathrm{Cu}$-catalyzed 1,2-addition route), respectively, were used to confirm the regioselectivity of the applied methods (Figure S1, A).

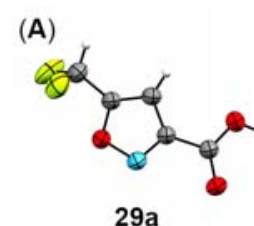

$29 a$

(B)

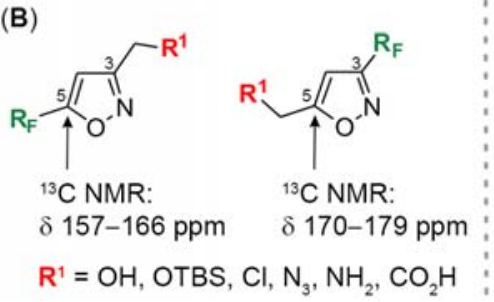

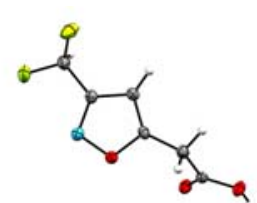

$35 \mathrm{~g}$

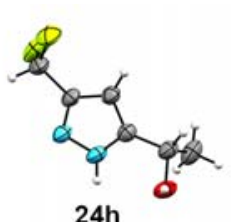

$24 \mathrm{~h}$

(C)

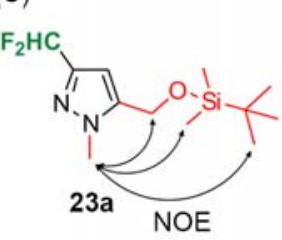

Figure S1. Oak ridge thermal ellipsoid plot (ORTEP) diagrams for compounds 29a, 35g and 24h (A). Thermal ellipsoids are shown at the $50 \%$ probability level. ${ }^{13} \mathrm{C}$ NMR shift regions of the C-5 atom of studied regioisomers $(\mathbf{B})$, and NOE correlations in 23a $(\mathbf{C})$.

In addition to that, ${ }^{13} \mathrm{C}$ NMR chemical shifts of the carbon atom adjacent to the isoxazole oxygen atom were also useful to distinguish two regioisomers (Figure S1, B). In particular, the ${ }^{13} \mathrm{C}$ NMR chemical shift of the aforementioned carbon in 5-fluoroalkyl substituted derivatives 17a,b, 17d-h, 21a-e, 21g,h, 38a,b,d, 40b,d, and 42a,b,d was found at 157-166 ppm. In the case of 3-fluoroalkyl substitutes derivatives 20a-c, 20g,h, 35g, 39a, and 41a, $\delta$ 170-179 ppm was a characteristic region for the C-5 carbon atoms. The structures of obtained $N$-non-substituted pyrazoles were confirmed by X-ray diffraction studies of the derivative $\mathbf{2 4 h}$. The assignment of the structures of major and minor isomers of $N$-methyl pyrazoles 23 and 27, respectively, was done by ${ }^{1} \mathrm{H}$ NOE NMR experiment for the compound 
23a (Figure S1, C), the analytical sample of which was obtained from the mixture of 23a and 27a mentioned in Table 5. 


\section{Experimental section}

General procedure for the preparation of the compounds $29,35,46$ and 47 . To a solution of the corresponding alcohol $(0.10 \mathrm{~mol})$ in acetone $(150 \mathrm{~mL})$, a solution of $\mathrm{CrO}_{3}(14.4 \mathrm{~g}, 0.144 \mathrm{~mol})$ and conc. $\mathrm{H}_{2} \mathrm{SO}_{4}(12.4 \mathrm{~mL})$ in $\mathrm{H}_{2} \mathrm{O}(50 \mathrm{~mL})$ was added slowly at $0{ }^{\circ} \mathrm{C}$, and the reaction was stirred overnight. After the reaction was complete (monitored by ${ }^{1} \mathrm{H}$ NMR analysis), the resulting mixture was concentrated under reduced pressure. The residue was dissolved in EtOAc $(150 \mathrm{~mL})$, then washed with $\mathrm{H}_{2} \mathrm{O}(70 \mathrm{~mL})$ and brine $(2 \times 70 \mathrm{~mL})$. The organic phase was dried over anhydrous $\mathrm{Na}_{2} \mathrm{SO}_{4}$ and concentrated under reduced pressure.

5-(Difluoromethyl)isoxazole-3-carboxylic acid (29a). Yield $65.7 \mathrm{~g} \mathrm{(90 \% )} \mathrm{from} 67.0 \mathrm{~g}$ of 21a. White solid, mp $=77-79{ }^{\circ} \mathrm{C}(\mathrm{dec}) .{ }^{1} \mathrm{H}$ NMR $\left(400 \mathrm{MHz}, \mathrm{D}_{2} \mathrm{O}\right): \delta 7.03(\mathrm{~s}, 1 \mathrm{H}), 6.95(\mathrm{t}, J=52.8 \mathrm{~Hz}, 1 \mathrm{H}) \mathrm{ppm}$. ${ }^{13} \mathrm{C}\left\{{ }^{1} \mathrm{H}\right\}$ NMR $\left(126 \mathrm{MHz}, \mathrm{D}_{2} \mathrm{O}\right): \delta 164.9(\mathrm{t}, J=30.2 \mathrm{~Hz}), 161.5,157.0,107.0(\mathrm{t}, J=237.8 \mathrm{~Hz}), 105.0$ ppm. ${ }^{19} \mathrm{~F}\left\{{ }^{1} \mathrm{H}\right\}$ NMR $\left(376 \mathrm{MHz}, \mathrm{D}_{2} \mathrm{O}\right): \delta-119.7 \mathrm{ppm}$. LC-MS $(\mathrm{m} / \mathrm{z}): 118\left(\mathrm{M}-\mathrm{H}^{+}-\mathrm{CO}_{2}\right)$. Anal. Calcd. for $\mathrm{C}_{5} \mathrm{H}_{3} \mathrm{~F}_{2} \mathrm{NO}_{3}$ : C, 36.83; H, 1.85; N, 8.59. Found: C, 36.75; H, 1.59; N, 8.78.

5-(Trifluoromethyl)isoxazole-3-carboxylic acid (29b). Yield $22.8 \mathrm{~g}(84 \%)$ from $25.0 \mathrm{~g}$ of 21 b. White solid, $\mathrm{mp}=75-77{ }^{\circ} \mathrm{C}(\mathrm{dec}) .{ }^{1} \mathrm{H}$ NMR $\left(400 \mathrm{MHz}, \mathrm{CDCl}_{3}\right): \delta 10.89$ (br s, $\left.1 \mathrm{H}\right), 7.19(\mathrm{~s}, 1 \mathrm{H}) \mathrm{ppm}$. ${ }^{13} \mathrm{C}\left\{{ }^{1} \mathrm{H}\right\} \operatorname{NMR}\left(101 \mathrm{MHz}, \mathrm{CDCl}_{3}\right): \delta 162.8,161.1(\mathrm{q}, J=43.8 \mathrm{~Hz}), 155.9,117.4(\mathrm{q}, J=271.0 \mathrm{~Hz}), 106.3$ ppm. ${ }^{19} \mathrm{~F}\left\{{ }^{1} \mathrm{H}\right\}$ NMR $\left(376 \mathrm{MHz}, \mathrm{CDCl}_{3}\right): \delta-64.5$ ppm. LC-MS $(\mathrm{m} / \mathrm{z}): 136\left(\mathrm{M}-\mathrm{H}^{+}-\mathrm{CO}_{2}\right)$. Anal. Calcd. for $\mathrm{C}_{5} \mathrm{H}_{2} \mathrm{~F}_{3} \mathrm{NO}_{3}:$ C, 33.17; H, 1.11; N, 7.74. Found: C, 32.94; H, 0.84; N, 7.42.

5-(Perfluoroethyl)isoxazole-3-carboxylic acid (29d). Yield $9.45 \mathrm{~g}(74 \%)$ from $12.0 \mathrm{~g}$ of 21d. White solid, mp $=114-116{ }^{\circ} \mathrm{C}$ (sublimation). ${ }^{1} \mathrm{H}$ NMR (400 MHz, DMSO-d $): \delta 13.77$ (br s, $\left.1 \mathrm{H}\right), 7.78(\mathrm{~s}, 1 \mathrm{H})$ ppm. ${ }^{13} \mathrm{C}\left\{{ }^{1} \mathrm{H}\right\}$ NMR (126 MHz, DMSO-d $\left.d_{6}\right): \delta 159.5,158.4,157.9(\mathrm{t}, J=31.8 \mathrm{~Hz}), 117.9$ (qt, $J=286.2$, $36.2 \mathrm{~Hz}), 109.4,108.0(\mathrm{tq}, J=254.6,41.0 \mathrm{~Hz}) \mathrm{ppm} .{ }^{19} \mathrm{~F}\left\{{ }^{1} \mathrm{H}\right\}$ NMR $\left(470 \mathrm{MHz}, \mathrm{DMSO}-d_{6}\right): \delta-84.5(\mathrm{~s}$, 3F), -115.1 (s, 2F) ppm. Anal. Calcd. for $\mathrm{C}_{6} \mathrm{H}_{2} \mathrm{~F}_{5} \mathrm{NO}_{3}: \mathrm{C}, 31.19 ; \mathrm{H}, 0.87 ; \mathrm{N}, 6.06$. Found: C, 30.96; $\mathrm{H}$, $1.07 ; \mathrm{N}, 6.43$. 
3-(Difluoromethyl)isoxazole-5-carboxylic acid (35a). Yield $24.3 \mathrm{~g}$ (89 \%) from $25.0 \mathrm{~g}$ of 20a. Beige solid, mp $111-112^{\circ} \mathrm{C}(\mathrm{dec})\left(\right.$ lit. $\left.112^{\circ} \mathrm{C}\right) .{ }^{1}$ For spectral and physical data, see ref. ${ }^{1}$

3-(Trifluoromethyl)isoxazole-5-carboxylic acid (35b). Yield $18.1 \mathrm{~g}$ (86 \%) from $19.4 \mathrm{~g}$ of 20b. White solid, $\mathrm{mp}=68-70{ }^{\circ} \mathrm{C}(\mathrm{dec}) .{ }^{1} \mathrm{H}$ NMR $\left(400 \mathrm{MHz}, \mathrm{DMSO}-d_{6}\right): \delta 13.72(\mathrm{br} \mathrm{s}, 1 \mathrm{H}), 7.85(\mathrm{~s}, 1 \mathrm{H})$ ppm. ${ }^{13} \mathrm{C}\left\{{ }^{1} \mathrm{H}\right\}$ NMR (126 MHz, DMSO-d $): \delta 164.2,156.6,155.4(\mathrm{q}, J=38.2 \mathrm{~Hz}), 119.2(\mathrm{q}, J=271.2$ Hz), 106.8 ppm. ${ }^{19} \mathrm{~F}\left\{{ }^{1} \mathrm{H}\right\}$ NMR (376 MHz, DMSO- $\left.d_{6}\right): \delta-62.6$ ppm. LC-MS (m/z): $180\left(\mathrm{M}-\mathrm{H}^{+}\right)$. Anal. Calcd. for $\mathrm{C}_{5} \mathrm{H}_{2} \mathrm{~F}_{3} \mathrm{NO}_{3}: \mathrm{C}, 33.17 ; \mathrm{H}, 1.11 ; \mathrm{N}, 7.74$. Found: C, 33.52; H, 0.99; N, 7.51.

3-(1,1-Difluoroethyl)isoxazole-5-carboxylic acid (35c). Yield $10.3 \mathrm{~g}$ (73\%) from $13.0 \mathrm{~g}$ of 20c. White solid, mp $=114-116{ }^{\circ} \mathrm{C} .{ }^{1} \mathrm{H}$ NMR $\left(400 \mathrm{MHz}, \mathrm{DMSO}-\mathrm{d}_{6}\right): \delta 14.41(\mathrm{br} \mathrm{s}, 1 \mathrm{H}), 7.42(\mathrm{~s}, 1 \mathrm{H}), 2.07$ (t, $J=19.2 \mathrm{~Hz}, 3 \mathrm{H}) \mathrm{ppm} .{ }^{13} \mathrm{C}\left\{{ }^{1} \mathrm{H}\right\} \mathrm{NMR}\left(126 \mathrm{MHz}, \mathrm{DMSO}-d_{6}\right): \delta 162.9,161.6(\mathrm{t}, J=33.0 \mathrm{~Hz}), 157.1$, $117.7(\mathrm{t}, J=236.1 \mathrm{~Hz}), 106.6,23.1(\mathrm{t}, J=25.4 \mathrm{~Hz}) \mathrm{ppm} .{ }^{19} \mathrm{~F}\left\{{ }^{1} \mathrm{H}\right\}$ NMR $\left(376 \mathrm{MHz}, \mathrm{DMSO}-d_{6}\right): \delta-86.4$ ppm. LC-MS (m/z): $176\left(\mathrm{M}-\mathrm{H}^{+}\right)$. Anal. Calcd. for $\mathrm{C}_{6} \mathrm{H}_{5} \mathrm{~F}_{2} \mathrm{NO}_{3}$ : C, 40.69; H, 2.85; N, 7.91. Found: C, $41.06 ; \mathrm{H}, 2.82 ; \mathrm{N}, 8.07$.

2-(3-(Difluoromethyl)isoxazol-5-yl)acetic acid (35g). Yield $2.01 \mathrm{~g}(43 \%)$ from $4.30 \mathrm{~g}$ of 20g. White solid, $\mathrm{mp}=90-92{ }^{\circ} \mathrm{C}(\mathrm{dec}) .{ }^{1} \mathrm{H} \mathrm{NMR}\left(400 \mathrm{MHz}, \mathrm{CDCl}_{3}\right): \delta 10.25$ (br s, $\left.1 \mathrm{H}\right), 6.76(\mathrm{t}, J=53.5 \mathrm{~Hz}, 1 \mathrm{H})$, $6.56(\mathrm{~s}, 1 \mathrm{H}), 3.96(\mathrm{~s}, 2 \mathrm{H}) \mathrm{ppm} .{ }^{13} \mathrm{C}\left\{{ }^{1} \mathrm{H}\right\} \mathrm{NMR}\left(126 \mathrm{MHz}, \mathrm{CDCl}_{3}\right): \delta 173.0,166.4,159.2(\mathrm{t}, J=30.1 \mathrm{~Hz})$, $108.9(\mathrm{t}, J=237.1 \mathrm{~Hz}), 101.0,32.4 \mathrm{ppm} .{ }^{19} \mathrm{~F}$ NMR (470 MHz, $\left.\mathrm{CDCl}_{3}\right): \delta-115.7(\mathrm{~d}, J=53.5 \mathrm{~Hz}) \mathrm{ppm}$. Anal. Calcd. for $\mathrm{C}_{6} \mathrm{H}_{5} \mathrm{~F}_{2} \mathrm{NO}_{3}:$ C, 40.69; H, 2.85; N, 7.91. Found: C, 40.89; H, 2.58; N, 7.53.

3-(Difluoromethyl)-1H-pyrazole-5-carboxylic acid (46a). Yield $40.8 \mathrm{~g}$ (82 \%) from $45.5 \mathrm{~g}$ of 24a. White solid, mp $175-177^{\circ} \mathrm{C} .{ }^{1} \mathrm{H}$ NMR (400 MHz, DMSO-d 6 ): $\delta 13.75$ (br s, 2H), 7.04 (t, $J=54.5 \mathrm{~Hz}$, 1H), 6.98 (s, 1H) ppm. ${ }^{13} \mathrm{C}\left\{{ }^{1} \mathrm{H}\right\}$ NMR (126 MHz, DMSO-d 6 ): $\delta 160.7,146.3(\mathrm{t}, J=27.0 \mathrm{~Hz}), 137.2$, $111.4(\mathrm{t}, J=232.4 \mathrm{~Hz}), 106.5 \mathrm{ppm} .{ }^{19} \mathrm{~F}$ NMR (376 MHz, DMSO- $\left.d_{6}\right): \delta-111.7(\mathrm{~d}, J=54.5 \mathrm{~Hz}) \mathrm{ppm}$. LC-MS $(\mathrm{m} / \mathrm{z})$ : $161\left(\mathrm{M}-\mathrm{H}^{+}\right)$. Anal. Calcd. for $\mathrm{C}_{5} \mathrm{H}_{4} \mathrm{~F}_{2} \mathrm{~N}_{2} \mathrm{O}_{2}$ : C, 37.05; H, 2.49; N, 17.28. Found: C, $37.41 ; \mathrm{H}, 2.12 ; \mathrm{N}, 17.11$. 
3-(Difluoromethyl)-1-methyl-1H-pyrazole-5-carboxylic acid (47a). ${ }^{2}$ Yield $55.7 \mathrm{~g}(85 \%)$ from 60.3 g of 25a. White solid, mp 179-181 ${ }^{\circ} \mathrm{C}$ (lit. $\left.180{ }^{\circ} \mathrm{C}\right)^{2} .{ }^{1} \mathrm{H}$ NMR $\left(500 \mathrm{MHz}, \mathrm{DMSO}-d_{6}\right): \delta 13.62(\mathrm{~s}, 1 \mathrm{H})$, $7.01(\mathrm{~s}, 1 \mathrm{H}), 7.00(\mathrm{t}, J=54.4 \mathrm{~Hz}, 1 \mathrm{H}), 4.11(\mathrm{~s}, 3 \mathrm{H}) \mathrm{ppm} .{ }^{13} \mathrm{C}\left\{{ }^{1} \mathrm{H}\right\} \mathrm{NMR}\left(101 \mathrm{MHz}, \mathrm{DMSO}-d_{6}\right): \delta 160.3$, $144.1(\mathrm{t}, J=28.6 \mathrm{~Hz}), 134.8,111.1(\mathrm{t}, J=232.4 \mathrm{~Hz}), 108.5,39.8 \mathrm{ppm} .{ }^{19} \mathrm{~F}\left\{{ }^{1} \mathrm{H}\right\} \mathrm{NMR}(376 \mathrm{MHz}$, DMSO-d $\left.{ }_{6}\right): \delta-112.1$ ppm. LC-MS $(\mathrm{m} / \mathrm{z}): 175\left(\mathrm{M}-\mathrm{H}^{+}\right)$. Anal. Calcd. for $\mathrm{C}_{6} \mathrm{H}_{6} \mathrm{~F}_{2} \mathrm{~N}_{2} \mathrm{O}_{2}: \mathrm{C}, 40.92 ; \mathrm{H}$, 3.43; N, 15.91. Found: C, 40.78; H, 3.37; N, 16.12 .

1-Methyl-3-(trifluoromethyl)-1H-pyrazole-5-carboxylic acid (47b). Yield $26.2 \mathrm{~g}(81 \%)$ from $30 \mathrm{~g}$ of 25b. White solid, mp $130-132{ }^{\circ} \mathrm{C}$ (lit. $\left.130-133{ }^{\circ} \mathrm{C}\right) .{ }^{3,4}$ For spectral and physical data, see refs. ${ }^{3,4}$

General procedure for the preparation of the compounds 40 and 41 . To a solution of alcohol 21 or 20 (0.10 mol) in $\mathrm{CH}_{2} \mathrm{Cl}_{2}(150 \mathrm{~mL})$, catalytic amount of DMF was added. $\mathrm{SOCl}_{2}(8.71 \mathrm{~mL}, 0.12 \mathrm{~mol})$ was added dropwise to the solution at $-10^{\circ} \mathrm{C}$, and the reaction mixture was stirred overnight. After the reaction was complete (monitored by ${ }^{1} \mathrm{H}$ NMR analysis), the resulting solution was concentrated under reduced pressure, and the crude product was purified by vacuum distillation.

3-(Chloromethyl)-5-(trifluoromethyl)isoxazole (40b). Yield $44.1 \mathrm{~g}(64 \%)$ from $62.0 \mathrm{~g}$ of $21 \mathrm{~b}$. Colorless liquid, bp $=37-39{ }^{\circ} \mathrm{C} / 10$ mbar. ${ }^{1} \mathrm{H}$ NMR $\left(400 \mathrm{MHz}, \mathrm{CDCl}_{3}\right): \delta 6.83(\mathrm{~s}, 1 \mathrm{H}), 4.63(\mathrm{~s}, 2 \mathrm{H}) \mathrm{ppm}$. ${ }^{13} \mathrm{C}\left\{{ }^{1} \mathrm{H}\right\} \mathrm{NMR}\left(126 \mathrm{MHz}, \mathrm{CDCl}_{3}\right): \delta 161.4,159.6(\mathrm{q}, J=42.9 \mathrm{~Hz}), 117.7(\mathrm{q}, J=270.2 \mathrm{~Hz}), 105.2,34.8$ ppm. ${ }^{19} \mathrm{~F}\left\{{ }^{1} \mathrm{H}\right\}$ NMR (376 MHz, $\mathrm{CDCl}_{3}$ ): $\delta-65.4$ ppm. Anal. Calcd. for $\mathrm{C}_{5} \mathrm{H}_{3} \mathrm{ClF}_{3} \mathrm{NO}: \mathrm{C}, 32.37 ; \mathrm{H}, 1.63$; N, 7.55; Cl, 19.11. Found: C, 32.08; H, 1.32; N, 7.33; Cl, 19.51.

3-(Chloromethyl)-5-(perfluoroethyl)isoxazole (40d). Yield $14.6 \mathrm{~g}$ (84\%) from $16.0 \mathrm{~g}$ of 21d. Colorless liquid, bp $=41-44{ }^{\circ} \mathrm{C} / 23$ mbar. ${ }^{1} \mathrm{H}$ NMR $\left(400 \mathrm{MHz}, \mathrm{CDCl}_{3}\right): \delta 6.89(\mathrm{~s}, 1 \mathrm{H}), 4.66(\mathrm{~s}, 2 \mathrm{H}) \mathrm{ppm}$. ${ }^{13} \mathrm{C}\left\{{ }^{1} \mathrm{H}\right\} \mathrm{NMR}\left(101 \mathrm{MHz}, \mathrm{CDCl}_{3}\right): \delta 161.5,159.2(\mathrm{t}, J=31.9 \mathrm{~Hz}), 118.1(\mathrm{qt}, J=286.2,35.9 \mathrm{~Hz}), 108.2$ (tq, $J=255.2,41.3 \mathrm{~Hz}), 107.0,34.9 \mathrm{ppm} .{ }^{19} \mathrm{~F}\left\{{ }^{1} \mathrm{H}\right\}$ NMR $\left(376 \mathrm{MHz}, \mathrm{CDCl}_{3}\right): \delta-84.8(\mathrm{~s}, 3 \mathrm{~F}),-115.8(\mathrm{~s}$, 2F) ppm. Anal. Calcd. for $\mathrm{C}_{6} \mathrm{H}_{3} \mathrm{ClF}_{5} \mathrm{NO}$ C, 30.60; H, 1.28; N, 5.95; Cl, 15.05. Found: C, 30.28; H, 1.36; $\mathrm{N}, 5.83 ; \mathrm{Cl}, 15.34$. 
5-(Chloromethyl)-3-(difluoromethyl)isoxazole (41a). Yield $79.6 \mathrm{~g}$ (60 \%) from $118 \mathrm{~g}$ of 20a. Colorless liquid, $\mathrm{bp}=50-52{ }^{\circ} \mathrm{C} / 10 \mathrm{mbar} .{ }^{1} \mathrm{H}$ NMR (500 MHz, DMSO- $\left.d_{6}\right): \delta 7.28(\mathrm{t}, J=53.0 \mathrm{~Hz}, 1 \mathrm{H})$, 6.97 (s, 1H), $5.02(\mathrm{~s}, 2 \mathrm{H}) \mathrm{ppm} .{ }^{13} \mathrm{C}\left\{{ }^{1} \mathrm{H}\right\}$ NMR (101 MHz, DMSO-d 6 ): $\delta 169.9,158.9$ (t, $\left.J=28.8 \mathrm{~Hz}\right)$, $109.3(\mathrm{t}, J=235.7 \mathrm{~Hz}), 101.6,33.9 \mathrm{ppm} .{ }^{19} \mathrm{~F}\left\{{ }^{1} \mathrm{H}\right\}$ NMR (376 MHz, DMSO-d 6 ): $\delta-116.9 \mathrm{ppm} . \mathrm{GC}-\mathrm{MS}$ $(\mathrm{m} / \mathrm{z}):$ 167/169 $\left(\mathrm{M}^{+}\right), 148 / 150\left(\mathrm{M}^{+}-\mathrm{F}\right), 132\left(\mathrm{M}^{+}-\mathrm{Cl}\right)$. Anal. Calcd. for $\mathrm{C}_{5} \mathrm{H}_{4} \mathrm{ClF}_{2} \mathrm{NO}: \mathrm{C}, 35.85 ; \mathrm{H}, 2.41$; N, 8.36; Cl, 21.16. Found: C, 36.24; H, 2.13; N, 8.57; Cl, 21.11.

General procedure for the preparation of the compounds 42 and 43 . To a suspension of $\mathrm{NaN}_{3}$ (19.5 g, $0.30 \mathrm{~mol})$ in DMF $(100 \mathrm{~mL})$, chloride 40 or $\mathbf{4 1}(0.10 \mathrm{~mol})$ was added, and the reaction was stirred overnight at rt. After the reaction was complete (monitored by ${ }^{1} \mathrm{H}$ NMR analysis), the resulting mixture was poured in the ice-cold water $(200 \mathrm{~mL})$, and the product was extracted with EtOAc $(3 \times 75$ $\mathrm{mL})$. The combined organic layers were washed with brine $(2 \times 50 \mathrm{~mL})$, dried over anhydrous $\mathrm{Na}_{2} \mathrm{SO}_{4}$, and concentrated under reduced pressure. The crude product was purified by vacuum distillation.

3-(Azidomethyl)-5-(difluoromethyl)isoxazole (42a). The compound was synthesized from (5(difluoromethyl)isoxazol-3-yl)methanol (21a) (68.5 g, $0.46 \mathrm{~mol})$, which was first converted to 3(chloromethyl)-5-(difluoromethyl)isoxazole (40a) by the general procedure for the preparation of the compounds 40 and 41 . The crude chloride 40a was subjected to vacuum distillation to give $73.2 \mathrm{~g}$ of product as a colorless liquid ( $\mathrm{bp}=63-65^{\circ} \mathrm{C} / 10 \mathrm{mbar}$ ). The purity of the obtained compound 40a was about $90-95 \%$, and it was used in the next step without additional purification. ${ }^{1} \mathrm{H}$ NMR (500 MHz, $\left.\mathrm{CDCl}_{3}\right): \delta 6.72(\mathrm{t}, J=53.5 \mathrm{~Hz}, 1 \mathrm{H}), 6.67(\mathrm{~s}, 1 \mathrm{H}), 4.59(\mathrm{~s}, 2 \mathrm{H}) \mathrm{ppm} .{ }^{13} \mathrm{C}\left\{{ }^{1} \mathrm{H}\right\} \mathrm{NMR}\left(126 \mathrm{MHz}, \mathrm{CDCl}_{3}\right): \delta$ $164.3(\mathrm{t}, J=30.9 \mathrm{~Hz}), 161.1,107.1(\mathrm{t}, J=239.4 \mathrm{~Hz}), 103.8(\mathrm{t}, J=2.6 \mathrm{~Hz}), 35.0 \mathrm{ppm} .{ }^{19} \mathrm{~F}\left\{{ }^{1} \mathrm{H}\right\} \mathrm{NMR}$ (376 MHz, $\left.\mathrm{CDCl}_{3}\right): \delta-118.7$ ppm. GC-MS (m/z): 167/169 $\left(\mathrm{M}^{+}\right), 148 / 150\left(\mathrm{M}^{+}-\mathrm{F}\right), 132\left(\mathrm{M}^{+}-\mathrm{Cl}\right)$, $116 / 118\left(\mathrm{M}^{+}-\mathrm{CHF}_{2}\right)$. The preparation of azide 42a was carried out by the general procedure for the preparation of the compounds 42 and 43 . Yield $60.0 \mathrm{~g}(75 \%)$ from $68.5 \mathrm{~g}$ of 21a. Colorless liquid, bp $=$ 58-60 ${ }^{\circ} \mathrm{C} / 3.5$ mbar. ${ }^{1} \mathrm{H}$ NMR $\left(400 \mathrm{MHz}, \mathrm{DMSO}_{-} \mathrm{d}_{6}\right): \delta 7.36(\mathrm{t}, J=52.6 \mathrm{~Hz}, 1 \mathrm{H}), 7.08(\mathrm{~s}, 1 \mathrm{H}), 4.67(\mathrm{~s}$, 
2H) ppm. ${ }^{13} \mathrm{C}\left\{{ }^{1} \mathrm{H}\right\}$ NMR (126 MHz, DMSO-d $): \delta 163.5(\mathrm{t}, J=28.9 \mathrm{~Hz}), 160.1,107.5(\mathrm{t}, J=236.6 \mathrm{~Hz})$, $104.8(\mathrm{t}, J=3.5 \mathrm{~Hz}), 44.6 \mathrm{ppm} .{ }^{19} \mathrm{~F}\left\{{ }^{1} \mathrm{H}\right\}$ NMR (376 MHz, DMSO- $\left.d_{6}\right): \delta-118.8 \mathrm{ppm} . \mathrm{GC}-\mathrm{MS}(\mathrm{m} / \mathrm{z})$ : $174\left(\mathrm{M}^{+}\right), 155\left(\mathrm{M}^{+}-\mathrm{F}\right), 146\left(\mathrm{M}^{+}-\mathrm{N}_{2}\right), 132\left(\mathrm{M}^{+}-\mathrm{N}_{3}\right)$. Anal. Calcd. for $\mathrm{C}_{5} \mathrm{H}_{4} \mathrm{~F}_{2} \mathrm{~N}_{4} \mathrm{O}: \mathrm{C}, 34.49 ; \mathrm{H}, 2.32$; N, 32.18. Found: C, 34.46; H, 2.53; N, 31.99.

3-(Azidomethyl)-5-(trifluoromethyl)isoxazole (42b). Yield $34.3 \mathrm{~g}$ (79\%) from $42.0 \mathrm{~g}$ of 40b. Colorless liquid, $\mathrm{bp}=51-53{ }^{\circ} \mathrm{C} / 10$ mbar. ${ }^{1} \mathrm{H}$ NMR (400 MHz, DMSO- $\left.d_{6}\right): \delta 7.54(\mathrm{~s}, 1 \mathrm{H}), 4.72(\mathrm{~s}, 2 \mathrm{H})$ ppm. ${ }^{13} \mathrm{C}\left\{{ }^{1} \mathrm{H}\right\}$ NMR $\left(126 \mathrm{MHz}, \mathrm{DMSO}-d_{6}\right): \delta 160.9,157.5$ (q, $\left.J=41.7 \mathrm{~Hz}\right), 117.8(\mathrm{q}, J=269.8 \mathrm{~Hz})$, 106.5, 44.6 ppm. ${ }^{19} \mathrm{~F}\left\{{ }^{1} \mathrm{H}\right\}$ NMR (376 MHz, DMSO-d $): \delta-64.4$ ppm. GC-MS $(\mathrm{m} / \mathrm{z}): 192\left(\mathrm{M}^{+}\right), 173\left(\mathrm{M}^{+}\right.$ - F), $164\left(\mathrm{M}^{+}-\mathrm{N}_{2}\right), 150\left(\mathrm{M}^{+}-\mathrm{N}_{3}\right)$. Anal. Calcd. for $\mathrm{C}_{5} \mathrm{H}_{3} \mathrm{~F}_{3} \mathrm{~N}_{4} \mathrm{O}$ : C, 31.26; H, 1.57; N, 29.17. Found: C, 31.16; H, 1.76; N, 29.14.

3-(Azidomethyl)-5-(perfluoroethyl)isoxazole (42d). Yield $12.2 \mathrm{~g}(88 \%)$ from $13.5 \mathrm{~g}$ of 40d. Colorless liquid, $\mathrm{bp}=59-61{ }^{\circ} \mathrm{C} / 23$ mbar. ${ }^{1} \mathrm{H}$ NMR $\left(500 \mathrm{MHz}, \mathrm{CDCl}_{3}\right): \delta 6.83(\mathrm{~s}, 1 \mathrm{H}), 4.54(\mathrm{~s}, 2 \mathrm{H}) \mathrm{ppm}$. ${ }^{13} \mathrm{C}\left\{{ }^{1} \mathrm{H}\right\} \mathrm{NMR}\left(101 \mathrm{MHz}, \mathrm{CDCl}_{3}\right): \delta 160.1,159.2(\mathrm{t}, J=31.9 \mathrm{~Hz}), 118.0(\mathrm{qt}, J=286.2,35.9 \mathrm{~Hz}), 108.2$ (tq, $J=255.2,41.3 \mathrm{~Hz}), 106.5,45.4 \mathrm{ppm} .{ }^{19} \mathrm{~F}\left\{{ }^{1} \mathrm{H}\right\}$ NMR $\left(376 \mathrm{MHz}, \mathrm{CDCl}_{3}\right): \delta-84.8(\mathrm{~s}, 3 \mathrm{~F}),-115.7(\mathrm{~s}$, 2F) ppm. Anal. Calcd. for $\mathrm{C}_{6} \mathrm{H}_{3} \mathrm{~F}_{5} \mathrm{~N}_{4} \mathrm{O}: \mathrm{C}, 29.77 ; \mathrm{H}, 1.25 ; \mathrm{N}, 23.14$. Found: C, 29.53; H, 1.62; N, 22.94.

General procedure for the preparation of the compounds 38 and 39 . To a solution of azide 42 or $43(0.10 \mathrm{~mol})$ in THF $(150 \mathrm{~mL}), \mathrm{PPh}_{3}(28.1 \mathrm{~g}, 0.107 \mathrm{~mol})$ was added in one portion at $0{ }^{\circ} \mathrm{C}$, and the reaction was stirred for $2 \mathrm{~h}$ at the same temperature. $\mathrm{H}_{2} \mathrm{O}(1.98 \mathrm{~mL}, 0.11 \mathrm{~mol})$ was added dropwise at 0 ${ }^{\circ} \mathrm{C}$, and the reaction was stirred overnight. After the reaction was complete (monitored by ${ }^{1} \mathrm{H}$ NMR analysis), the organic solvent was evaporated and the solid residue was suspended in $t$-BuOMe $(30 \mathrm{~mL})$. The obtained suspension was filtered; precipitate was additionally washed with cold $t$-BuOMe $(2 \times 30$ $\mathrm{mL}$ ) and the mother liquor was concentrated under reduced pressure. The obtained products were purified by vacuum distillation. 
(5-(Difluoromethyl)isoxazol-3-yl)methanamine (38a). Yield $38.1 \mathrm{~g}$ (76\%) from $59.0 \mathrm{~g}$ of 42a. Colorless liquid, $\mathrm{bp}=69-72{ }^{\circ} \mathrm{C} / 10 \mathrm{mbar} .{ }^{1} \mathrm{H}$ NMR $\left(400 \mathrm{MHz}, \mathrm{CDCl}_{3}\right): \delta 6.73(\mathrm{t}, J=53.8 \mathrm{~Hz}, 1 \mathrm{H}), 6.58$ (s, 1H), 3.99 (s, 2H), $1.53(\mathrm{~s}, 2 \mathrm{H}) \mathrm{ppm} .{ }^{13} \mathrm{C}\left\{{ }^{1} \mathrm{H}\right\}$ NMR (101 MHz, $\left.\mathrm{CDCl}_{3}\right): \delta 165.3,163.3(\mathrm{t}, J=30.6$ $\mathrm{Hz}), 107.2(\mathrm{t}, J=238.8 \mathrm{~Hz}), 102.8(\mathrm{t}, J=2.3 \mathrm{~Hz}), 37.7 \mathrm{ppm} .{ }^{19} \mathrm{~F}\left\{{ }^{1} \mathrm{H}\right\}$ NMR $\left(376 \mathrm{MHz}, \mathrm{CDCl}_{3}\right): \delta-$ 118.5 ppm. LC-MS (m/z): $149\left(\mathrm{M}+\mathrm{H}^{+}\right)$. Anal. Calcd. for $\mathrm{C}_{5} \mathrm{H}_{6} \mathrm{~F}_{2} \mathrm{~N}_{2} \mathrm{O}: \mathrm{C}, 40.55 ; \mathrm{H}, 4.08 ; \mathrm{N}, 18.91$. Found: C, 40.61; H, 4.21; N, 18.65.

(5-(Trifluoromethyl)isoxazol-3-yl)methanamine (38b). Yield $26.6 \mathrm{~g}$ (92\%) from $33.5 \mathrm{~g}$ of 42b. Yellow liquid, $\mathrm{bp}=65-68{ }^{\circ} \mathrm{C} / 10 \mathrm{mbar} .{ }^{1} \mathrm{H}$ NMR $\left(400 \mathrm{MHz}, \mathrm{CDCl}_{3}\right): \delta 6.71(\mathrm{~s}, 1 \mathrm{H}), 4.00(\mathrm{~s}, 2 \mathrm{H}), 1.56$ (s, 2H) ppm. ${ }^{13} \mathrm{C}\left\{{ }^{1} \mathrm{H}\right\} \operatorname{NMR}\left(126 \mathrm{MHz}, \mathrm{CDCl}_{3}\right): \delta 165.5,158.9$ (q, $\left.J=42.5 \mathrm{~Hz}\right), 118.0(\mathrm{q}, J=270.1 \mathrm{~Hz})$, 104.4, 38.0 ppm. ${ }^{19} \mathrm{~F}\left\{{ }^{1} \mathrm{H}\right\}$ NMR (376 MHz, $\left.\mathrm{CDCl}_{3}\right): \delta-64.7$ ppm. LC-MS $(\mathrm{m} / \mathrm{z}): 167\left(\mathrm{M}+\mathrm{H}^{+}\right)$. Anal. Calcd. for $\mathrm{C}_{5} \mathrm{H}_{5} \mathrm{~F}_{3} \mathrm{~N}_{2} \mathrm{O}: \mathrm{C}, 36.16 ; \mathrm{H}, 3.03 ; \mathrm{N}, 16.87$. Found: C, 35.96; H, 2.98; N, 16.59.

(5-(Perfluoroethyl)isoxazol-3-yl)methanamine (38d). Yield $8.62 \mathrm{~g}(84 \%)$ from $11.5 \mathrm{~g}$ of 42d. Colorless liquid, $\mathrm{bp}=60-61{ }^{\circ} \mathrm{C} / 23$ mbar. ${ }^{1} \mathrm{H}$ NMR $\left(500 \mathrm{MHz}, \mathrm{CDCl}_{3}\right): \delta 6.78(\mathrm{~s}, 1 \mathrm{H}), 4.03(\mathrm{~s}, 2 \mathrm{H}), 1.51$ (s, 2H) ppm. ${ }^{13} \mathrm{C}\left\{{ }^{1} \mathrm{H}\right\}$ NMR $\left(126 \mathrm{MHz}, \mathrm{CDCl}_{3}\right): \delta 165.7,158.2(\mathrm{t}, J=31.5 \mathrm{~Hz}), 118.1$ (qt, $J=286.2$, $36.1 \mathrm{~Hz}), 108.3(\mathrm{tq}, J=254.5,40.9 \mathrm{~Hz}), 106.2,37.9 \mathrm{ppm} .{ }^{19} \mathrm{~F}\left\{{ }^{1} \mathrm{H}\right\}$ NMR $\left(376 \mathrm{MHz}, \mathrm{CDCl}_{3}\right): \delta-84.8(\mathrm{~s}$, 3F), -115.7 (s, 2F) ppm. LC-MS (m/z): $217\left(\mathrm{M}+\mathrm{H}^{+}\right)$. Anal. Calcd. for $\mathrm{C}_{6} \mathrm{H}_{5} \mathrm{~F}_{5} \mathrm{~N}_{2} \mathrm{O}: \mathrm{C}, 33.35 ; \mathrm{H}, 2.33$; N, 12.96. Found: C, 33.56; H, 2.37; N, 13.27.

(3-(Difluoromethyl)isoxazol-5-yl)methanamine (39a). The compound was obtained from 5(chloromethyl)-3-(difluoromethyl)isoxazole (41a) $(78.5 \mathrm{~g}, 0.469 \mathrm{~mol})$, which was first converted to 5(azidomethyl)-3-(difluoromethyl)isoxazole (43a) by the general procedure for the preparation of the compounds 42 or 43 . The crude azide 43a was subjected to vacuum distillation to give $72.6 \mathrm{~g}$ of product as a colorless liquid $\left(\mathrm{bp}=37-39{ }^{\circ} \mathrm{C} / 1 \mathrm{mbar}\right.$ ). The purity of the obtained compound 43a was about $90 \%$, and it was used in the next step without additional purification. The next reduction of 43a by the general procedure for the preparation of the compounds $\mathbf{3 8}$ and $\mathbf{3 9}$ resulted in the target product 39a. Yield 52.0 
$\mathrm{g}(75 \%)$ from $78.5 \mathrm{~g}$ of 41a. Colorless liquid, $\mathrm{bp}=70-72{ }^{\circ} \mathrm{C} / 10 \mathrm{mbar} .{ }^{1} \mathrm{H}$ NMR $\left(400 \mathrm{MHz}, \mathrm{CDCl}_{3}\right): \delta$ $6.70(\mathrm{t}, J=53.8 \mathrm{~Hz}, 1 \mathrm{H}), 6.34(\mathrm{~s}, 1 \mathrm{H}), 3.97(\mathrm{~s}, 2 \mathrm{H}), 1.50(\mathrm{~s}, 2 \mathrm{H}) \mathrm{ppm} .{ }^{13} \mathrm{C}\left\{{ }^{1} \mathrm{H}\right\} \mathrm{NMR}(126 \mathrm{MHz}$, $\left.\mathrm{CDCl}_{3}\right): \delta 175.9,158.6(\mathrm{t}, J=29.9 \mathrm{~Hz}), 109.0(\mathrm{t}, J=236.4 \mathrm{~Hz}), 97.4,38.0 \mathrm{ppm} .{ }^{19} \mathrm{~F}\left\{{ }^{1} \mathrm{H}\right\} \mathrm{NMR}(376$ $\left.\mathrm{MHz}, \mathrm{CDCl}_{3}\right): \delta-116.1$ ppm. LC-MS $(\mathrm{m} / \mathrm{z}): 149\left(\mathrm{M}+\mathrm{H}^{+}\right)$. Anal. Calcd. for $\mathrm{C}_{5} \mathrm{H}_{6} \mathrm{~F}_{2} \mathrm{~N}_{2} \mathrm{O}: \mathrm{C}, 40.55 ; \mathrm{H}$, 4.08; N, 18.91. Found: C, 40.83; H, 3.79; N, 18.67.

5-(Difluoromethyl)isoxazole-3-carbaldehyde (44a). Pyridinium chlorochromate (7.50 g, $51.0 \mathrm{mmol})$ and $\mathrm{SiO}_{2}$ (ca. $20 \mathrm{~g}$ ) were suspended in $\mathrm{CH}_{2} \mathrm{Cl}_{2}(100 \mathrm{~mL})$. The resulting mechanically stirred solution was cooled to $-10{ }^{\circ} \mathrm{C}$ and alcohol 21a $(5.07 \mathrm{~g}, 34.0 \mathrm{mmol})$ in $\mathrm{CH}_{2} \mathrm{Cl}_{2}(100 \mathrm{~mL})$ was added dropwise, maintaining the temperature from $-10{ }^{\circ} \mathrm{C}$ to $-5{ }^{\circ} \mathrm{C}$. The resulting mixture was stirred overnight at $\mathrm{rt}$. After the reaction was complete (monitored by ${ }^{1} \mathrm{H}$ NMR analysis), the obtained solution was filtered through a silica gel and evaporated in under reduced pressure. The obtained residue was redissolved in $t$ BuOMe, filtered through a silica plug, and concentrated in vacuo. The obtained crude product was subjected to vacuum distillation (fractions were collected in the cooled condenser). Yield $2.15 \mathrm{~g}(43 \%)$. Colorless liquid, bp $=40-41{ }^{\circ} \mathrm{C} / 30$ mbar. ${ }^{1} \mathrm{H}$ NMR (500 MHz, $\left.\mathrm{CDCl}_{3}\right): \delta 10.17(\mathrm{~s}, 1 \mathrm{H}), 6.97(\mathrm{~s}, 1 \mathrm{H})$, $6.83(\mathrm{t}, J=53.2 \mathrm{~Hz}, 1 \mathrm{H}) \mathrm{ppm} .{ }^{13} \mathrm{C}\left\{{ }^{1} \mathrm{H}\right\} \mathrm{NMR}\left(126 \mathrm{MHz}, \mathrm{CDCl}_{3}\right): \delta 183.6,165.3(\mathrm{t}, J=31.3 \mathrm{~Hz}), 161.8$, $106.8(\mathrm{t}, J=240.0 \mathrm{~Hz}), 101.5(\mathrm{t}, J=2.5 \mathrm{~Hz}) \mathrm{ppm} .{ }^{19} \mathrm{~F}$ NMR $\left(470 \mathrm{MHz}, \mathrm{CDCl}_{3}\right): \delta-118.5(\mathrm{~d}, J=53.2$ Hz) ppm. GC-MS $(\mathrm{m} / \mathrm{z}): 147\left(\mathrm{M}^{+}\right), 128\left(\mathrm{M}^{+}-\mathrm{F}\right), 119\left(\mathrm{M}^{+}-\mathrm{CO}\right)$. Anal. Calcd. for $\mathrm{C}_{5} \mathrm{H}_{3} \mathrm{~F}_{2} \mathrm{NO}_{2}: \mathrm{C}$, 40.83; H, 2.06; N, 9.52. Found: C, 41.15; H, 1.97; N, 9.44.

3-(Difluoromethyl)isoxazole-5-carbaldehyde (45a). The compound was obtained by the same procedure described for the preparation of 44a. Yield $1.37 \mathrm{~g}(41 \%)$ from $3.38 \mathrm{~g}$ of 20a. Colorless liquid, bp $=38-40{ }^{\circ} \mathrm{C} / 1$ mbar. ${ }^{1} \mathrm{H}$ NMR $\left(500 \mathrm{MHz}, \mathrm{CDCl}_{3}\right): \delta 10.03(\mathrm{~s}, 1 \mathrm{H}), 7.20(\mathrm{~s}, 1 \mathrm{H}), 6.88(\mathrm{t}, J=53.4 \mathrm{~Hz}$, 1H) ppm. ${ }^{13} \mathrm{C}\left\{{ }^{1} \mathrm{H}\right\}$ NMR $\left(101 \mathrm{MHz}, \mathrm{CDCl}_{3}\right): \delta 177.7,166.7,159.5(\mathrm{t}, J=30.6 \mathrm{~Hz}), 108.4(\mathrm{t}, J=237.9$ Hz), 106.0 ppm. ${ }^{19} \mathrm{~F}\left\{{ }^{1} \mathrm{H}\right\}$ NMR (376 MHz, $\left.\mathrm{CDCl}_{3}\right): \delta-116.4$ ppm. GC-MS $(\mathrm{m} / \mathrm{z}): 147\left(\mathrm{M}^{+}\right), 128\left(\mathrm{M}^{+}-\right.$ F). Anal. Calcd. for $\mathrm{C}_{5} \mathrm{H}_{3} \mathrm{~F}_{2} \mathrm{NO}_{2}$ : C, 40.83; H, 2.06; N, 9.52. Found: C, 40.74; H, 2.37; N, 9.42. 
1-(5-(Difluoromethyl)isoxazol-3-yl)ethanone (44h). To a solution of alcohol $\mathbf{2 1 h}(1.00 \mathrm{~g}, 6.21$ mmol), $\mathrm{NEt}_{3}(2.77 \mathrm{~mL}, 1.99 \mathrm{~mol})$ and $\mathrm{DMSO}(2.20 \mathrm{~mL}, 31.1 \mathrm{mmol})$ in $\mathrm{CH}_{2} \mathrm{Cl}_{2}(10 \mathrm{~mL}), \mathrm{Py} \cdot \mathrm{SO}_{3}(2.96$ g, $18.6 \mathrm{mmol}$ ) was added under argon flow at $0{ }^{\circ} \mathrm{C}$. Then, the solution was allowed to warm to $\mathrm{rt}$ and stirred for $1 \mathrm{~h}$. The reaction mixture was poured in ice-cold water $(10 \mathrm{~mL})$, and two layers were separated. The organic phase was concentrated in vacuo, whereas the aqueous layer was extracted with EtOAc $(2 \times 7 \mathrm{~mL})$. The organic phase was combined with the residue obtained after concentration, washed with brine $(7 \mathrm{~mL})$, dried over anhydrous $\mathrm{Na}_{2} \mathrm{SO}_{4}$, filtered through a silica plug and concentrated under reduced pressure. Yield $849 \mathrm{mg}(86 \%)$. Colorless liquid, bp $68-69{ }^{\circ} \mathrm{C} / 8$ mbar. ${ }^{1} \mathrm{H}$ NMR $(400$ $\left.\mathrm{MHz}, \mathrm{CDCl}_{3}\right): \delta 6.92(\mathrm{~s}, 1 \mathrm{H}), 6.78(\mathrm{t}, J=53.4 \mathrm{~Hz}, 1 \mathrm{H}), 2.66(\mathrm{~s}, 3 \mathrm{H}) \mathrm{ppm} .{ }^{13} \mathrm{C}\left\{{ }^{1} \mathrm{H}\right\} \mathrm{NMR}(101 \mathrm{MHz}$, $\left.\mathrm{CDCl}_{3}\right): \delta 190.9,164.9(\mathrm{t}, J=31.1 \mathrm{~Hz}), 161.8,106.9(\mathrm{t}, J=239.8 \mathrm{~Hz}), 102.7(\mathrm{t}, J=2.5 \mathrm{~Hz}), 27.3 \mathrm{ppm}$. ${ }^{19} \mathrm{~F}\left\{{ }^{1} \mathrm{H}\right\}$ NMR $\left(376 \mathrm{MHz}, \mathrm{CDCl}_{3}\right): \delta-118.8 \mathrm{ppm}$. GC-MS $(\mathrm{m} / \mathrm{z}): 146\left(\mathrm{M}^{+}-\mathrm{CH}_{3}\right), 142\left(\mathrm{M}^{+}-\mathrm{F}\right)$. Anal. Calcd. for $\mathrm{C}_{6} \mathrm{H}_{5} \mathrm{~F}_{2} \mathrm{NO}_{2}$ : C, 44.73; H, 3.13; N, 8.69. Found: C, 44.36; H, 3.34; N, 9.01.

General procedure for the preparation of the compounds 48, 49 and 50 . The corresponding alkyne $(6.60 \mathrm{mmol})$ and $\mathrm{Cu}(\mathrm{OAc})_{2} \cdot \mathrm{H}_{2} \mathrm{O}(57.3 \mathrm{mg}, 0.287 \mathrm{mmol})$ were added to a solution of azide 42a or 43a (5.74 mmol) in $\mathrm{CHCl}_{3}(10 \mathrm{~mL})$, and the mixture was stirred overnight. After the reaction was complete (monitored by ${ }^{1} \mathrm{H}$ NMR analysis), the solution was concentrated in vacuo. The residue was dissolved in EtOAc $(10 \mathrm{~mL})$, filtered through a silica plug, and concentrated under reduced pressure.

Methyl 1-((5-(difluoromethyl)isoxazol-3-yl)methyl)-1H-1,2,3-triazole-4-carboxylate (48). The compound was synthesized from methyl propiolate $(554 \mathrm{mg}, 6.60 \mathrm{mmol})$ and azide 42a $(1.00 \mathrm{~g}, 5.74$ mmol). Yield $1.17 \mathrm{~g}(79 \%)$. White solid, mp 89-91 ${ }^{\circ} \mathrm{C} .{ }^{1} \mathrm{H}$ NMR (400 MHz, DMSO-d 6 ): $\delta 8.95$ (s, 1H), $7.33(\mathrm{t}, J=52.6 \mathrm{~Hz}, 1 \mathrm{H}), 7.07(\mathrm{~s}, 1 \mathrm{H}), 5.92(\mathrm{~s}, 2 \mathrm{H}), 3.84(\mathrm{~s}, 3 \mathrm{H}) \mathrm{ppm} .{ }^{13} \mathrm{C}\left\{{ }^{1} \mathrm{H}\right\} \mathrm{NMR}(101 \mathrm{MHz}$, DMSO- $\left.d_{6}\right): \delta 163.6(\mathrm{t}, J=28.8 \mathrm{~Hz}), 160.6,159.5,138.9,130.0,107.4(\mathrm{t}, J=236.6 \mathrm{~Hz}), 105.0(\mathrm{t}, J=3.6$ Hz), 51.8, 44.9 ppm. ${ }^{19} \mathrm{~F}\left\{{ }^{1} \mathrm{H}\right\}$ NMR (376 MHz, DMSO- $\left.d_{6}\right): \delta-118.8$ ppm. LC-MS $(\mathrm{m} / \mathrm{z}): 259\left(\mathrm{M}+\mathrm{H}^{+}\right)$. Anal. Calcd. for $\mathrm{C}_{9} \mathrm{H}_{8} \mathrm{~F}_{2} \mathrm{~N}_{4} \mathrm{O}_{3}:$ C, 41.87; H, 3.12; N, 21.70. Found: C, 41.99; H, 2.77; N, 21.49. 
Ethyl 1-((3-(difluoromethyl)isoxazol-5-yl)methyl)-1H-1,2,3-triazole-4-carboxylate (49). The compound was synthesized from ethyl propiolate $(712 \mathrm{mg}, 7.27 \mathrm{mmol}$ ) and 5-(azidomethyl)-3(difluoromethyl)isoxazole (43a). Azide 43a was in turn prepared from 5-(chloromethyl)-3(difluoromethyl)isoxazole (41a) $(1.19 \mathrm{~g}, 7.11 \mathrm{mmol})$ by the general procedure for the preparation of the compounds 42 and 43. The crude intermediate 43a was subjected to vacuum distillation to give $1.10 \mathrm{~g}$ of the product as colorless liquid ( $\mathrm{bp}=37-39{ }^{\circ} \mathrm{C} / 1 \mathrm{mbar}$ ). The purity of the compound 43a was about $90 \%$, and it was used in the next 'click' reaction with ethyl propiolate without additional purification. Yield $1.45 \mathrm{~g}(75 \%)$ from $1.19 \mathrm{~g}$ of 41a. White solid, mp $103-105{ }^{\circ} \mathrm{C} .{ }^{1} \mathrm{H} \mathrm{NMR}\left(400 \mathrm{MHz}, \mathrm{CDCl}_{3}\right): \delta 8.25(\mathrm{~s}$, $1 \mathrm{H}), 6.75(\mathrm{t}, J=53.5 \mathrm{~Hz}, 1 \mathrm{H}), 6.58(\mathrm{~s}, 1 \mathrm{H}), 5.81(\mathrm{~s}, 2 \mathrm{H}), 4.42(\mathrm{q}, J=7.1 \mathrm{~Hz}, 2 \mathrm{H}), 1.40(\mathrm{t}, J=7.1 \mathrm{~Hz}$, 3H) ppm. ${ }^{13} \mathrm{C}\left\{{ }^{1} \mathrm{H}\right\}$ NMR $\left(126 \mathrm{MHz}, \mathrm{CDCl}_{3}\right): \delta 166.3,160.2,159.1(\mathrm{t}, \mathrm{J}=30.0 \mathrm{~Hz}), 140.6,128.3,108.5$ $(\mathrm{t}, J=237.6 \mathrm{~Hz}), 101.6,61.3,45.0,14.0 \mathrm{ppm} .{ }^{19} \mathrm{~F}\left\{{ }^{1} \mathrm{H}\right\}$ NMR $\left(376 \mathrm{MHz}, \mathrm{CDCl}_{3}\right): \delta-116.4 \mathrm{ppm} . \mathrm{LC}-\mathrm{MS}$ $(\mathrm{m} / \mathrm{z}): 273\left(\mathrm{M}+\mathrm{H}^{+}\right)$. Anal. Calcd. for $\mathrm{C}_{10} \mathrm{H}_{10} \mathrm{~F}_{2} \mathrm{~N}_{4} \mathrm{O}_{3}: \mathrm{C}, 44.12 ; \mathrm{H}, 3.70 ; \mathrm{N}, 20.58$. Found: $\mathrm{C}, 44.28 ; \mathrm{H}$, $3.58 ; \mathrm{N}, 20.76$.

\section{5-((1H-1,2,3-Triazol-1-yl)methyl)-3-(difluoromethyl)isoxazole (50).}

The compound was synthesized from trimethylsilylacetylene $(712 \mathrm{mg}, 7.27 \mathrm{mmol})$ and 5(azidomethyl)-3-(difluoromethyl)isoxazole (43a). Azide 43a was in turn prepared from 5(chloromethyl)-3-(difluoromethyl)isoxazole (41a) $(1.19 \mathrm{~g}, 7.11 \mathrm{mmol})$ by the general procedure for the preparation of the compounds 42 and 43. The crude intermediate 43a was subjected to vacuum distillation to give $1.10 \mathrm{~g}$ of the product as colorless liquid $\left(\mathrm{bp}=37-39^{\circ} \mathrm{C} / 1 \mathrm{mbar}\right.$ ). The purity of the compound 43a was about $90 \%$, and it was used in the next 'click' reaction with trimethylsilylacetylene without additional purification. The resulting 3-(difluoromethyl)-5-((4-(trimethylsilyl)-1H-1,2,3-triazol1-yl)methyl)isoxazole was dissolved in $\mathrm{MeOH}-\mathrm{H}_{2} \mathrm{O}(9: 1)$ mixture $(15 \mathrm{~mL}), \mathrm{KHF}_{2}(247 \mathrm{mg}, 3.16$ mmol) was added and the mixture was stirred overnight. After the reaction was complete (monitored by ${ }^{1} \mathrm{H}$ NMR), the solution concentrated under reduced pressure. The residue was dissolved in EtOAc (15 
$\mathrm{mL})$, washed with brine $(2 \times 5 \mathrm{~mL})$, filtered through a silica plug and concentrated in vacuo. Yield $1.05 \mathrm{~g}$ (74\%) from $1.19 \mathrm{~g}$ of 41a. White solid, mp $101-103{ }^{\circ} \mathrm{C}(\mathrm{dec}) .{ }^{1} \mathrm{H}$ NMR $\left(400 \mathrm{MHz}, \mathrm{CDCl}_{3}\right): \delta 7.78(\mathrm{~s}$, 1H), $7.73(\mathrm{~s}, 1 \mathrm{H}), 6.74(\mathrm{t}, J=53.5 \mathrm{~Hz}, 1 \mathrm{H}), 6.50(\mathrm{~s}, 1 \mathrm{H}), 5.77(\mathrm{~s}, 2 \mathrm{H}) \mathrm{ppm} .{ }^{13} \mathrm{C}\left\{{ }^{1} \mathrm{H}\right\} \mathrm{NMR}(126 \mathrm{MHz}$, $\left.\mathrm{CDCl}_{3}\right): \delta 167.0,159.0(\mathrm{t}, J=30.1 \mathrm{~Hz}), 134.4,124.3,108.6(\mathrm{t}, J=237.5 \mathrm{~Hz}), 101.1,44.7 \mathrm{ppm} .{ }^{19} \mathrm{~F}\left\{{ }^{1} \mathrm{H}\right\}$ NMR (376 MHz, $\left.\mathrm{CDCl}_{3}\right): \delta-116.3$ ppm. LC-MS (m/z): $201\left(\mathrm{M}+\mathrm{H}^{+}\right)$. Anal. Calcd. for $\mathrm{C}_{7} \mathrm{H}_{6} \mathrm{~F}_{2} \mathrm{~N}_{4} \mathrm{O}: \mathrm{C}$, 42.01; H, 3.02; N, 27.99. Found: C, 42.33; H, 2.75; N, 28.11.

1-((5-(Difluoromethyl)isoxazol-3-yl)methyl)-1H-1,2,3-triazole-4-carboxylic acid (51). To a solution of $48(1.10 \mathrm{~g}, 4.26 \mathrm{mmol})$ in $\mathrm{MeOH}(11 \mathrm{~mL})$, a solution of $\mathrm{NaOH}(188 \mathrm{mg}, 4.69 \mathrm{mmol})$ in $\mathrm{H}_{2} \mathrm{O}$ $(1.9 \mathrm{~mL})$ was added, and the reaction was stirred overnight. The resulting solution was concentrated in vacuo, and the residue was dissolved in 1:1 mixture of $\mathrm{H}_{2} \mathrm{O}$ and EtOAc $(10 \mathrm{~mL}) . \mathrm{NaHSO}_{4}(562 \mathrm{mg}, 4.69$ mmol) was added to the solution, the mixture was shaken and two layers were separated. The aqueous phase was extracted with EtOAc $(5 \mathrm{~mL})$. The combined organic phase was washed with brine $(5 \mathrm{~mL})$, dried over anhydrous $\mathrm{Na}_{2} \mathrm{SO}_{4}$, filtered through a silica plug and concentrated under reduced pressure. Yield $862 \mathrm{mg}(83 \%)$. White solid, mp $184-186{ }^{\circ} \mathrm{C} .{ }^{1} \mathrm{H}$ NMR (400 MHz, DMSO-d 6 ): $\delta 13.15$ (br s, $\left.1 \mathrm{H}\right)$, $8.84(\mathrm{~s}, 1 \mathrm{H}), 7.33(\mathrm{t}, J=52.6 \mathrm{~Hz}, 1 \mathrm{H}), 7.07(\mathrm{~s}, 1 \mathrm{H}), 5.90(\mathrm{~s}, 2 \mathrm{H}) \mathrm{ppm} .{ }^{13} \mathrm{C}\left\{{ }^{1} \mathrm{H}\right\} \mathrm{NMR}(126 \mathrm{MHz}$, DMSO- $\left.d_{6}\right): \delta 163.6(\mathrm{t}, J=28.7 \mathrm{~Hz}), 161.6,159.7,140.1,129.9,107.5(\mathrm{t}, J=236.6 \mathrm{~Hz}), 105.1(\mathrm{t}, J=3.6$ Hz), 44.8 ppm. ${ }^{19} \mathrm{~F}\left\{{ }^{1} \mathrm{H}\right\}$ NMR (376 MHz, $\left.\mathrm{CDCl}_{3}\right): \delta-118.2$ ppm. LC-MS $(\mathrm{m} / \mathrm{z}): 243\left(\mathrm{M}-\mathrm{H}^{+}\right)$. Anal. Calcd. for $\mathrm{C}_{8} \mathrm{H}_{6} \mathrm{~F}_{2} \mathrm{~N}_{4} \mathrm{O}_{3}:$ C, 39.35; H, 2.48; N, 22.95. Found: C, 39.50; H, 2.40; N, 22.59.

5-((1H-1,2,3-Triazol-1-yl)methyl)-4-chloro-3-(difluoromethyl)isoxazole (52). To a solution of 5((1H-1,2,3-triazol-1-yl)methyl)-3-(difluoromethyl)isoxazole (50) $(0.50 \mathrm{~g}, 2.50 \mathrm{mmol})$ in $\mathrm{CH}_{2} \mathrm{Cl}_{2}(5 \mathrm{~mL})$, $\mathrm{SO}_{2} \mathrm{Cl}_{2}(0.62 \mathrm{~mL}, 2.75 \mathrm{mmol})$ was added dropwise at $0{ }^{\circ} \mathrm{C}$, and the reaction mixture was left to stir at $\mathrm{rt}$ overnight. After the reaction was complete (monitored by ${ }^{1} \mathrm{H}$ NMR analysis), the resulting solution was concentrated in vacuo. Yield $493 \mathrm{mg}(84 \%)$. White solid, mp $133-135{ }^{\circ} \mathrm{C}$ (dec). ${ }^{1} \mathrm{H}$ NMR (400 MHz, $\left.\mathrm{CDCl}_{3}\right): \delta 7.77(\mathrm{~s}, 1 \mathrm{H}), 7.73(\mathrm{~s}, 1 \mathrm{H}), 6.77(\mathrm{t}, J=52.4 \mathrm{~Hz}, 1 \mathrm{H}), 5.77(\mathrm{~s}, 2 \mathrm{H}) \mathrm{ppm} .{ }^{13} \mathrm{C}\left\{{ }^{1} \mathrm{H}\right\} \mathrm{NMR}(126$ 
$\left.\mathrm{MHz}, \mathrm{CDCl}_{3}\right): \delta 162.4,155.7(\mathrm{t}, J=28.8 \mathrm{~Hz}), 134.5,124.3,108.3(\mathrm{t}, J=239.4 \mathrm{~Hz}), 107.9,42.5 \mathrm{ppm}$. ${ }^{19} \mathrm{~F}\left\{{ }^{1} \mathrm{H}\right\}$ NMR $\left(376 \mathrm{MHz}, \mathrm{CDCl}_{3}\right): \delta-119.6 \mathrm{ppm}$. LC-MS $(\mathrm{m} / \mathrm{z}): 235 / 237\left(\mathrm{M}+\mathrm{H}^{+}\right)$. Anal. Calcd. for $\mathrm{C}_{7} \mathrm{H}_{5} \mathrm{ClF}_{2} \mathrm{~N}_{4} \mathrm{O}: \mathrm{C}, 35.84 ; \mathrm{H}, 2.15 ; \mathrm{N}, 23.88 ; \mathrm{Cl}, 15.11$. Found: $\mathrm{C}, 36.17 ; \mathrm{H}, 2.45 ; \mathrm{N}, 24.06 ; \mathrm{Cl}, 15.33$.

\section{REFERENCES}

(1) Khutorianskyi, A.; Chalyk, B.; Borysko, P.; Kondratiuk, A.; Mykhailiuk, P. K. Difluoromethyl Nitrile Oxide ( $\left.\mathrm{CF}_{2} \mathrm{HCNO}\right)$ : A Neglected Chemical Reagent. Eur. J. Org. Chem. 2017, 2017 (27), 3935-3940.

(2) Herrera, A. G.; Schmitt, E.; Panossian, A.; Vors, J.-P.; Pazenok, S.; Leroux, F. R. New Synthetic Access to 3-Fluoroalkyl-5-Pyrazolecarboxylates and Carboxylic Acids. J. Fluor. Chem. 2018, $214,17-23$.

(3) Fustero, S.; Román, R.; Sanz-Cervera, J. F.; Simón-Fuentes, A.; Cuñat, A. C.; Villanova, S.; Murguía, M. Improved Regioselectivity in Pyrazole Formation through the Use of Fluorinated Alcohols as Solvents: Synthesis and Biological Activity of Fluorinated Tebufenpyrad Analogs. $J$. Org. Chem. 2008, 73 (9), 3523-3529.

(4) Li, F.; Nie, J.; Sun, L.; Zheng, Y.; Ma, J.-A. Silver-Mediated Cycloaddition of Alkynes with $\mathrm{CF}_{3} \mathrm{CHN}_{2}$ : Highly Regioselective Synthesis of 3-Trifluoromethylpyrazoles. Angew. Chem., Int. Ed. 2013, 52 (24), 6255-6258. 
${ }^{1} \mathrm{H}$ NMR spectrum of the compound 13a.

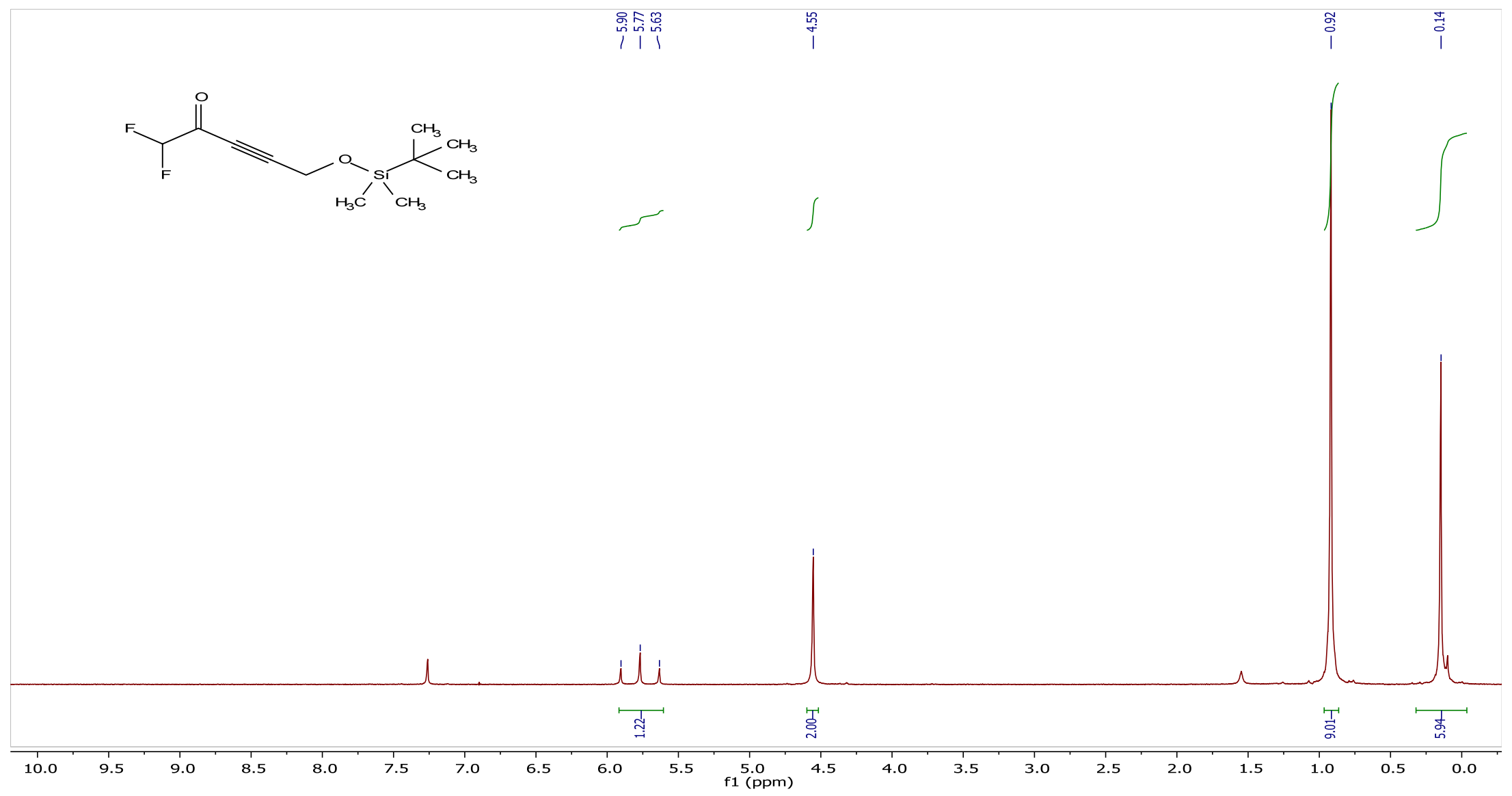


${ }^{13} \mathrm{C}\left\{{ }^{1} \mathrm{H}\right\}$ NMR spectrum of the compound 13a.

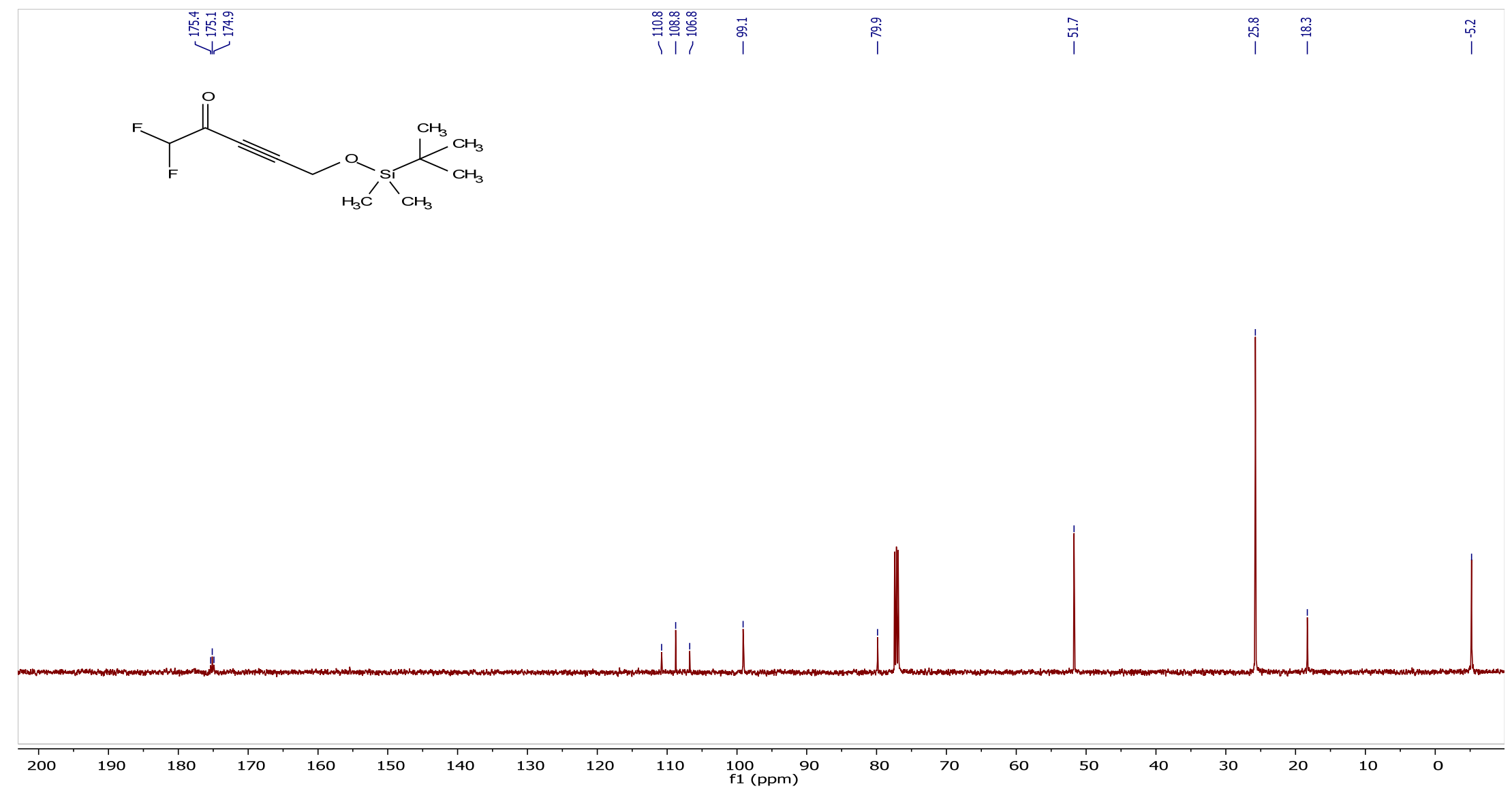


${ }^{19} \mathrm{~F}$ NMR spectrum of the compound 13a.

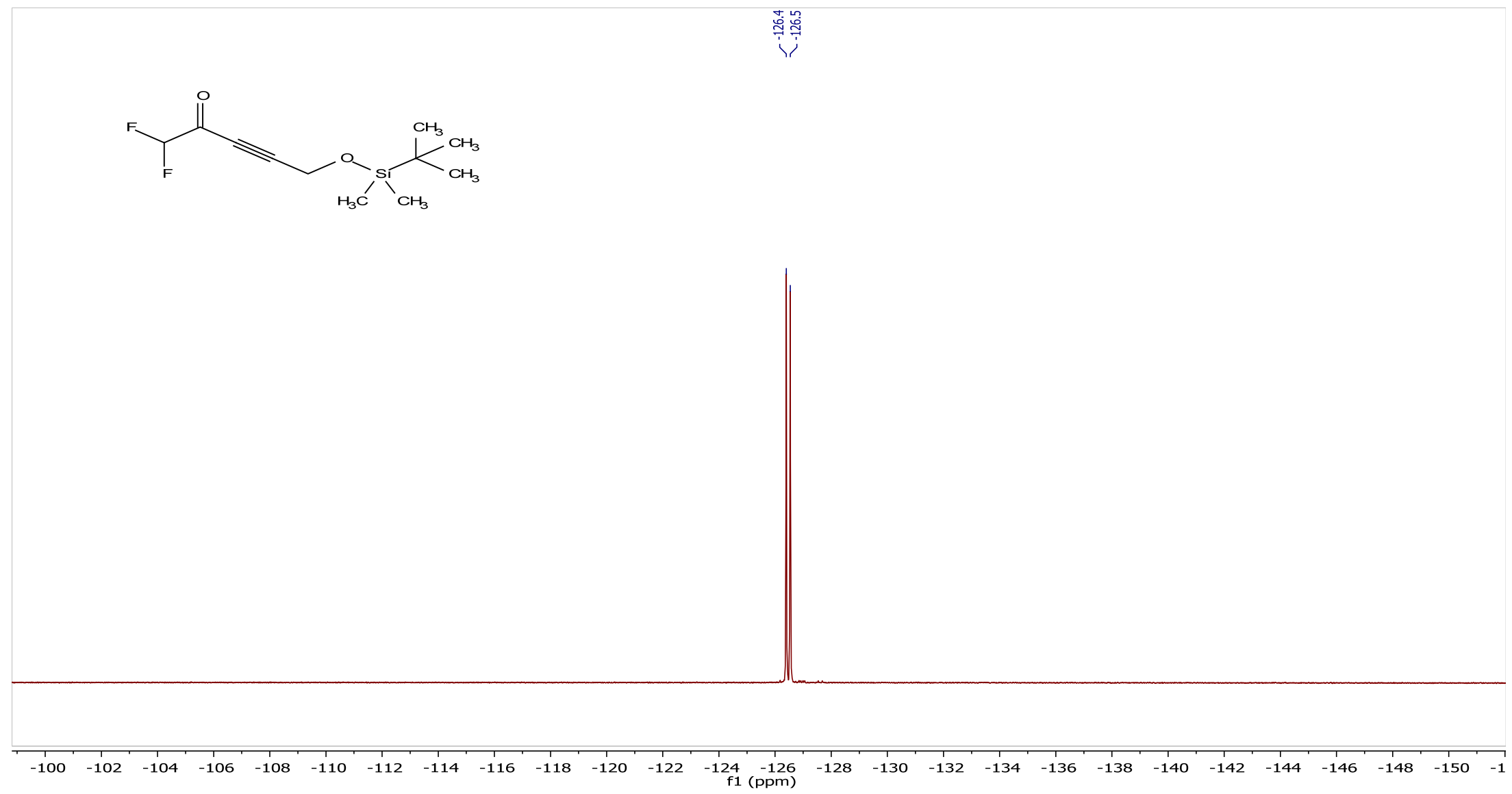


${ }^{1} \mathrm{H}$ NMR spectrum of the compound $\mathbf{1 3 b}$.

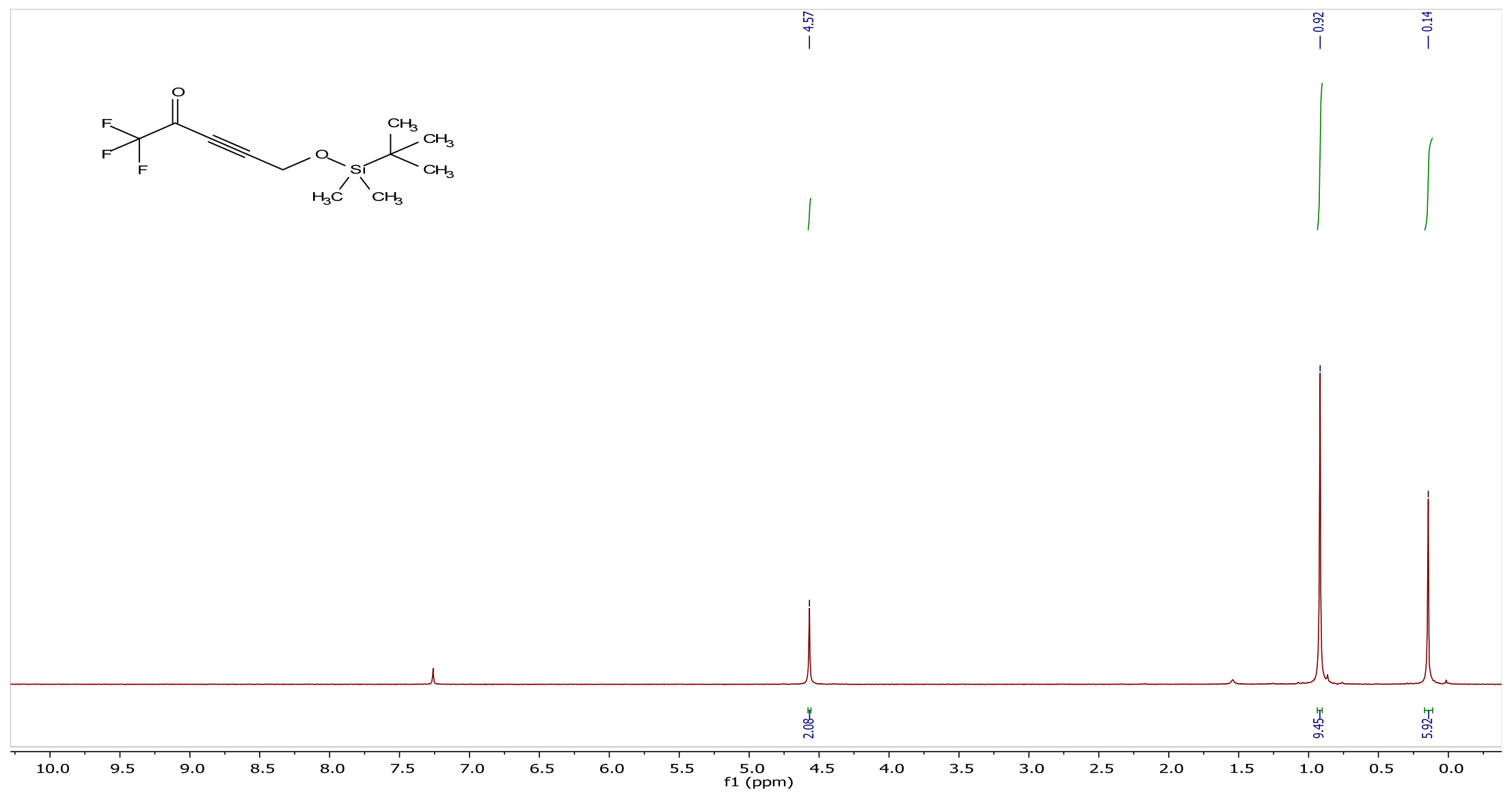


${ }^{13} \mathrm{C}\left\{{ }^{1} \mathrm{H}\right\}$ NMR spectrum of the compound $\mathbf{1 3 b}$.

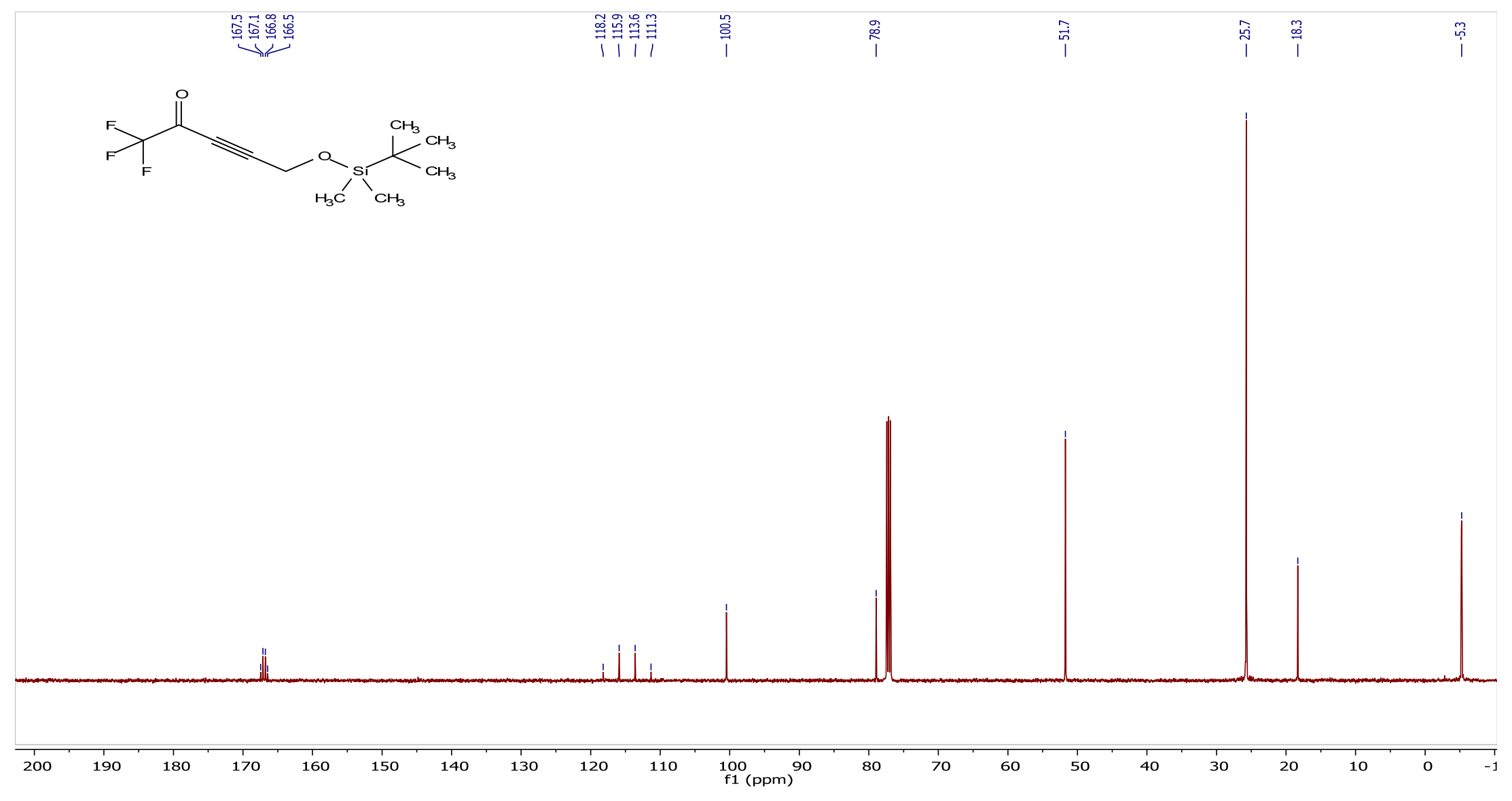


${ }^{19} \mathrm{~F}\left\{{ }^{1} \mathrm{H}\right\}$ NMR spectrum of the compound $\mathbf{1 3 b}$.

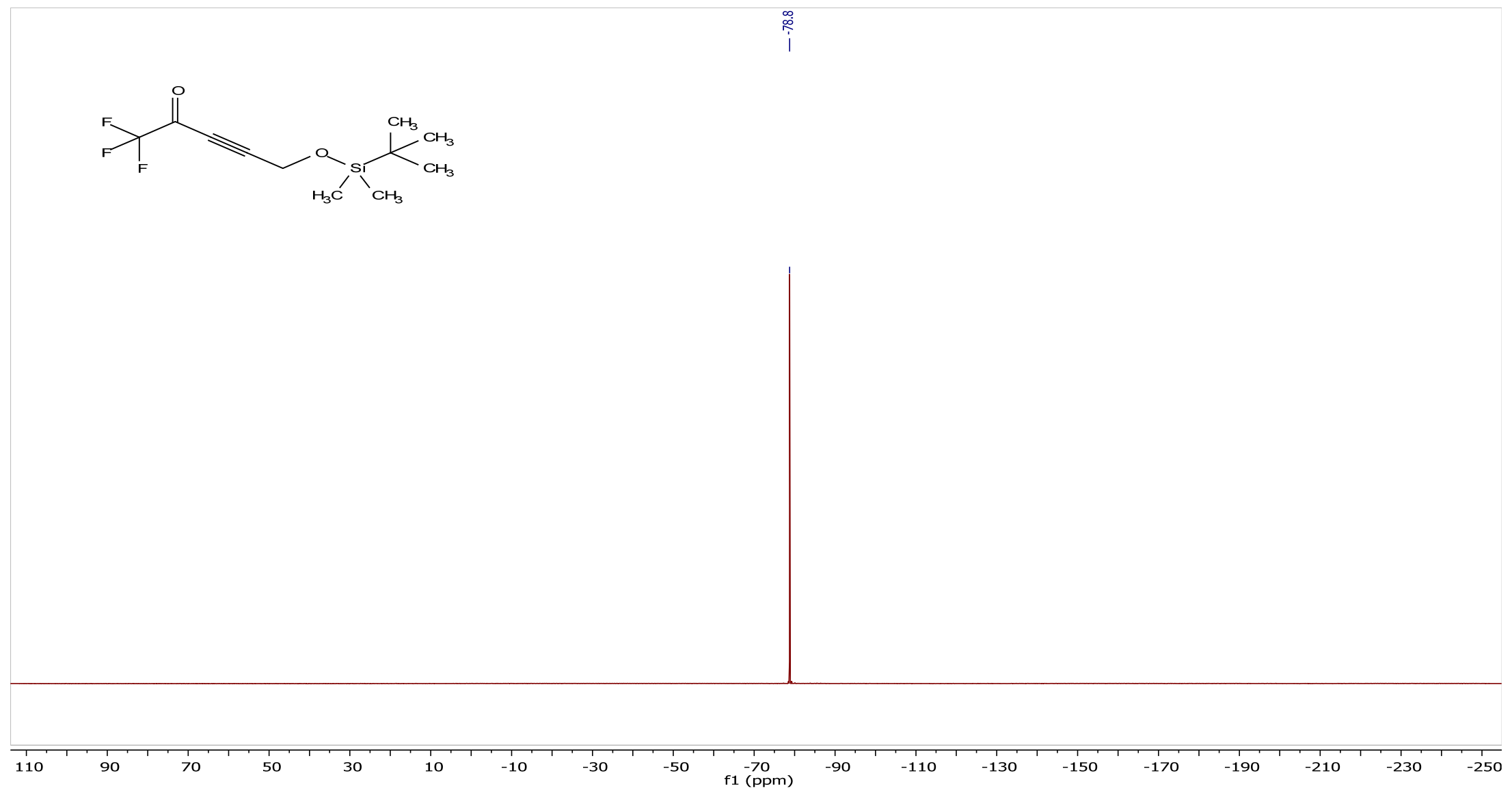


${ }^{1} \mathrm{H}$ NMR spectrum of the compound 13c.

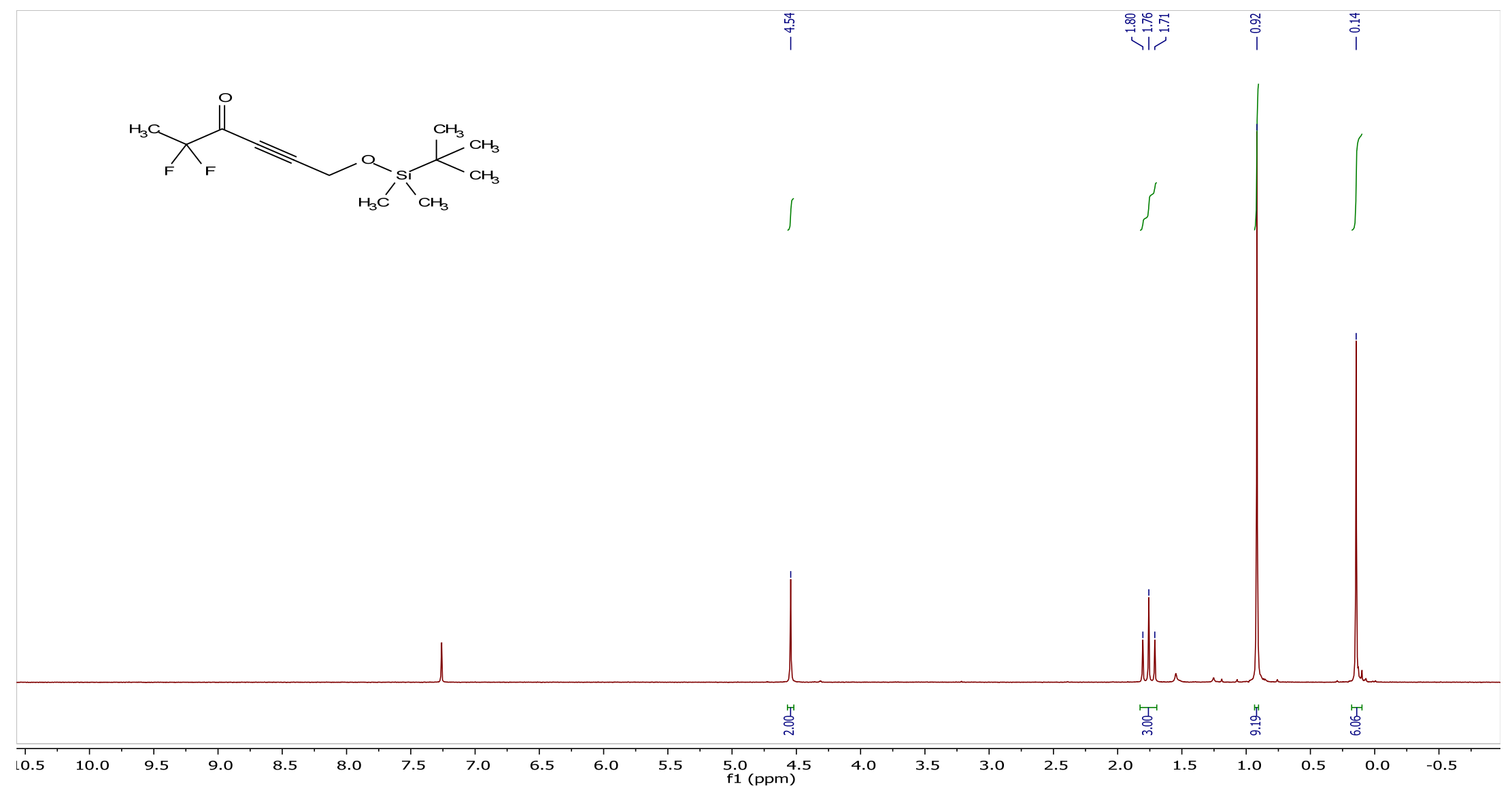


${ }^{13} \mathrm{C}\left\{{ }^{1} \mathrm{H}\right\}$ NMR spectrum of the compound 13c.

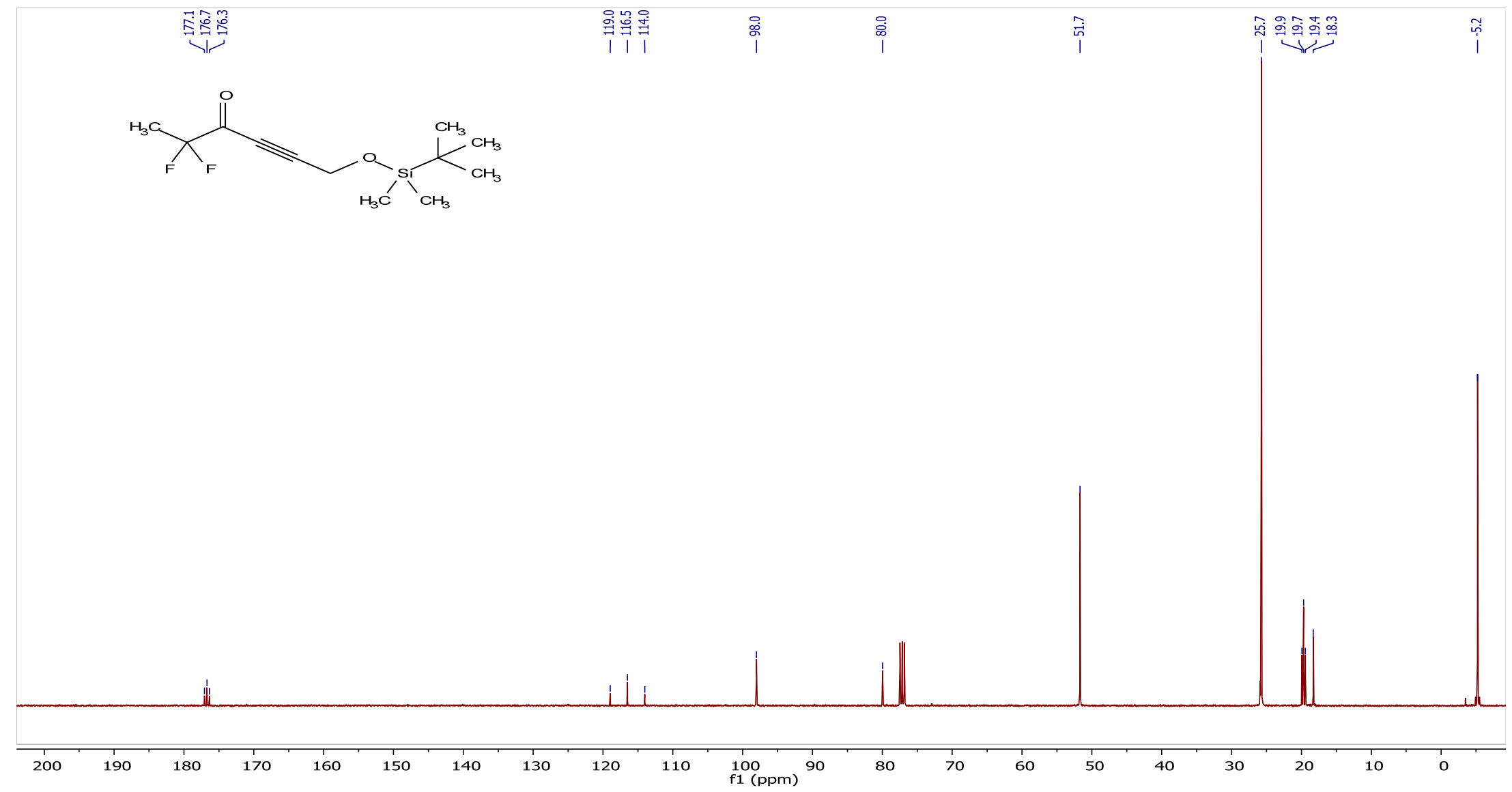


${ }^{19} \mathrm{~F}\left\{{ }^{1} \mathrm{H}\right\}$ NMR spectrum of the compound 13c.

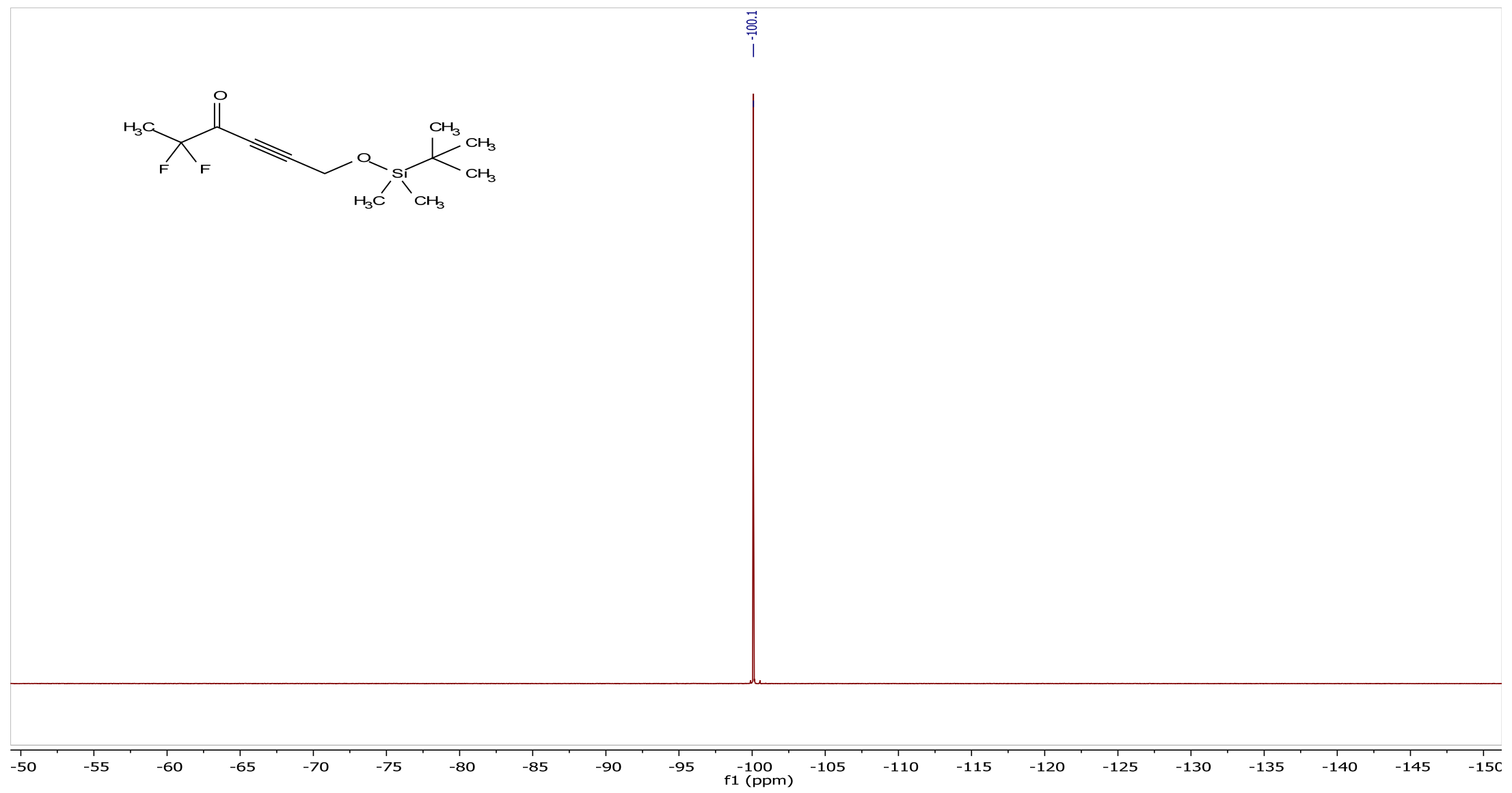


${ }^{1} \mathrm{H}$ NMR spectrum of the compound 13d.

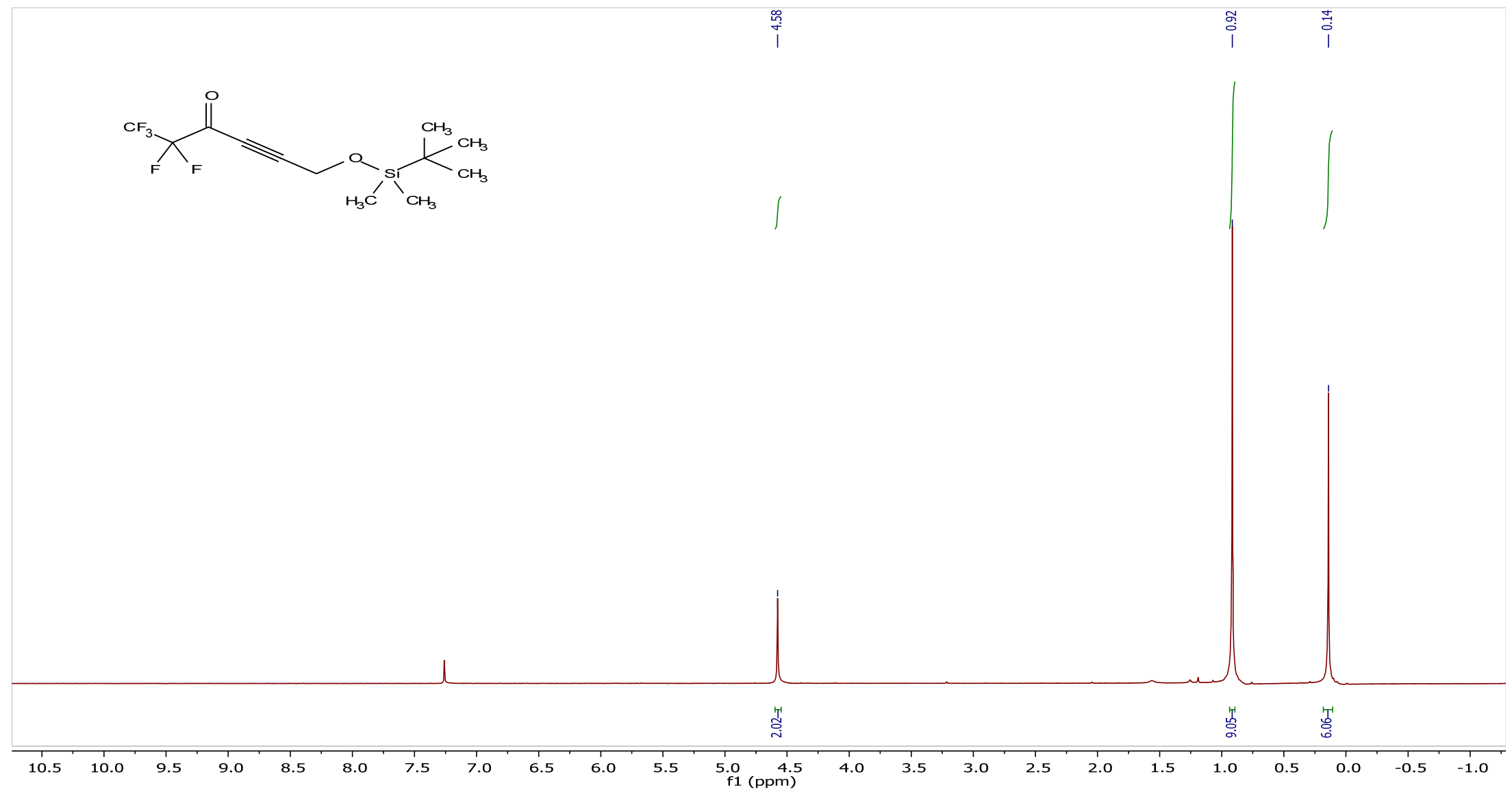


${ }^{13} \mathrm{C}\left\{{ }^{1} \mathrm{H}\right\}$ NMR spectrum of the compound 13d.

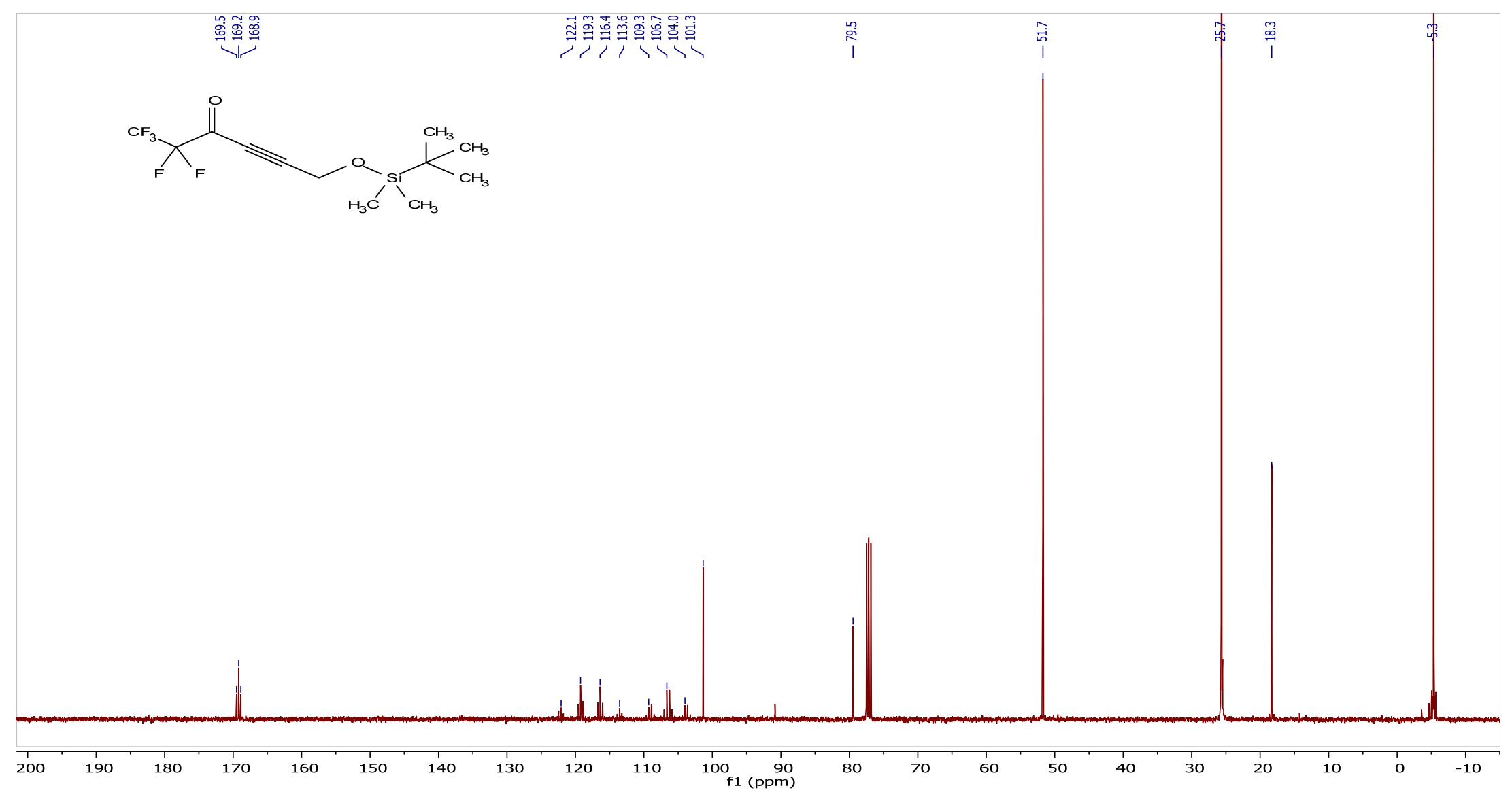


${ }^{19} \mathrm{~F}\left\{{ }^{1} \mathrm{H}\right\}$ NMR spectrum of the compound 13d.

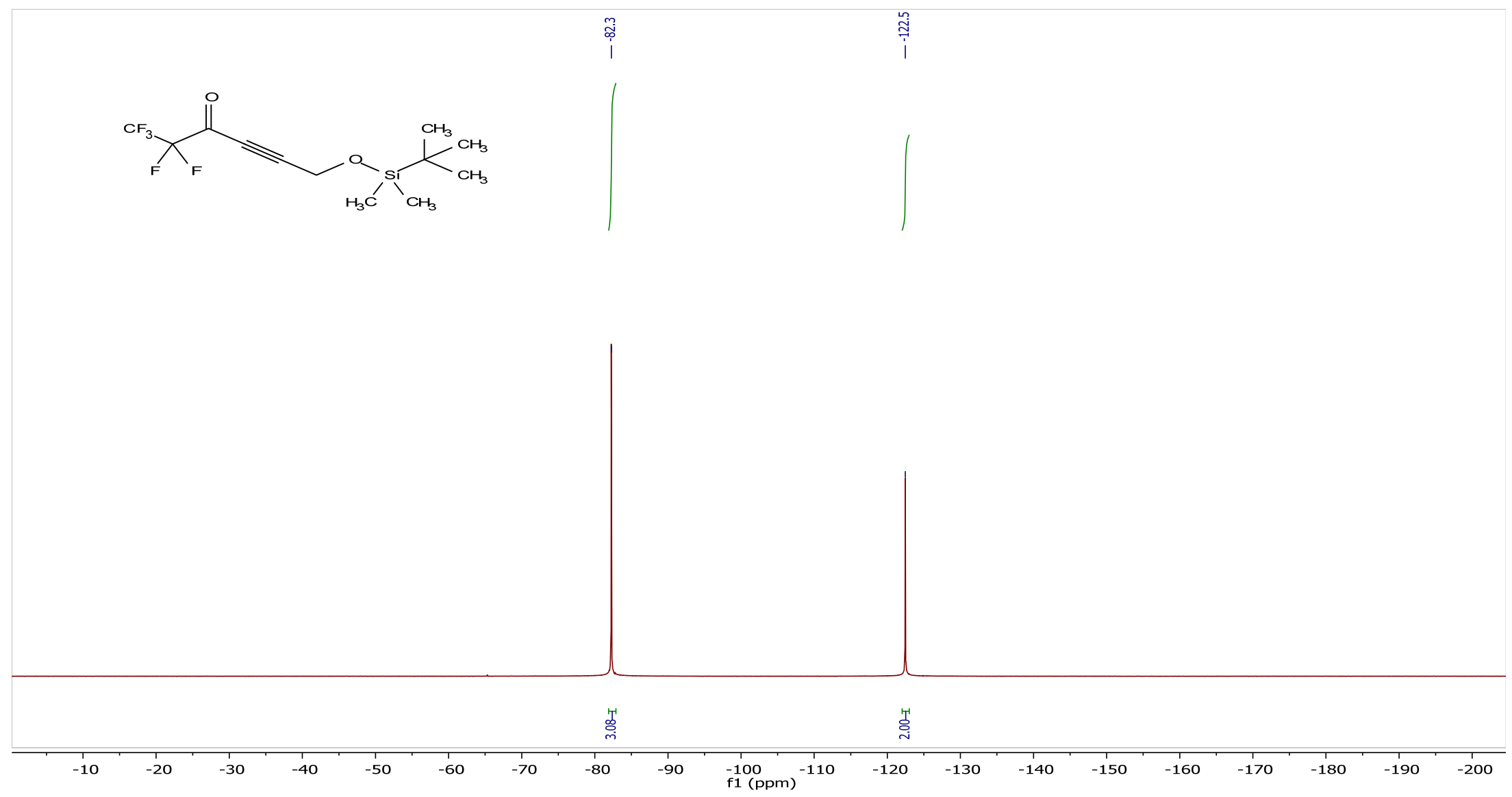


${ }^{1} \mathrm{H}$ NMR spectrum of the compound $13 \mathbf{e}$.

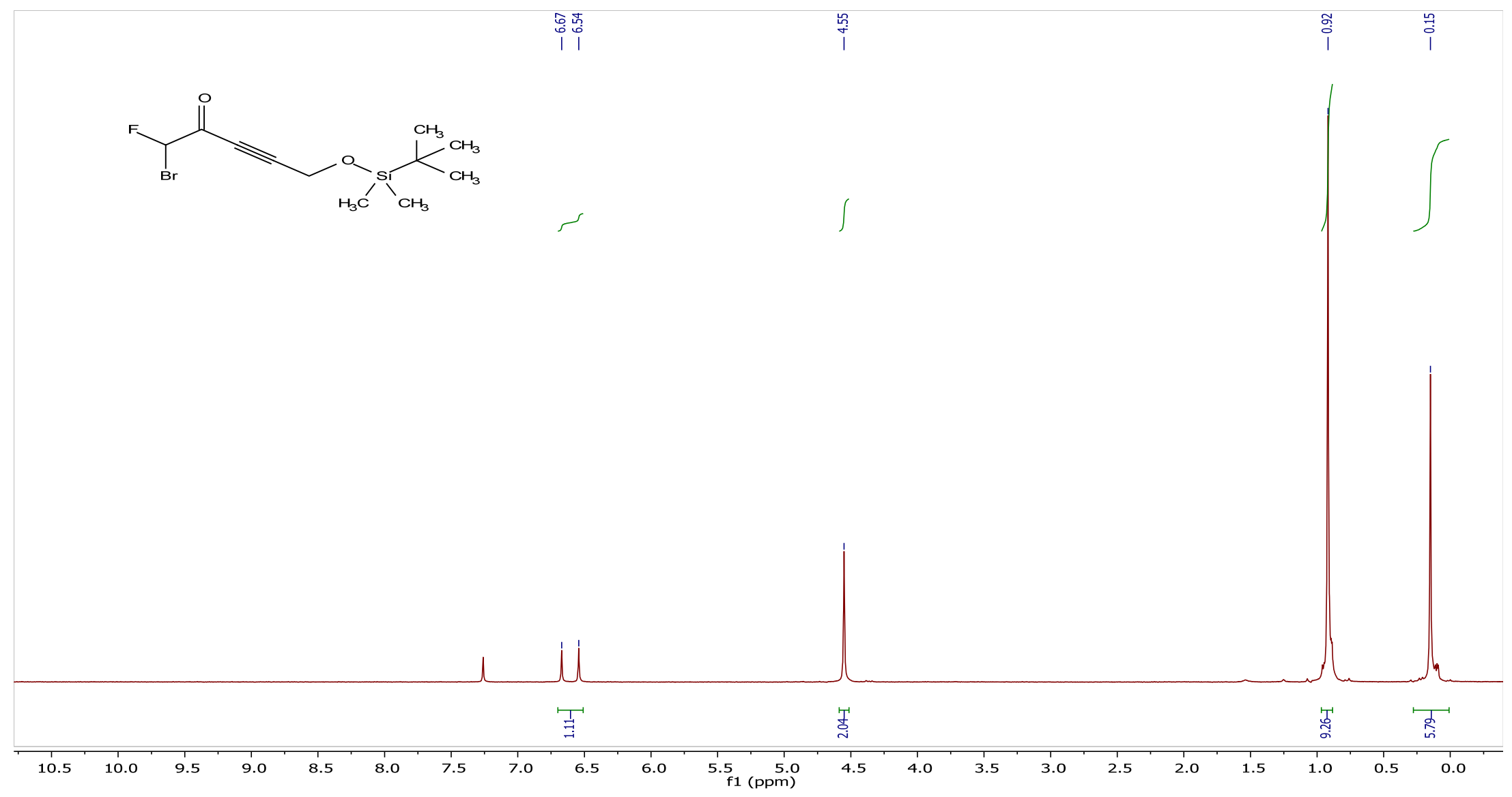


${ }^{13} \mathrm{C}\left\{{ }^{1} \mathrm{H}\right\}$ NMR spectrum of the compound $\mathbf{1 3 e}$.

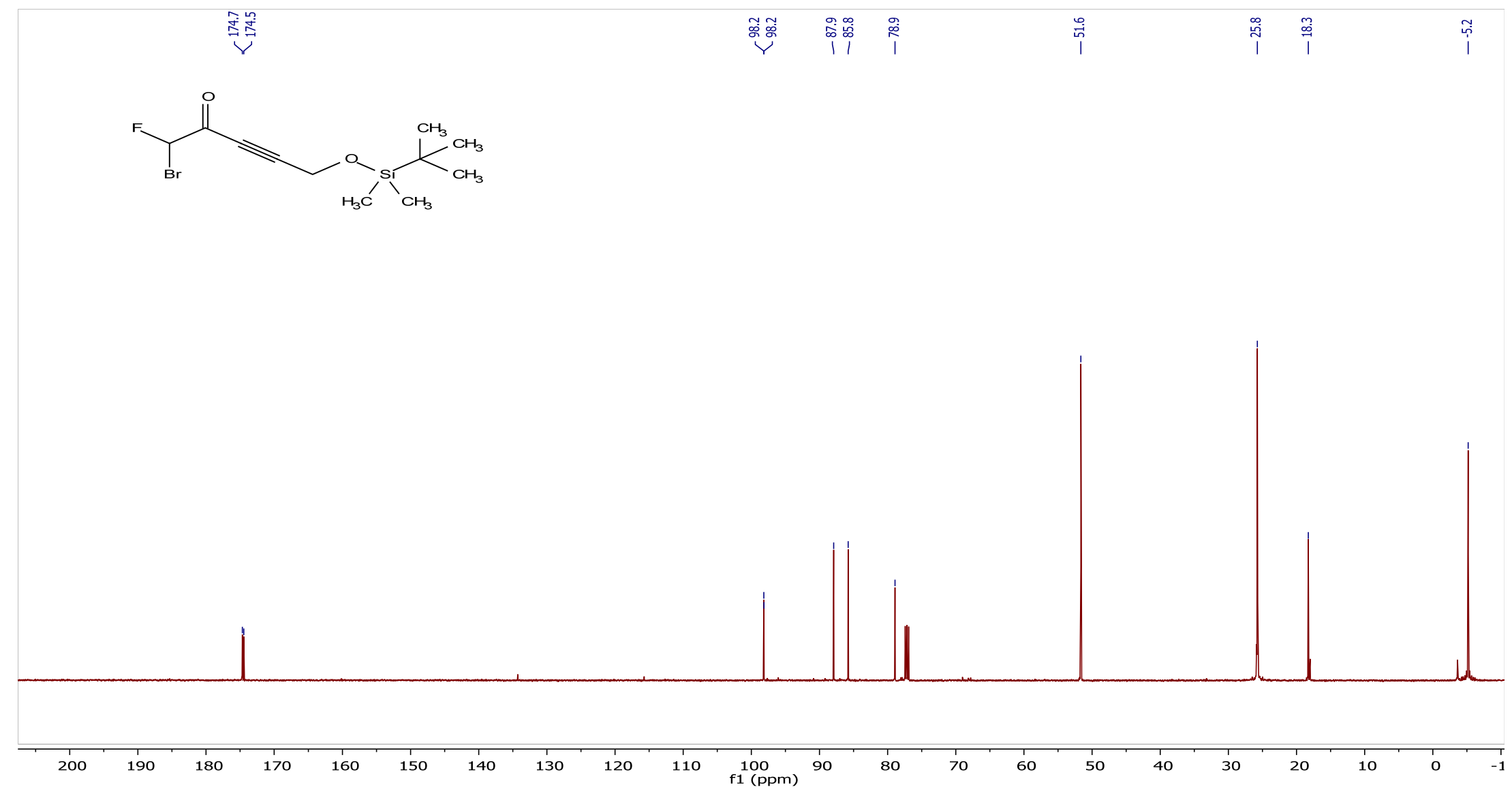


${ }^{19} \mathrm{~F}\left\{{ }^{1} \mathrm{H}\right\}$ NMR spectrum of the compound $13 \mathbf{e}$.

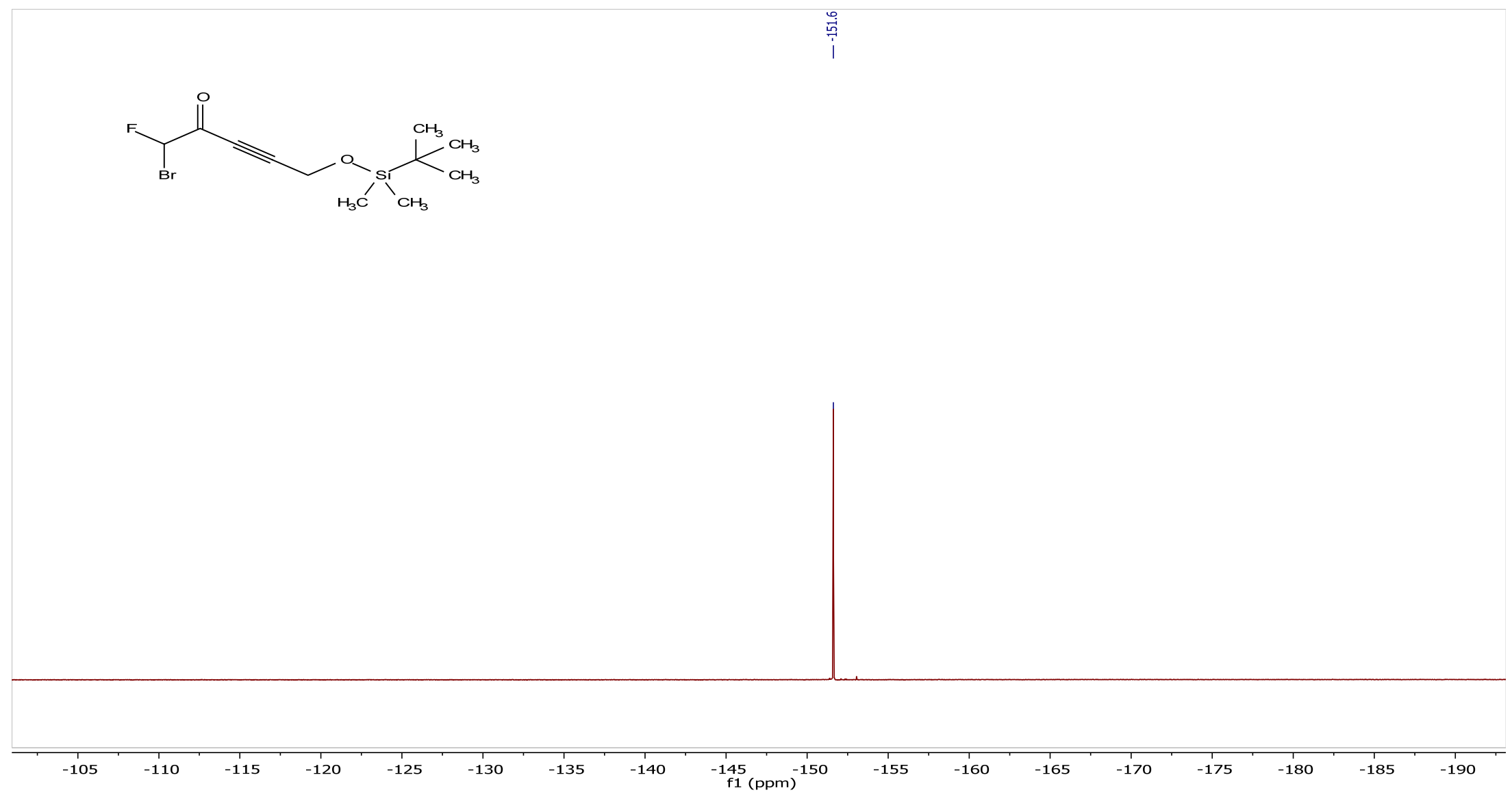


${ }^{1} \mathrm{H}$ NMR spectrum of the compound $13 \mathbf{1 3 f}$

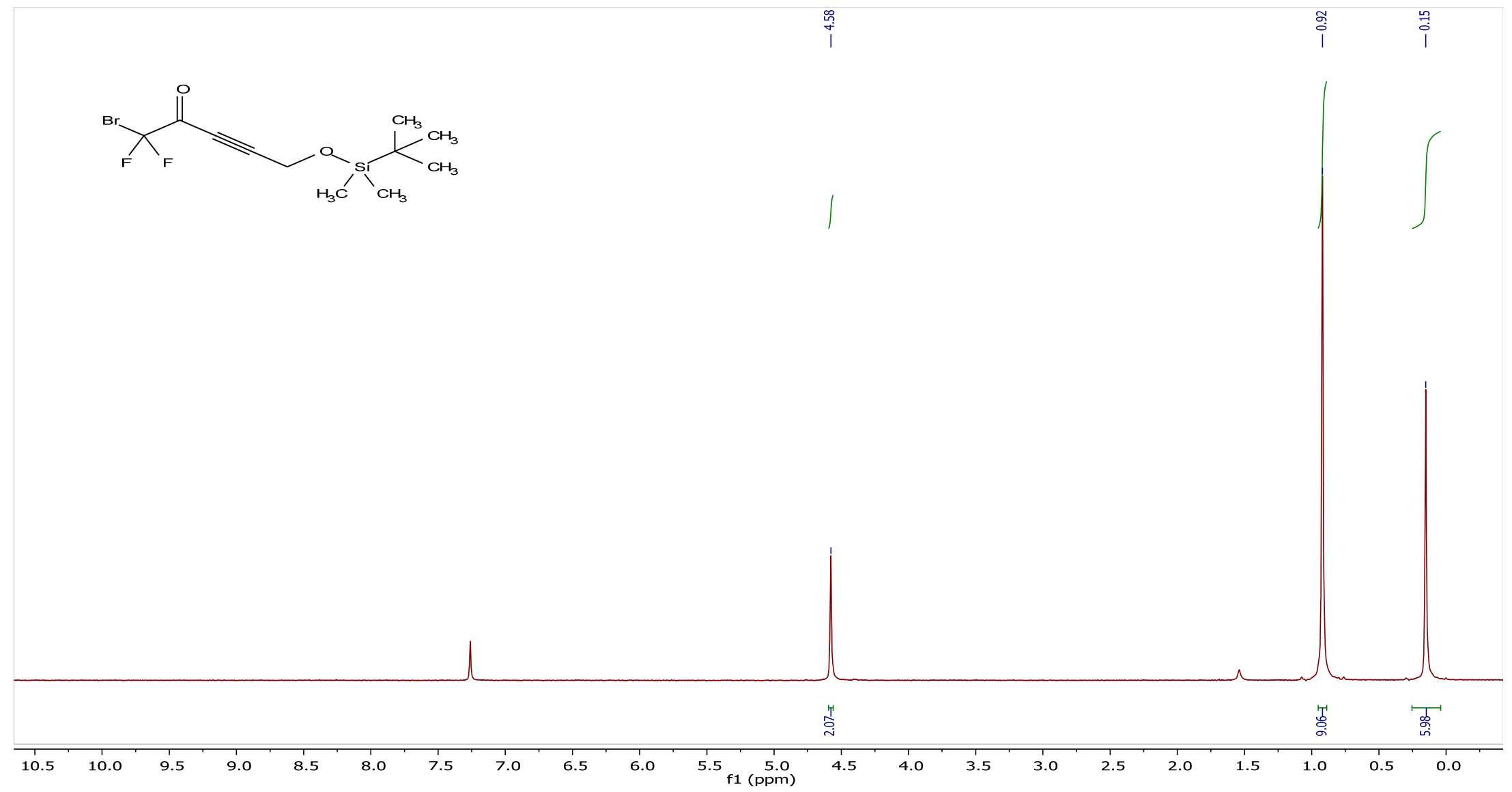


${ }^{13} \mathrm{C}\left\{{ }^{1} \mathrm{H}\right\}$ NMR spectrum of the compound $\mathbf{1 3 f}$.

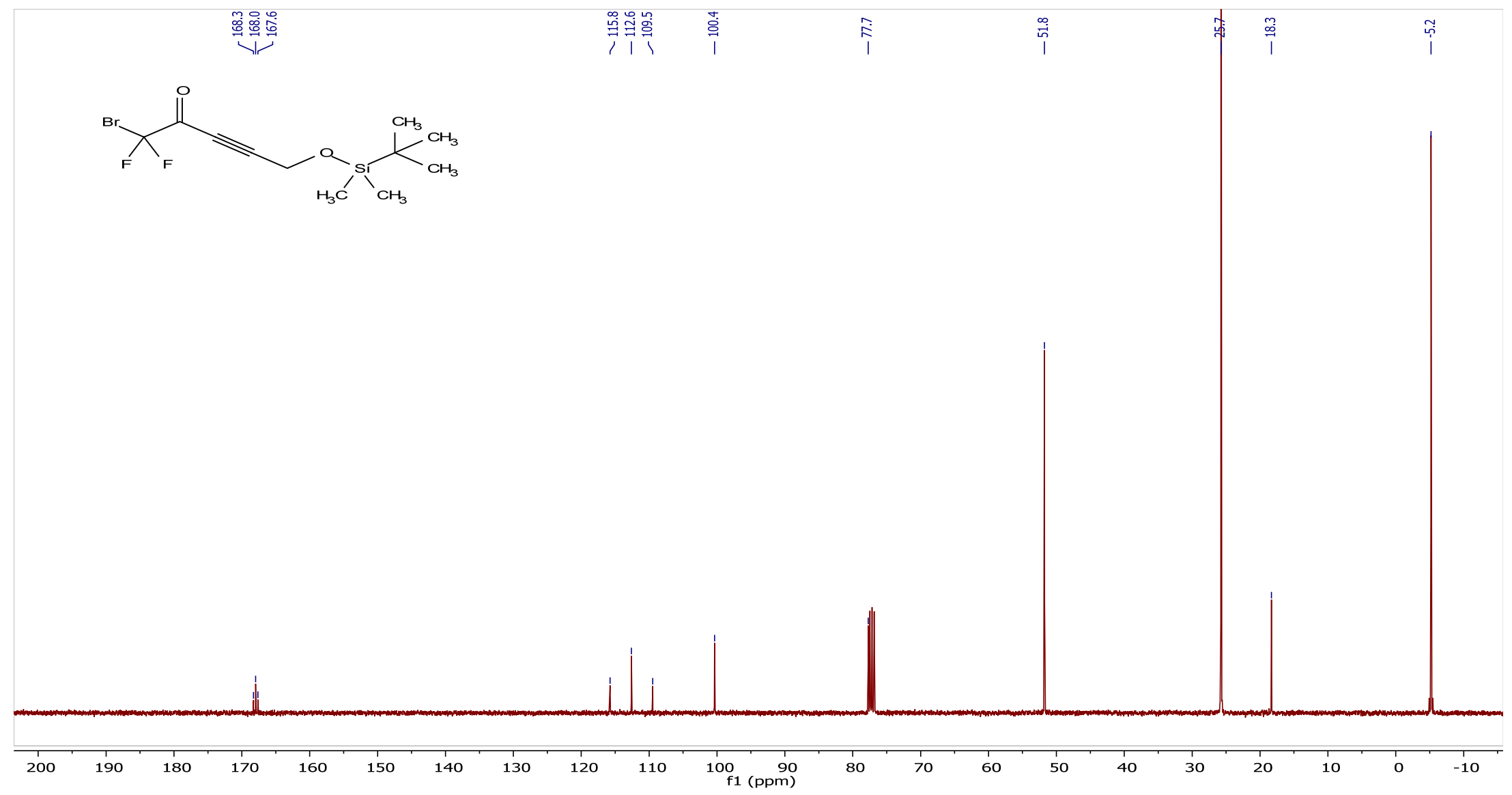


${ }^{19} \mathrm{~F}\left\{{ }^{1} \mathrm{H}\right\}$ NMR spectrum of the compound $\mathbf{1 3 f}$.

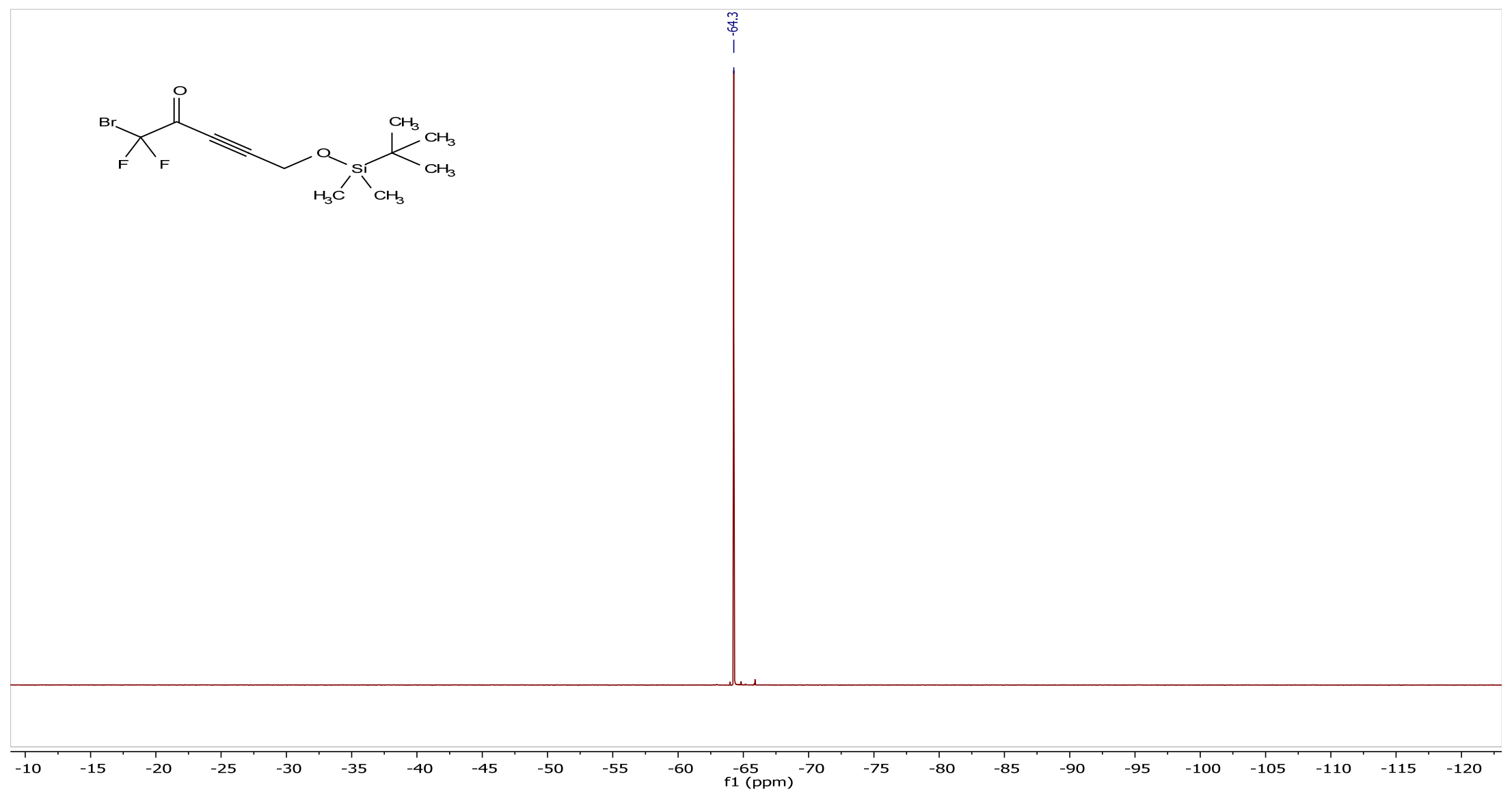


${ }^{1} \mathrm{H}$ NMR spectrum of the compound $\mathbf{1 3 g}$.

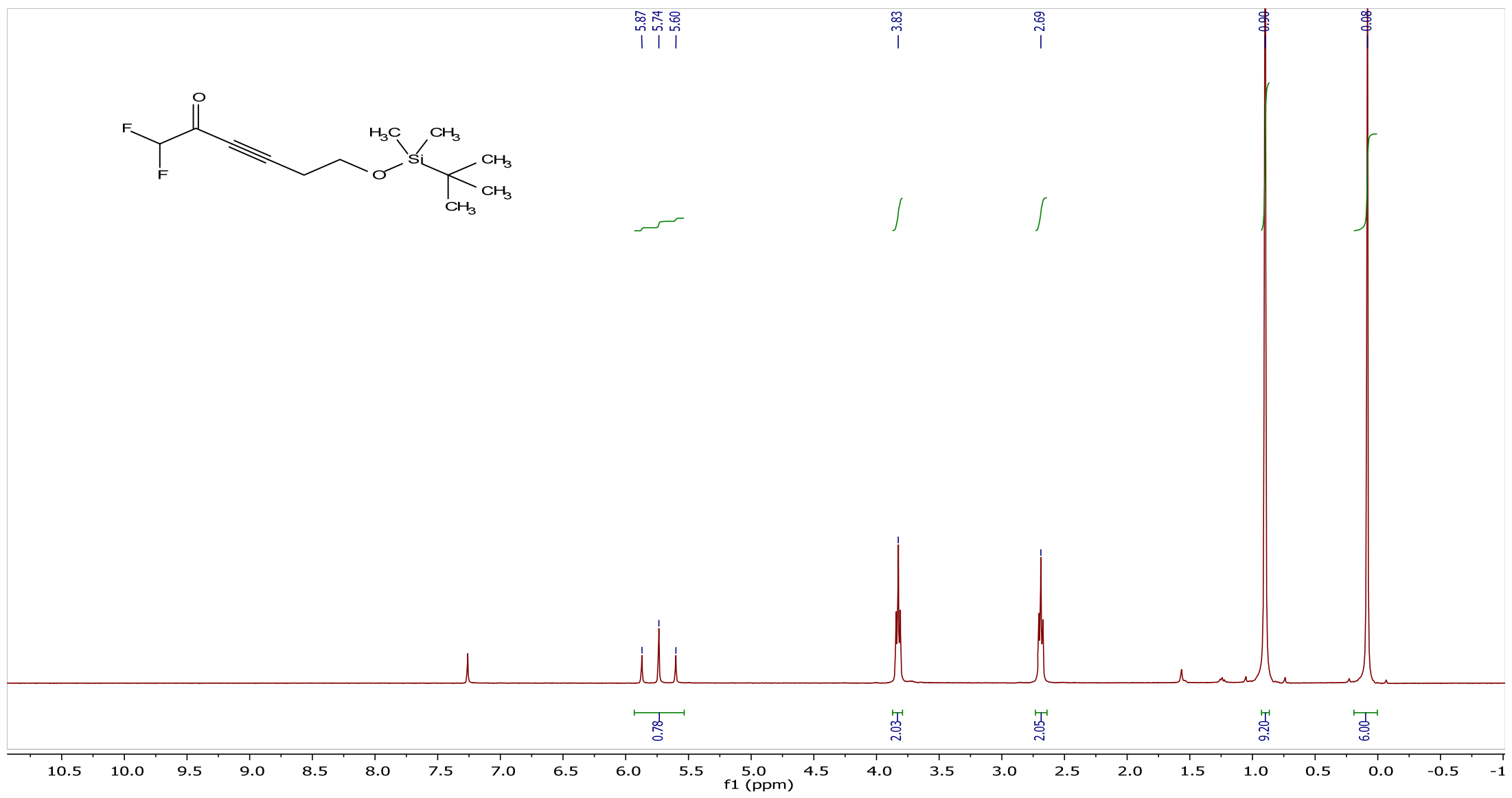

S47 
${ }^{13} \mathrm{C}\left\{{ }^{1} \mathrm{H}\right\}$ NMR spectrum of the compound $\mathbf{1 3 g}$.

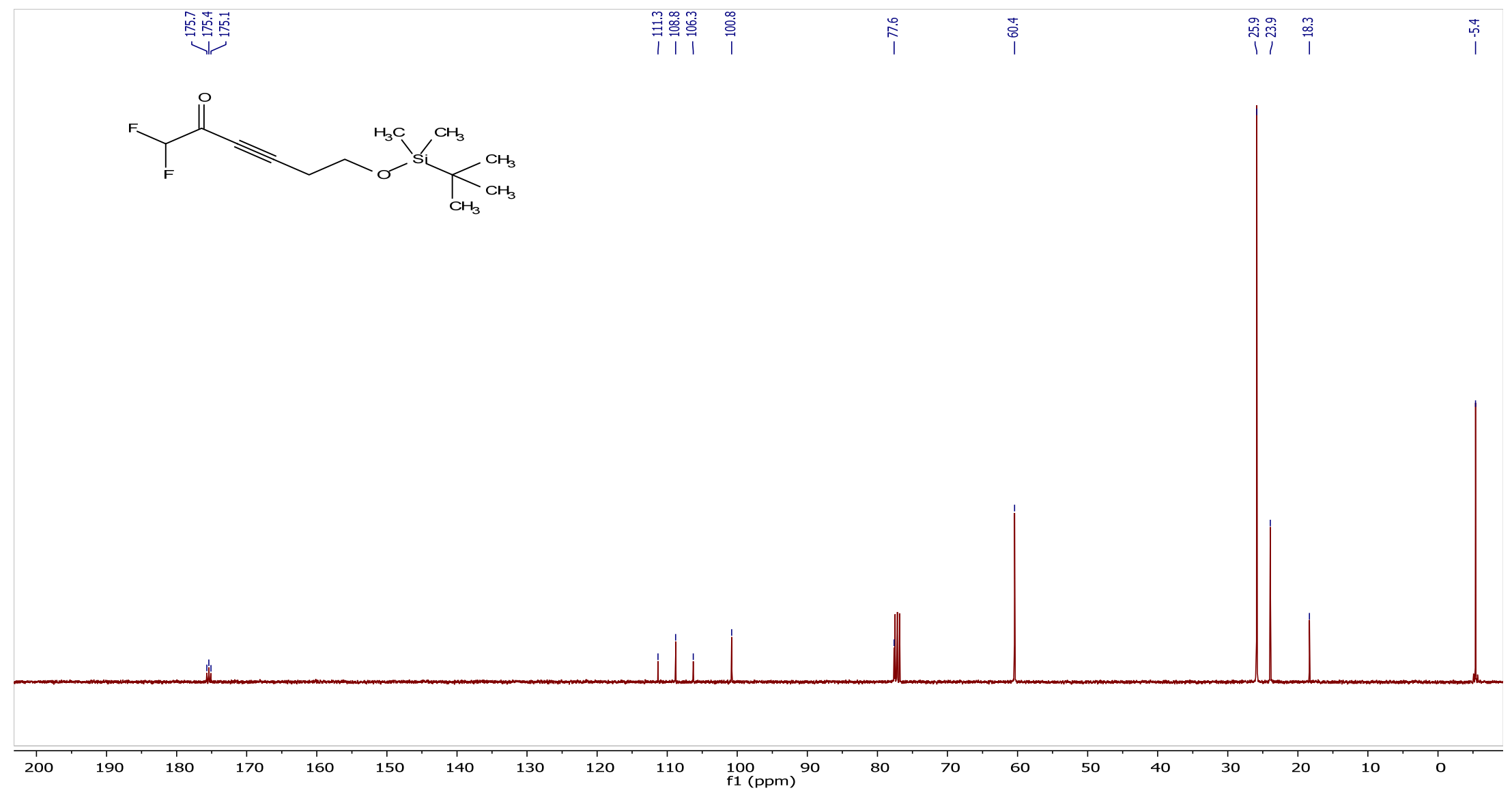


${ }^{19} \mathrm{~F}\left\{{ }^{1} \mathrm{H}\right\}$ NMR spectrum of the compound 13g.

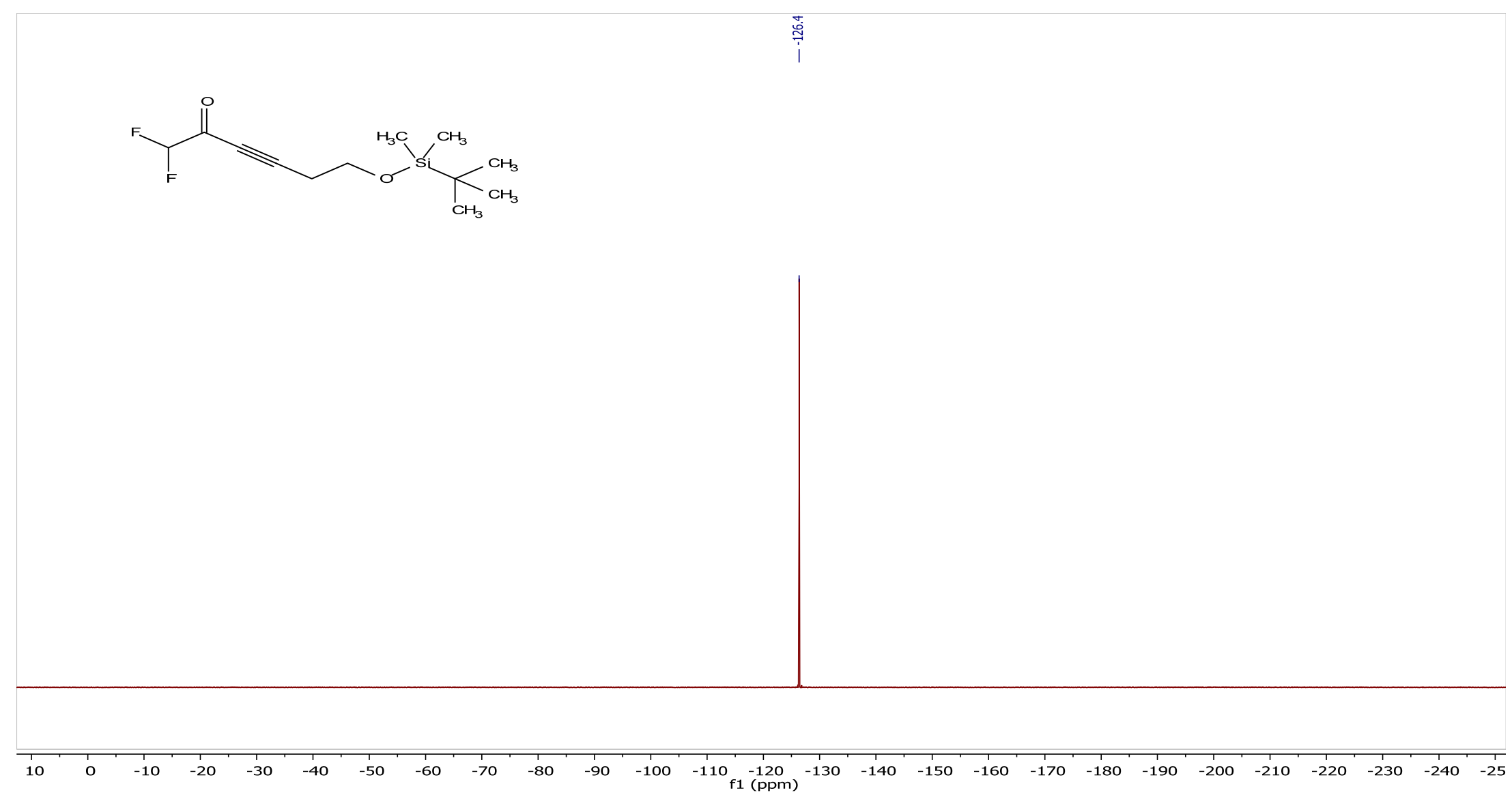


${ }^{1} \mathrm{H}$ NMR spectrum of the compound $\mathbf{1 3 h}$.

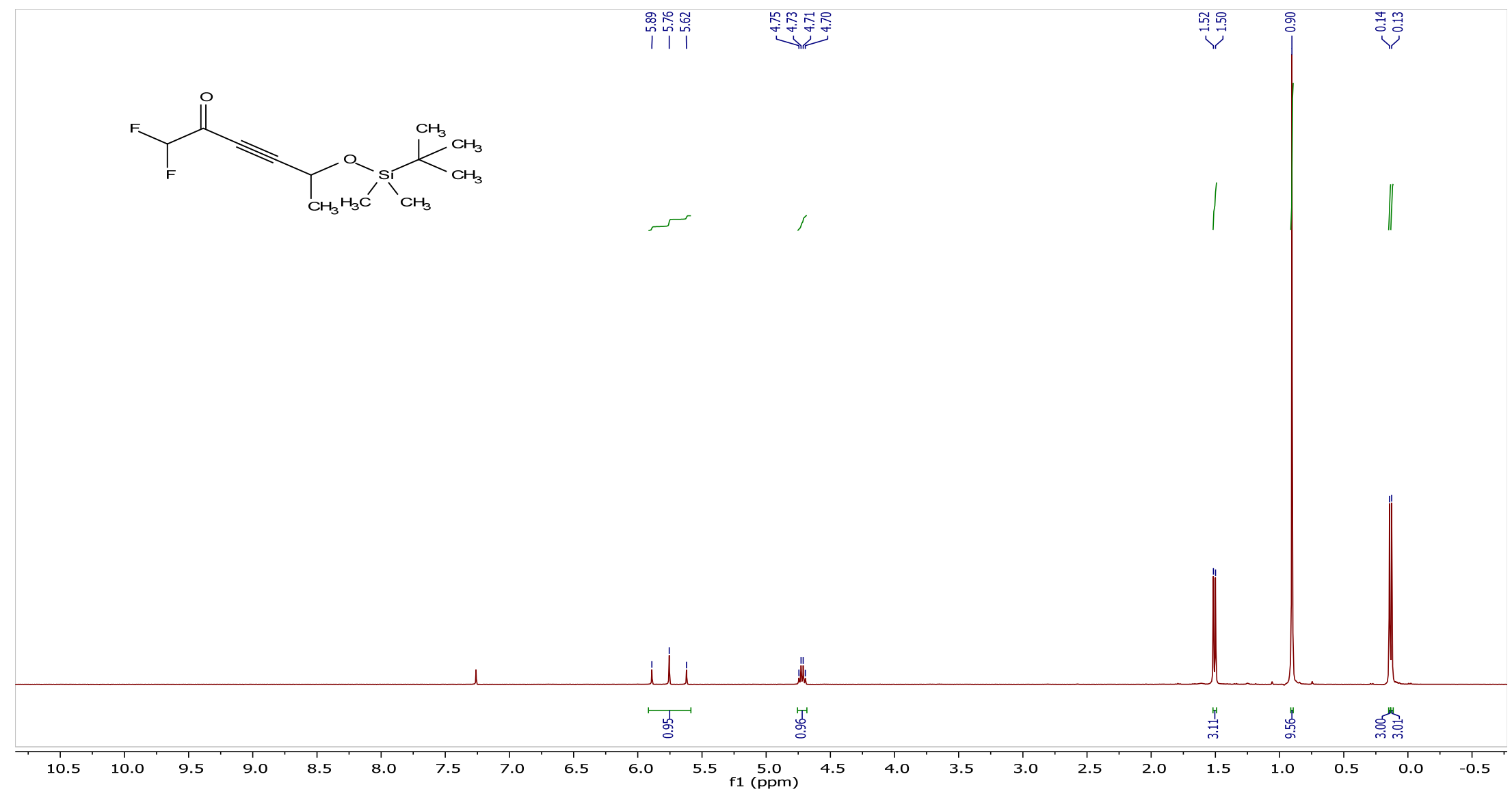


${ }^{13} \mathrm{C}\left\{{ }^{1} \mathrm{H}\right\}$ NMR spectrum of the compound $\mathbf{1 3 h}$.

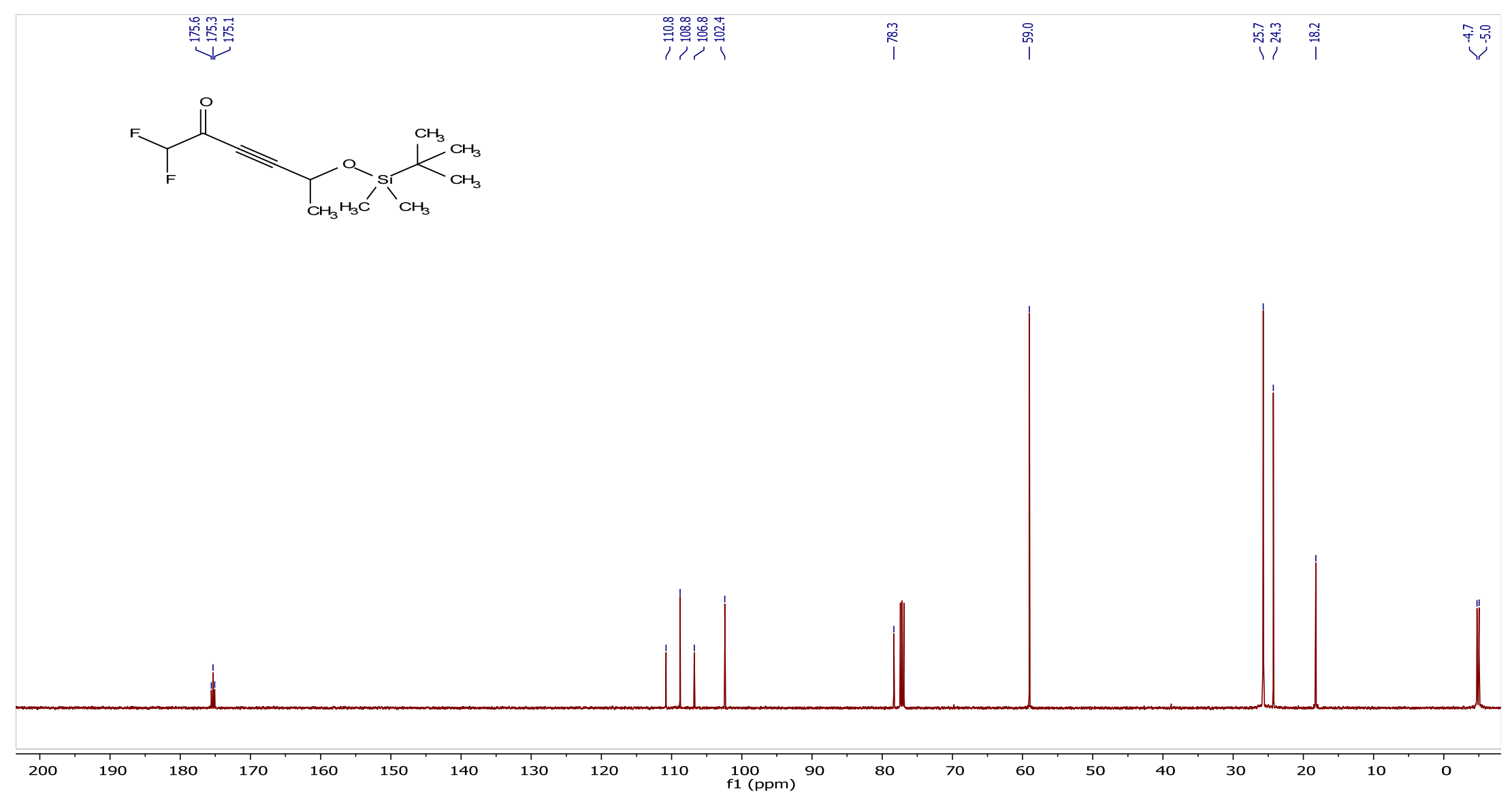


${ }^{19} \mathrm{~F}\left\{{ }^{1} \mathrm{H}\right\}$ NMR spectrum of the compound $\mathbf{1 3 h}$.

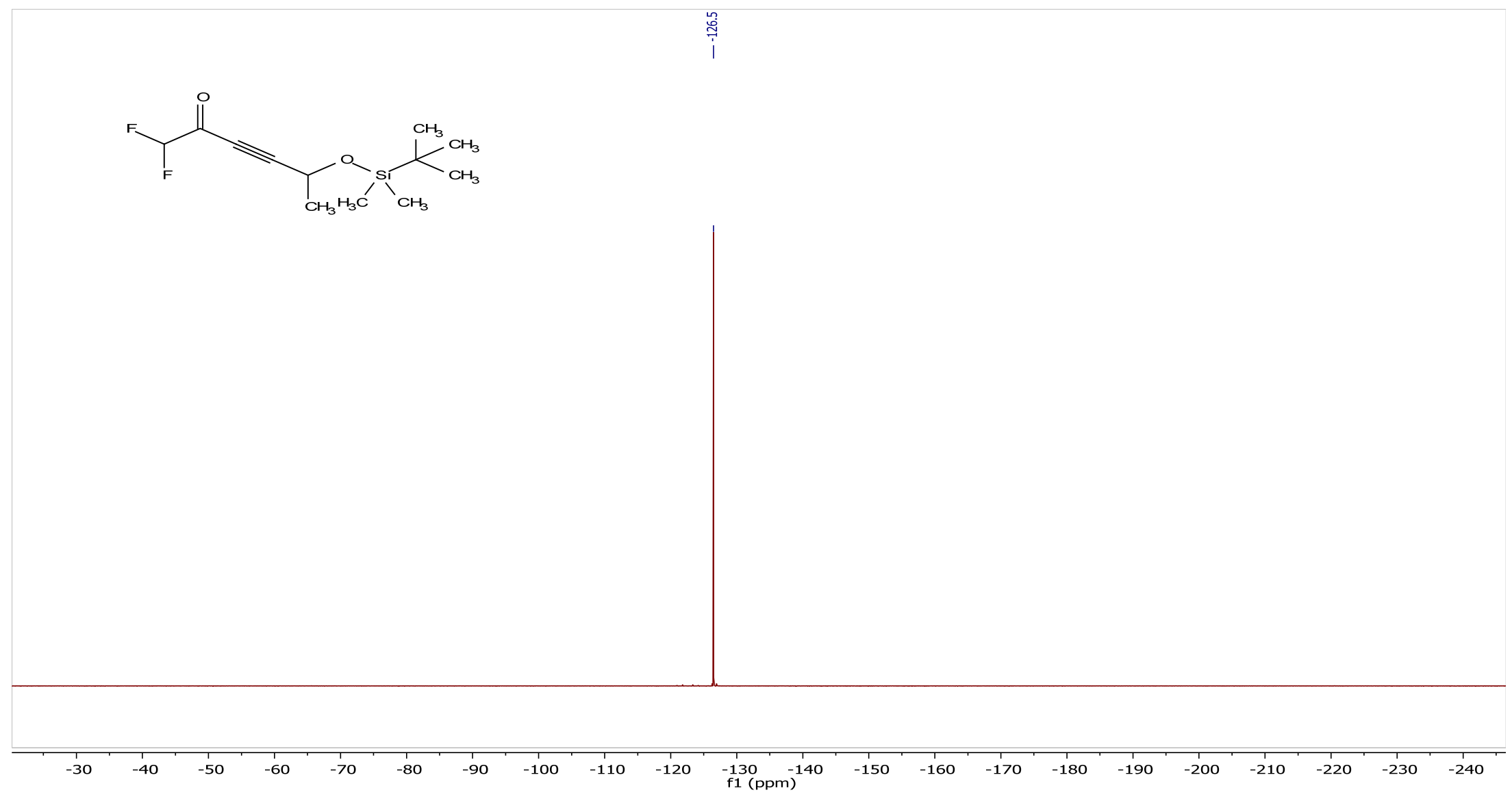


${ }^{1} \mathrm{H}$ NMR spectrum of the compound $\mathbf{1 6 a}$.

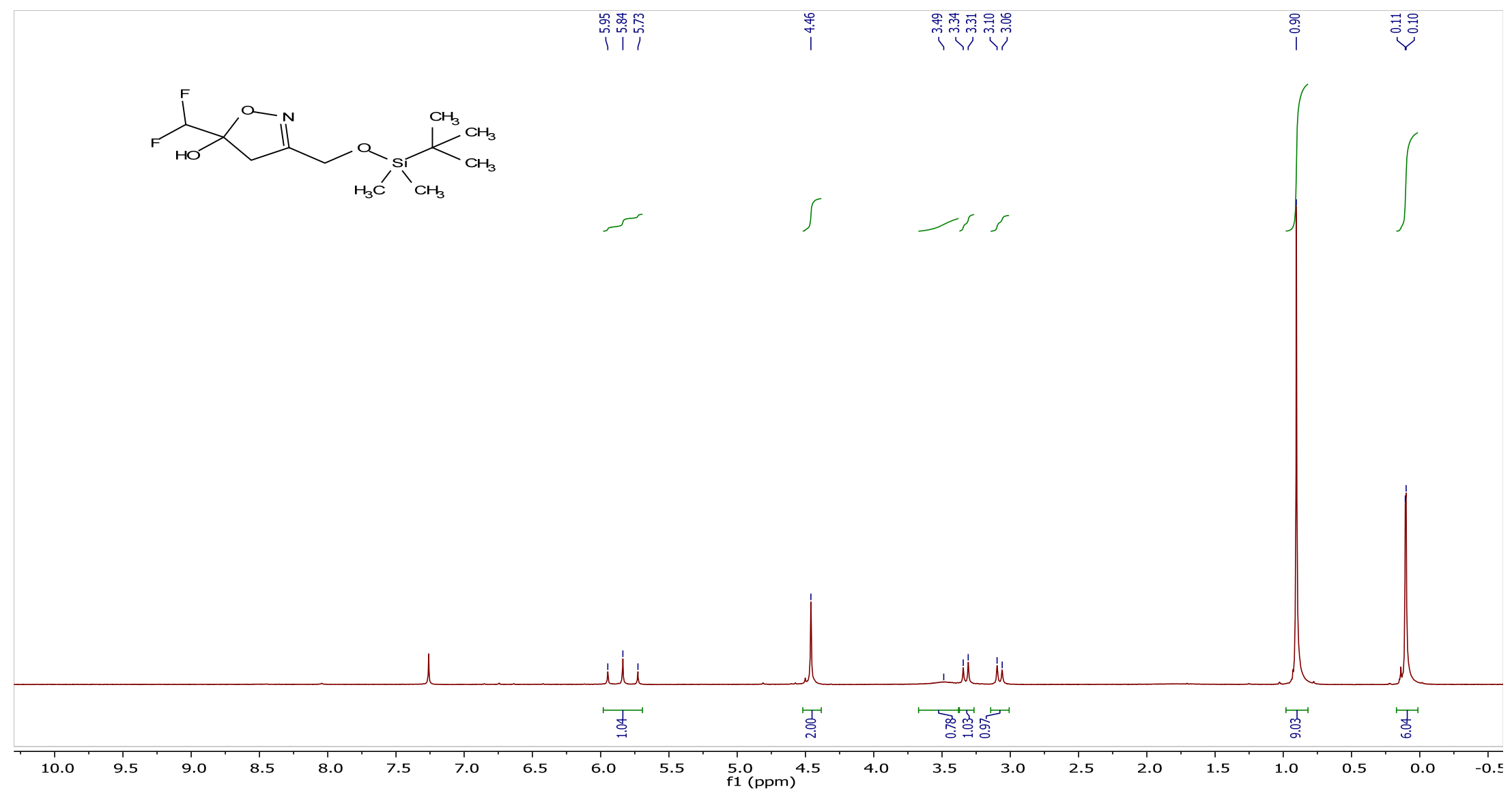


${ }^{13} \mathrm{C}\left\{{ }^{1} \mathrm{H}\right\}$ NMR spectrum of the compound 16a.

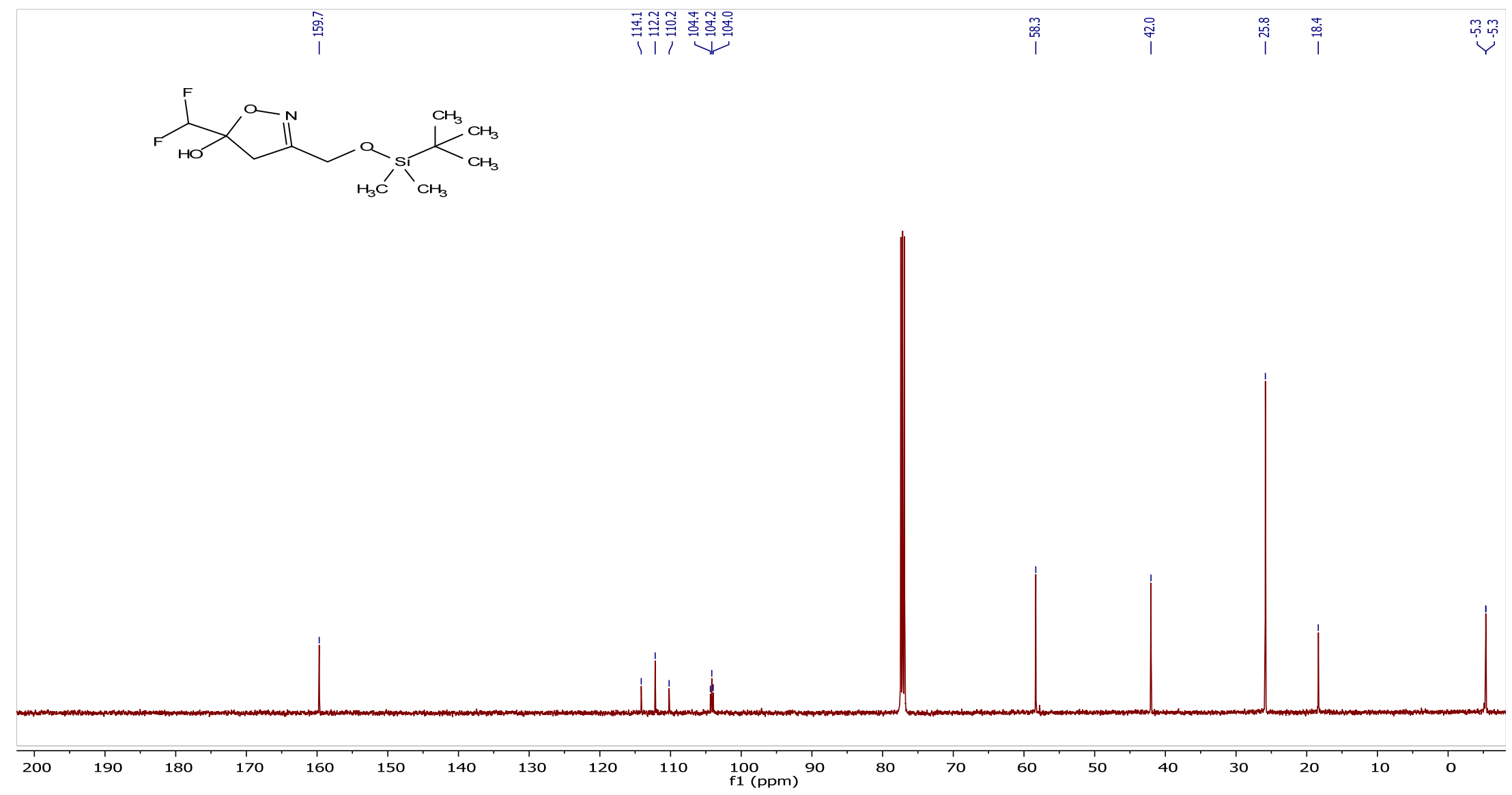


${ }^{19}$ F NMR spectrum of the compound $\mathbf{1 6 a}$.

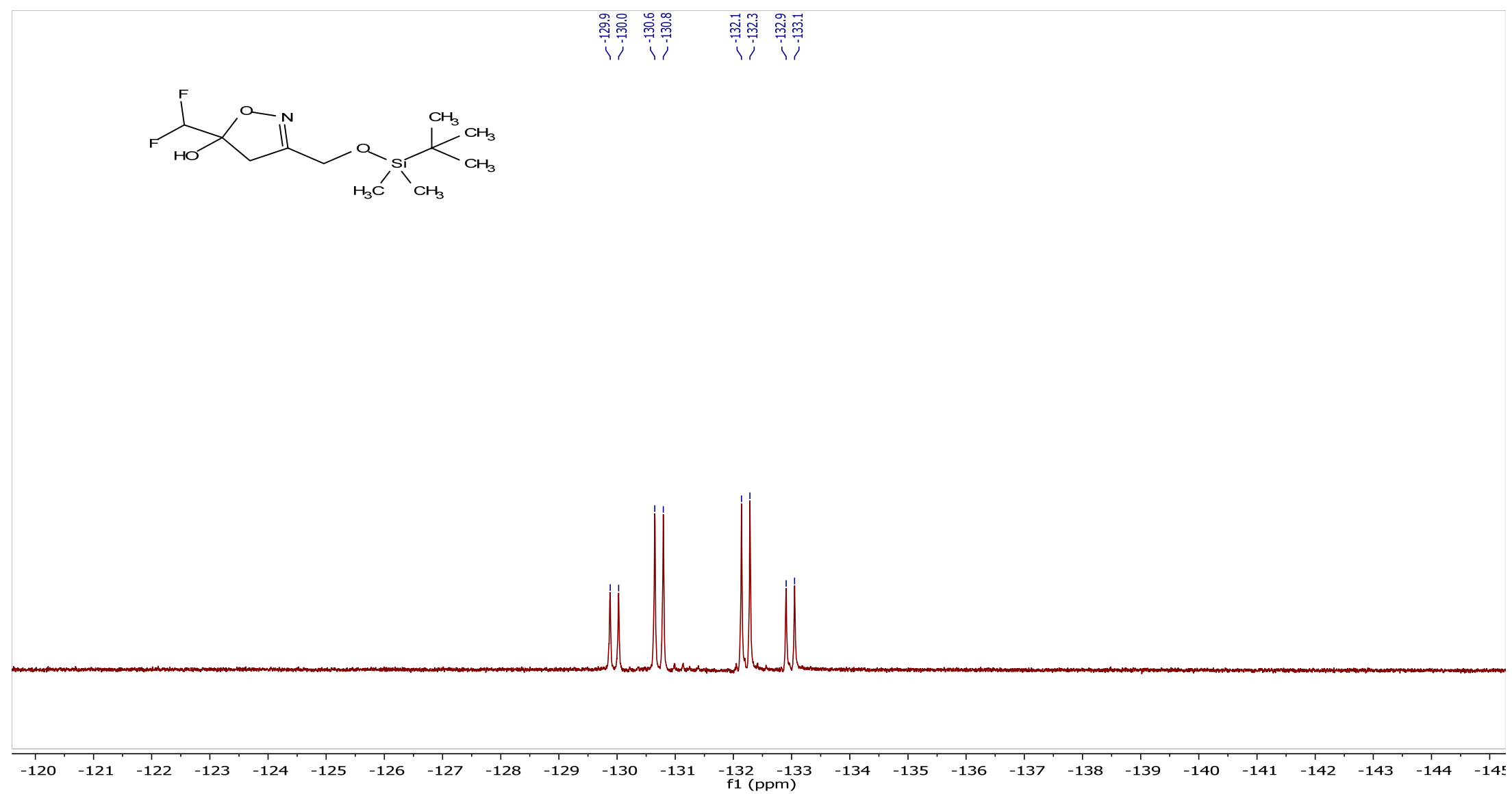


${ }^{1} \mathrm{H}$ NMR spectrum of the compound $\mathbf{1 6 b}$.

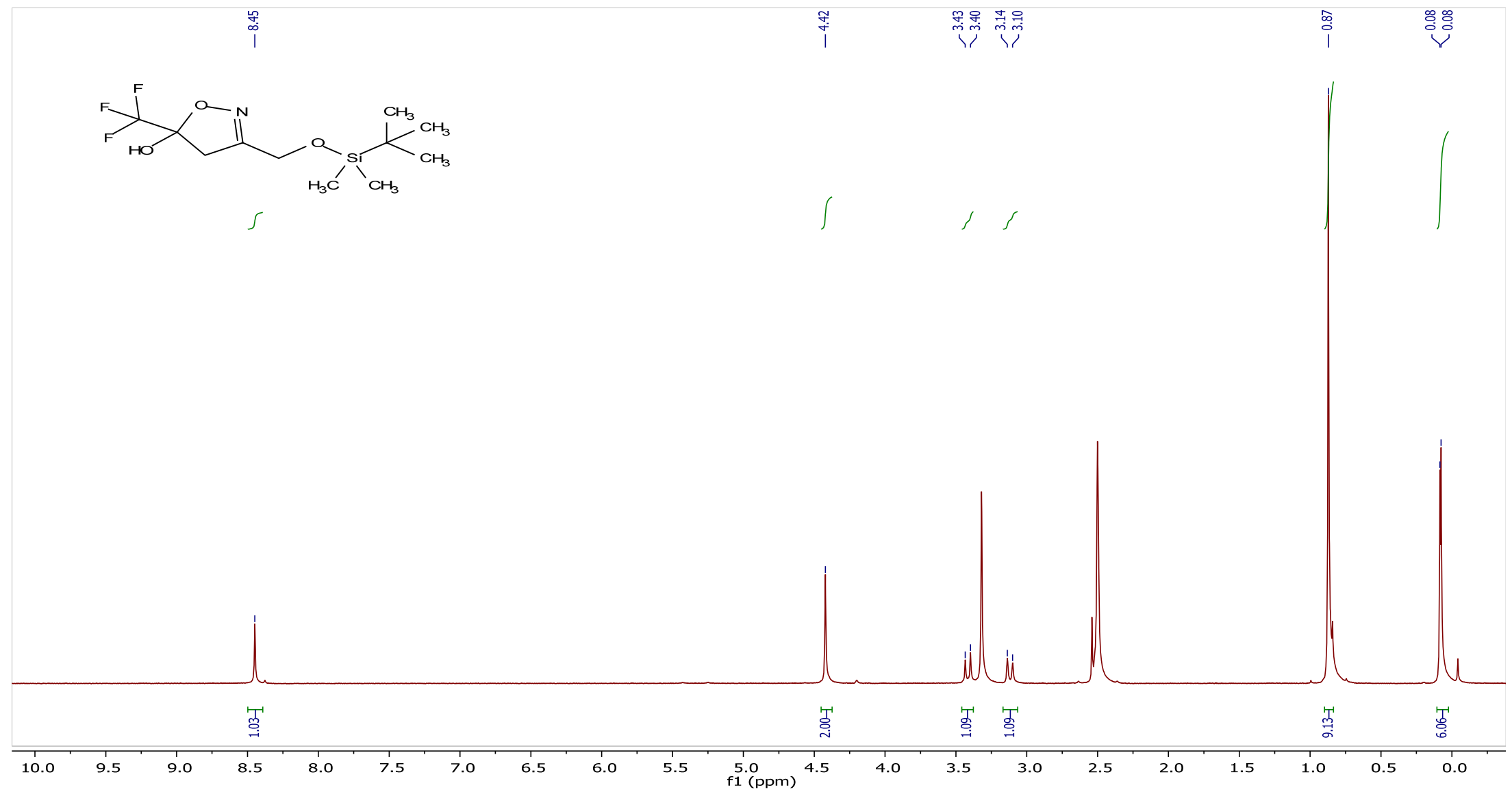


${ }^{13} \mathrm{C}\left\{{ }^{1} \mathrm{H}\right\}$ NMR spectrum of the compound $\mathbf{1 6 b}$.

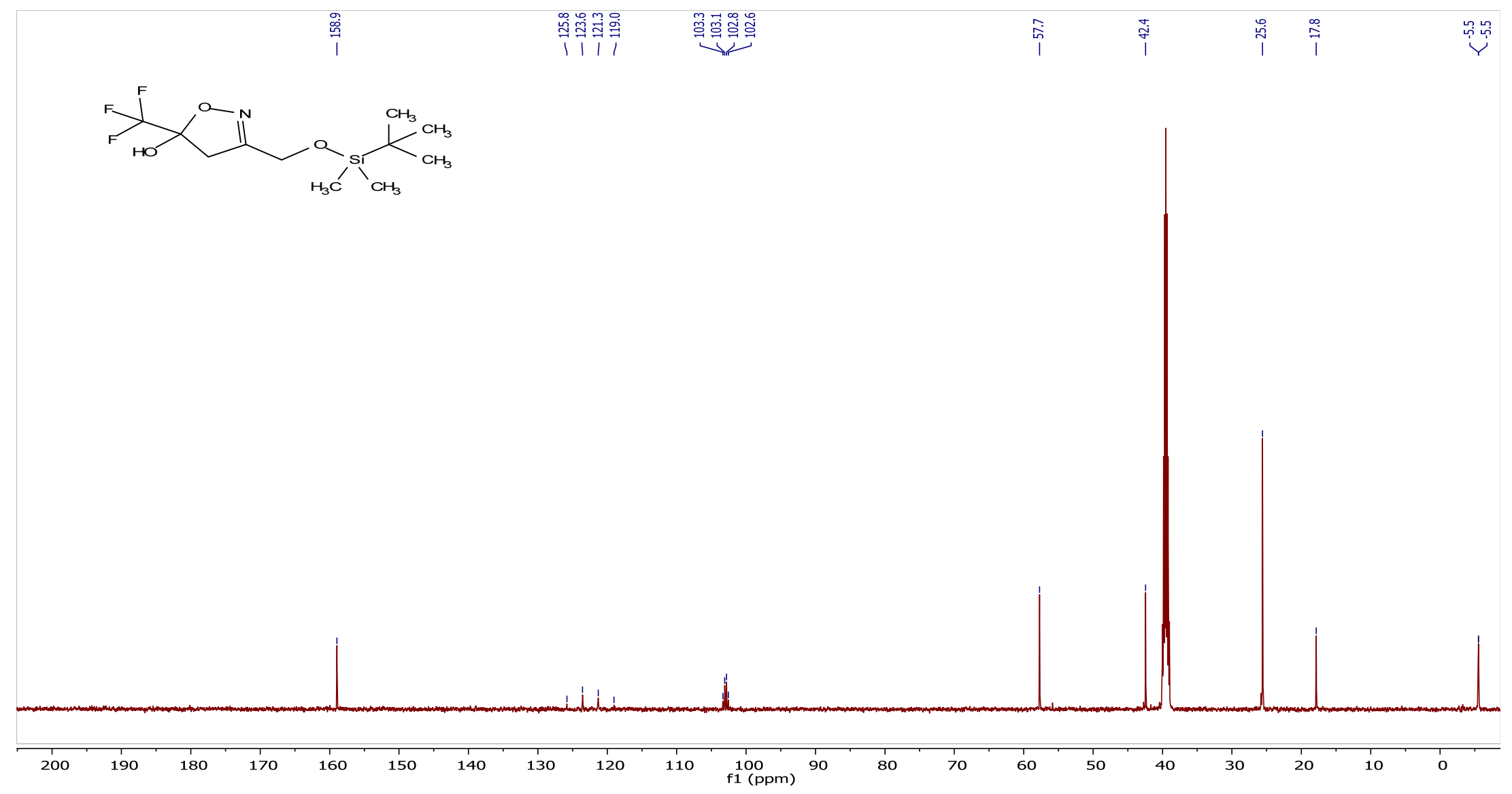


${ }^{19} \mathrm{~F}\left\{{ }^{1} \mathrm{H}\right\}$ NMR spectrum of the compound $\mathbf{1 6 b}$.

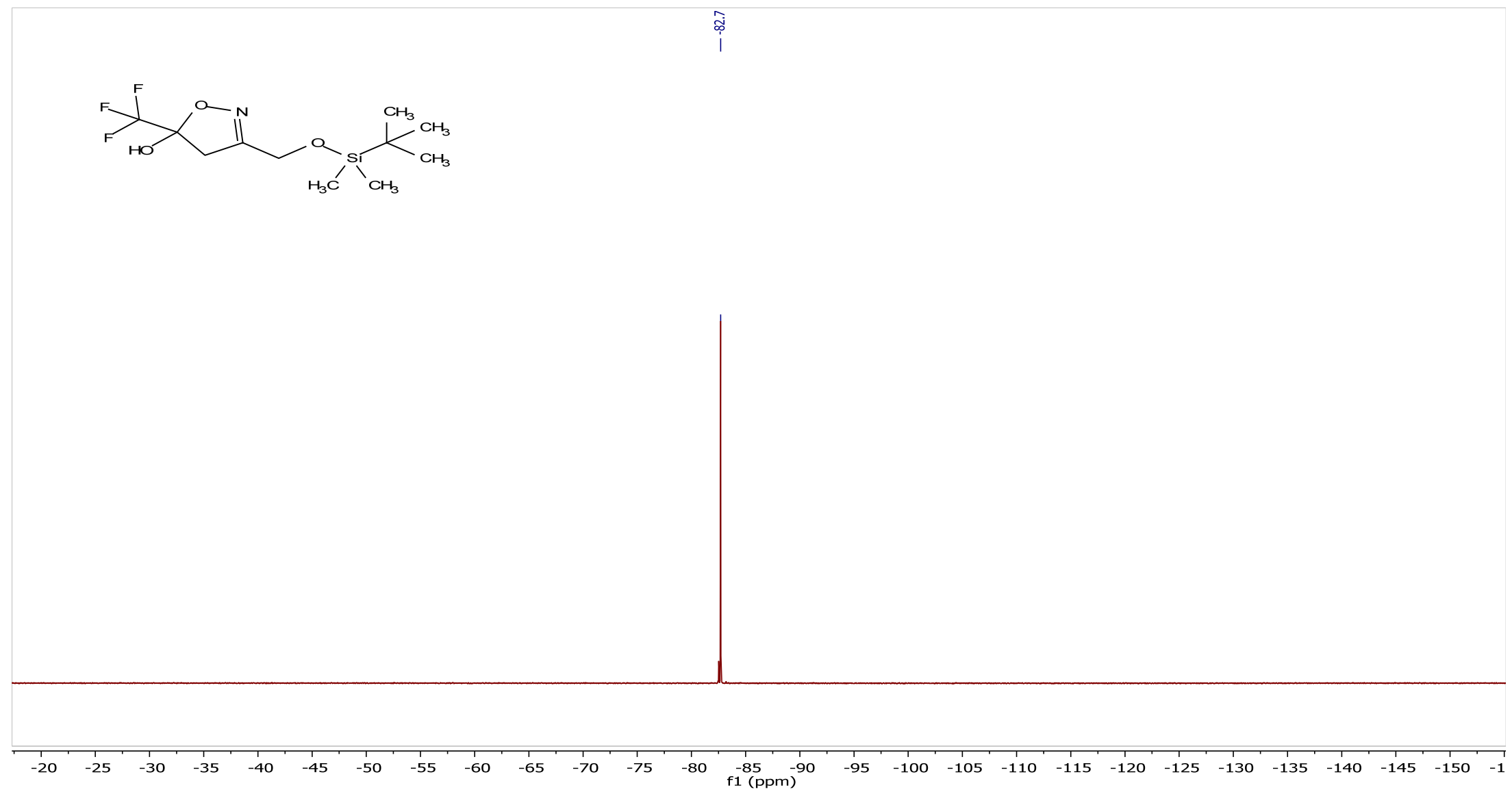


${ }^{1} \mathrm{H}$ NMR spectrum of the compound 16c.

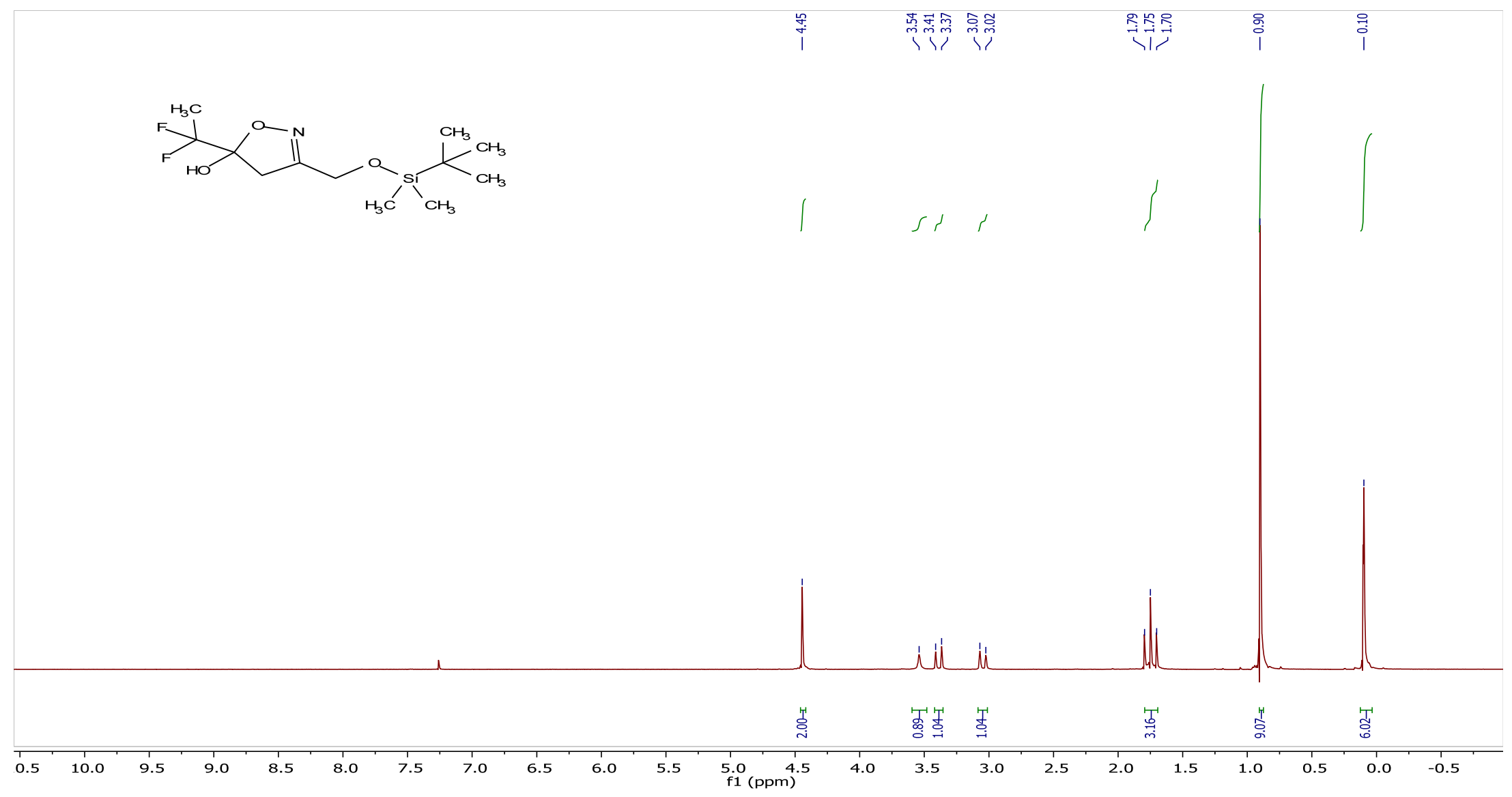


${ }^{13} \mathrm{C}\left\{{ }^{1} \mathrm{H}\right\}$ NMR spectrum of the compound 16c.

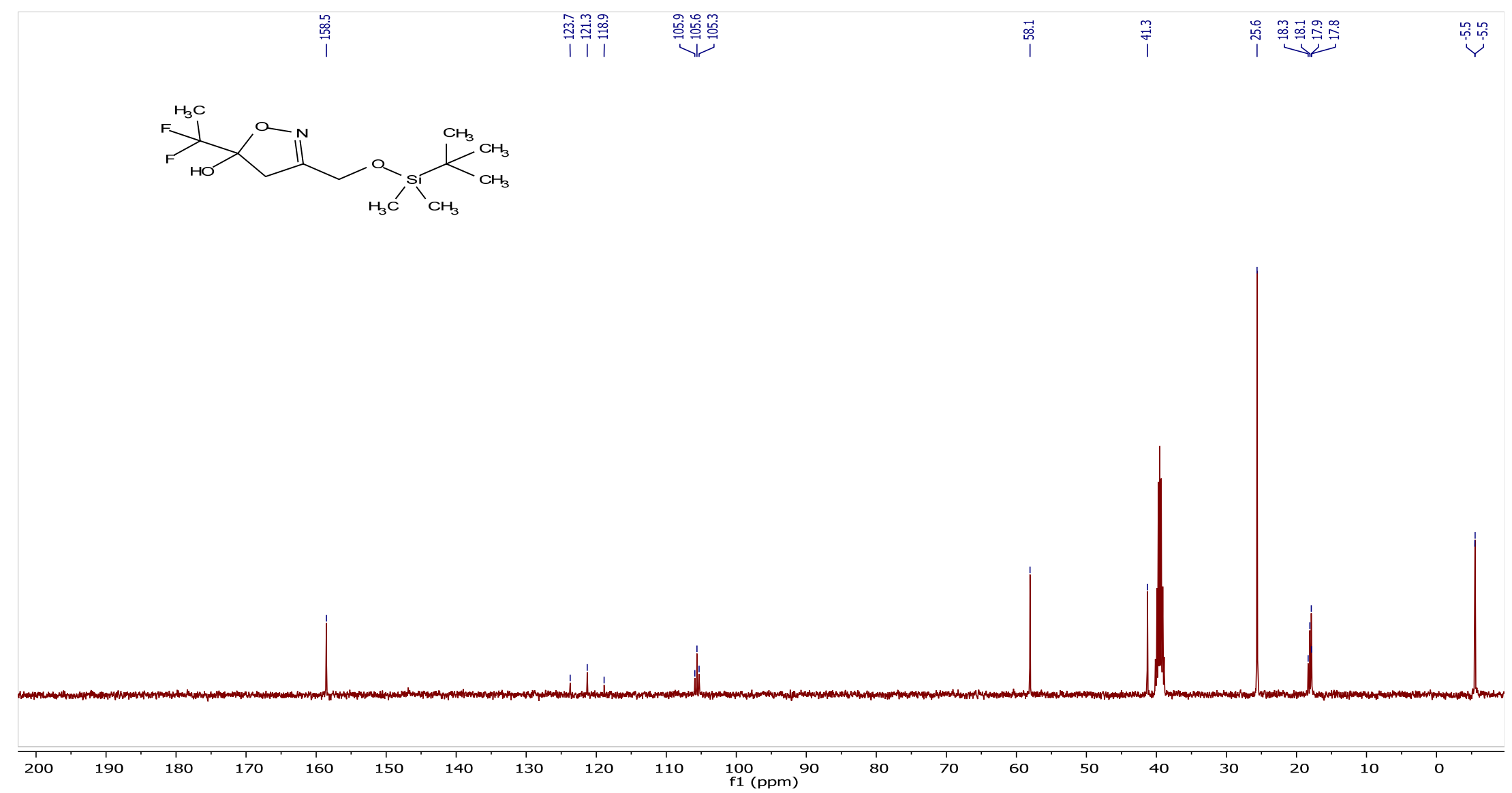


${ }^{19} \mathrm{~F}\left\{{ }^{1} \mathrm{H}\right\}$ NMR spectrum of the compound 16c.

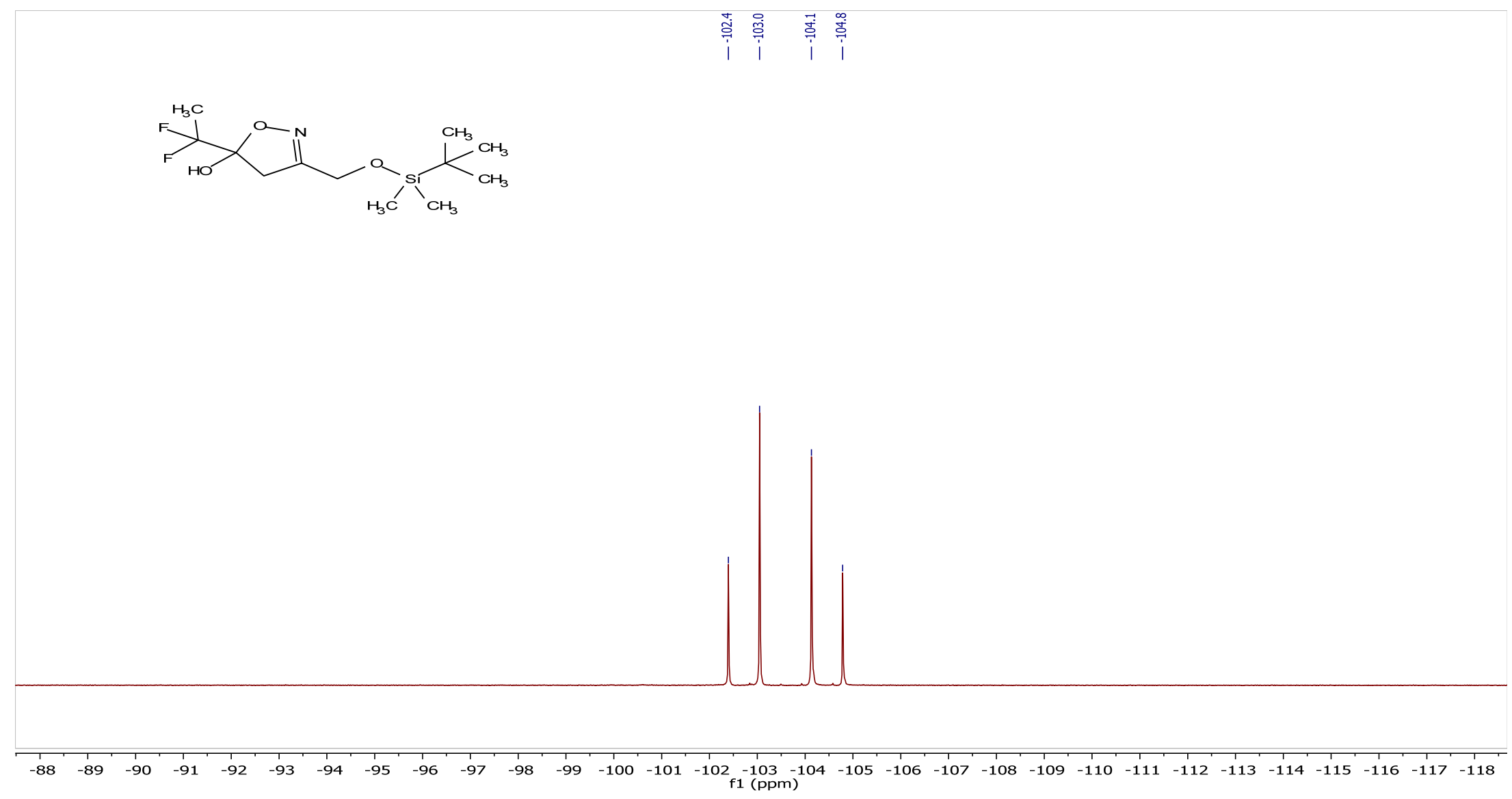


${ }^{1} \mathrm{H}$ NMR spectrum of the compound $\mathbf{1 6 d}$.

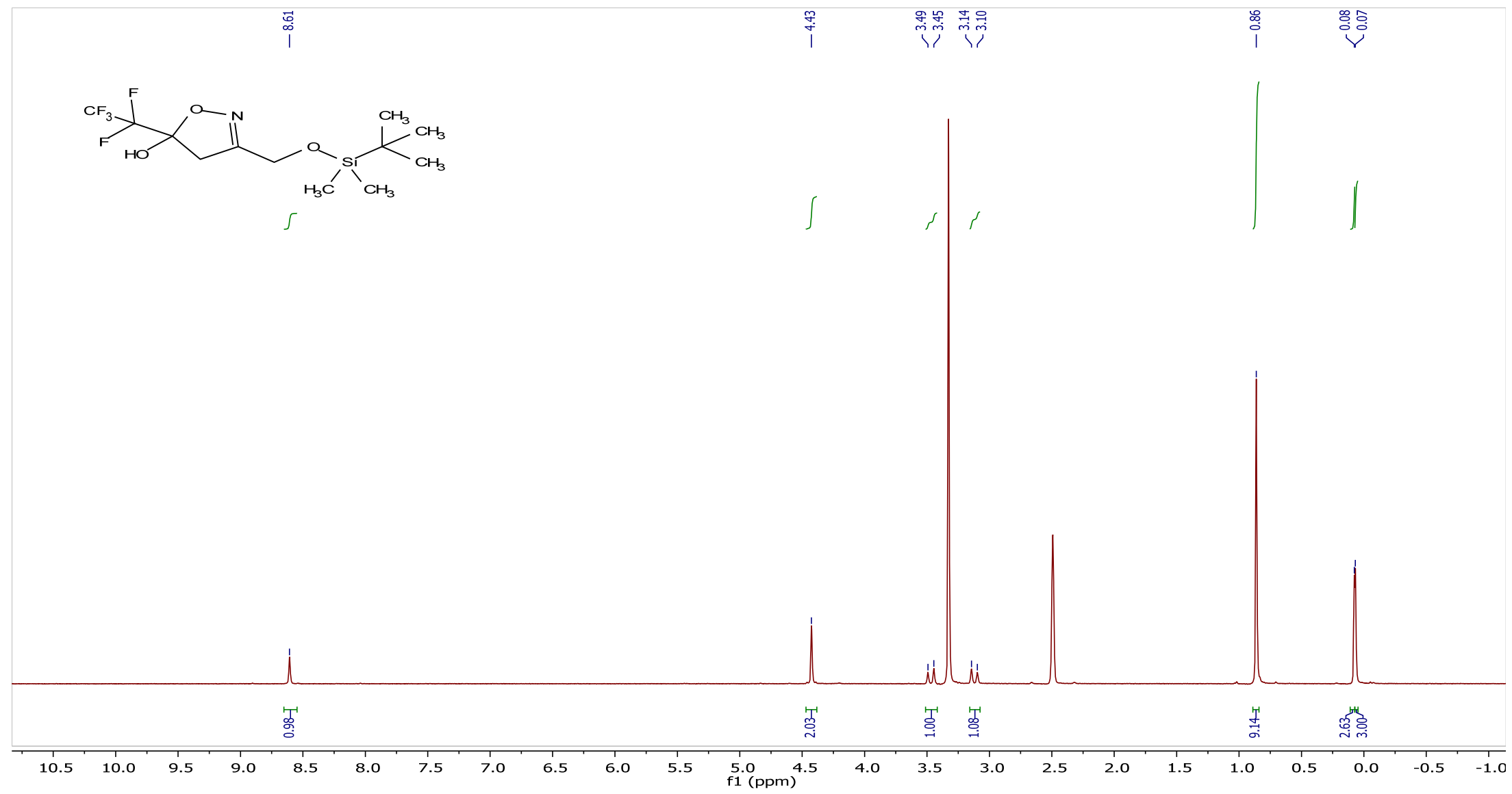


${ }^{13} \mathrm{C}\left\{{ }^{1} \mathrm{H}\right\}$ NMR spectrum of the compound 16d.

遮
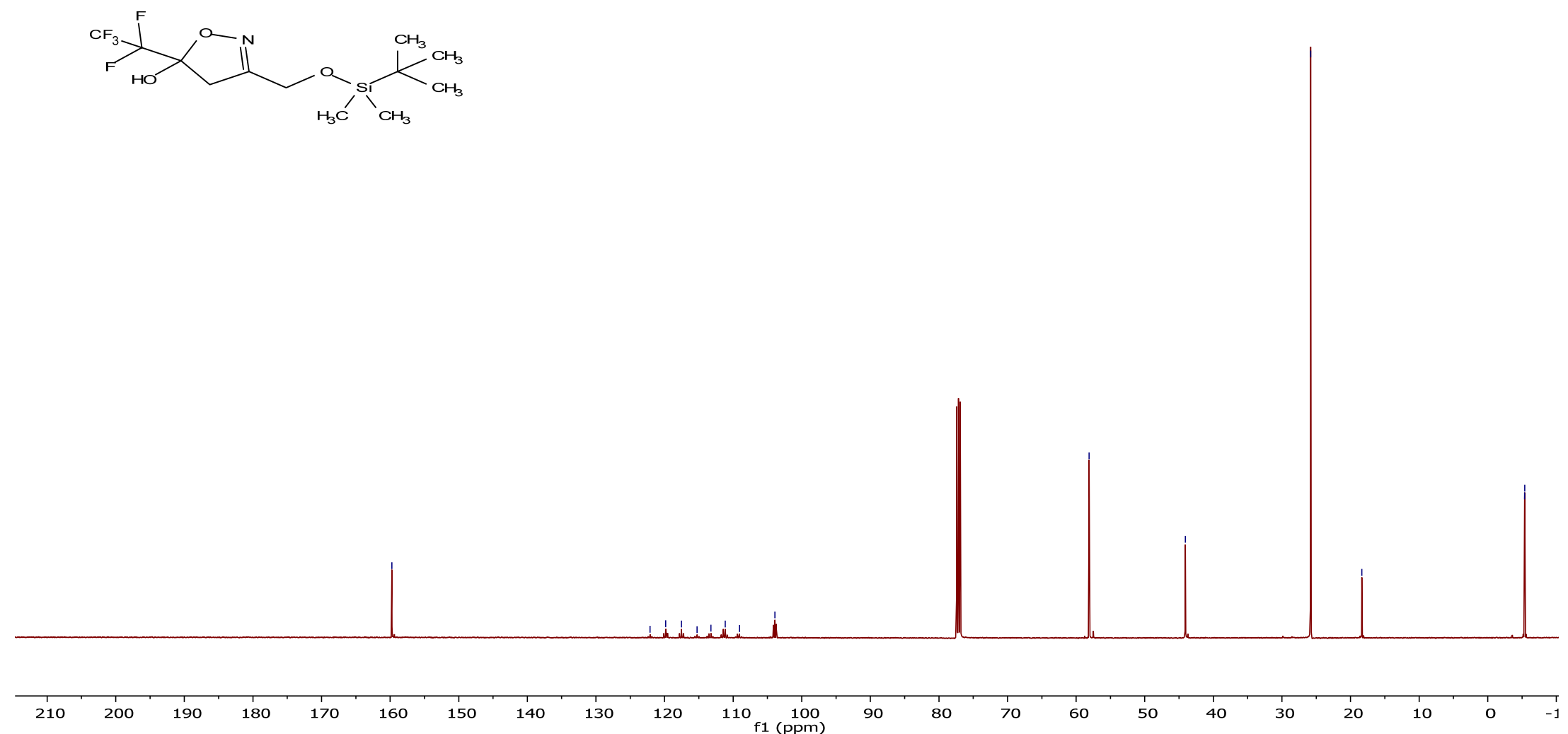
${ }^{19} \mathrm{~F}\left\{{ }^{1} \mathrm{H}\right\}$ NMR spectrum of the compound $\mathbf{1 6 d}$.

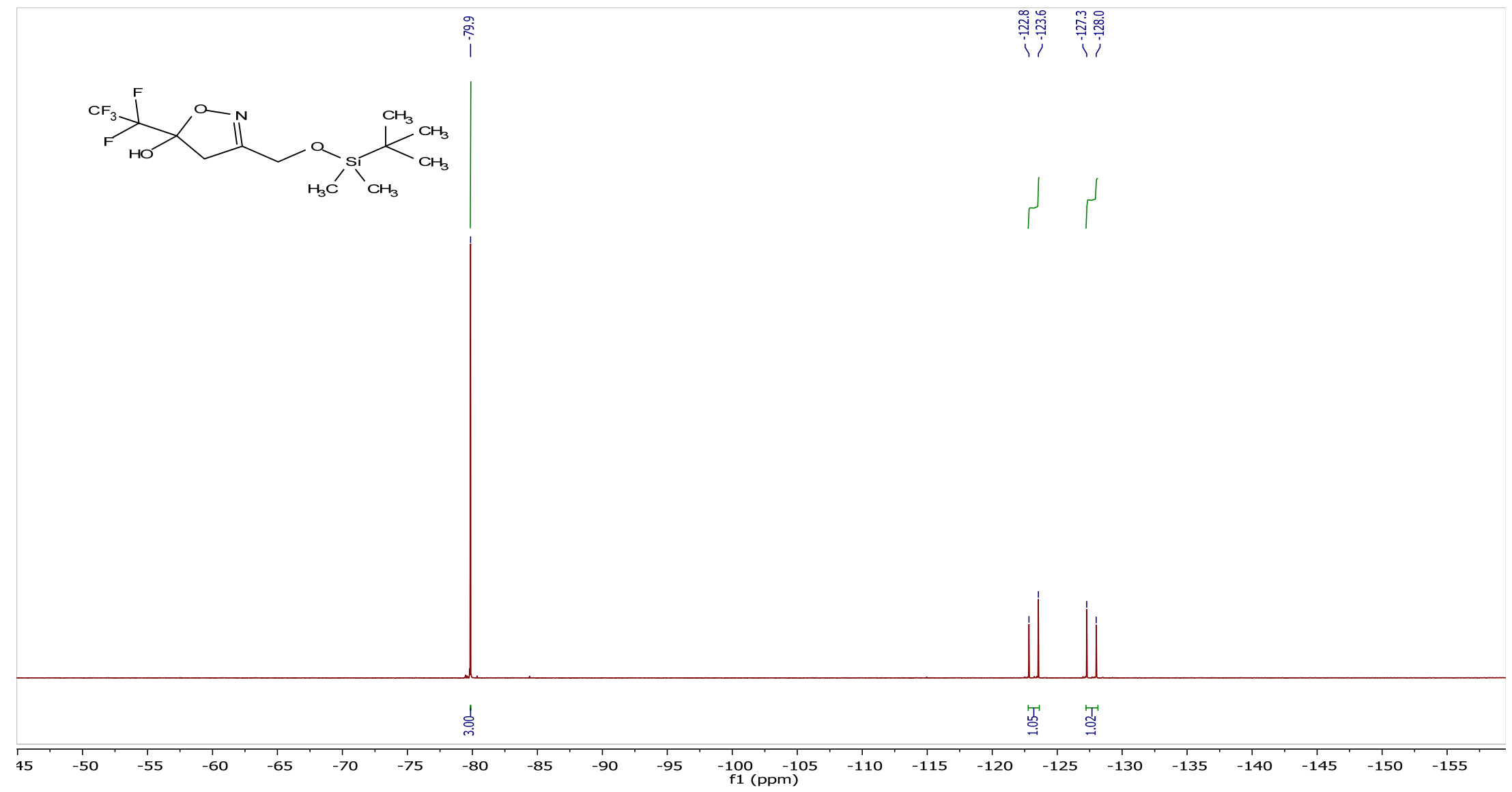


${ }^{1} \mathrm{H}$ NMR spectrum of the compound $\mathbf{1 6 e}$.

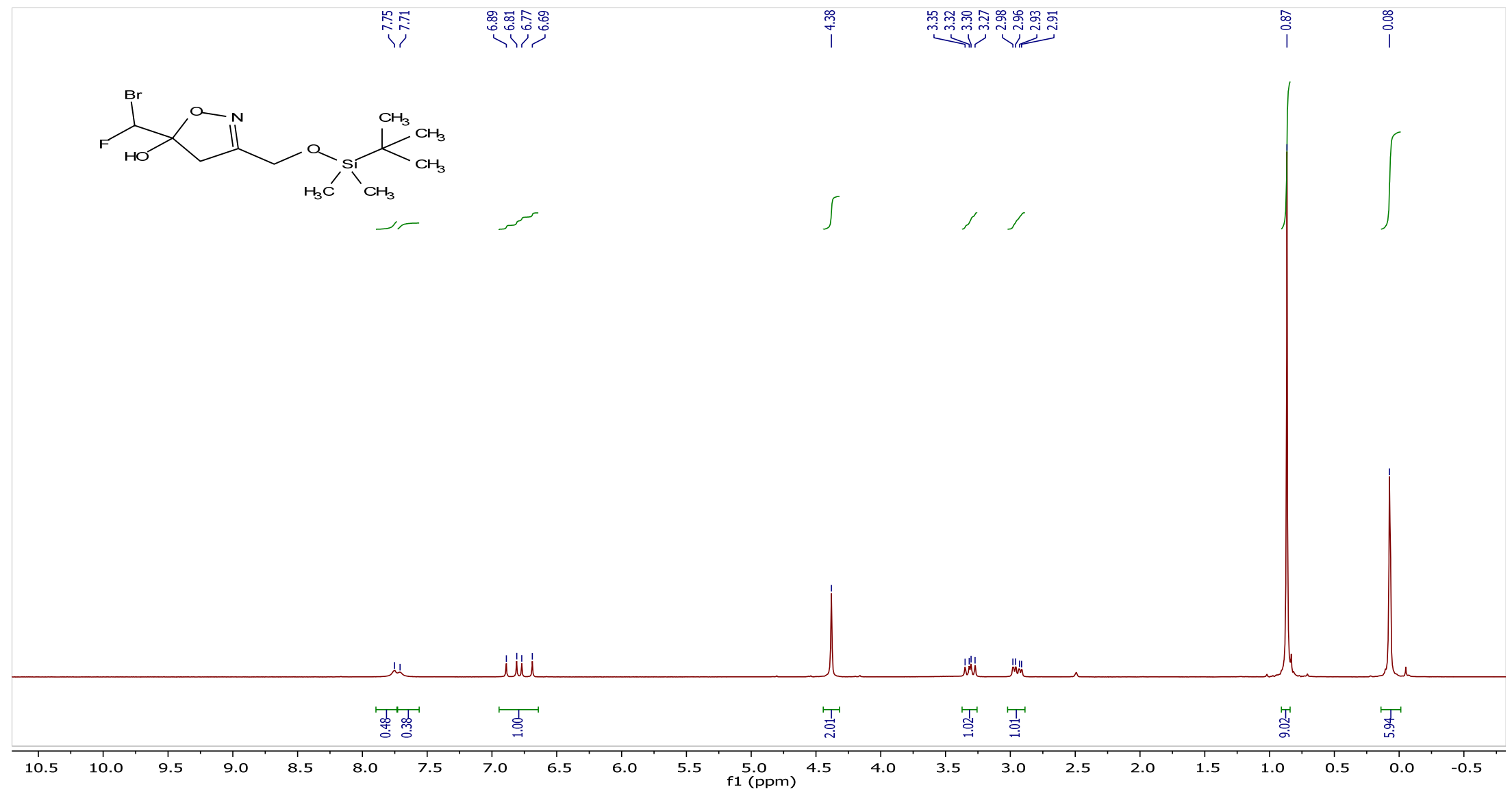


${ }^{13} \mathrm{C}\left\{{ }^{1} \mathrm{H}\right\}$ NMR spectrum of the compound $\mathbf{1 6 e}$.

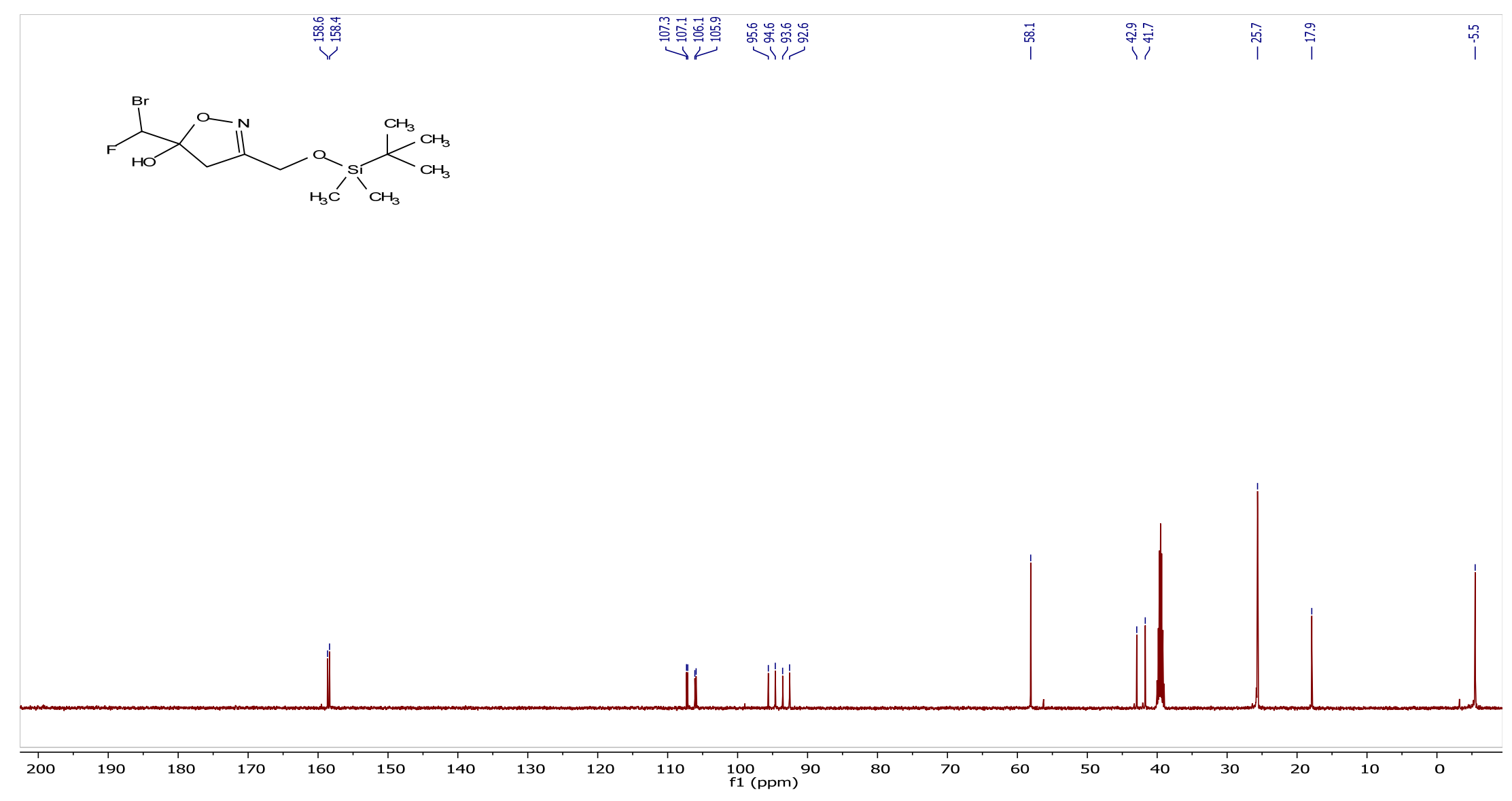


${ }^{19} \mathrm{~F}\left\{{ }^{1} \mathrm{H}\right\}$ NMR spectrum of the compound 16e.

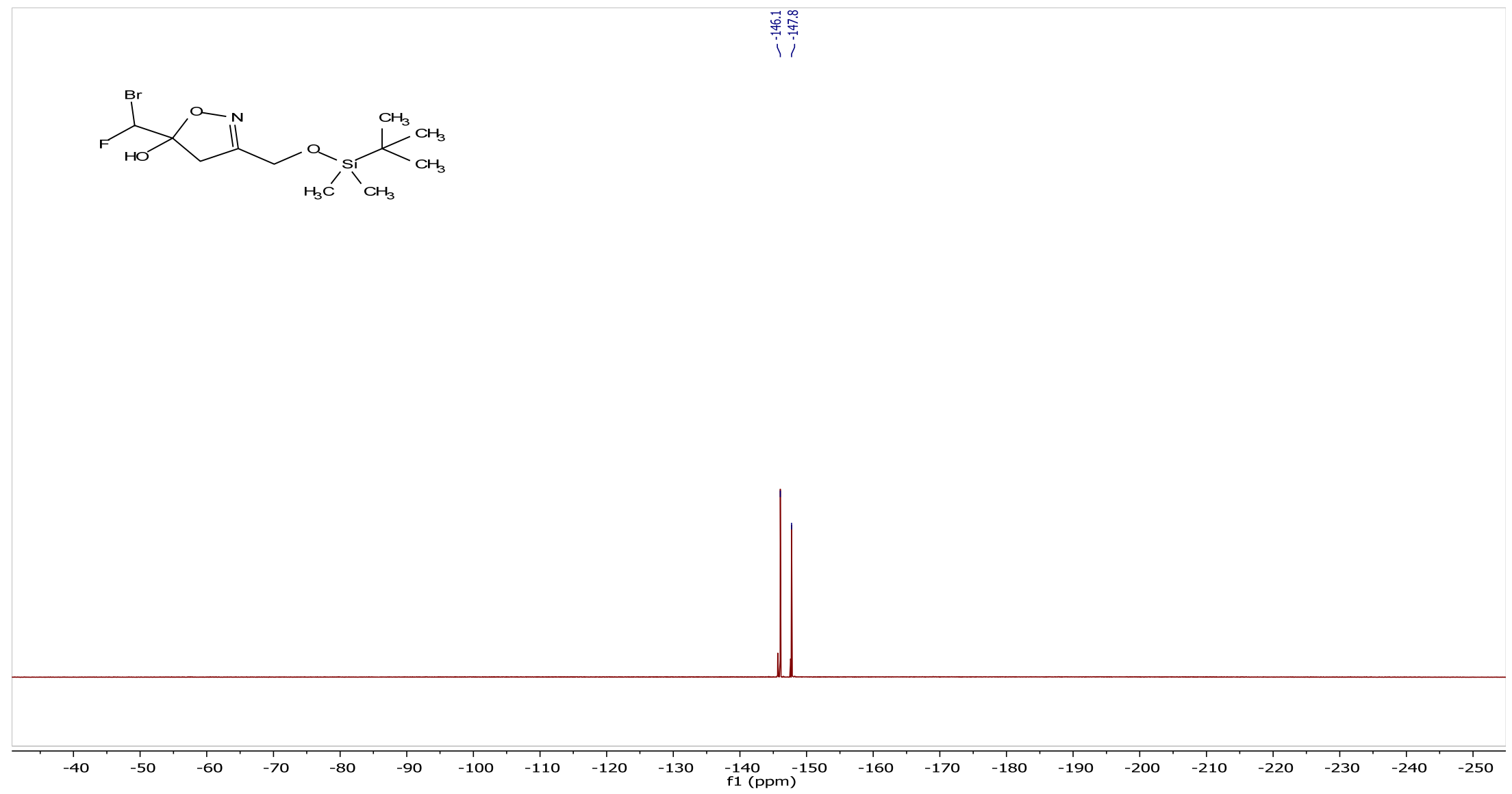


${ }^{1} \mathrm{H}$ NMR spectrum of the compound $\mathbf{1 6 f}$.

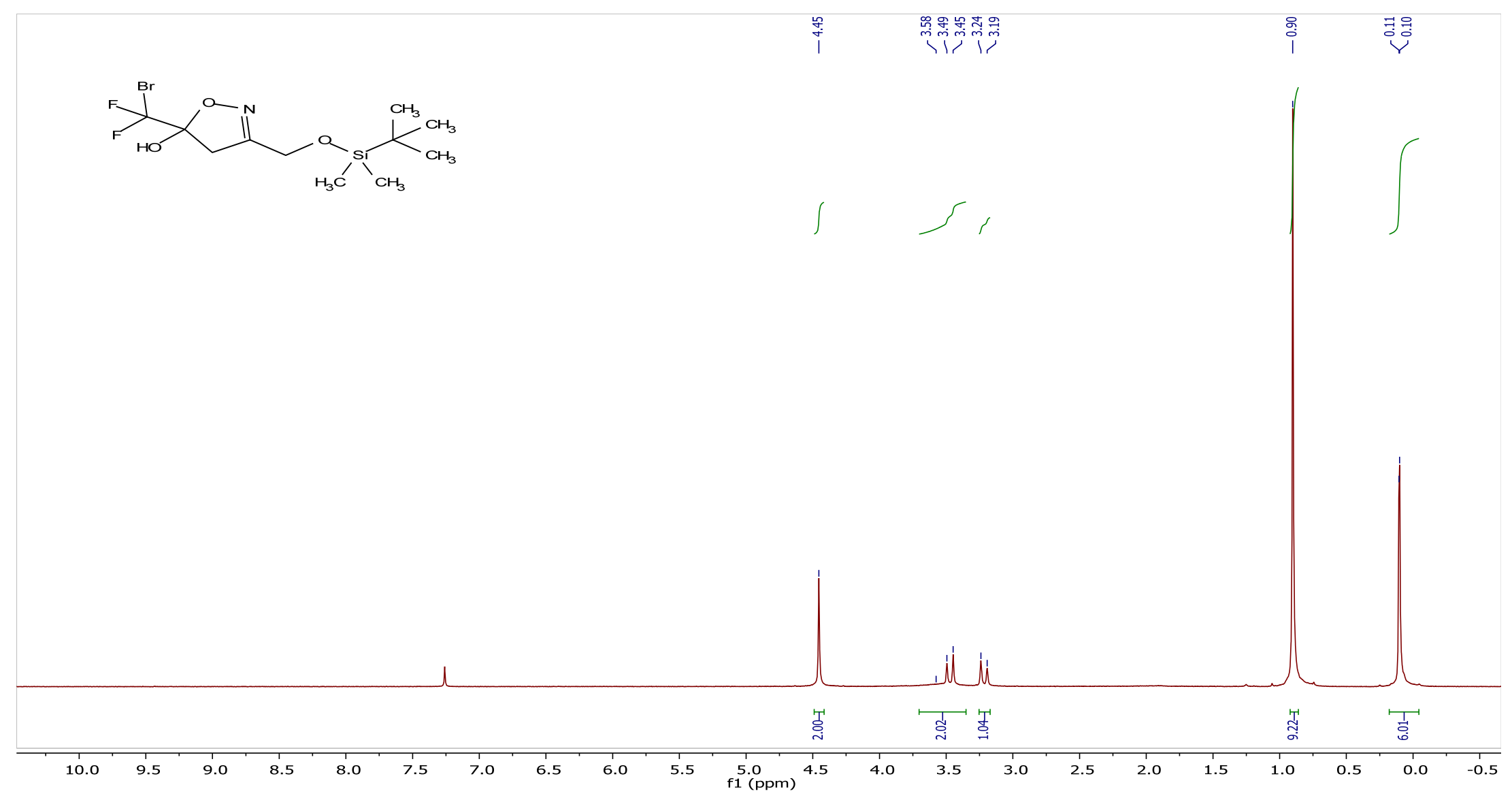


${ }^{13} \mathrm{C}\left\{{ }^{1} \mathrm{H}\right\}$ NMR spectrum of the compound $\mathbf{1 6 f}$.

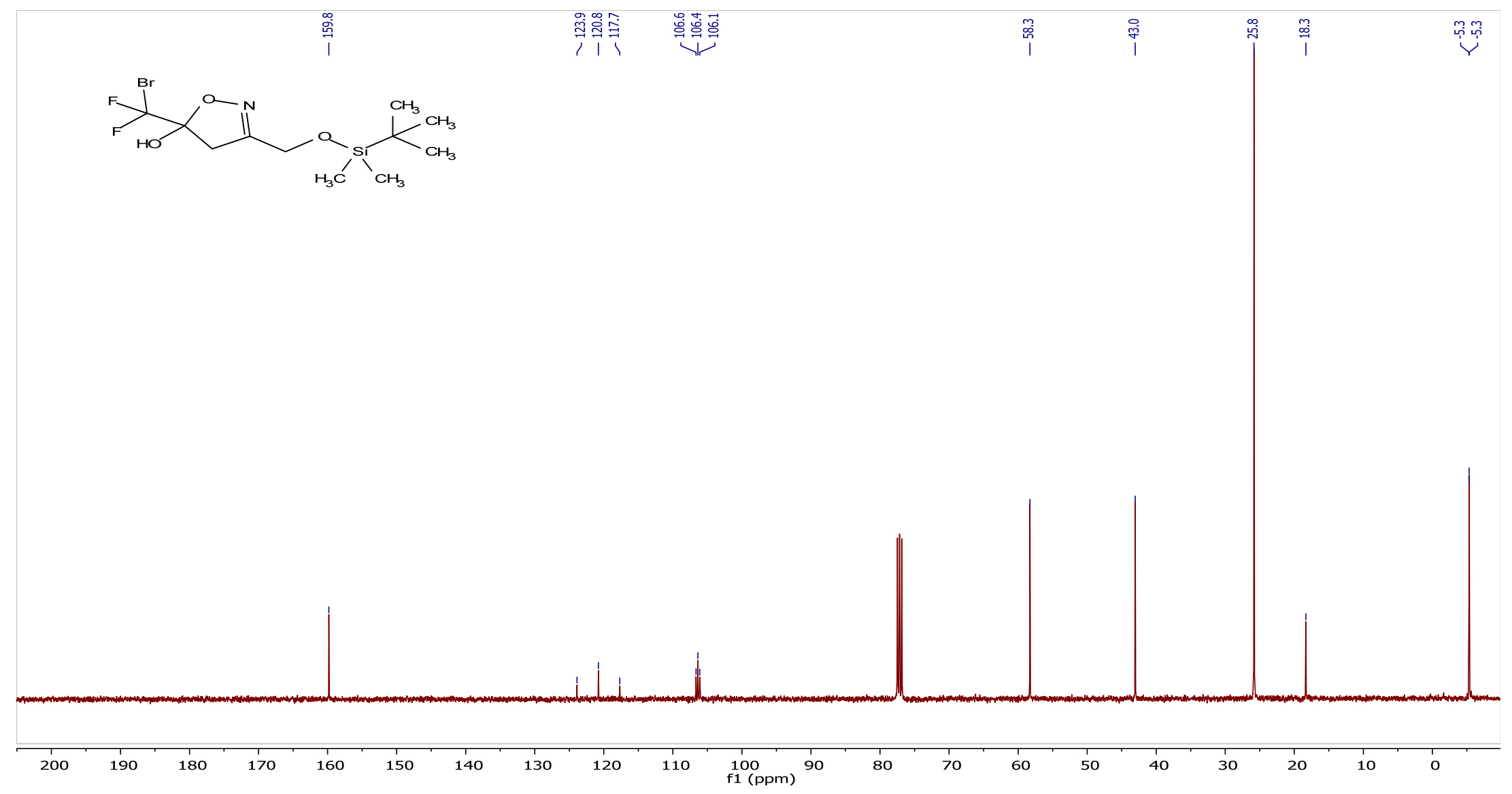


${ }^{19} \mathrm{~F}\left\{{ }^{1} \mathrm{H}\right\}$ NMR spectrum of the compound $\mathbf{1 6 f}$.

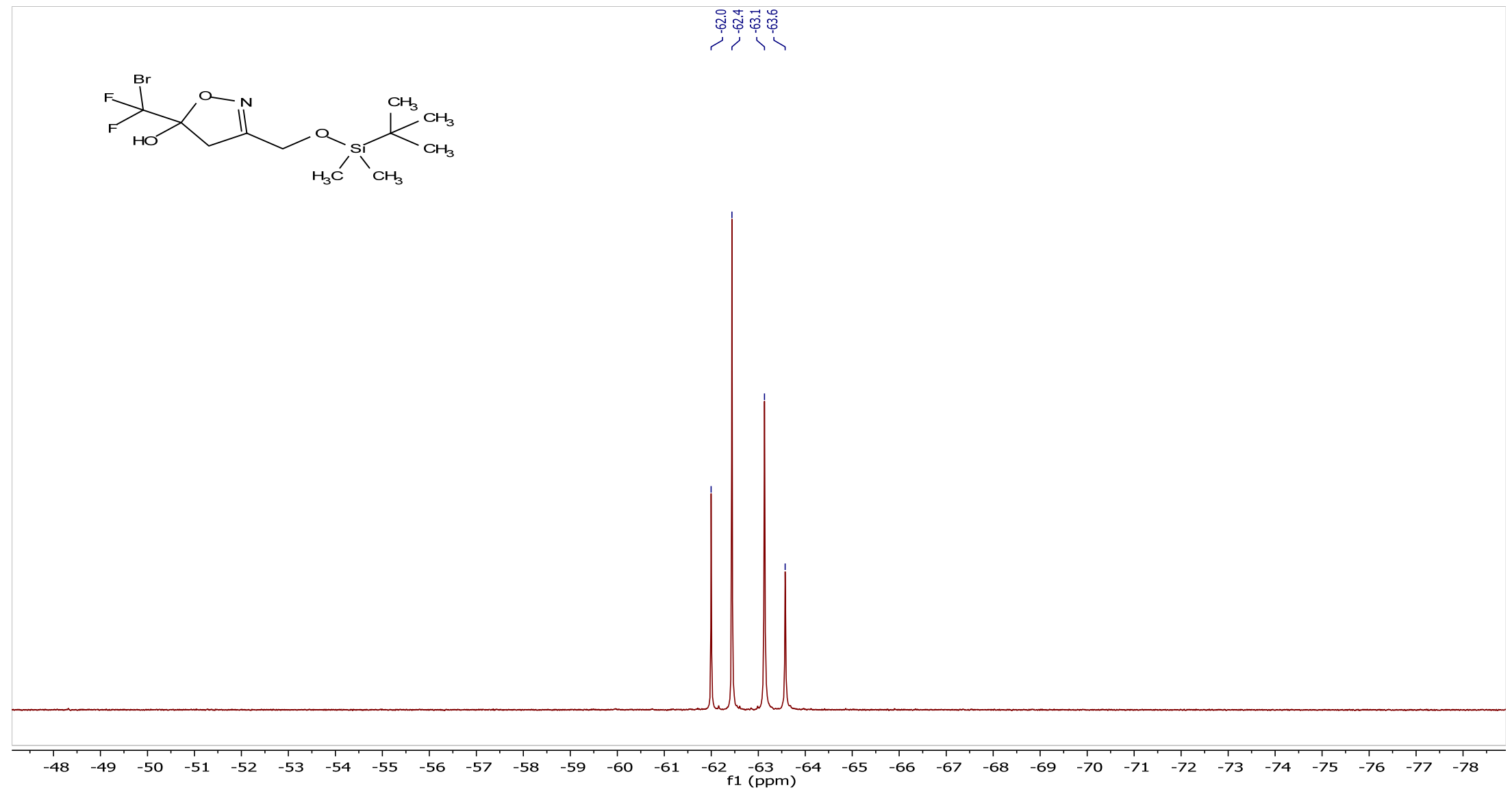


${ }^{1} \mathrm{H}$ NMR spectrum of the compound 16g.

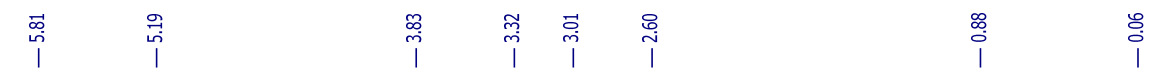
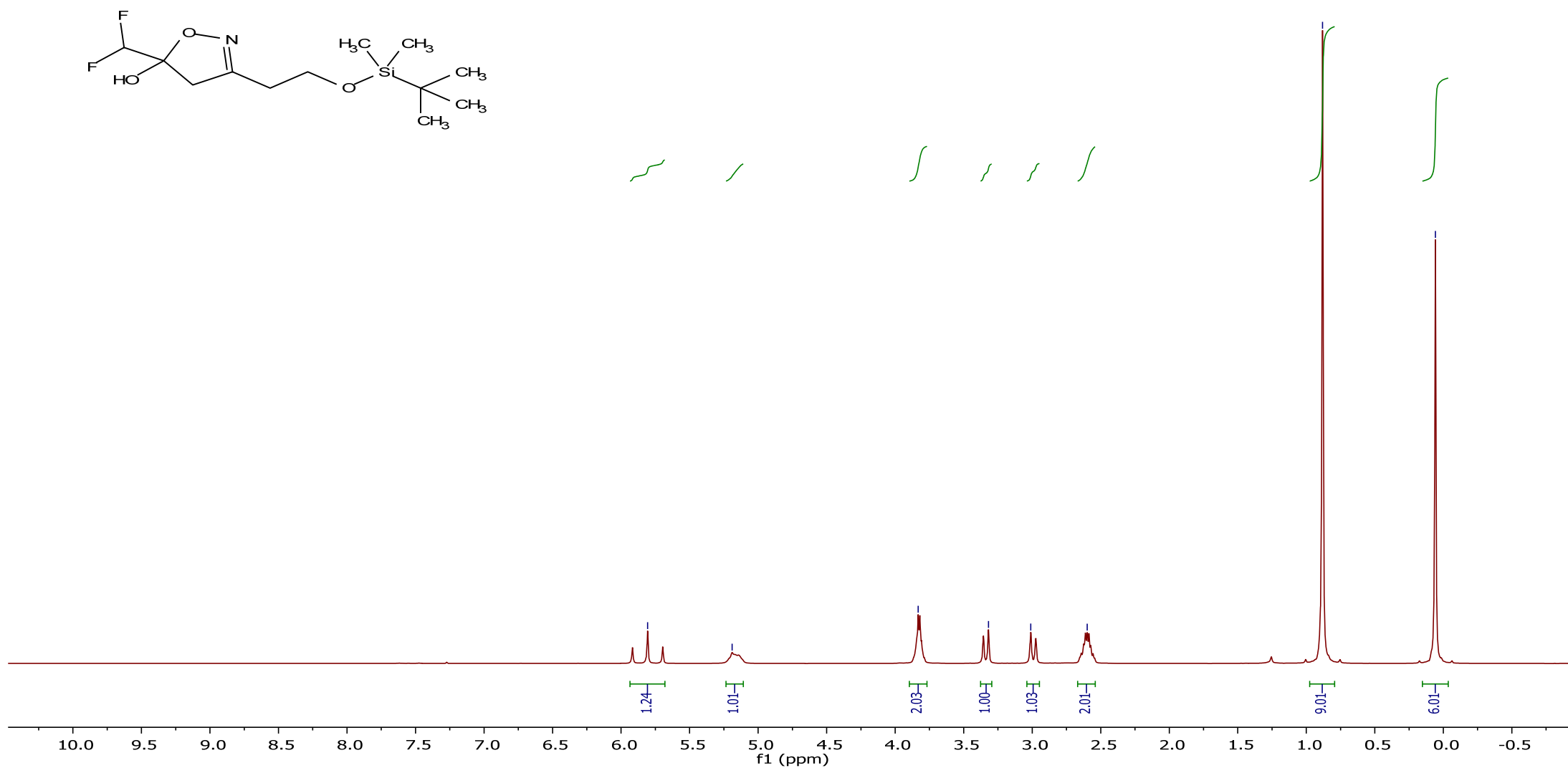
${ }^{13} \mathrm{C}\left\{{ }^{1} \mathrm{H}\right\}$ NMR spectrum of the compound $\mathbf{1 6 g}$.

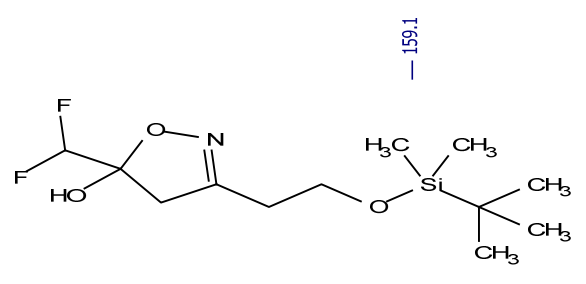

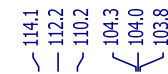

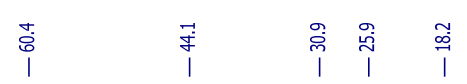

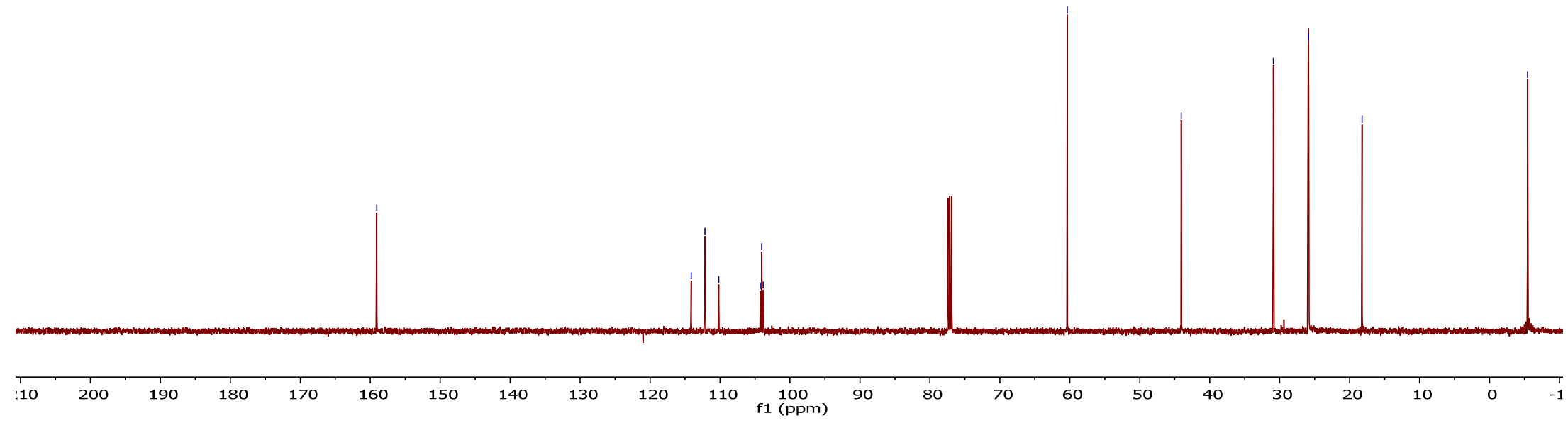


${ }^{19}$ F NMR spectrum of the compound 16g.

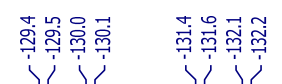
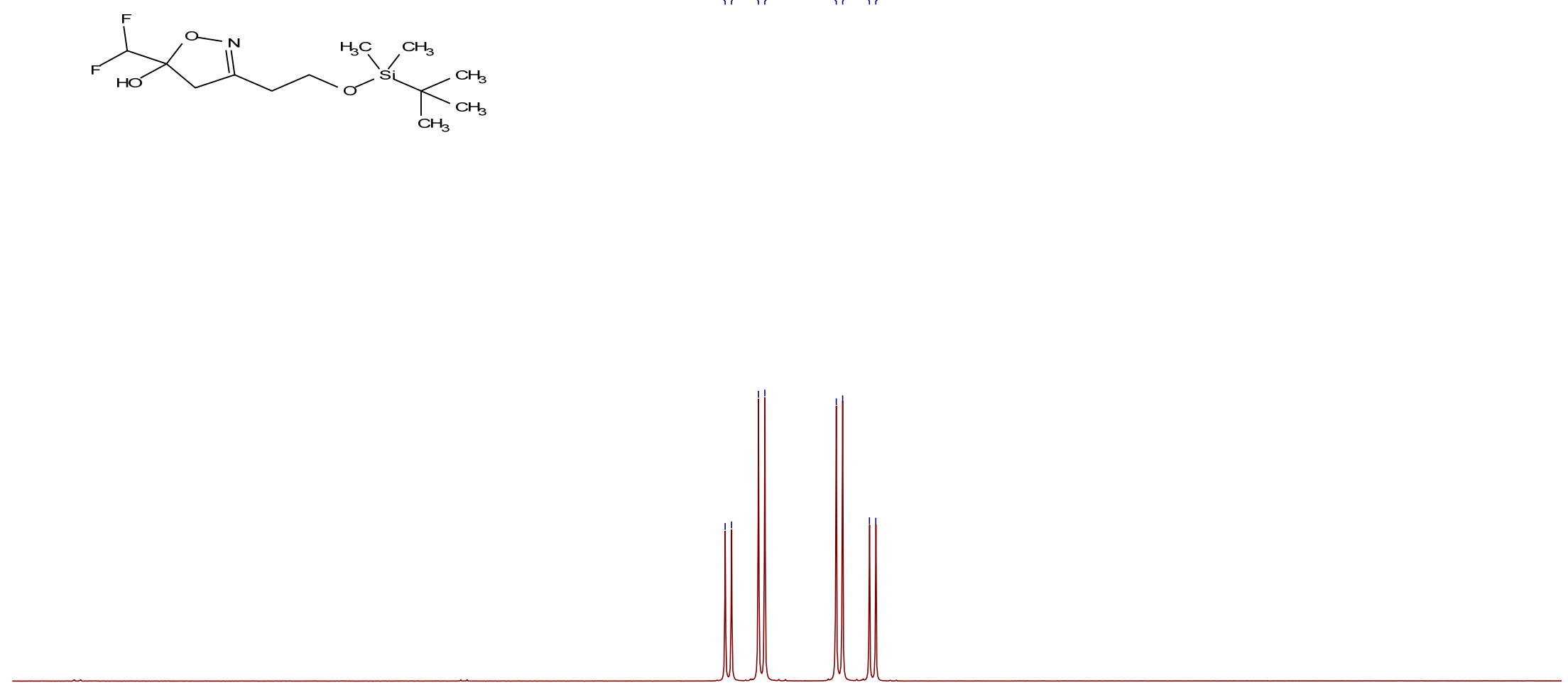

$\begin{array}{lllllllllllllllllllllllllllllllll}-117 & -118 & -119 & -120 & -121 & -122 & -123 & -124 & -125 & -126 & -127 & -128 & -129 & -130 & -131 & -132 & -133 & -134 & -135 & -136 & -137 & -138 & -139 & -140 & -141 & -142 & -143 & -144\end{array}$ 
${ }^{1} \mathrm{H}$ NMR spectrum of the compound $\mathbf{1 8 g}$.

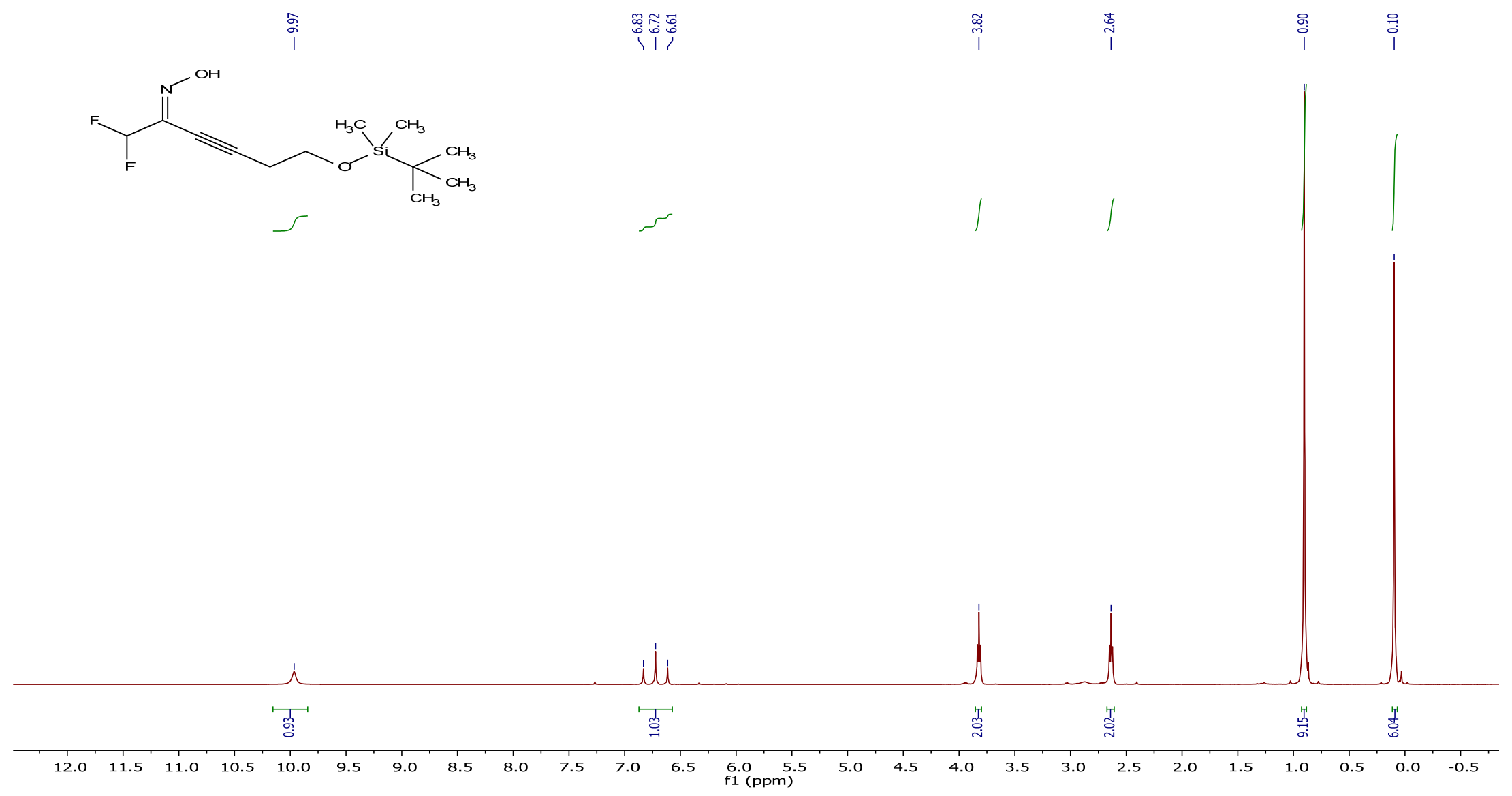




\section{${ }^{13} \mathrm{C}\left\{{ }^{1} \mathrm{H}\right\}$ NMR spectrum of the compound $\mathbf{1 8 g}$.}

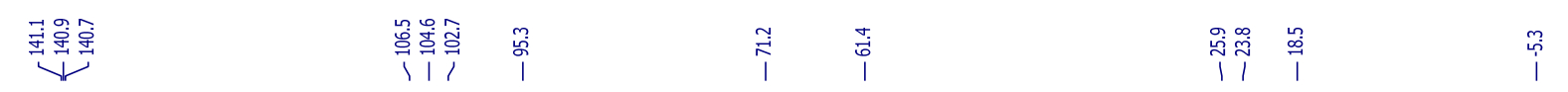
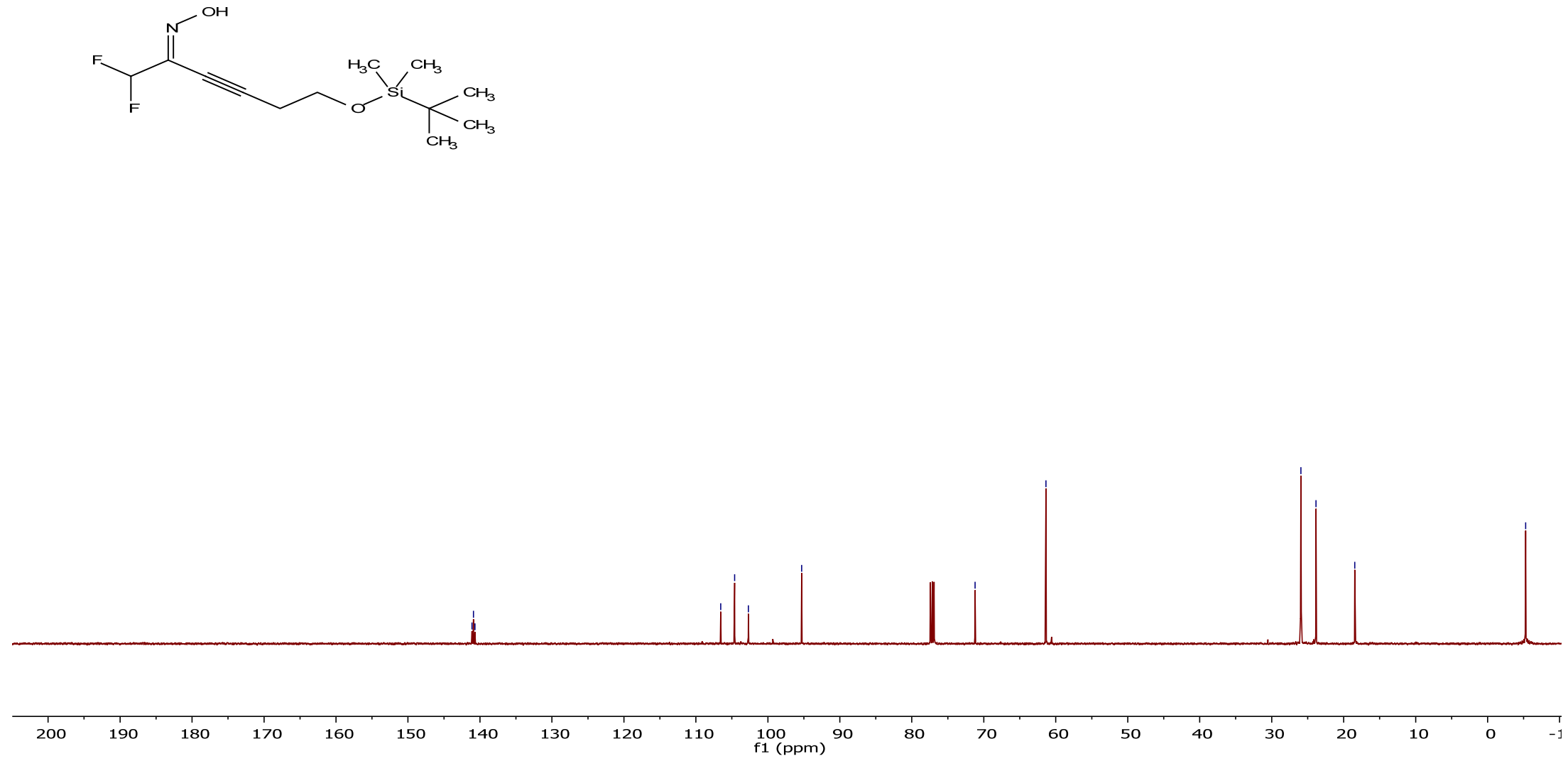
${ }^{19} \mathrm{~F}\left\{{ }^{1} \mathrm{H}\right\}$ NMR spectrum of the compound $\mathbf{1 8 g}$.

$\overrightarrow{\stackrel{i}{i}}$
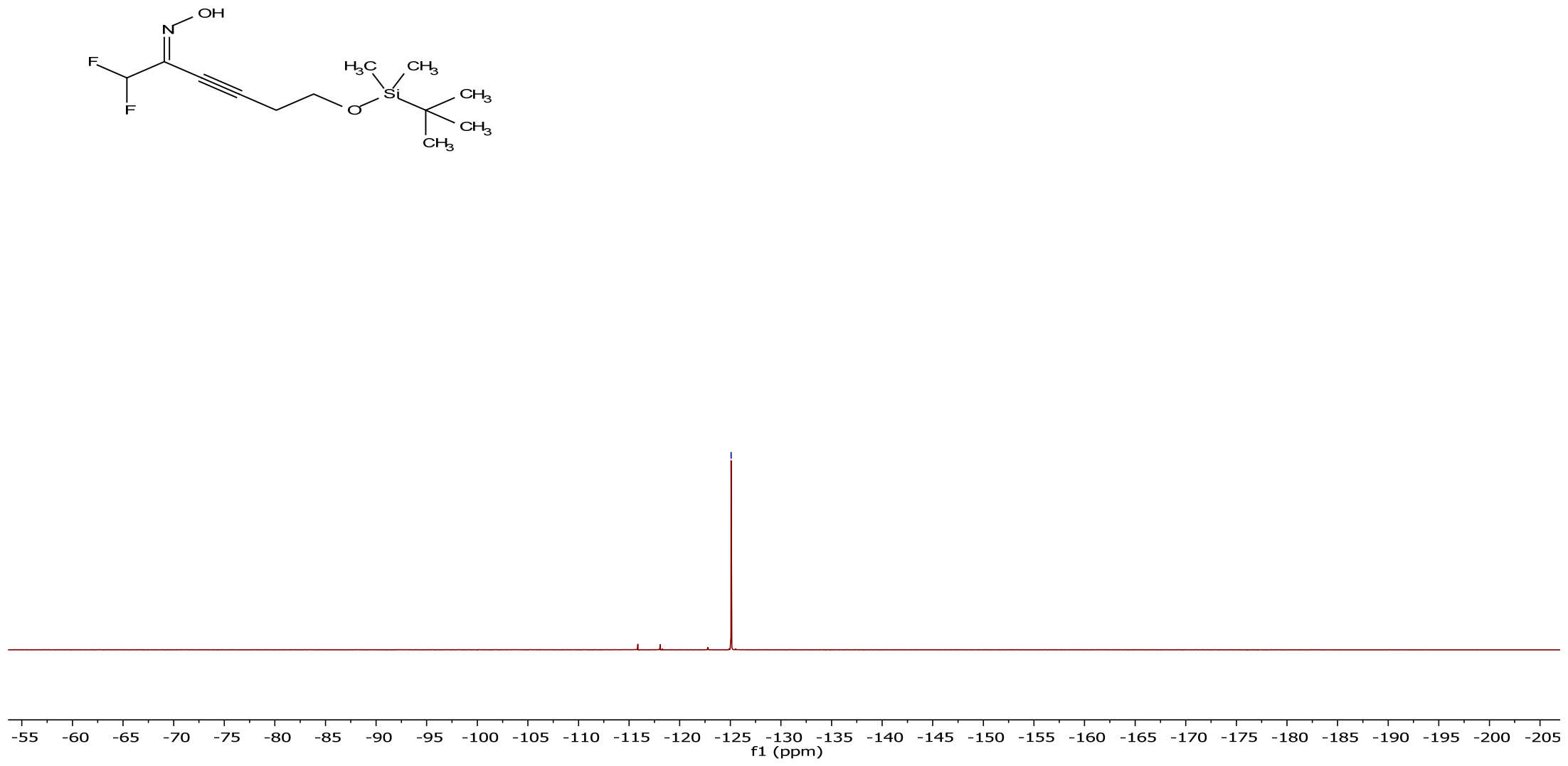
${ }^{1} \mathrm{H}$ NMR spectrum of the compound $\mathbf{1 6 h}$.

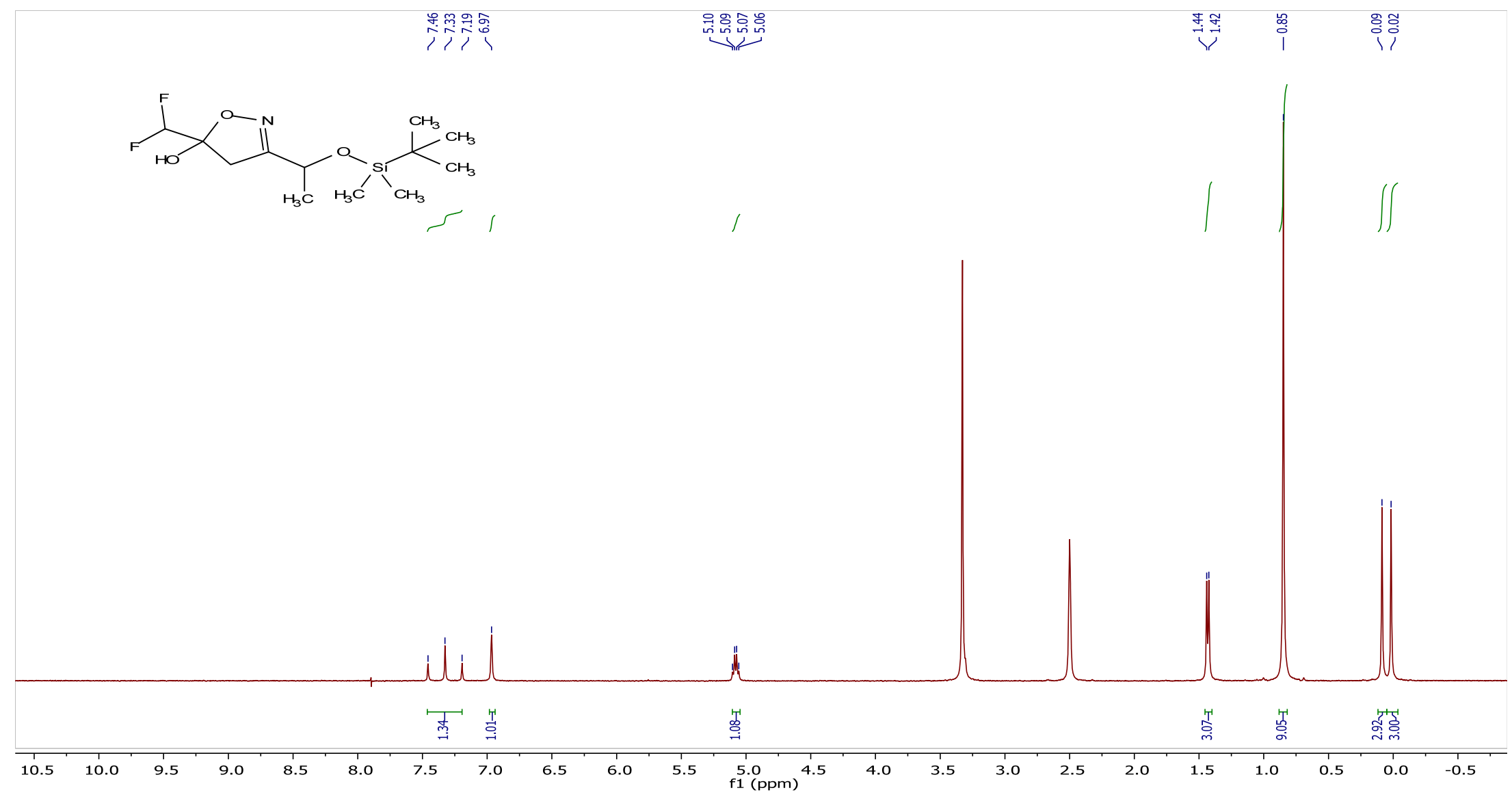


${ }^{13} \mathrm{C}\left\{{ }^{1} \mathrm{H}\right\}$ NMR spectrum of the compound $\mathbf{1 6 h}$.

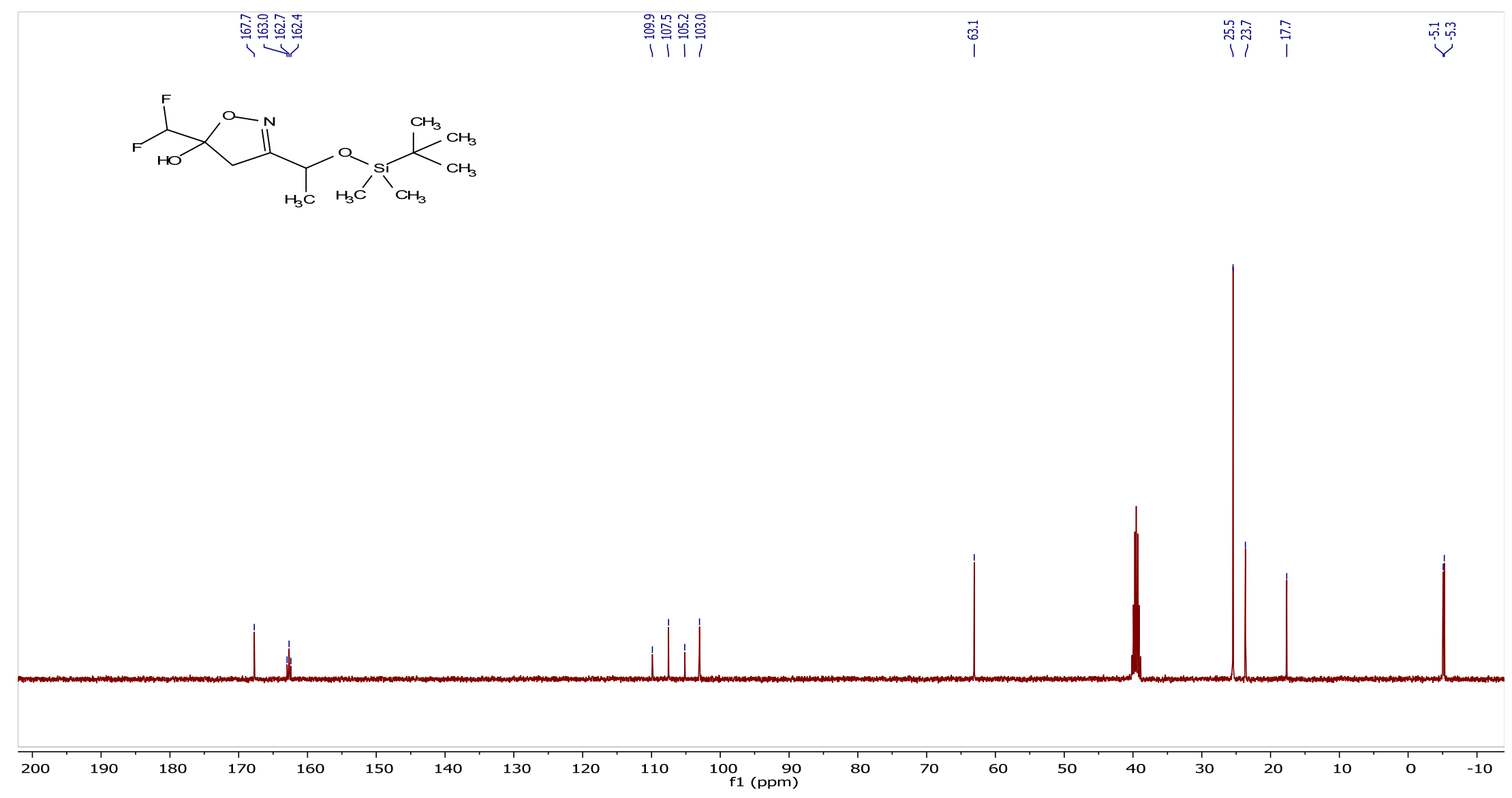


${ }^{19} \mathrm{~F}\left\{{ }^{1} \mathrm{H}\right\}$ NMR spectrum of the compound $\mathbf{1 6 h}$.

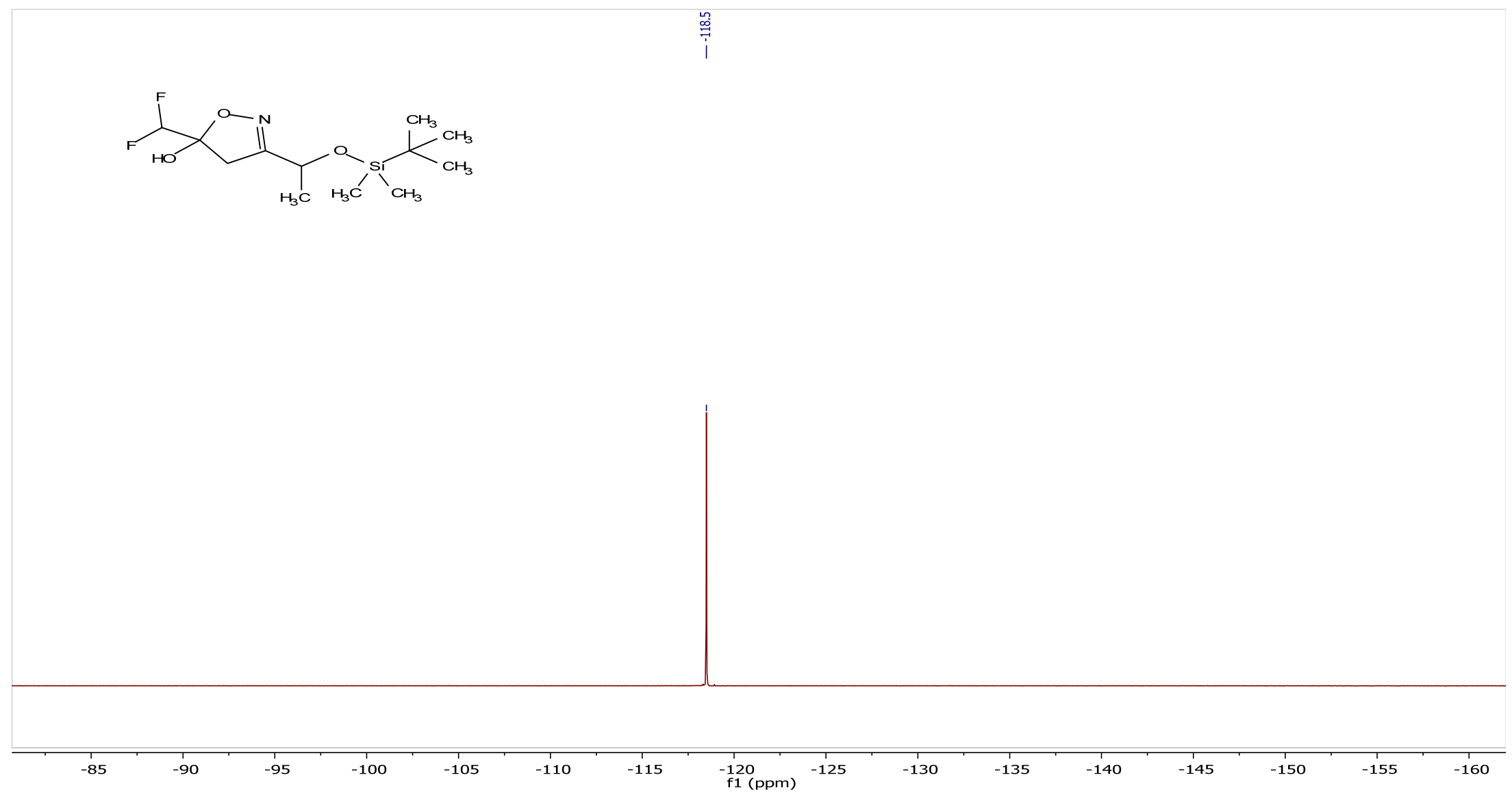


${ }^{1} \mathrm{H}$ NMR spectrum of the compound 17a.

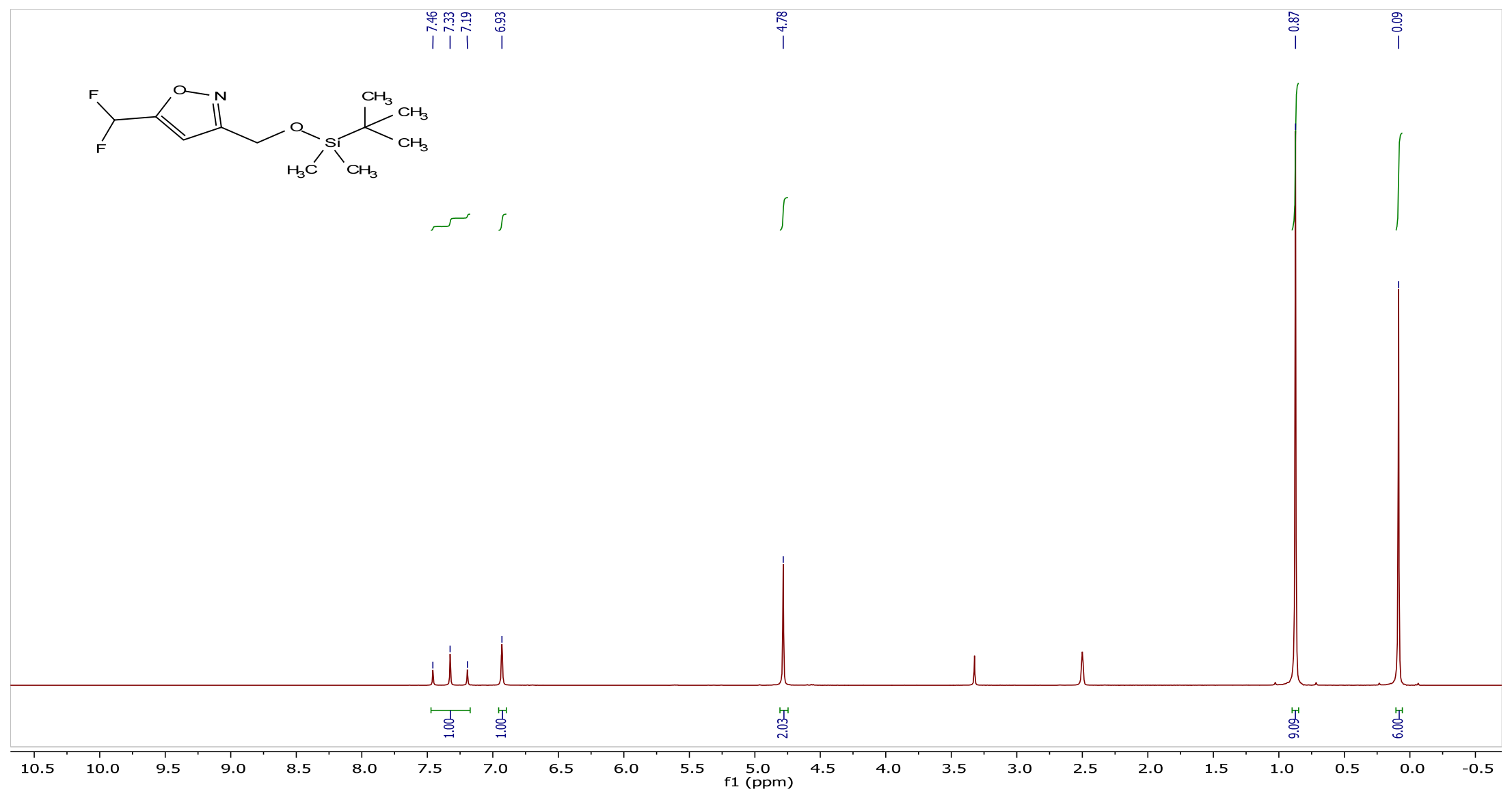


${ }^{13} \mathrm{C}\left\{{ }^{1} \mathrm{H}\right\}$ NMR spectrum of the compound 17a.

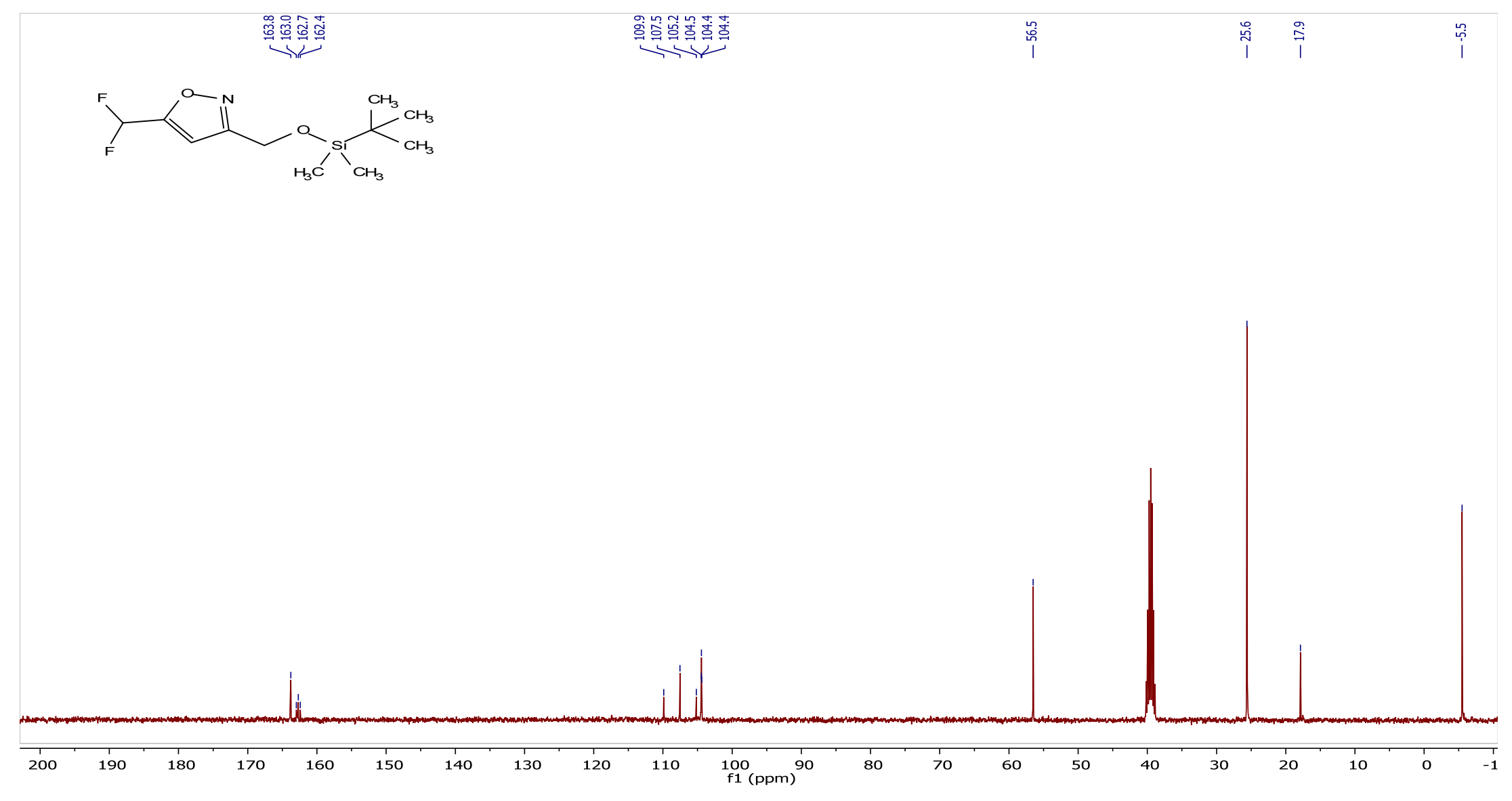


${ }^{19} \mathrm{~F}$ NMR spectrum of the compound 17a.

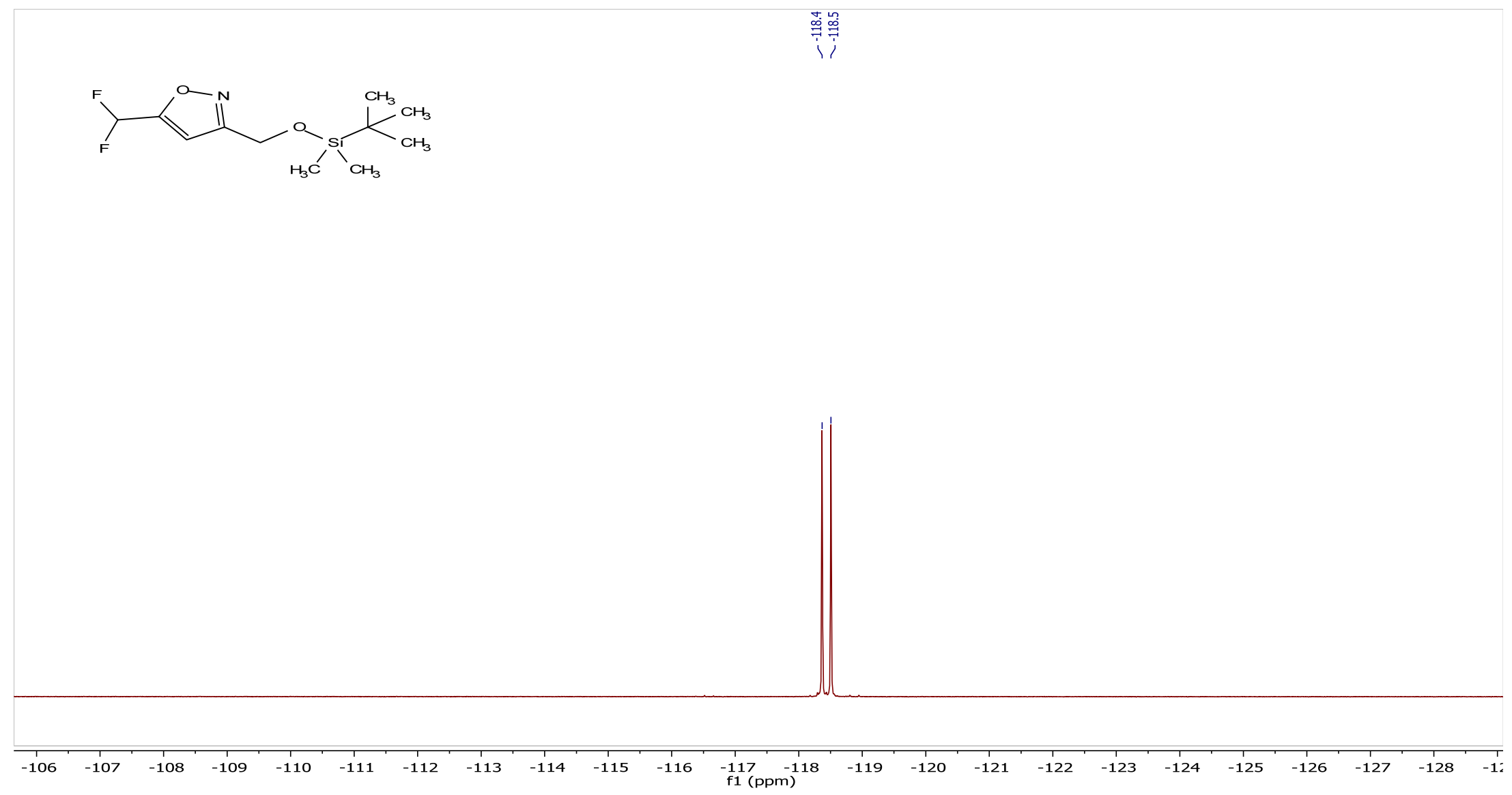


${ }^{1} \mathrm{H}$ NMR spectrum of the compound $\mathbf{1 7 b}$.

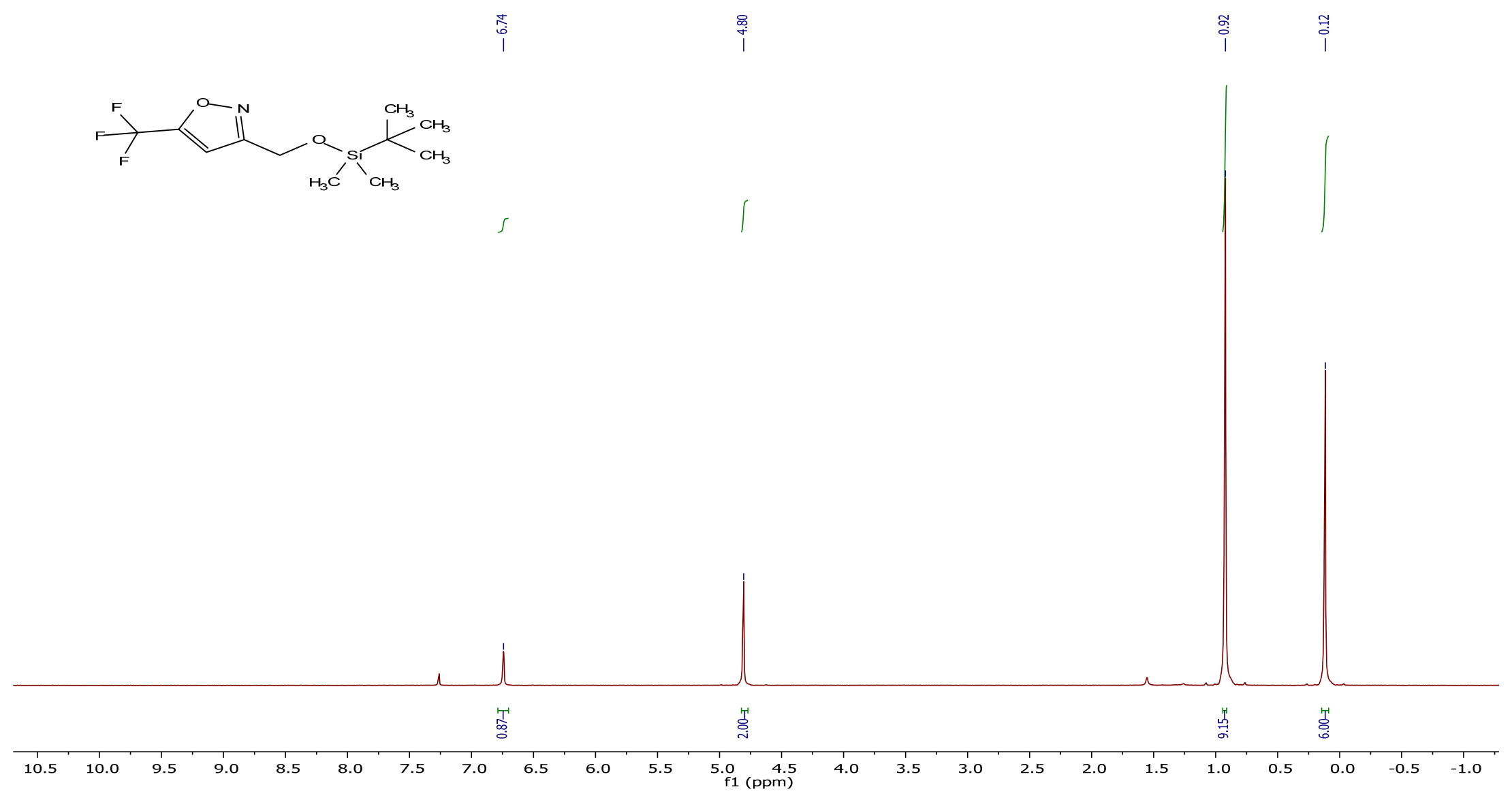


${ }^{13} \mathrm{C}\left\{{ }^{1} \mathrm{H}\right\}$ NMR spectrum of the compound $\mathbf{1 7 b}$.

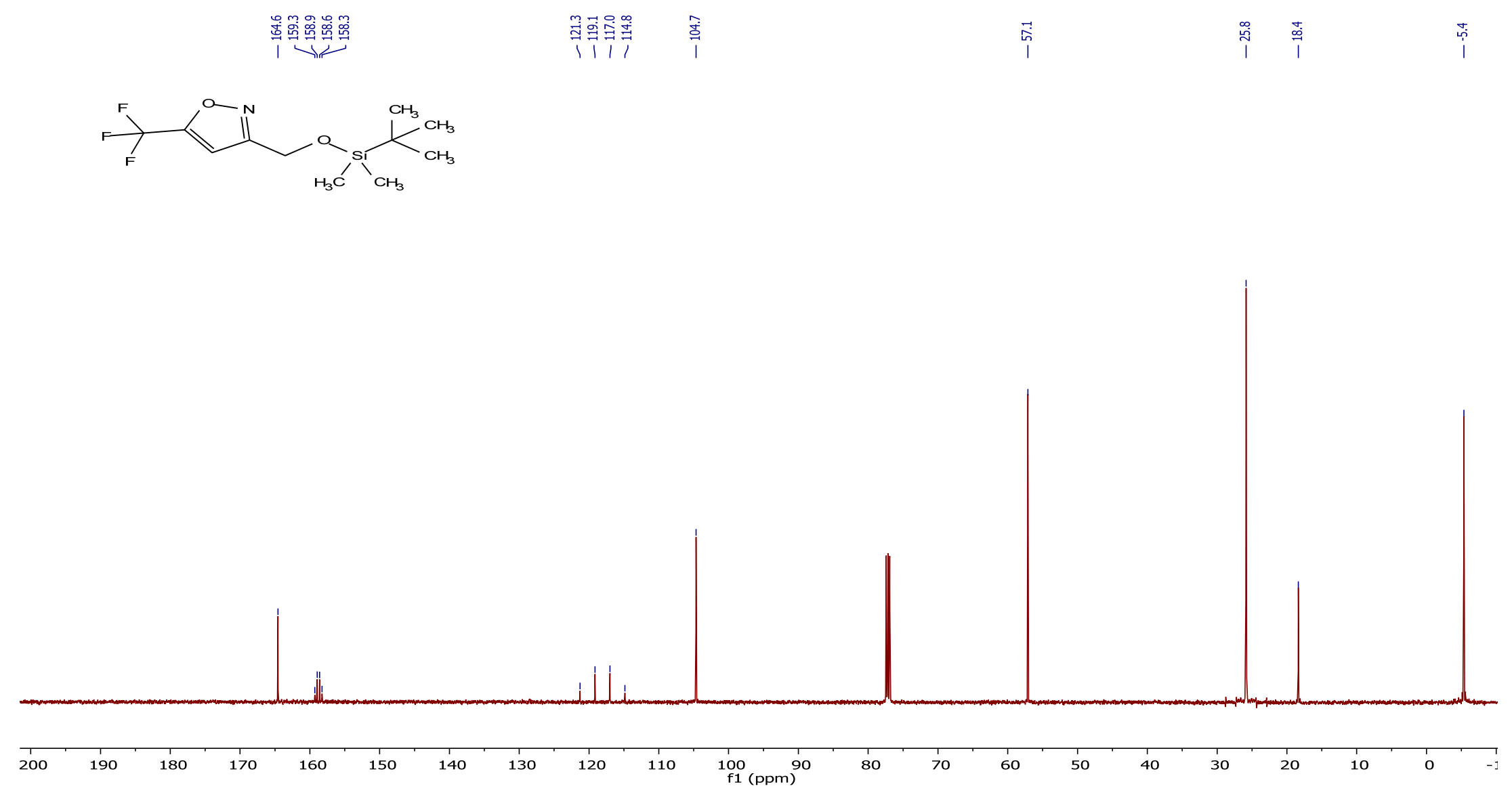


${ }^{19} \mathrm{~F}\left\{{ }^{1} \mathrm{H}\right\}$ NMR spectrum of the compound $\mathbf{1 7 b}$.

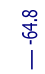
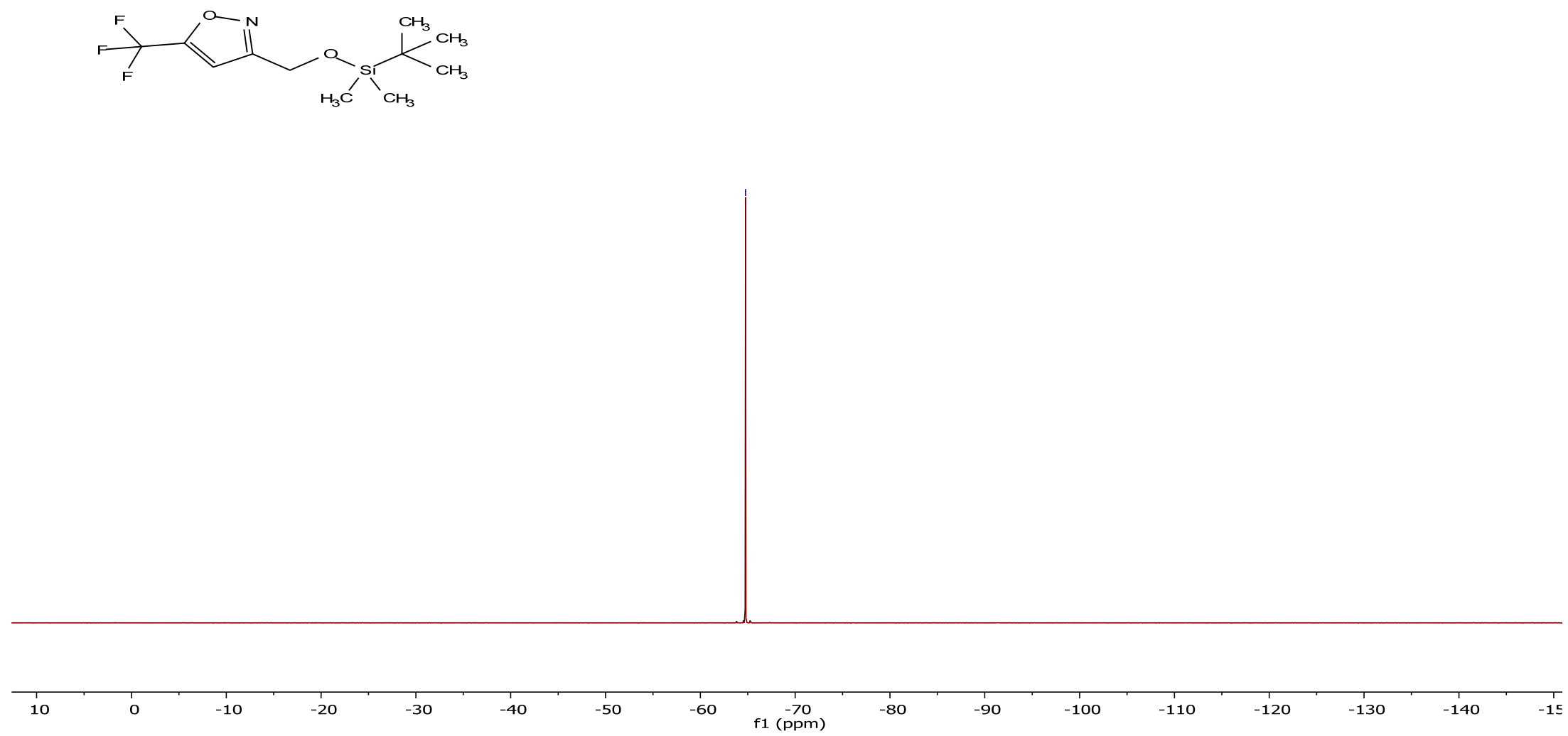
${ }^{1} \mathrm{H}$ NMR spectrum of the compound 17c.

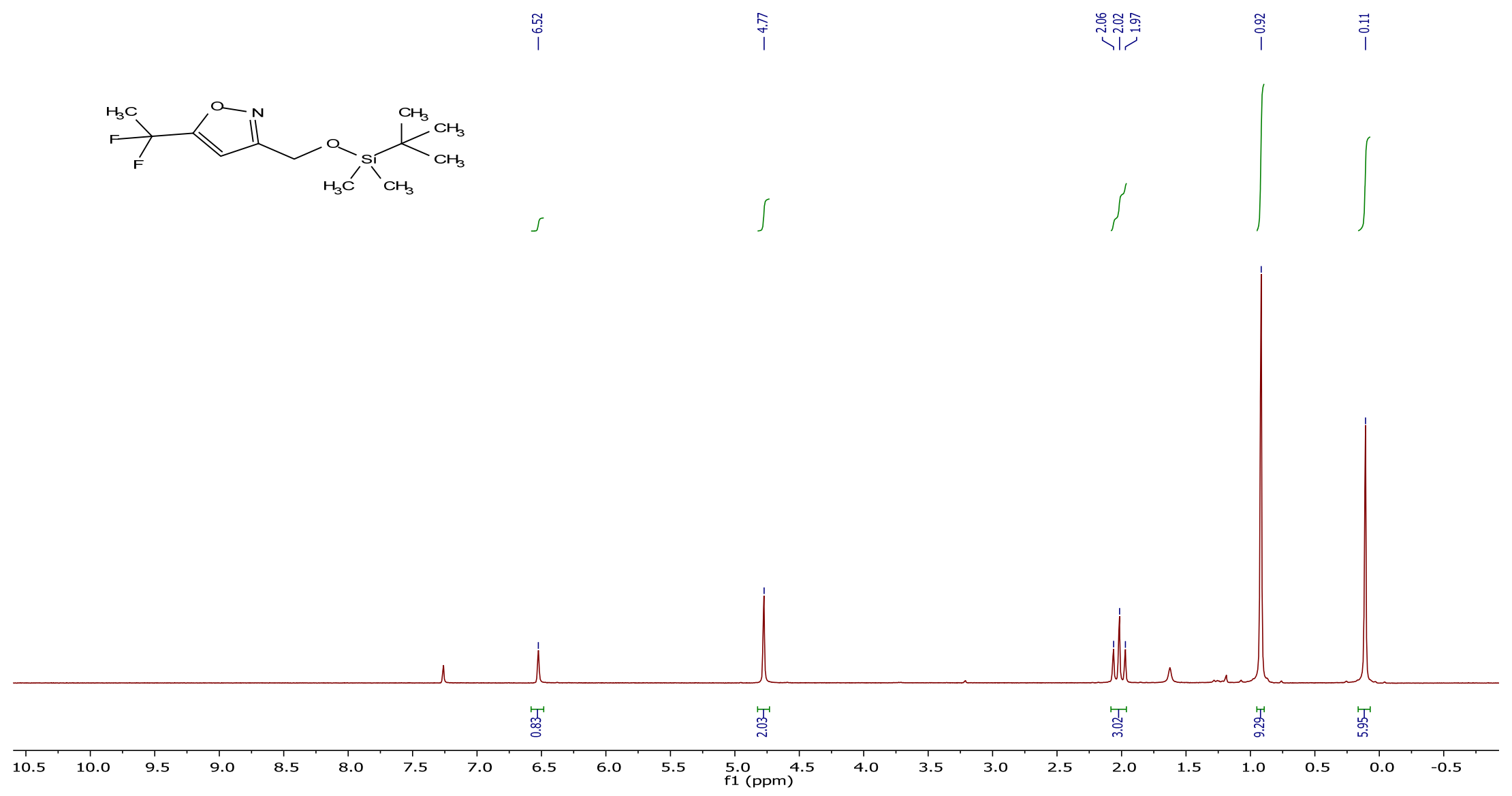


${ }^{19} \mathrm{~F}\left\{{ }^{1} \mathrm{H}\right\}$ NMR spectrum of the compound 17c.

$\stackrel{m}{\substack{p \\ i}}$
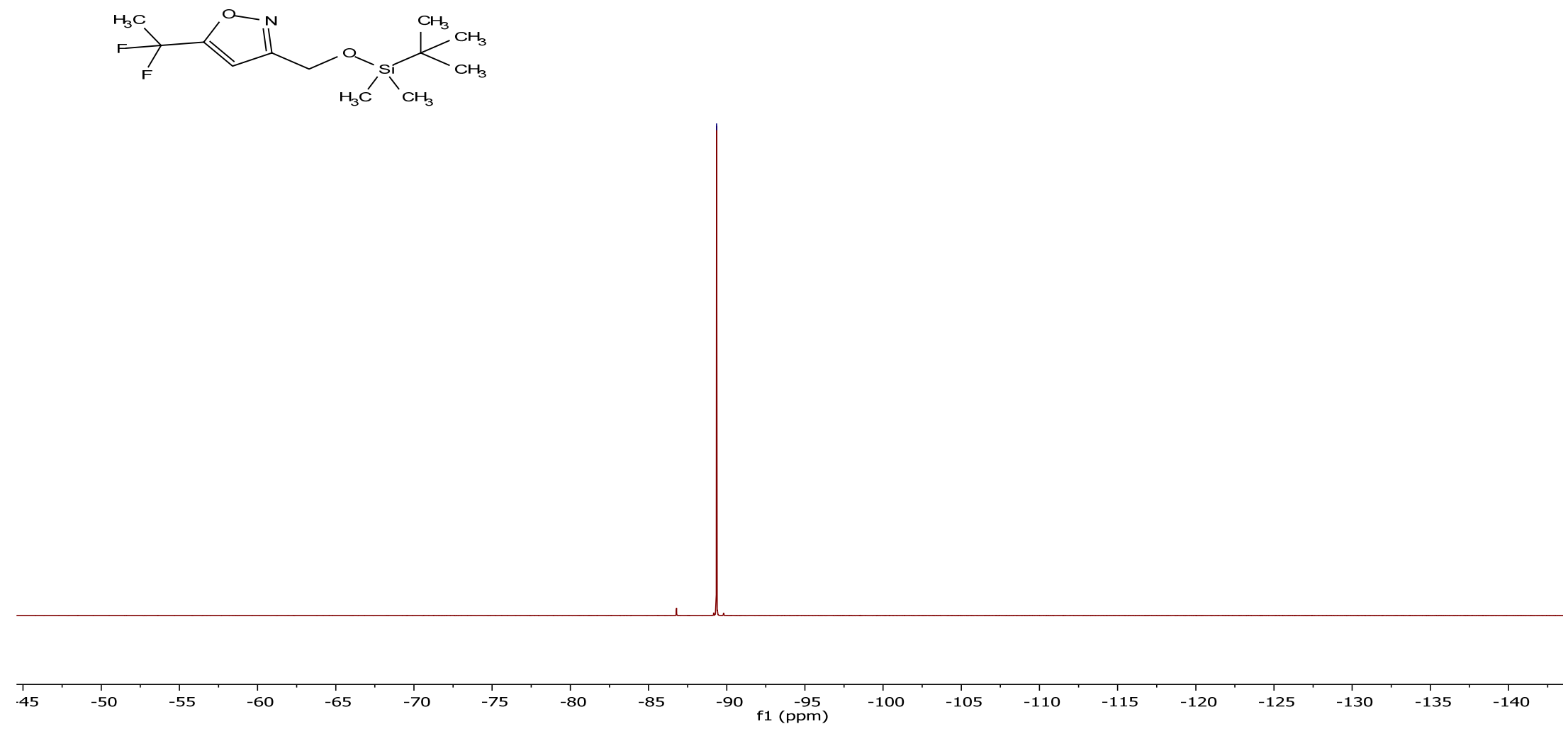
${ }^{1} \mathrm{H}$ NMR spectrum of the compound $\mathbf{1 7 d}$.

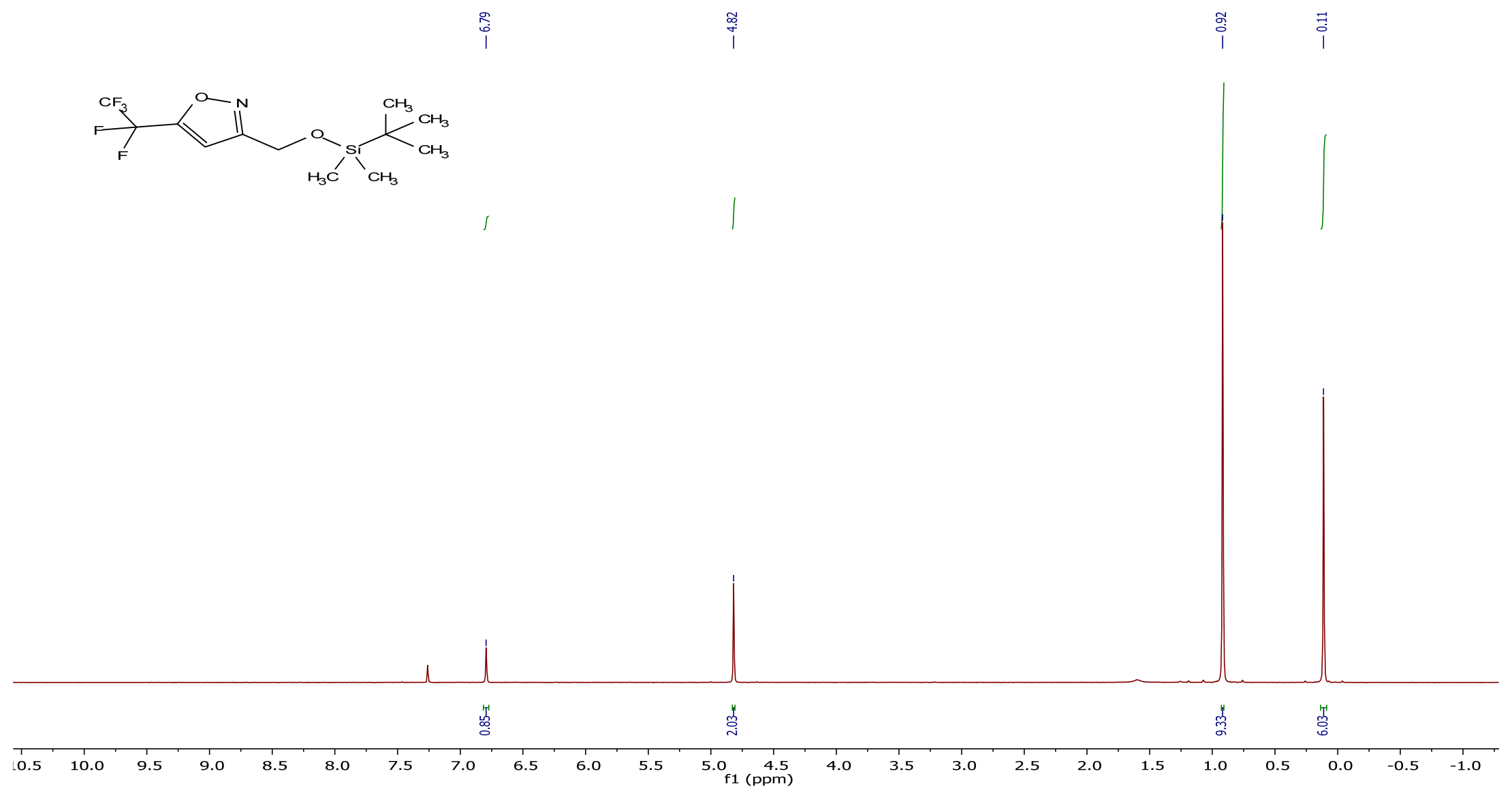


${ }^{13} \mathrm{C}\left\{{ }^{1} \mathrm{H}\right\}$ NMR spectrum of the compound 17d.

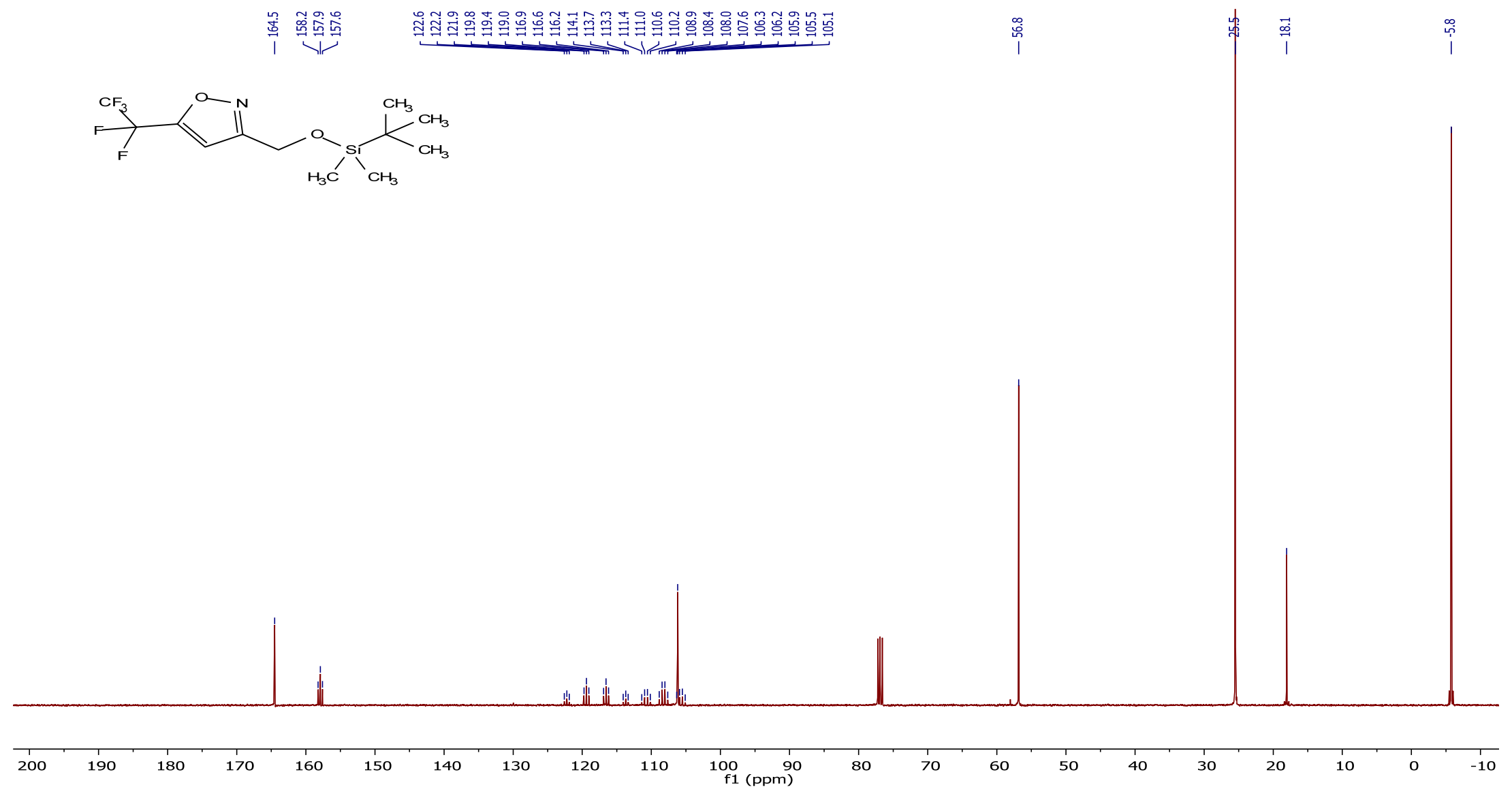


${ }^{19} \mathrm{~F}\left\{{ }^{1} \mathrm{H}\right\}$ NMR spectrum of the compound 17d.
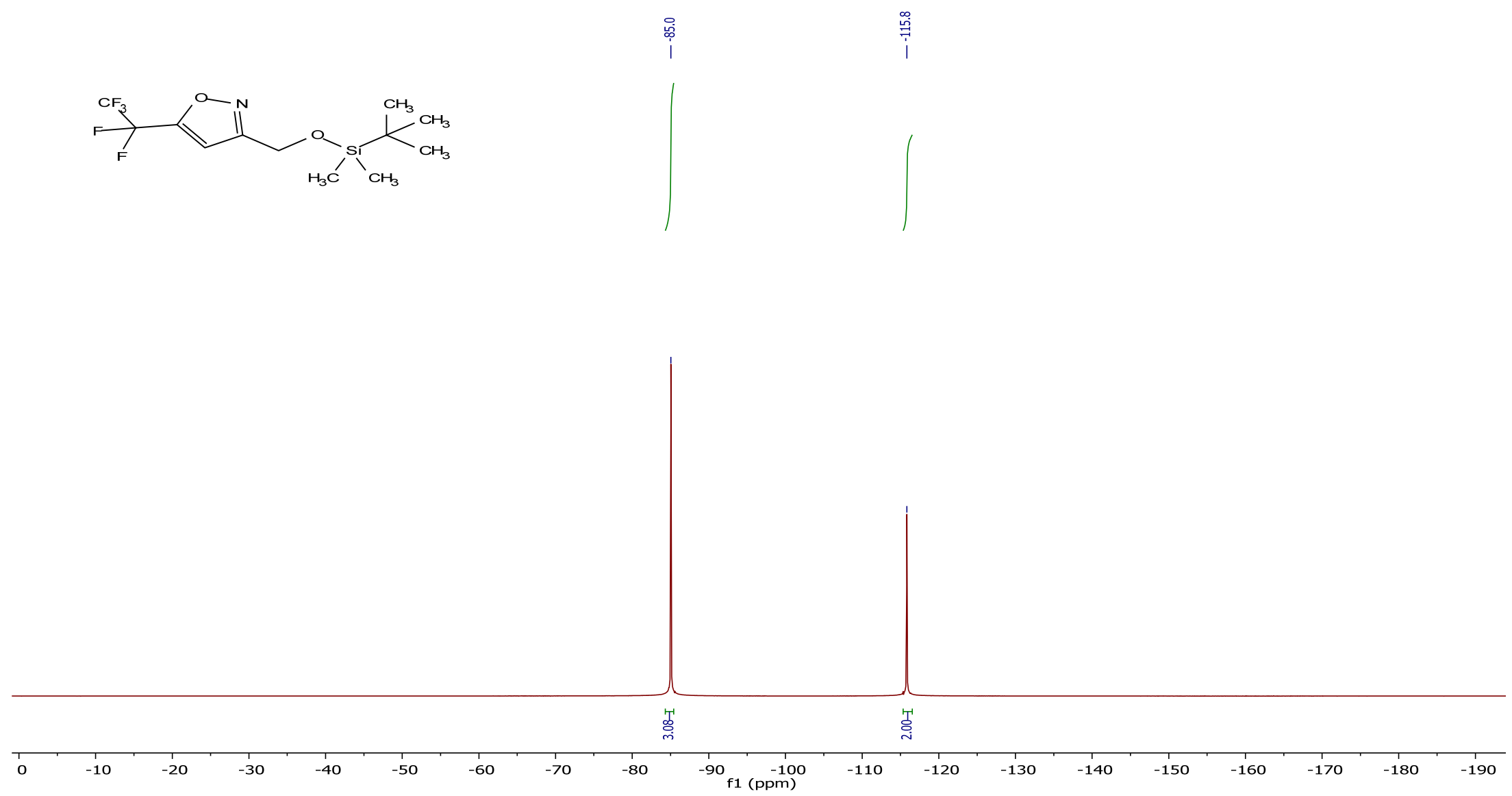
${ }^{1} \mathrm{H}$ NMR spectrum of the compound $\mathbf{1 7 e}$.

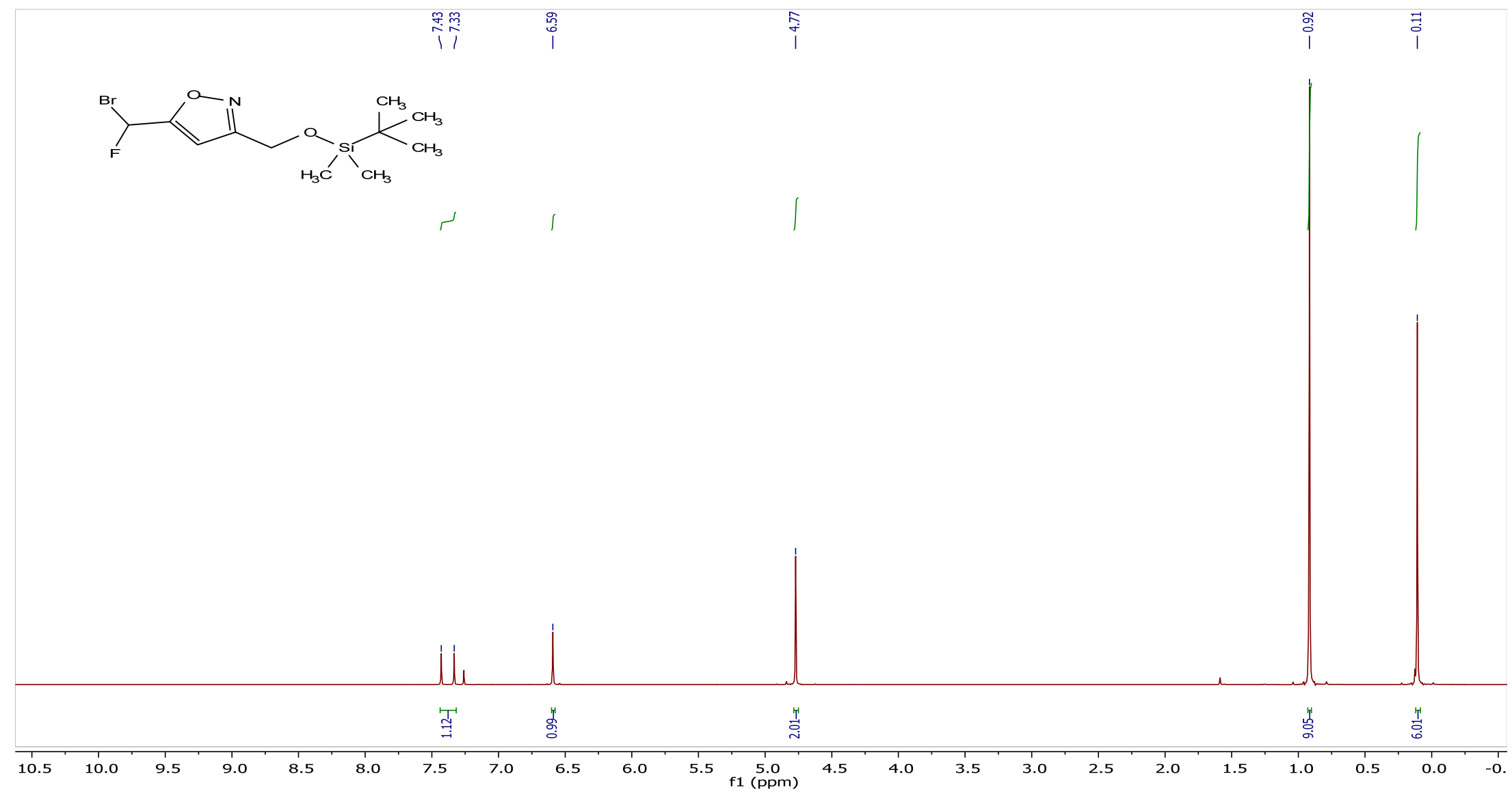


${ }^{13} \mathrm{C}\left\{{ }^{1} \mathrm{H}\right\}$ NMR spectrum of the compound 17e.

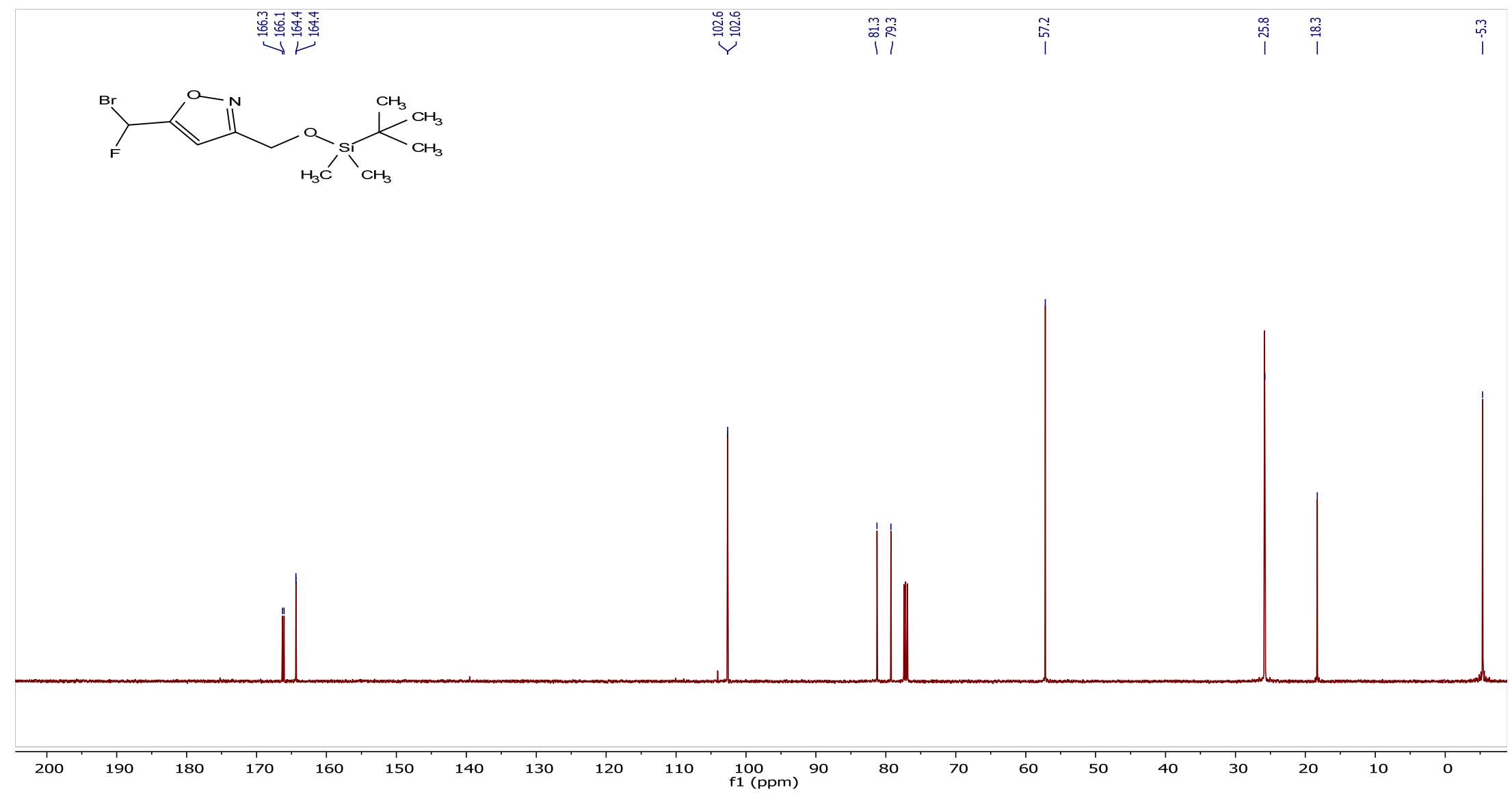


${ }^{19} \mathrm{~F}\left\{{ }^{1} \mathrm{H}\right\}$ NMR spectrum of the compound 17e.

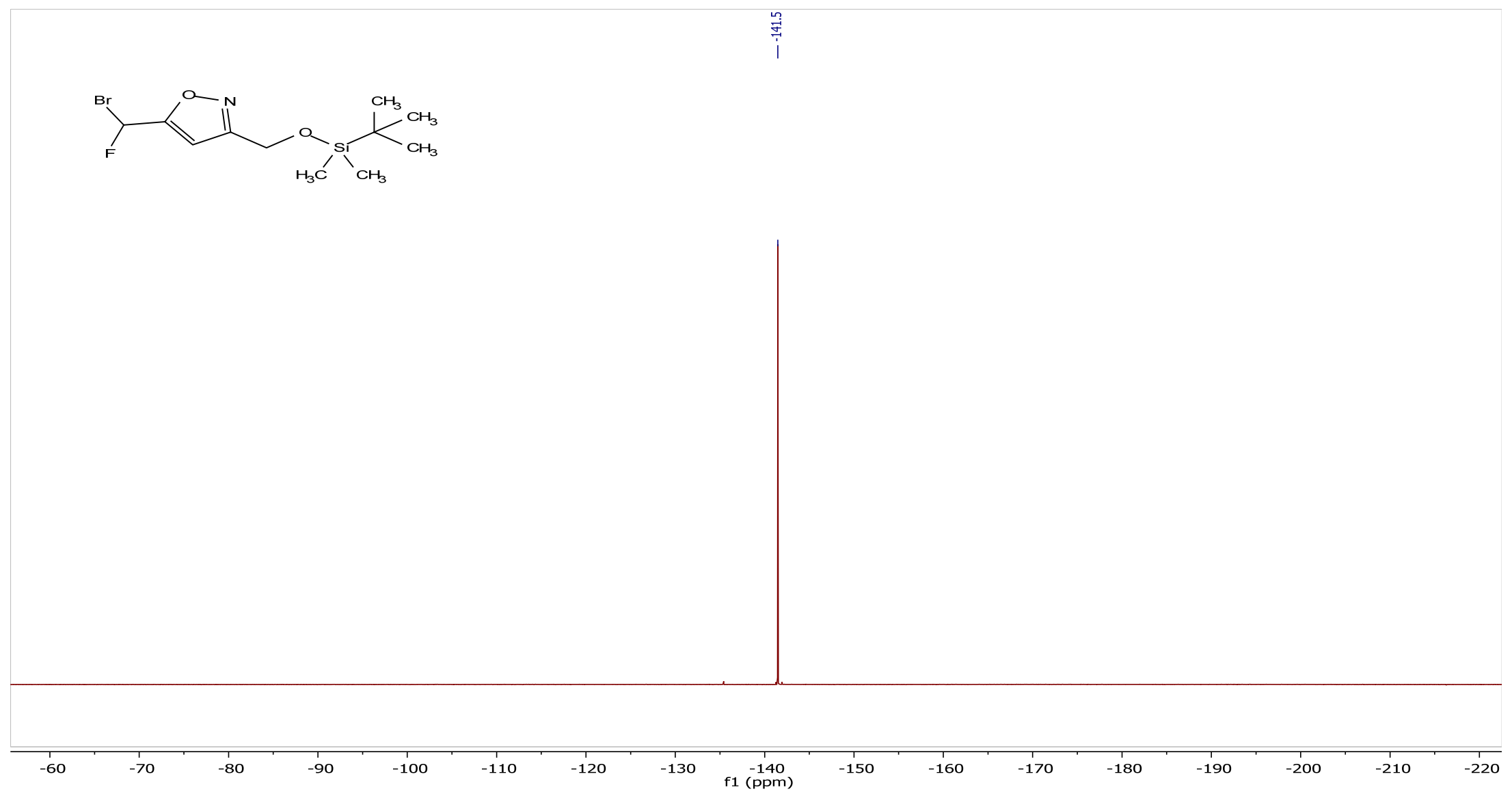




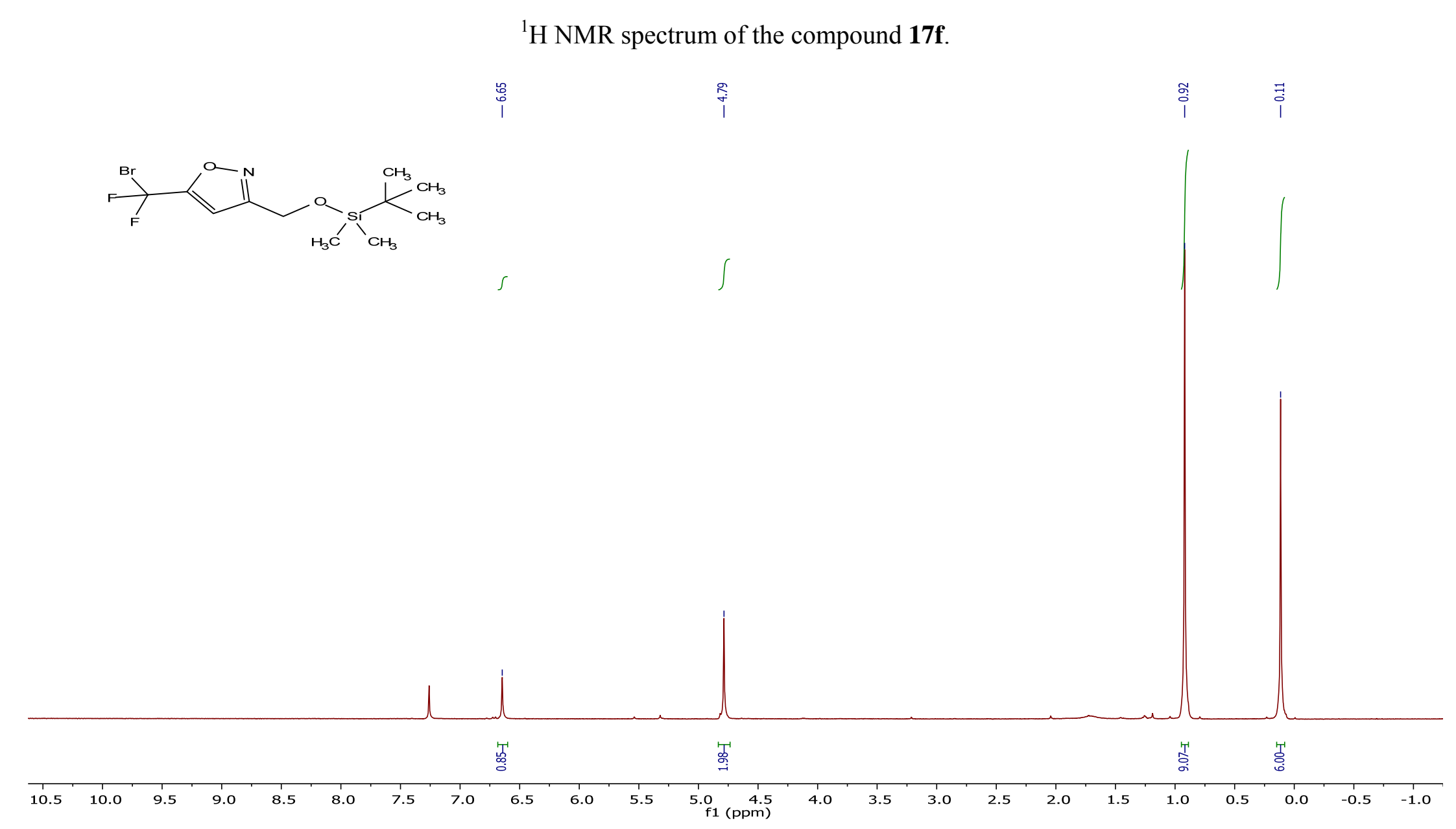


${ }^{13} \mathrm{C}\left\{{ }^{1} \mathrm{H}\right\}$ NMR spectrum of the compound $\mathbf{1 7 f}$.
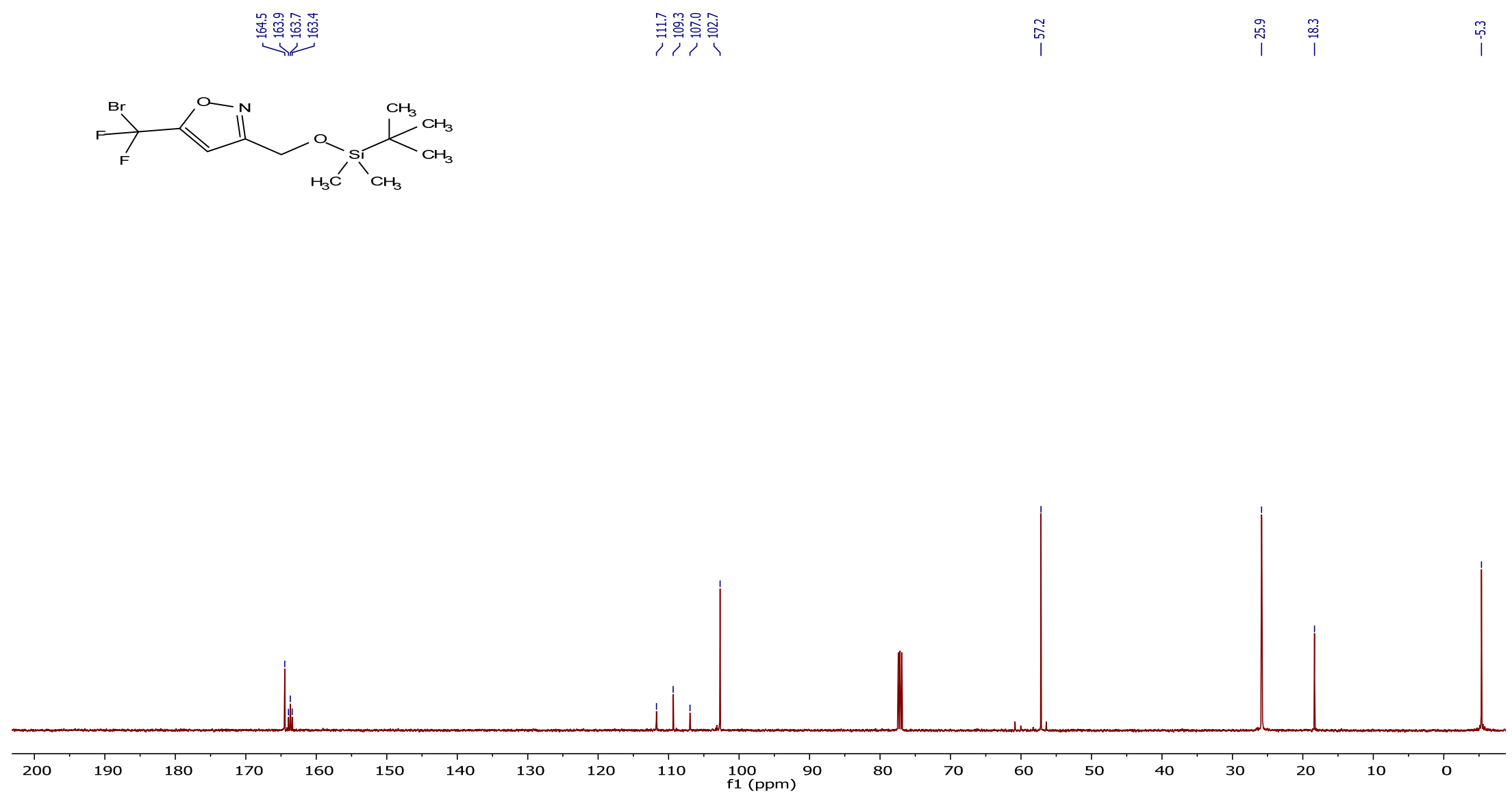
${ }^{19} \mathrm{~F}\left\{{ }^{1} \mathrm{H}\right\}$ NMR spectrum of the compound $\mathbf{1 7 f}$.

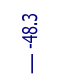
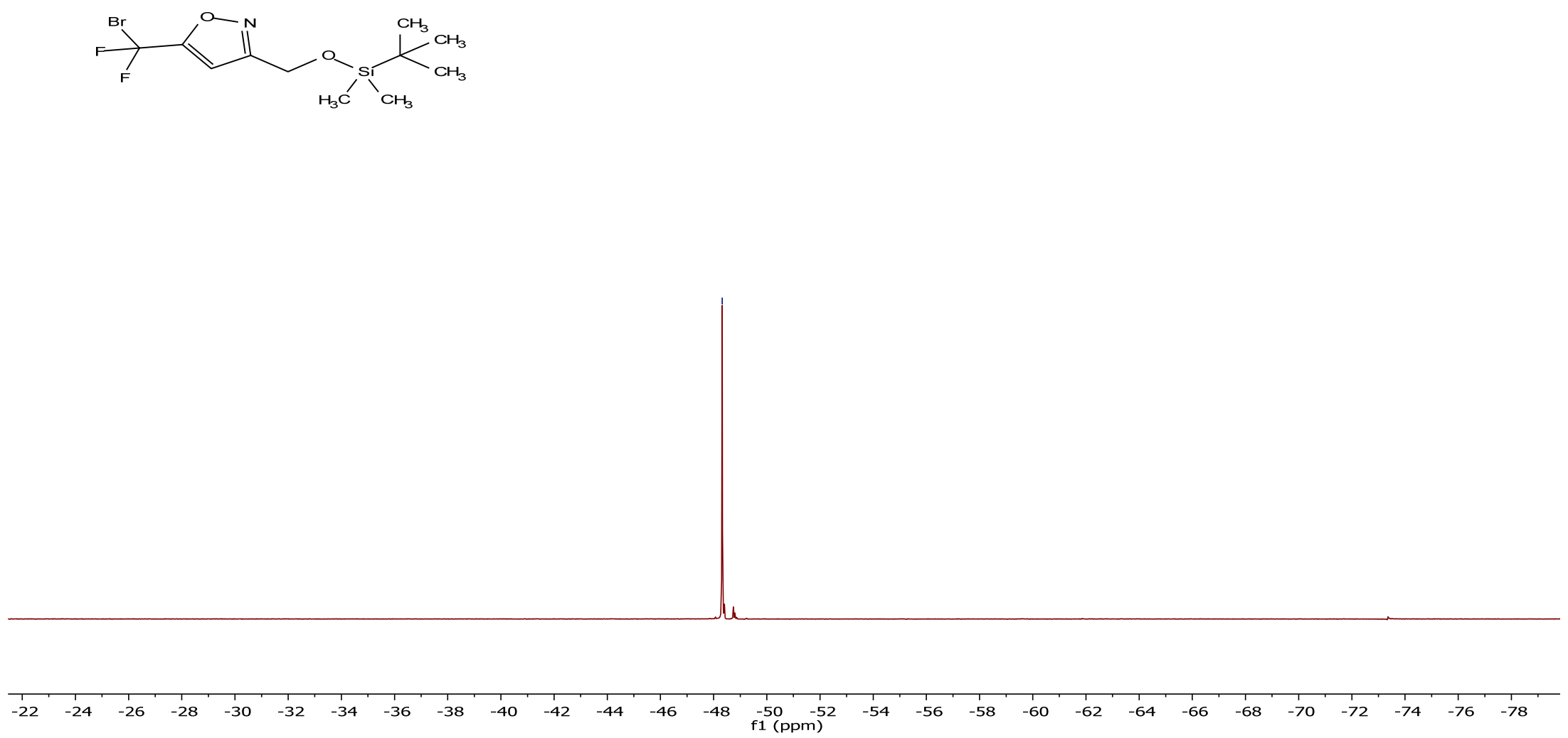
${ }^{1} \mathrm{H}$ NMR spectrum of the compound $\mathbf{1 7 g}$.

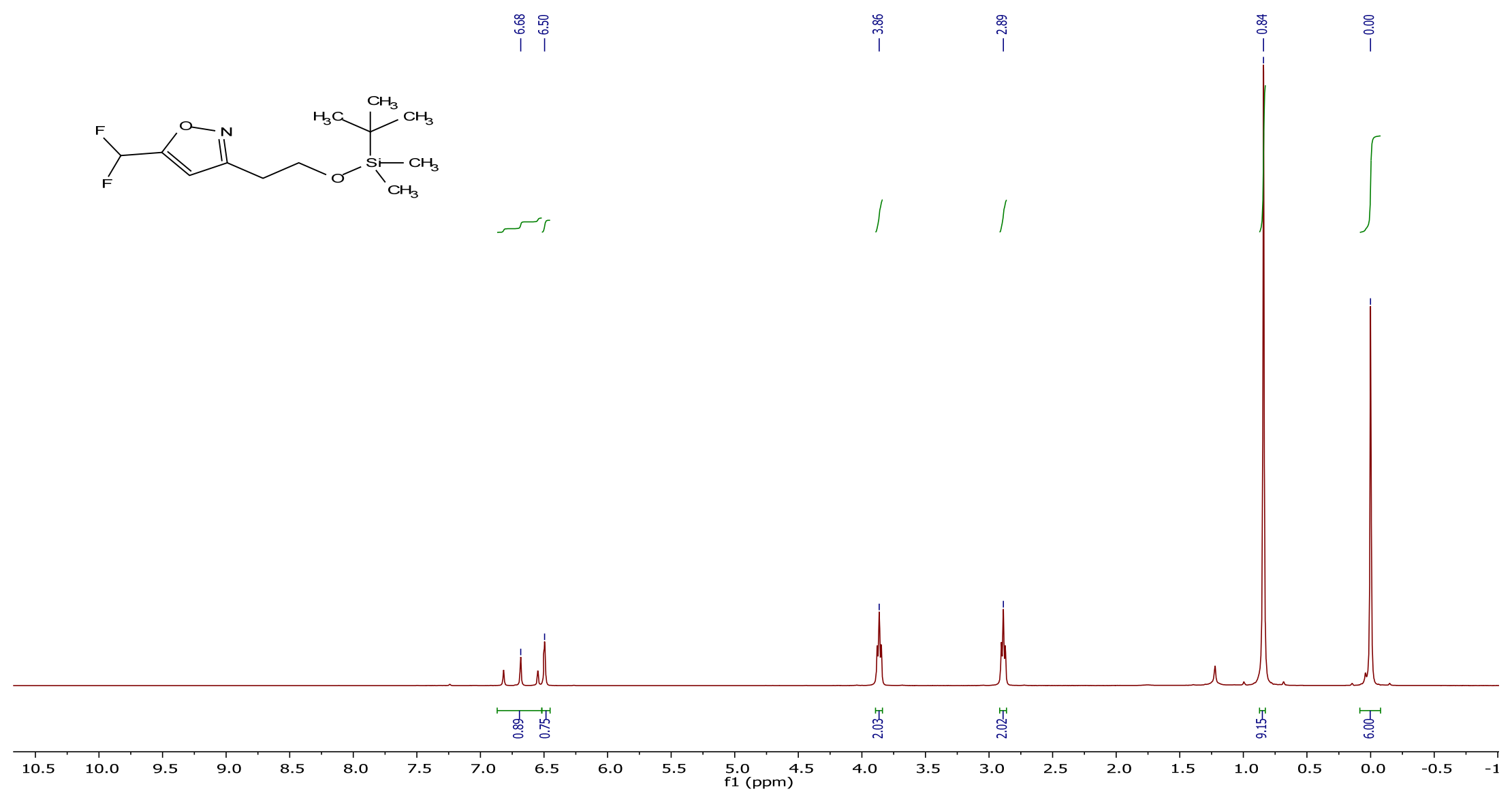


${ }^{13} \mathrm{C}\left\{{ }^{1} \mathrm{H}\right\}$ NMR spectrum of the compound $\mathbf{1 7 g}$.

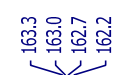

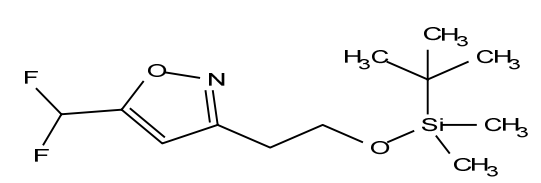

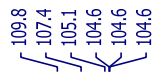

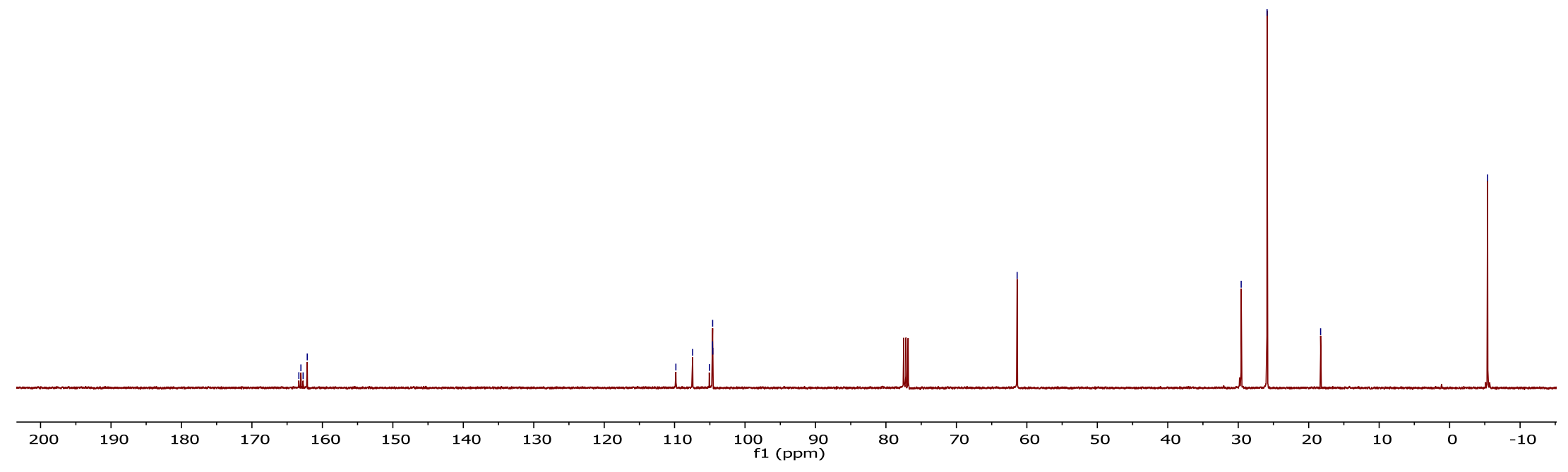


${ }^{19} \mathrm{~F}\left\{{ }^{1} \mathrm{H}\right\}$ NMR spectrum of the compound $\mathbf{1 7 g}$.

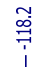
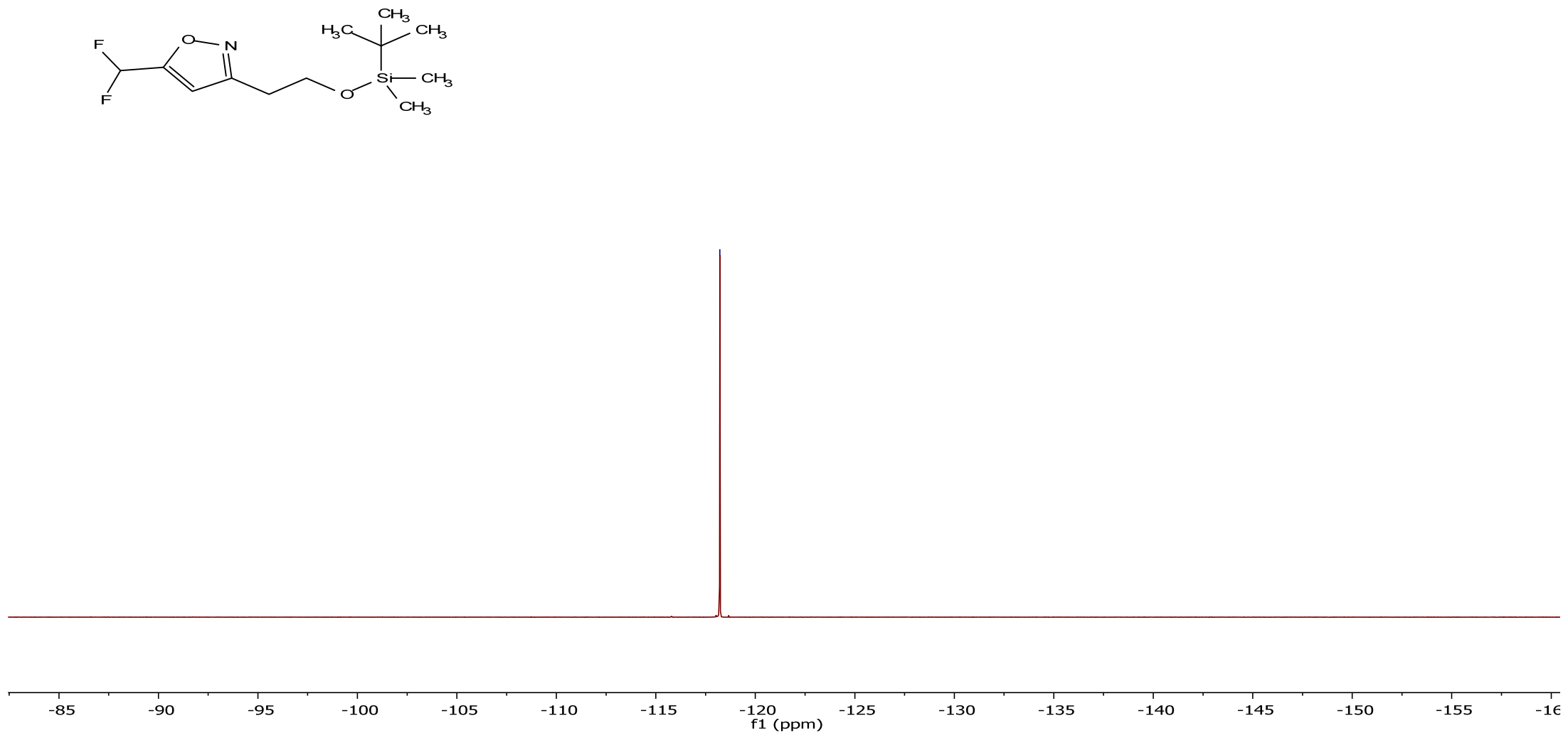
${ }^{1} \mathrm{H}$ NMR spectrum of the compound $\mathbf{1 7 h}$.

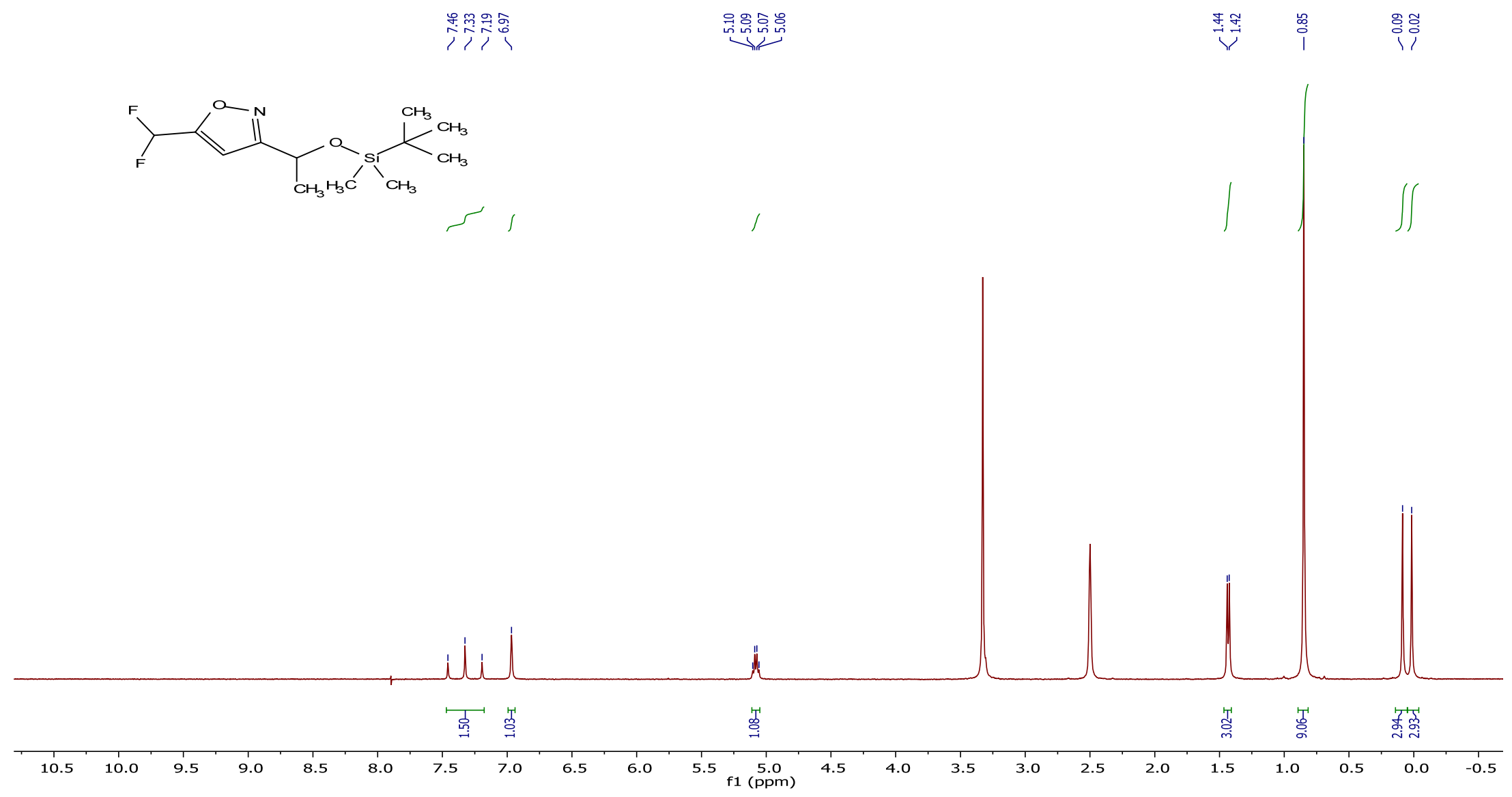


${ }^{13} \mathrm{C}\left\{{ }^{1} \mathrm{H}\right\}$ NMR spectrum of the compound $\mathbf{1 7 h}$.

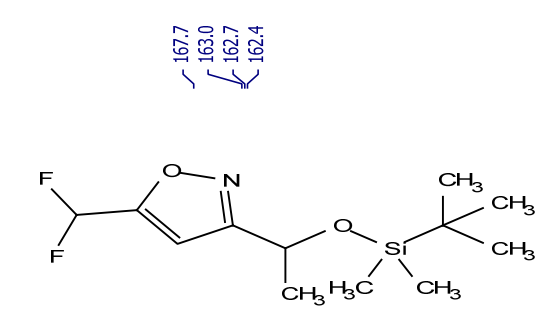

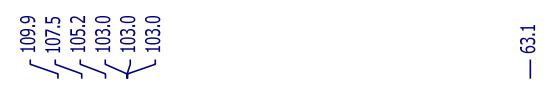

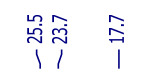

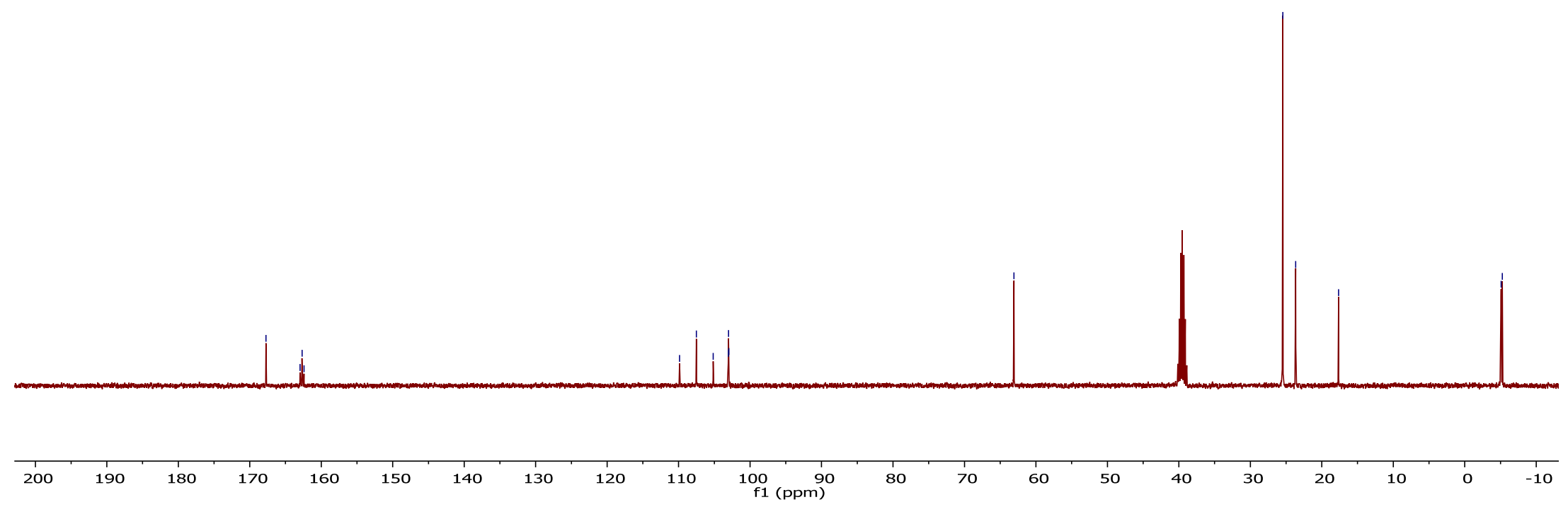


${ }^{19} \mathrm{~F}\left\{{ }^{1} \mathrm{H}\right\}$ NMR spectrum of the compound $\mathbf{1 7 h}$.

$\stackrel{9}{\stackrel{9}{\longrightarrow}}$
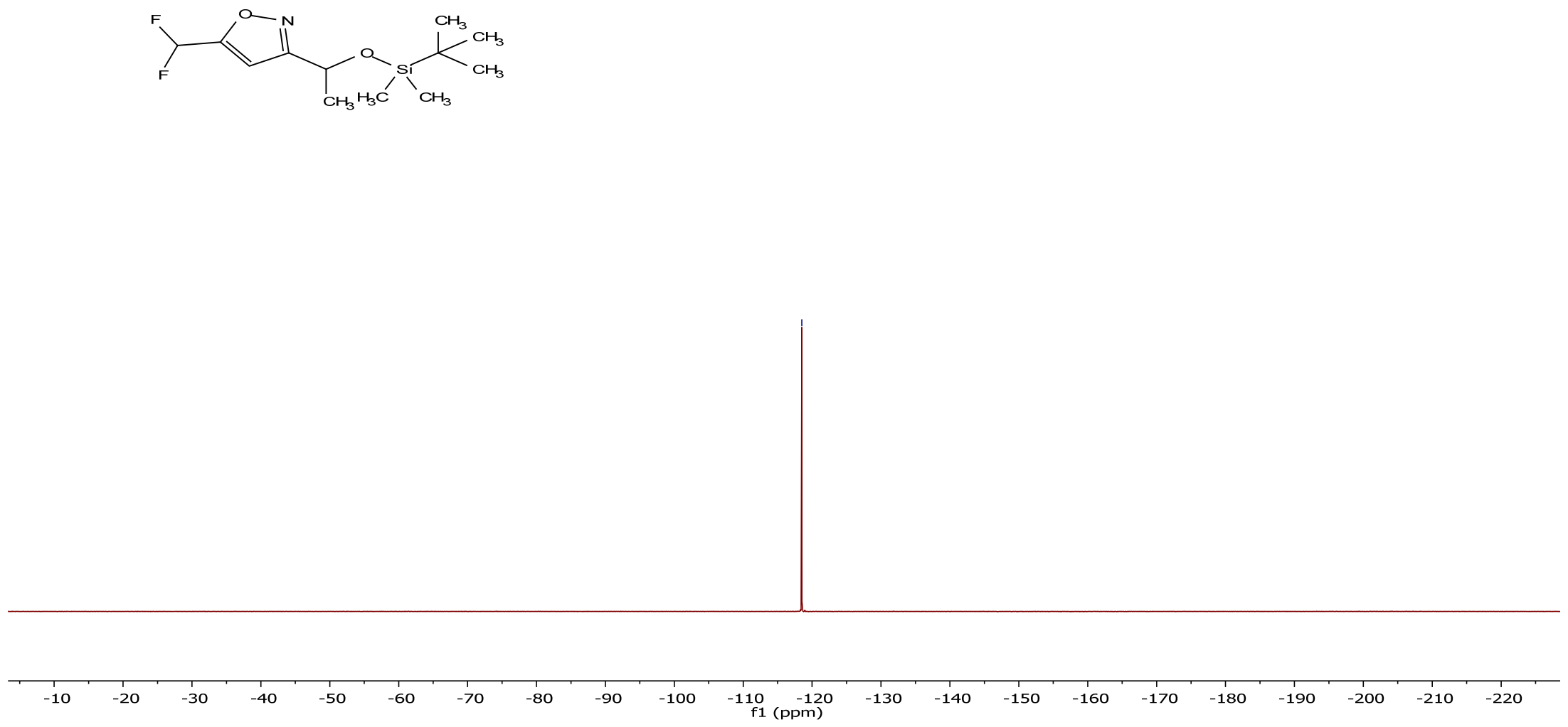
${ }^{1} \mathrm{H}$ NMR spectrum of the compound 21a.
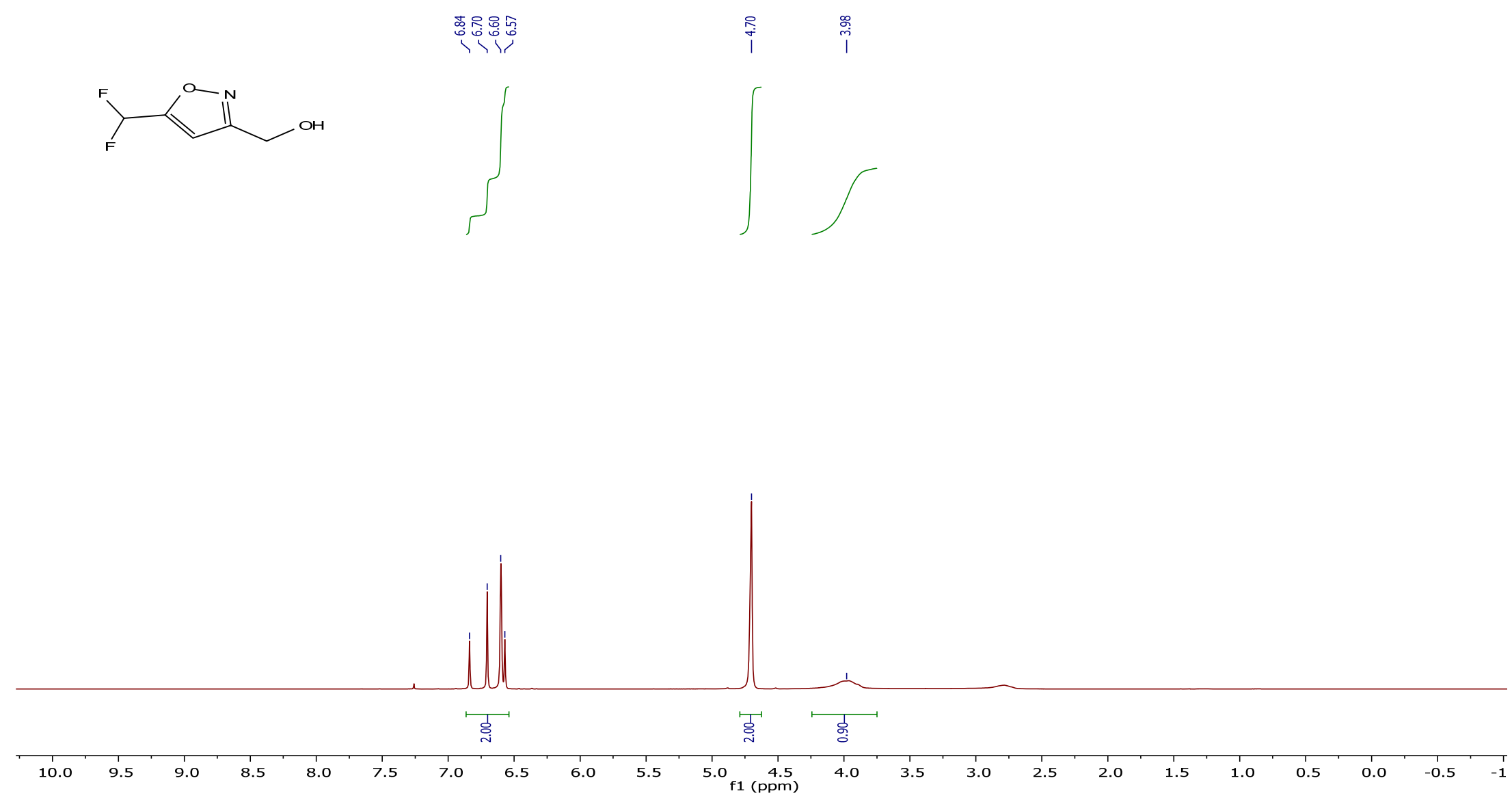
${ }^{13} \mathrm{C}\left\{{ }^{1} \mathrm{H}\right\}$ NMR spectrum of the compound 21a.

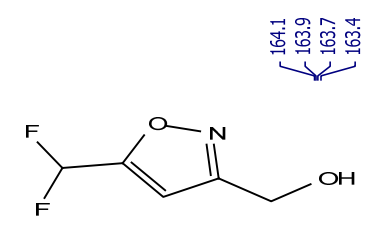

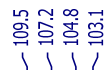

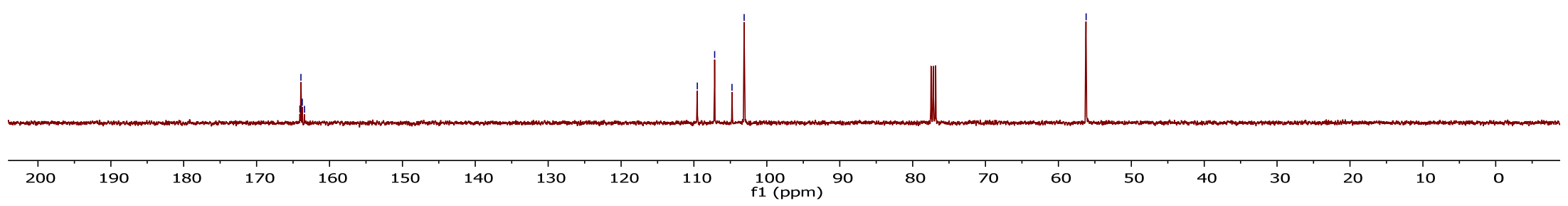


${ }^{19}$ F NMR spectrum of the compound 21a.

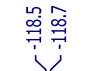
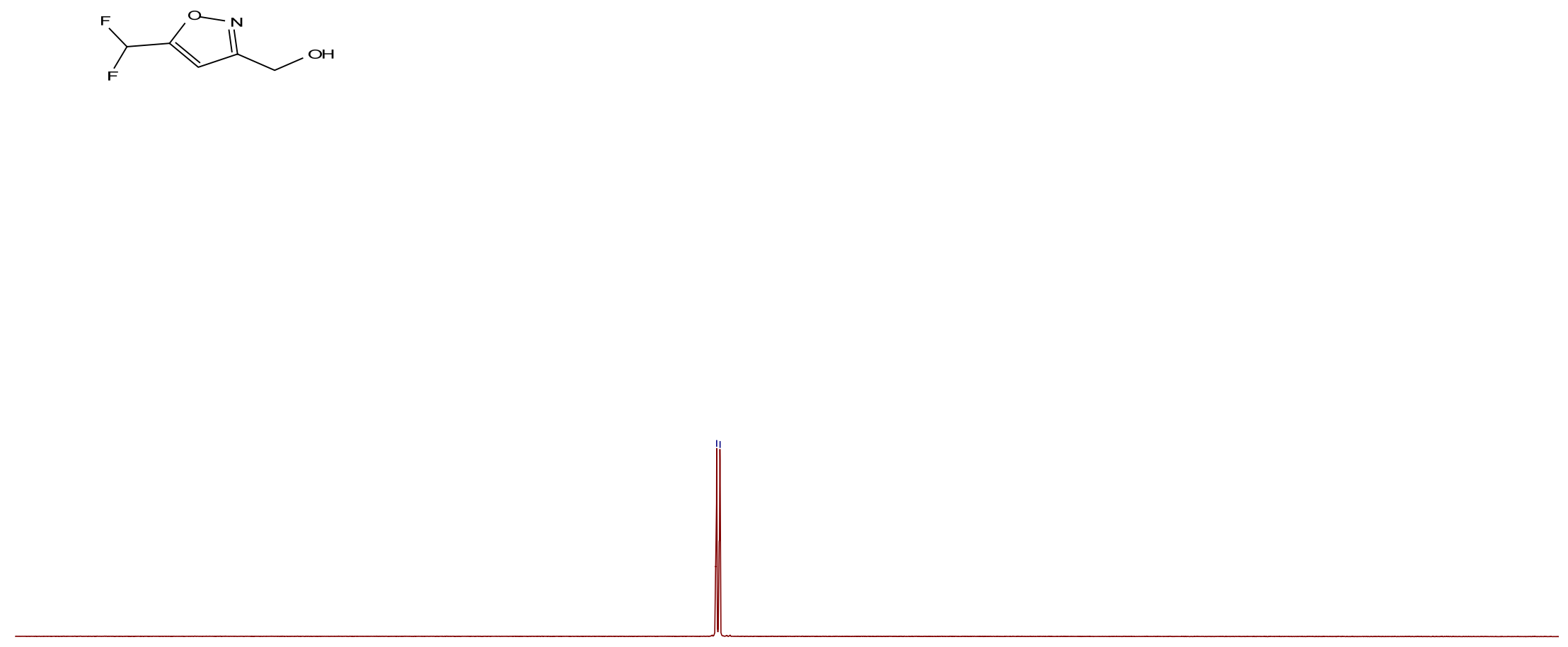

$-90-92-94-96-98-100-102-104-106-108-110-112-114-116-118-120-122-124-126-128-130-132-134-136-138-140-142-144-146-148-150-152-154$ 
${ }^{1} \mathrm{H}$ NMR spectrum of the compound $21 \mathbf{b}$.
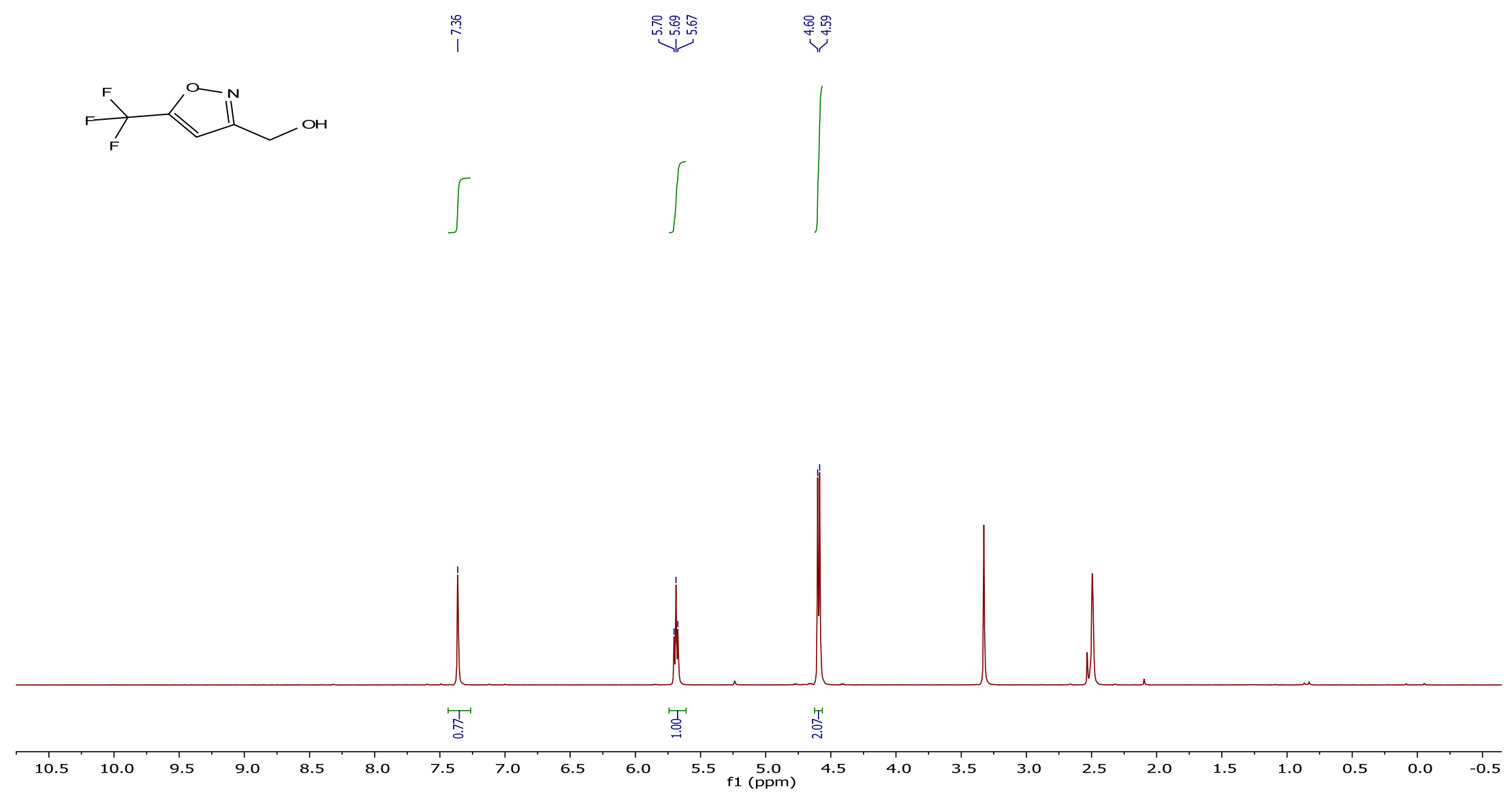
${ }^{13} \mathrm{C}\left\{{ }^{1} \mathrm{H}\right\}$ NMR spectrum of the compound 21b.

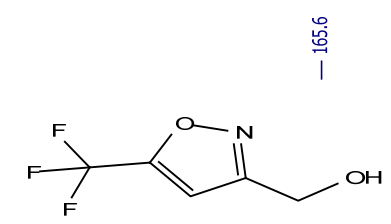

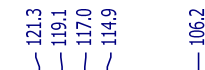

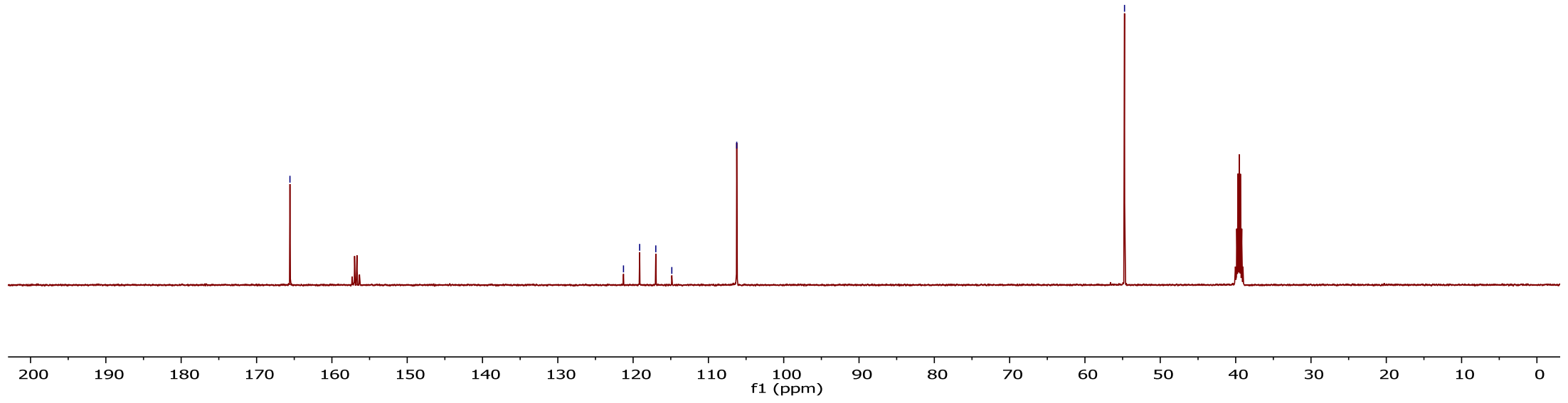


${ }^{19} \mathrm{~F}\left\{{ }^{1} \mathrm{H}\right\}$ NMR spectrum of the compound 21b.

草
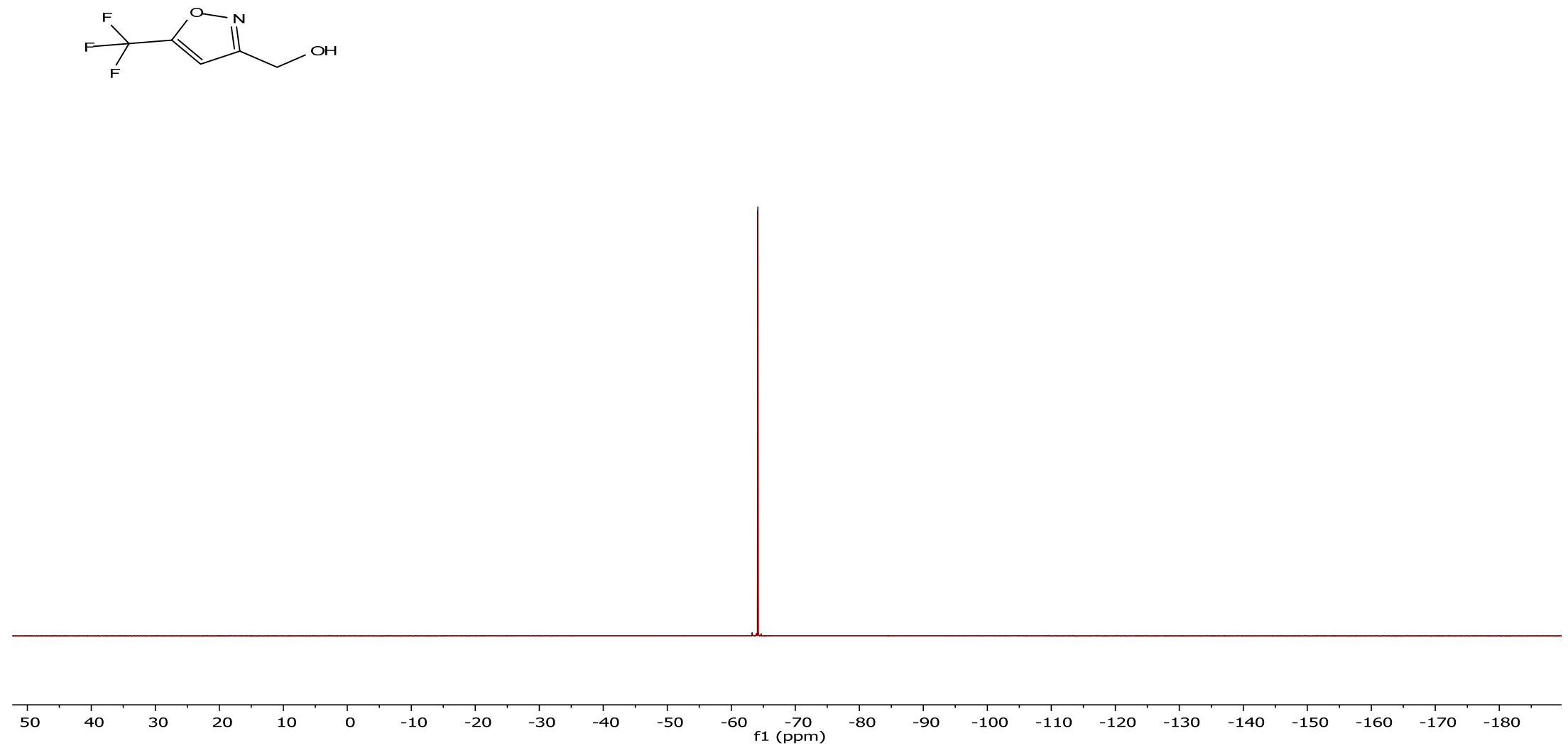
${ }^{1} \mathrm{H}$ NMR spectrum of the compound 21c.

每<smiles>CC(F)(F)c1cc(CO)no1</smiles><smiles>[C]1C=CC=C1</smiles>

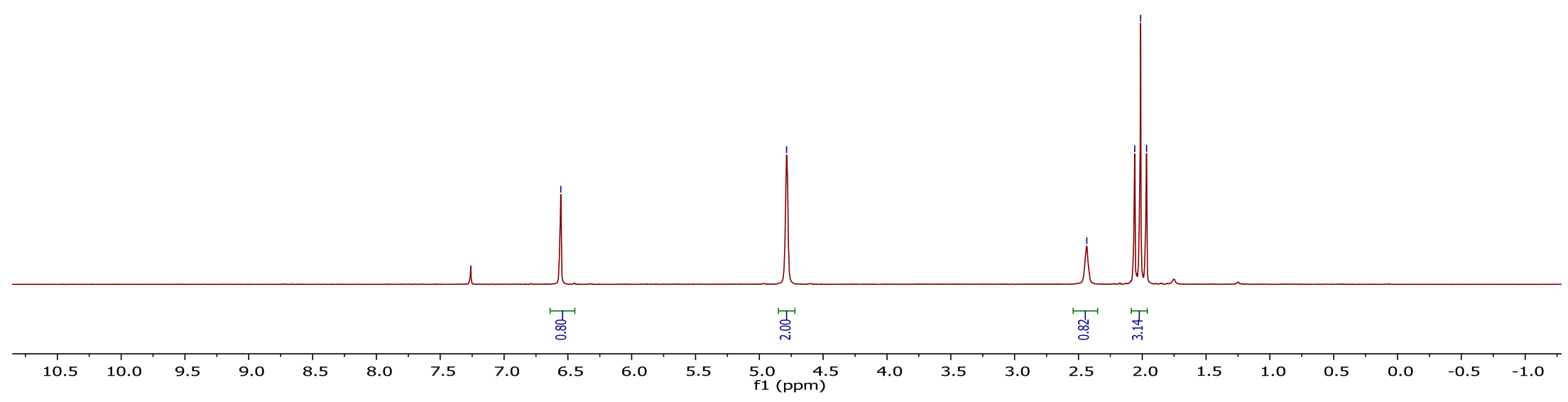


${ }^{13} \mathrm{C}\left\{{ }^{1} \mathrm{H}\right\}$ NMR spectrum of the compound 21c.

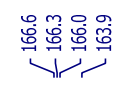

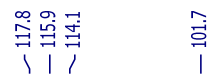

i

ํํำ
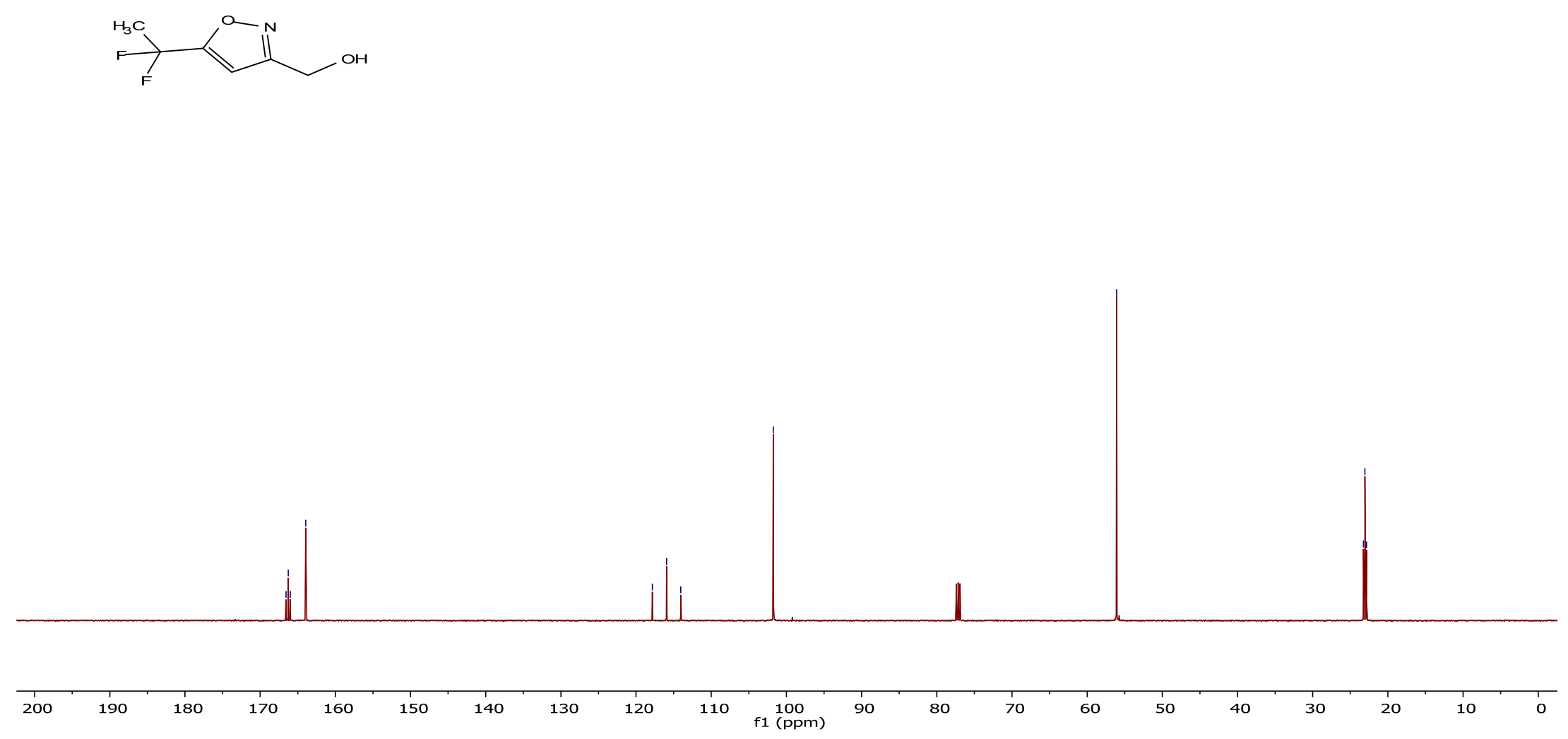
${ }^{19} \mathrm{~F}\left\{{ }^{1} \mathrm{H}\right\}$ NMR spectrum of the compound 21c.

$\stackrel{+}{\circ}$
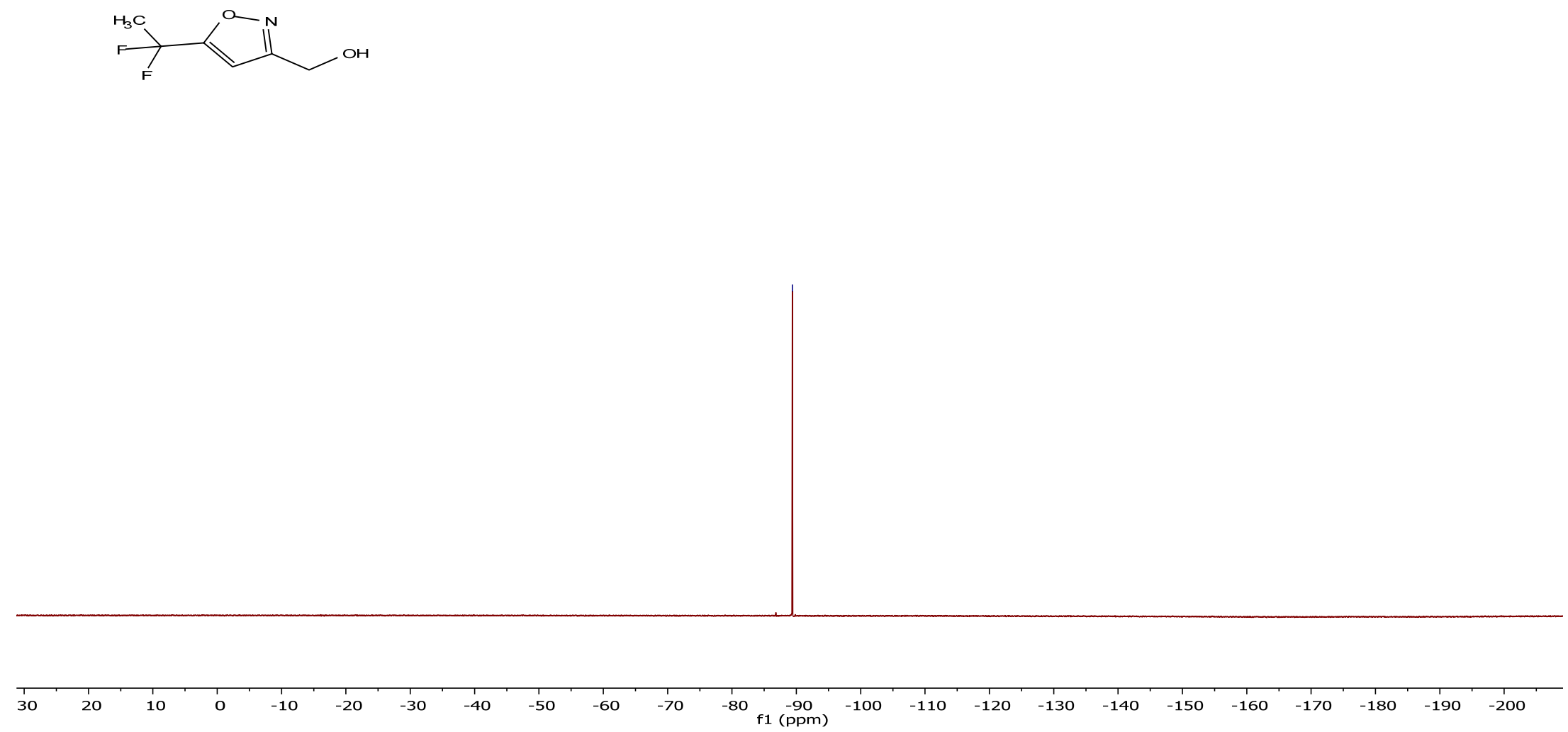
${ }^{1} \mathrm{H}$ NMR spectrum of the compound 21d.
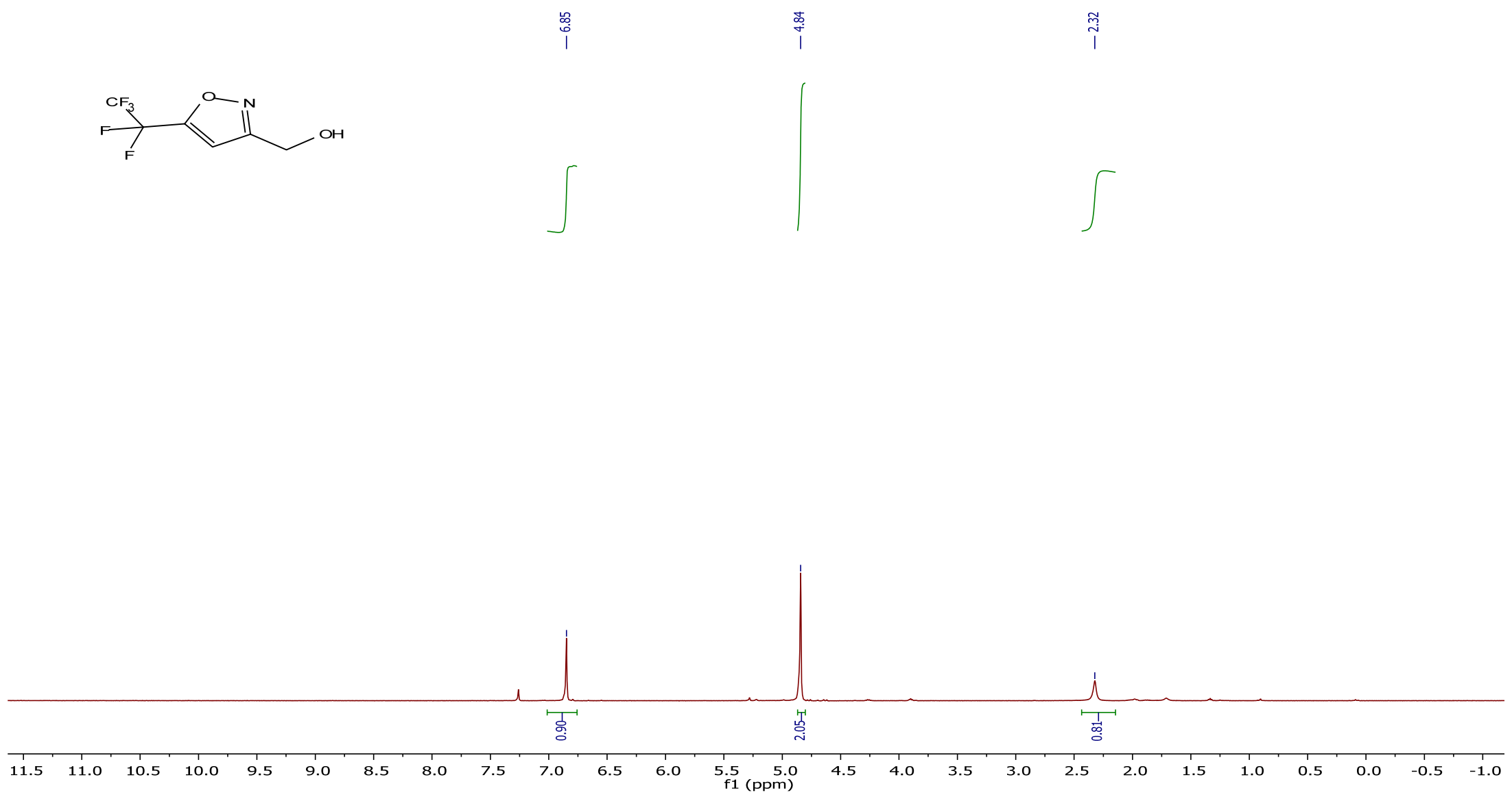
<smiles>OCc1cc(C(F)(F)F)on1</smiles>

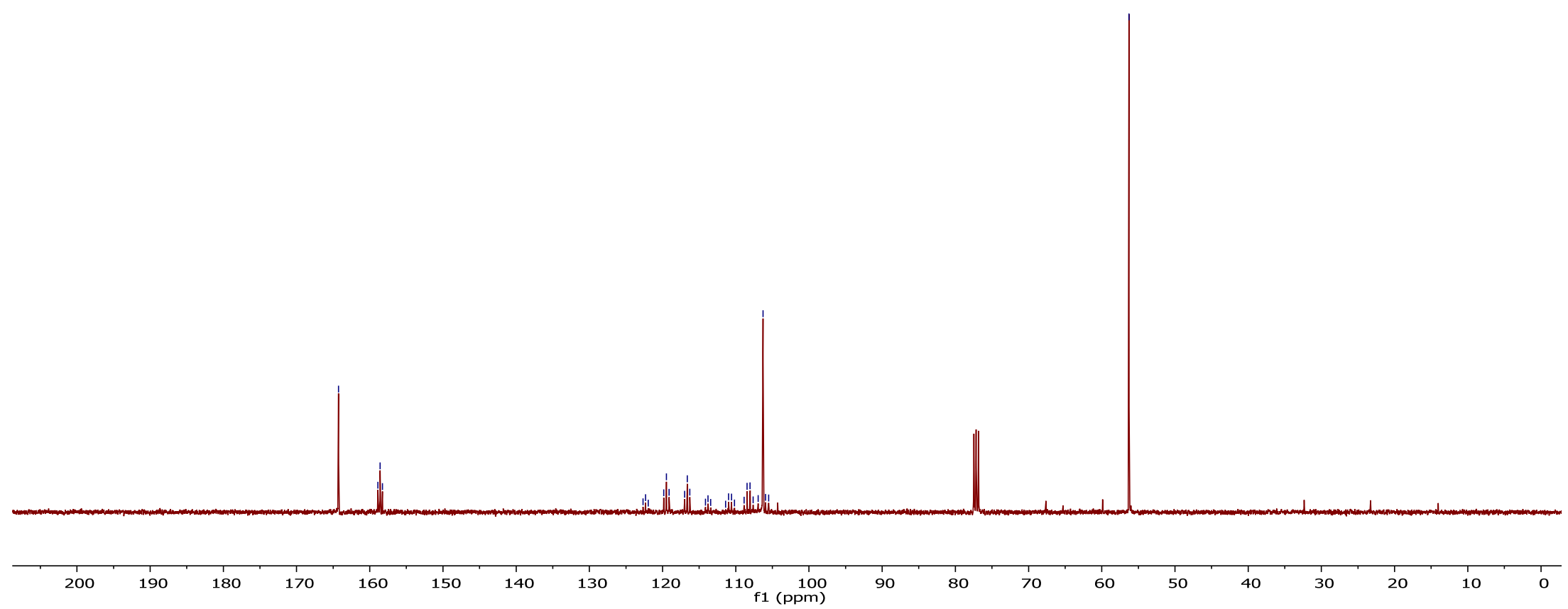


${ }^{19} \mathrm{~F}\left\{{ }^{1} \mathrm{H}\right\}$ NMR spectrum of the compound 21d.
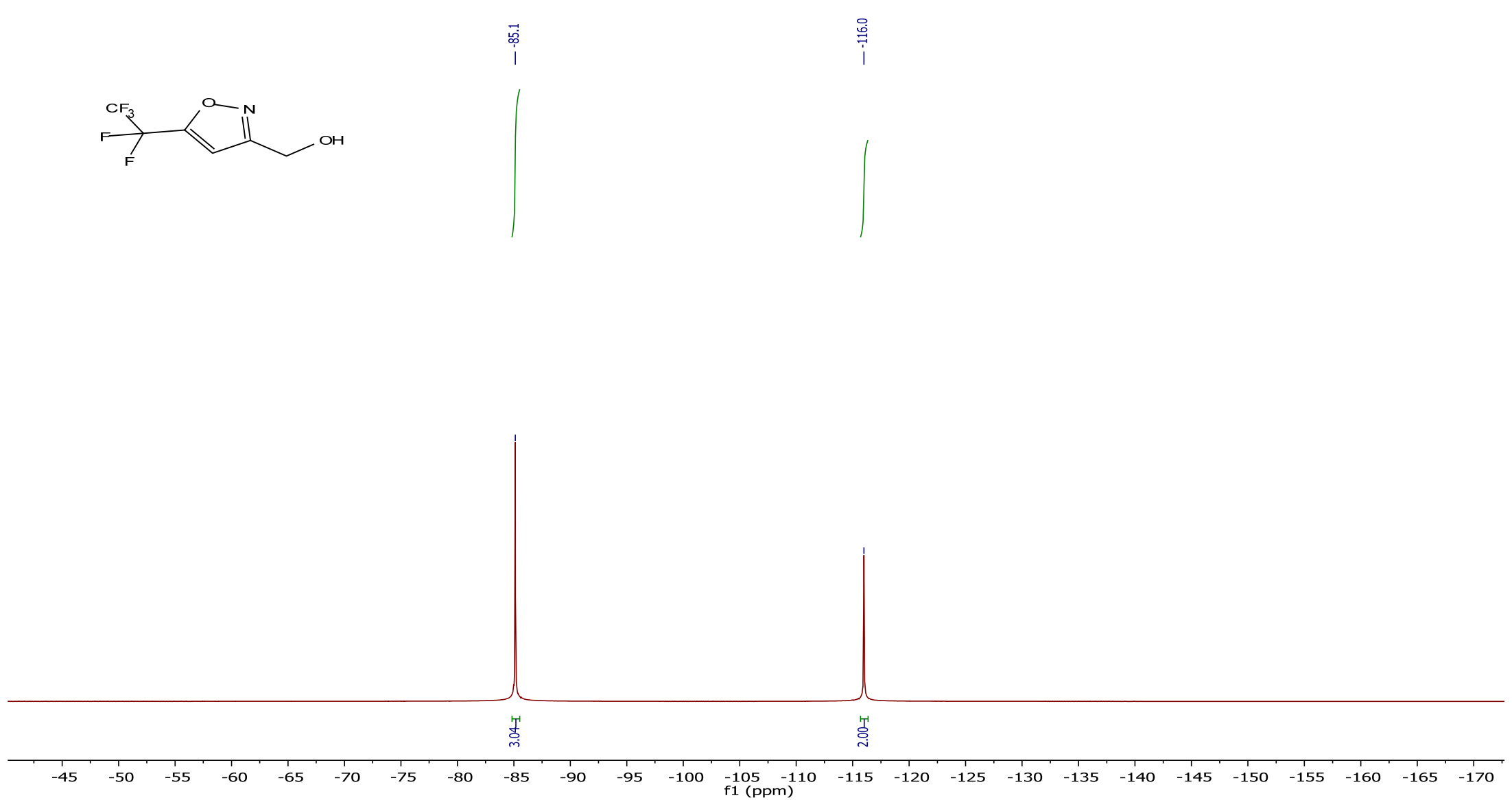
${ }^{1} \mathrm{H}$ NMR spectrum of the compound 21 .

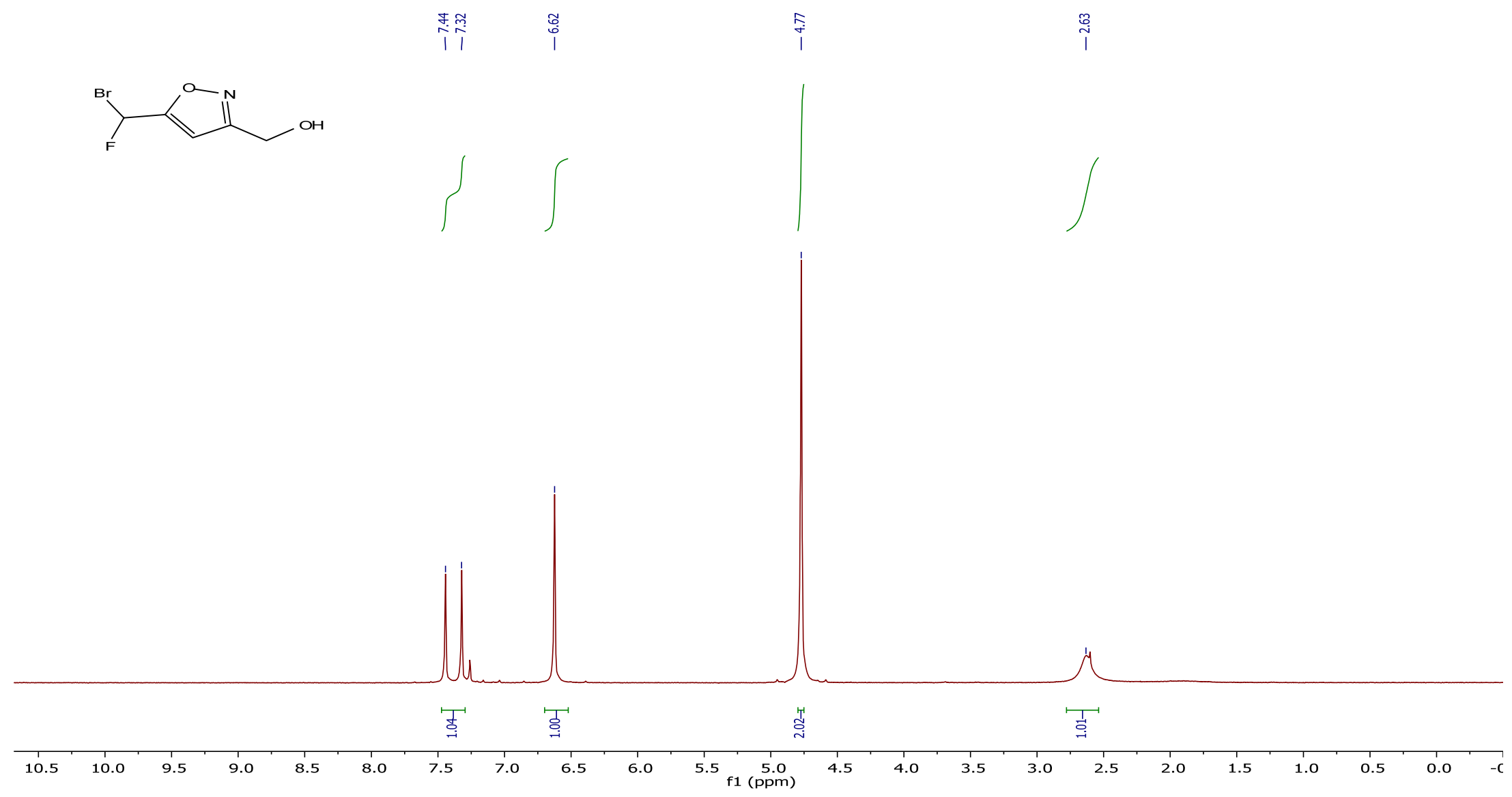


${ }^{13} \mathrm{C}\left\{{ }^{1} \mathrm{H}\right\}$ NMR spectrum of the compound 21e.

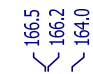

$>^{\mathrm{Br}}{ }^{\mathrm{N}}$
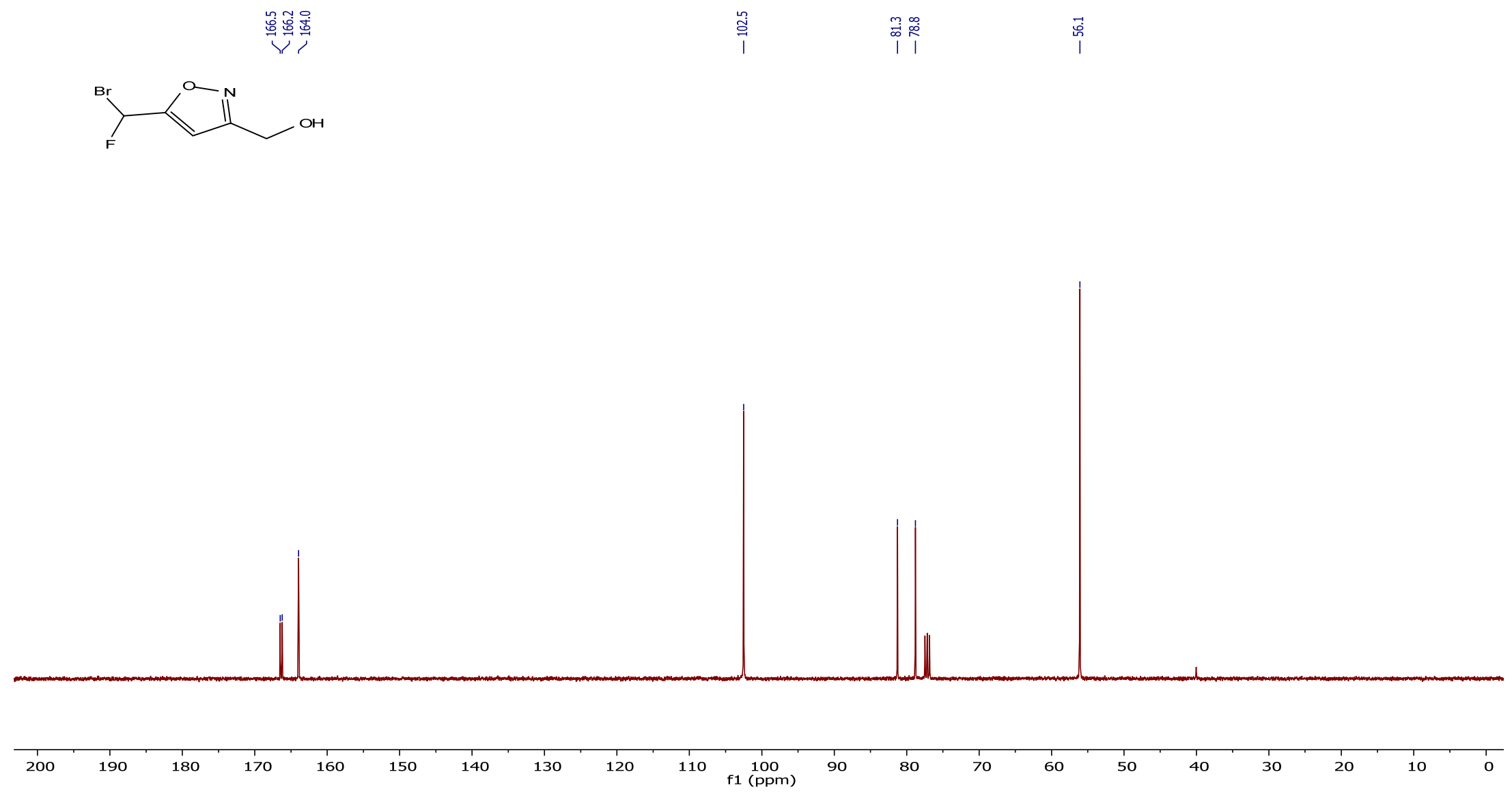
${ }^{19} \mathrm{~F}\left\{{ }^{1} \mathrm{H}\right\}$ NMR spectrum of the compound 21e.

$$
\stackrel{9}{\overrightarrow{7}}
$$<smiles>[B]C([B])c1cc(CO)no1</smiles>

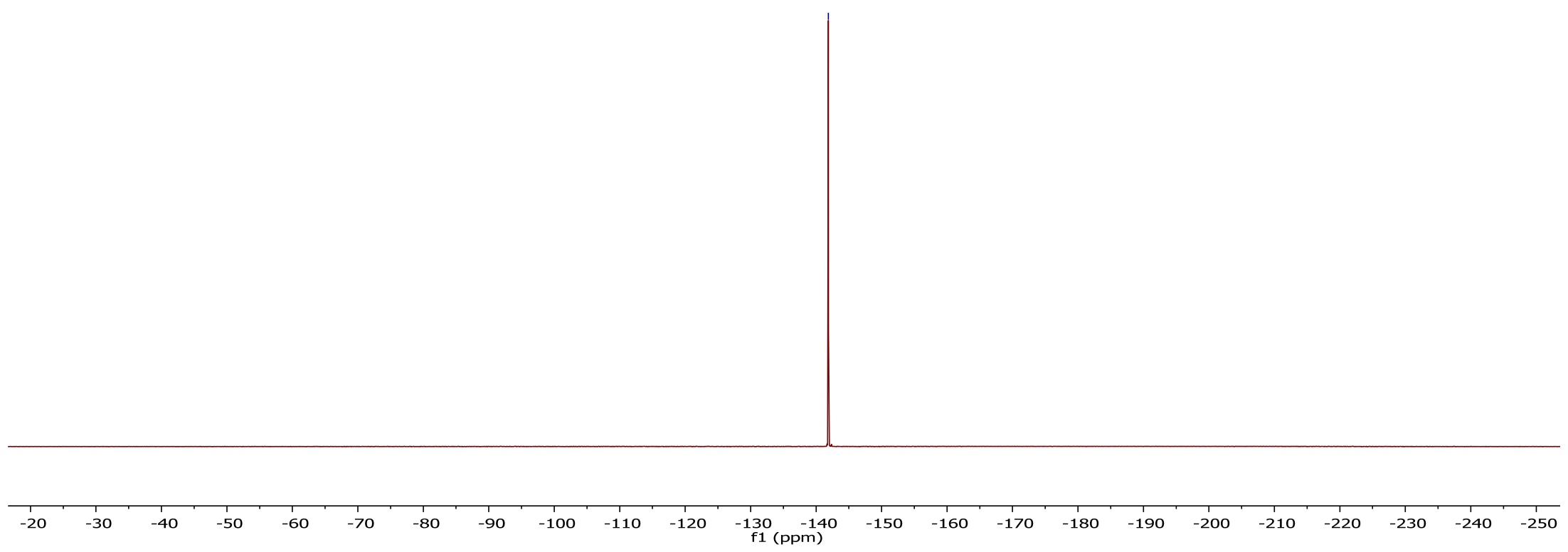


${ }^{1} \mathrm{H}$ NMR spectrum of the compound 21g.
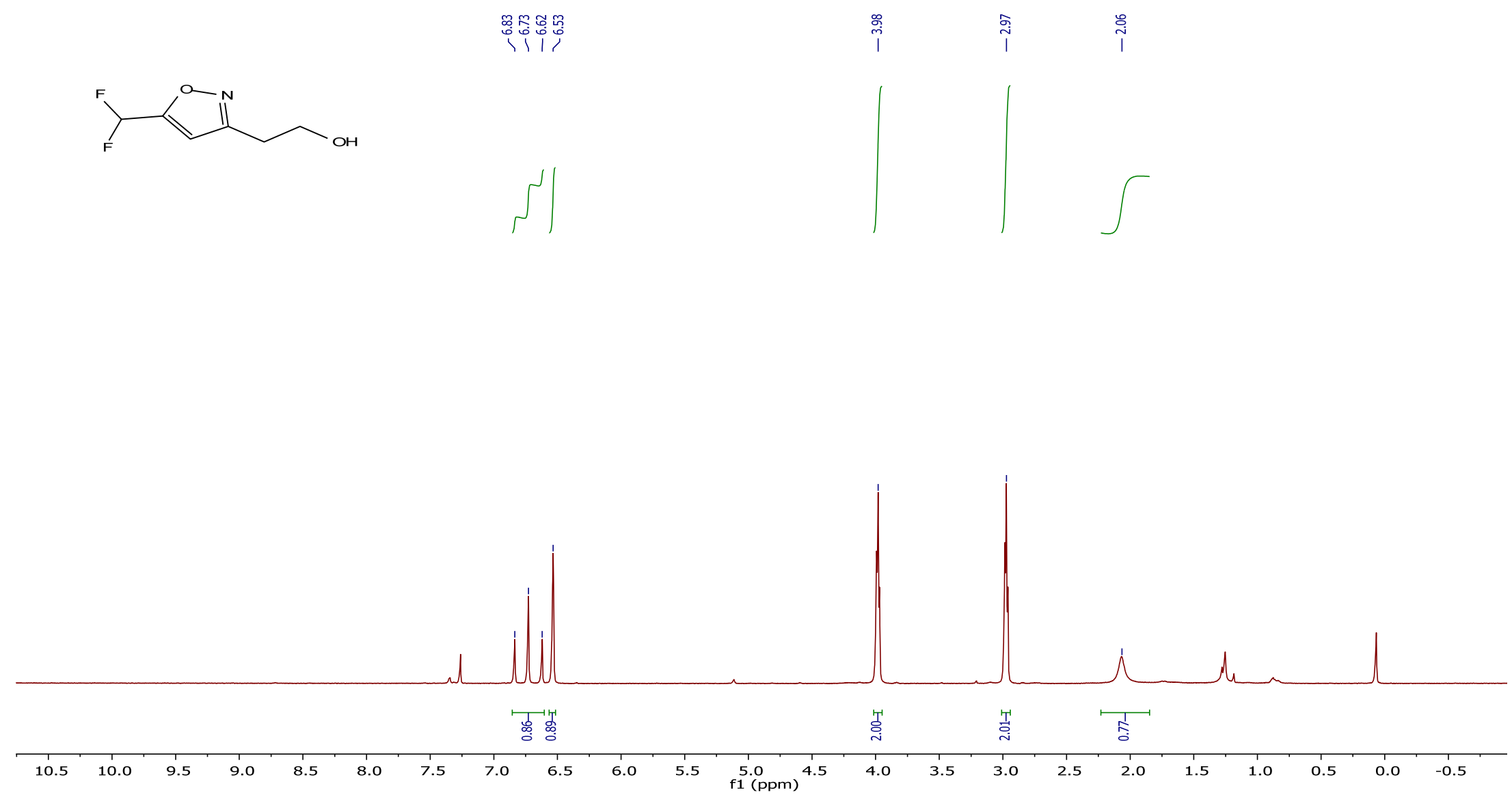
${ }^{13} \mathrm{C}\left\{{ }^{1} \mathrm{H}\right\}$ NMR spectrum of the compound 21g.
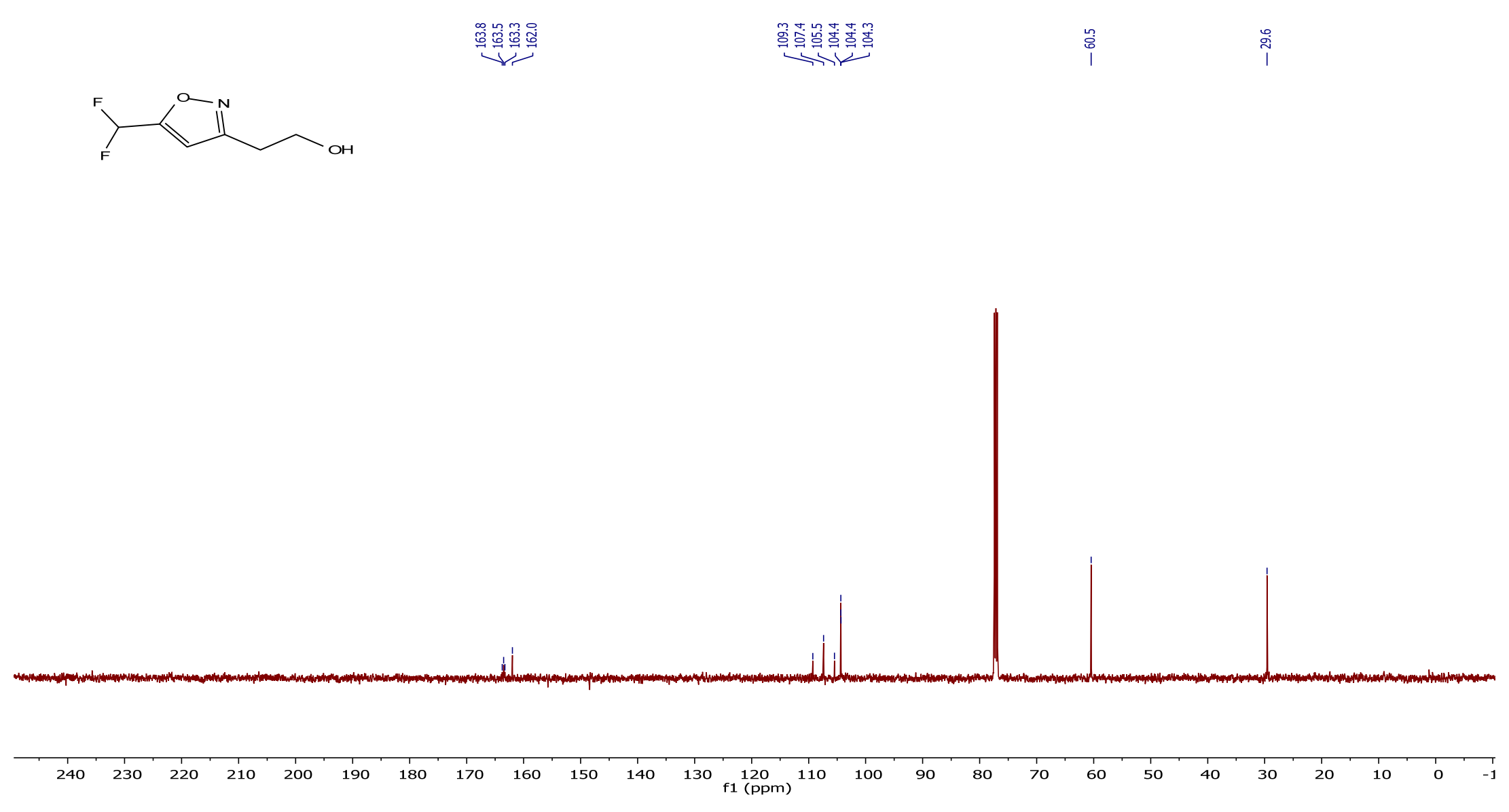
${ }^{19} \mathrm{~F}\left\{{ }^{1} \mathrm{H}\right\}$ NMR spectrum of the compound 21g.

$\stackrel{\infty}{\rightrightarrows}$
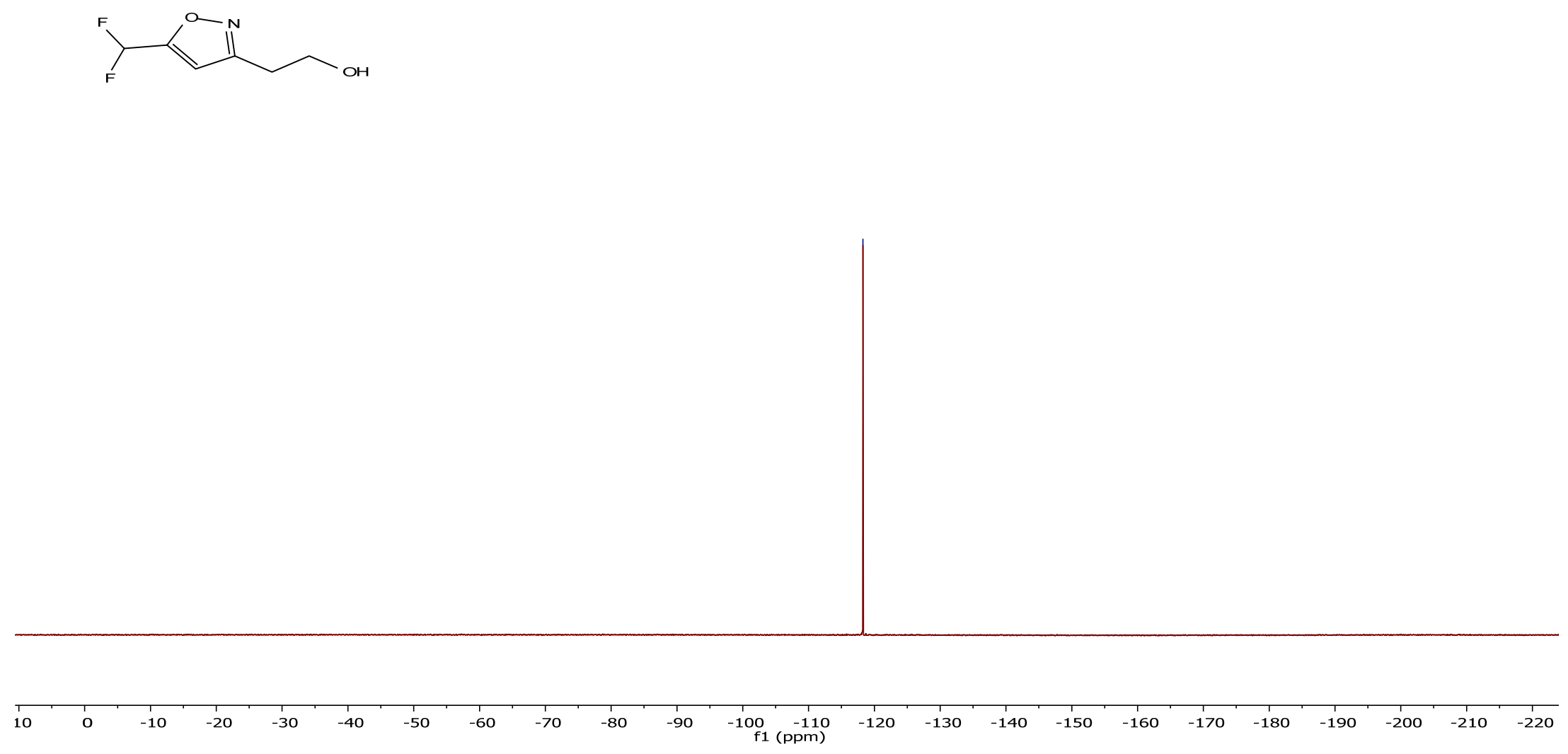
${ }^{1} \mathrm{H}$ NMR spectrum of the compound $\mathbf{2 1 h}$.
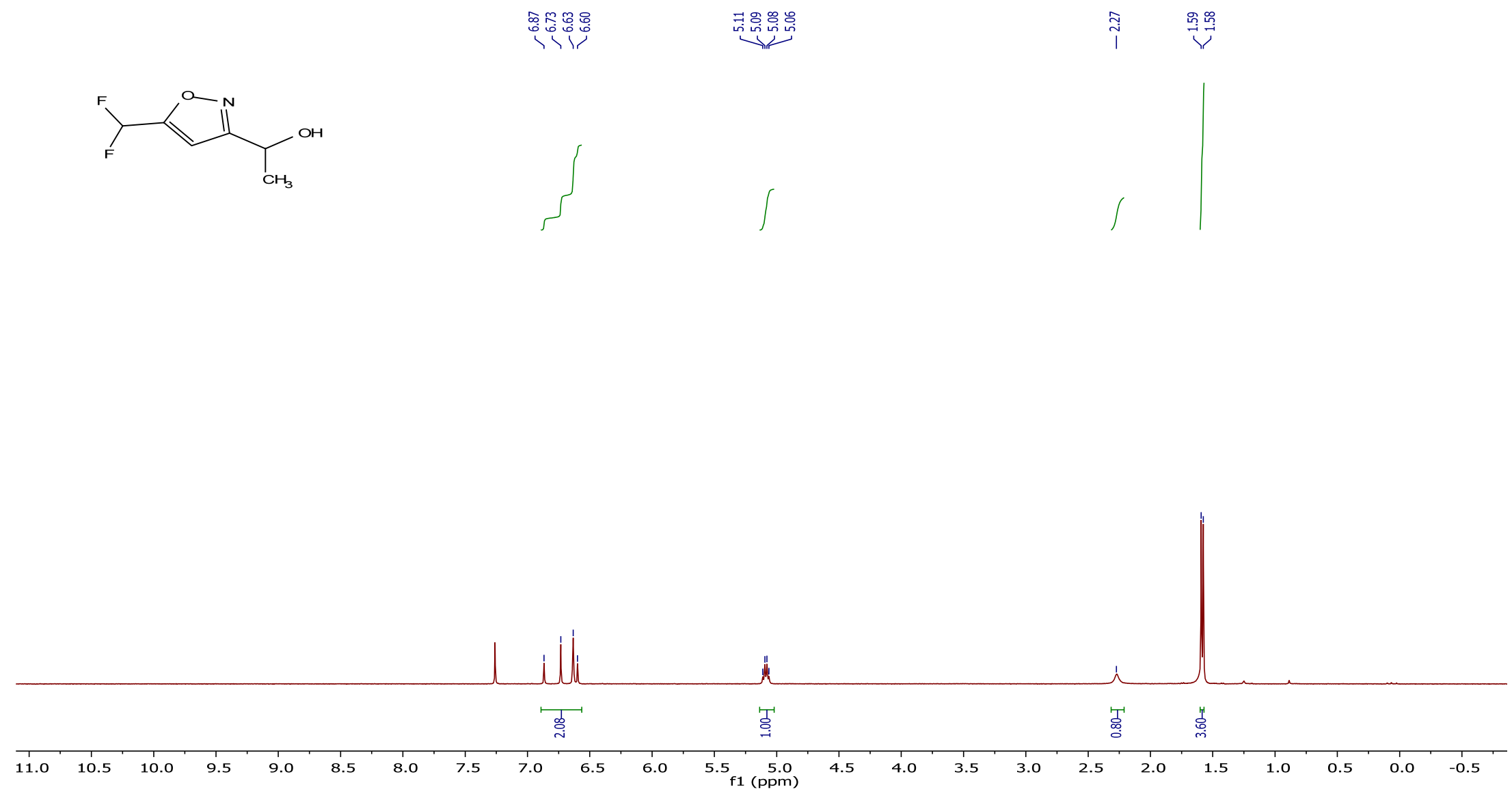
${ }^{13} \mathrm{C}\left\{{ }^{1} \mathrm{H}\right\}$ NMR spectrum of the compound 21h.

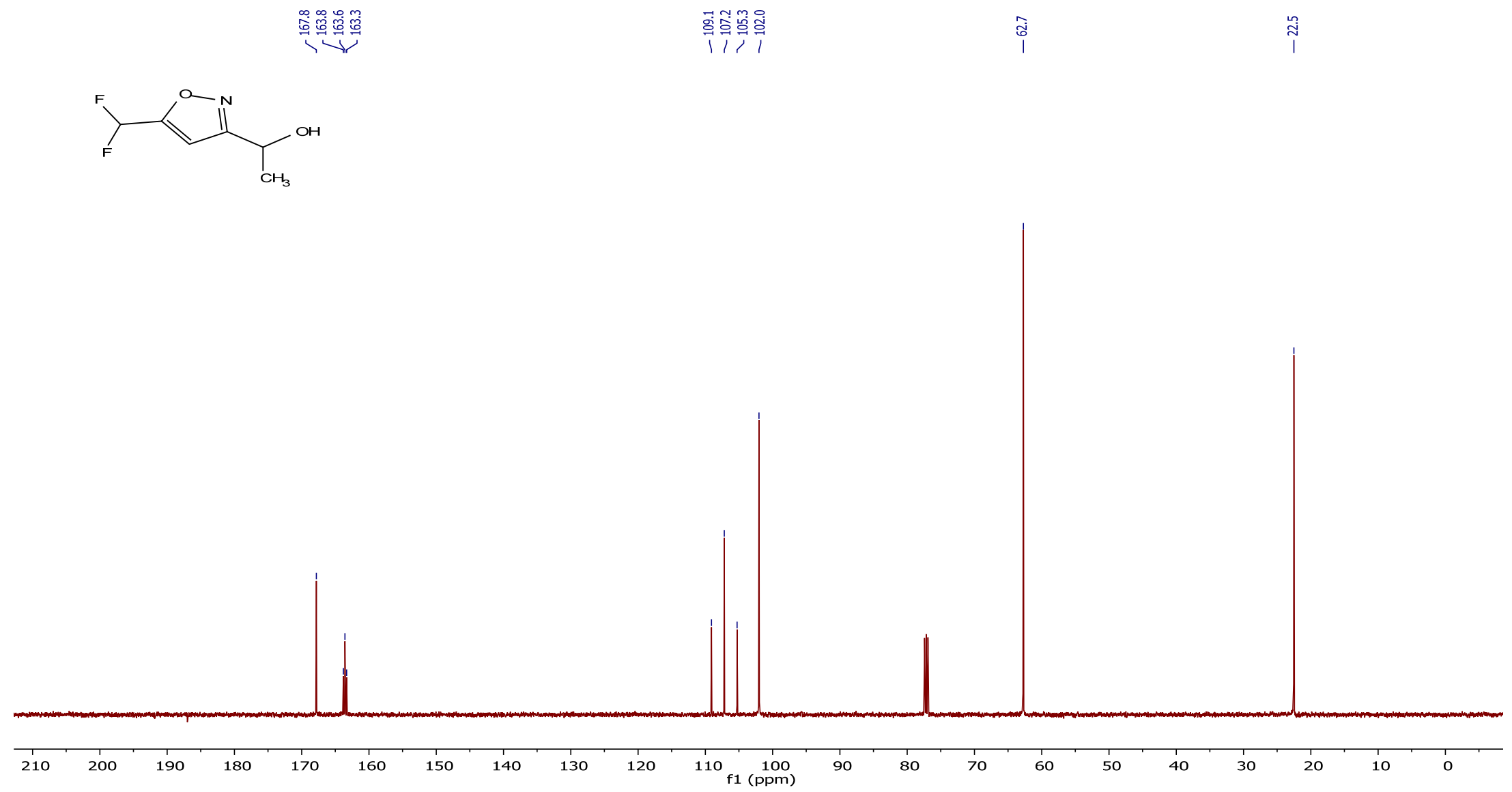


${ }^{19} \mathrm{~F}$ NMR spectrum of the compound $\mathbf{2 1 h}$.

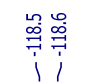
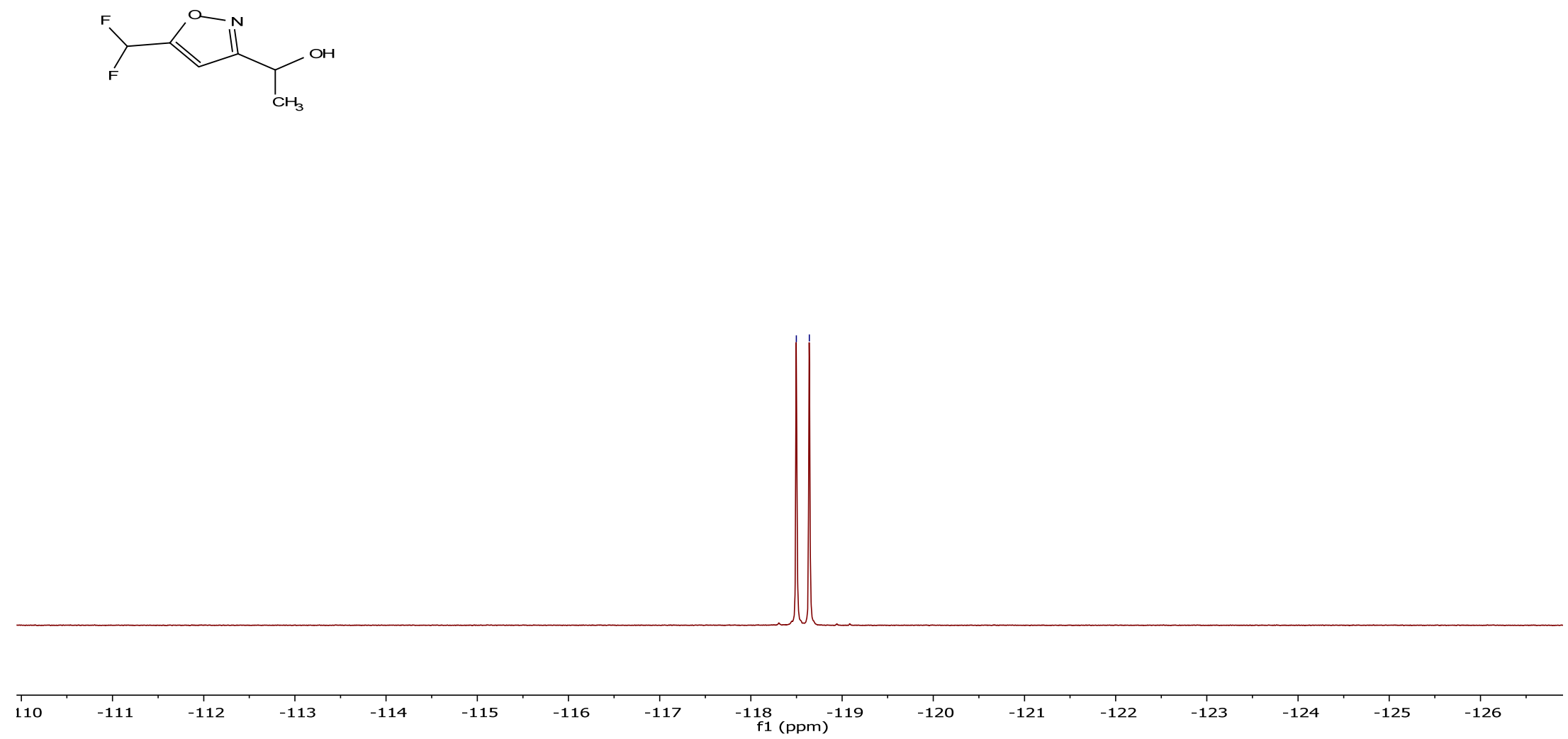
${ }^{1} \mathrm{H}$ NMR spectrum of the compound $20 \mathbf{b}$.

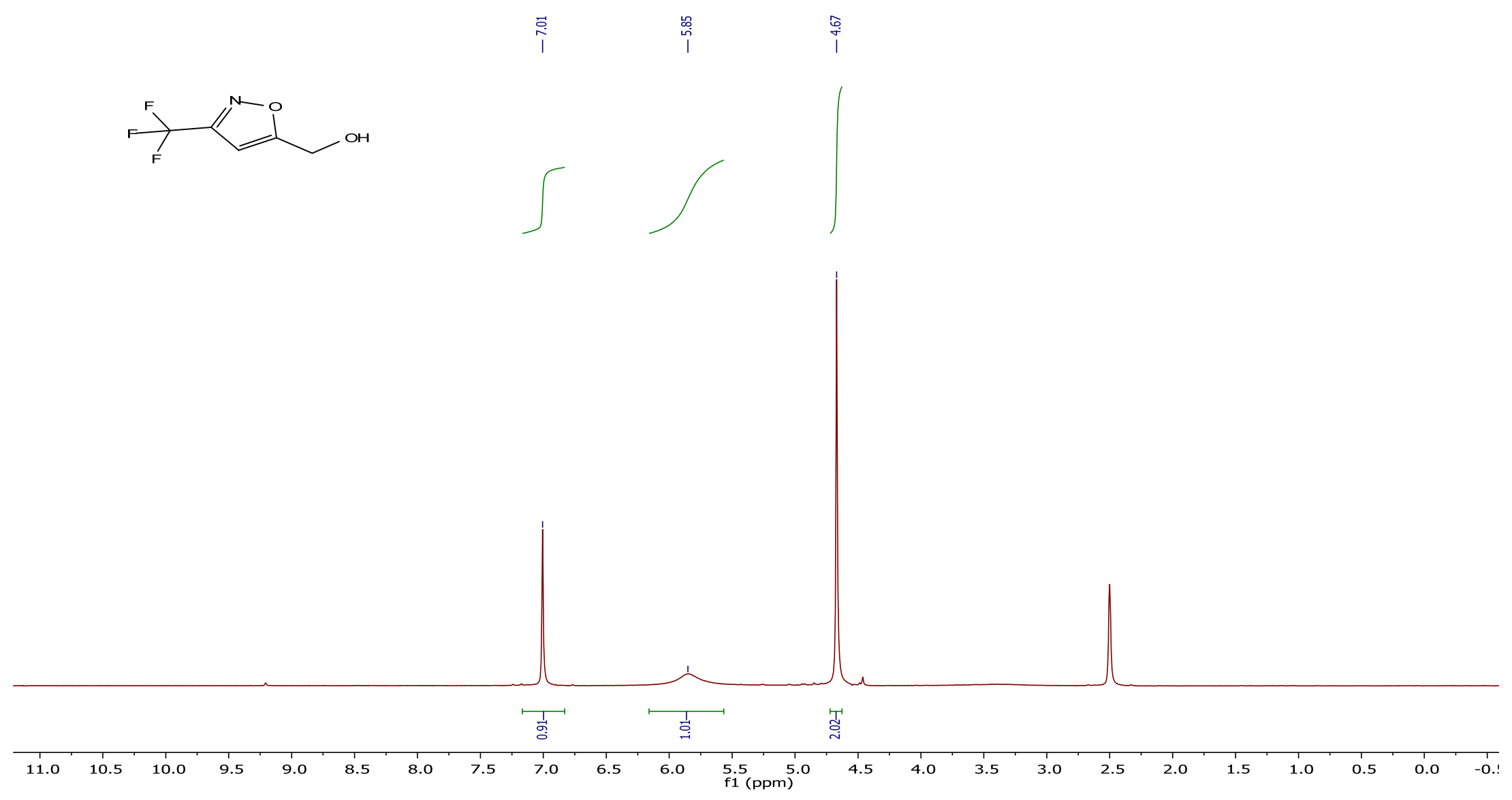


${ }^{13} \mathrm{C}\left\{{ }^{1} \mathrm{H}\right\}$ NMR spectrum of the compound 20b.
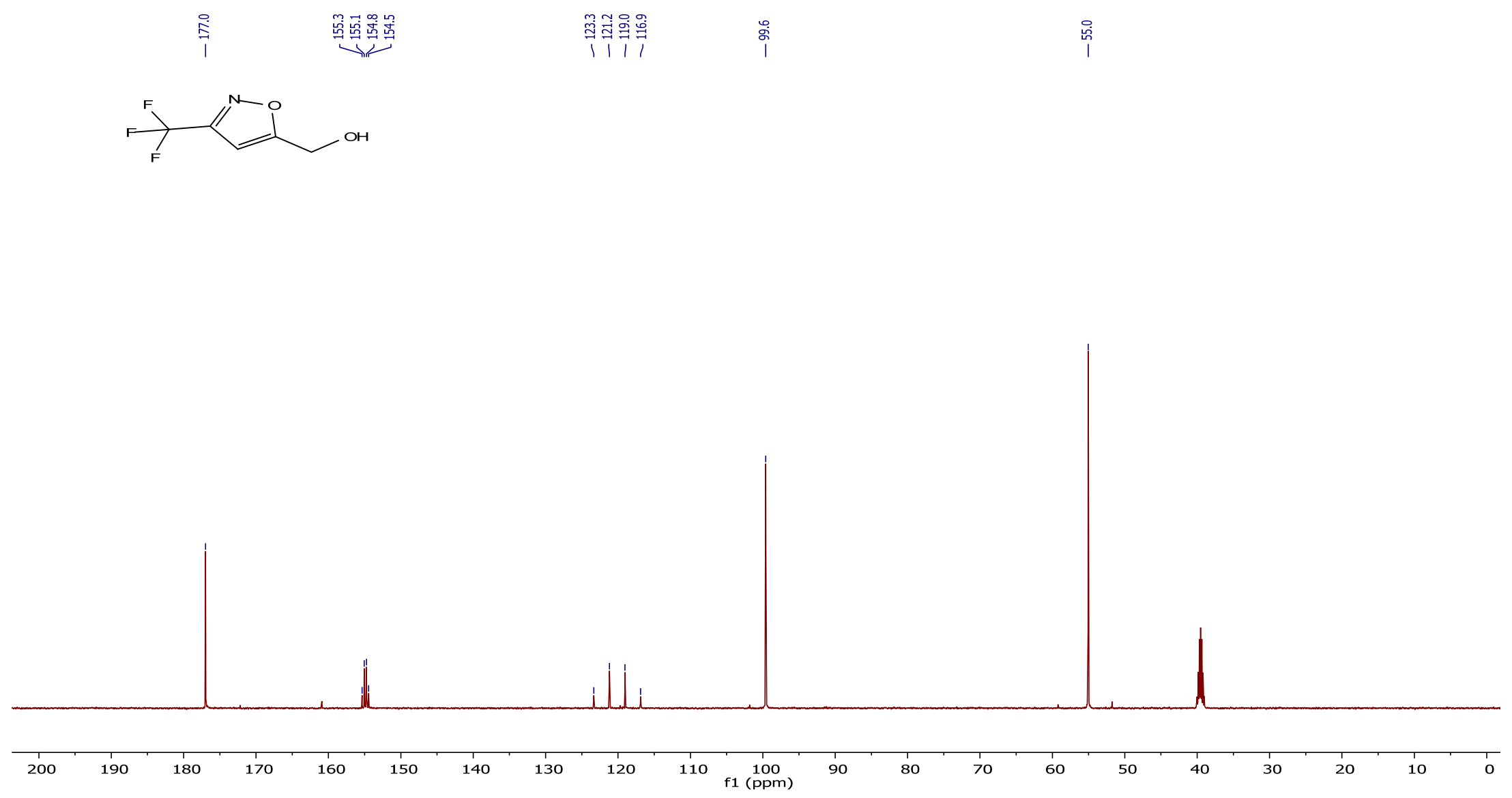
${ }^{19} \mathrm{~F}\left\{{ }^{1} \mathrm{H}\right\}$ NMR spectrum of the compound $20 \mathbf{b}$.

ఢ⿳亠口冋
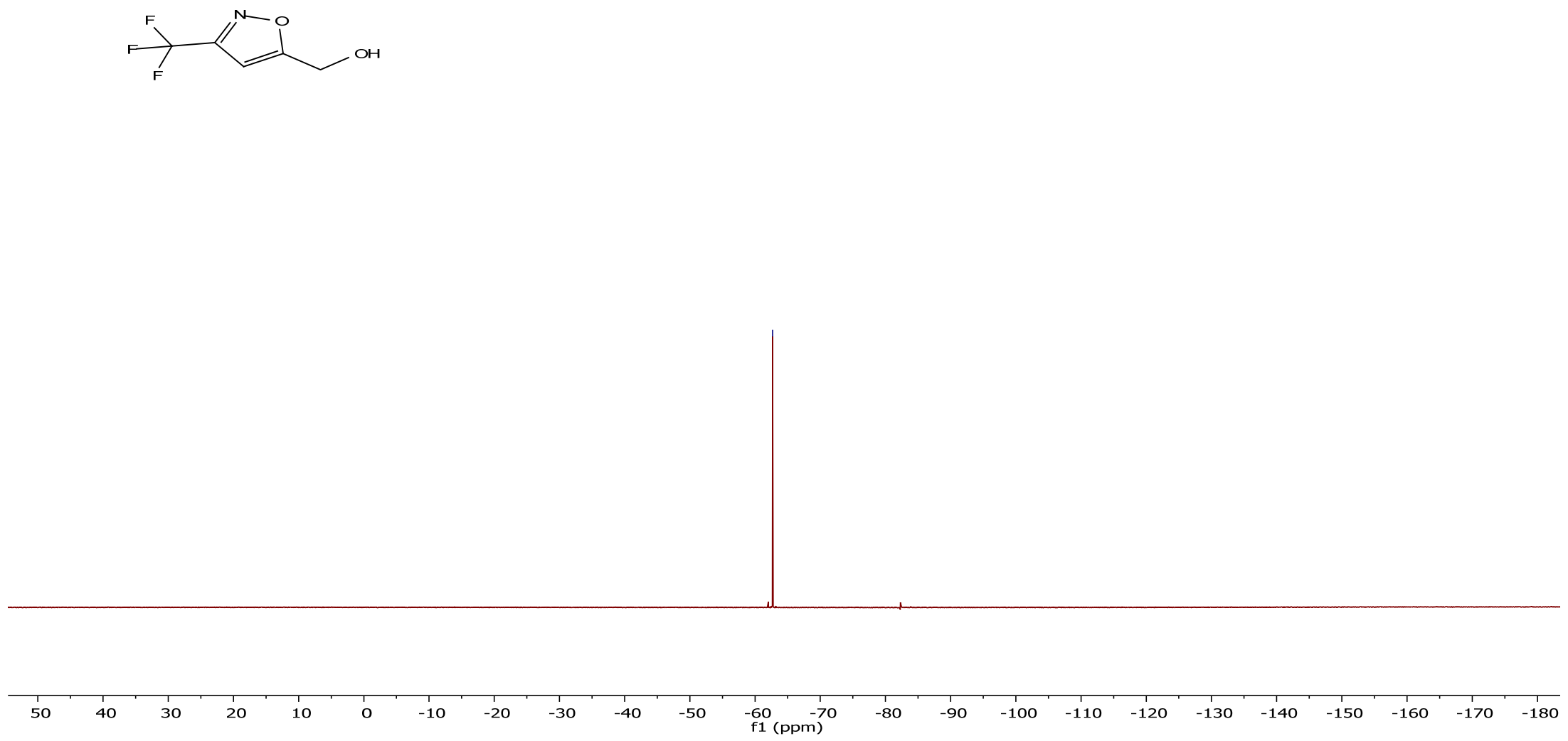
${ }^{1} \mathrm{H}$ NMR spectrum of the compound 20c.

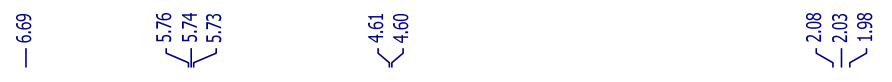

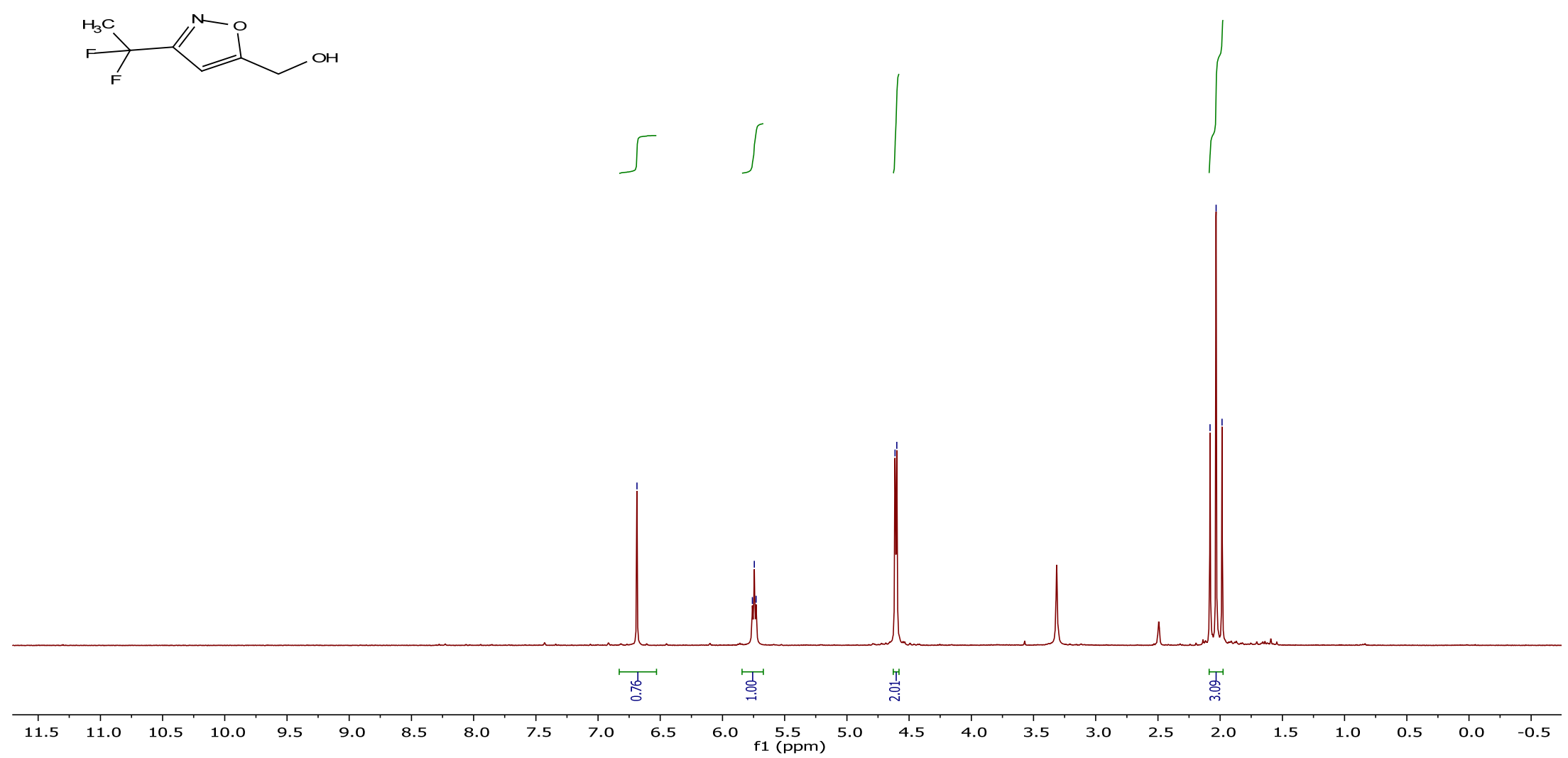


${ }^{13} \mathrm{C}\left\{{ }^{1} \mathrm{H}\right\}$ NMR spectrum of the compound 20c.
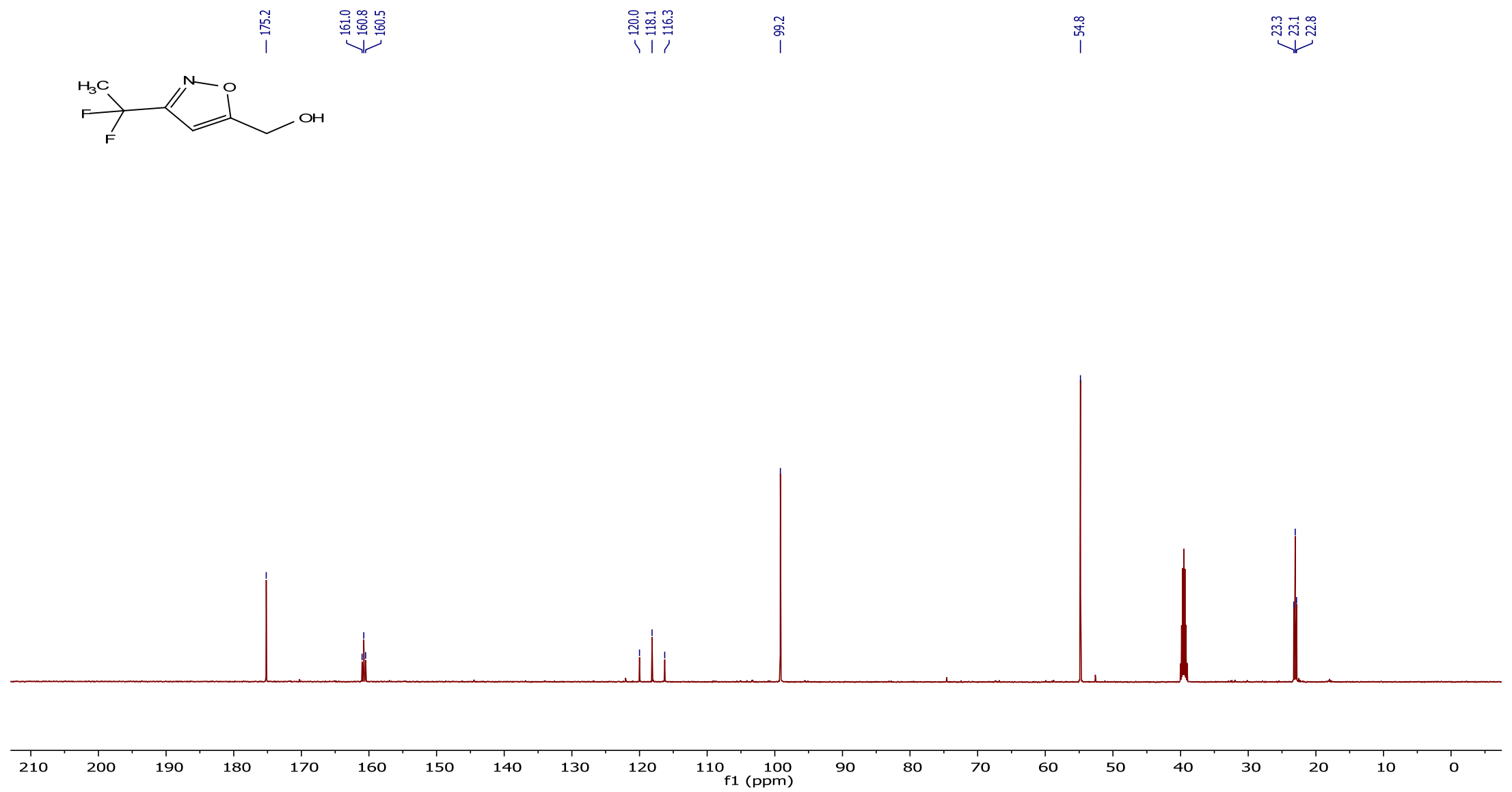
${ }^{19} \mathrm{~F}\left\{{ }^{1} \mathrm{H}\right\}$ NMR spectrum of the compound 20c.

$\widetilde{\tilde{p}^{\circ}}$

$\mathrm{F}_{\mathrm{F}}^{\mathrm{N}}$

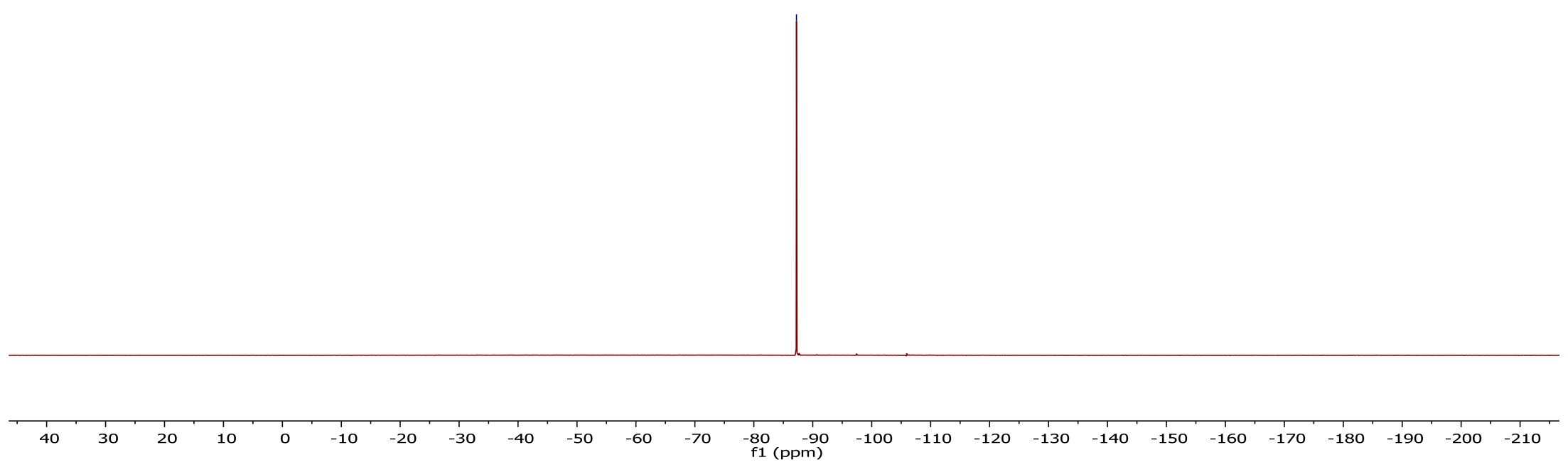


${ }^{1} \mathrm{H}$ NMR spectrum of the compound $20 \mathrm{f}$.

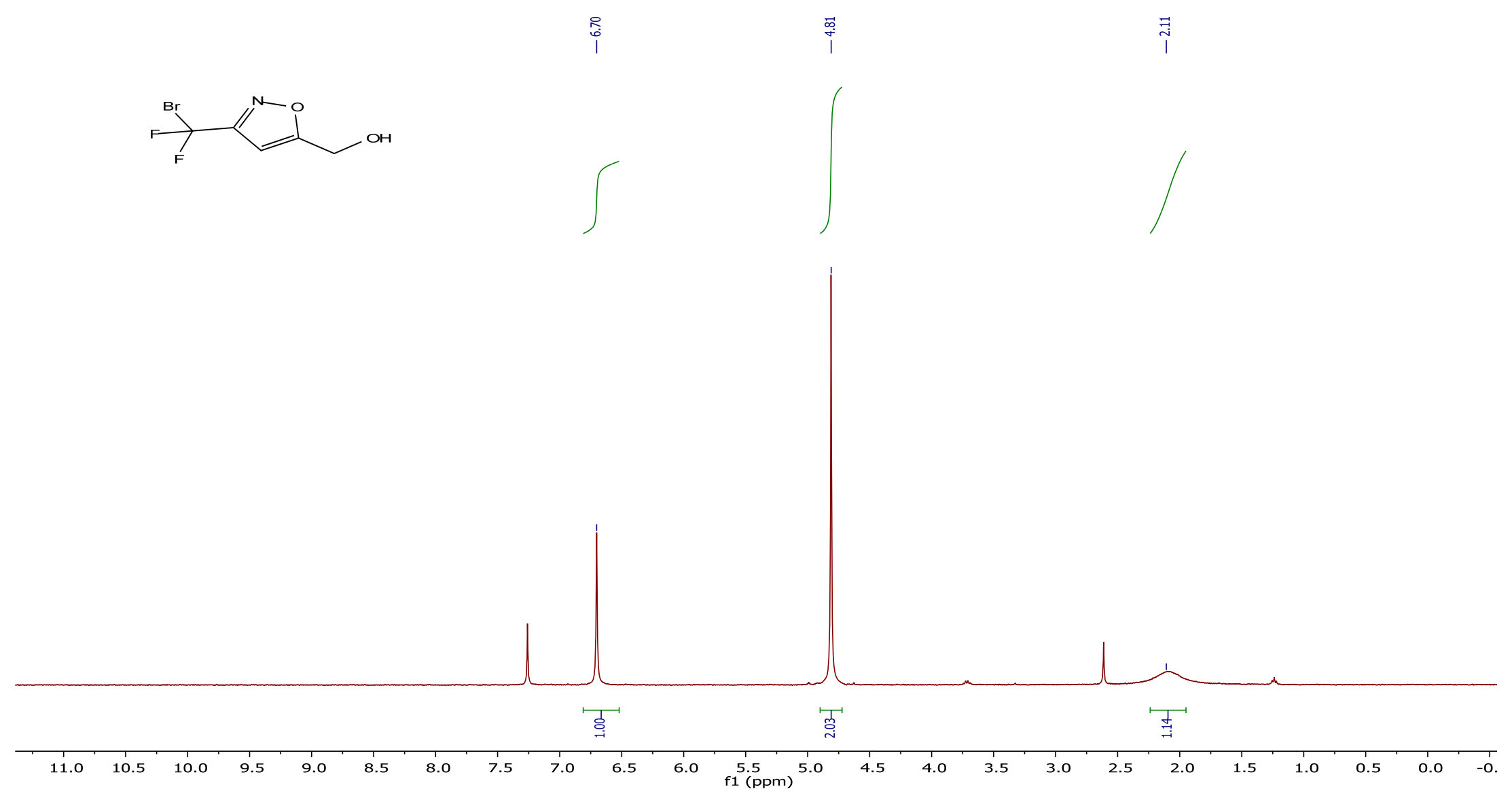


${ }^{13} \mathrm{C}\left\{{ }^{1} \mathrm{H}\right\}$ NMR spectrum of the compound $20 \mathrm{f}$.
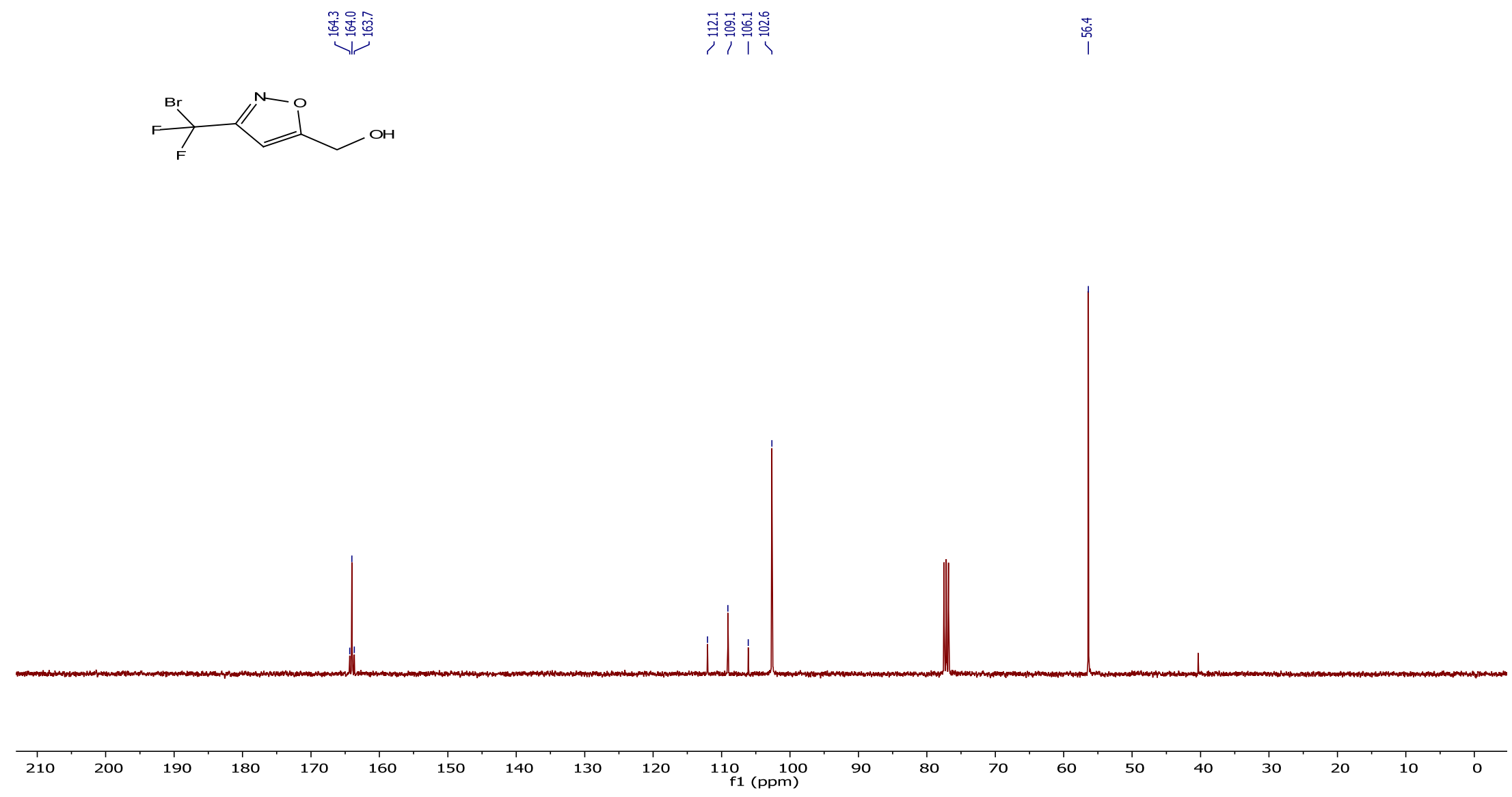
${ }^{19} \mathrm{~F}\left\{{ }^{1} \mathrm{H}\right\}$ NMR spectrum of the compound $20 \mathrm{f}$.

$\stackrel{\stackrel{\%}{\%}}{i}$
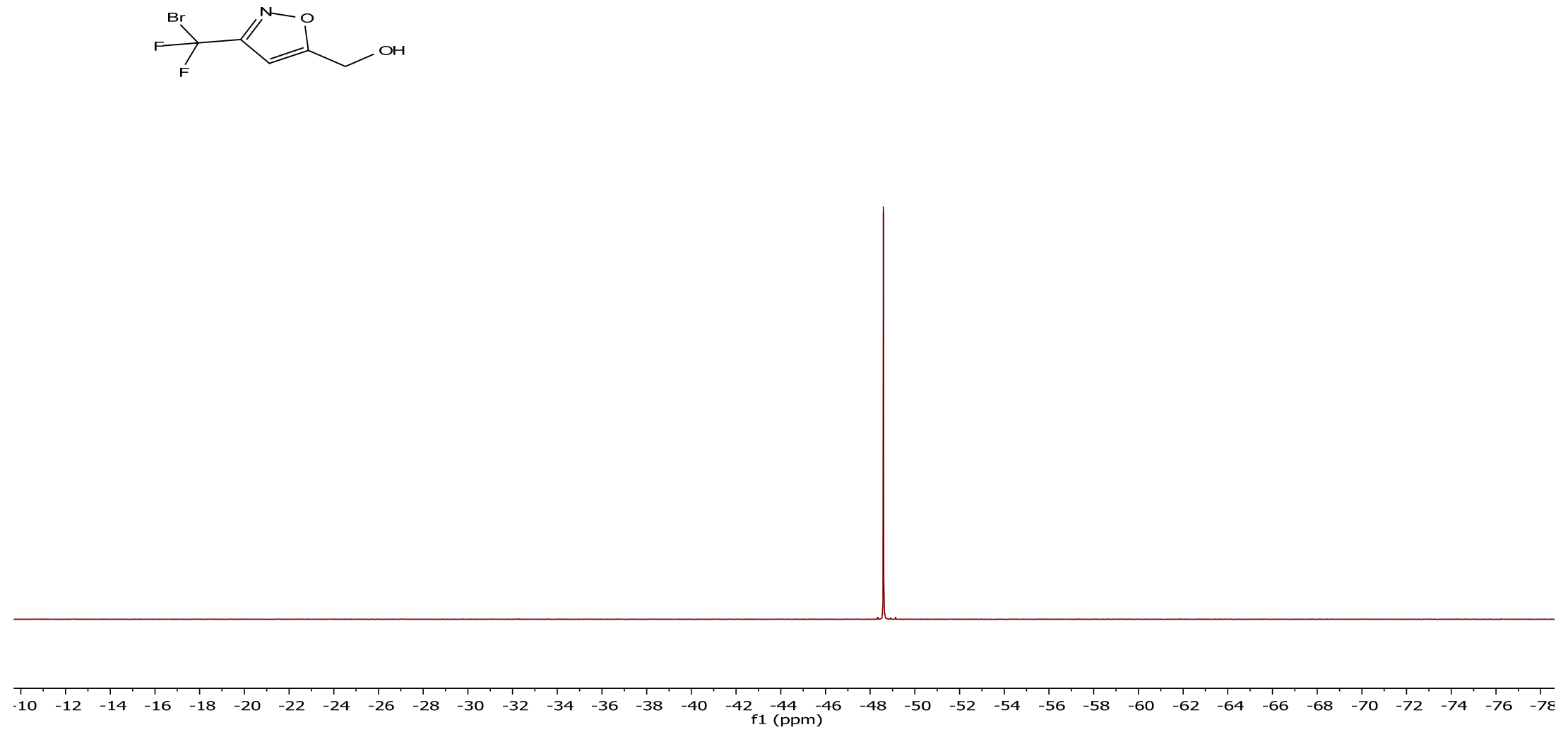


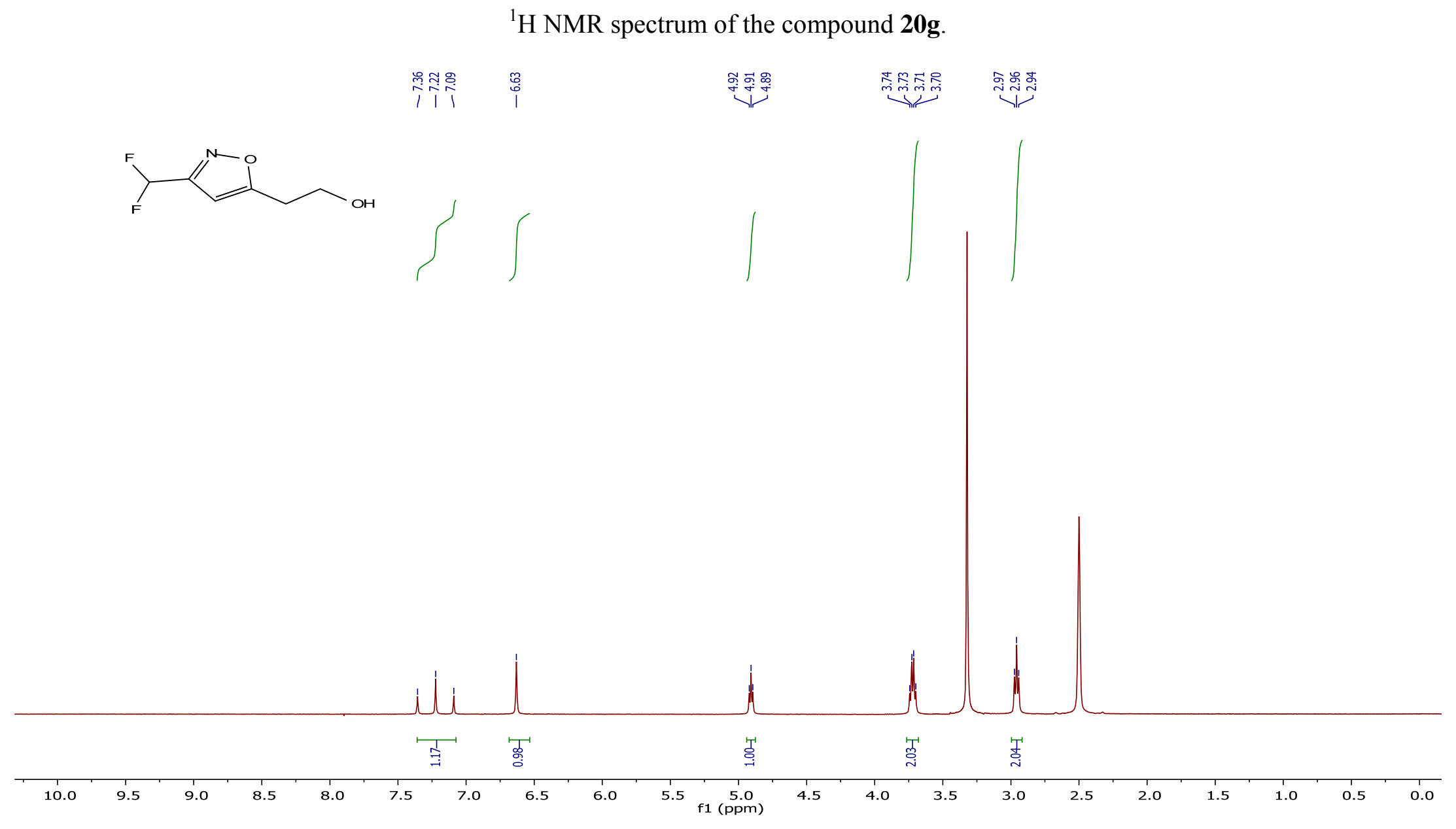


${ }^{13} \mathrm{C}\left\{{ }^{1} \mathrm{H}\right\}$ NMR spectrum of the compound $\mathbf{2 0 g}$.

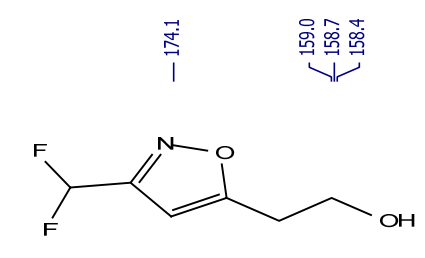

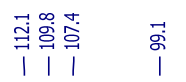

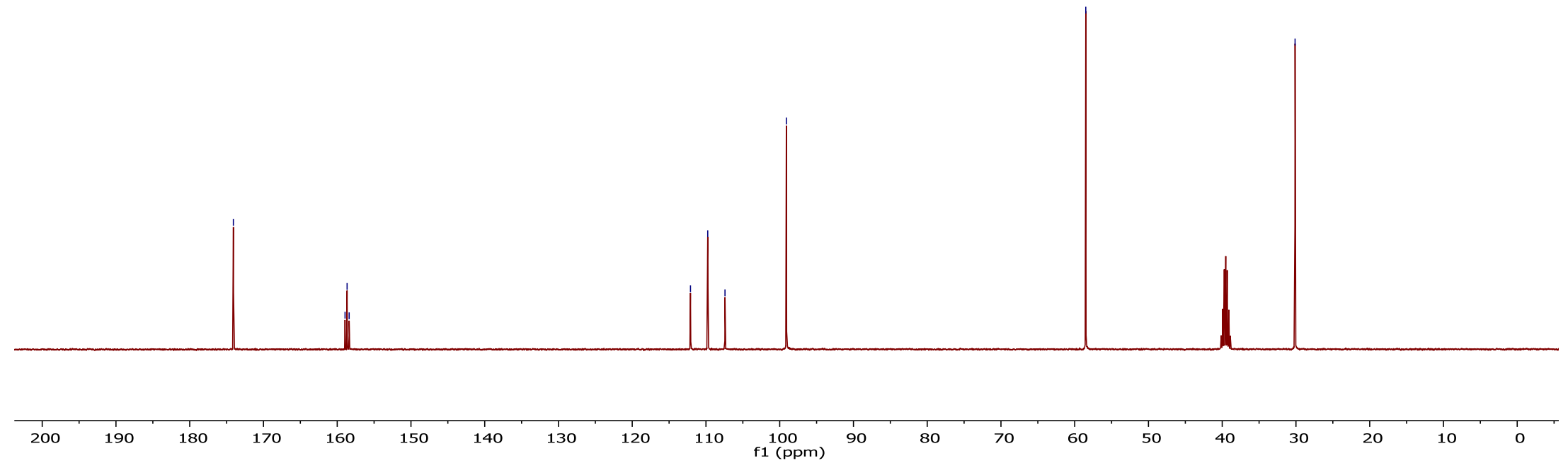


${ }^{19} \mathrm{~F}\left\{{ }^{1} \mathrm{H}\right\}$ NMR spectrum of the compound $\mathbf{2 0 g}$.

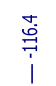
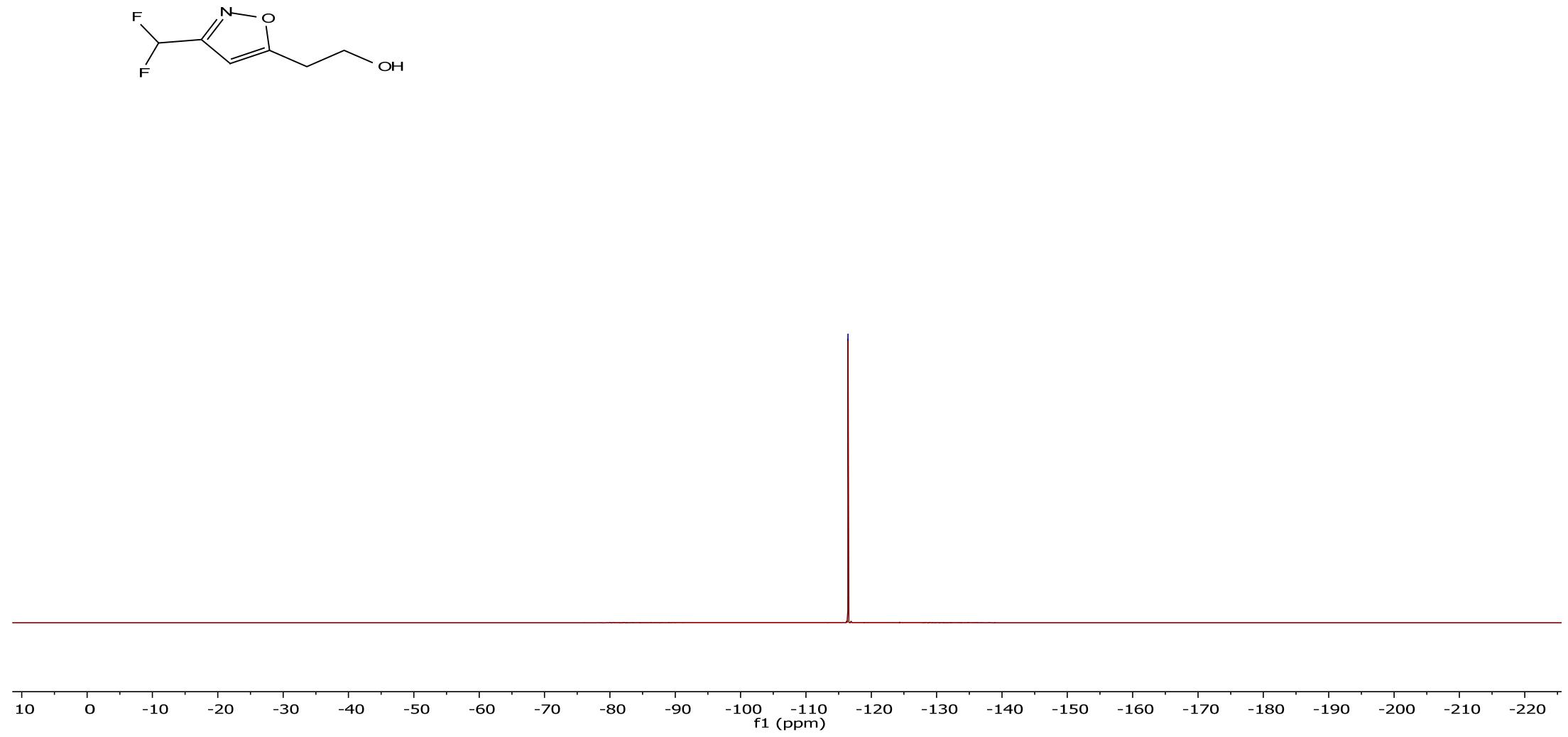
${ }^{1} \mathrm{H}$ NMR spectrum of the compound $20 \mathbf{h}$.

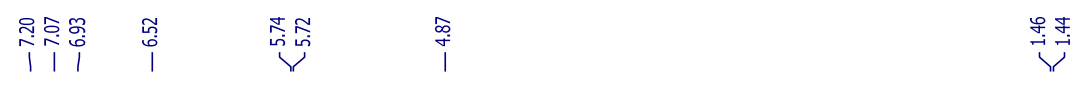<smiles>CC(O)c1cc(C(F)F)no1</smiles>
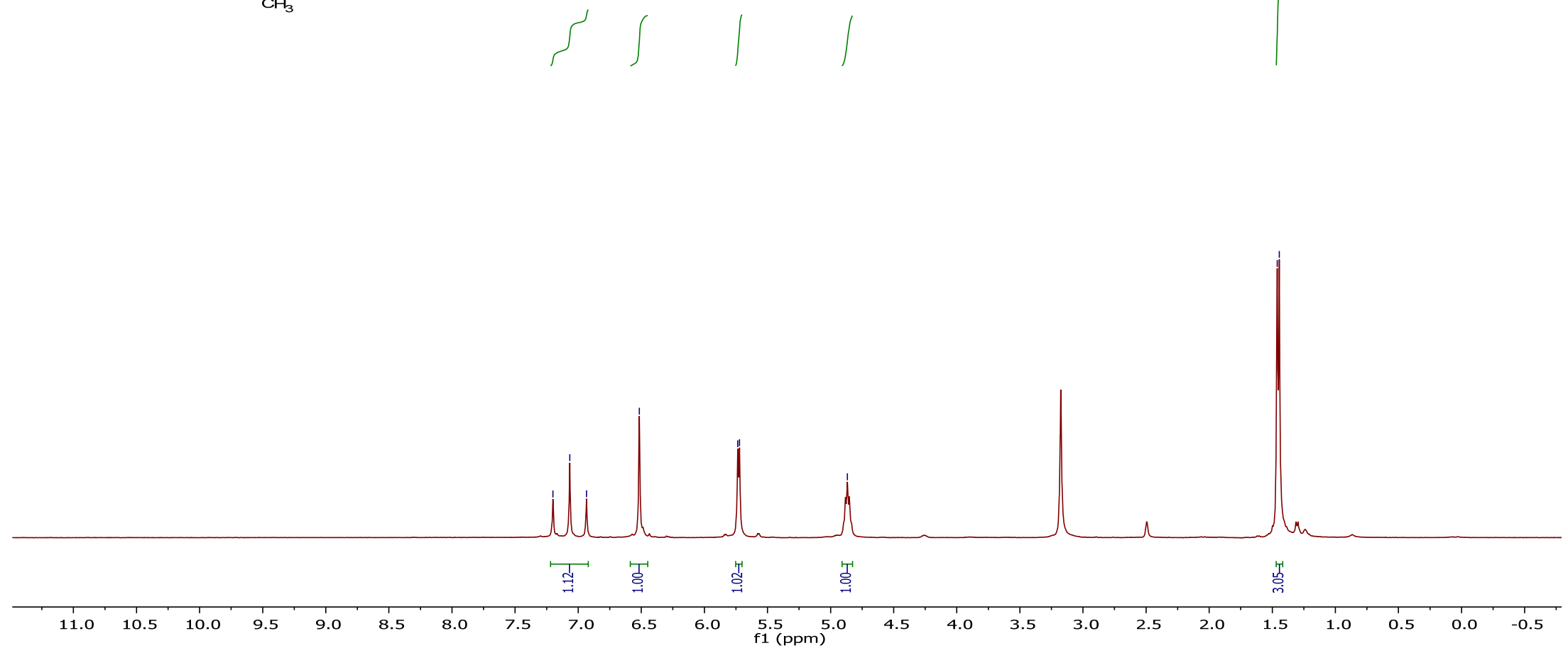
${ }^{13} \mathrm{C}\left\{{ }^{1} \mathrm{H}\right\}$ NMR spectrum of the compound 20h.

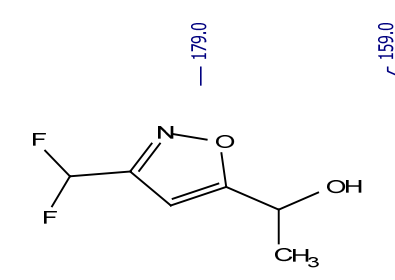

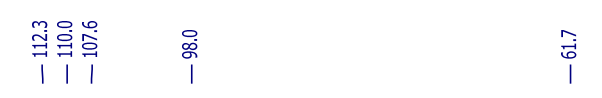

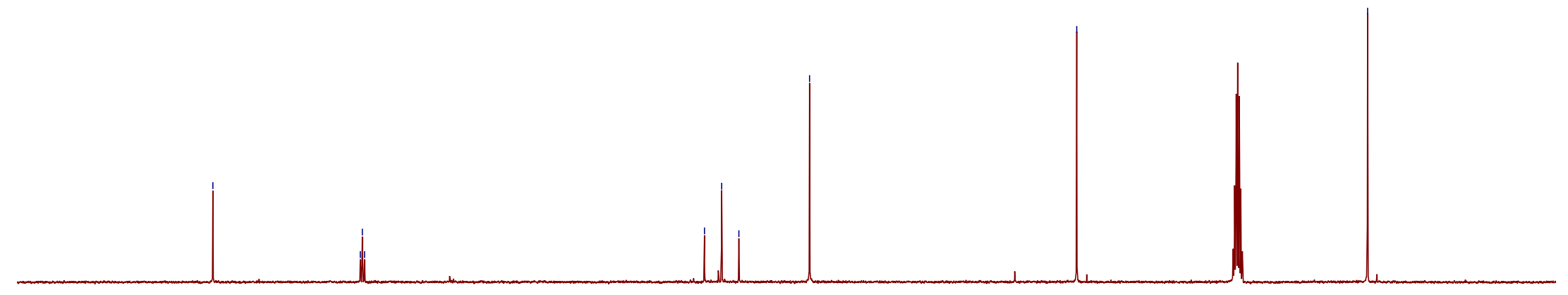

200

$190 \quad 180 \quad 170$

160

150

140

130

$110 \quad \begin{gathered}100 \\ \text { f1 }(\mathrm{ppm})\end{gathered}$ 
${ }^{19} \mathrm{~F}\left\{{ }^{1} \mathrm{H}\right\}$ NMR spectrum of the compound $20 \mathbf{h}$.

芦
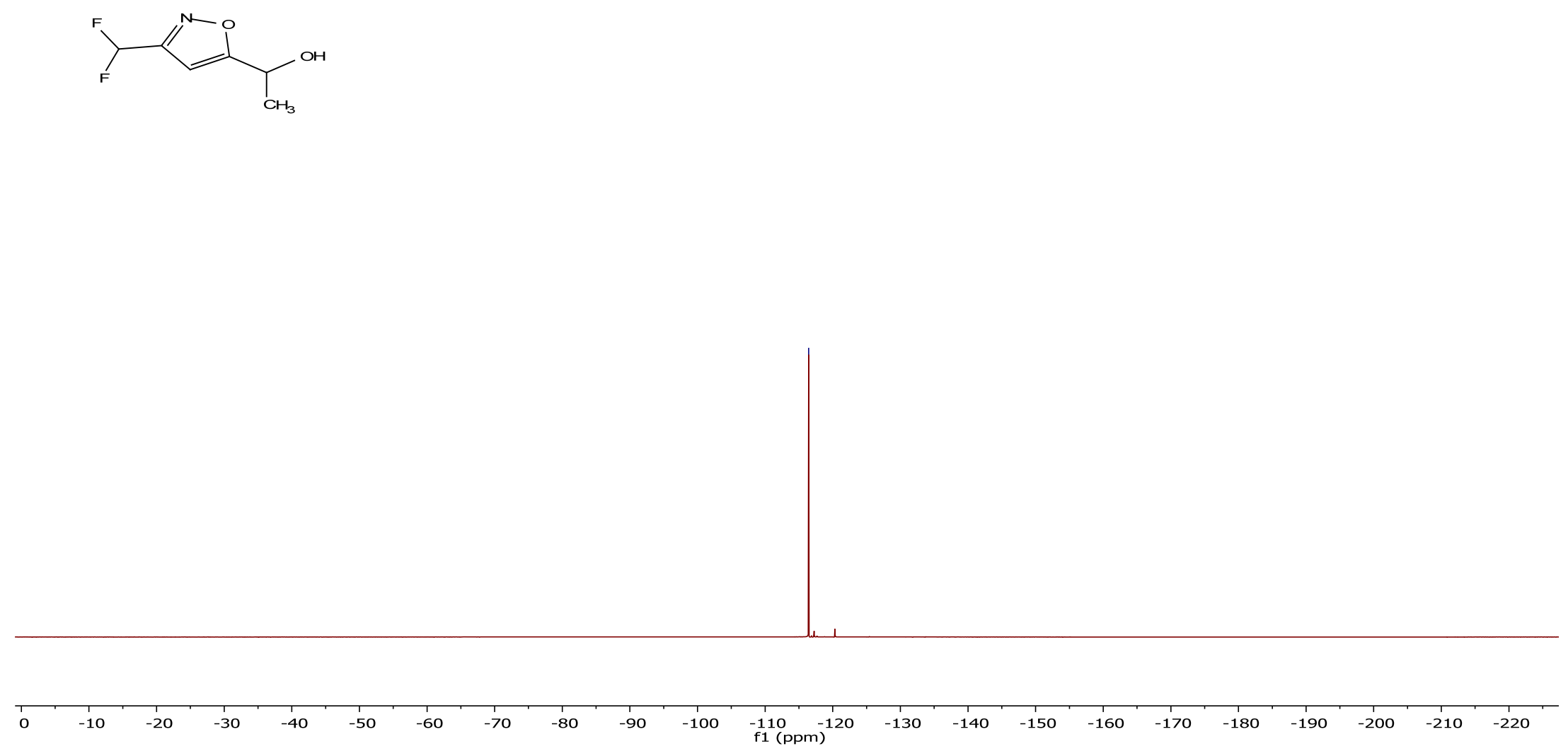
${ }^{1} \mathrm{H}$ NMR spectrum of the compound 22a.

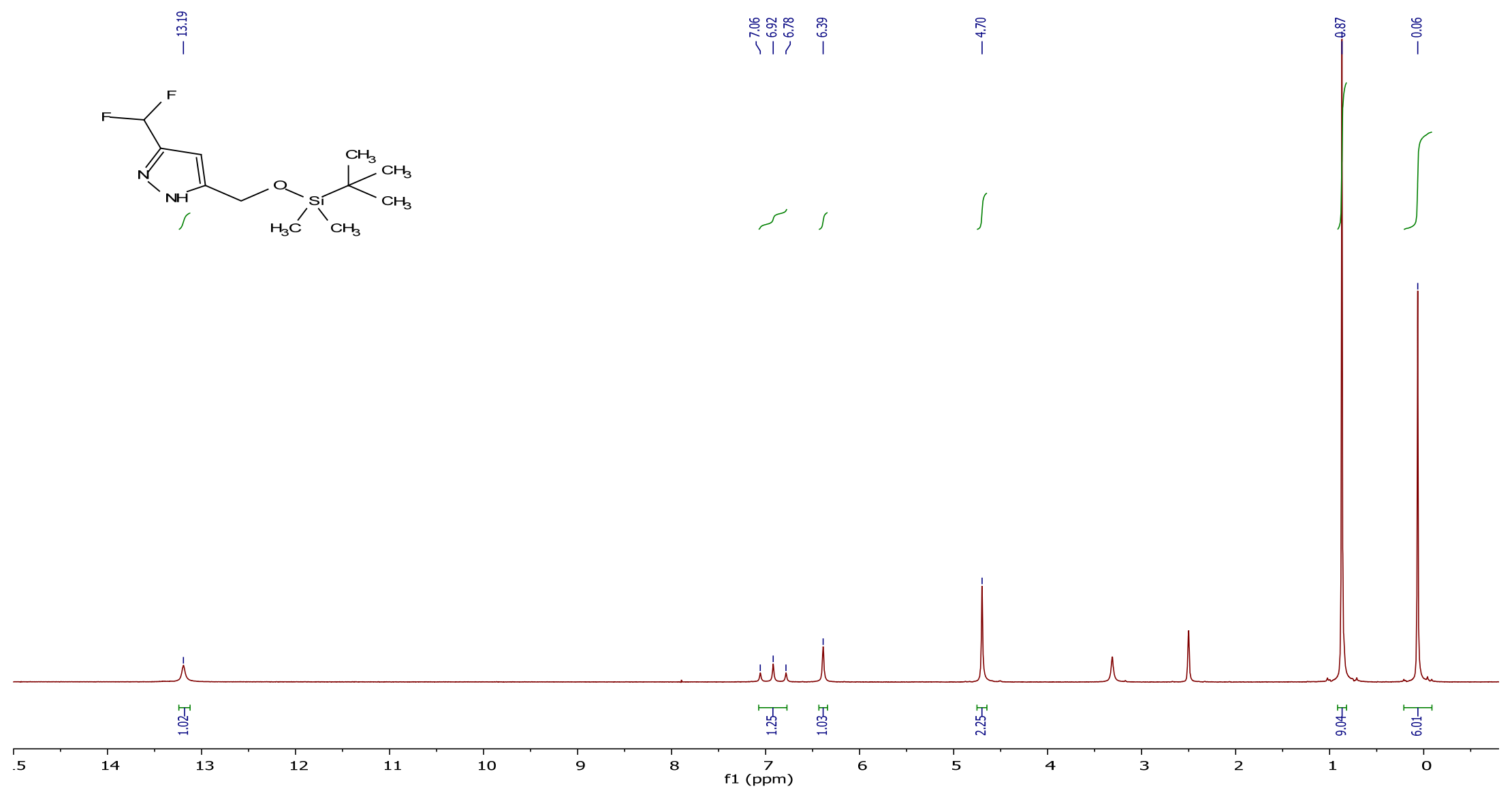


${ }^{13} \mathrm{C}\left\{{ }^{1} \mathrm{H}\right\}$ NMR spectrum of the compound 22a.

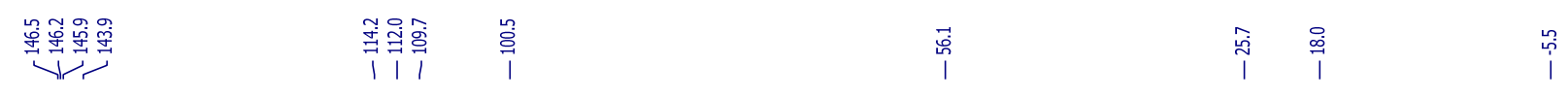

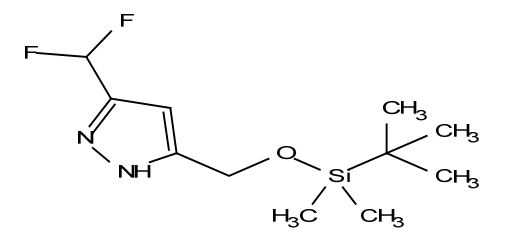


${ }^{19}$ F NMR spectrum of the compound 22a.

ㅎํㄱㅇํㅇ
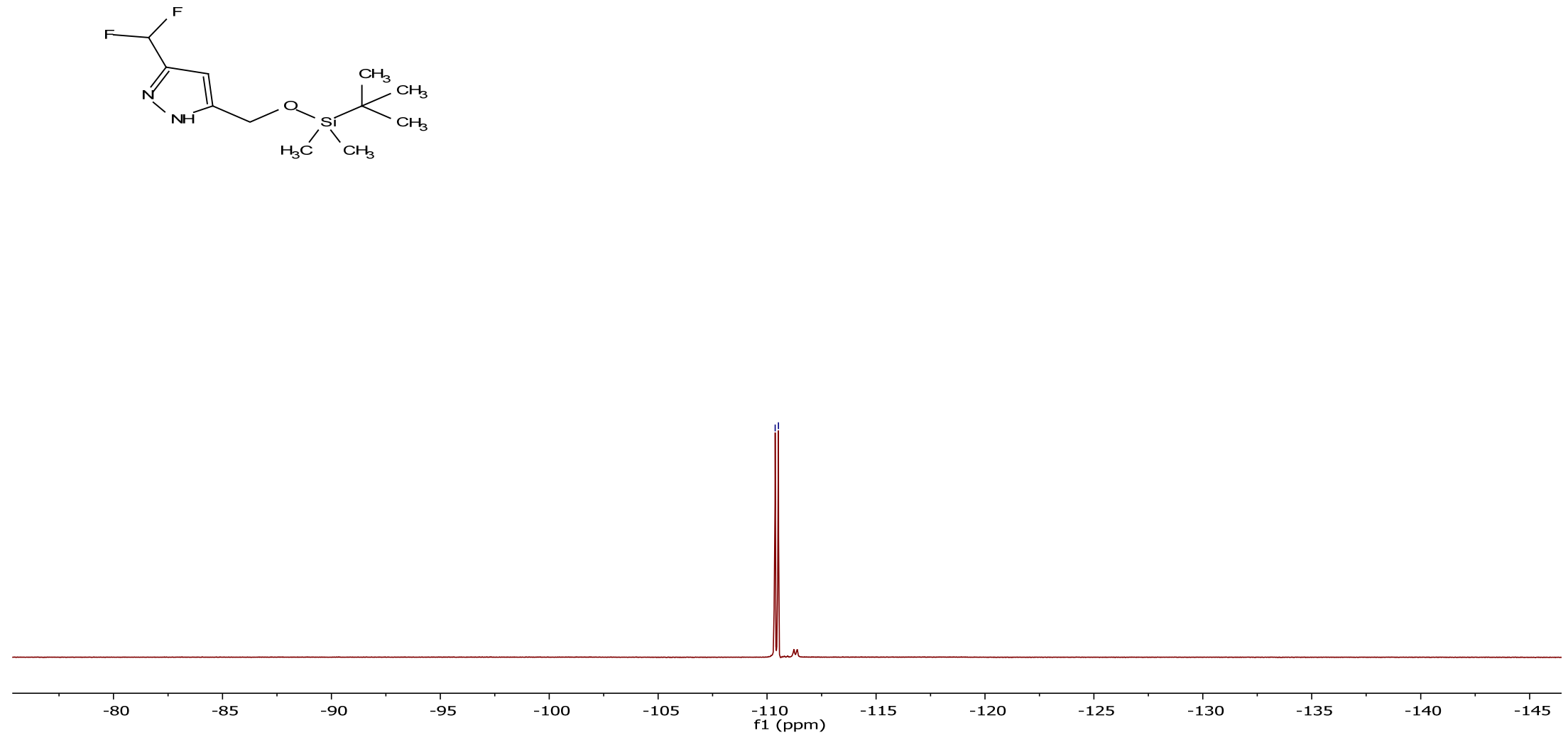
${ }^{1} \mathrm{H}$ NMR spectrum of the compound 28c.

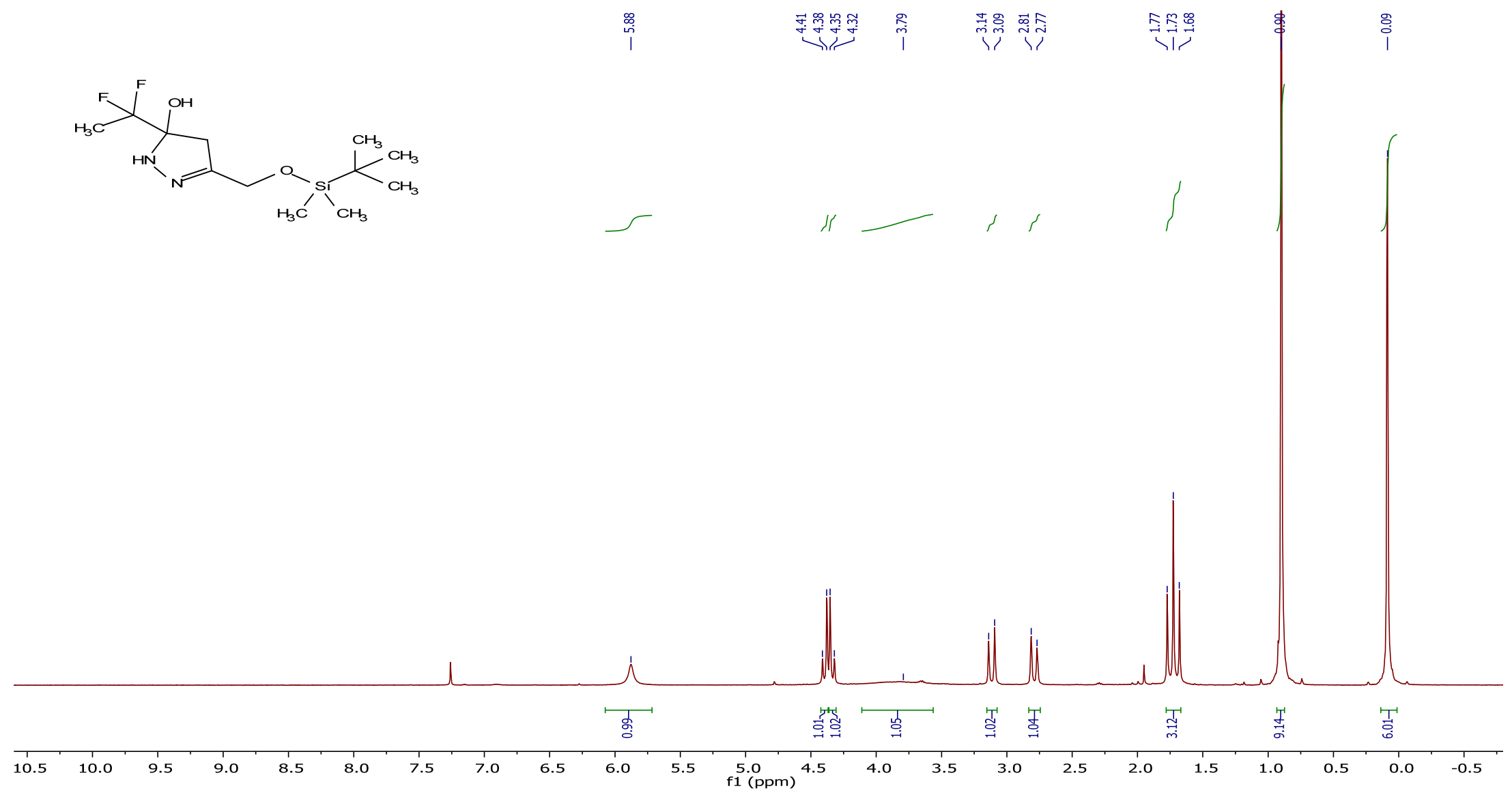


${ }^{13} \mathrm{C}\left\{{ }^{1} \mathrm{H}\right\}$ NMR spectrum of the compound 28c.

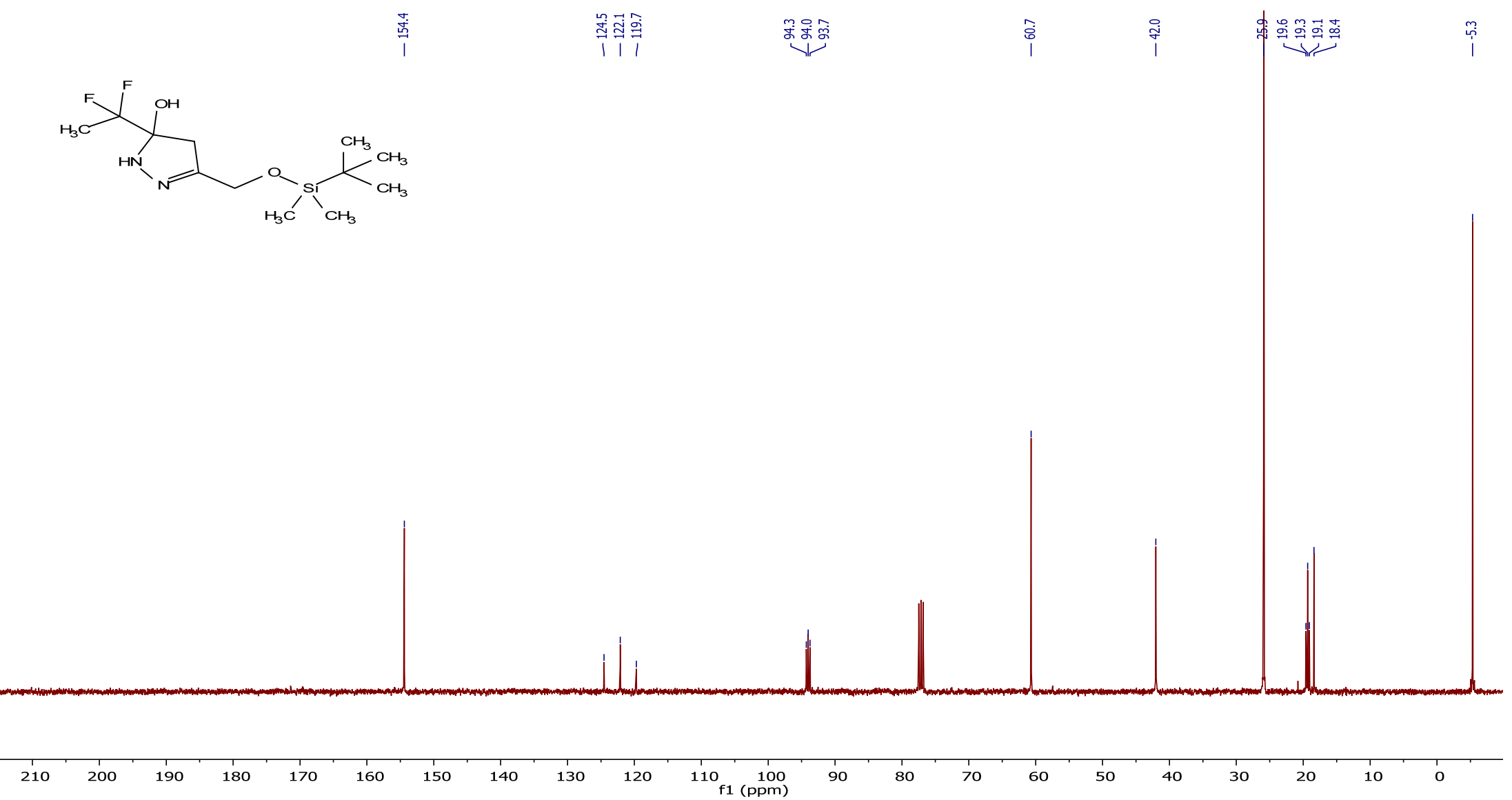


${ }^{19} \mathrm{~F}\left\{{ }^{1} \mathrm{H}\right\}$ NMR spectrum of the compound 28c.

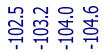
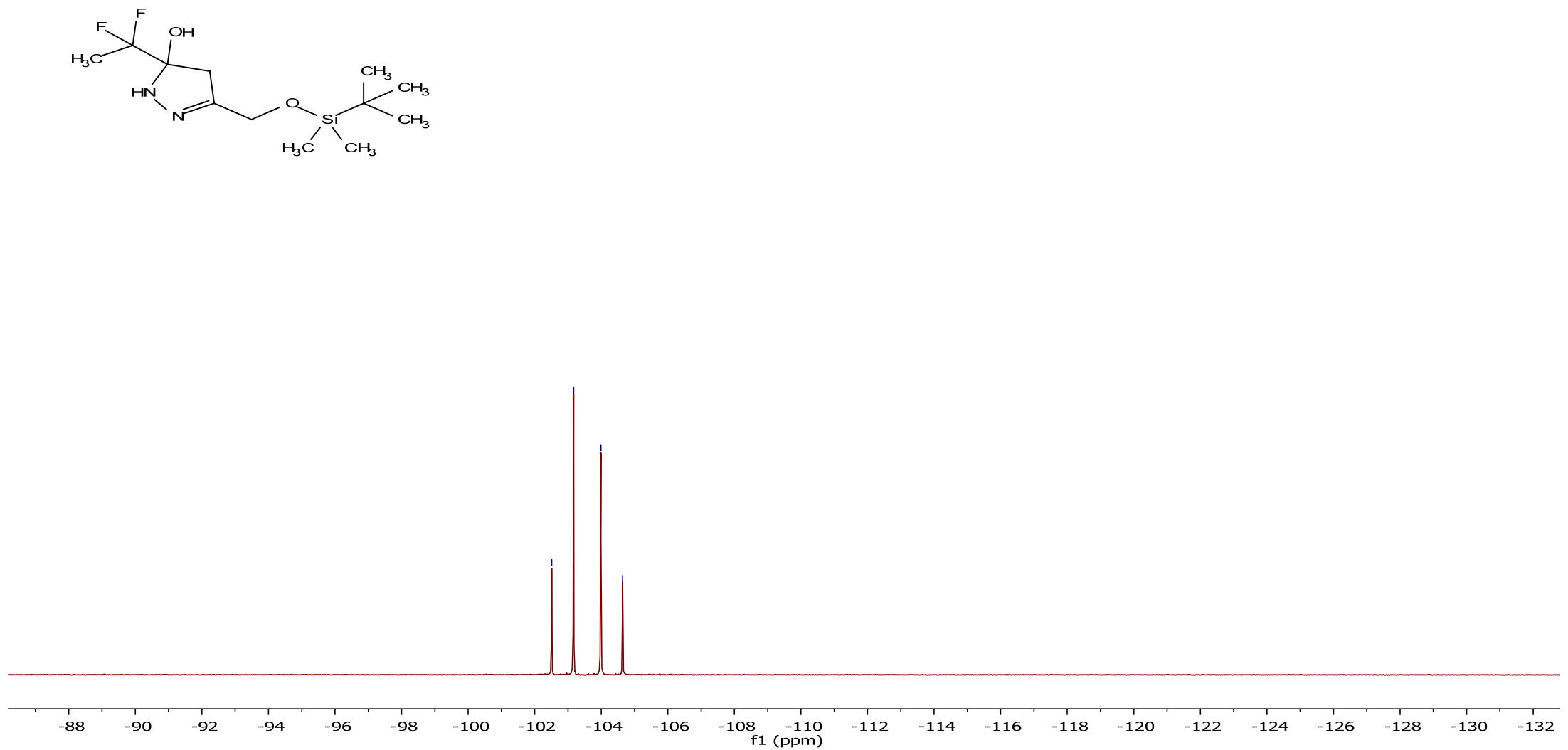
${ }^{1} \mathrm{H}$ NMR spectrum of the compound 22c.

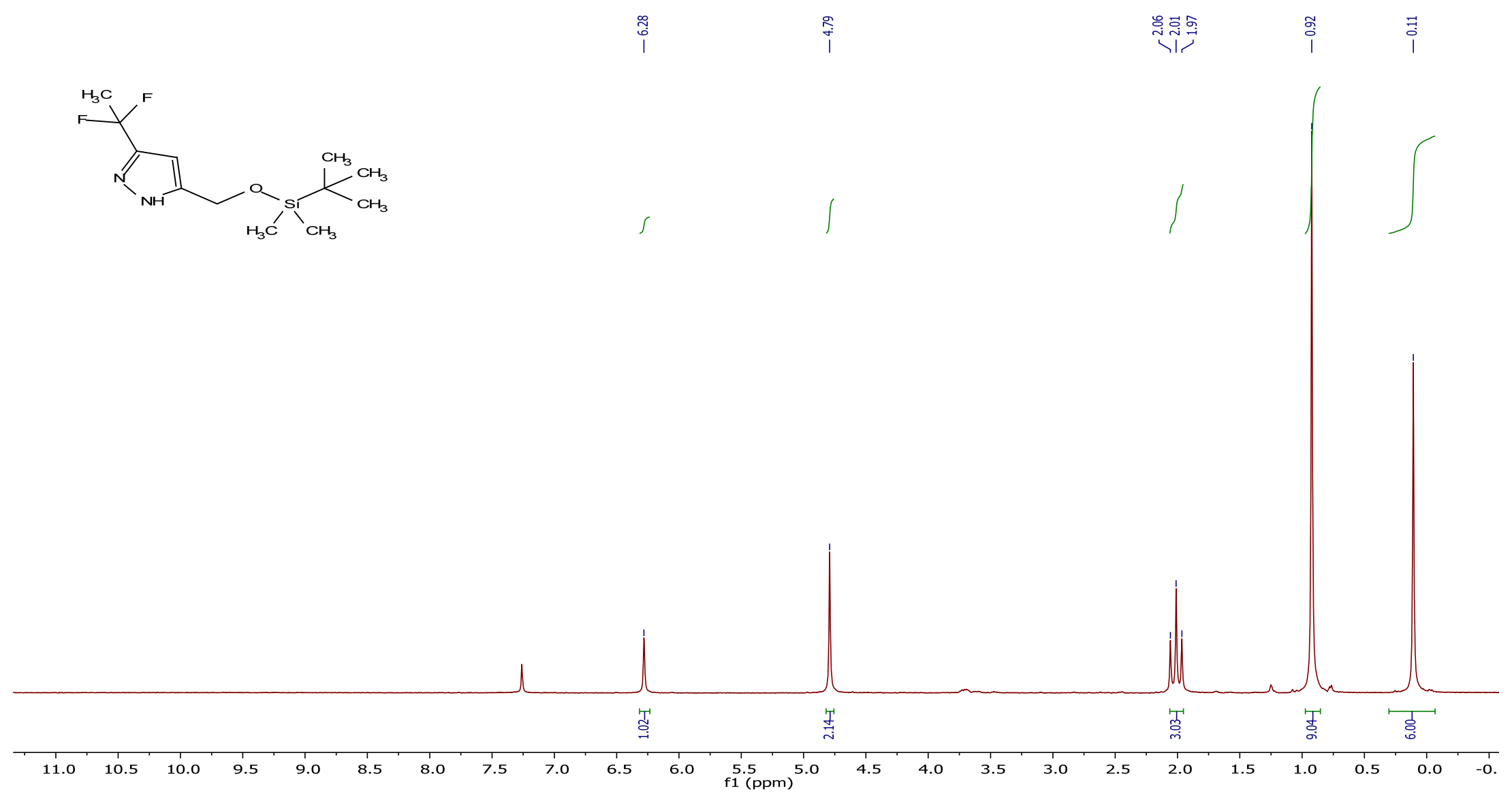


${ }^{13} \mathrm{C}\left\{{ }^{1} \mathrm{H}\right\}$ NMR spectrum of the compound 22c.

等

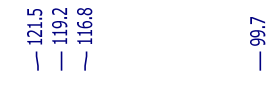

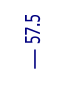

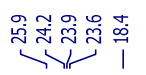
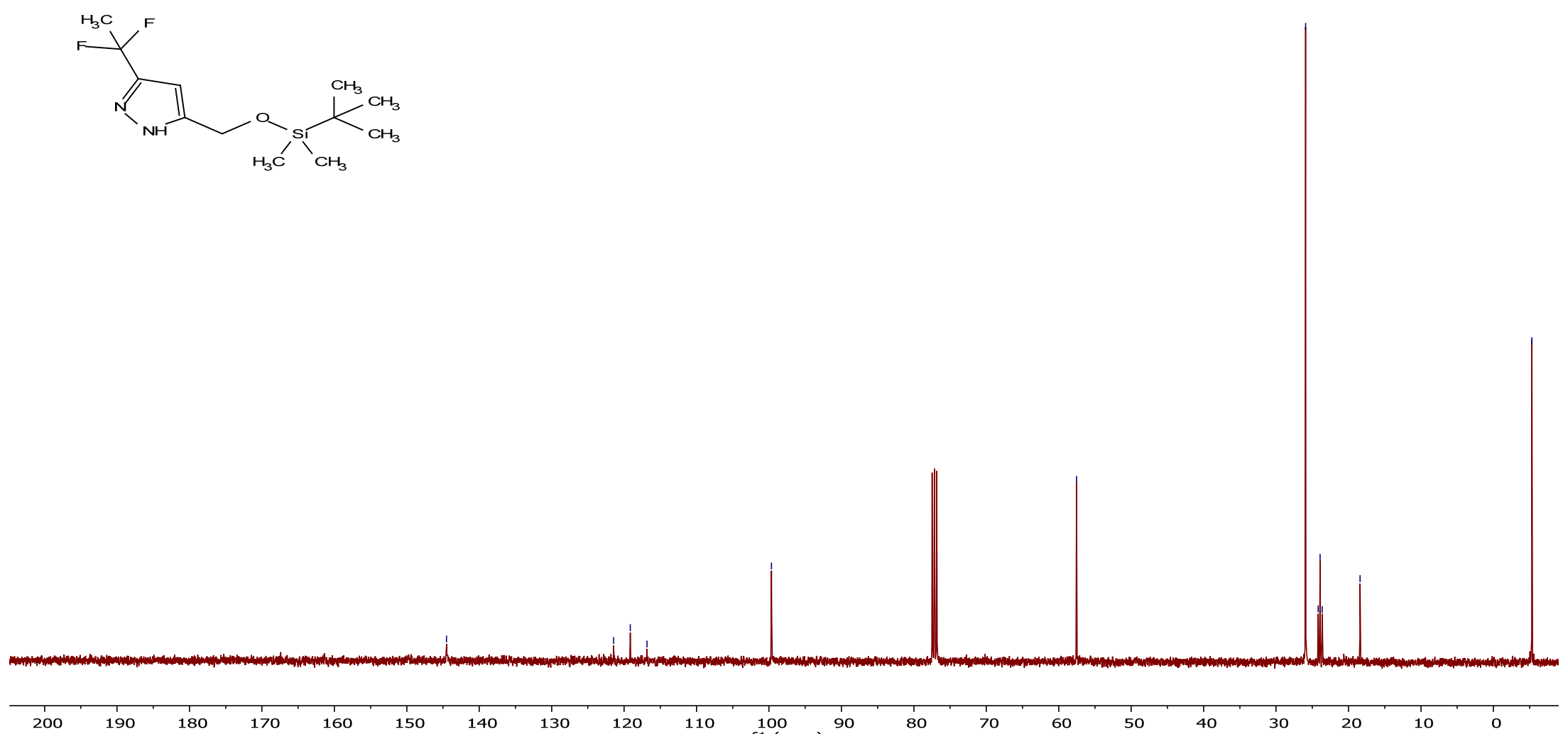
${ }^{19} \mathrm{~F}\left\{{ }^{1} \mathrm{H}\right\}$ NMR spectrum of the compound 22c.
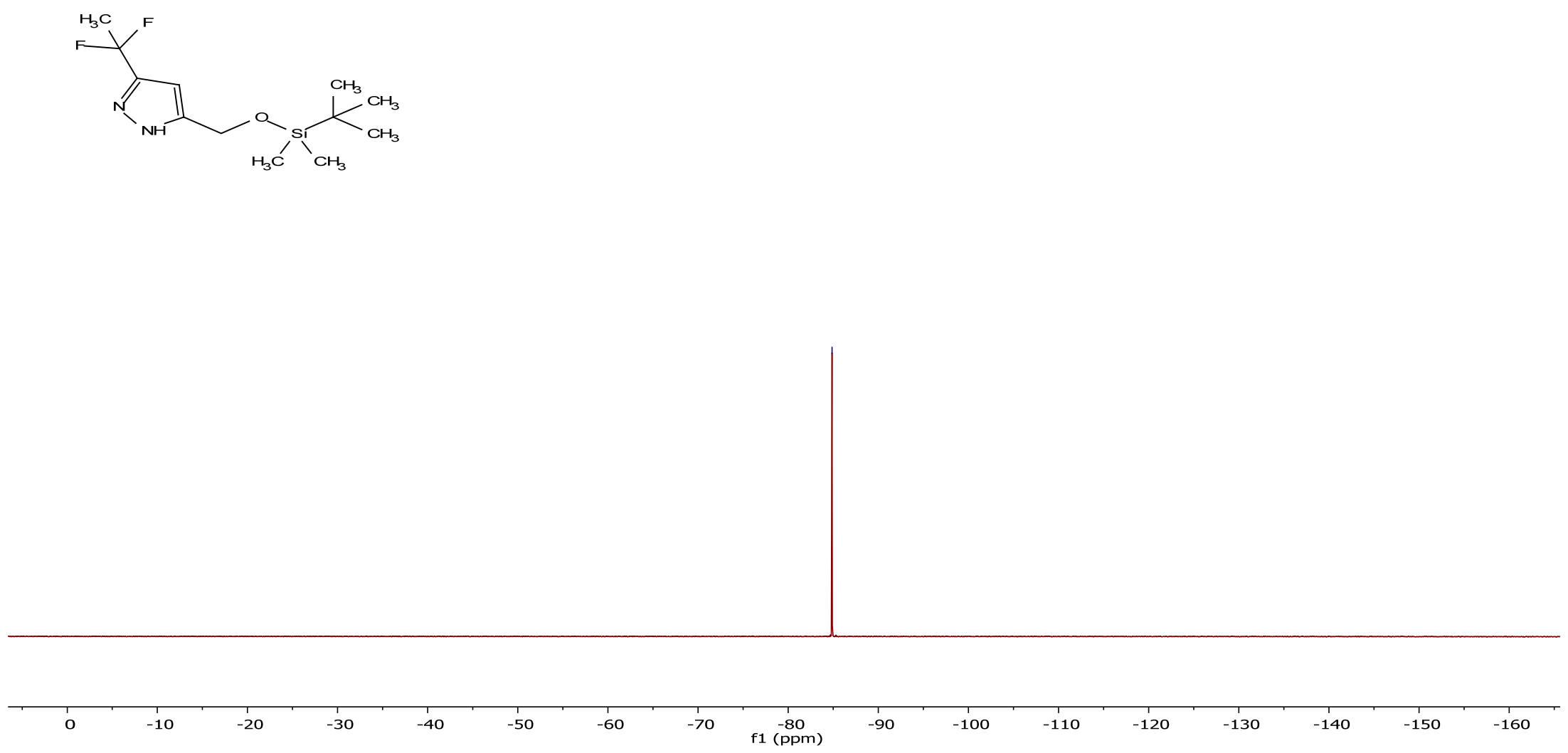
${ }^{1} \mathrm{H}$ NMR spectrum of the compound $\mathbf{2 2 h}$.

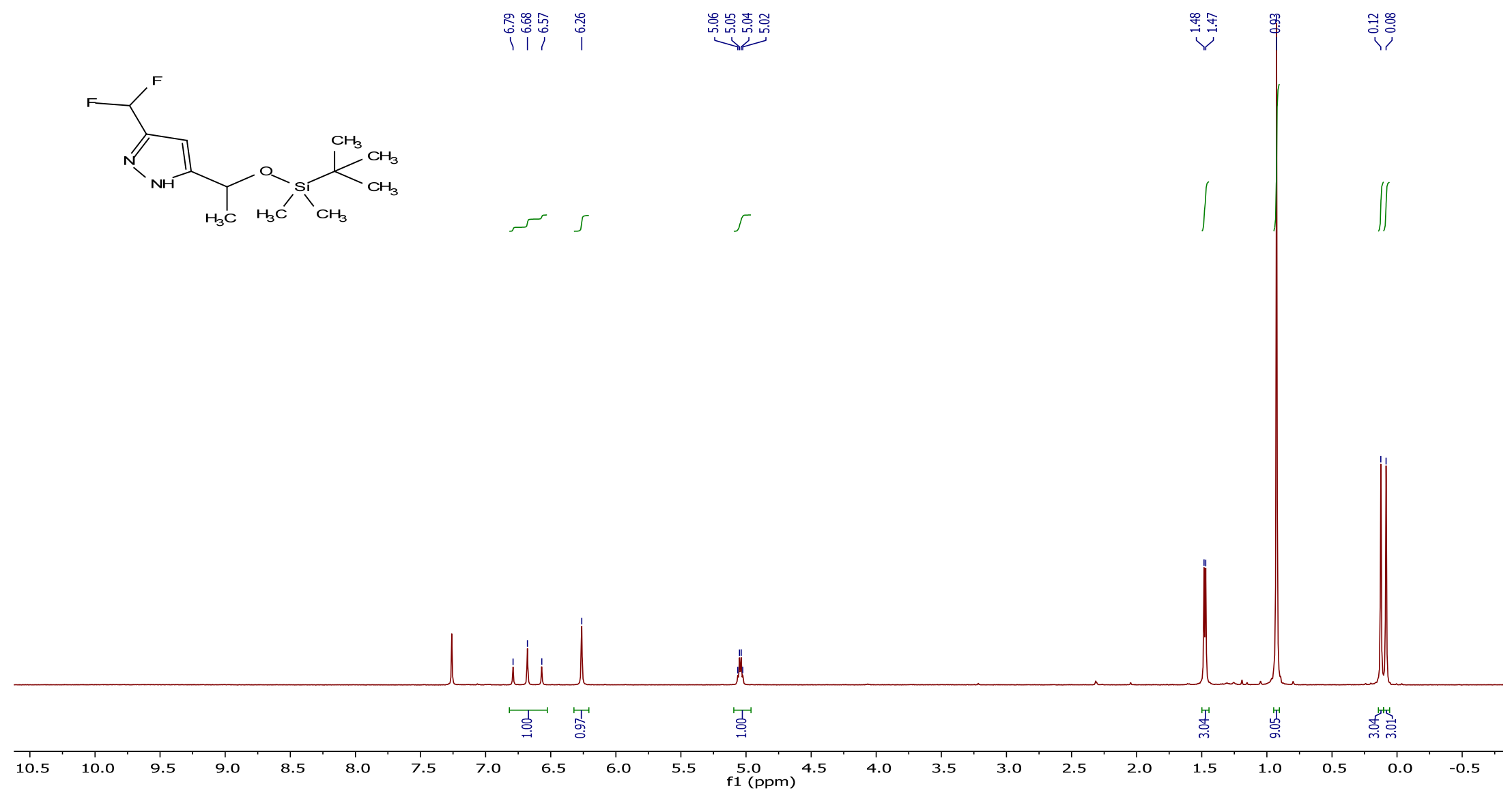


${ }^{13} \mathrm{C}\left\{{ }^{1} \mathrm{H}\right\}$ NMR spectrum of the compound $\mathbf{2 2} \mathbf{h}$.

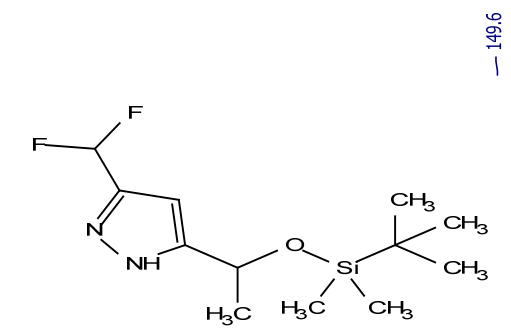

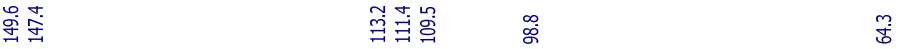

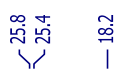

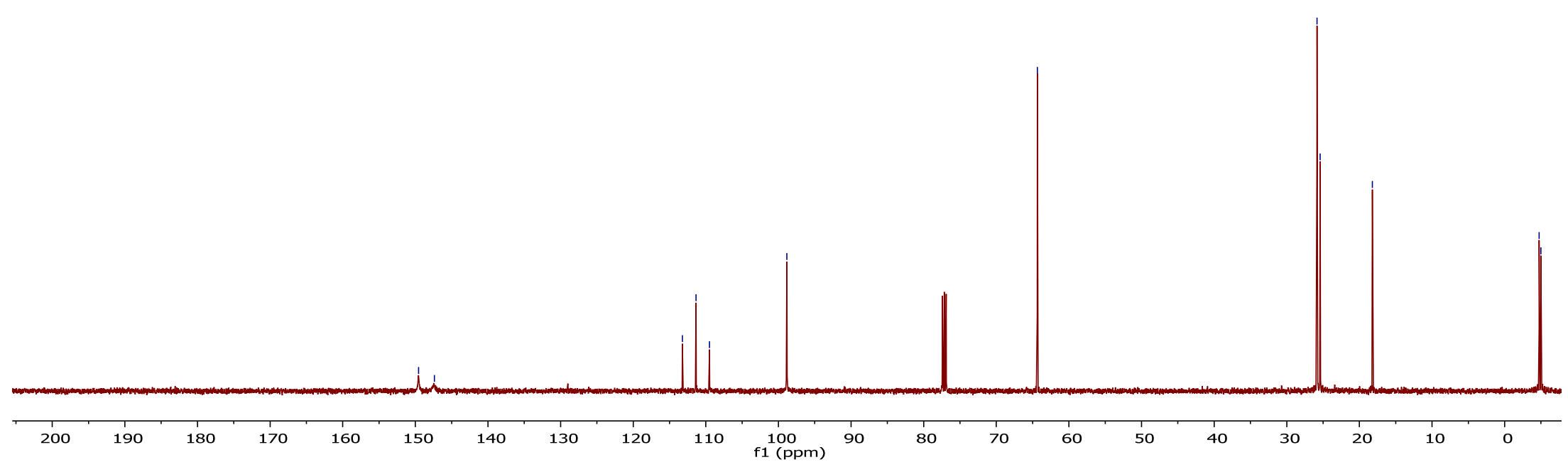


${ }^{19}$ F NMR spectrum of the compound $22 \mathbf{h}$.

쿡국
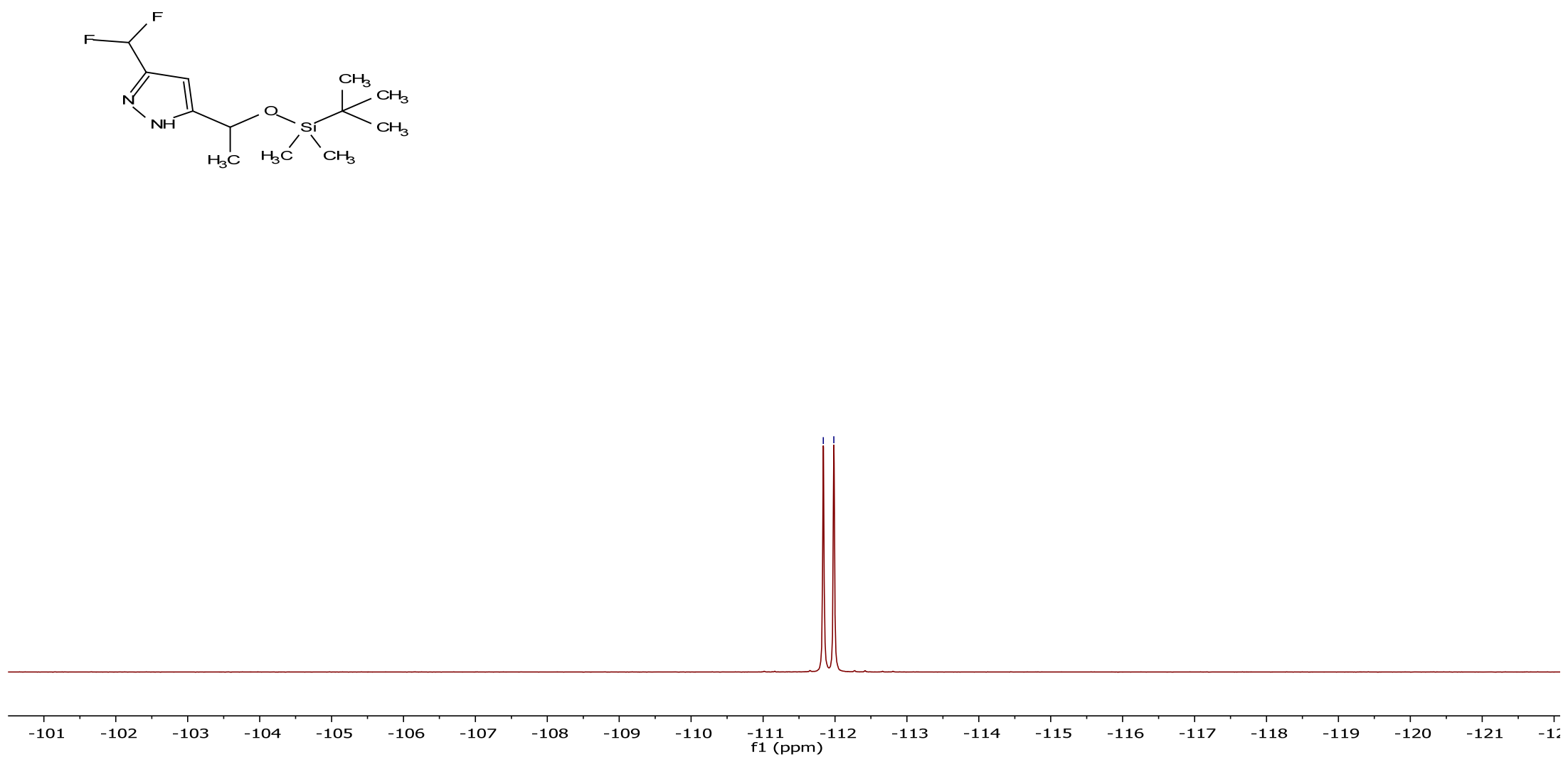
${ }^{1} \mathrm{H}$ NMR spectrum of the compound 23a.

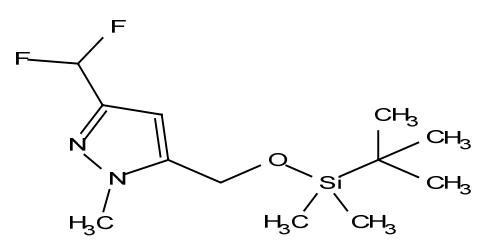

8.980

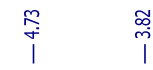

i

s
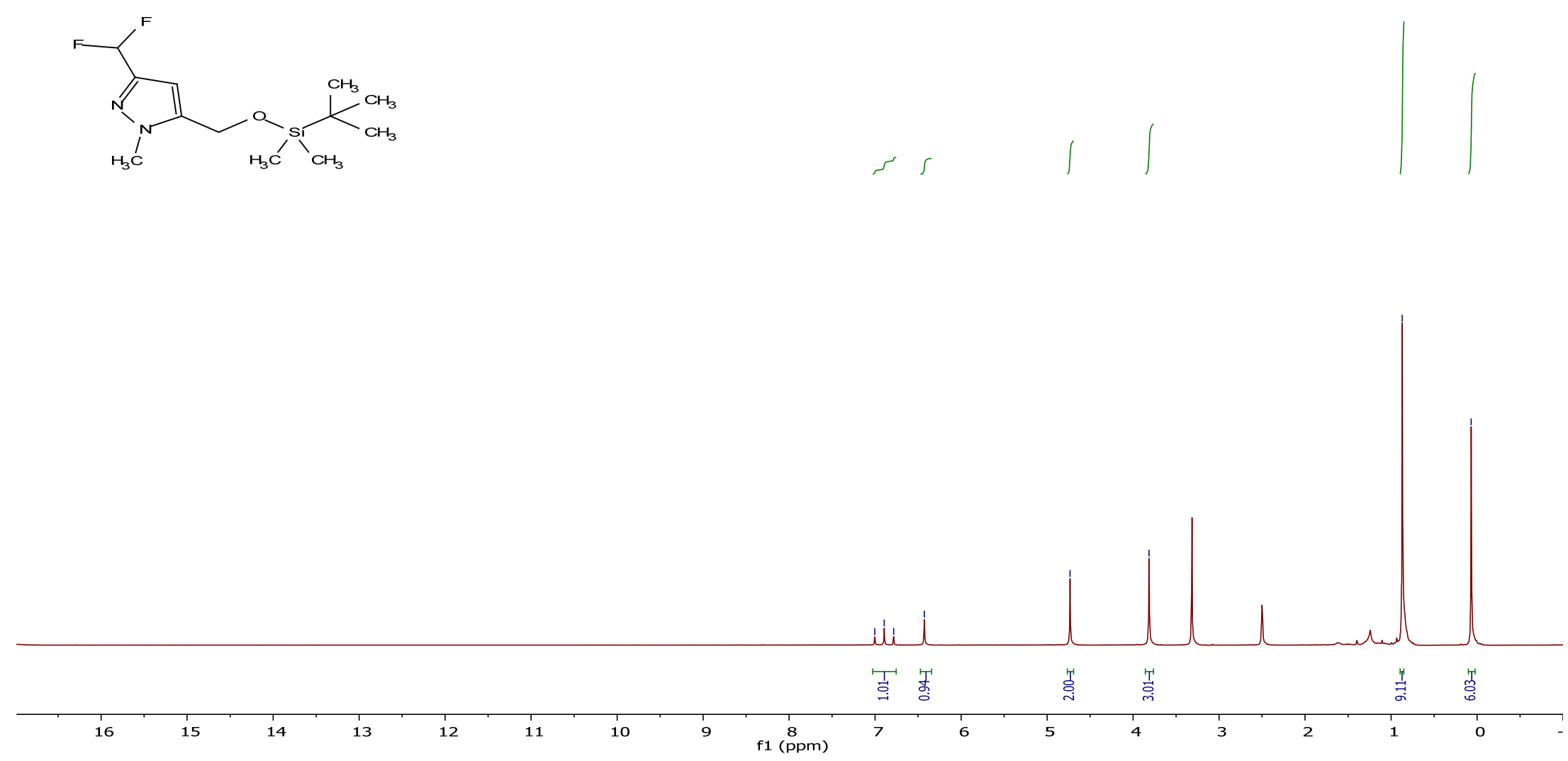
${ }^{13} \mathrm{C}\left\{{ }^{1} \mathrm{H}\right\}$ NMR spectrum of the compound 23a.

\begin{tabular}{|c|c|}
\hline 等寿喿 & 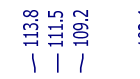 \\
\hline
\end{tabular}<smiles>Cn1nc(C(F)F)cc1CO[Si](C)(C)C(C)(C)C</smiles>
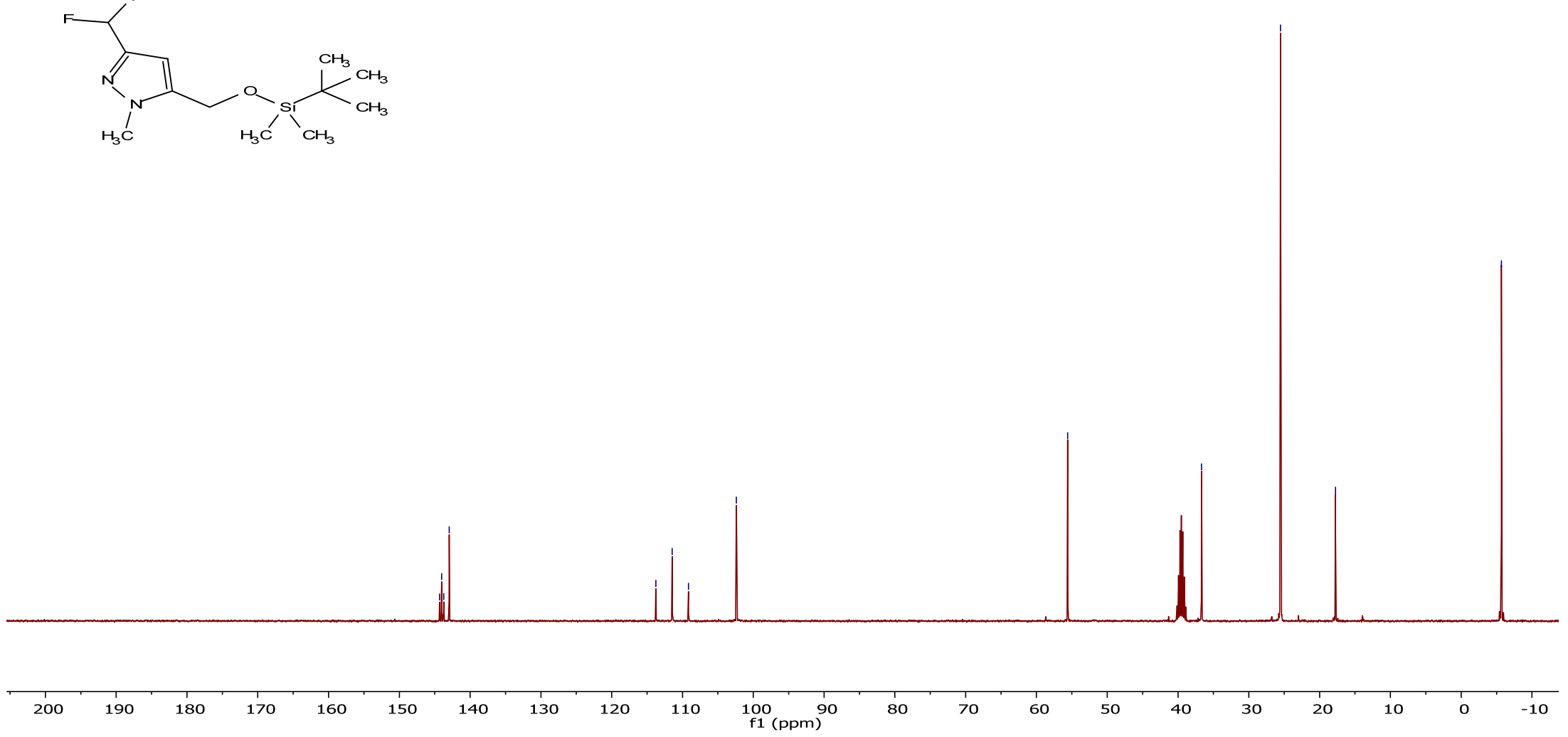
${ }^{19}$ F NMR spectrum of the compound 23a.

$\stackrel{\infty}{\stackrel{\infty}{i} \bar{j}}$
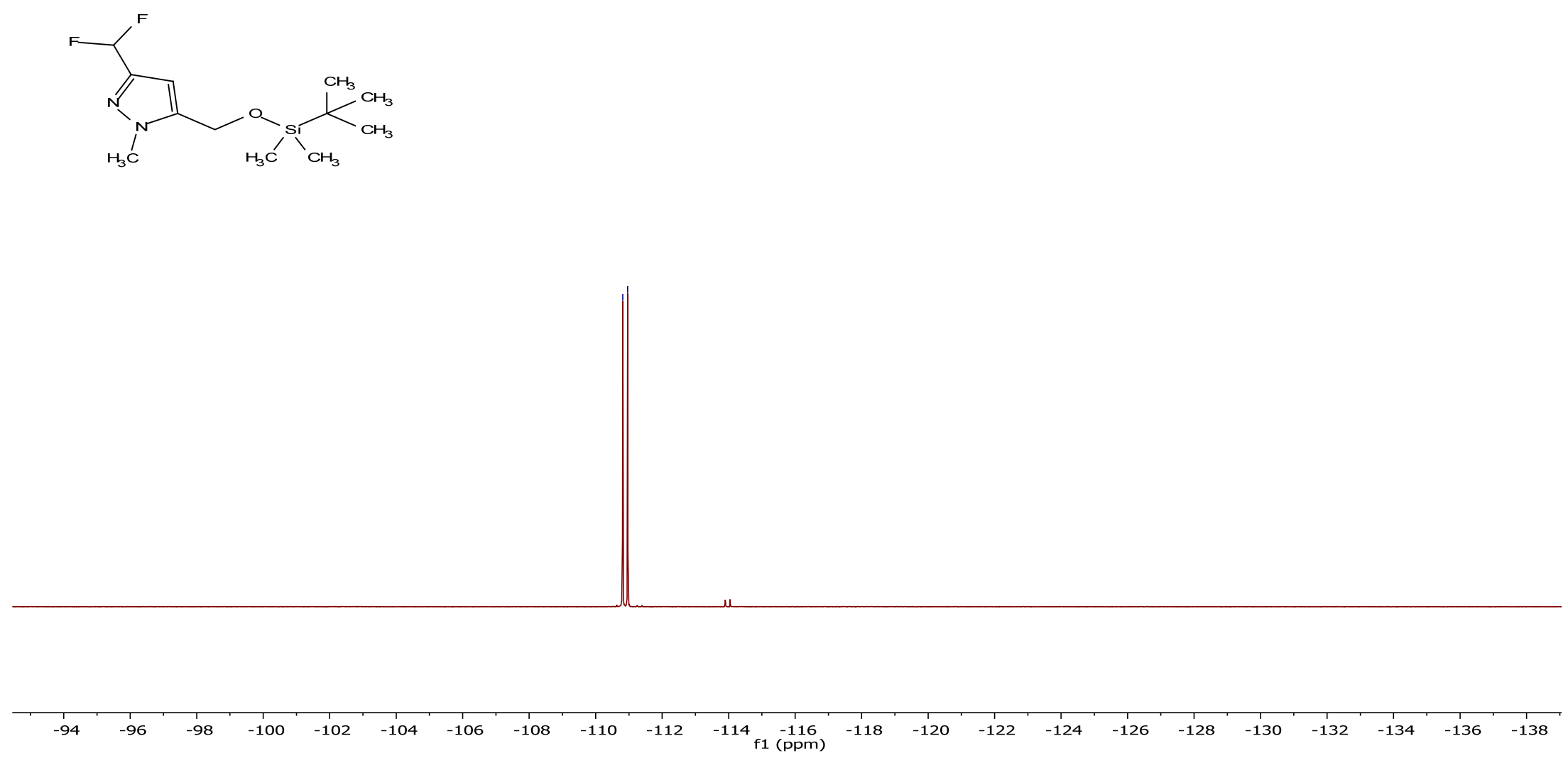
${ }^{1}$ H NOE NMR spectrum of the compound 23a.
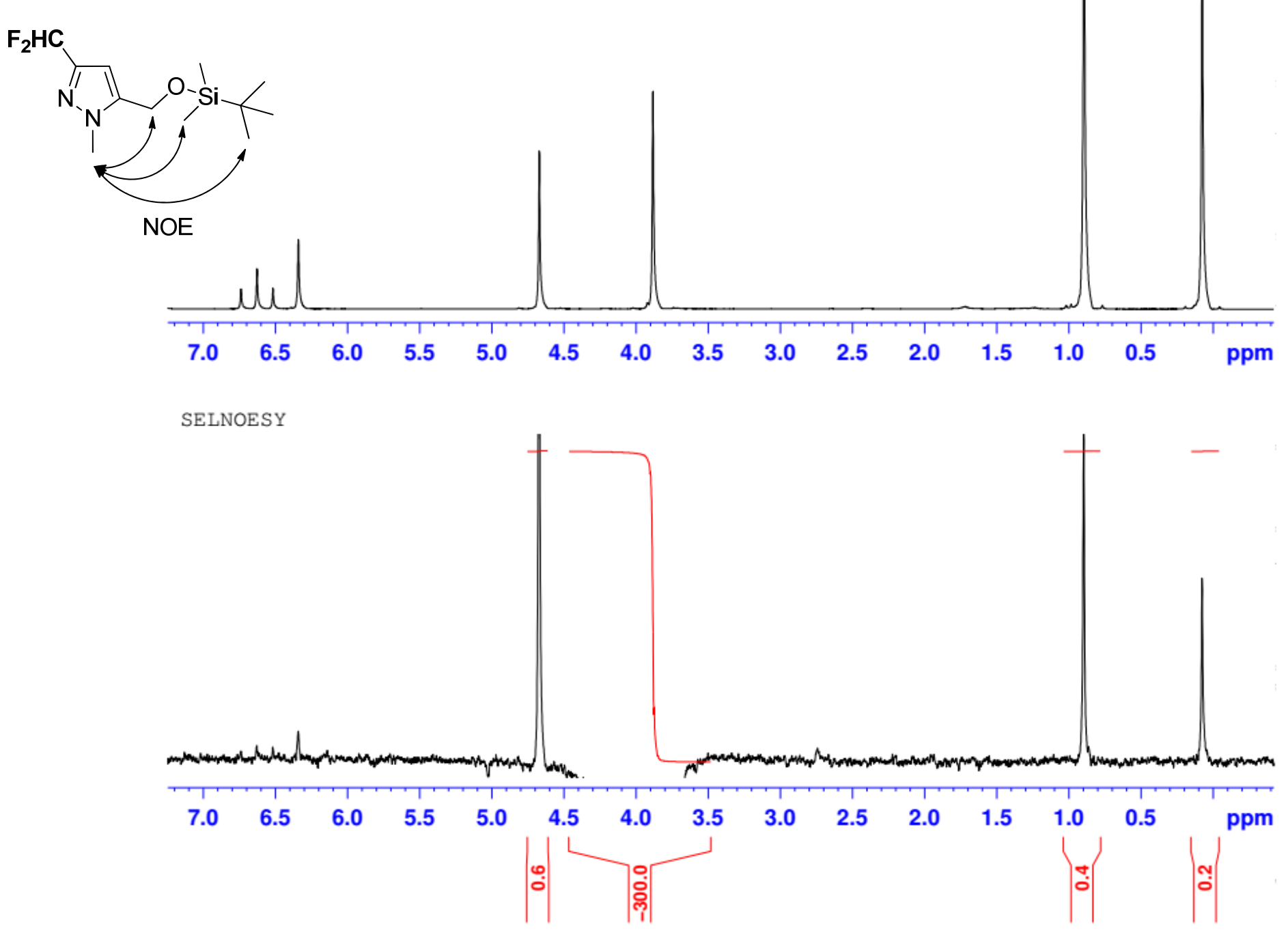
${ }^{1} \mathrm{H}$ NMR spectrum of the compound $23 \mathbf{b}$.

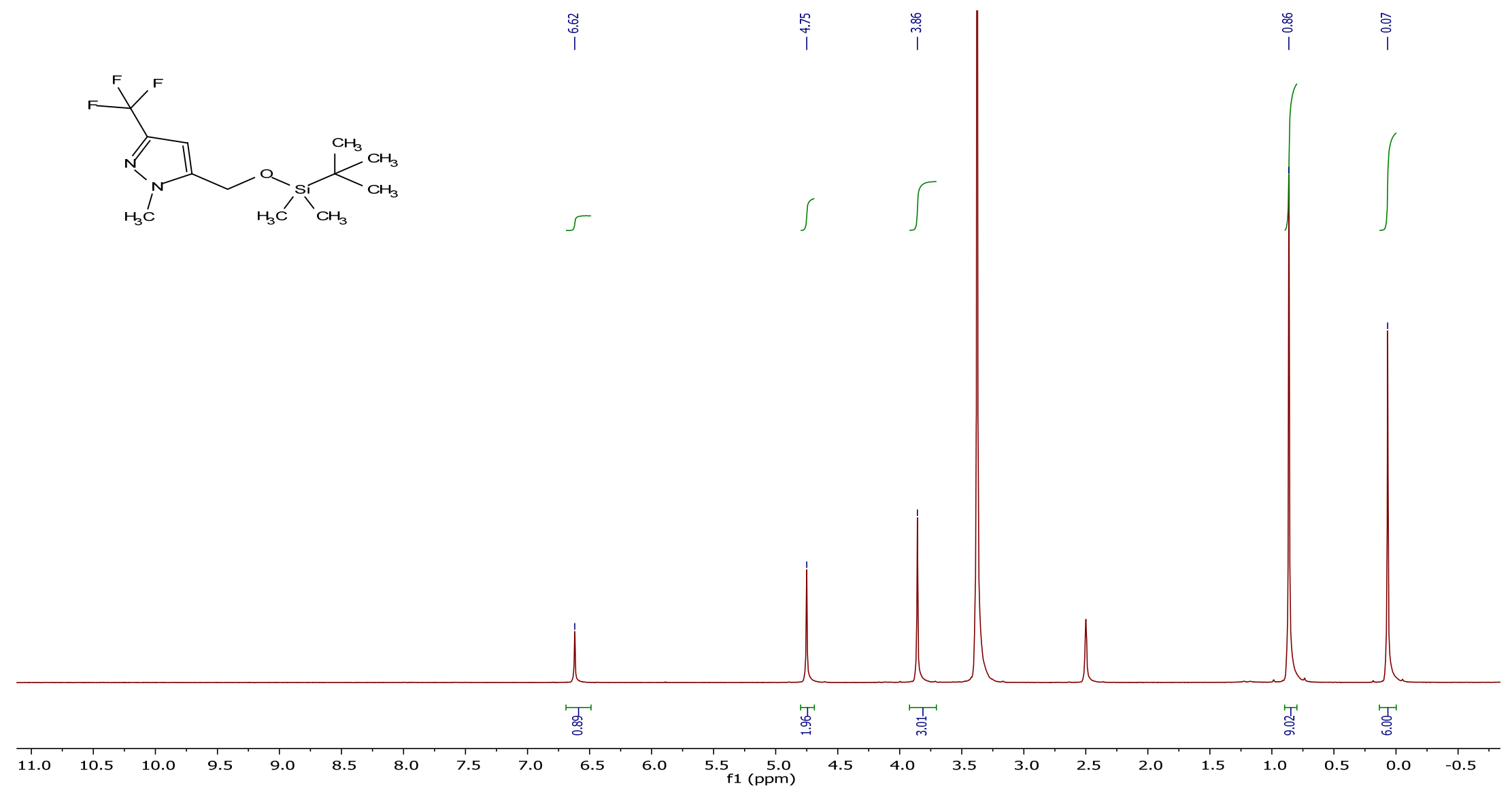


${ }^{13} \mathrm{C}\left\{{ }^{1} \mathrm{H}\right\}$ NMR spectrum of the compound 23b.

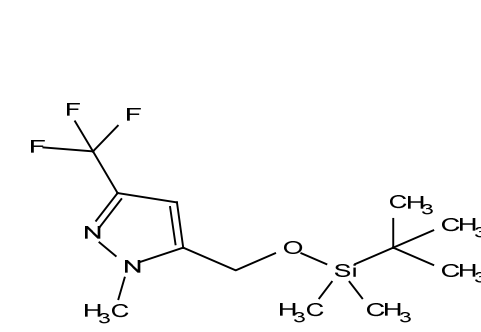

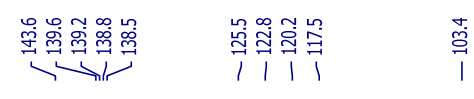

前

莴

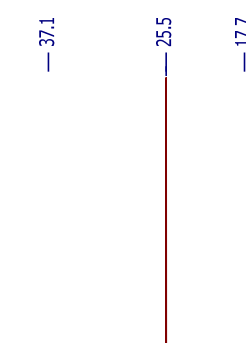

$\stackrel{\leftrightarrow}{i}$

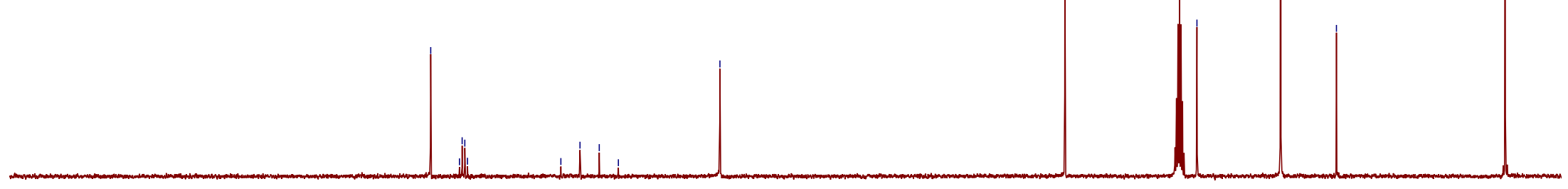

200 
${ }^{19} \mathrm{~F}\left\{{ }^{1} \mathrm{H}\right\}$ NMR spectrum of the compound 23b.
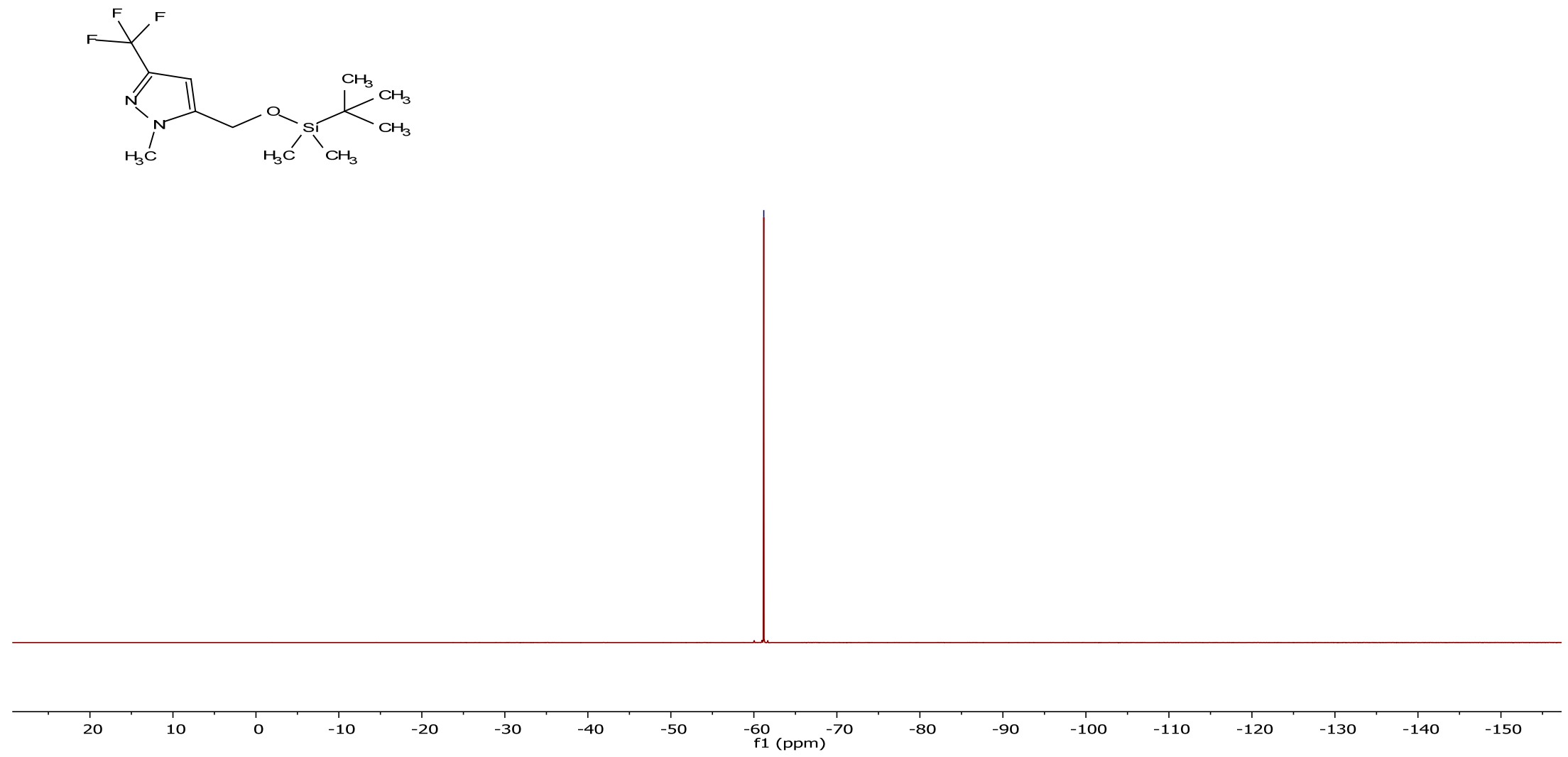
${ }^{1} \mathrm{H}$ NMR spectrum of the compound $\mathbf{2 6 \mathbf { b }}$.

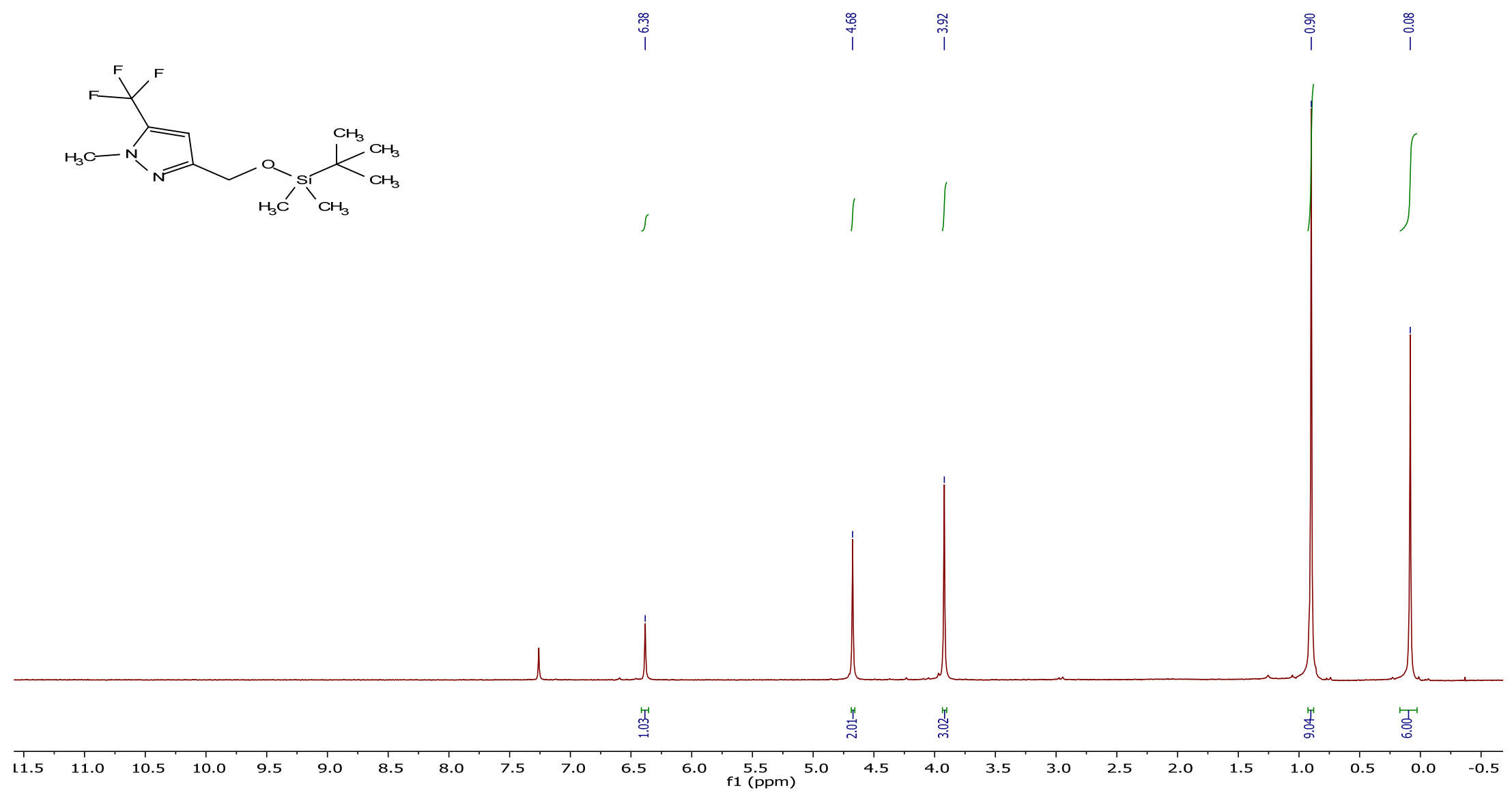


${ }^{13} \mathrm{C}\left\{{ }^{1} \mathrm{H}\right\}$ NMR spectrum of the compound $\mathbf{2 6 \mathbf { b }}$.

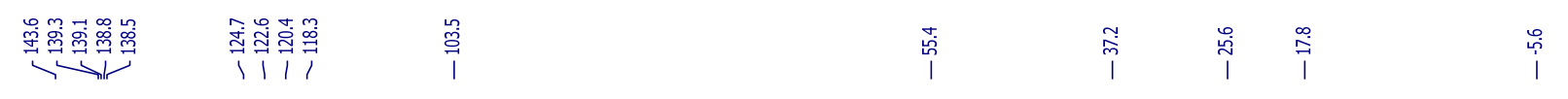
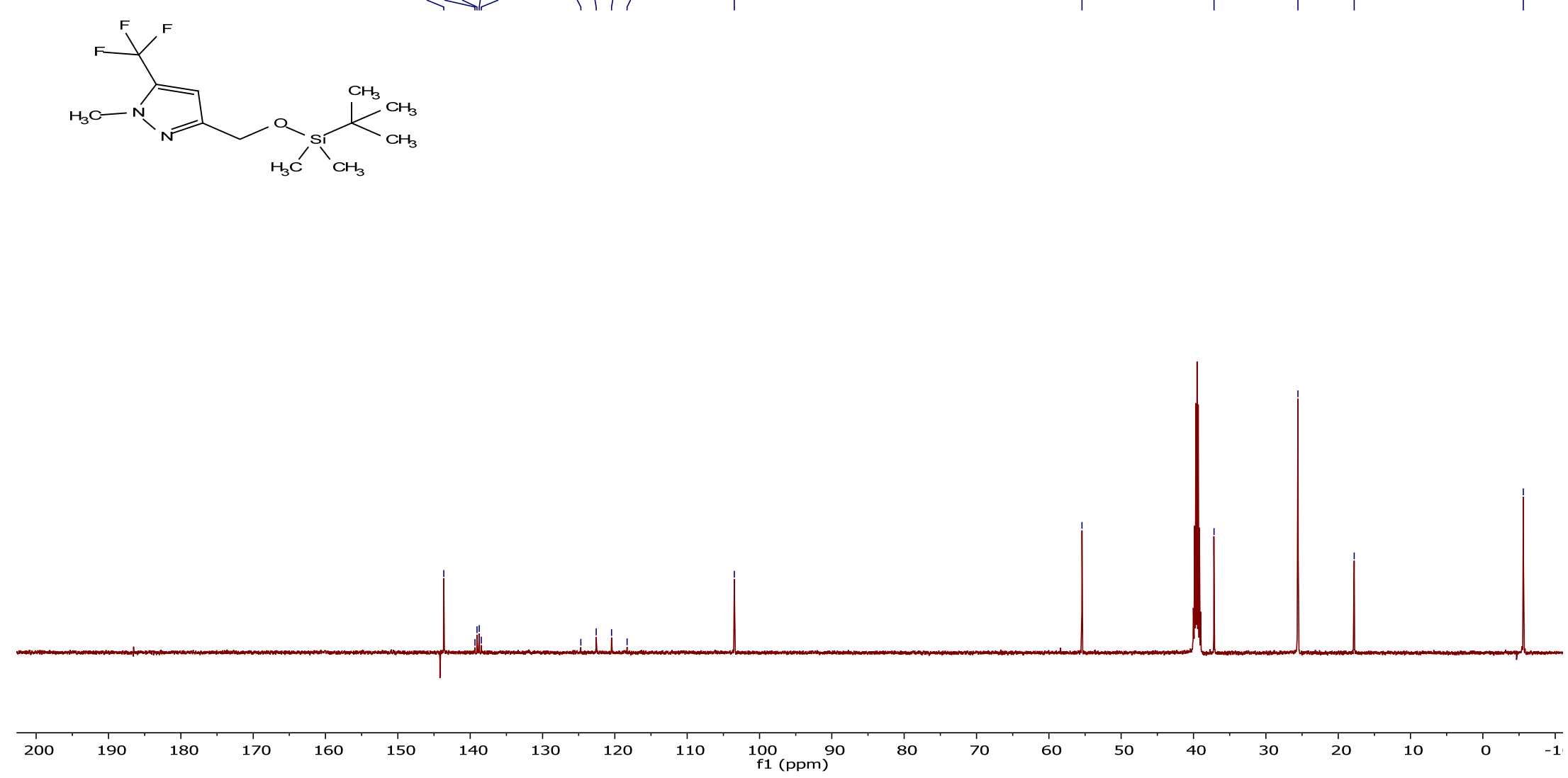
${ }^{19} \mathrm{~F}\left\{{ }^{1} \mathrm{H}\right\}$ NMR spectrum of the compound $26 \mathbf{b}$.

$\overrightarrow{\mathrm{i}}$
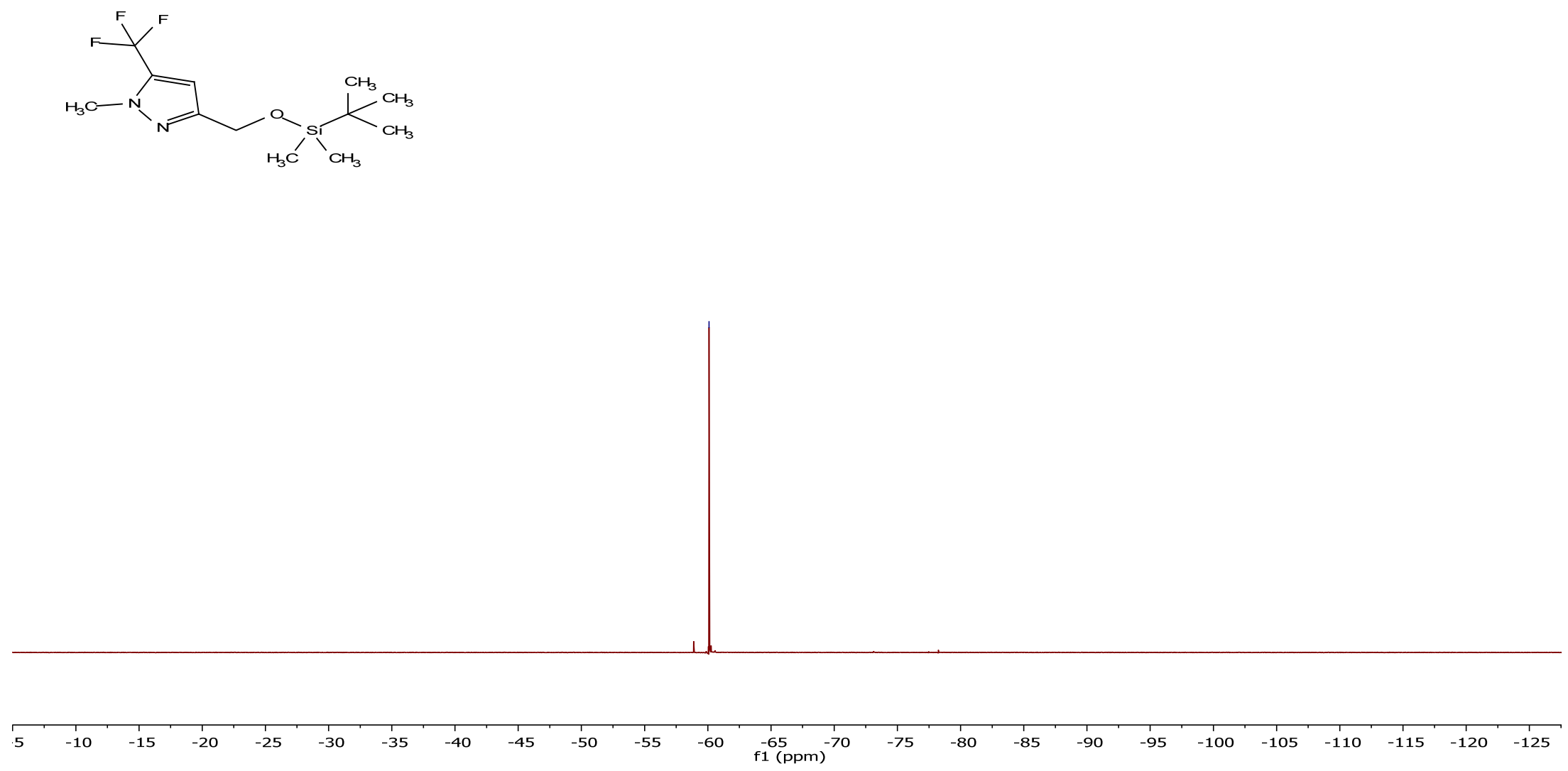
${ }^{1} \mathrm{H}$ NMR spectrum of the compound 23c.

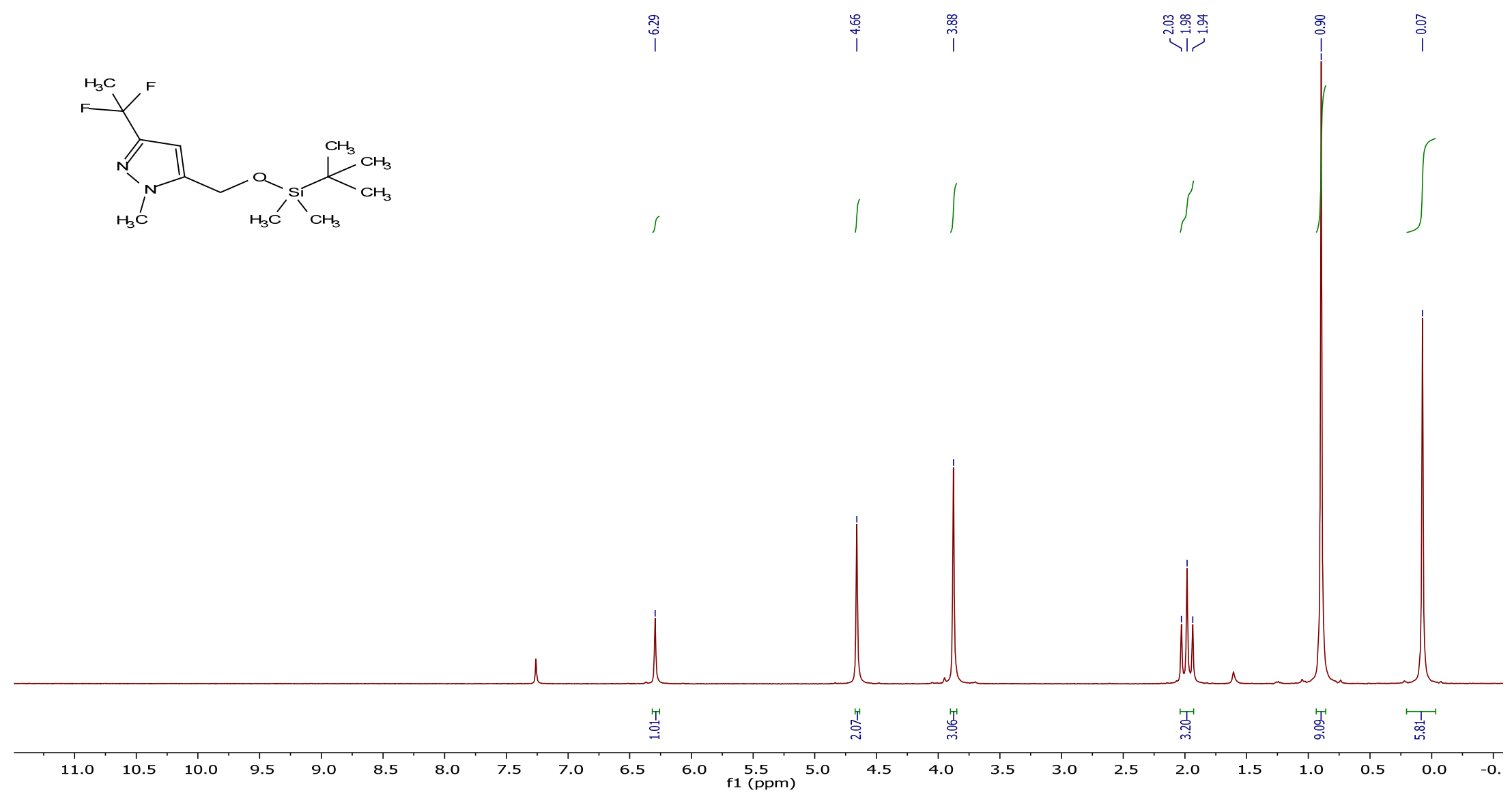


${ }^{13} \mathrm{C}\left\{{ }^{1} \mathrm{H}\right\}$ NMR spectrum of the compound 23c.

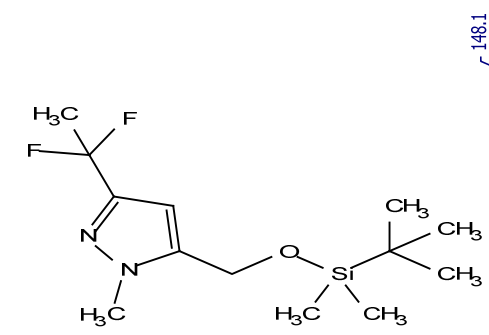

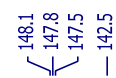

影唦

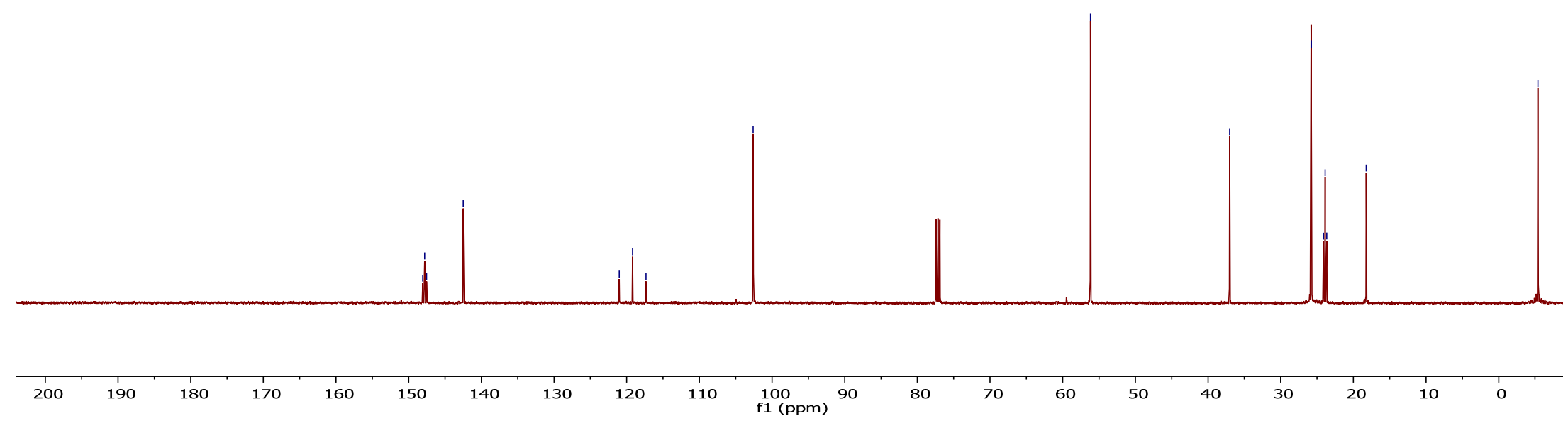


${ }^{19} \mathrm{~F}\left\{{ }^{1} \mathrm{H}\right\}$ NMR spectrum of the compound 23c.

市
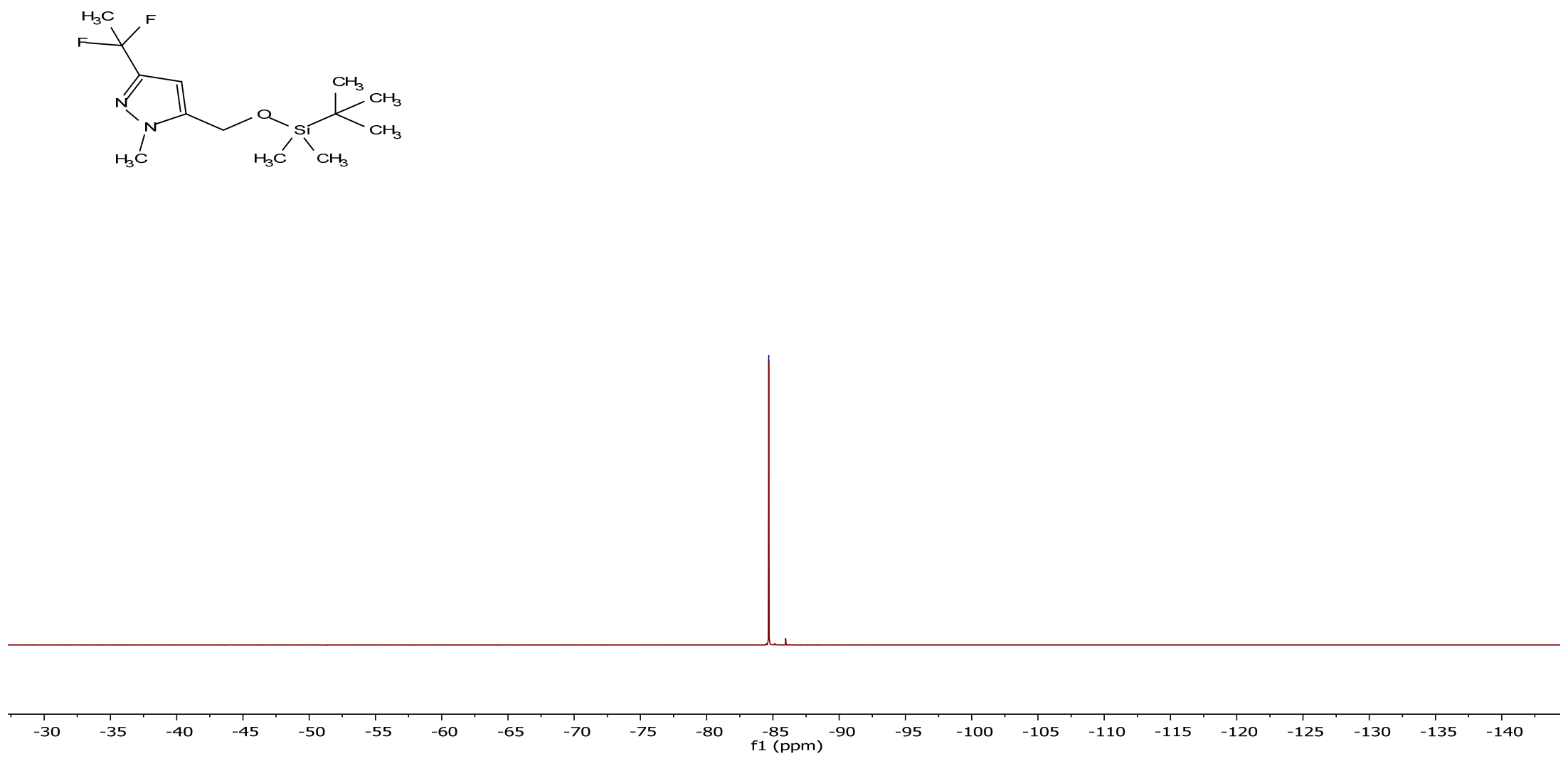
${ }^{1} \mathrm{H}$ NMR spectrum of the compound 23d.

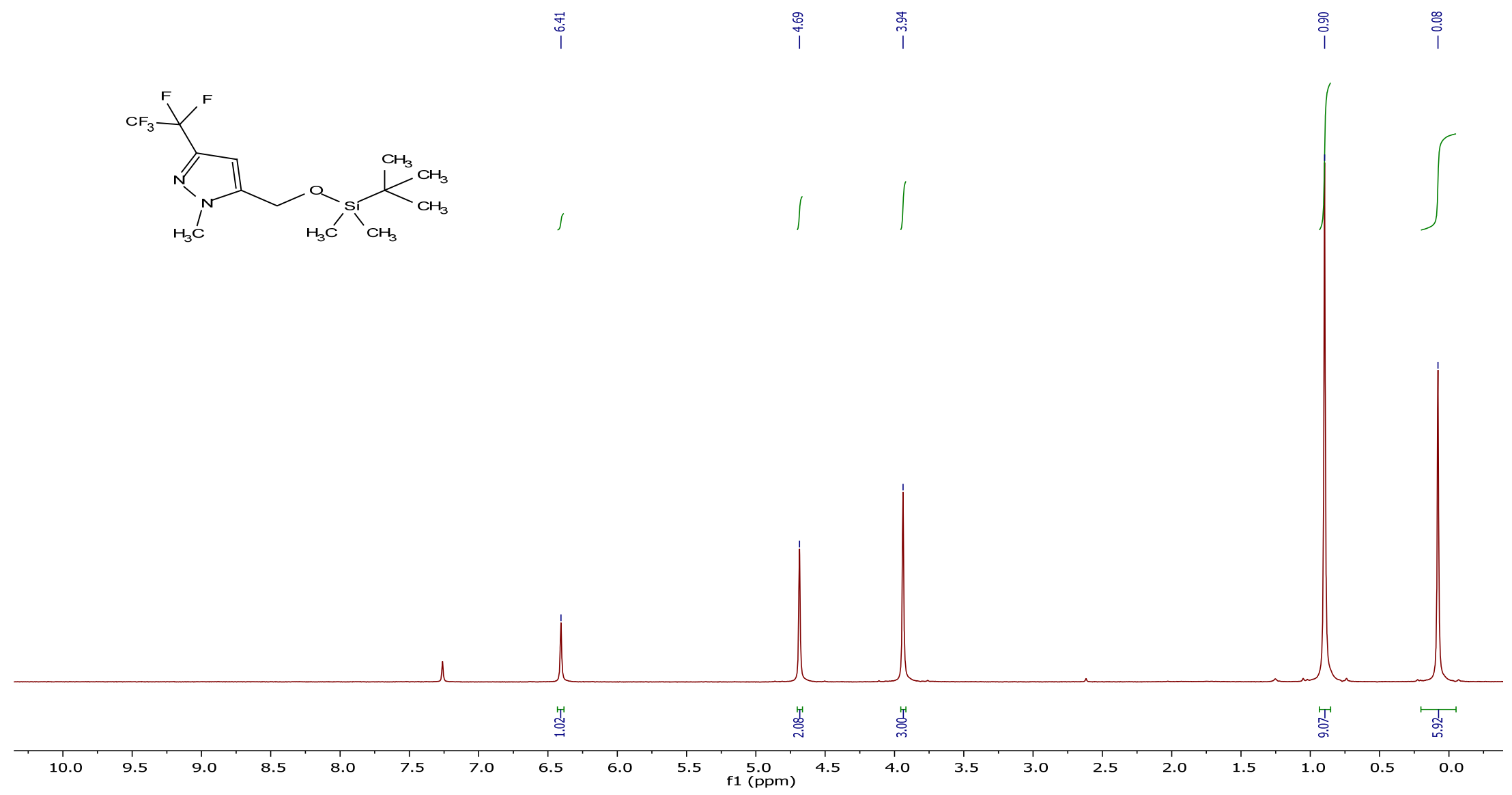




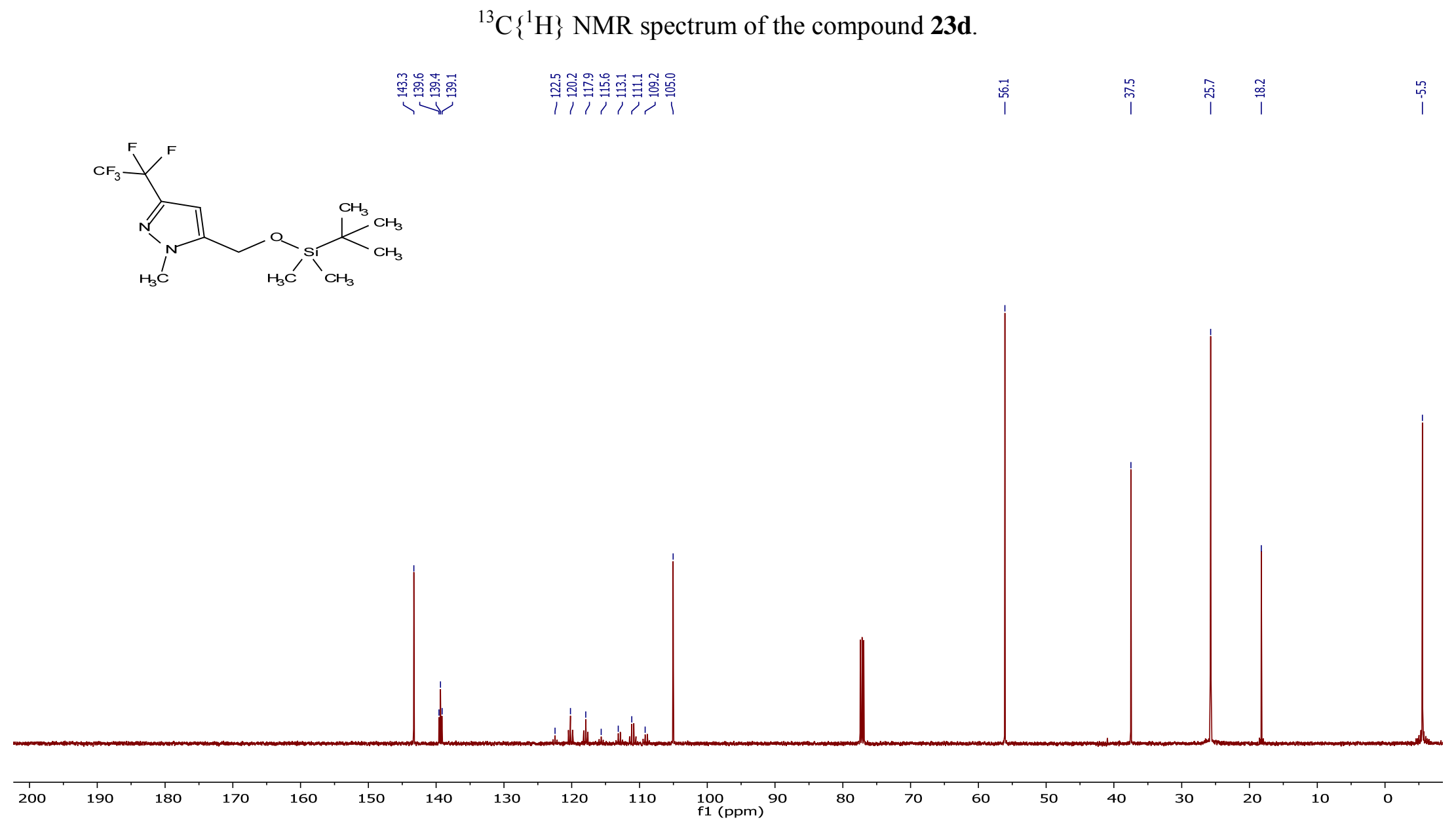


${ }^{19} \mathrm{~F}\left\{{ }^{1} \mathrm{H}\right\}$ NMR spectrum of the compound 23d.

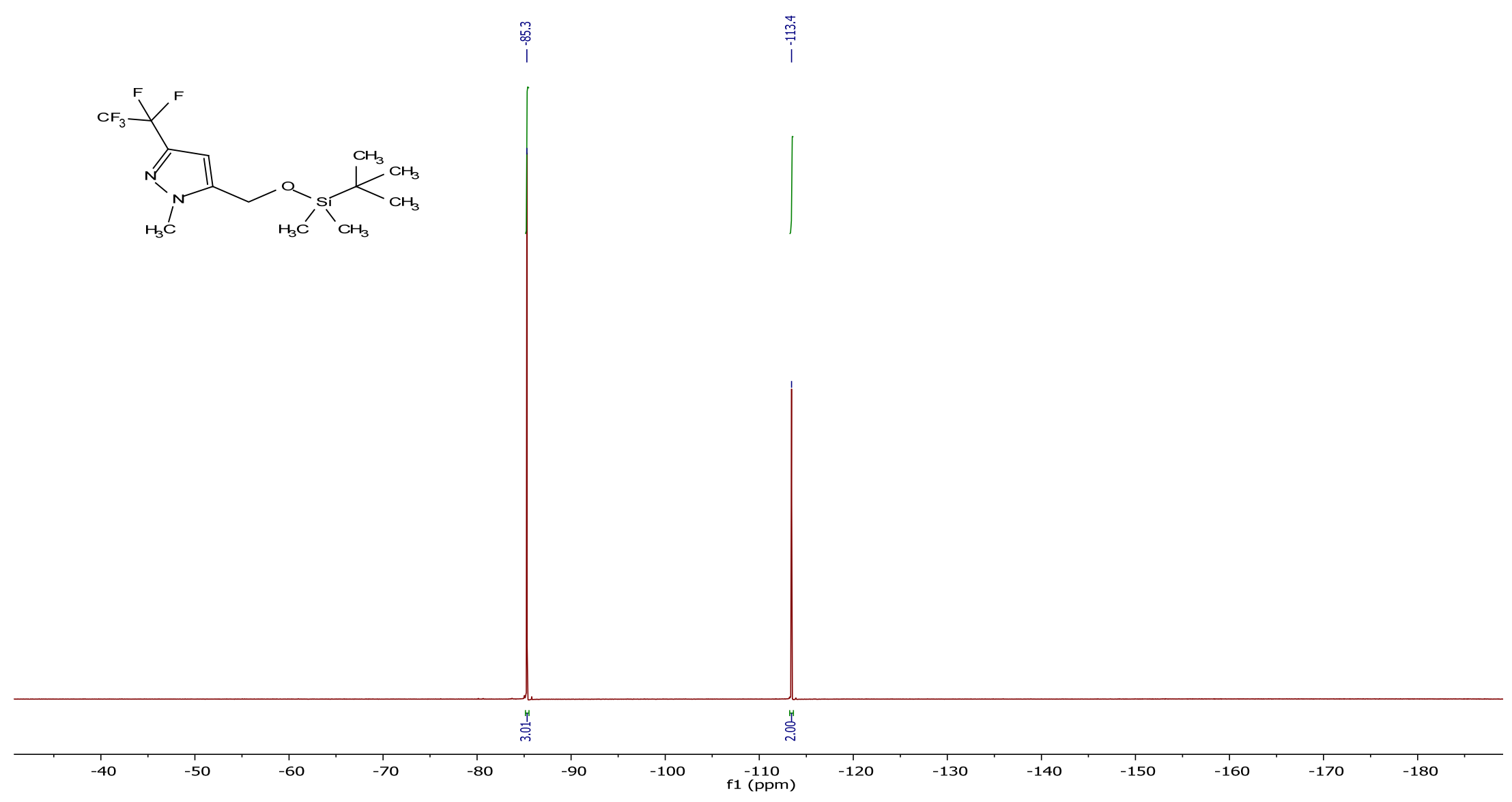


${ }^{1} \mathrm{H}$ NMR spectrum of the compound $23 \mathbf{e}$.

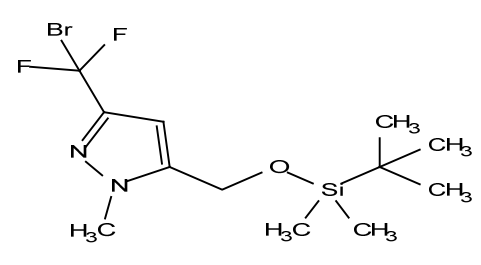

冚 1

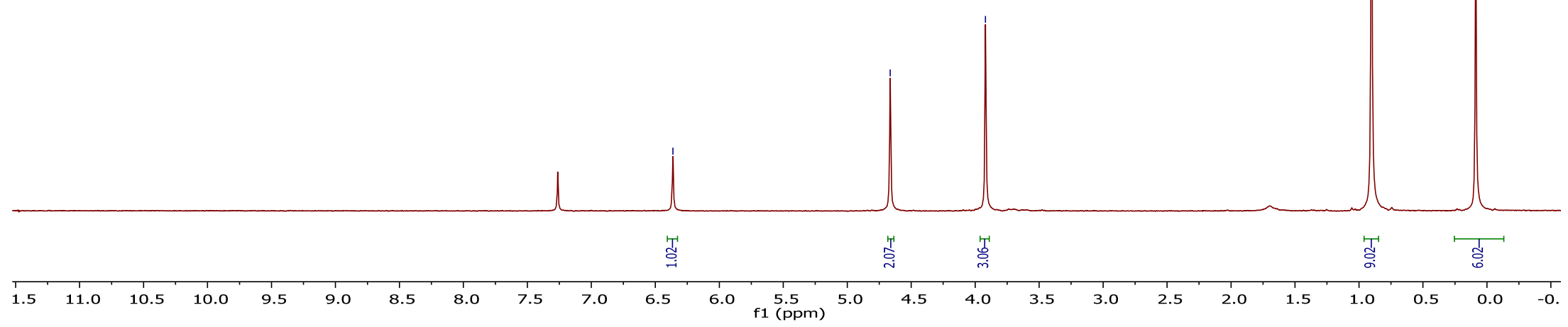


${ }^{13} \mathrm{C}\left\{{ }^{1} \mathrm{H}\right\}$ NMR spectrum of the compound 23e.
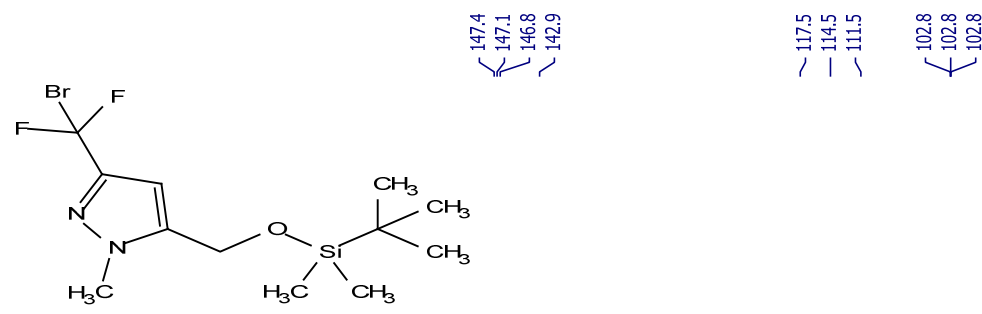

通

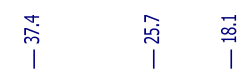

$\mathrm{H}_{3} \mathrm{C} \quad \mathrm{H}_{3} \mathrm{C} \quad \mathrm{CH}_{3}$

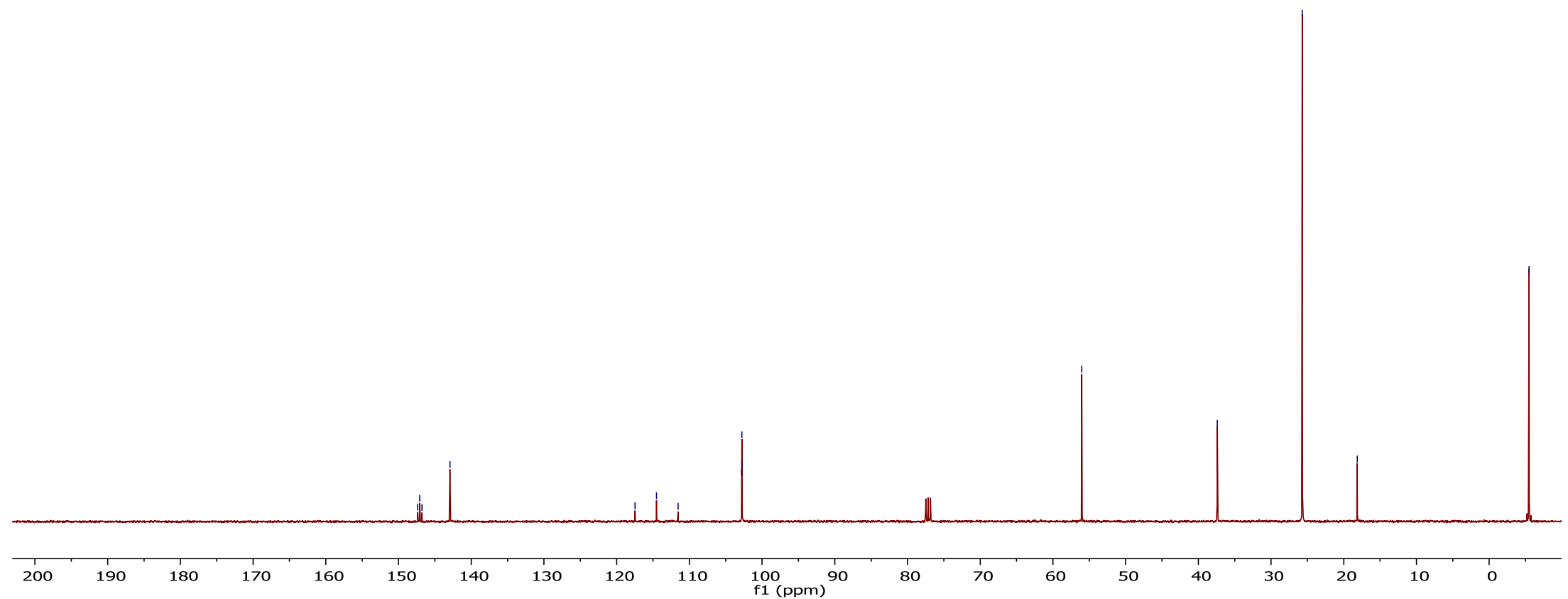


${ }^{19} \mathrm{~F}\left\{{ }^{1} \mathrm{H}\right\}$ NMR spectrum of the compound 23e.

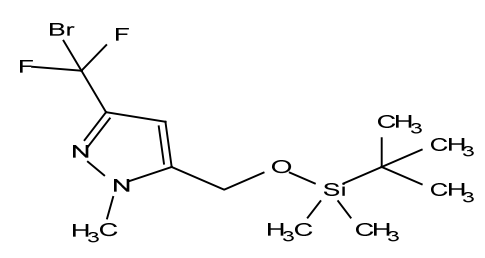

ఫี

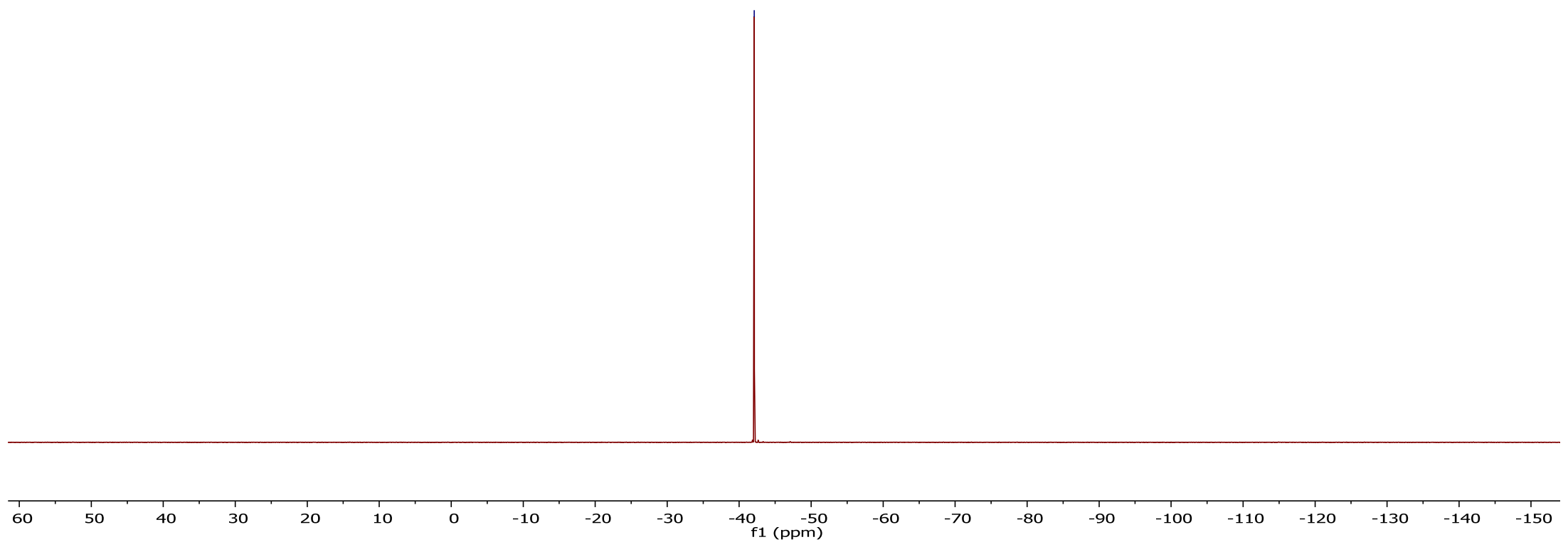


${ }^{1}$ H NMR spectrum of the compound $\mathbf{2 3 h}$.

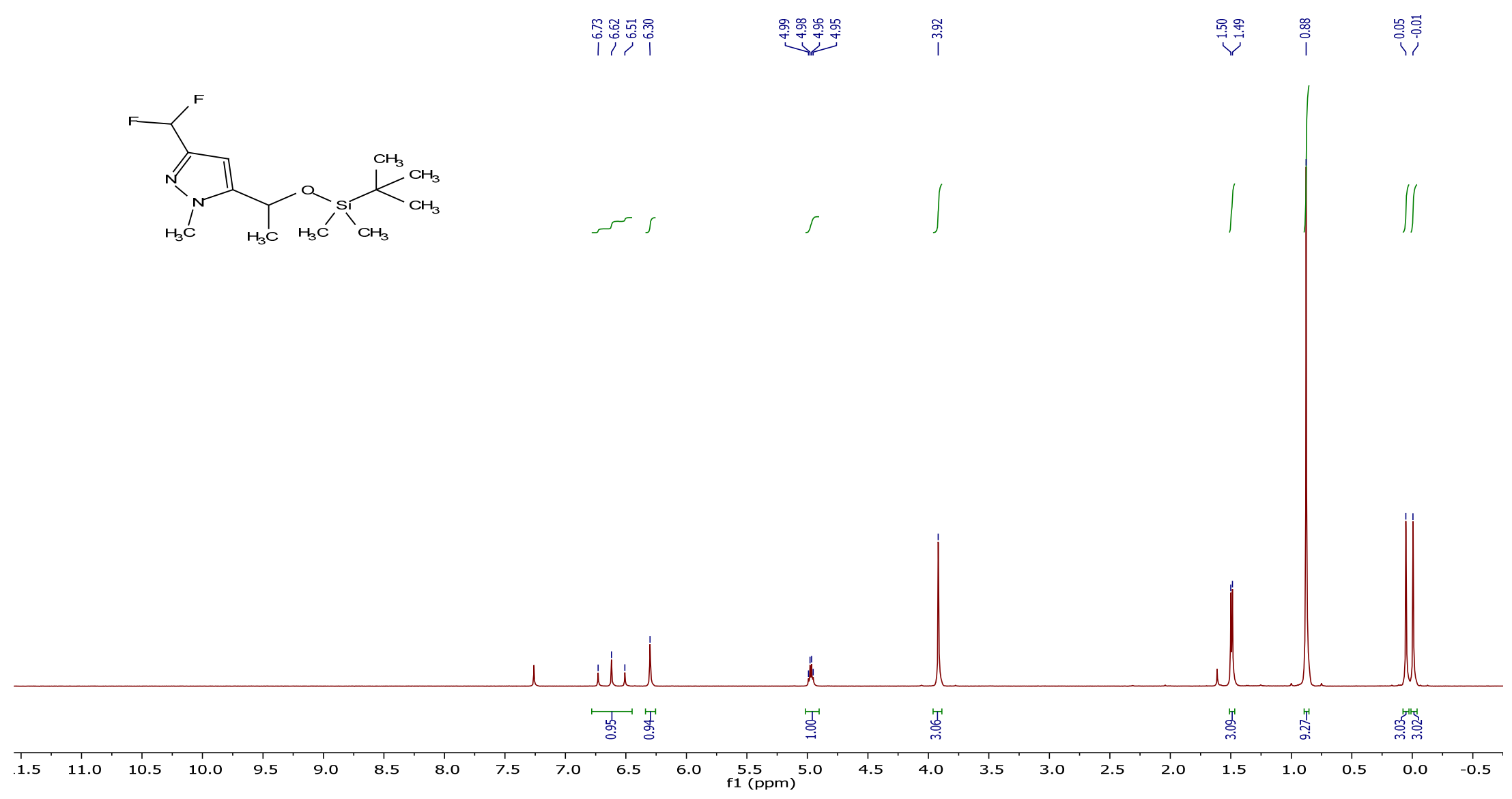


${ }^{13} \mathrm{C}\left\{{ }^{1} \mathrm{H}\right\}$ NMR spectrum of the compound 23h.

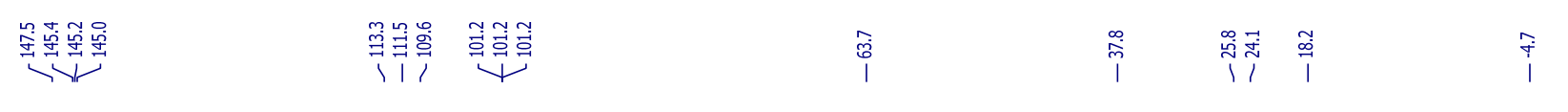
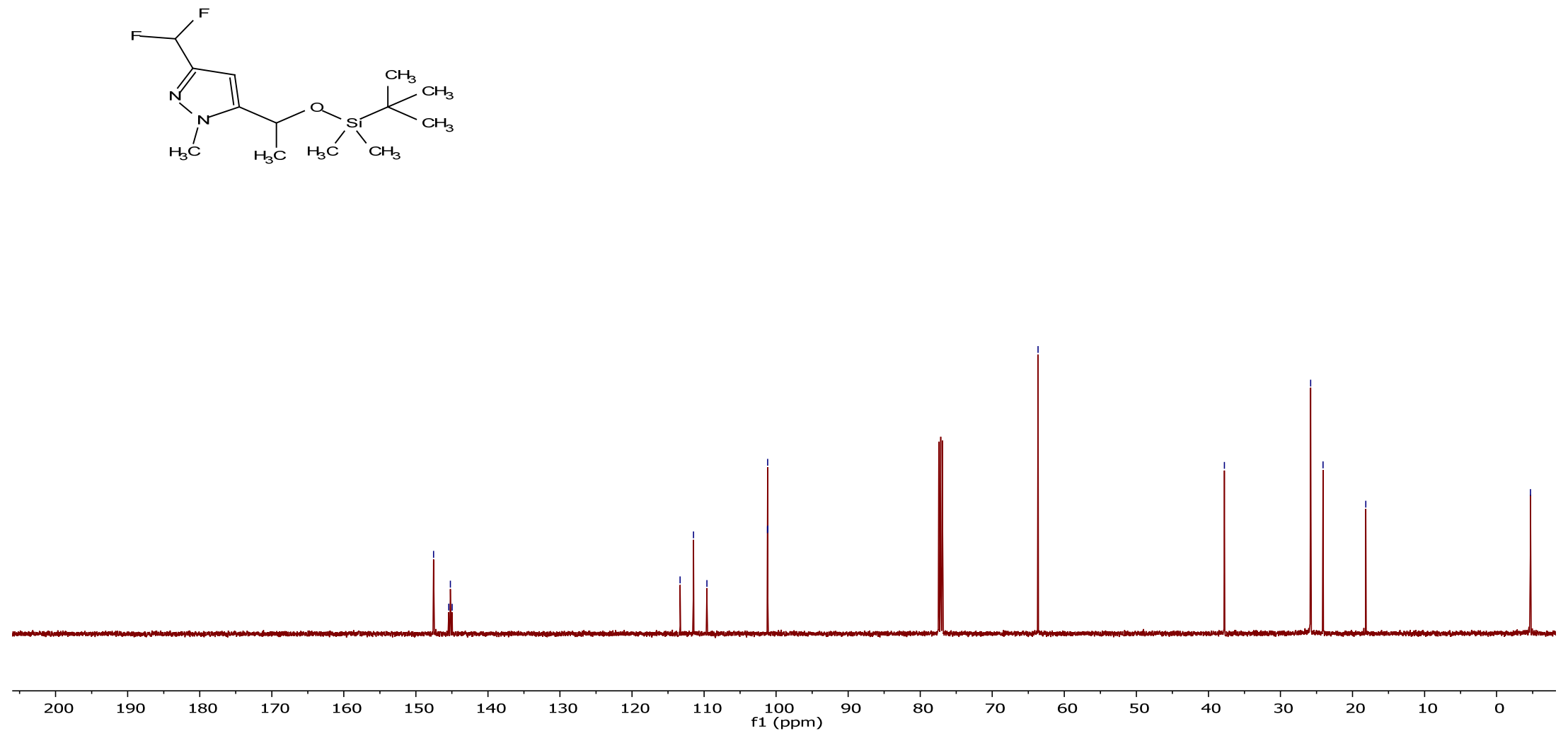
${ }^{19}$ F NMR spectrum of the compound $\mathbf{2 3 h}$.

刍 辛
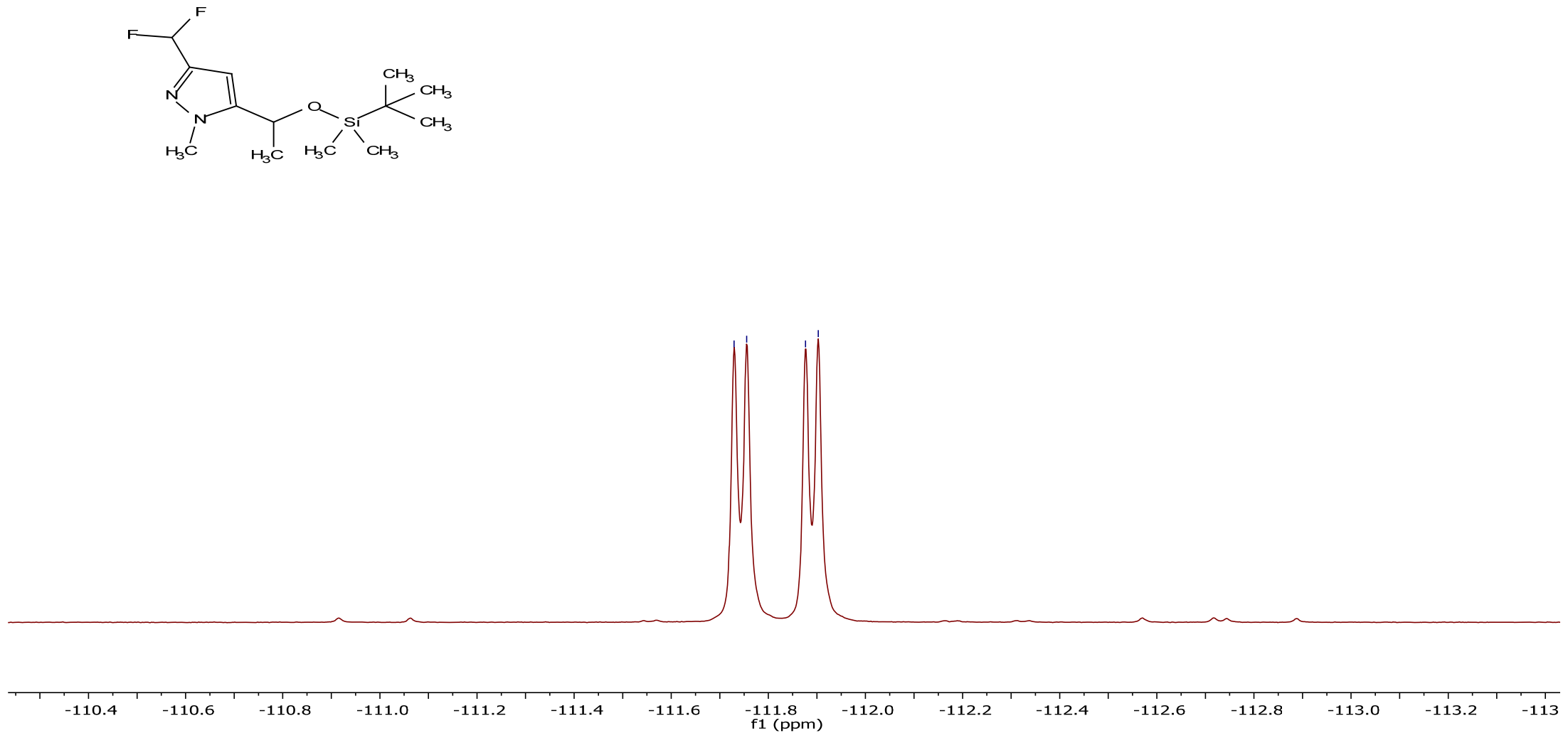
${ }^{1} \mathrm{H}$ NMR spectrum of the compound $\mathbf{2 4 a}$.
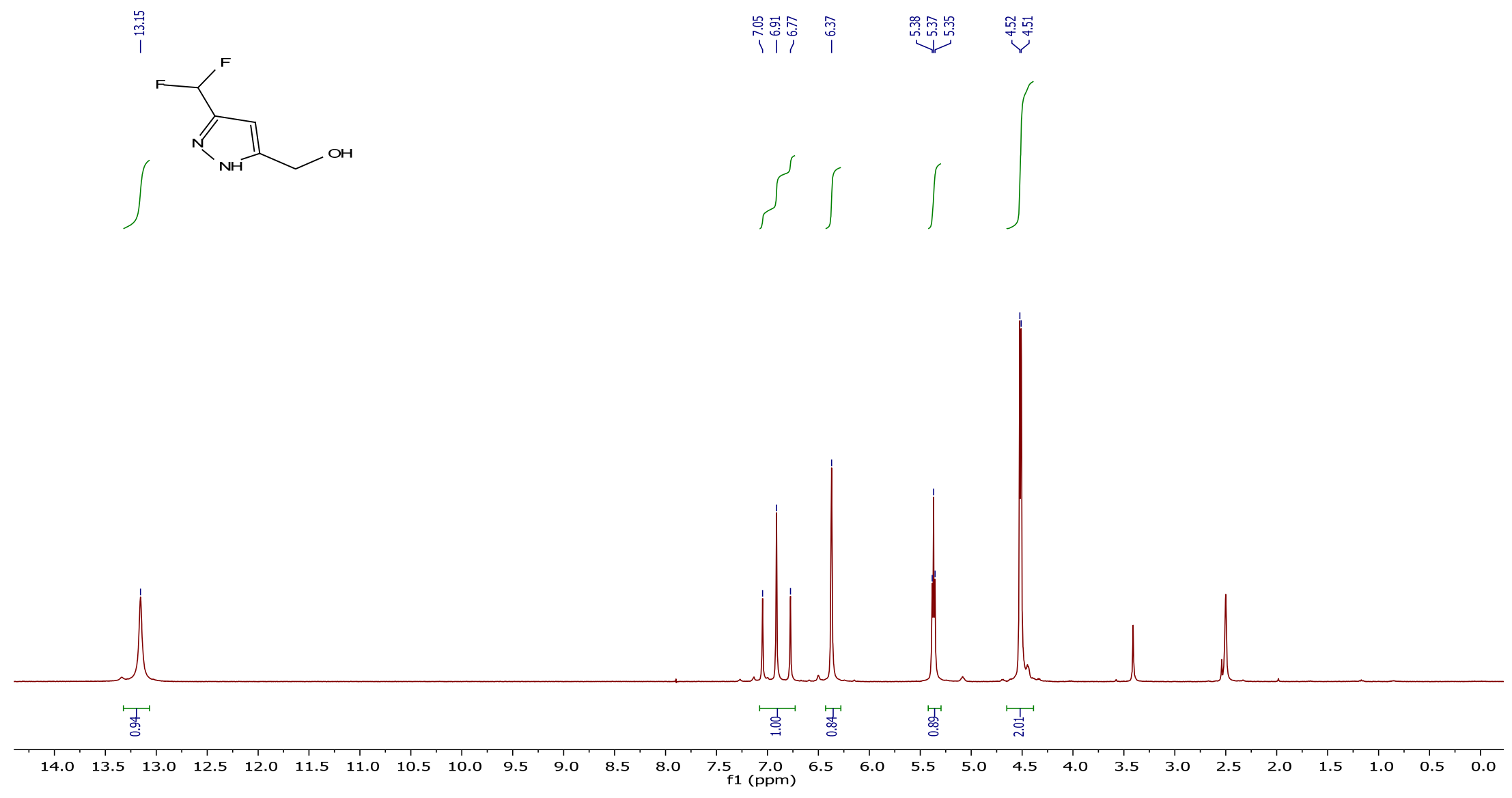
${ }^{13} \mathrm{C}\left\{{ }^{1} \mathrm{H}\right\}$ NMR spectrum of the compound 24a.
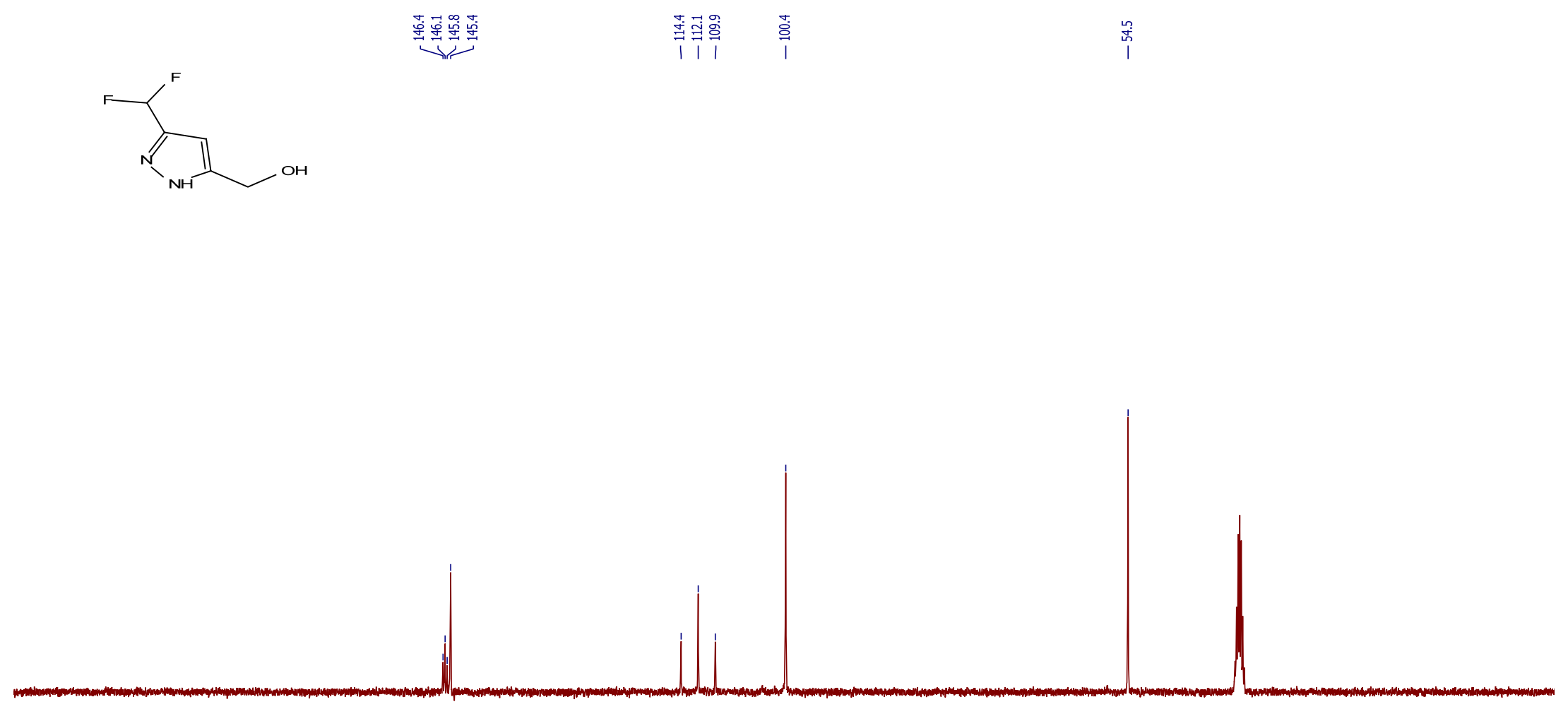

200

190

180

160 
${ }^{19}$ F NMR spectrum of the compound $24 a$.

产芦
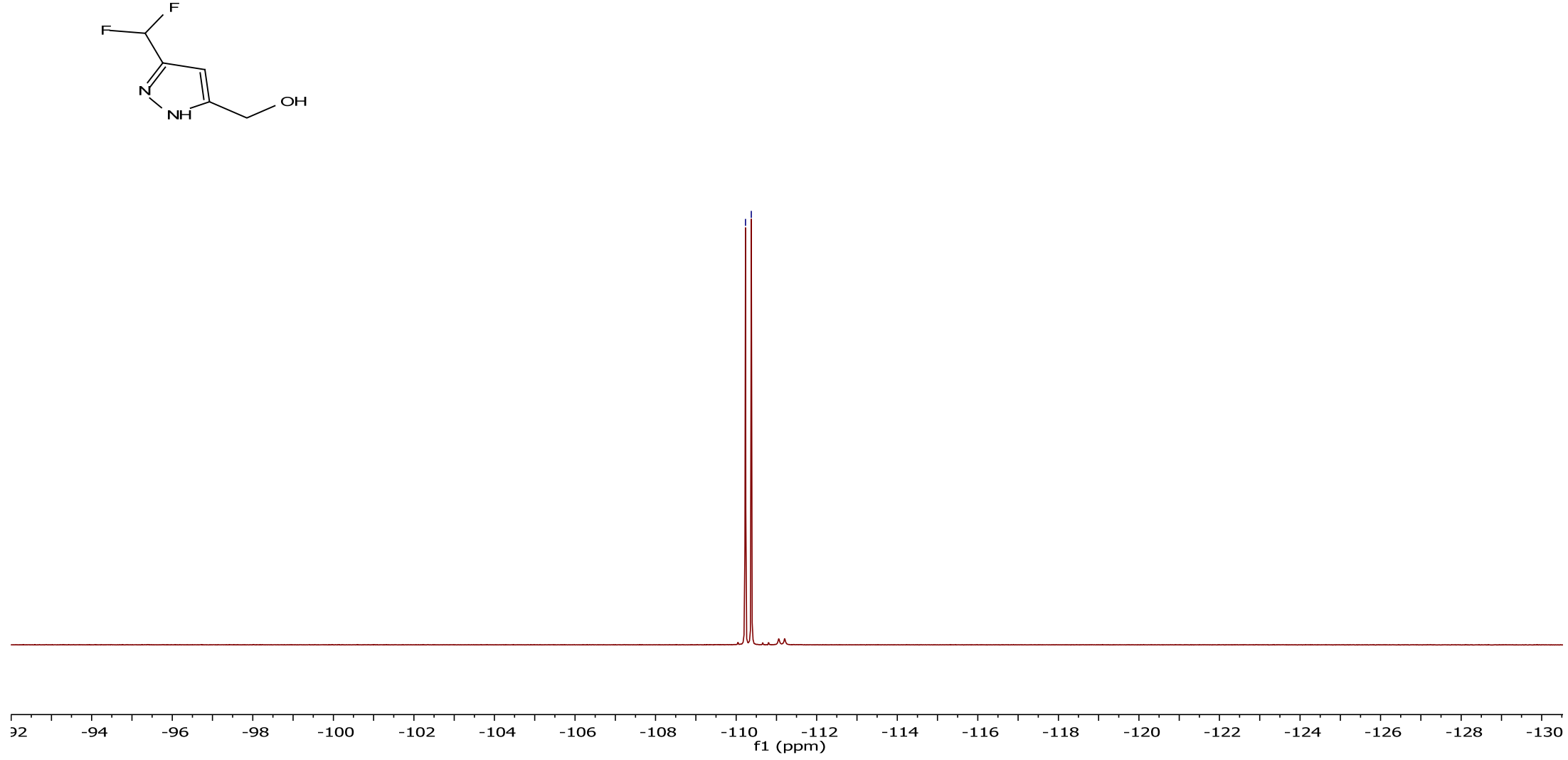
${ }^{1} \mathrm{H}$ NMR spectrum of the compound 24c.
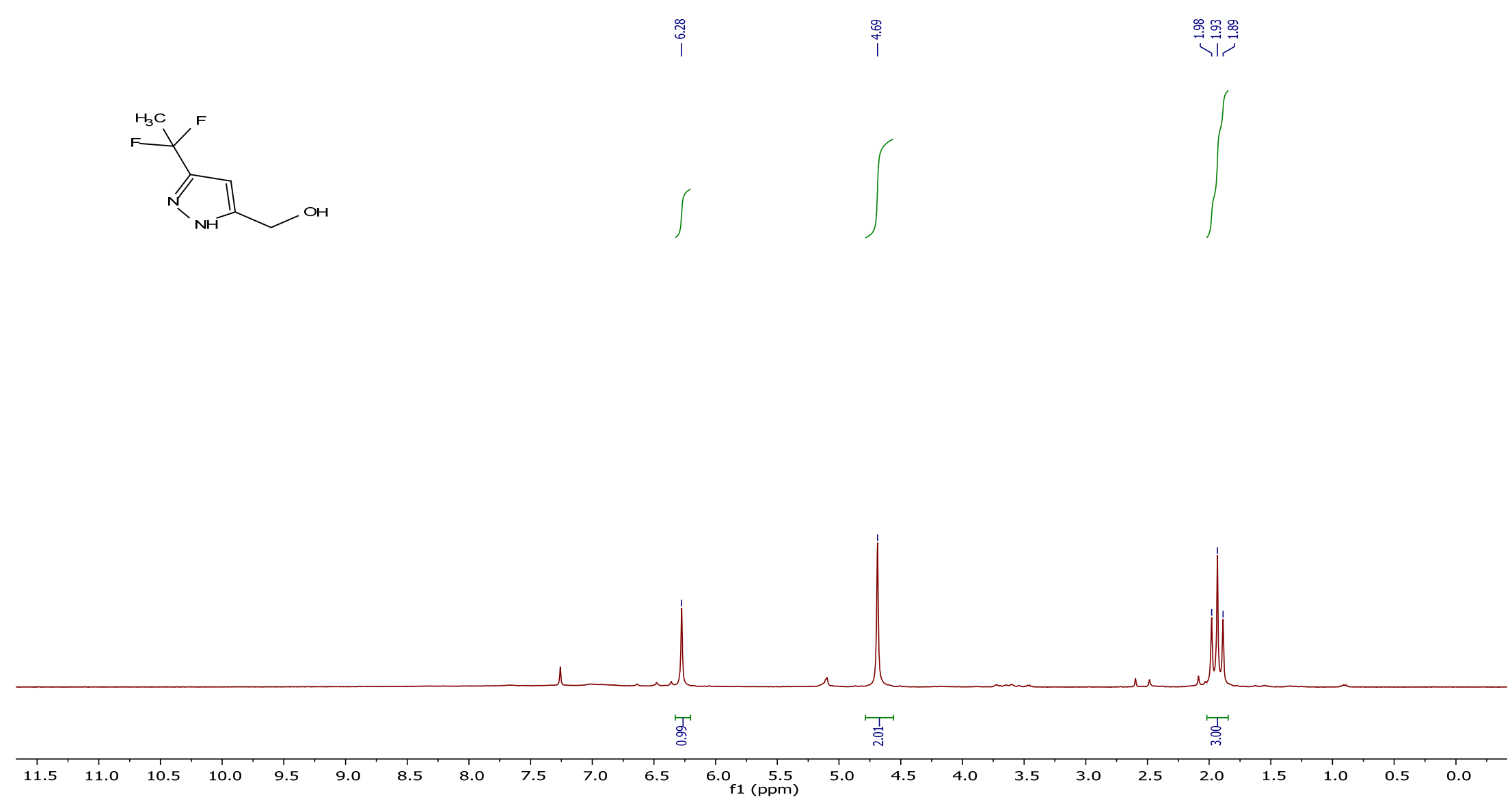
${ }^{13} \mathrm{C}\left\{{ }^{1} \mathrm{H}\right\}$ NMR spectrum of the compound 24c.

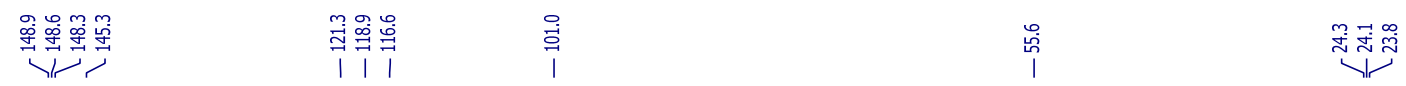<smiles>CC(F)(F)c1cc(CO)[nH]n1</smiles>

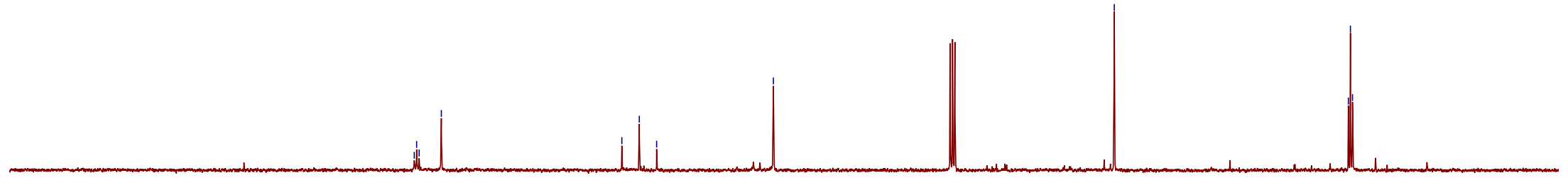


${ }^{19} \mathrm{~F}\left\{{ }^{1} \mathrm{H}\right\}$ NMR spectrum of the compound 24c.

$\dddot{\oplus}$

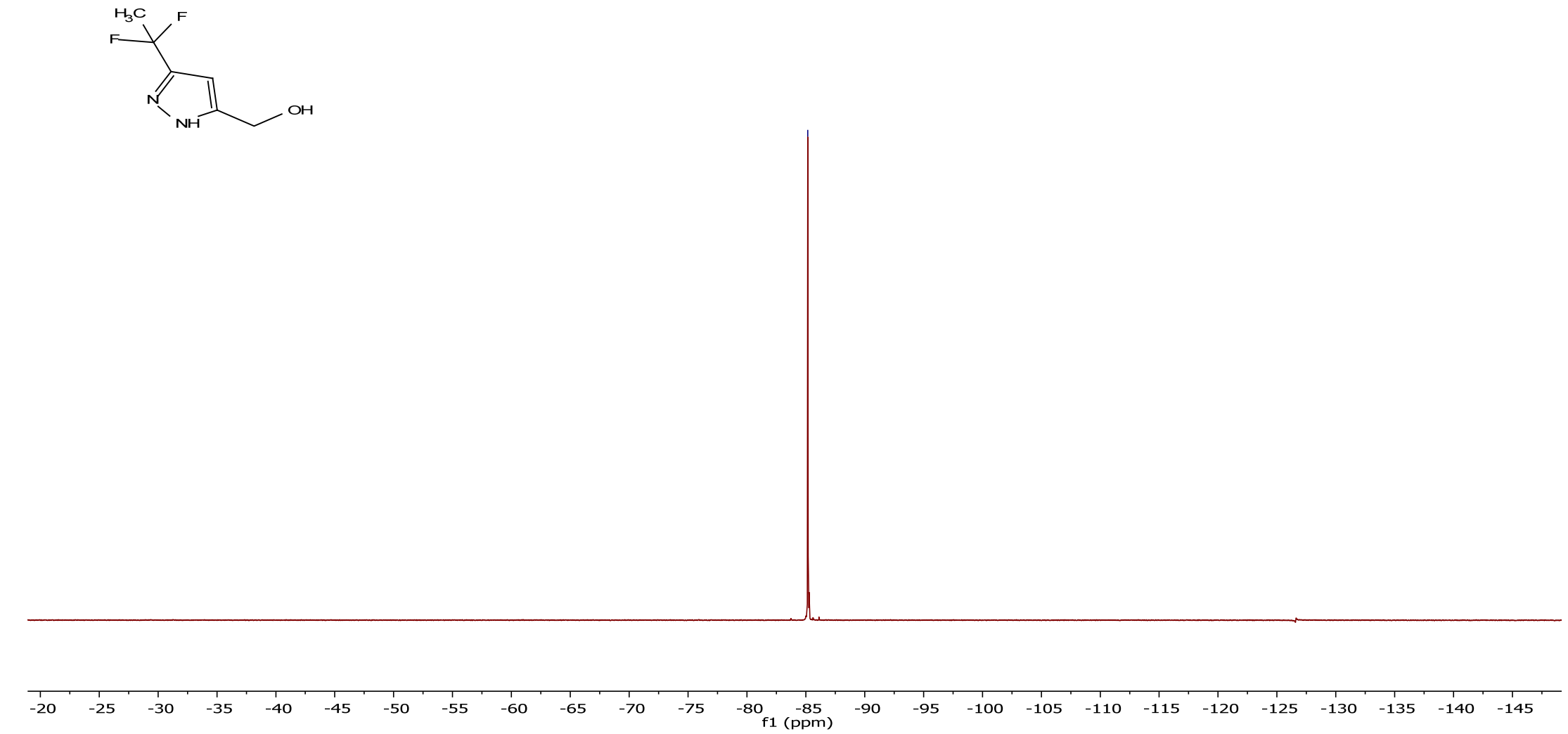


${ }^{1} \mathrm{H}$ NMR spectrum of the compound $\mathbf{2 4 g}$.

产

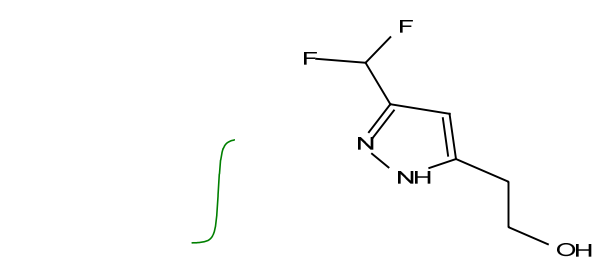

il

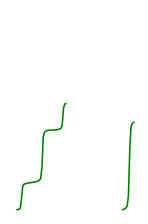

$\stackrel{2}{i}$

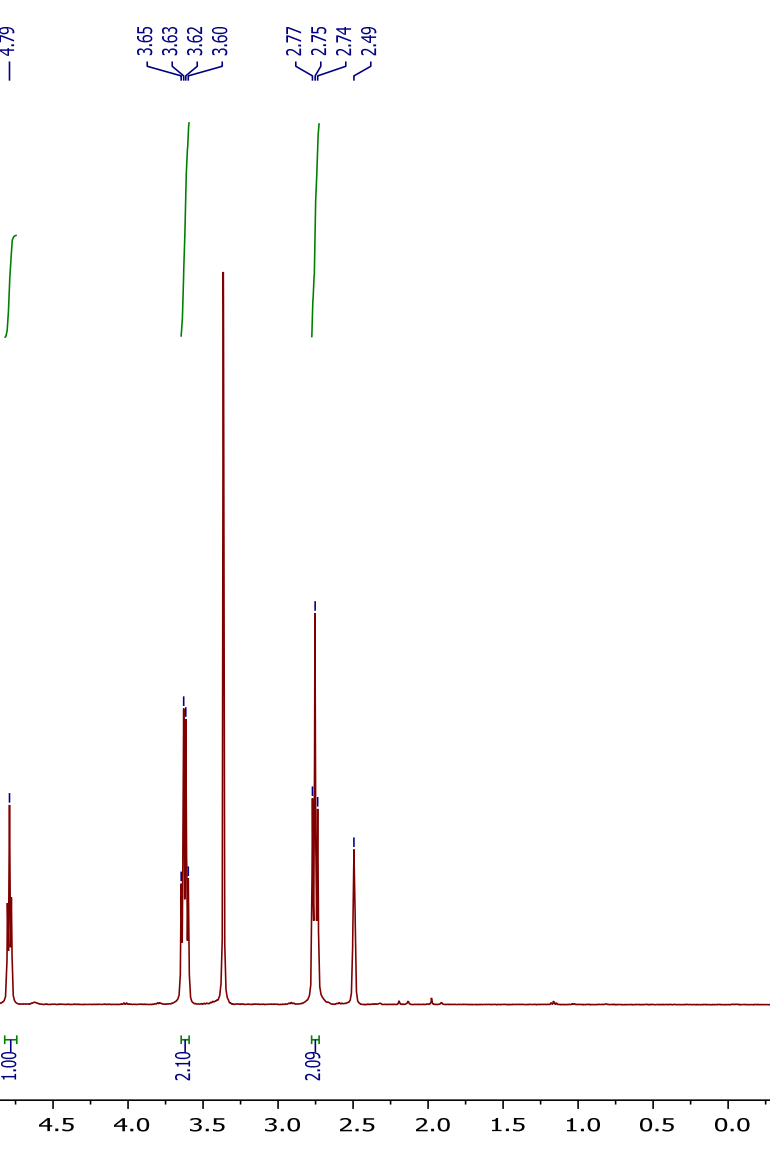

S179 
${ }^{13} \mathrm{C}\left\{{ }^{1} \mathrm{H}\right\}$ NMR spectrum of the compound 24g.

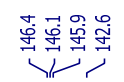

哭茟要

is

$\stackrel{\infty}{i}$
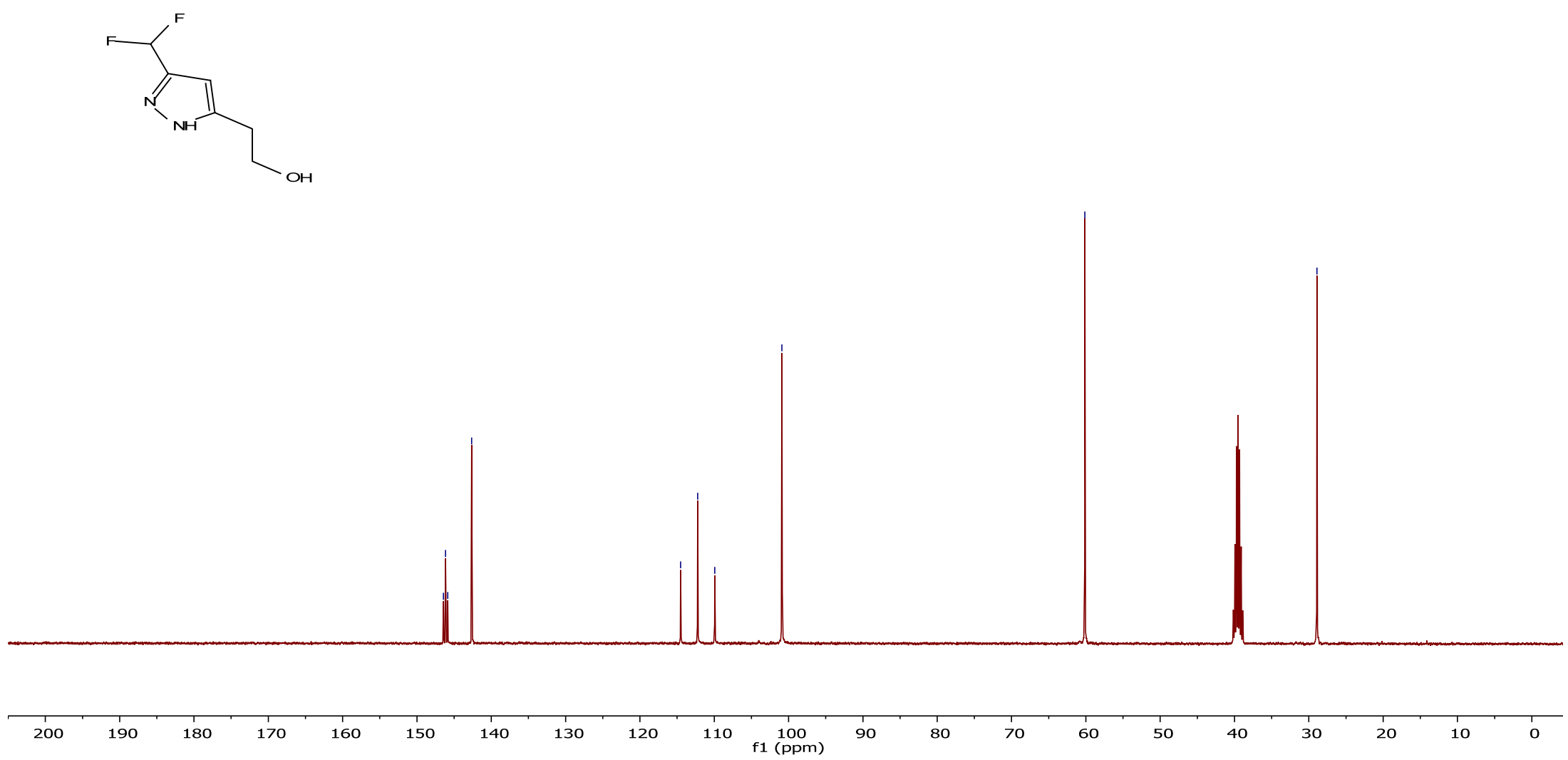

S180 
${ }^{19}$ F NMR spectrum of the compound $\mathbf{2 4 g}$.

苛羿
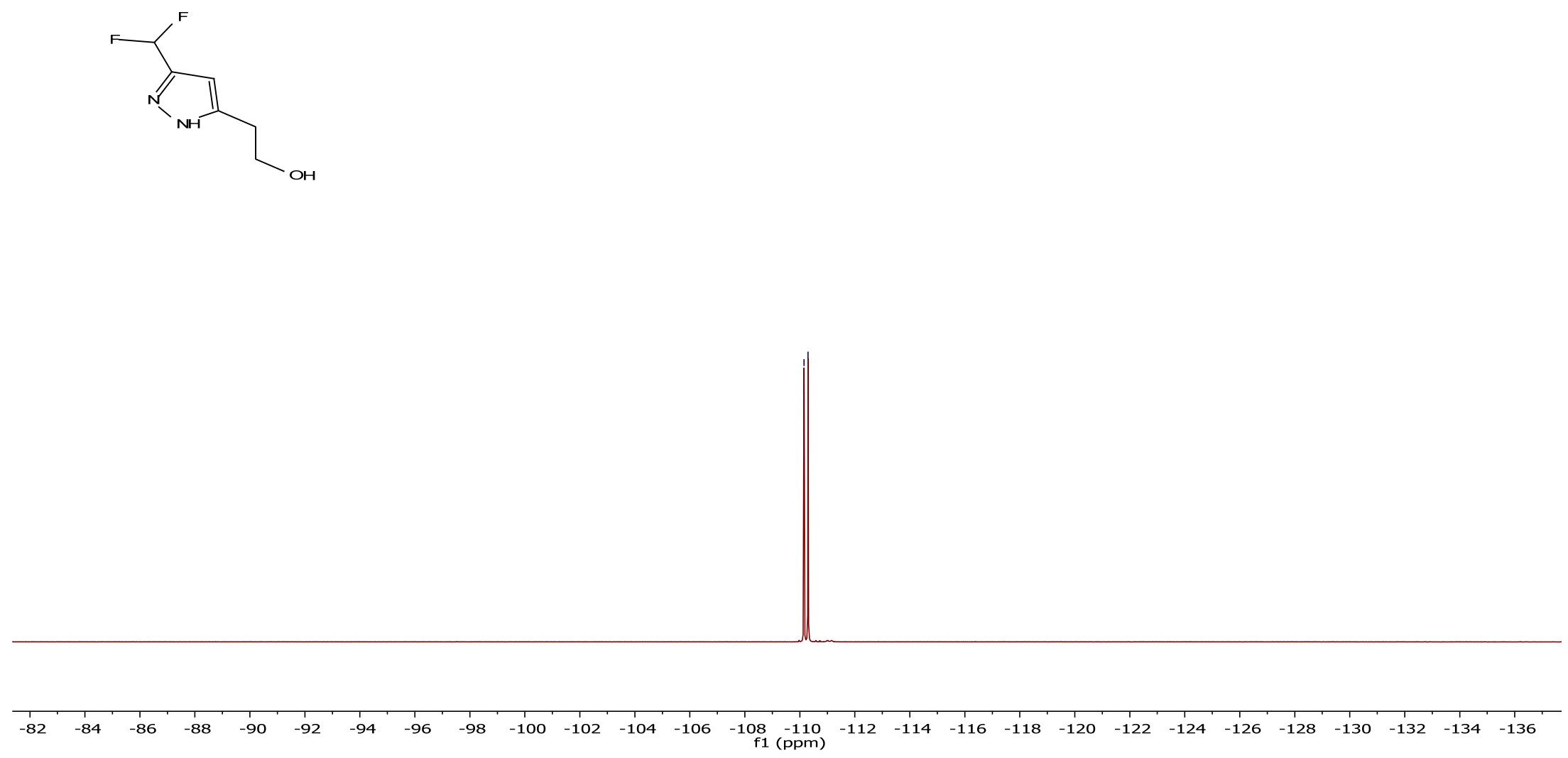
${ }^{1} \mathrm{H}$ NMR spectrum of the compound $\mathbf{2 4 h}$.

$\stackrel{\circ}{9}$

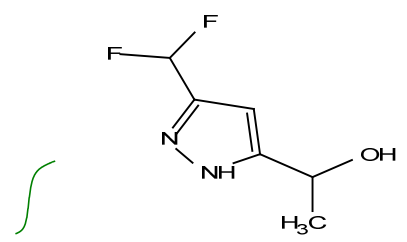

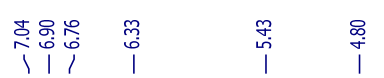

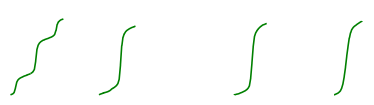

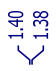

$\int$

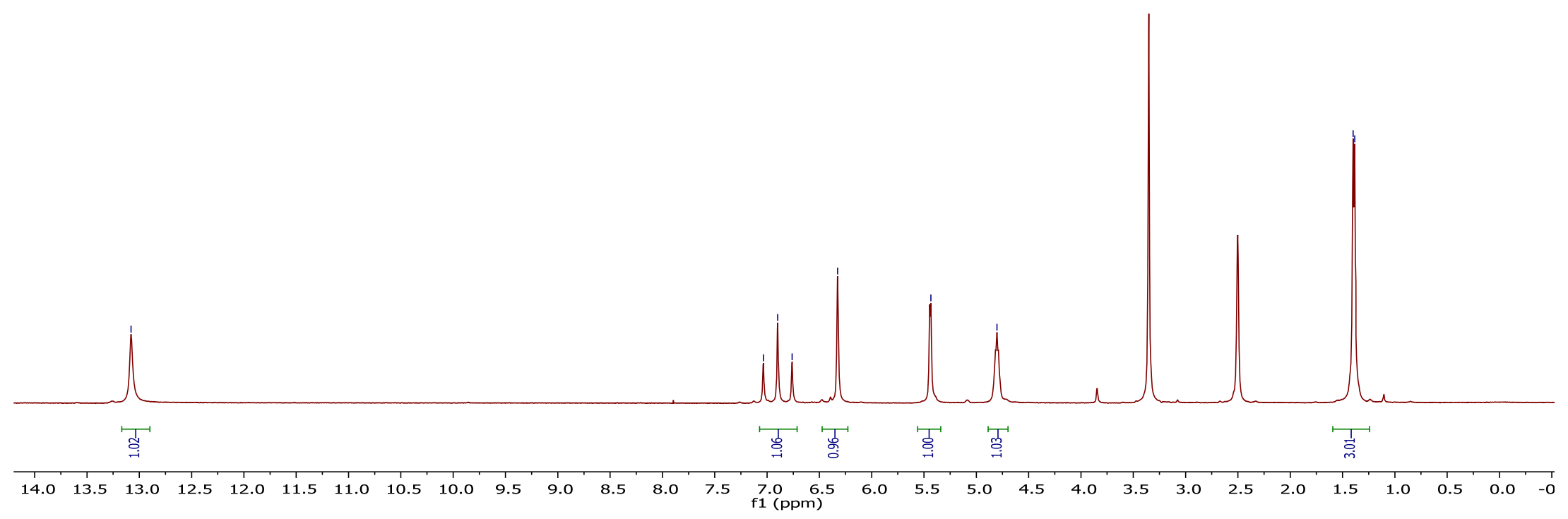


${ }^{13} \mathrm{C}\left\{{ }^{1} \mathrm{H}\right\}$ NMR spectrum of the compound $\mathbf{2 4 h}$.

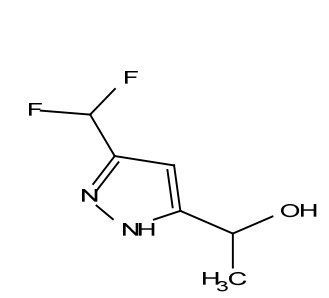

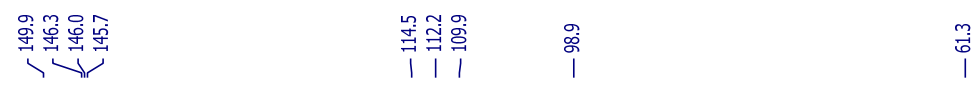

$\stackrel{\infty}{i}$

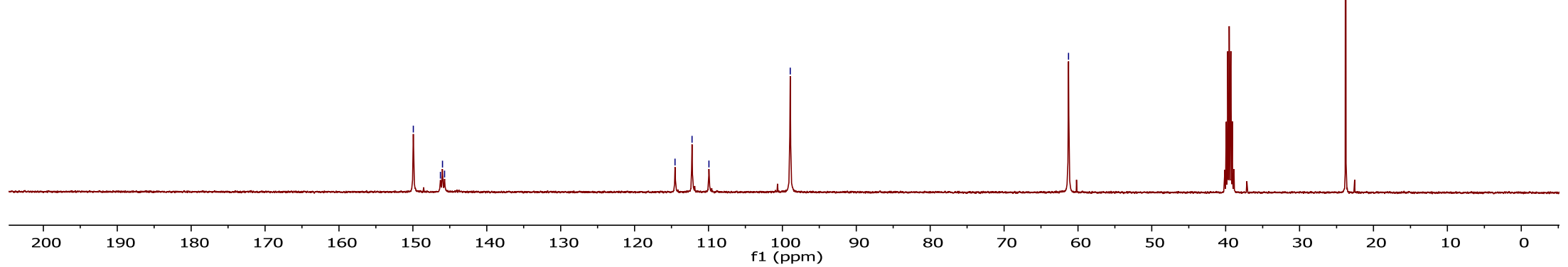


${ }^{19} \mathrm{~F}\left\{{ }^{1} \mathrm{H}\right\}$ NMR spectrum of the compound $\mathbf{2 4 h}$.
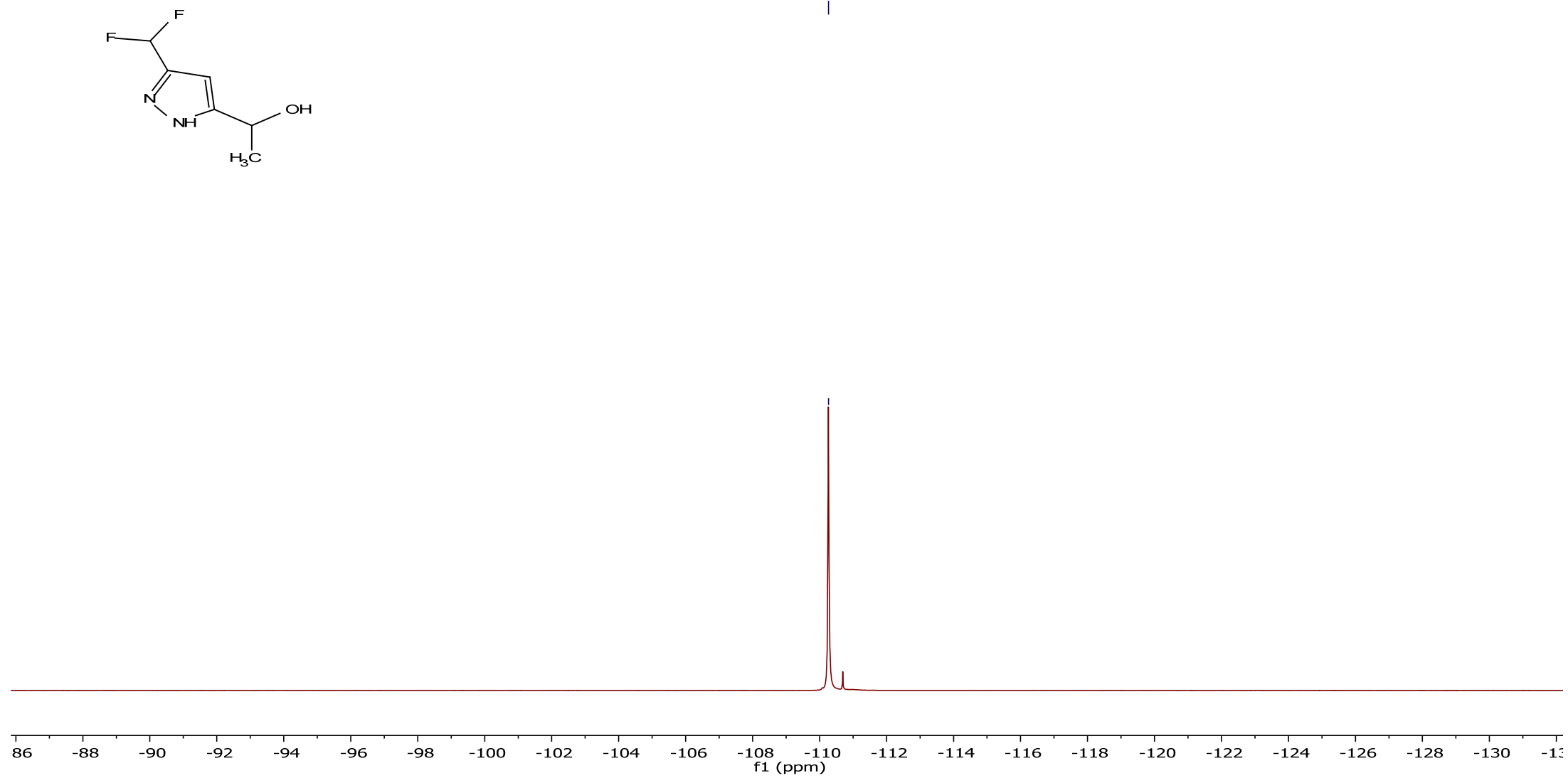
${ }^{1} \mathrm{H}$ NMR spectrum of the compound 25a.

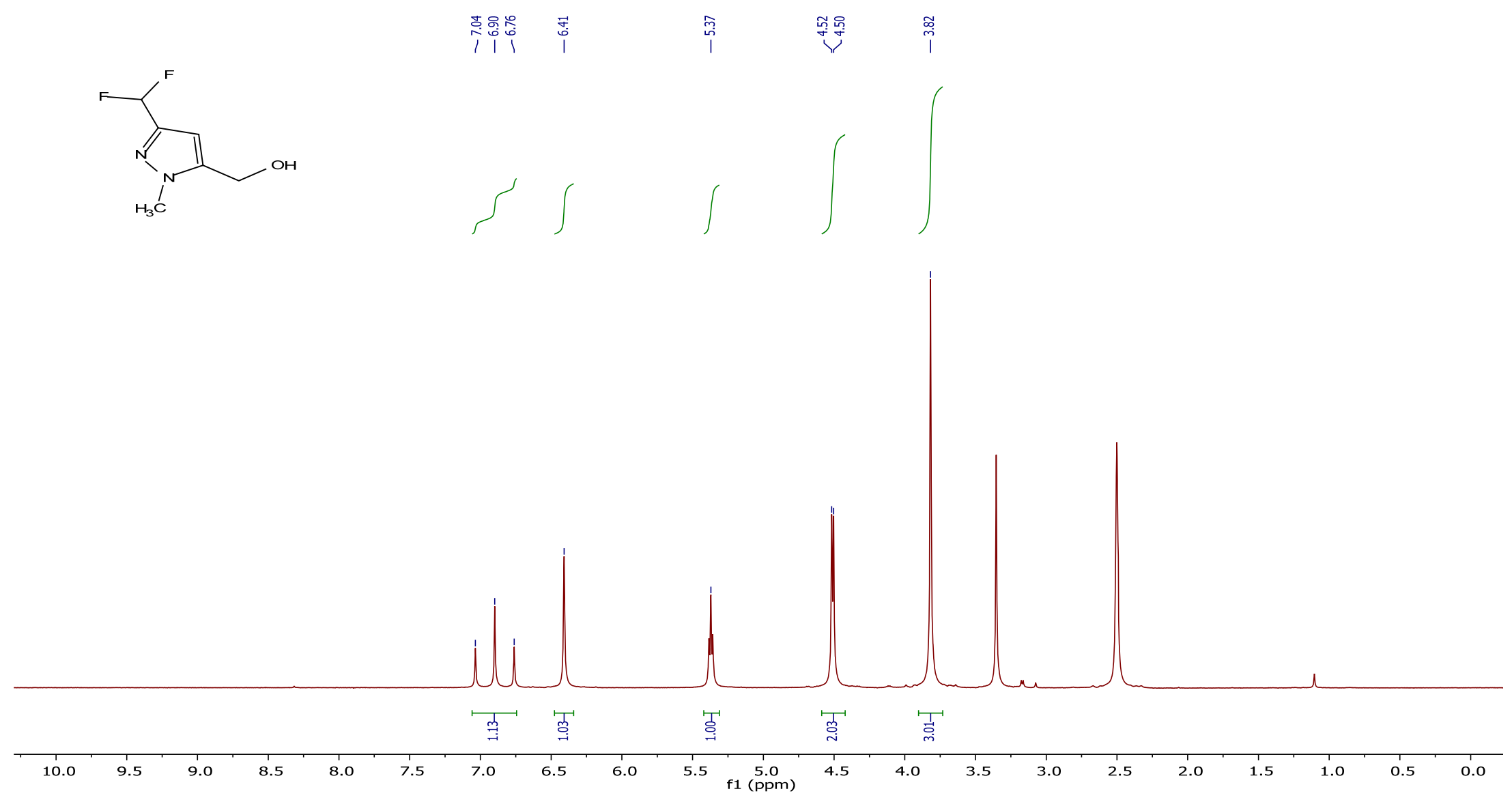


${ }^{13} \mathrm{C}\left\{{ }^{1} \mathrm{H}\right\}$ NMR spectrum of the compound 25a.
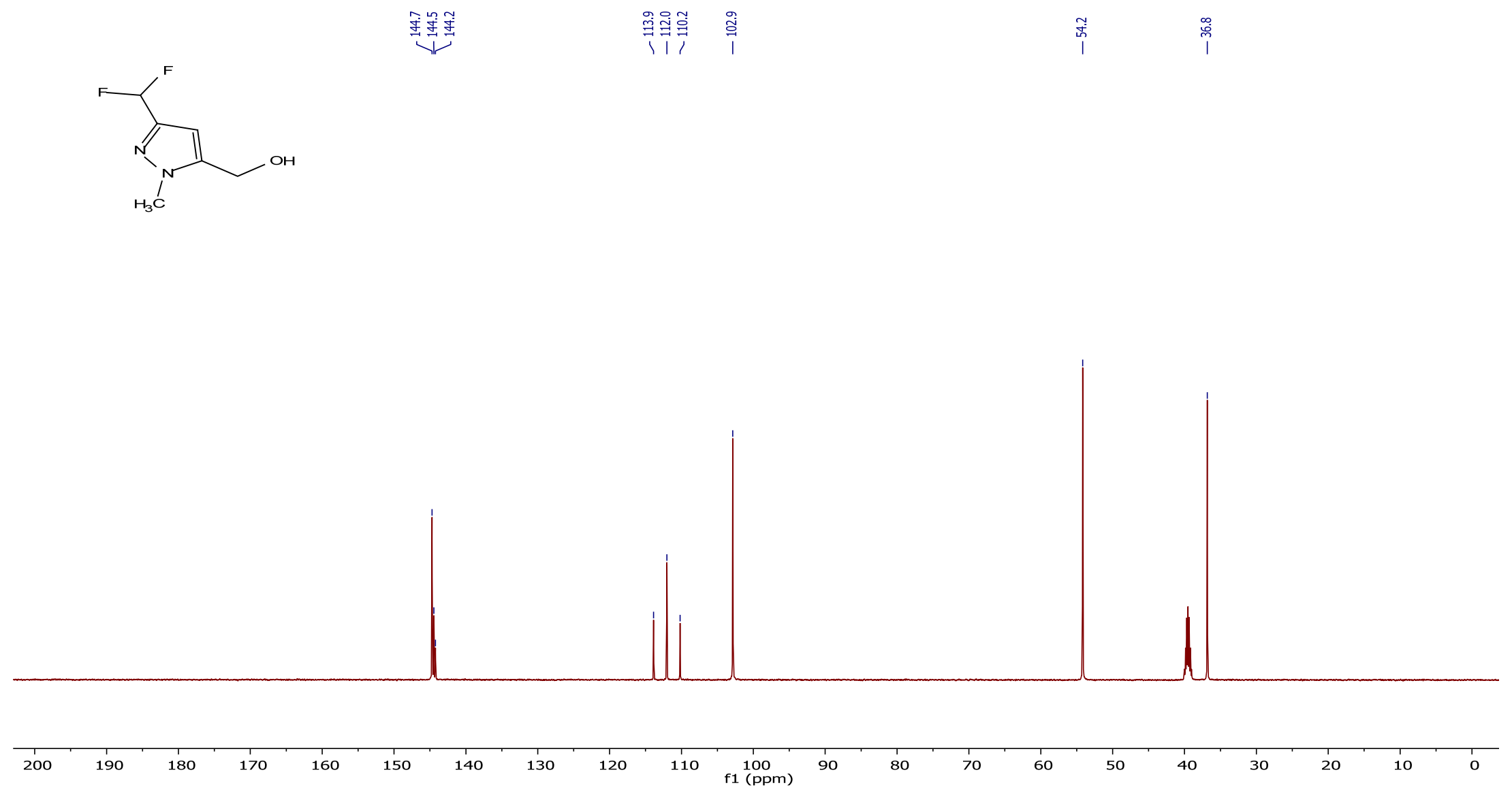
${ }^{19}$ F NMR spectrum of the compound 25a.

刍高
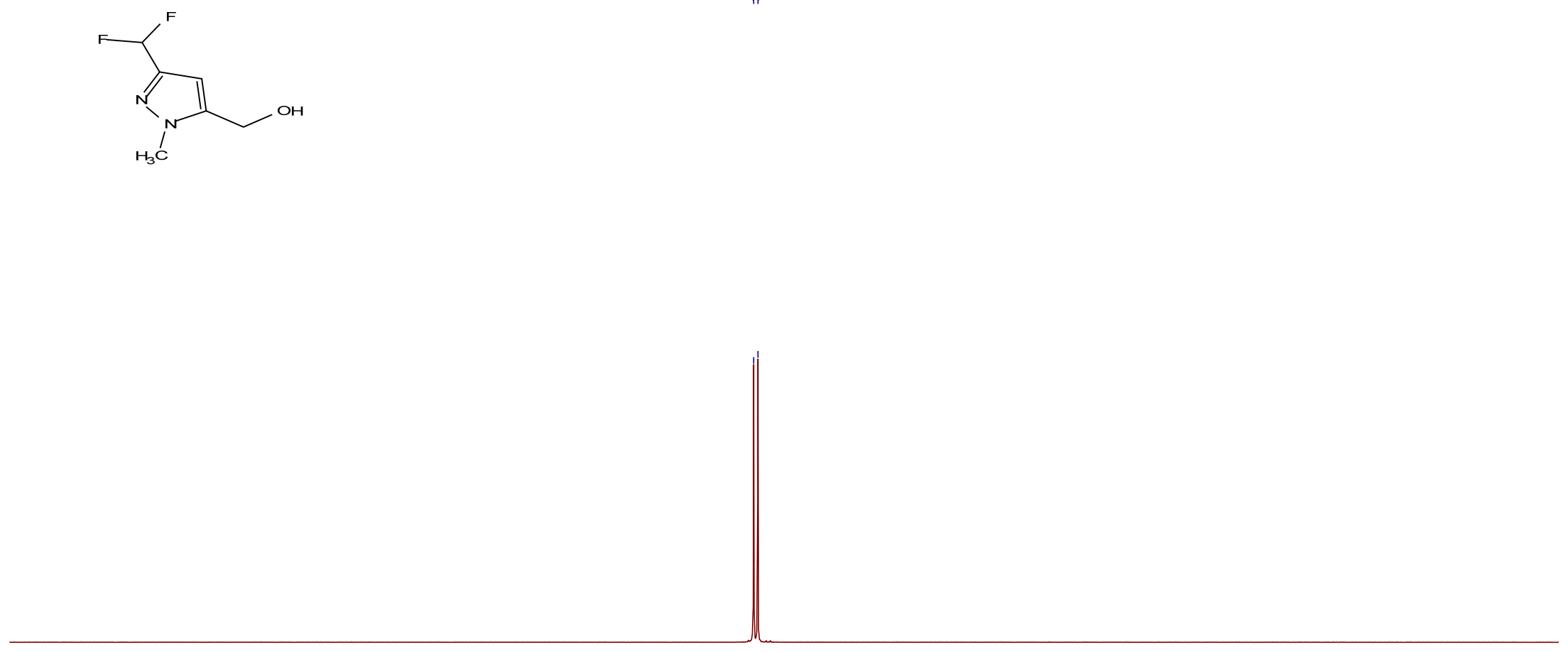
${ }^{1} \mathrm{H}$ NMR spectrum of the compound 27a.

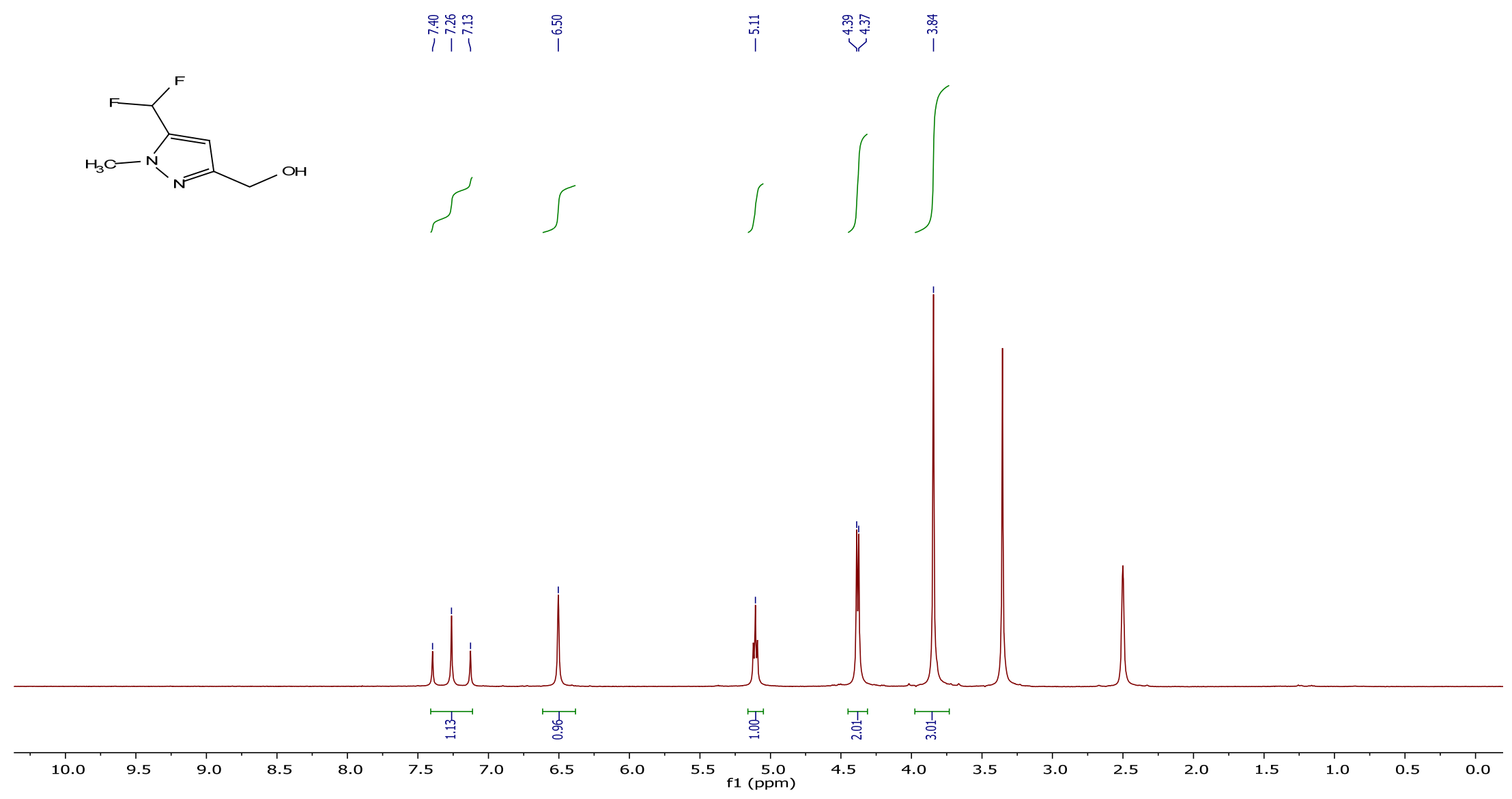


${ }^{13} \mathrm{C}\left\{{ }^{1} \mathrm{H}\right\}$ NMR spectrum of the compound 27a.
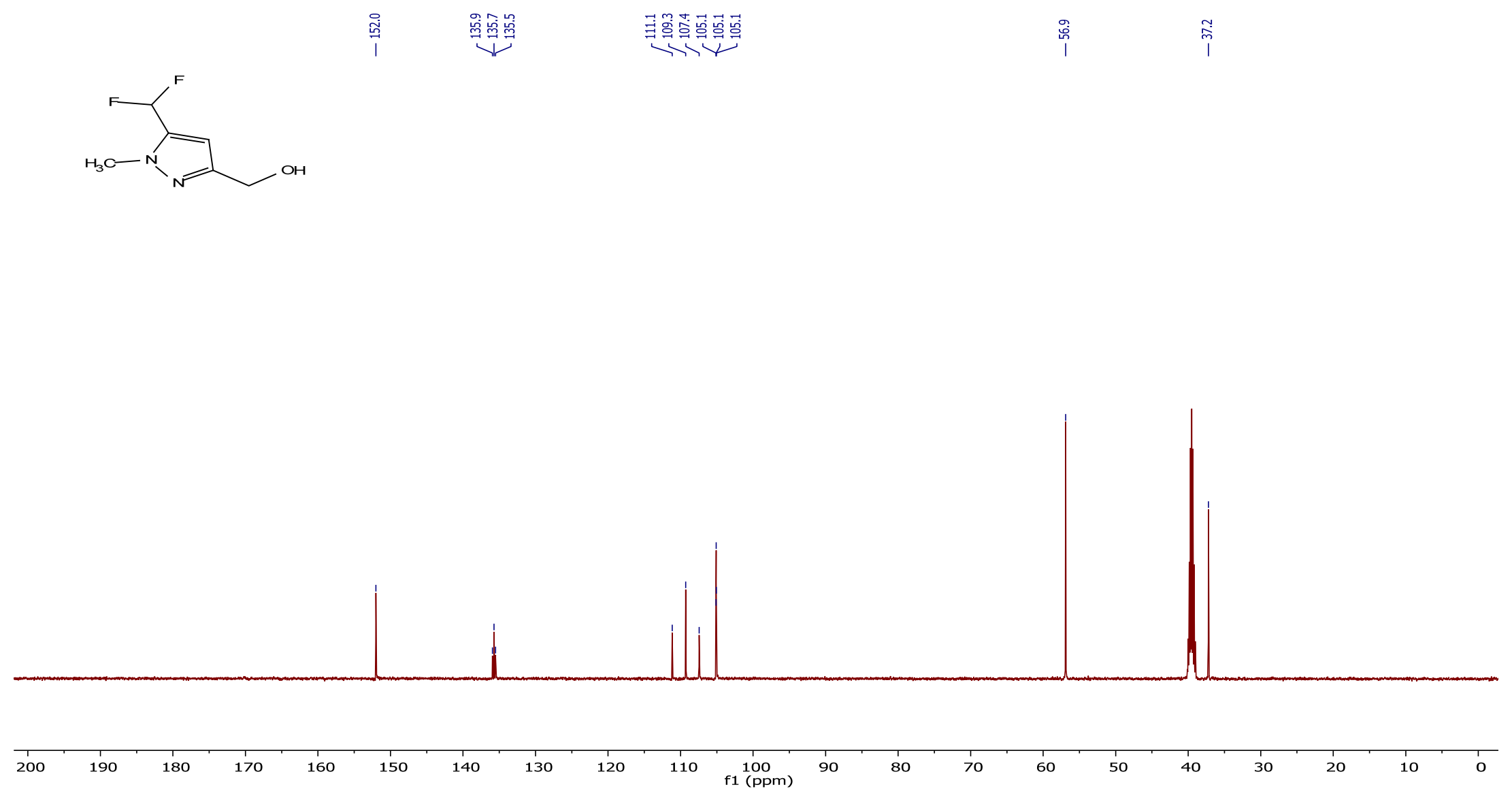
${ }^{19}$ F NMR spectrum of the compound 27a.

$\stackrel{\circ}{\mathrm{g}}$
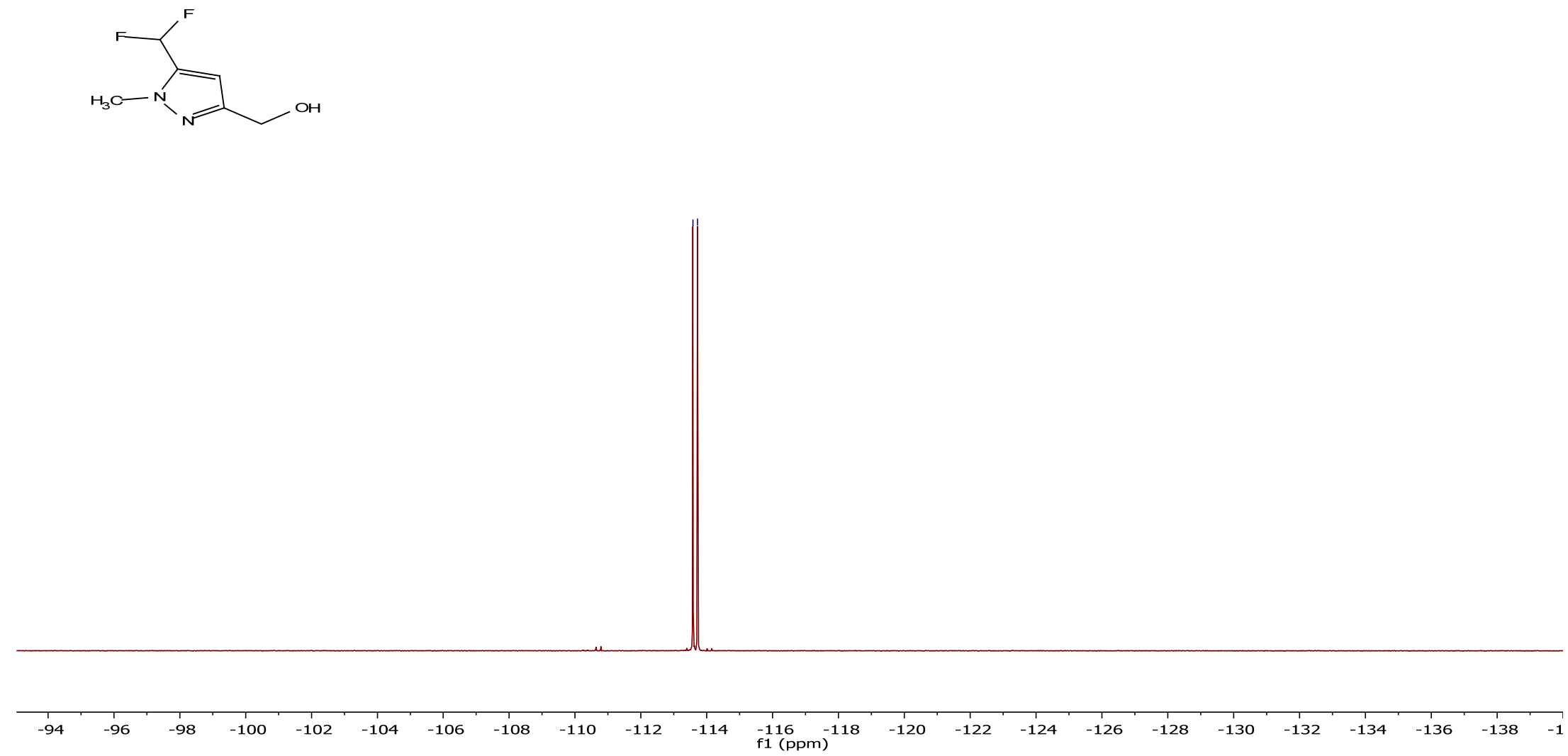
${ }^{1} \mathrm{H}$ NMR spectrum of the compound 25b.

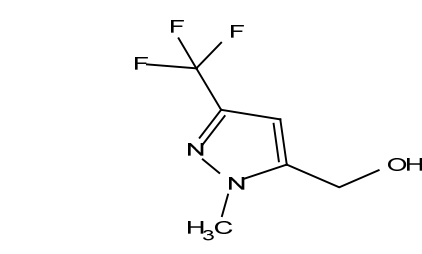

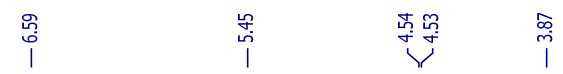

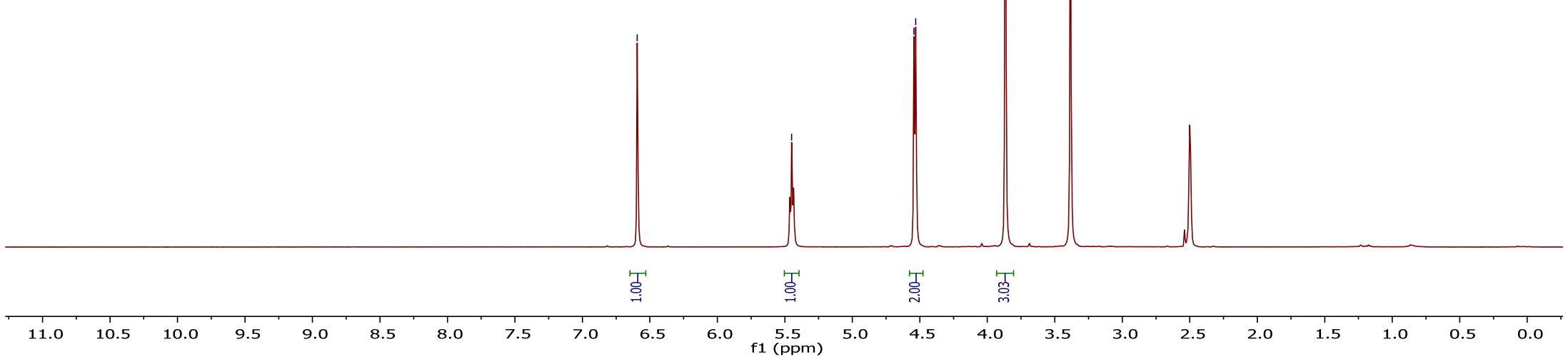


${ }^{13} \mathrm{C}\left\{{ }^{1} \mathrm{H}\right\}$ NMR spectrum of the compound 25b.

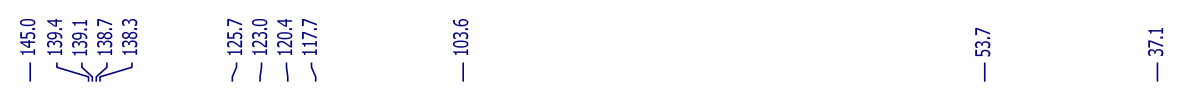
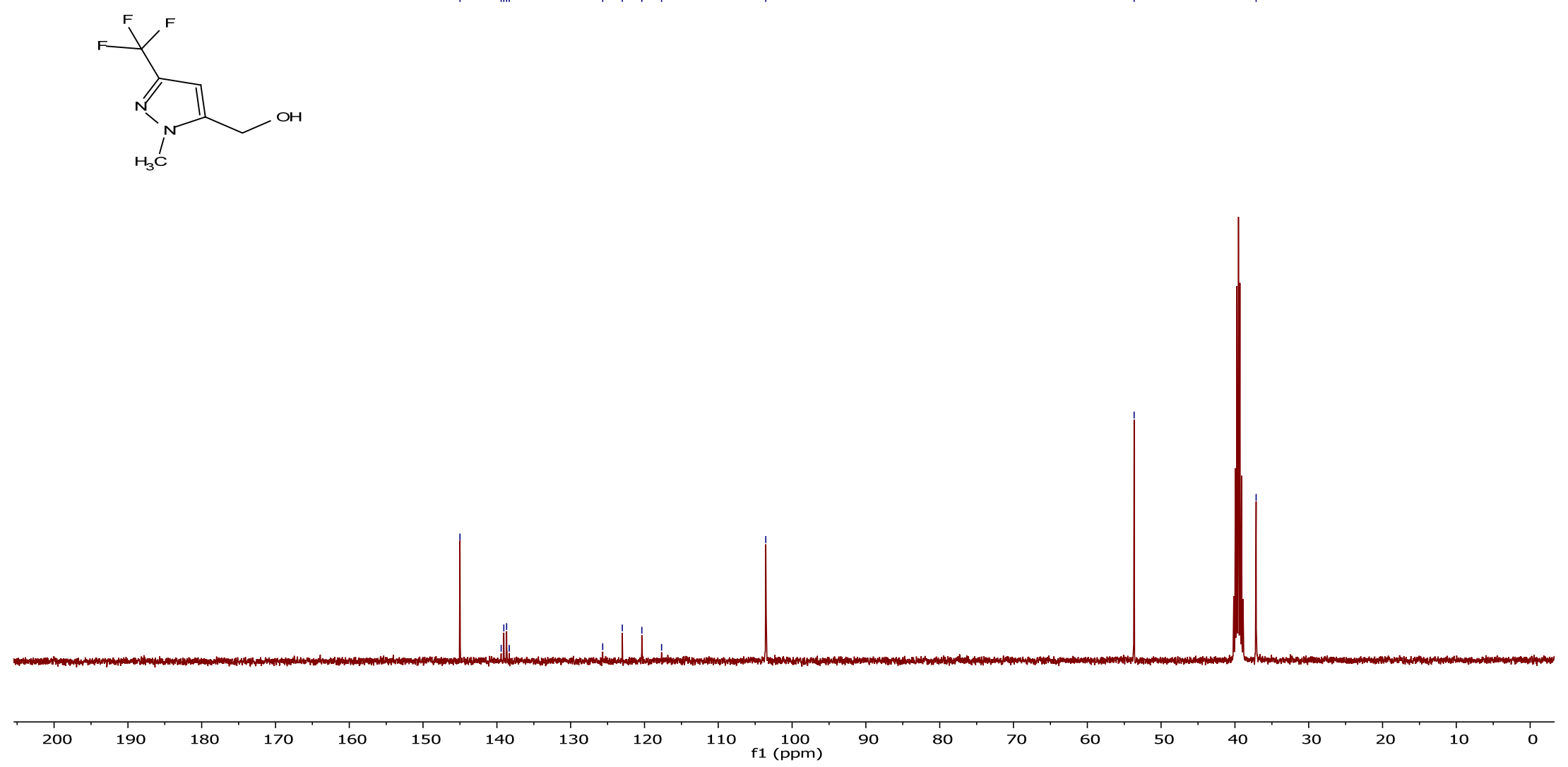
${ }^{19} \mathrm{~F}\left\{{ }^{1} \mathrm{H}\right\}$ NMR spectrum of the compound 25b.
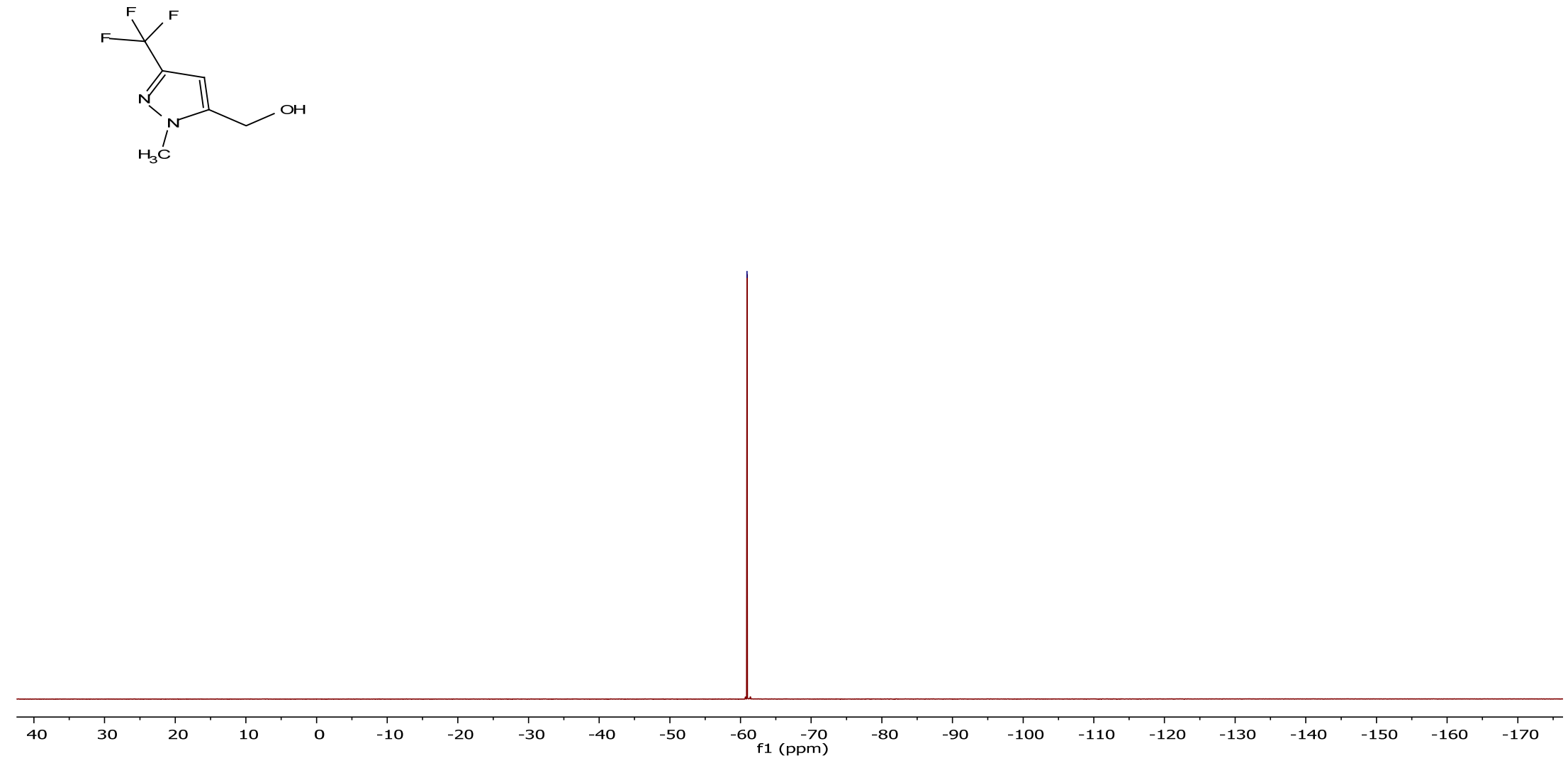
${ }^{1} \mathrm{H}$ NMR spectrum of the compound 25g.
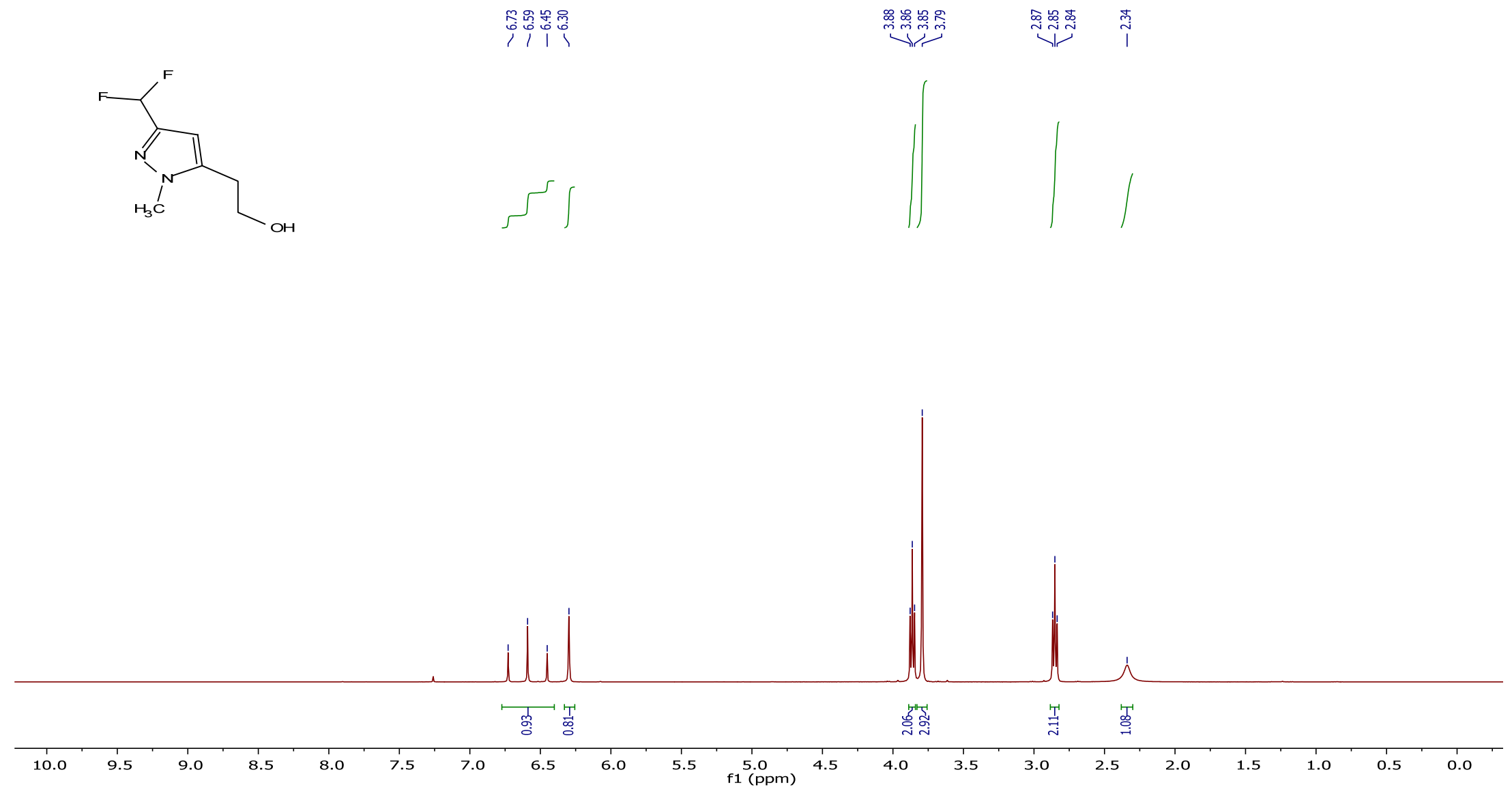
${ }^{13} \mathrm{C}\left\{{ }^{1} \mathrm{H}\right\}$ NMR spectrum of the compound 25g.
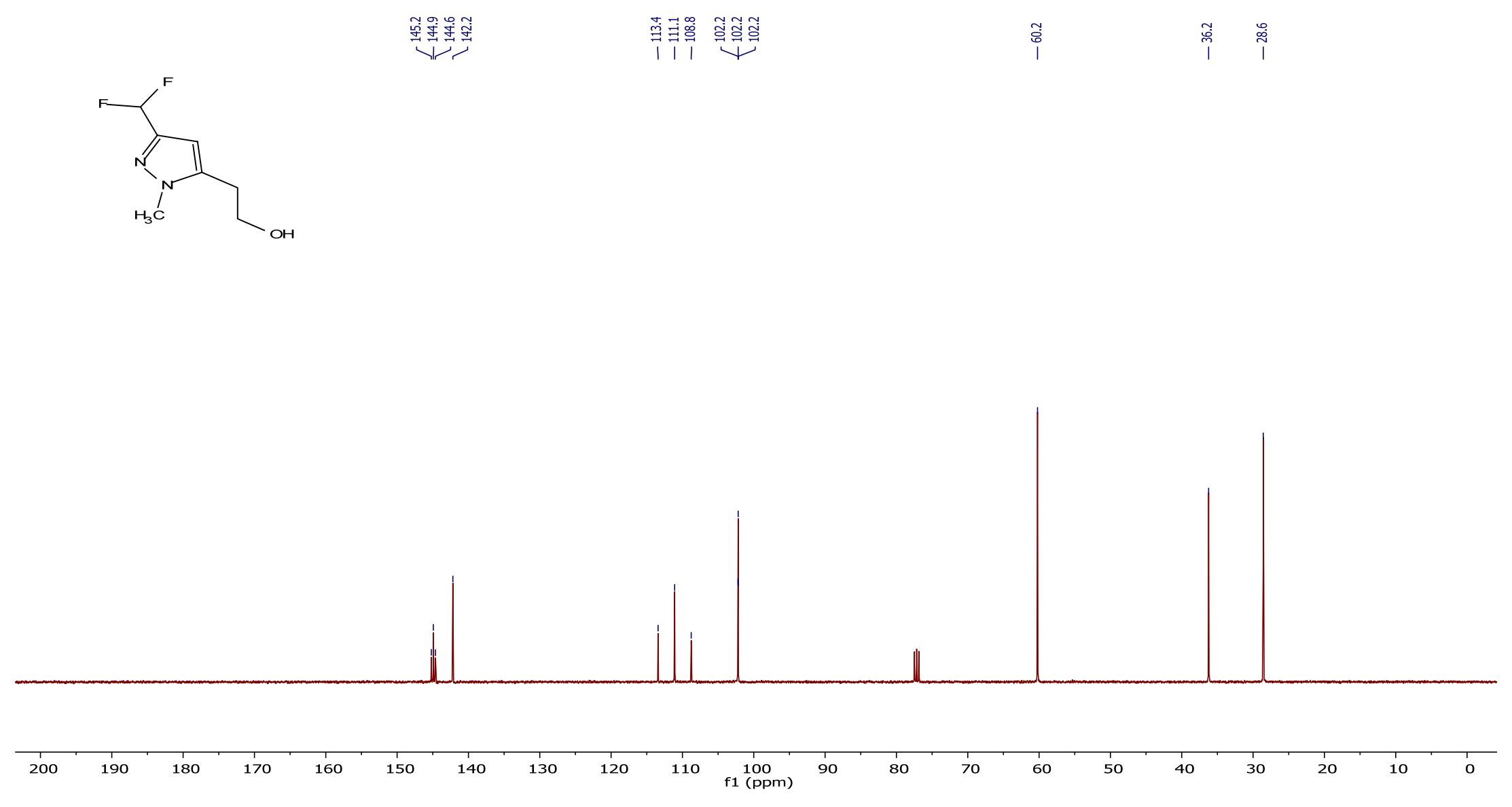
${ }^{19}$ F NMR spectrum of the compound 25g.

잋
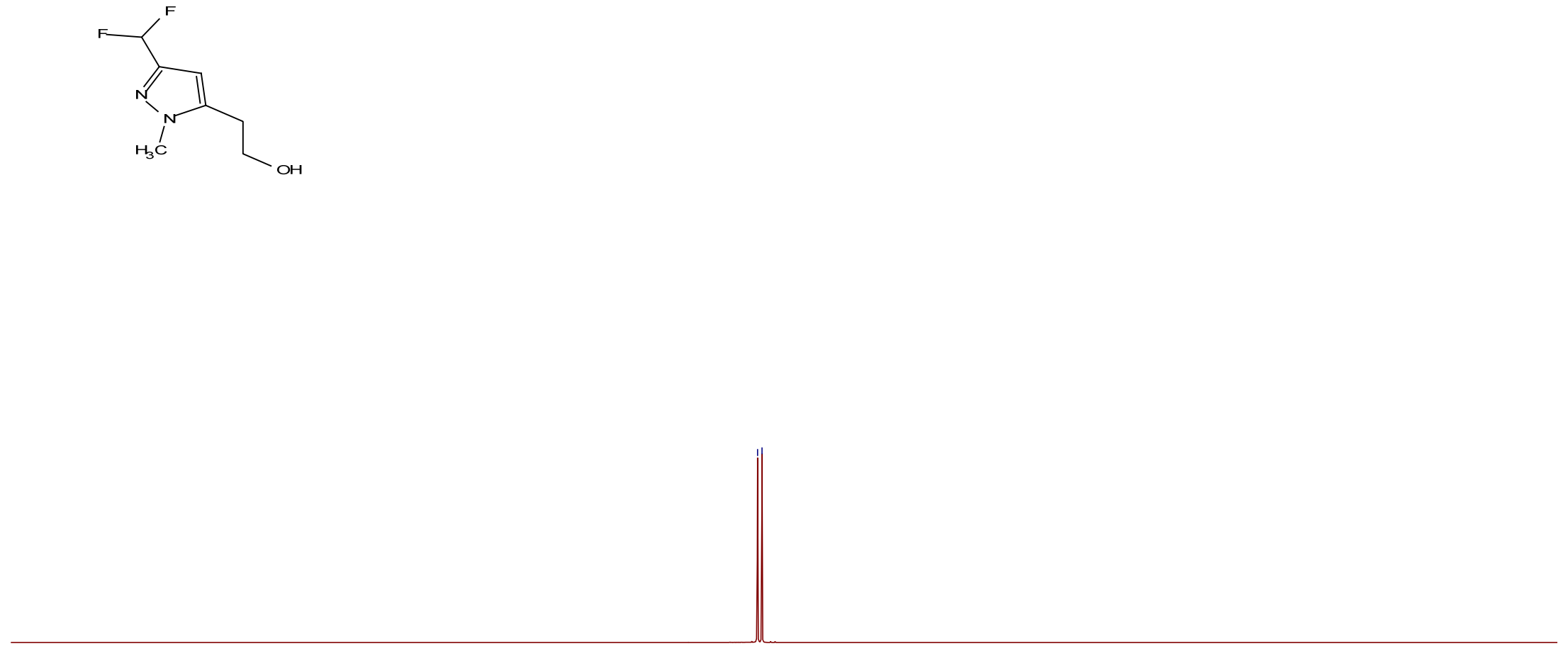

$-88 \quad-90$

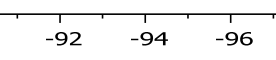

$\begin{array}{lll}-98 & -100 & -102\end{array}$

$-104-106-108$

$-112-114$
$\mathrm{f1}(\mathrm{ppm})$ 
${ }^{1} \mathrm{H}$ NMR spectrum of the compound $25 \mathbf{h}$.

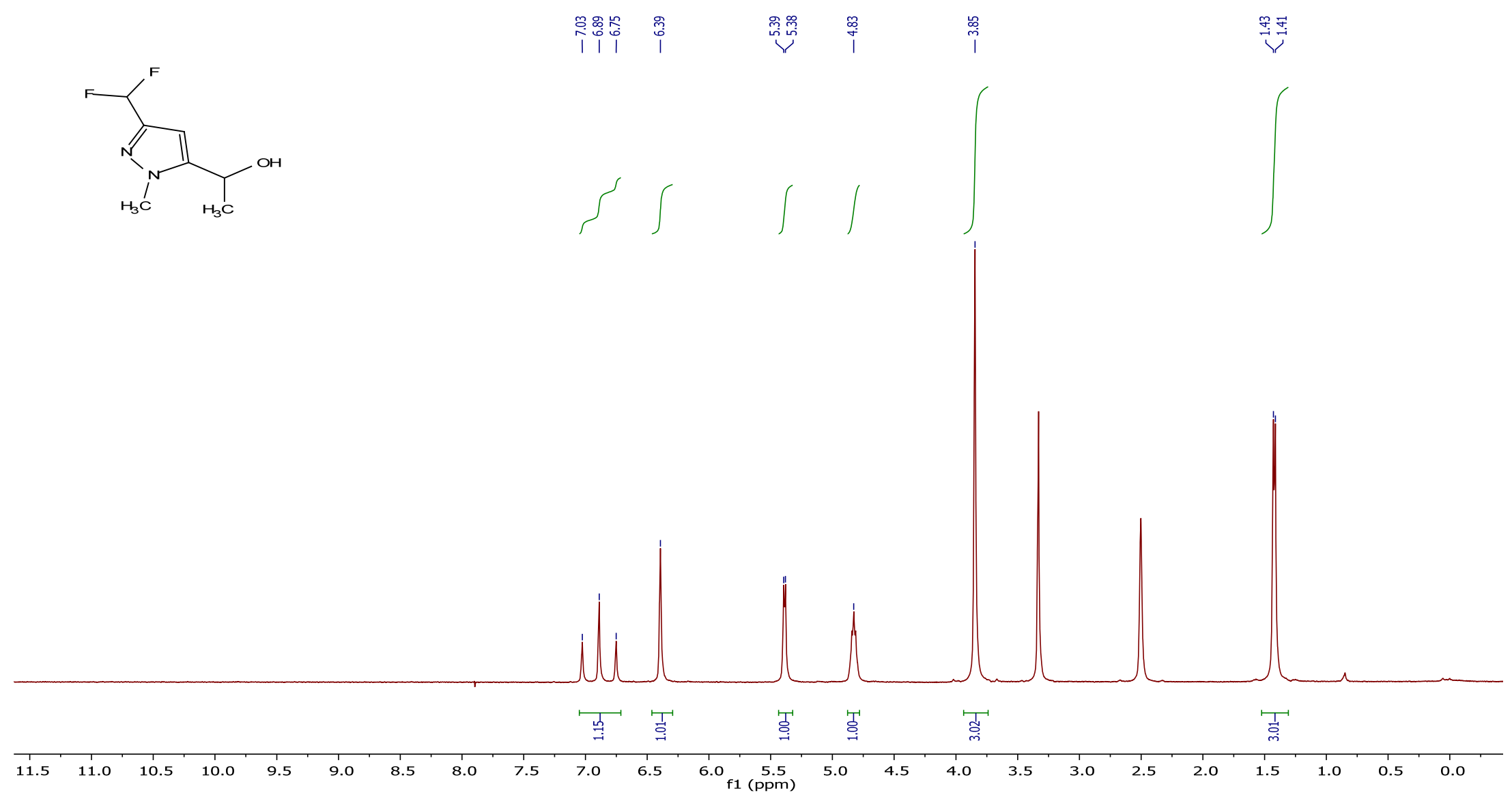


${ }^{13} \mathrm{C}\left\{{ }^{1} \mathrm{H}\right\}$ NMR spectrum of the compound 25h.
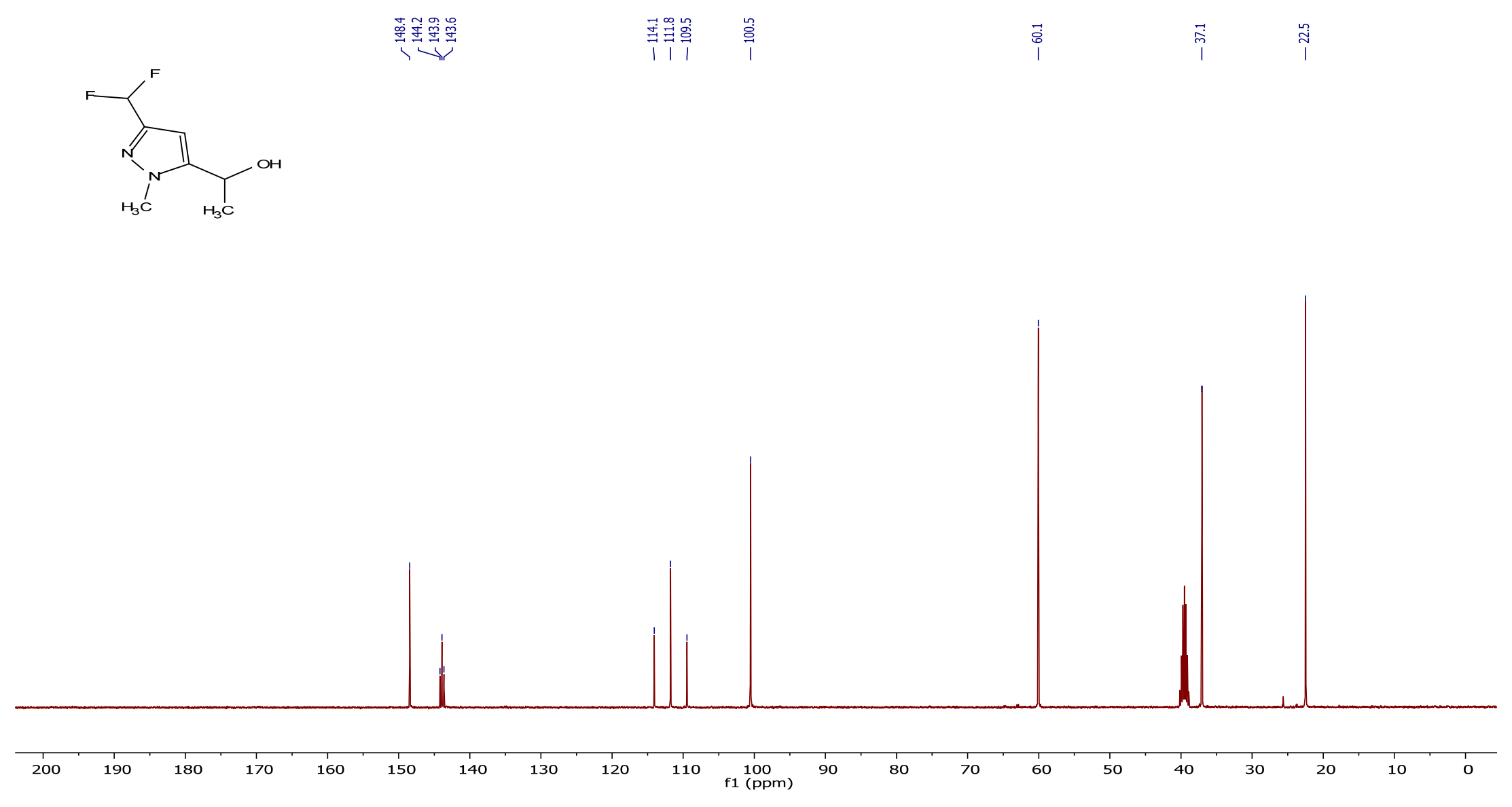
${ }^{19} \mathrm{~F}\left\{{ }^{1} \mathrm{H}\right\}$ NMR spectrum of the compound $\mathbf{2 5 h}$.

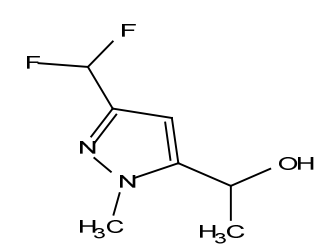

$\hat{\mathrm{i}}$

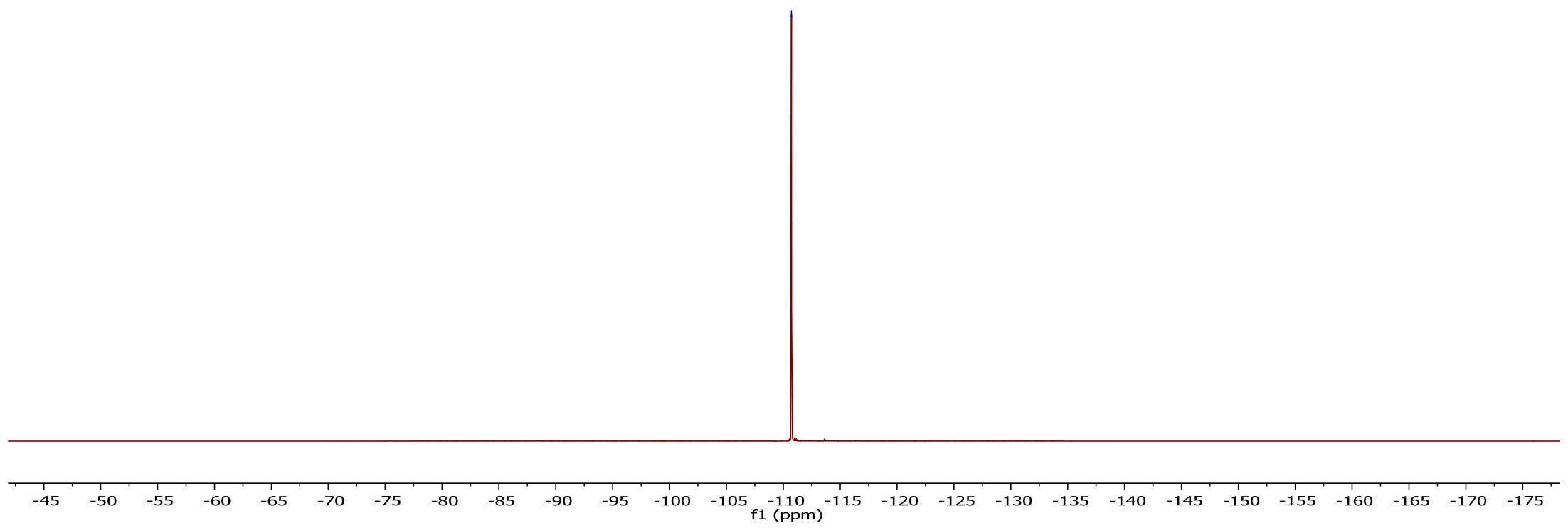


${ }^{1} \mathrm{H}$ NMR spectrum of the compound 29a.
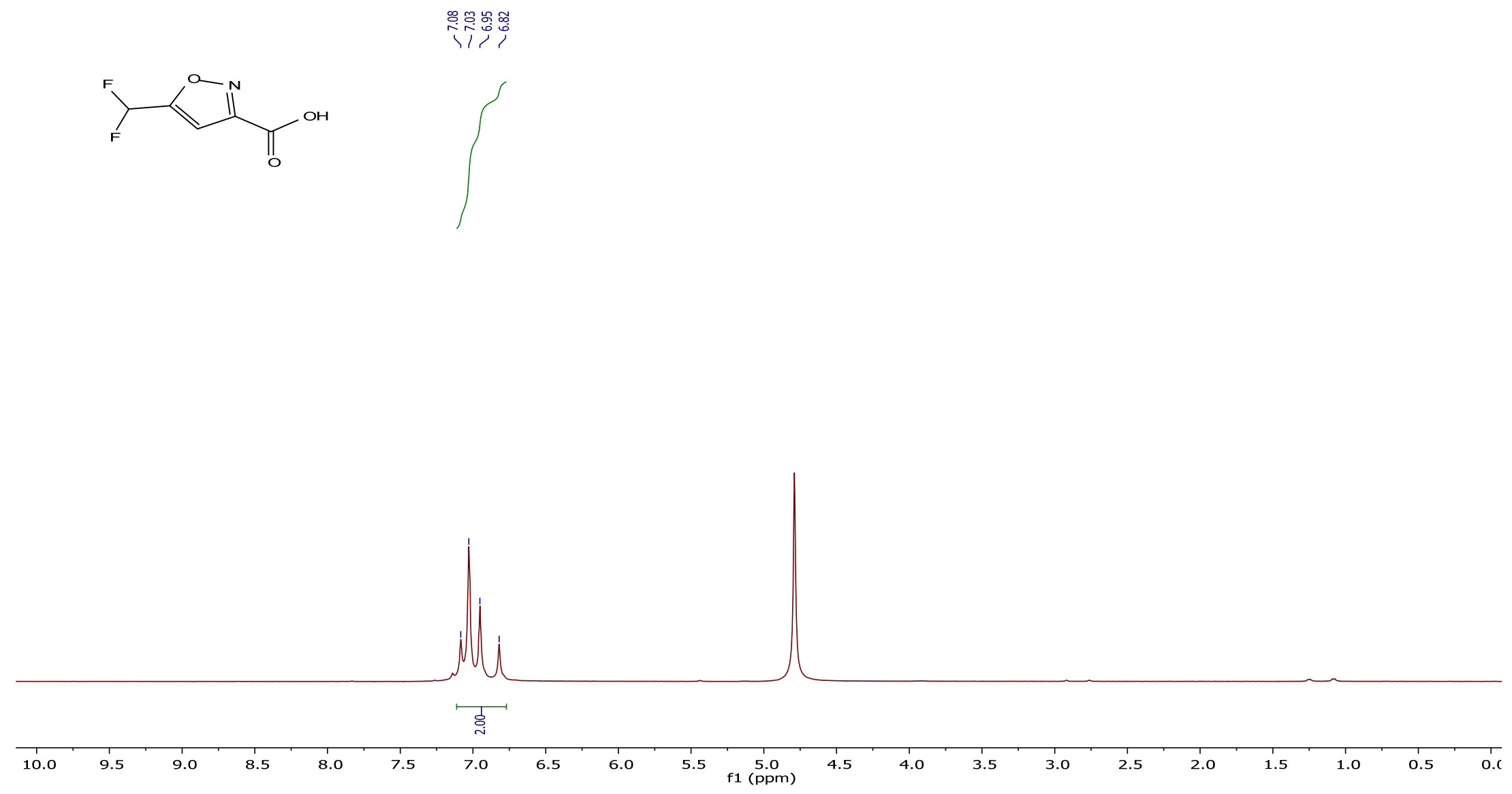


\section{${ }^{13} \mathrm{C}\left\{{ }^{1} \mathrm{H}\right\}$ NMR spectrum of the compound 29a.}

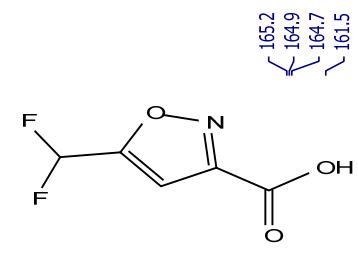

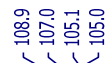

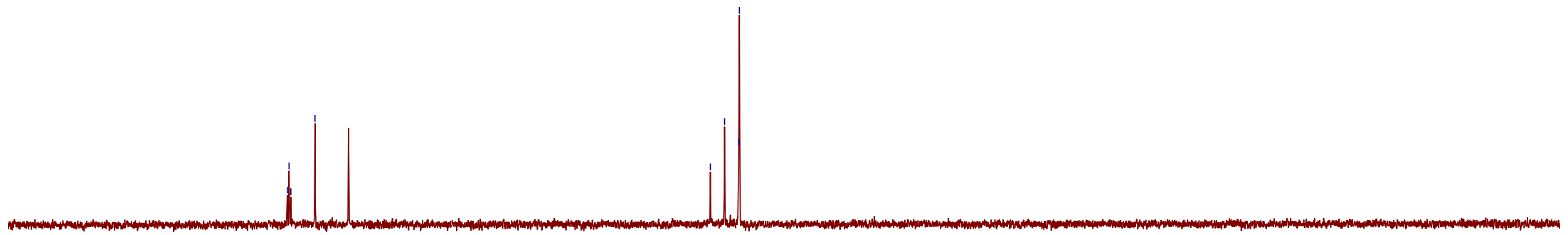

200 
${ }^{19} \mathrm{~F}\left\{{ }^{1} \mathrm{H}\right\}$ NMR spectrum of the compound 29a.
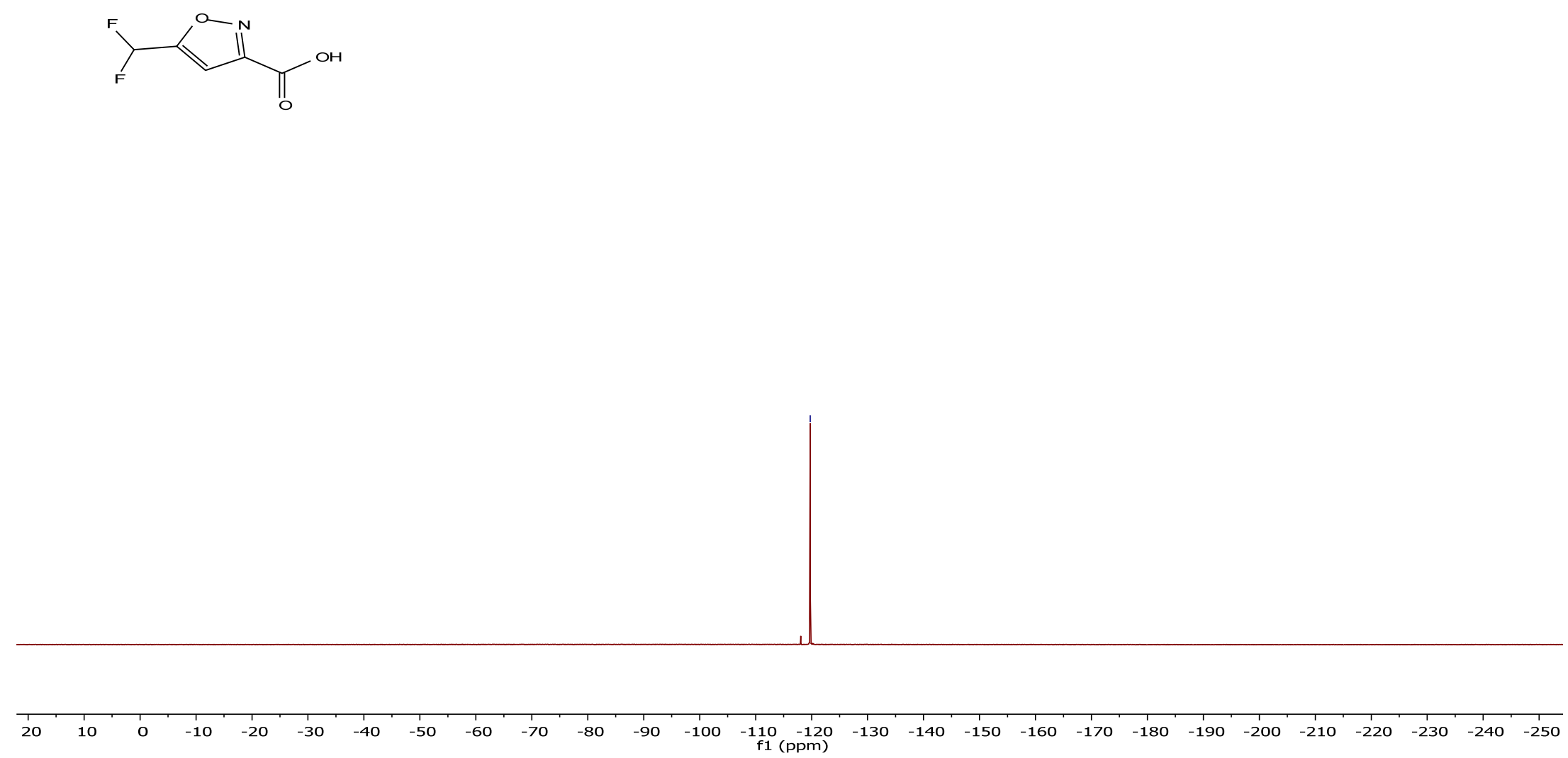
${ }^{1} \mathrm{H}$ NMR spectrum of the compound $29 \mathbf{b}$.
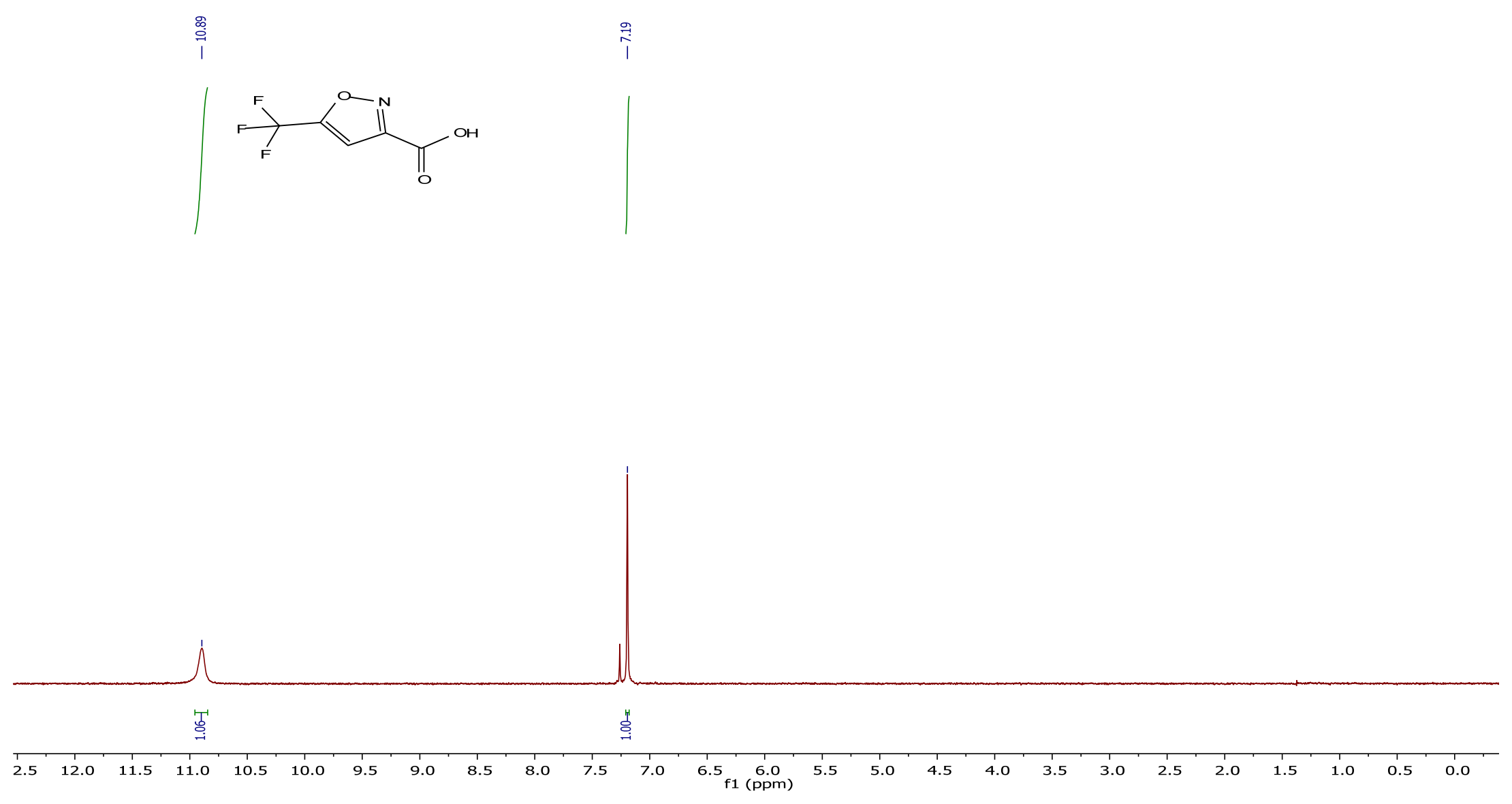


\section{${ }^{13} \mathrm{C}\left\{{ }^{1} \mathrm{H}\right\}$ NMR spectrum of the compound 29b.}

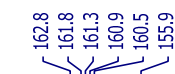

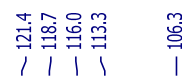<smiles>O=C(O)c1cc(C(F)(F)F)on1</smiles>

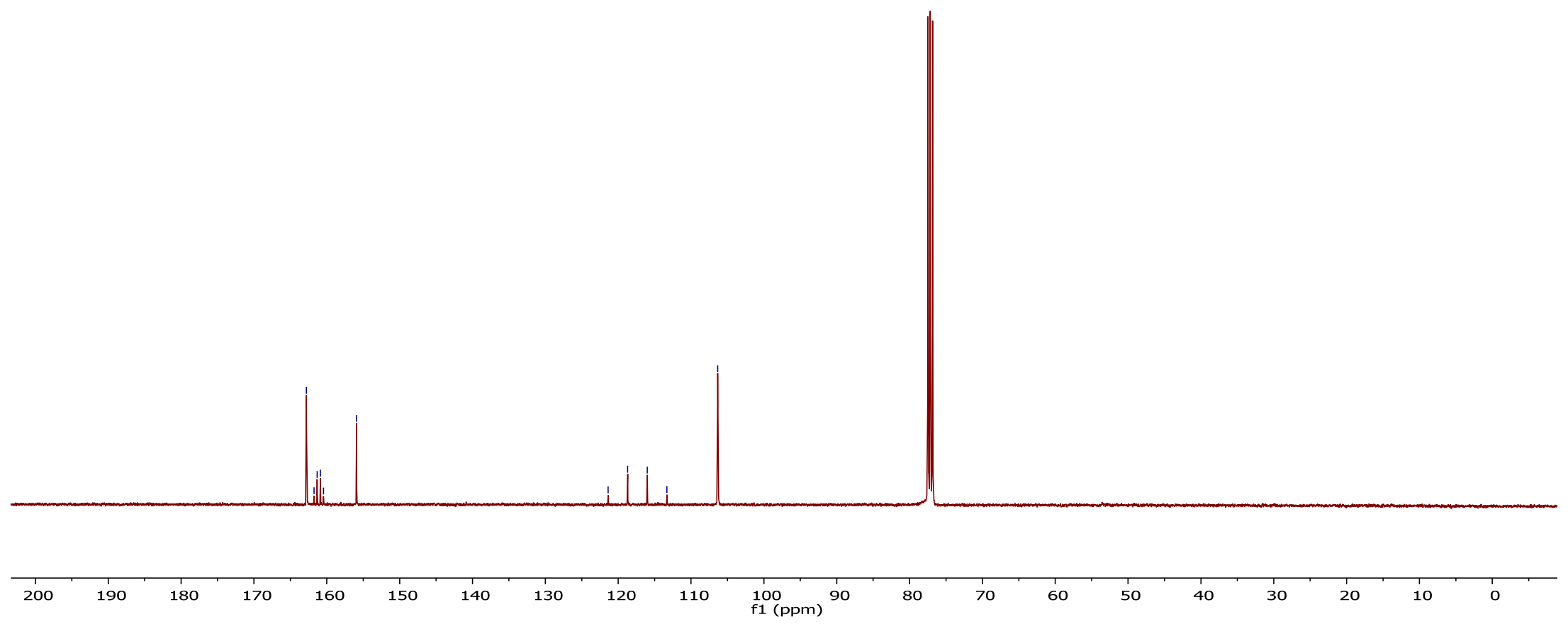


${ }^{19} \mathrm{~F}\left\{{ }^{1} \mathrm{H}\right\}$ NMR spectrum of the compound 29b.

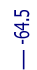<smiles>O=C(O)c1cc(C(F)(F)F)on1</smiles>

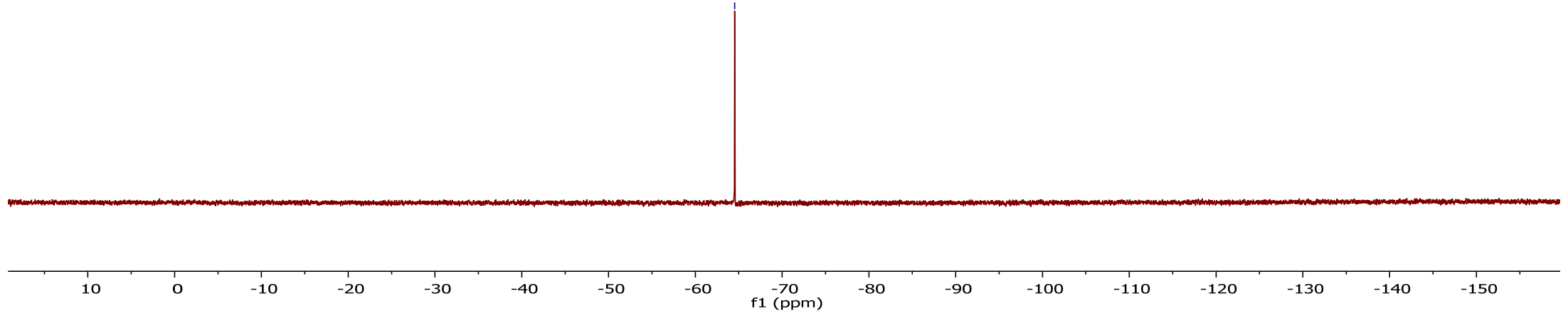


${ }^{1} \mathrm{H}$ NMR spectrum of the compound 29d.
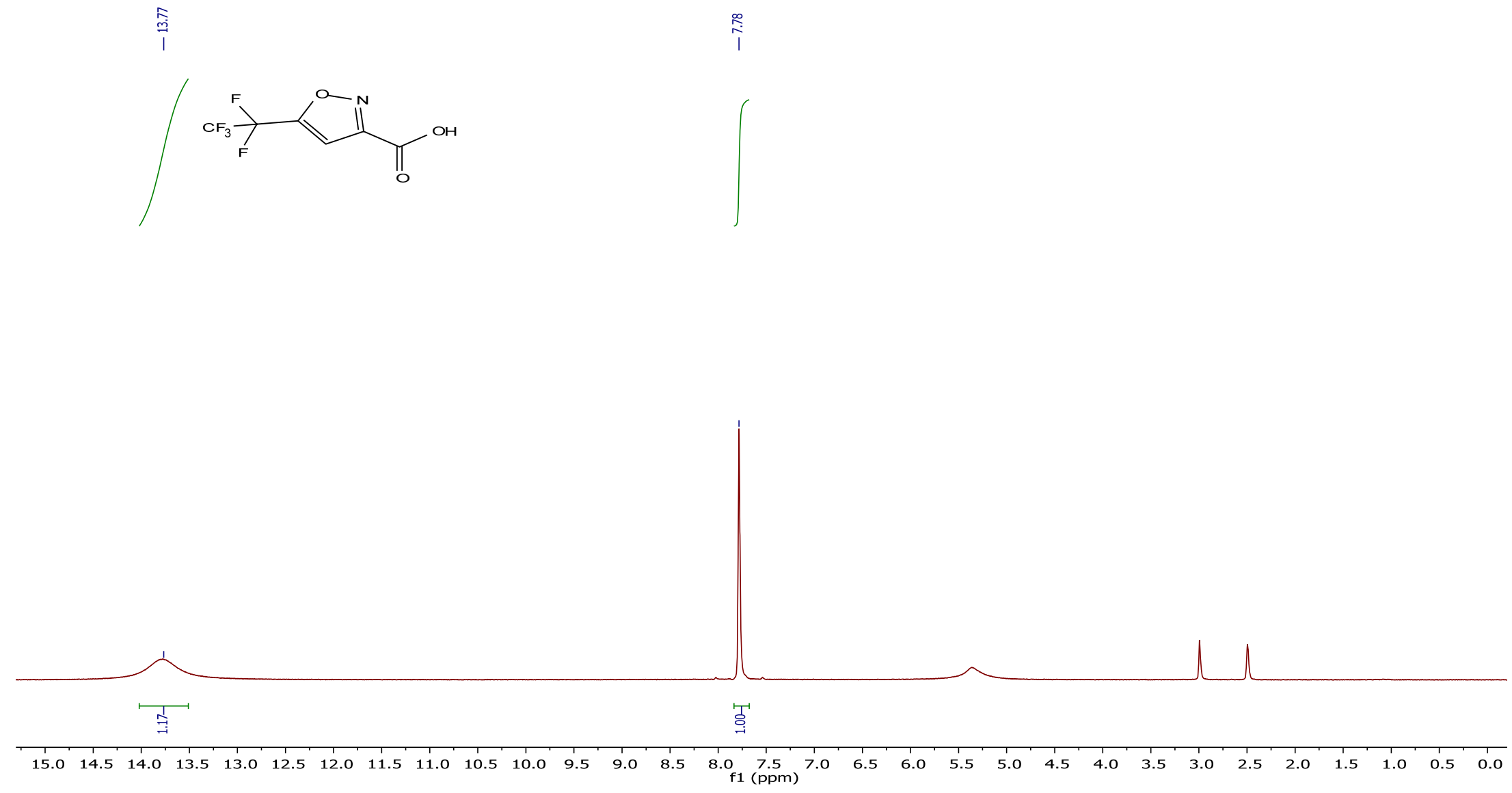
${ }^{13} \mathrm{C}\left\{{ }^{1} \mathrm{H}\right\}$ NMR spectrum of the compound 29d.

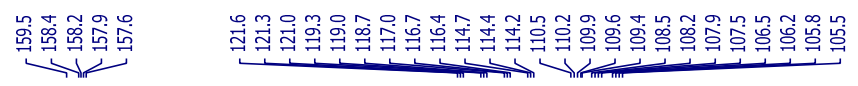<smiles>O=C(O)c1cc(C(F)(F)F)on1</smiles>

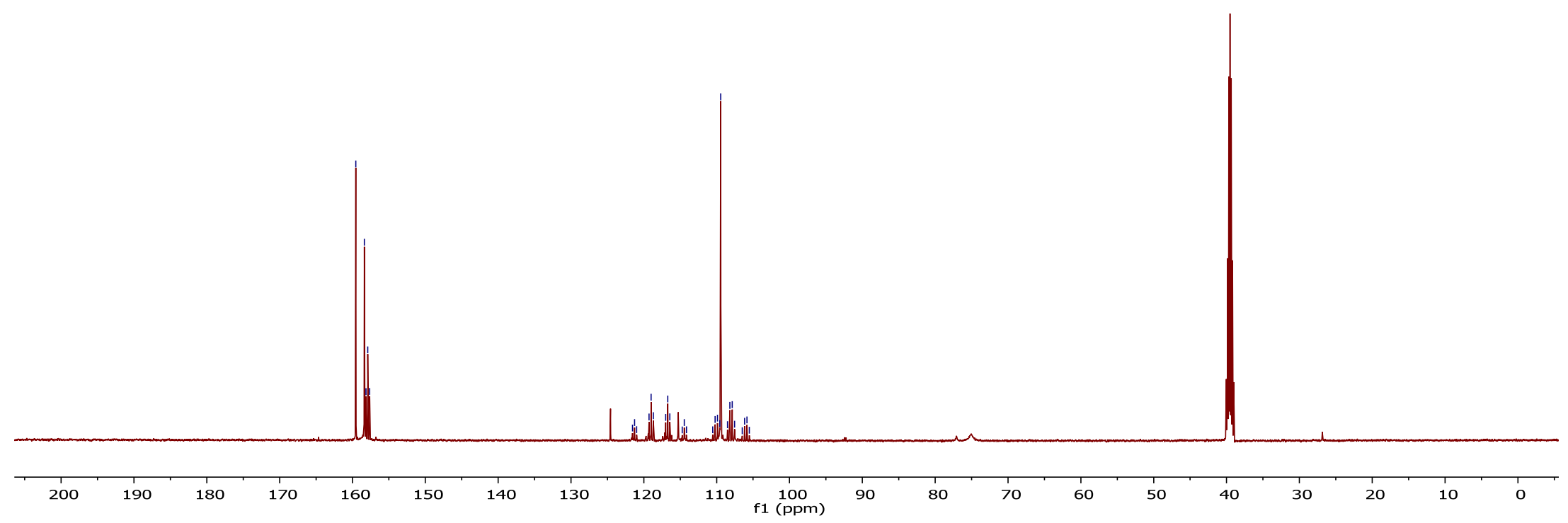


${ }^{19} \mathrm{~F}\left\{{ }^{1} \mathrm{H}\right\}$ NMR spectrum of the compound 29d.
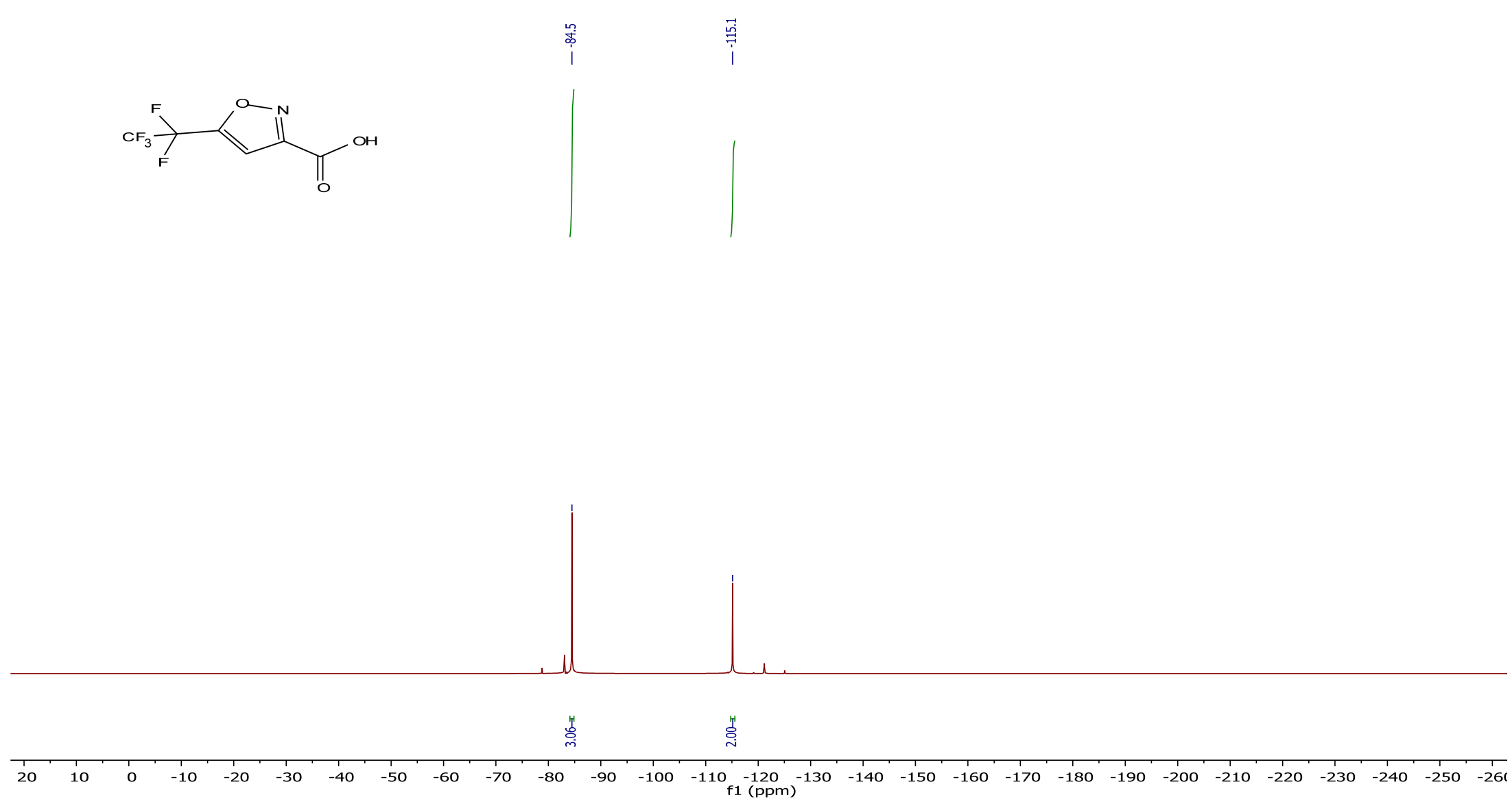
${ }^{1} \mathrm{H}$ NMR spectrum of the compound 30.

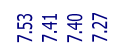

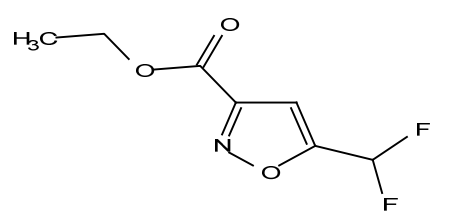

等昌举兴

旁兽学
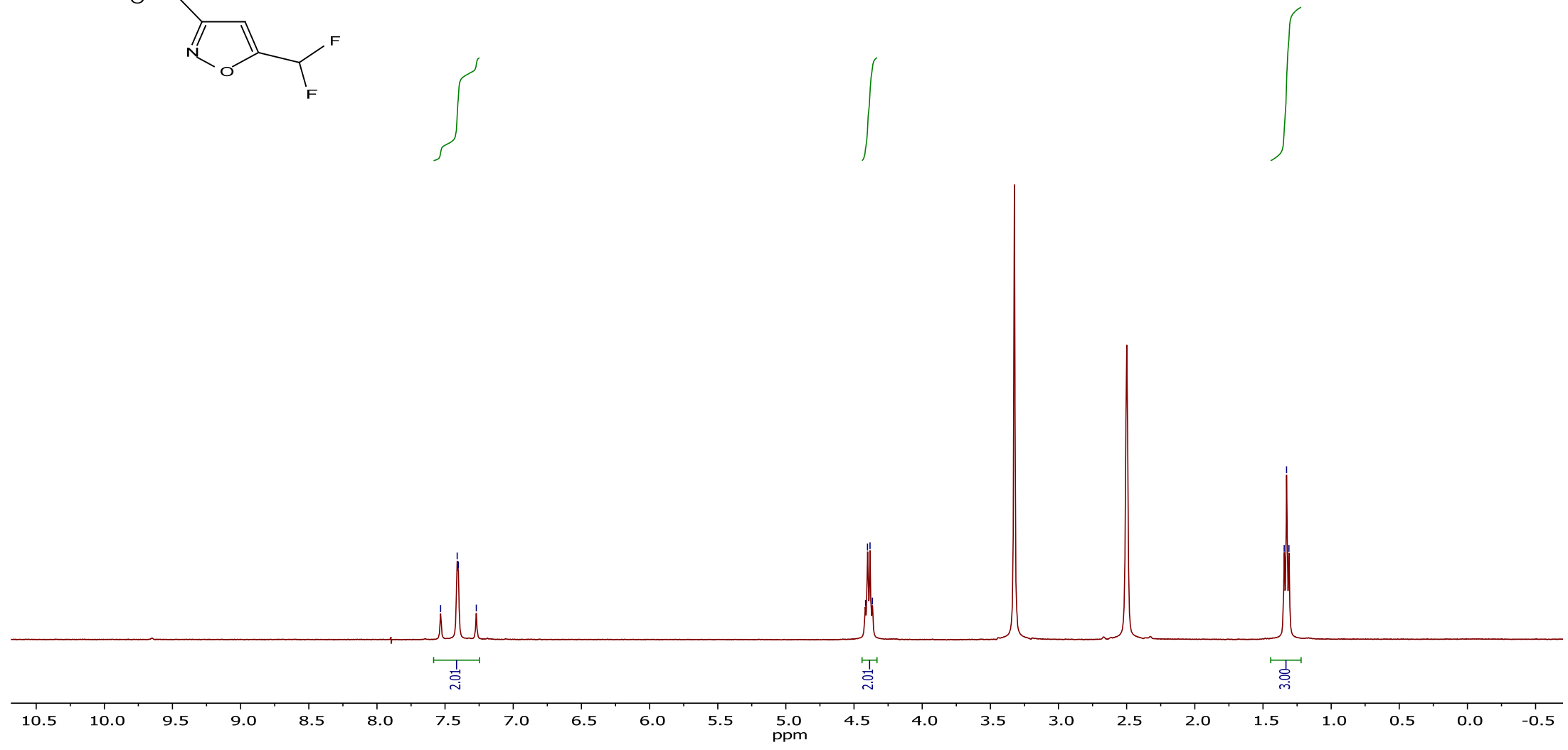

S209 
${ }^{13} \mathrm{C}\left\{{ }^{1} \mathrm{H}\right\}$ NMR spectrum of the compound $\mathbf{3 0}$.
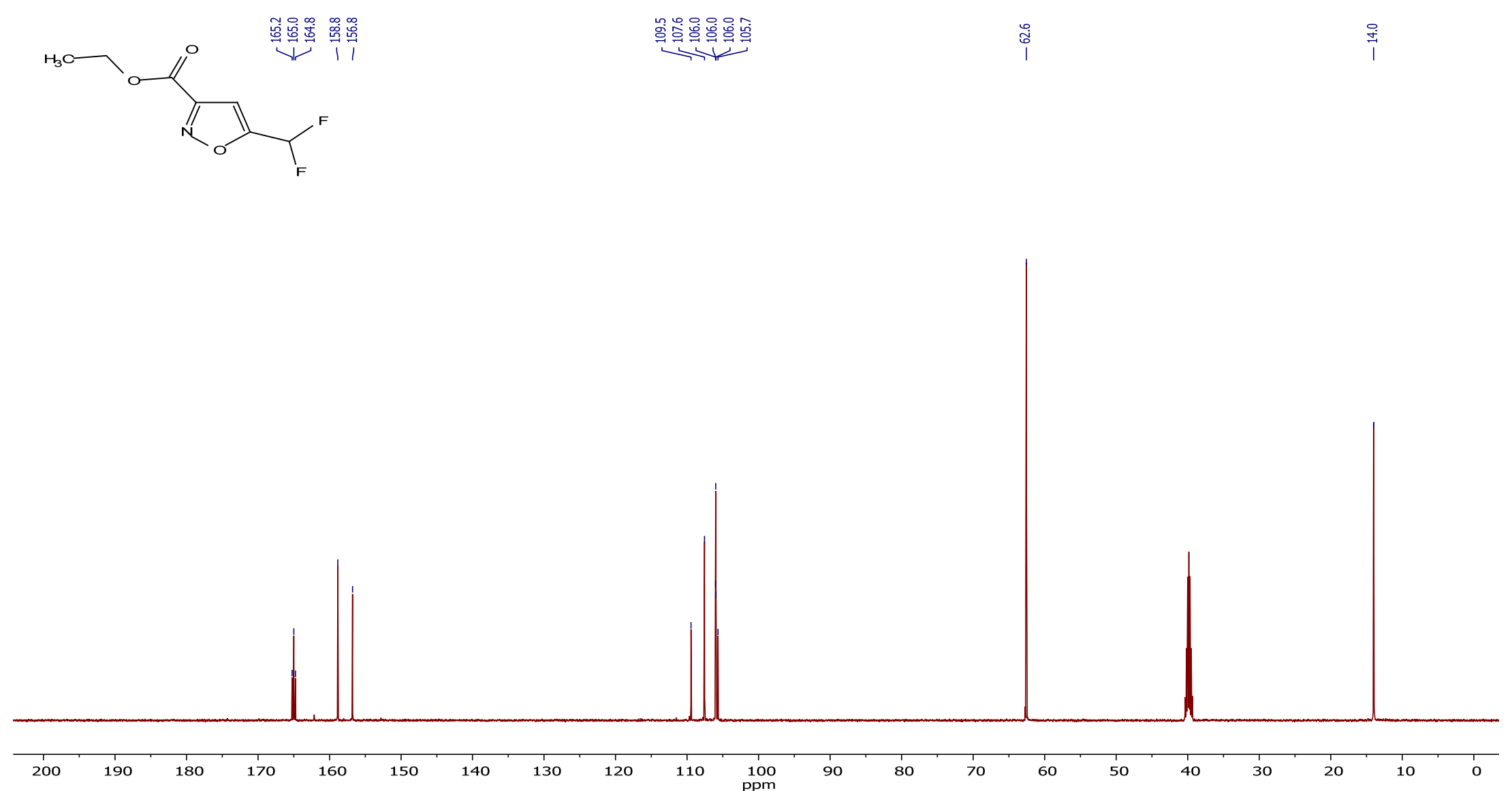
${ }^{19} \mathrm{~F}\left\{{ }^{1} \mathrm{H}\right\}$ NMR spectrum of the compound 30.

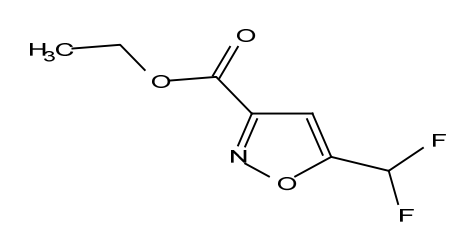

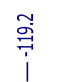

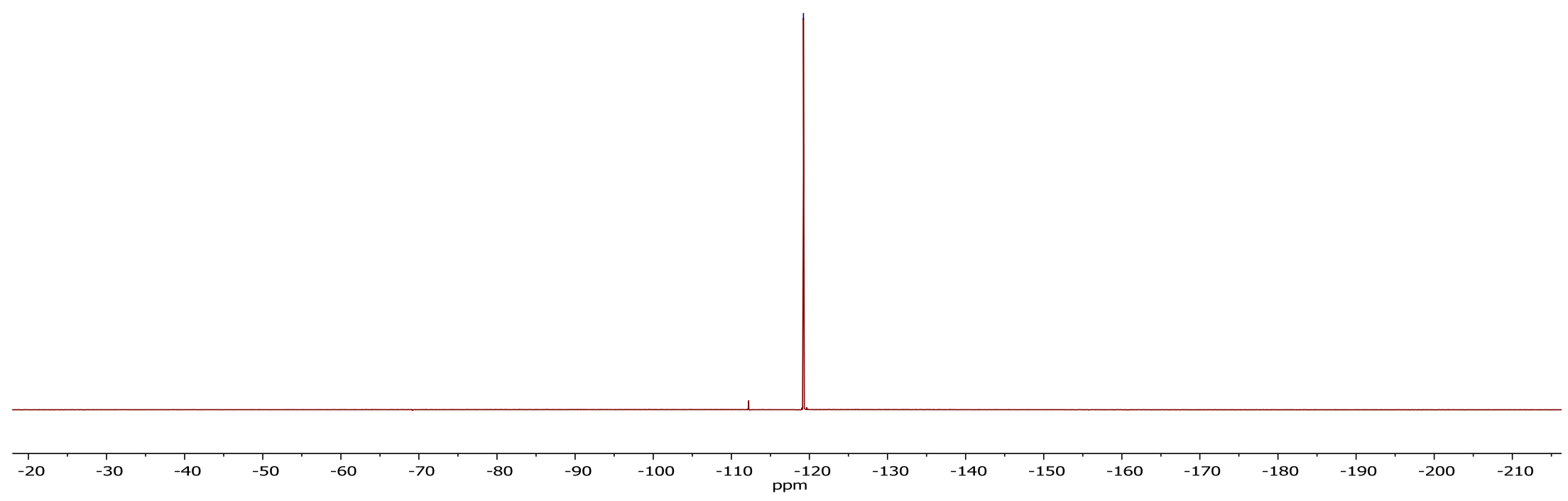


${ }^{1} \mathrm{H}$ NMR spectrum of the compound 33.

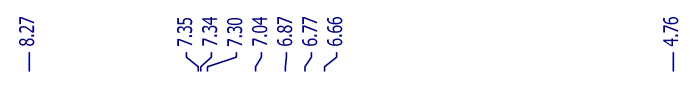

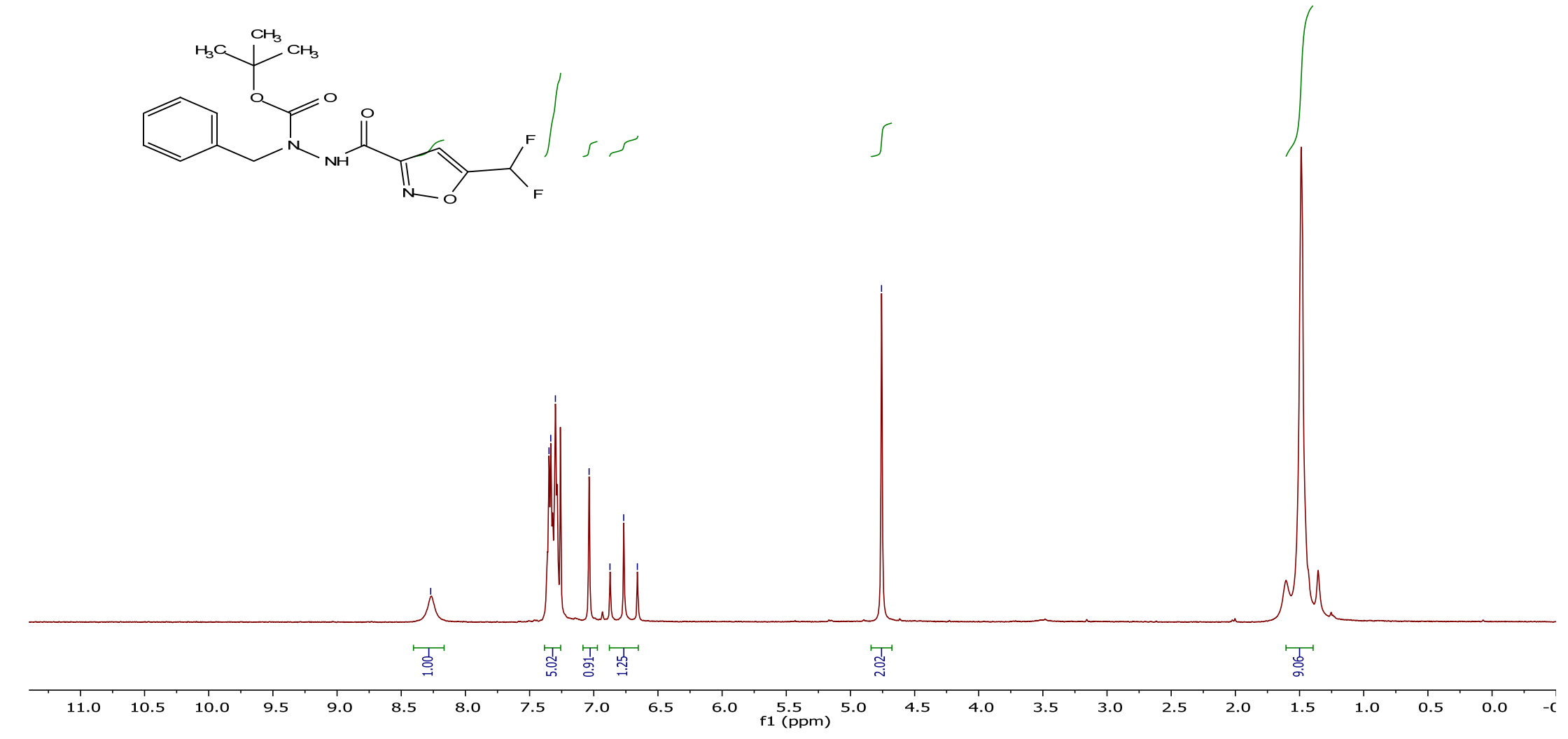




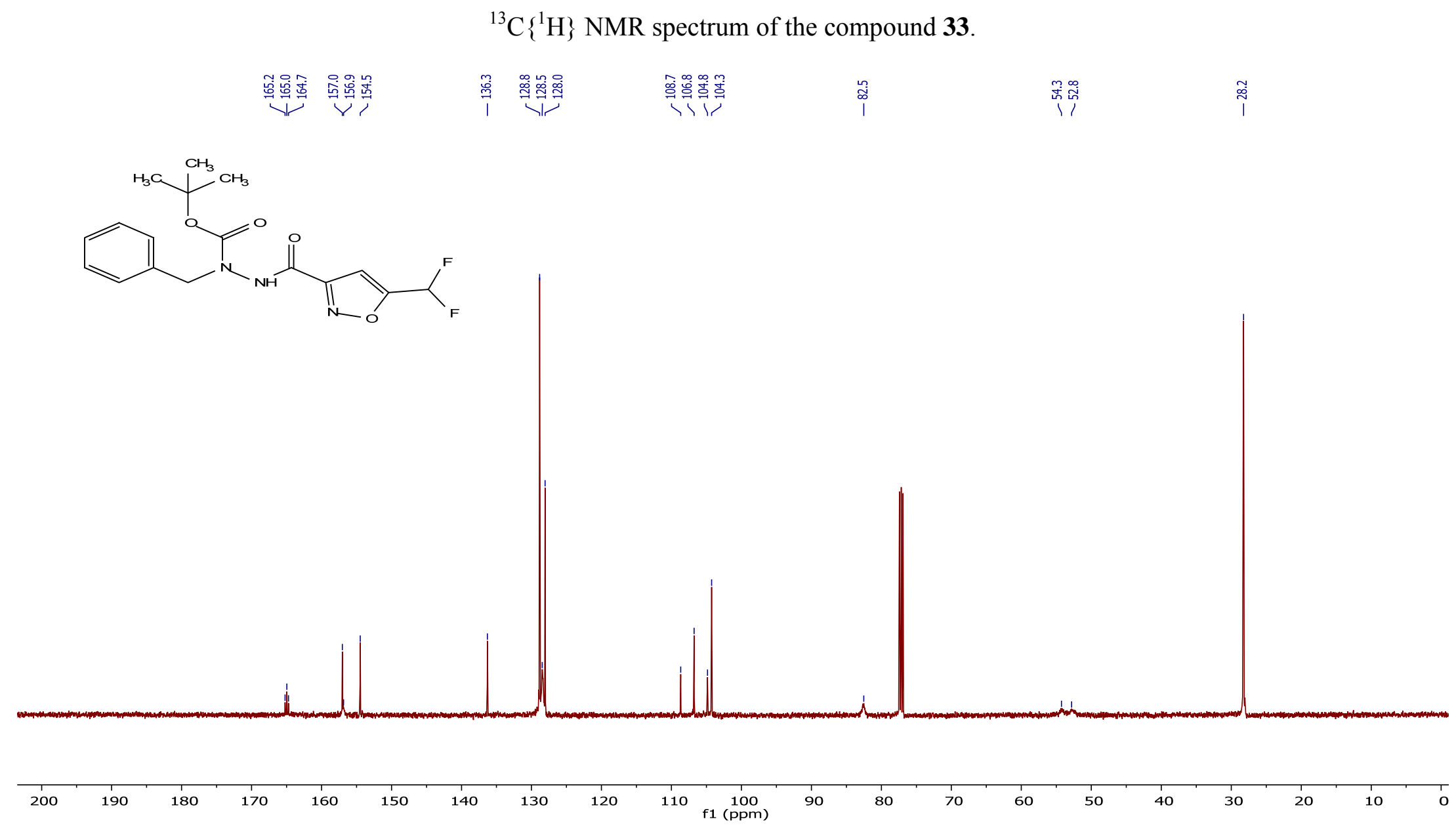


${ }^{19}$ F NMR spectrum of the compound 33.

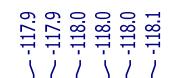
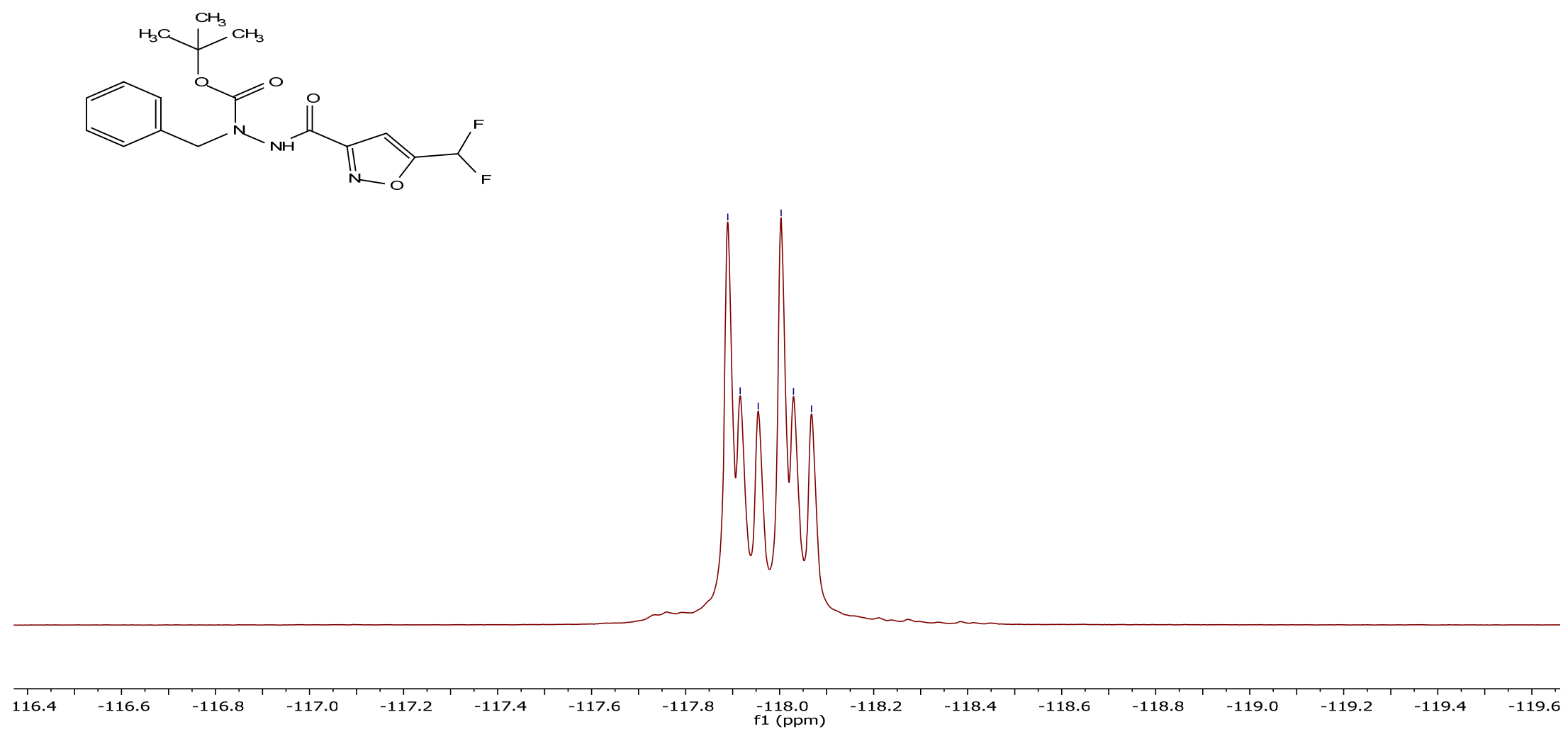
${ }^{1} \mathrm{H}$ NMR spectrum of the compound 37.

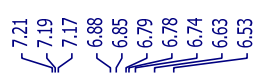
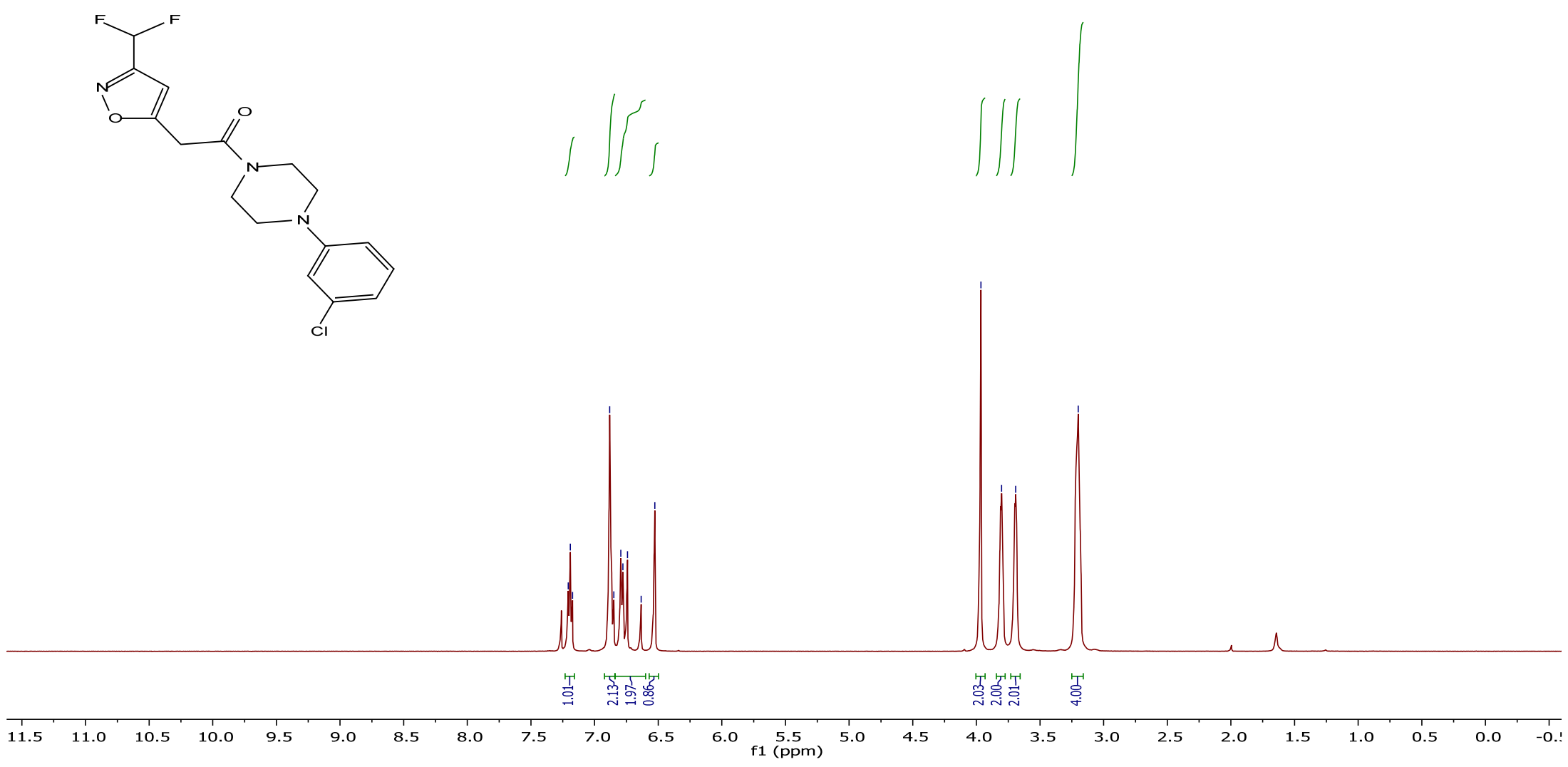

S215 


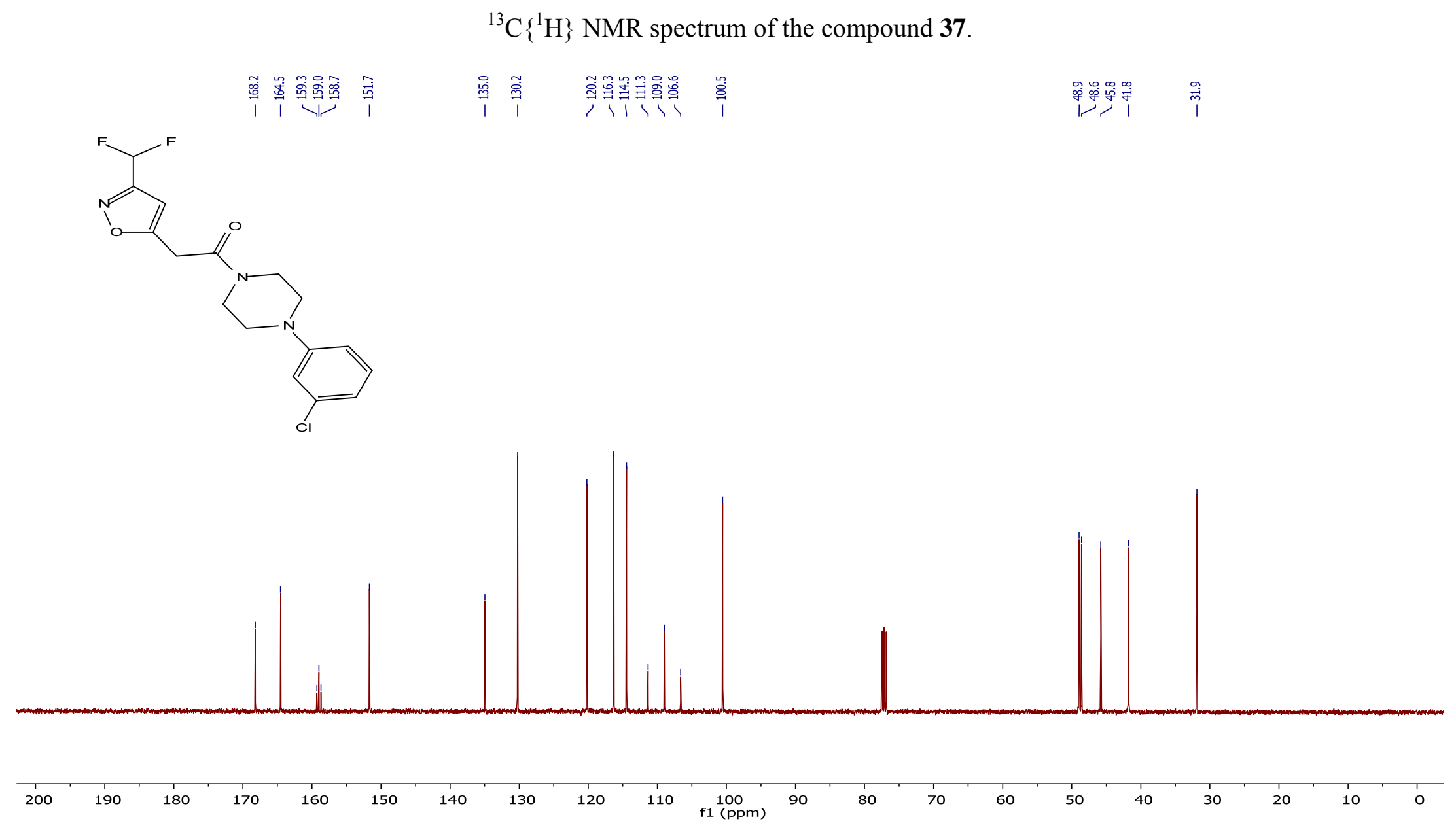


${ }^{19} \mathrm{~F}\left\{{ }^{1} \mathrm{H}\right\}$ NMR spectrum of the compound 37.

$\stackrel{\circ}{\mathrm{P}}$

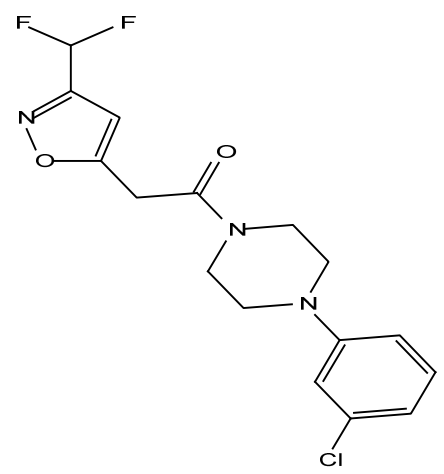

$-10$

$-20$

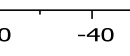

$-50 \quad-60$

$-70 \quad-80$

$-90$

$-100$

-110
$\mathrm{f1}(\mathrm{ppm})$ 
${ }^{1} \mathrm{H}$ NMR spectrum of the compound 34.
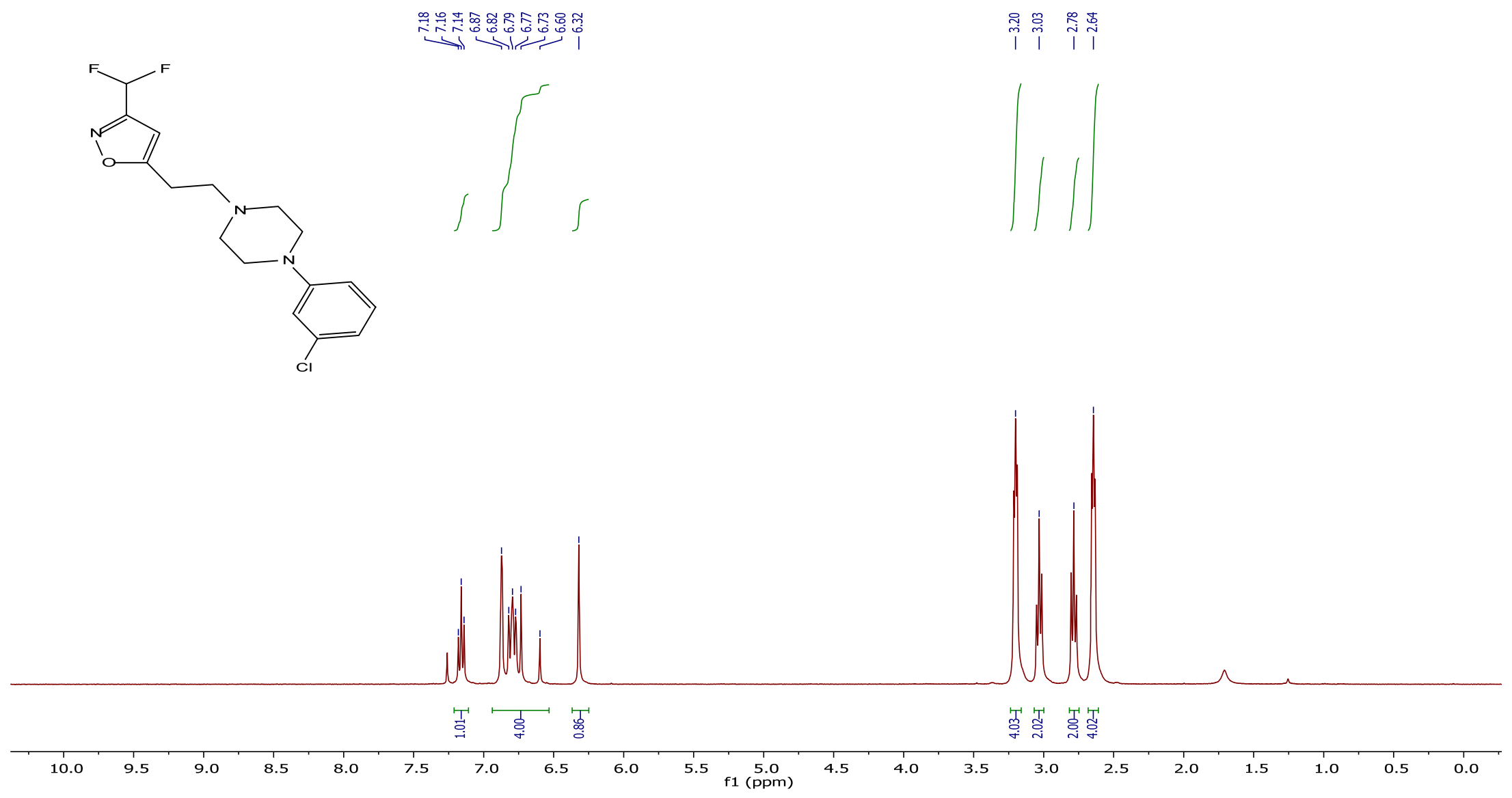
${ }^{13} \mathrm{C}\left\{{ }^{1} \mathrm{H}\right\}$ NMR spectrum of the compound 34.

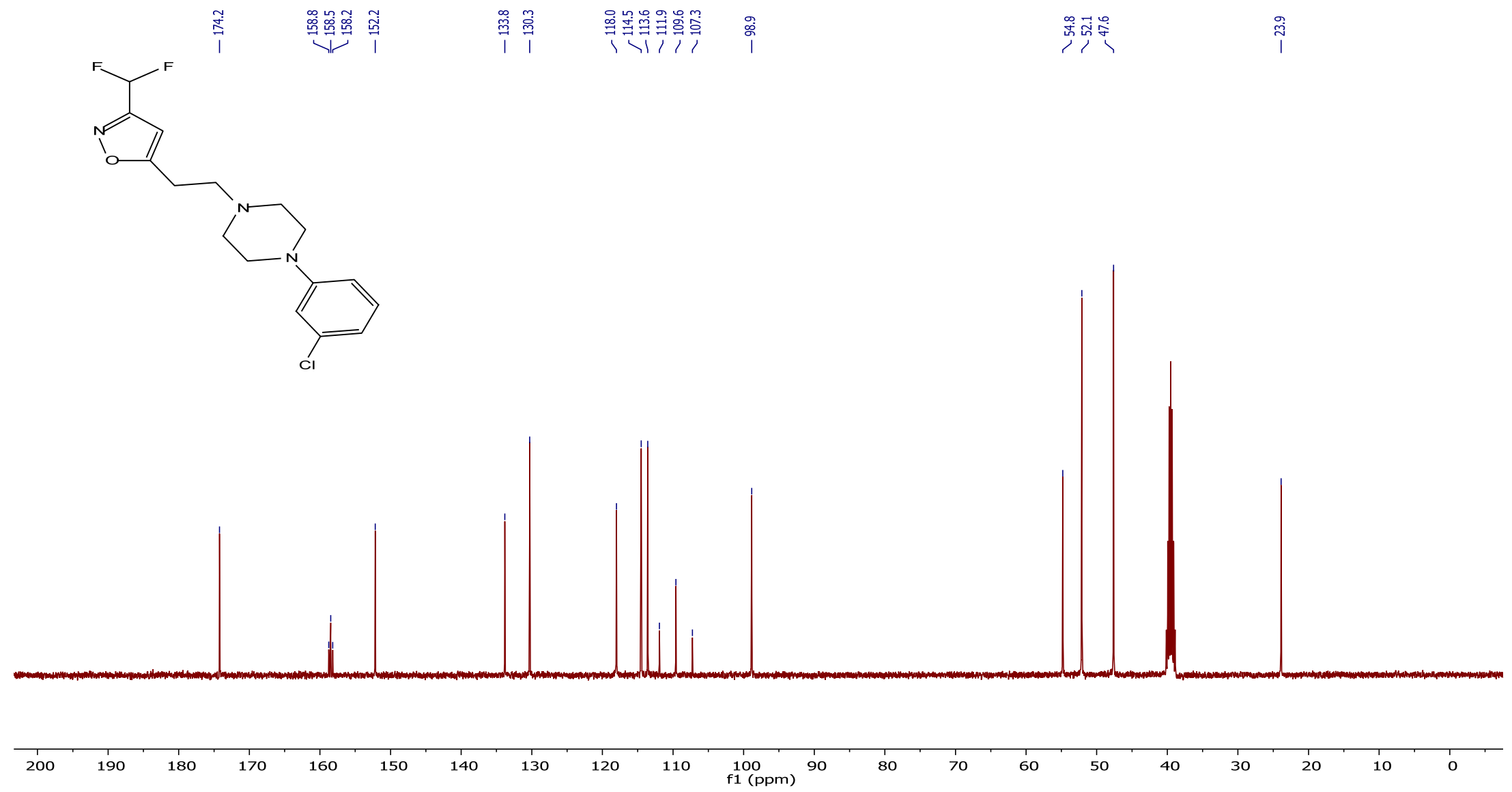


${ }^{19} \mathrm{~F}\left\{{ }^{1} \mathrm{H}\right\}$ NMR spectrum of the compound 34 .

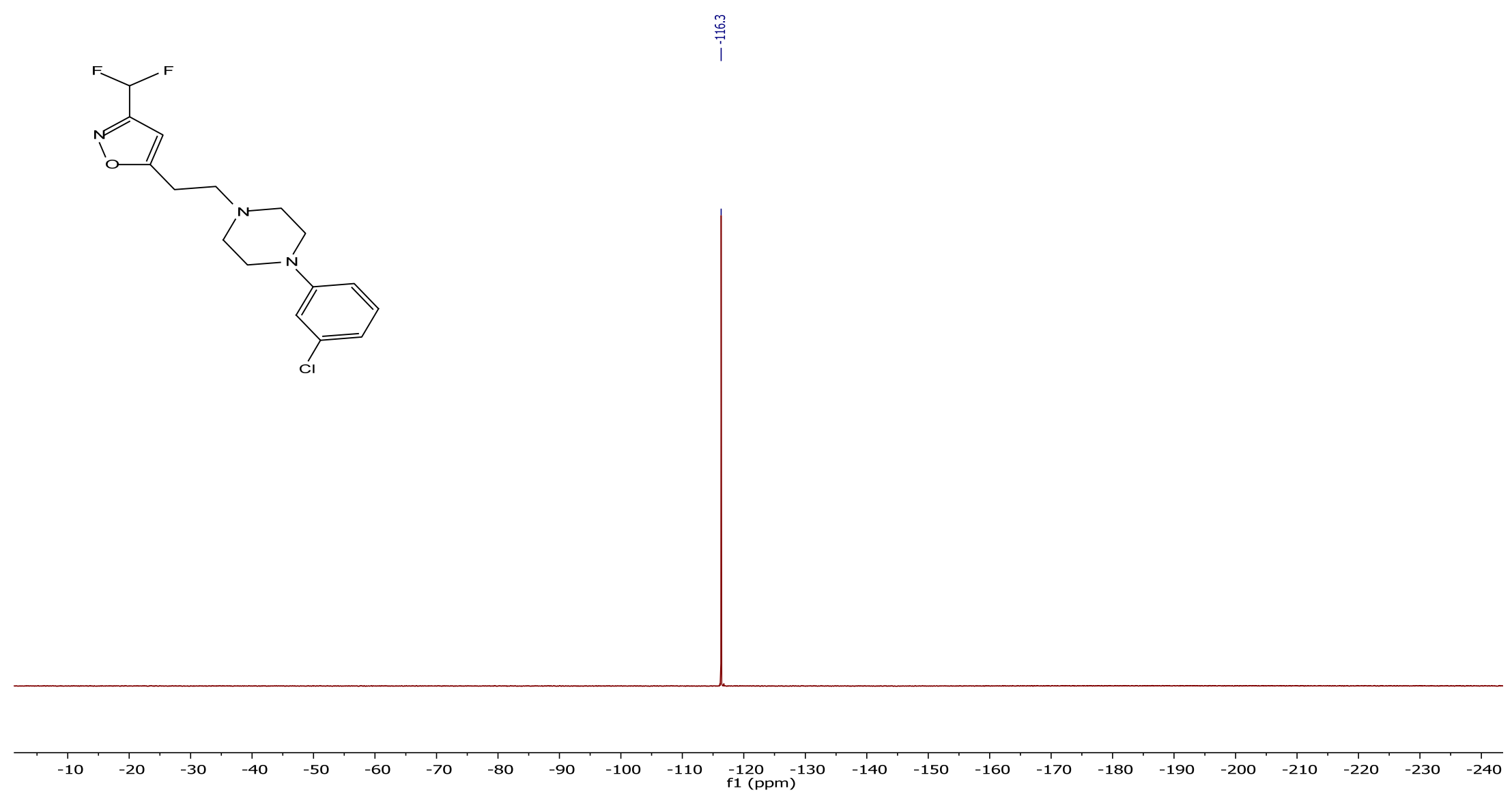


${ }^{1} \mathrm{H}$ NMR spectrum of the compound $35 \mathbf{b}$.

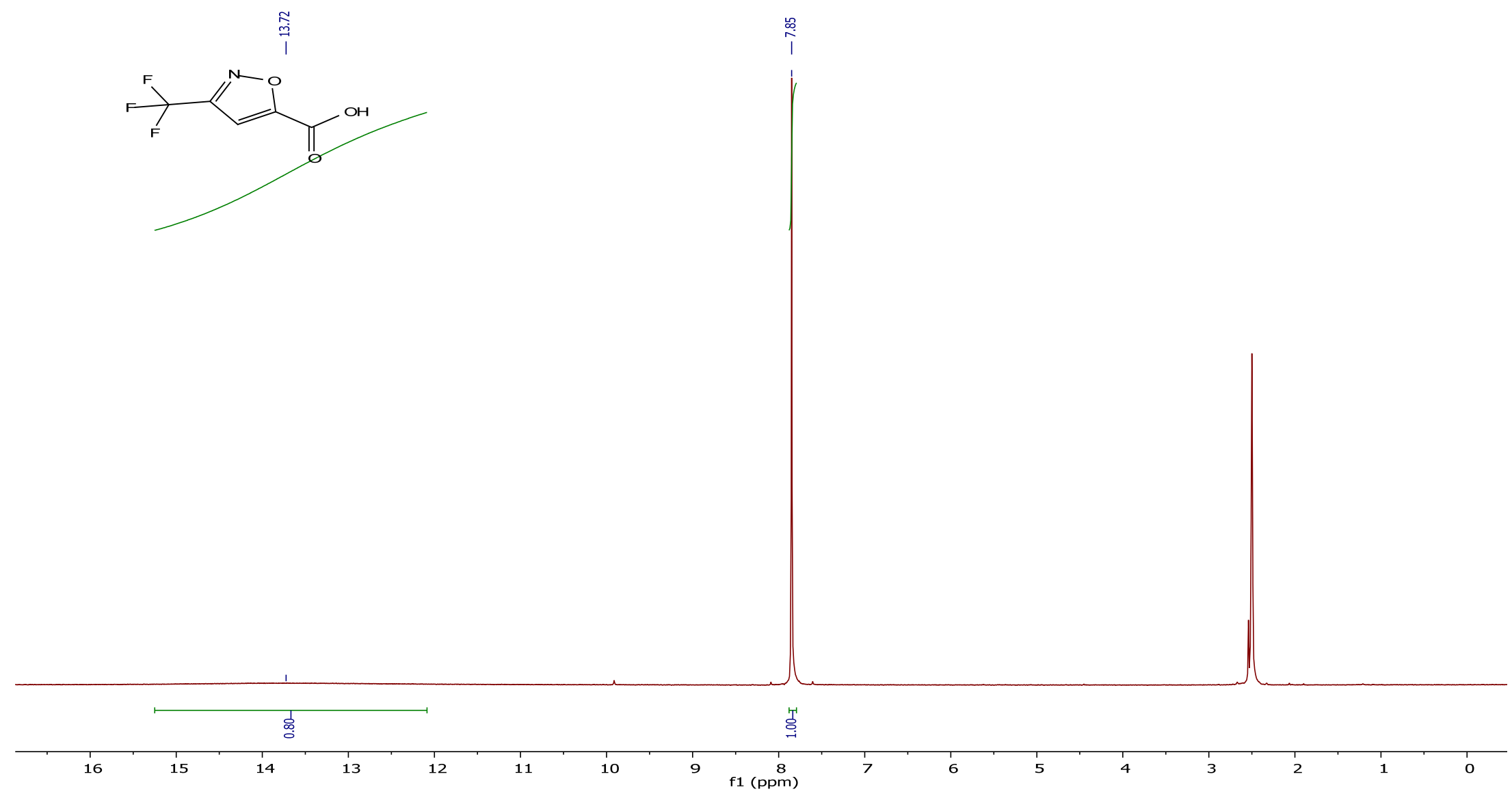


${ }^{13} \mathrm{C}\left\{{ }^{1} \mathrm{H}\right\}$ NMR spectrum of the compound 35b.

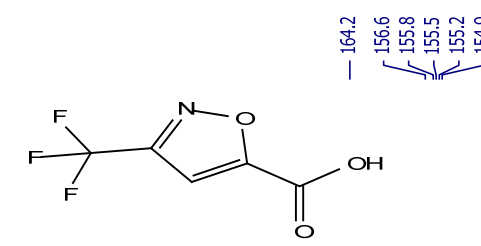

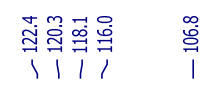

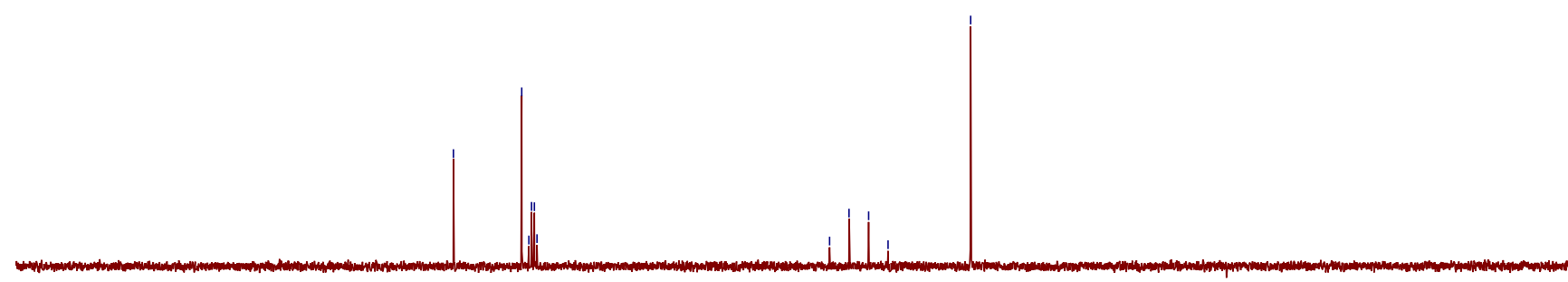


${ }^{19} \mathrm{~F}\left\{{ }^{1} \mathrm{H}\right\}$ NMR spectrum of the compound 35b.

i̊<smiles>O=C(O)c1cc(C(F)(F)F)no1</smiles>

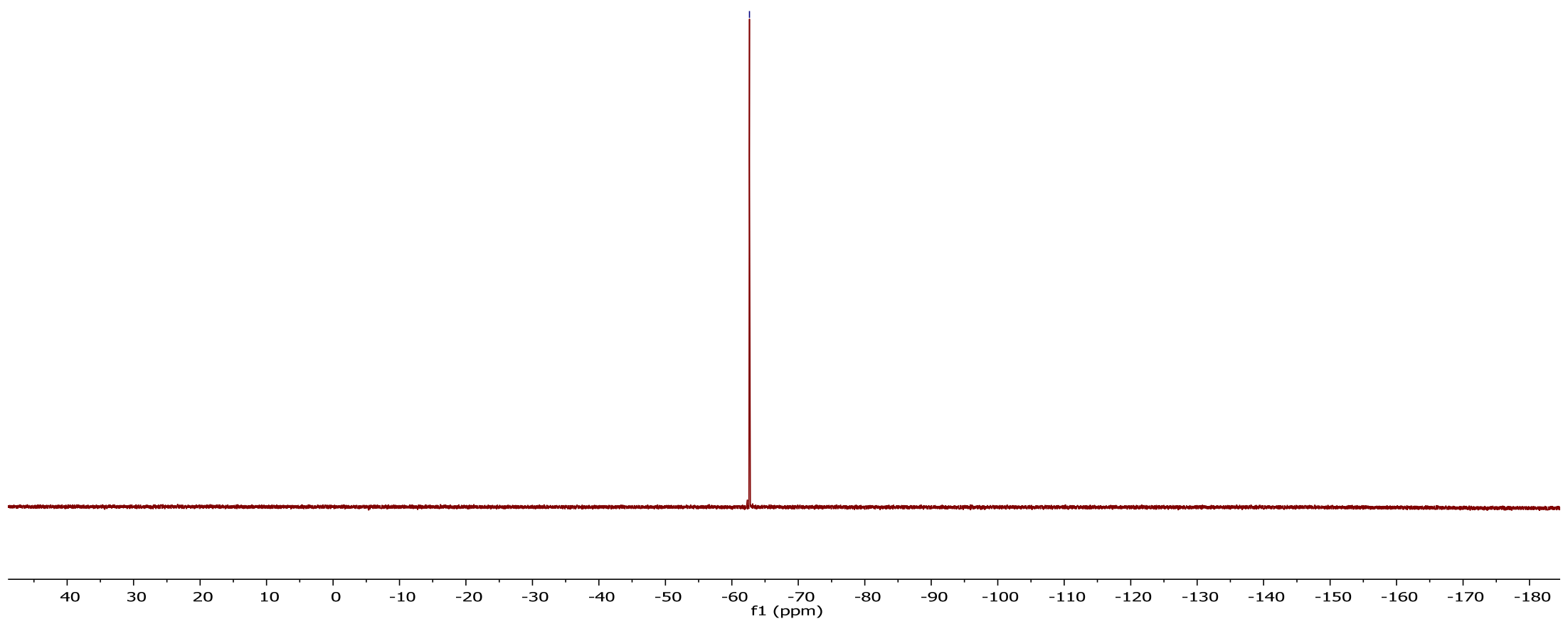


${ }^{1} \mathrm{H}$ NMR spectrum of the compound 35c.

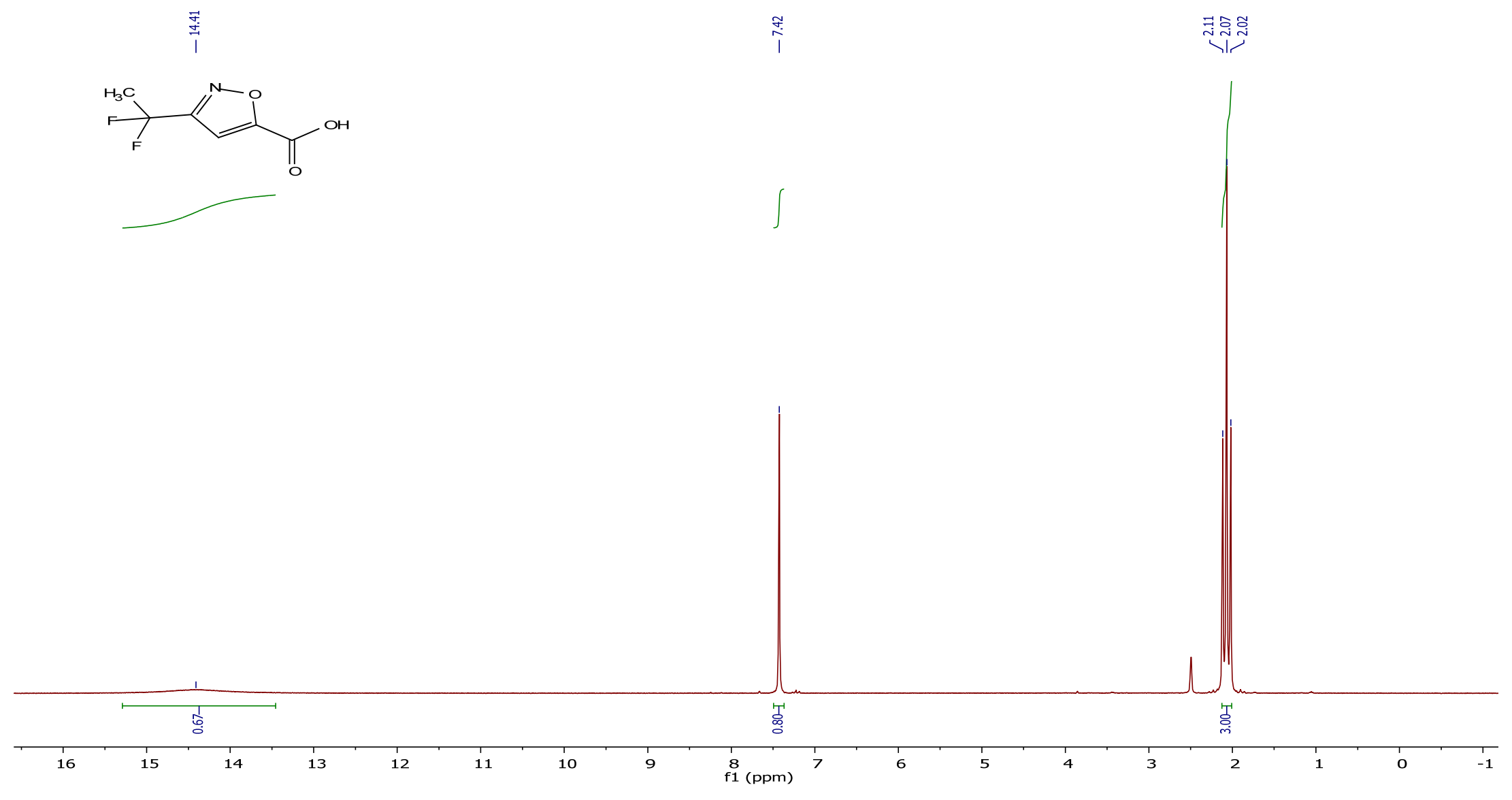


${ }^{13} \mathrm{C}\left\{{ }^{1} \mathrm{H}\right\}$ NMR spectrum of the compound 35c.
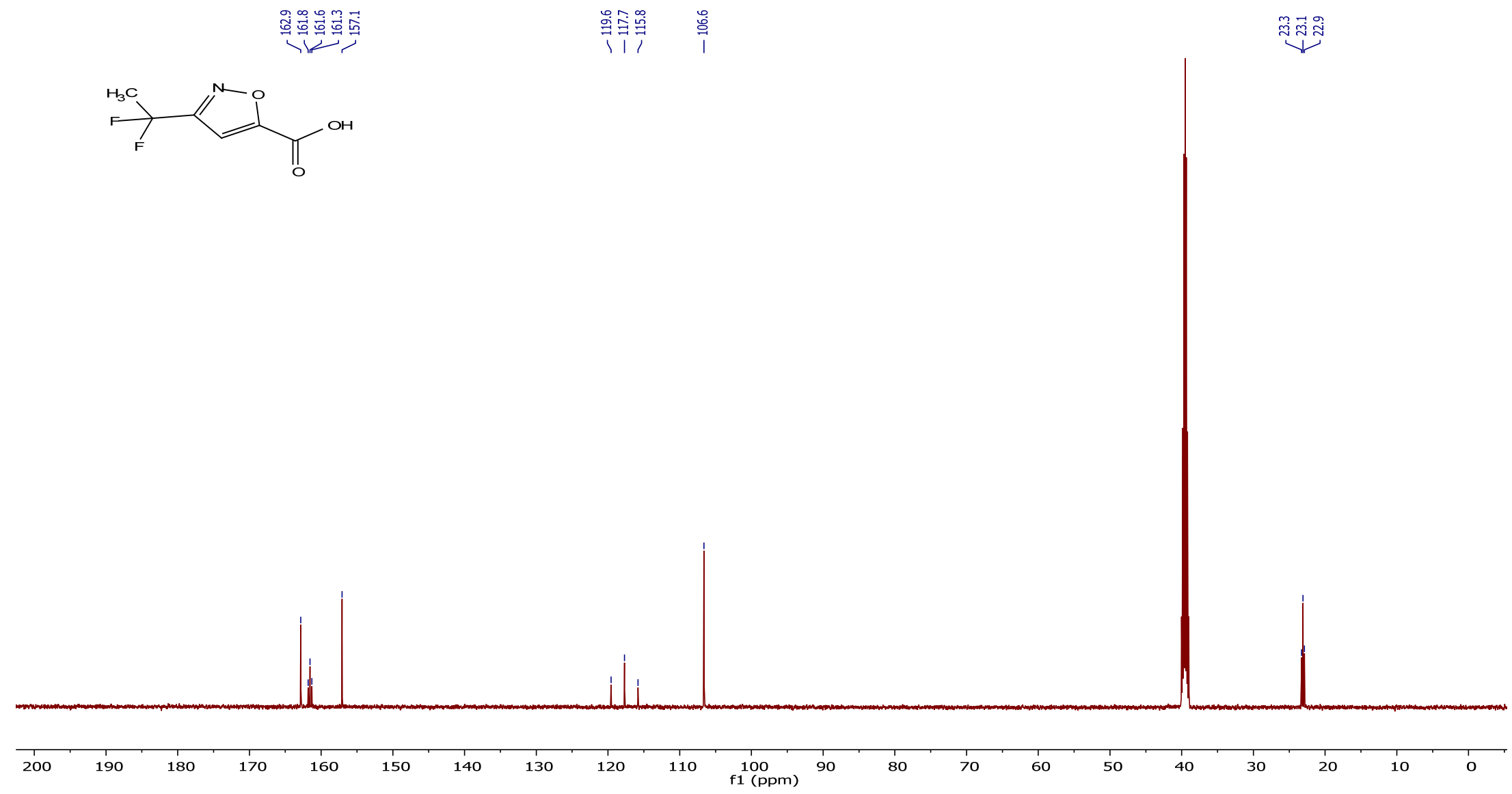
${ }^{19} \mathrm{~F}\left\{{ }^{1} \mathrm{H}\right\}$ NMR spectrum of the compound 35c.

$$
\text { 产 }
$$<smiles>CC(F)(F)c1cc(C(=O)O)on1</smiles>

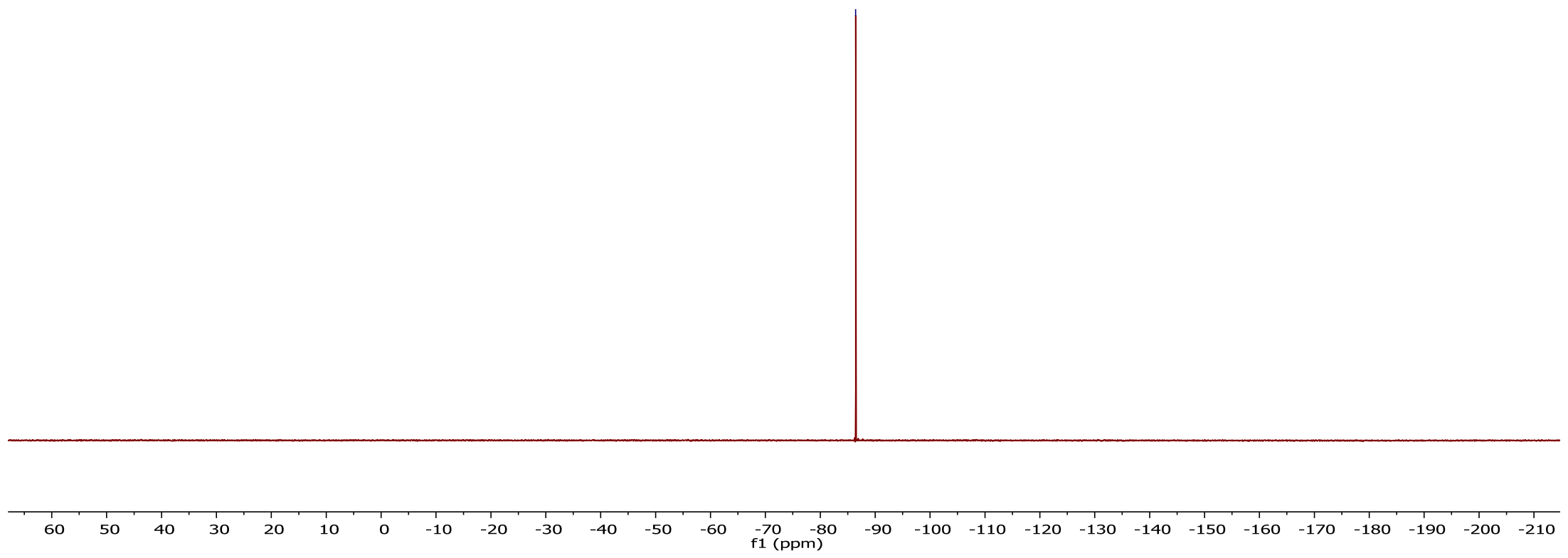


${ }^{1} \mathrm{H}$ NMR spectrum of the compound 35g.

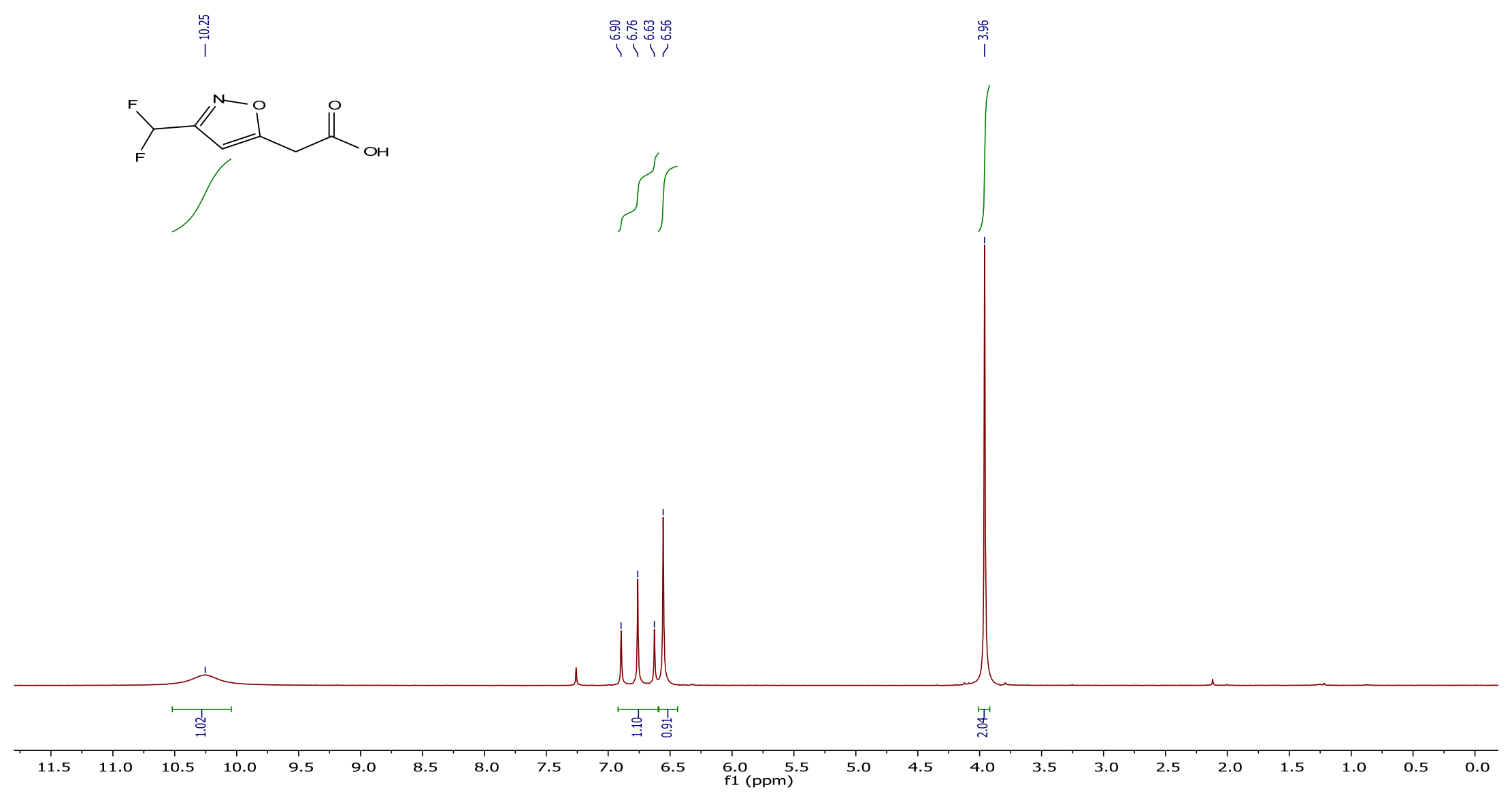


${ }^{13} \mathrm{C}\left\{{ }^{1} \mathrm{H}\right\}$ NMR spectrum of the compound 35g.

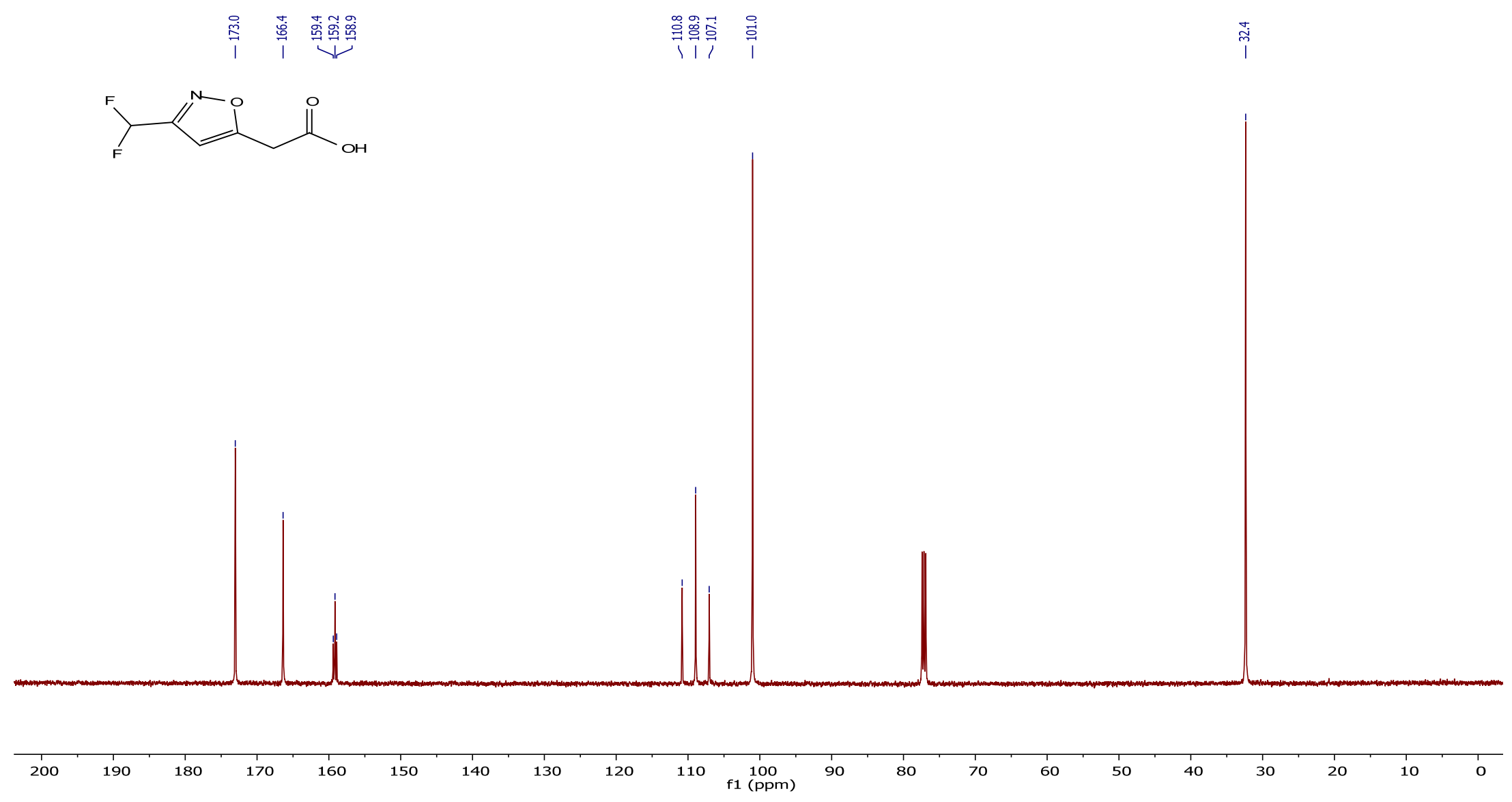


${ }^{19}$ F NMR spectrum of the compound 35g.

ตำำ

$>_{\mathrm{F}}^{\mathrm{F}}$

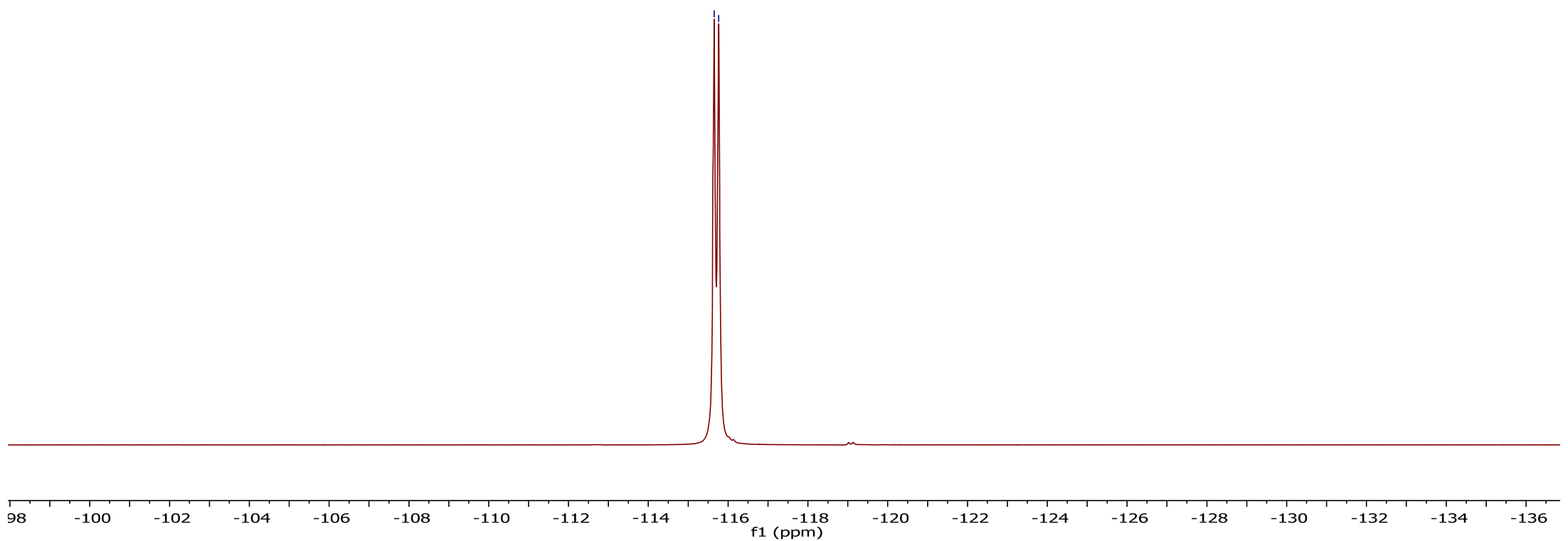


${ }^{1} \mathrm{H}$ NMR spectrum of the compound 40a.

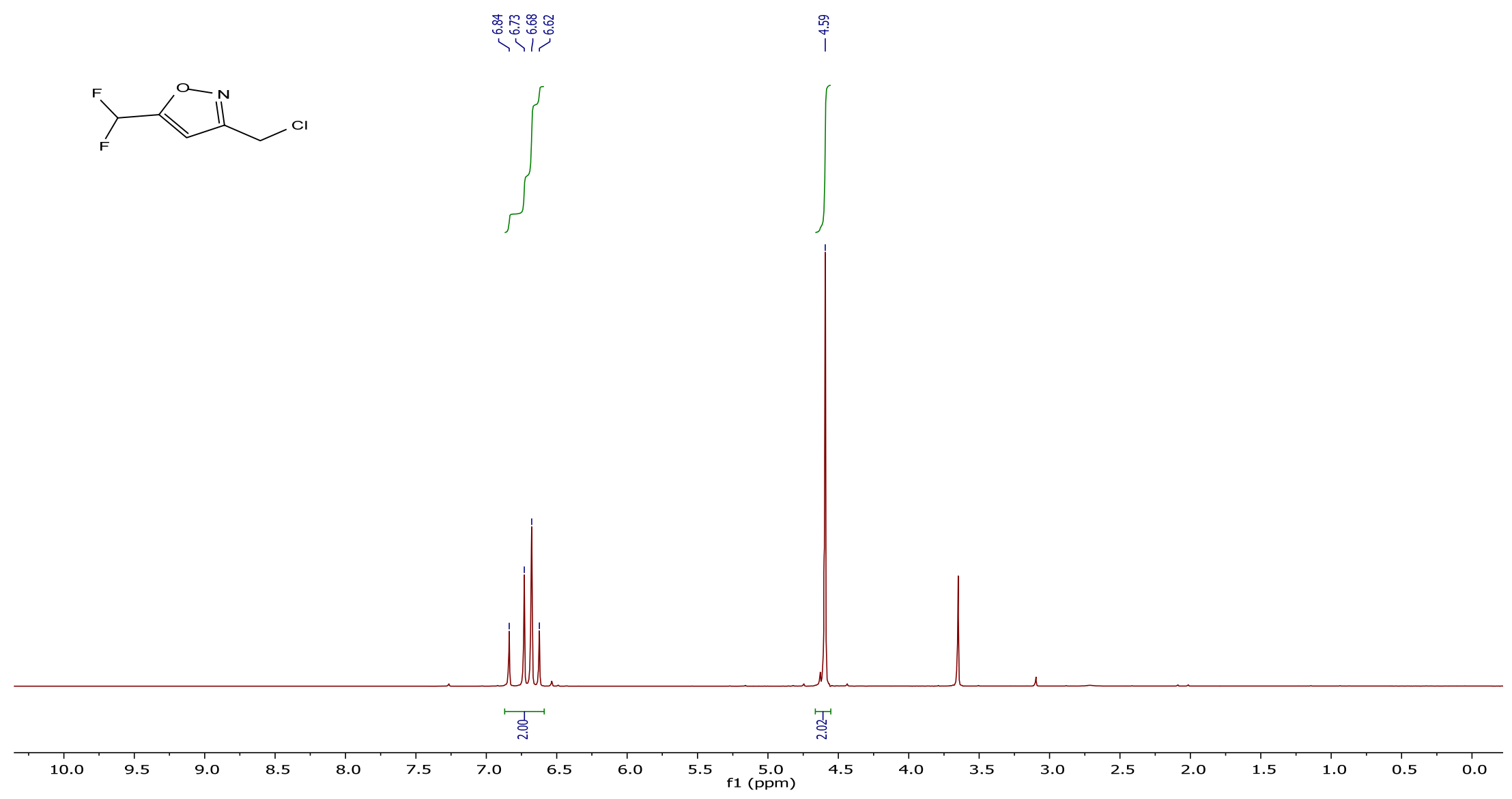


${ }^{13} \mathrm{C}\left\{{ }^{1} \mathrm{H}\right\}$ NMR spectrum of the compound 40a.

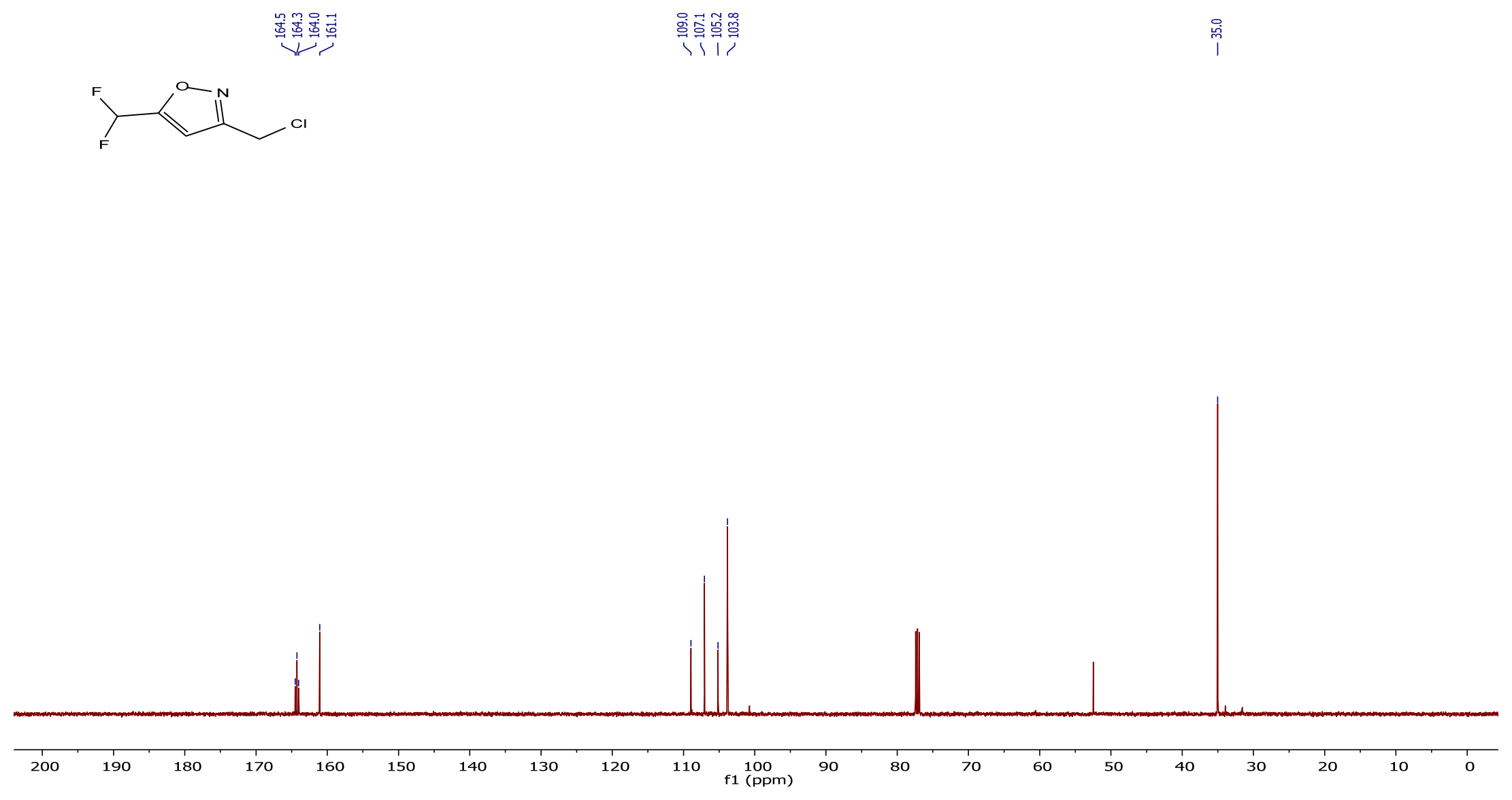


${ }^{19} \mathrm{~F}\left\{{ }^{1} \mathrm{H}\right\}$ NMR spectrum of the compound 40a.
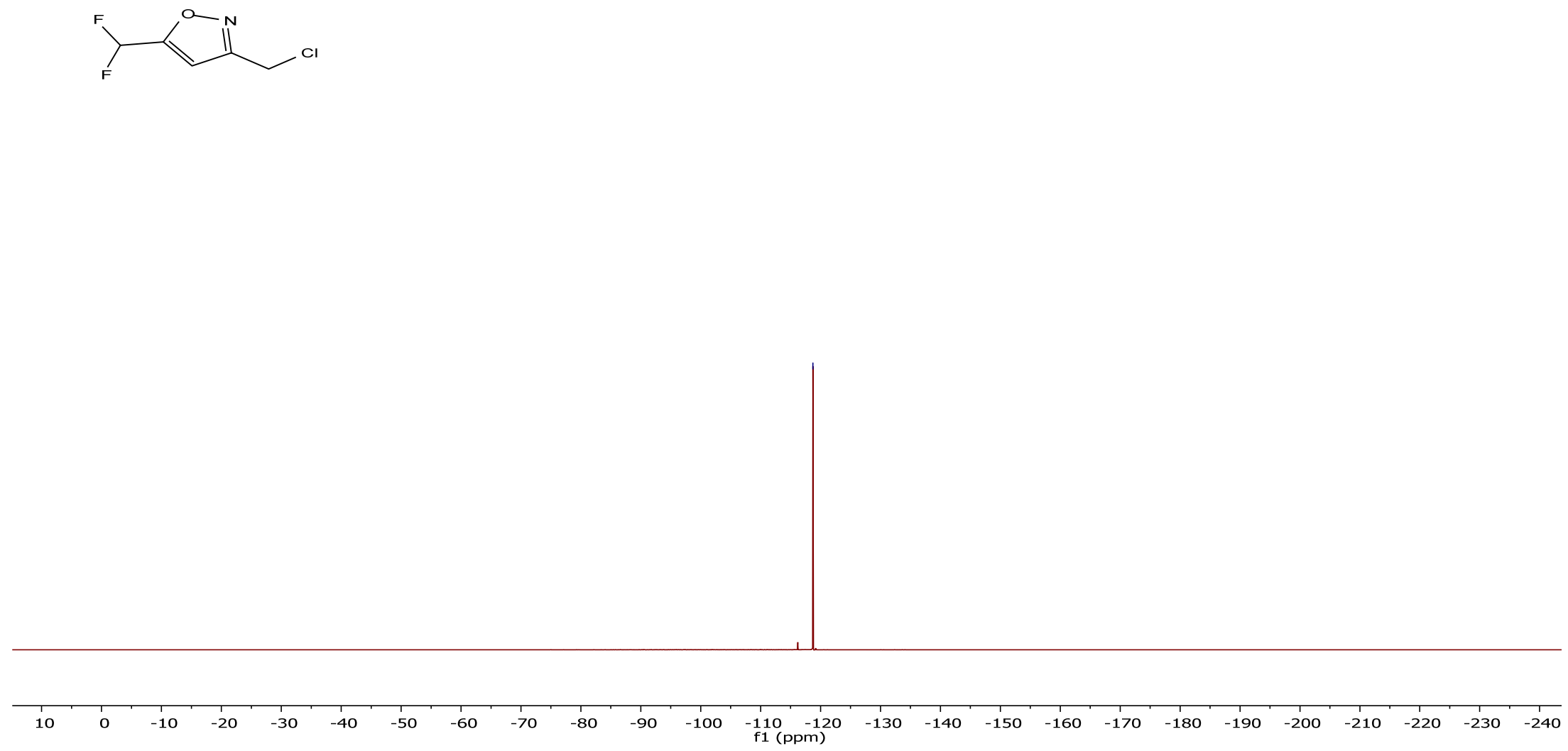
${ }^{1} \mathrm{H}$ NMR spectrum of the compound $40 \mathrm{~b}$.
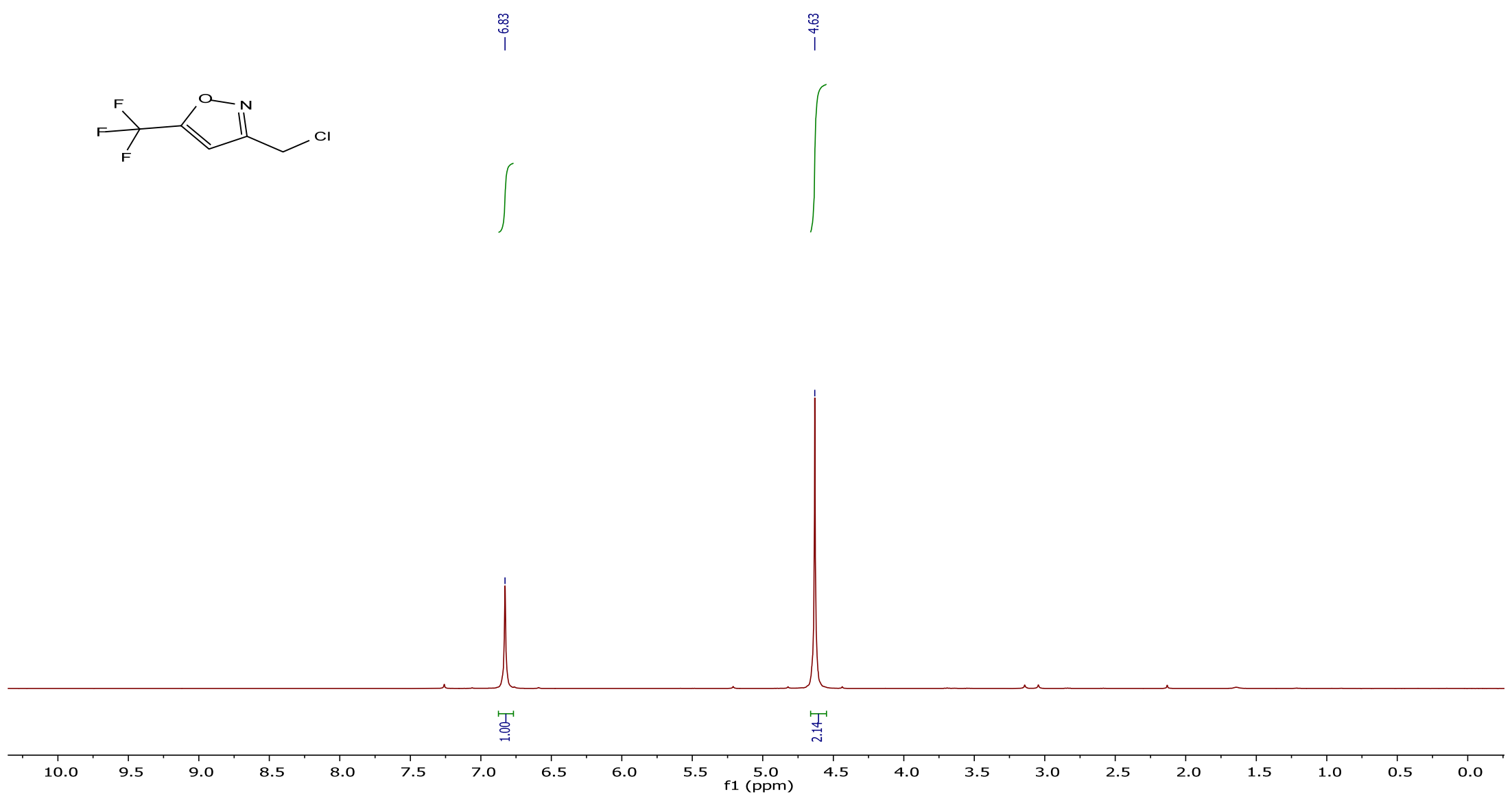
${ }^{13} \mathrm{C}\left\{{ }^{1} \mathrm{H}\right\}$ NMR spectrum of the compound $\mathbf{4 0 b}$.

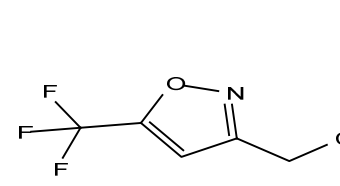

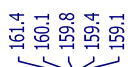

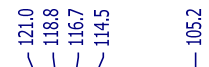

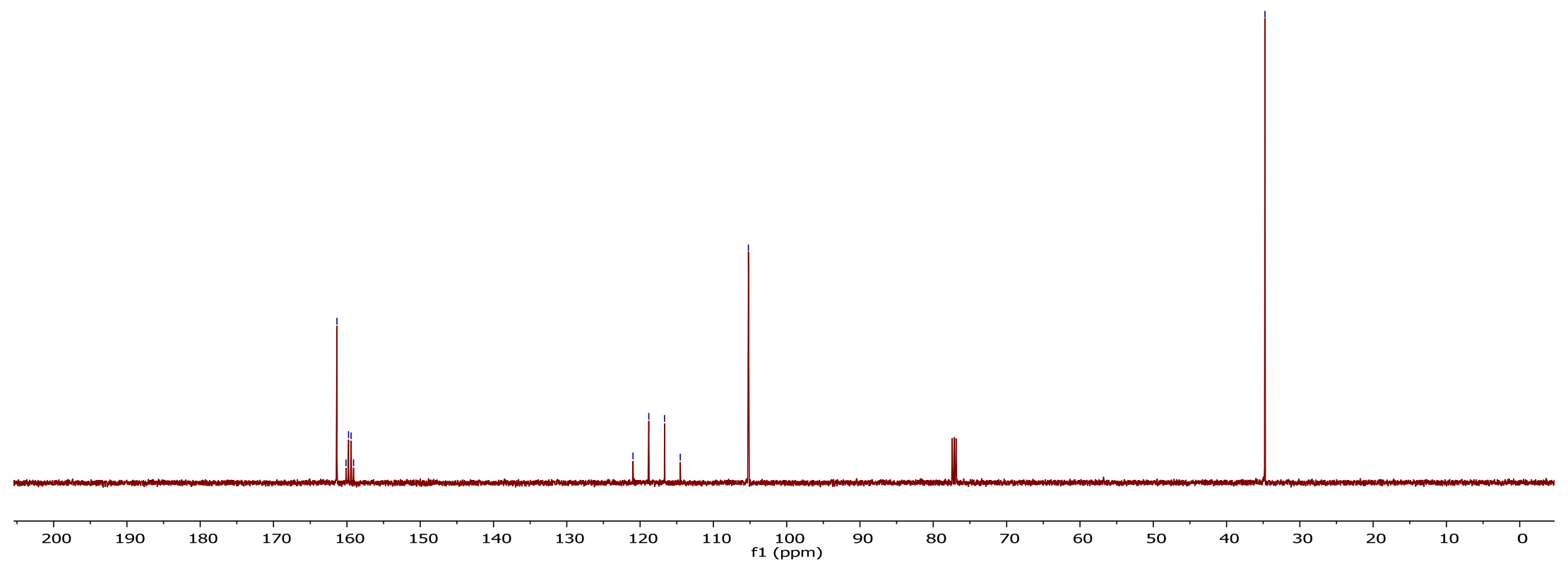


${ }^{19} \mathrm{~F}\left\{{ }^{1} \mathrm{H}\right\}$ NMR spectrum of the compound 40b.

$\stackrel{\text { 草 }}{i}$
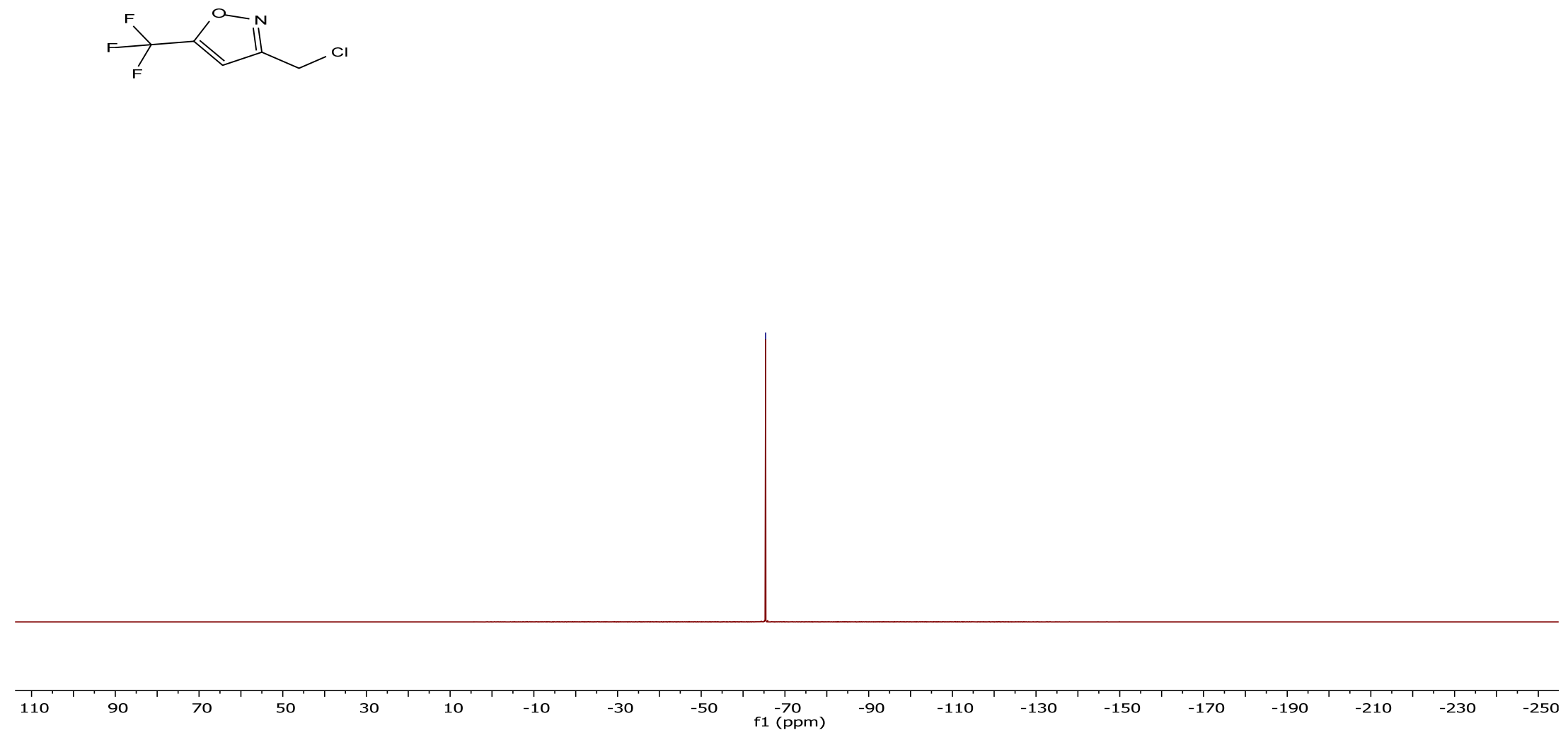
${ }^{1} \mathrm{H}$ NMR spectrum of the compound 40d.
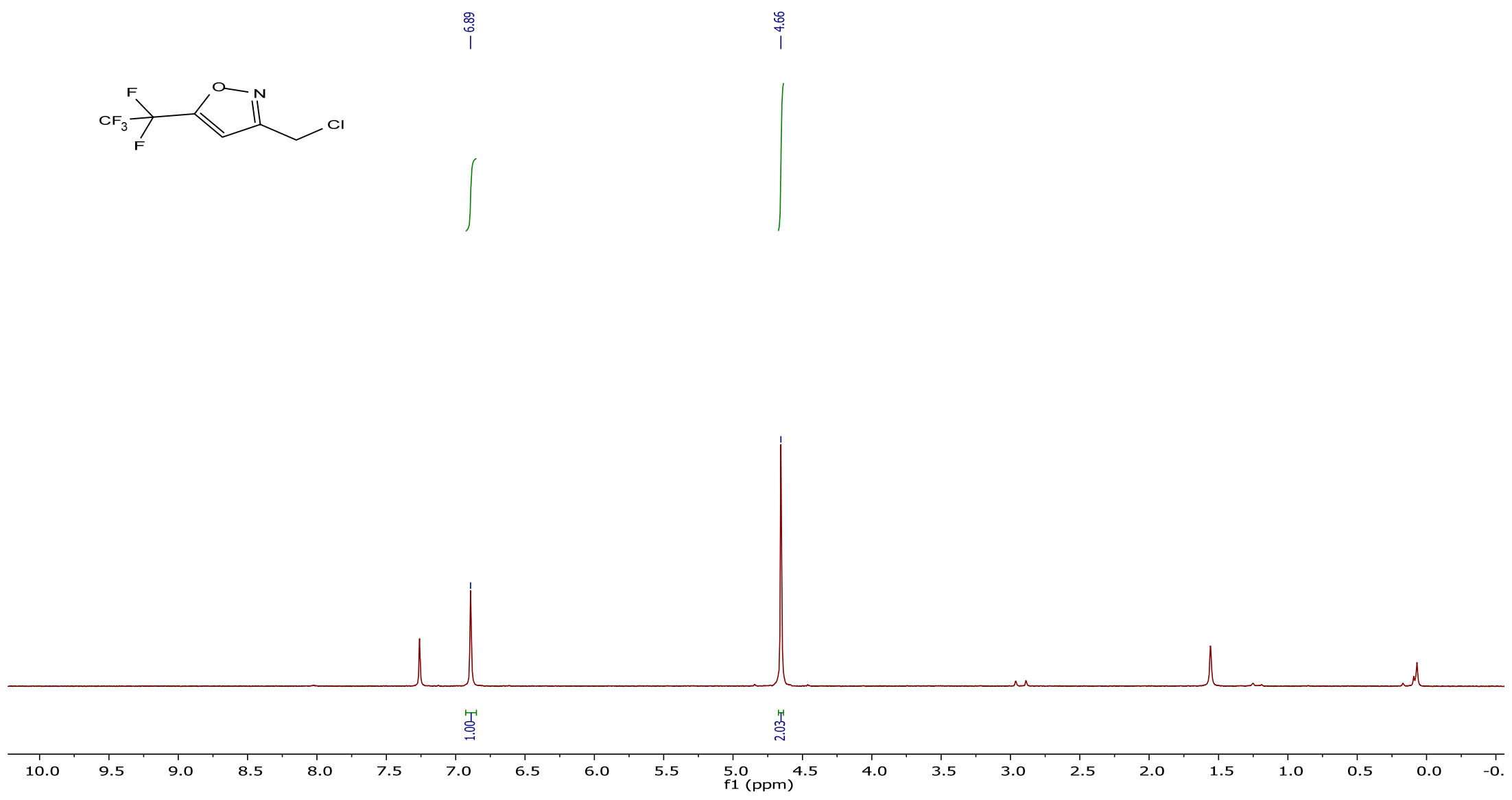
${ }^{13} \mathrm{C}\left\{{ }^{1} \mathrm{H}\right\}$ NMR spectrum of the compound $\mathbf{4 0 d}$.

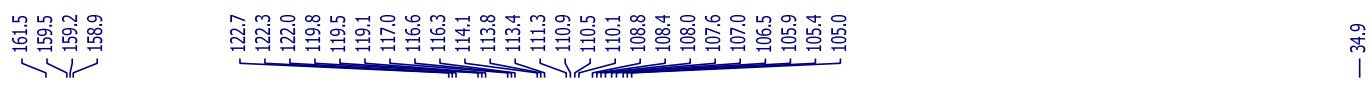
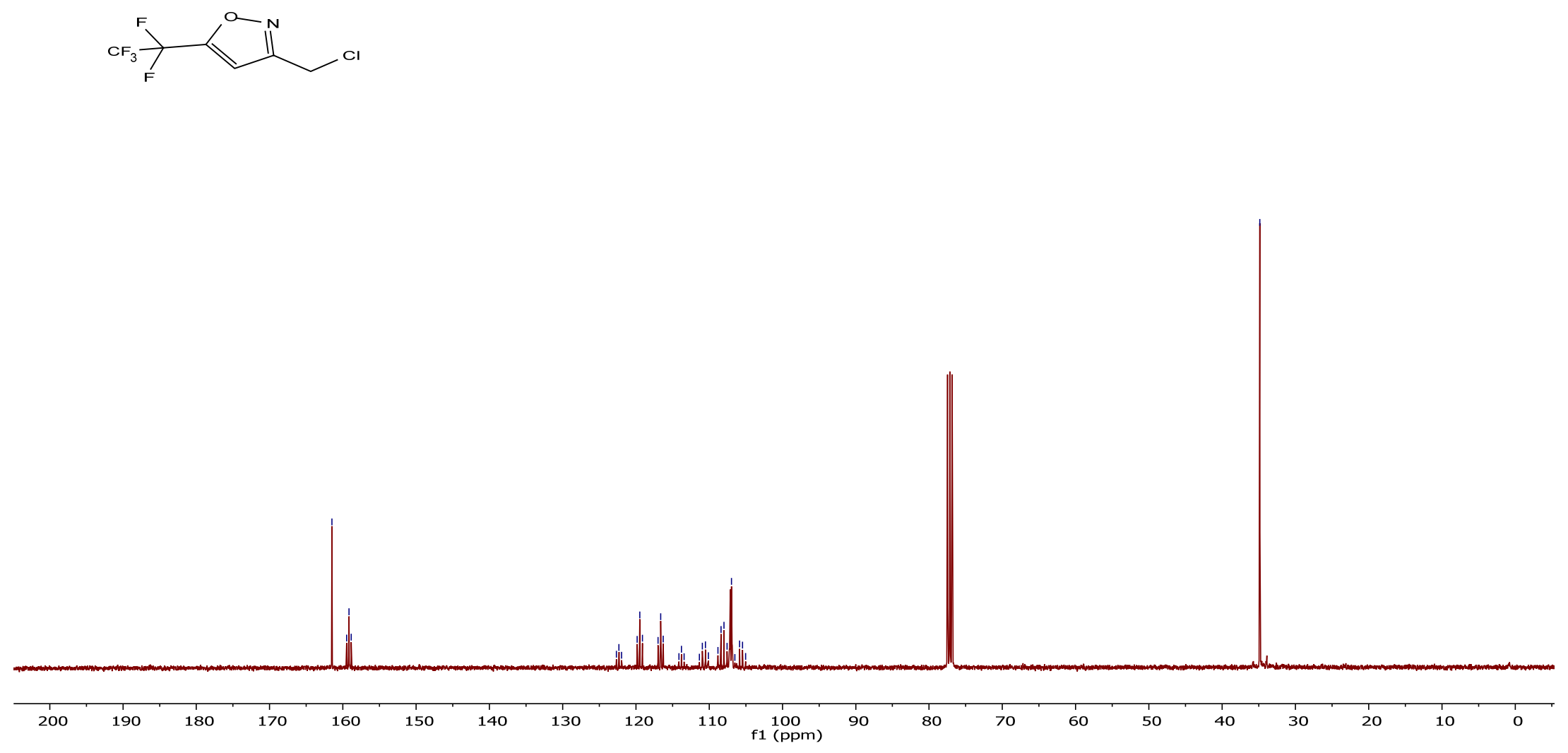
${ }^{19} \mathrm{~F}\left\{{ }^{1} \mathrm{H}\right\}$ NMR spectrum of the compound 40d.

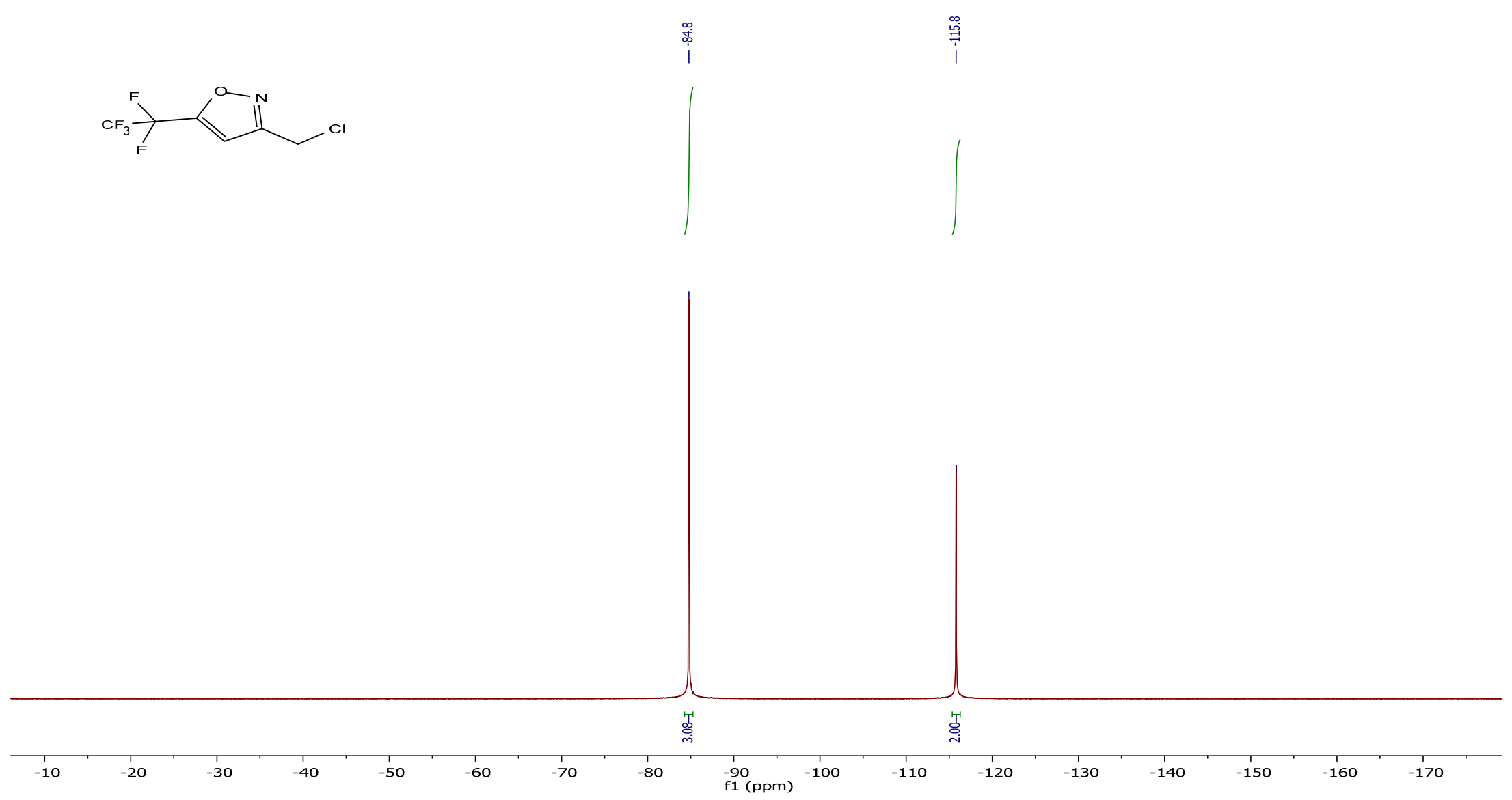


${ }^{1} \mathrm{H}$ NMR spectrum of the compound 41a.
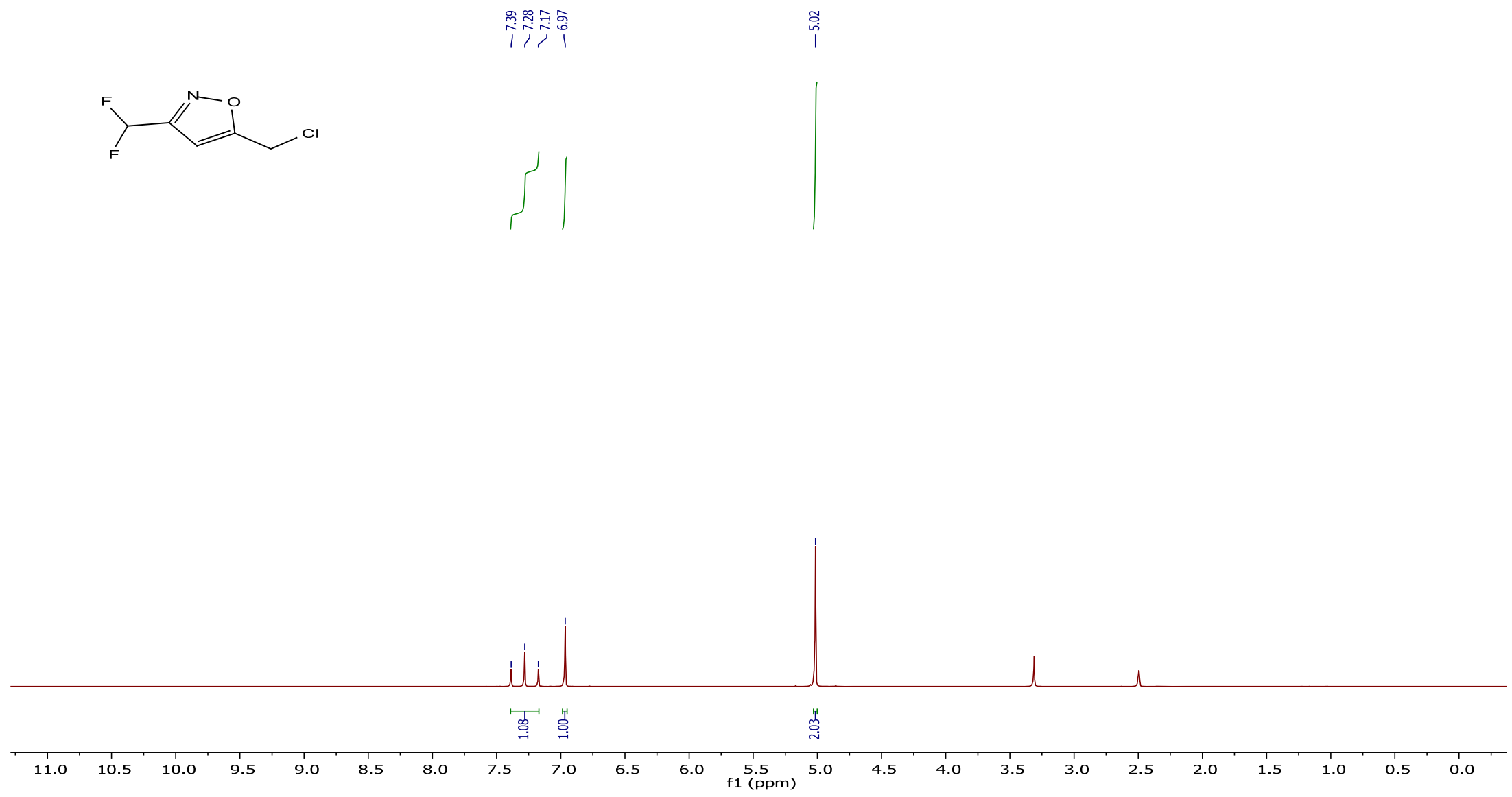
${ }^{13} \mathrm{C}\left\{{ }^{1} \mathrm{H}\right\}$ NMR spectrum of the compound 41a.

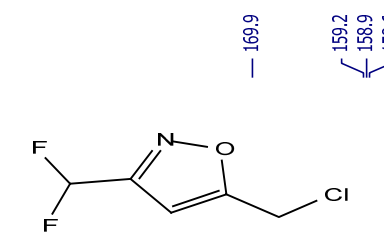

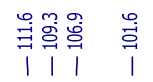

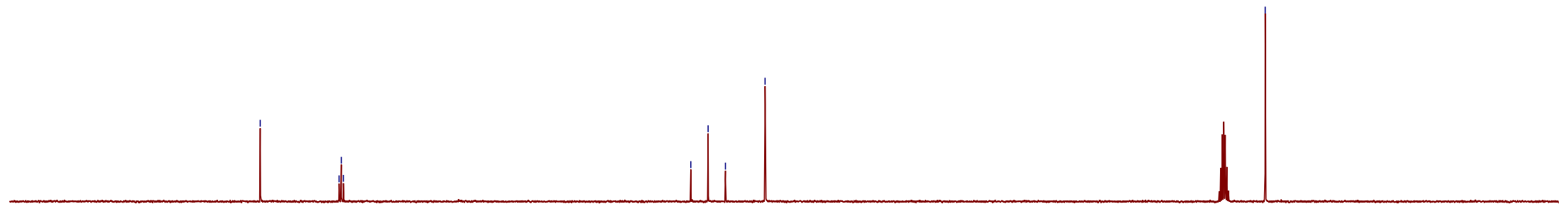

$\frac{1}{200}$

$190 \quad 180$

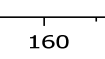

140

$130 \quad 120$

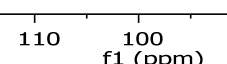

f1 100 
${ }^{19} \mathrm{~F}\left\{{ }^{1} \mathrm{H}\right\}$ NMR spectrum of the compound 41a.

\section{:}
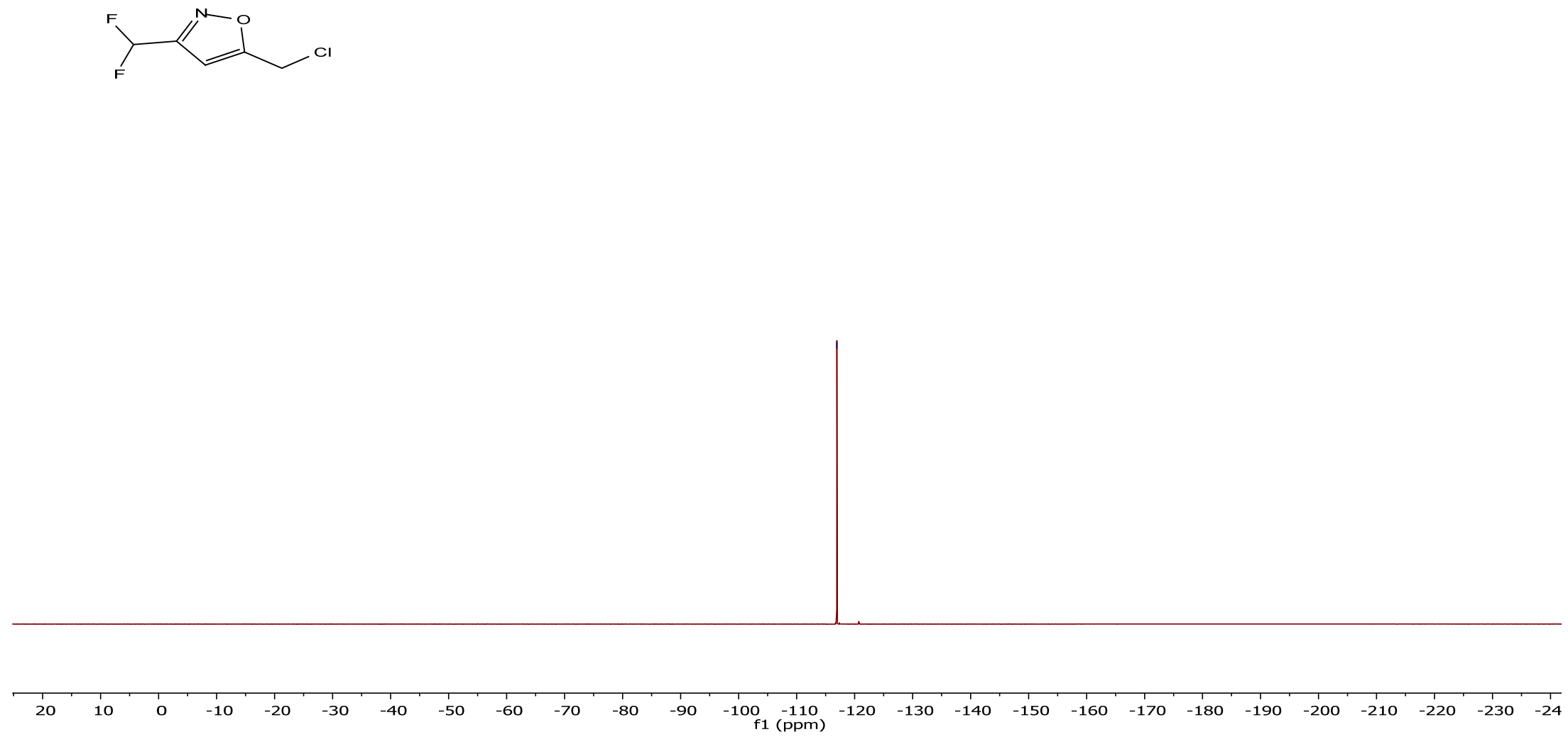
${ }^{1} \mathrm{H}$ NMR spectrum of the compound 42a.

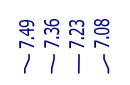

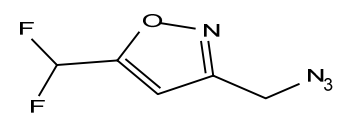

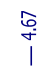
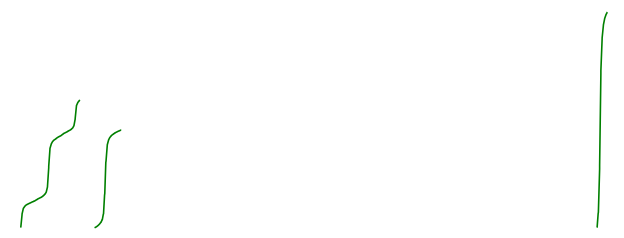

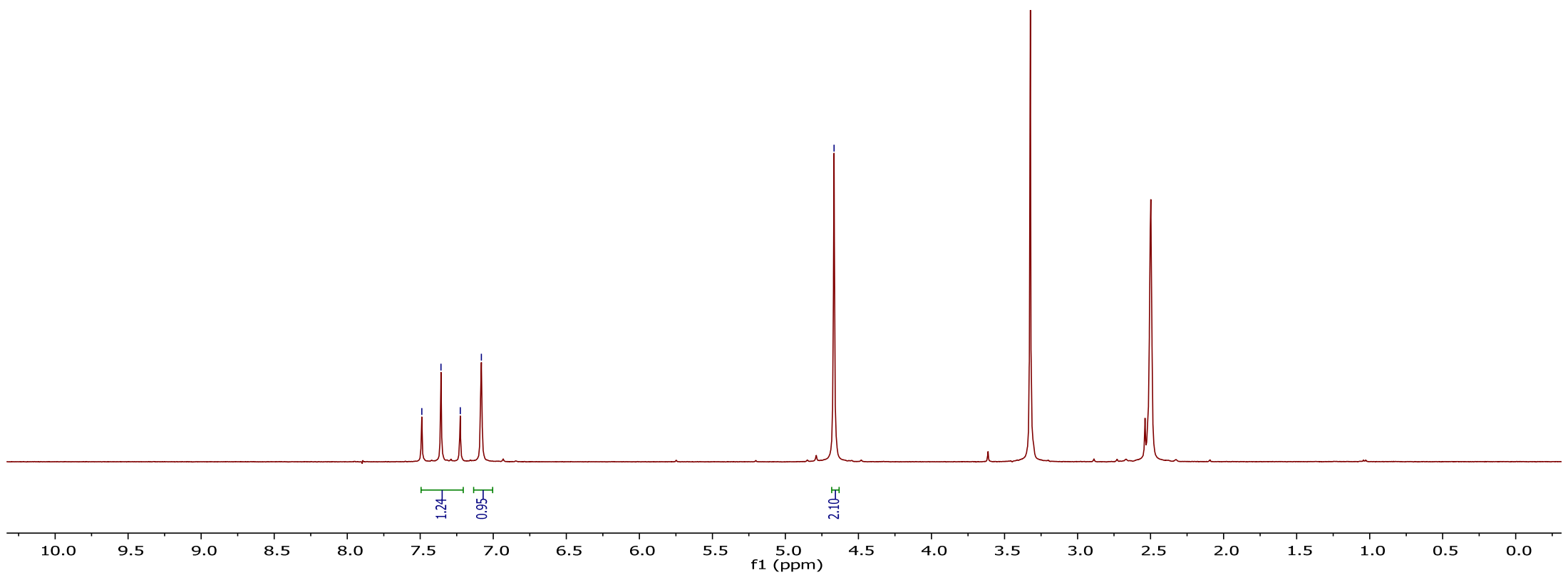

S242 
${ }^{13} \mathrm{C}\left\{{ }^{1} \mathrm{H}\right\}$ NMR spectrum of the compound 42a.

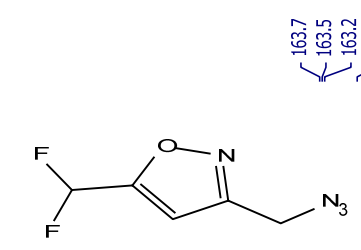

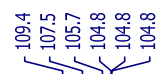

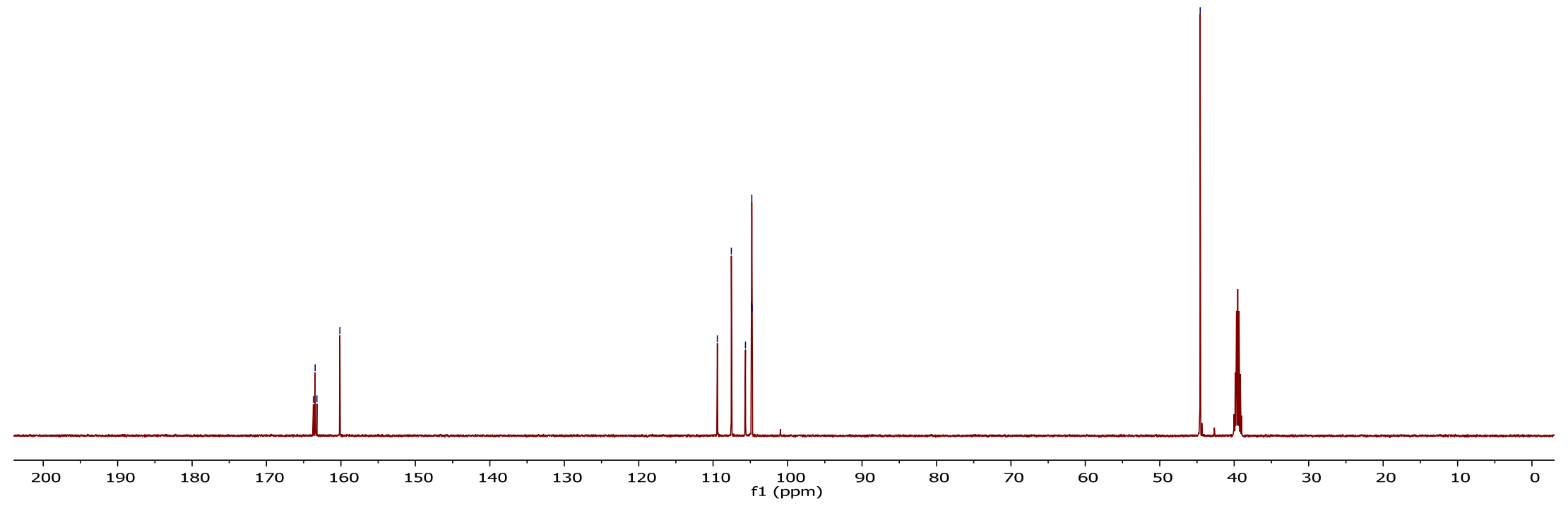


${ }^{19} \mathrm{~F}\left\{{ }^{1} \mathrm{H}\right\}$ NMR spectrum of the compound 42a.

$\stackrel{\infty}{\stackrel{\infty}{\longrightarrow}}$
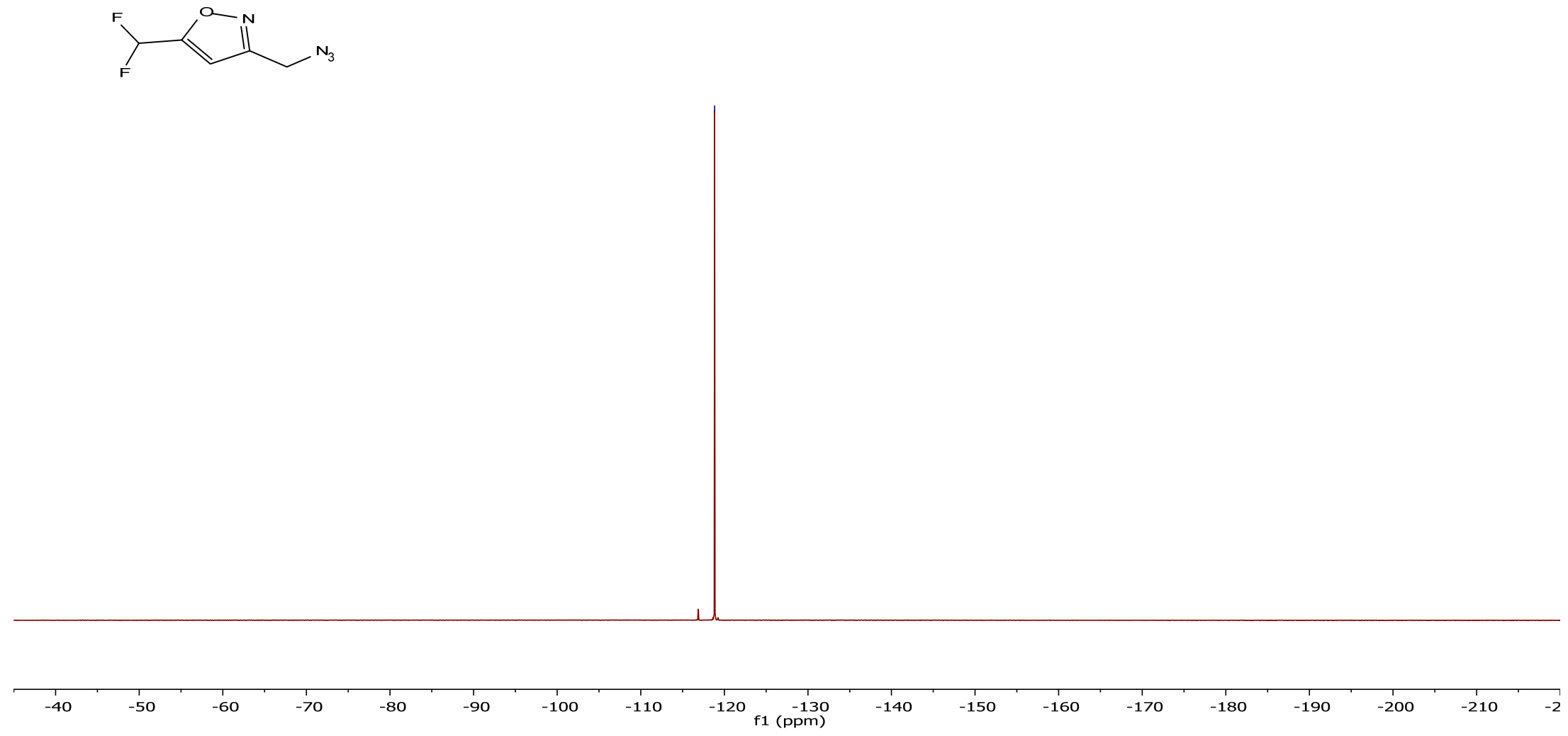
${ }^{1} \mathrm{H}$ NMR spectrum of the compound $\mathbf{4 2 b}$.

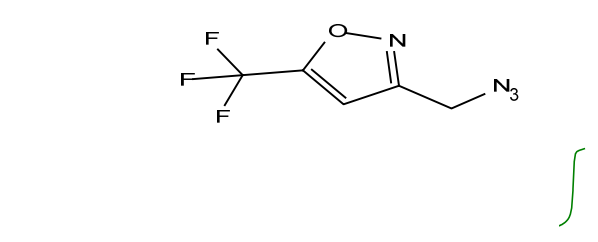

$\stackrel{\text { 兌 }}{i}$

竞

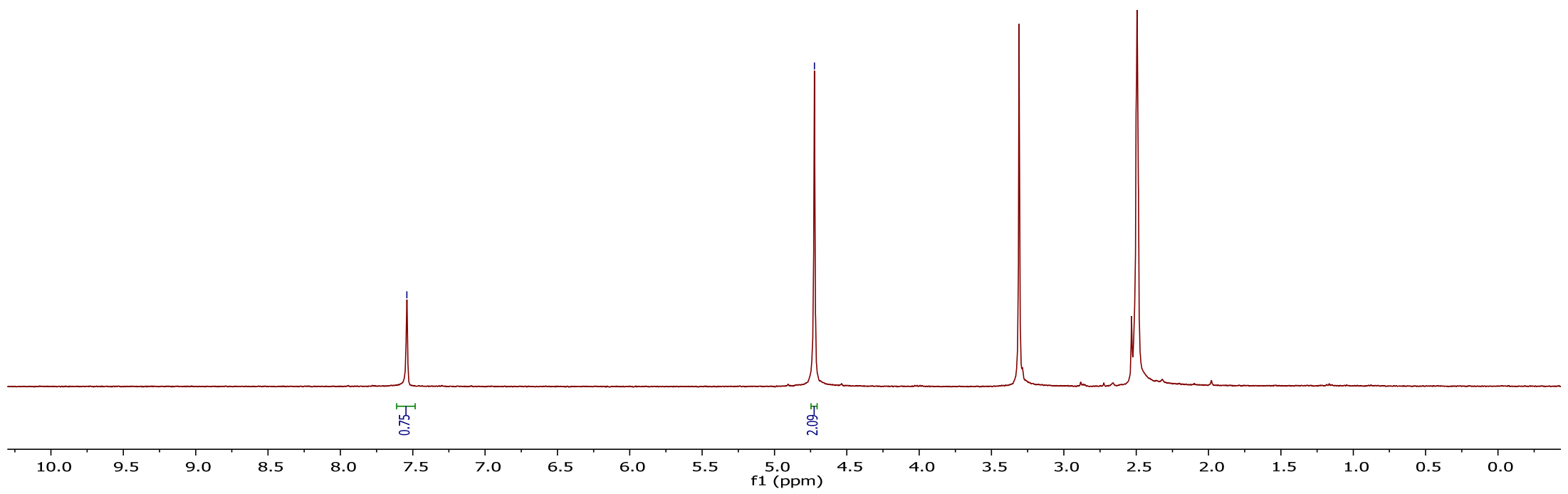

S245 
${ }^{13} \mathrm{C}\left\{{ }^{1} \mathrm{H}\right\}$ NMR spectrum of the compound $\mathbf{4 2 b}$.

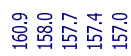

군

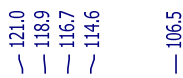
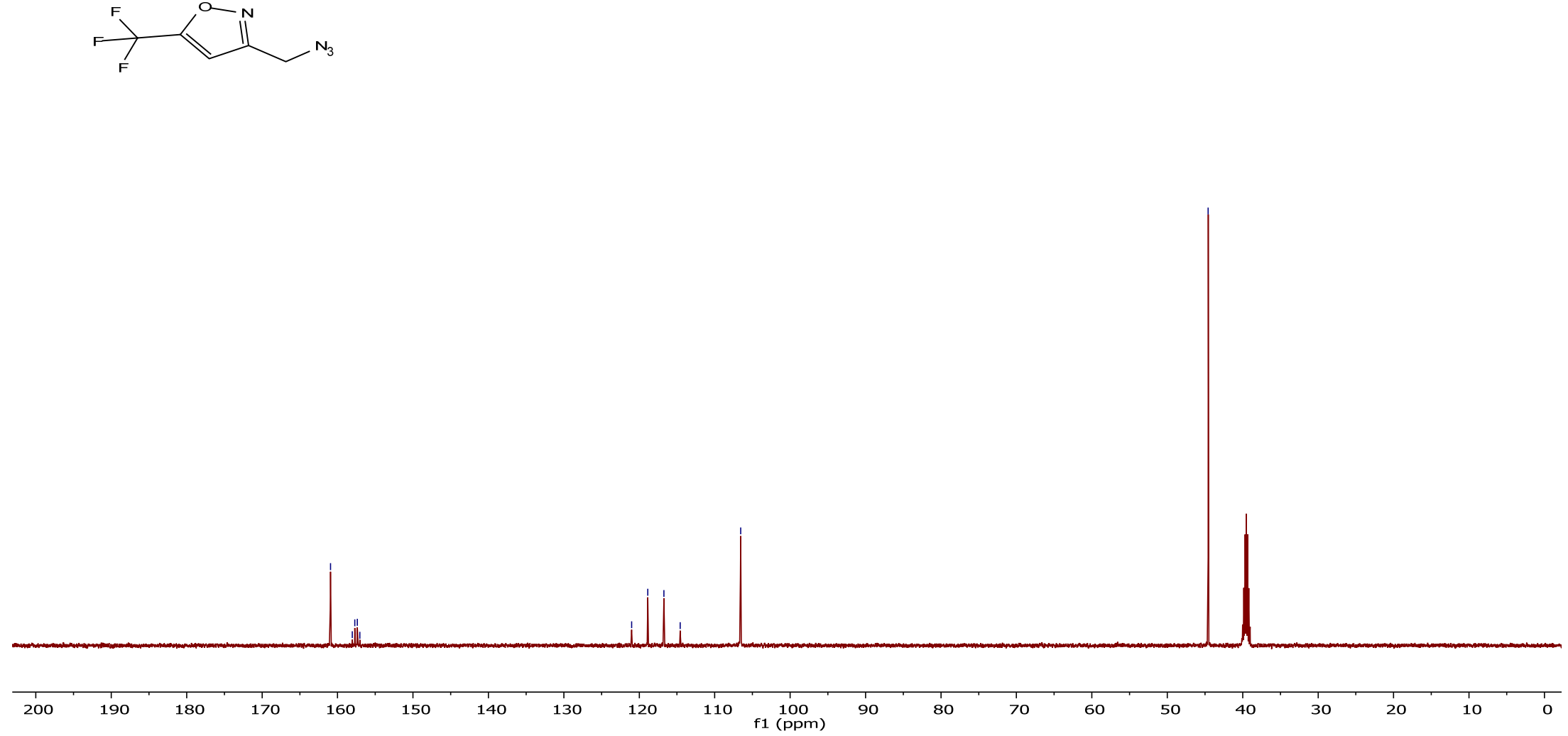
${ }^{19} \mathrm{~F}\left\{{ }^{1} \mathrm{H}\right\}$ NMR spectrum of the compound $\mathbf{4 2 b}$.

蒂
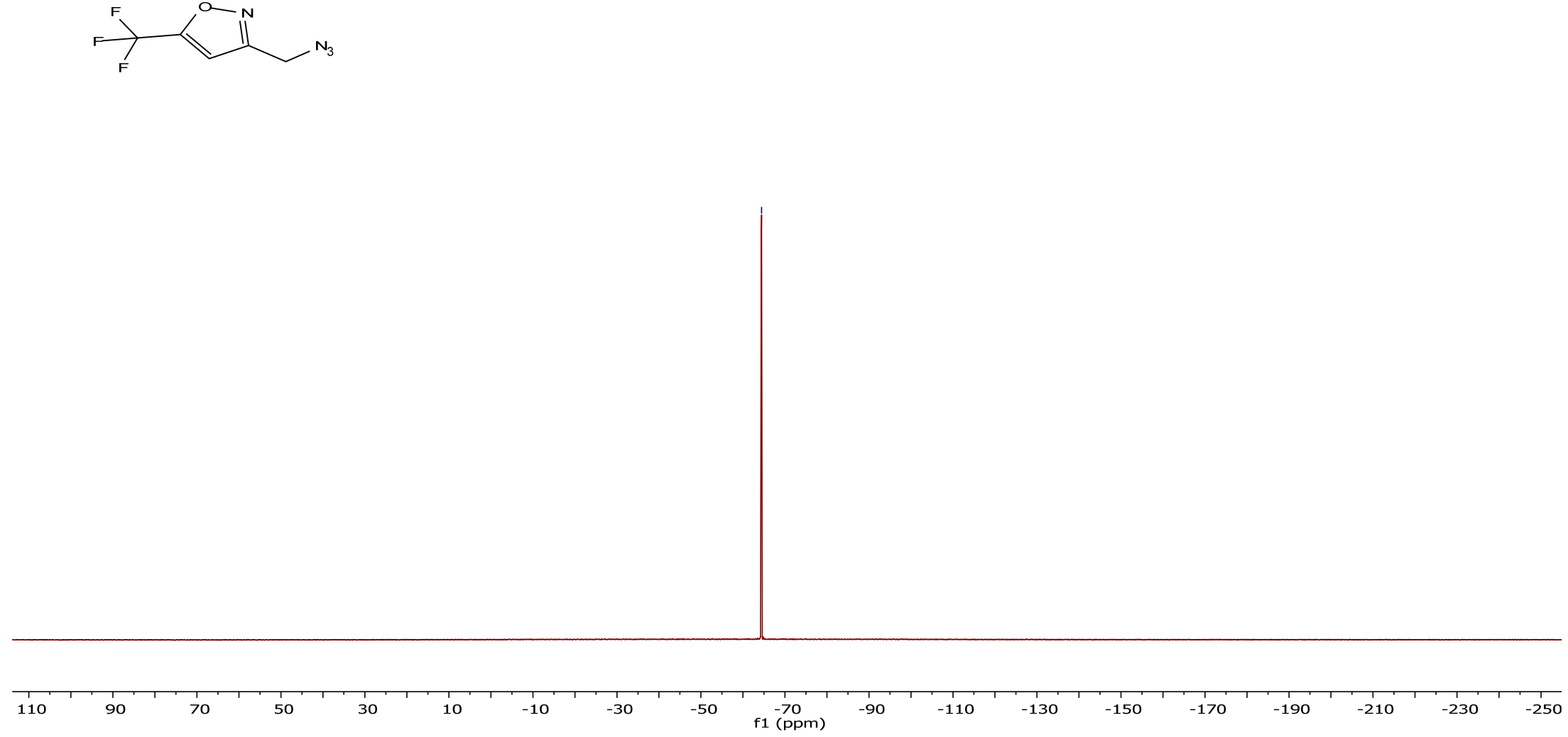
${ }^{1} \mathrm{H}$ NMR spectrum of the compound 42d.

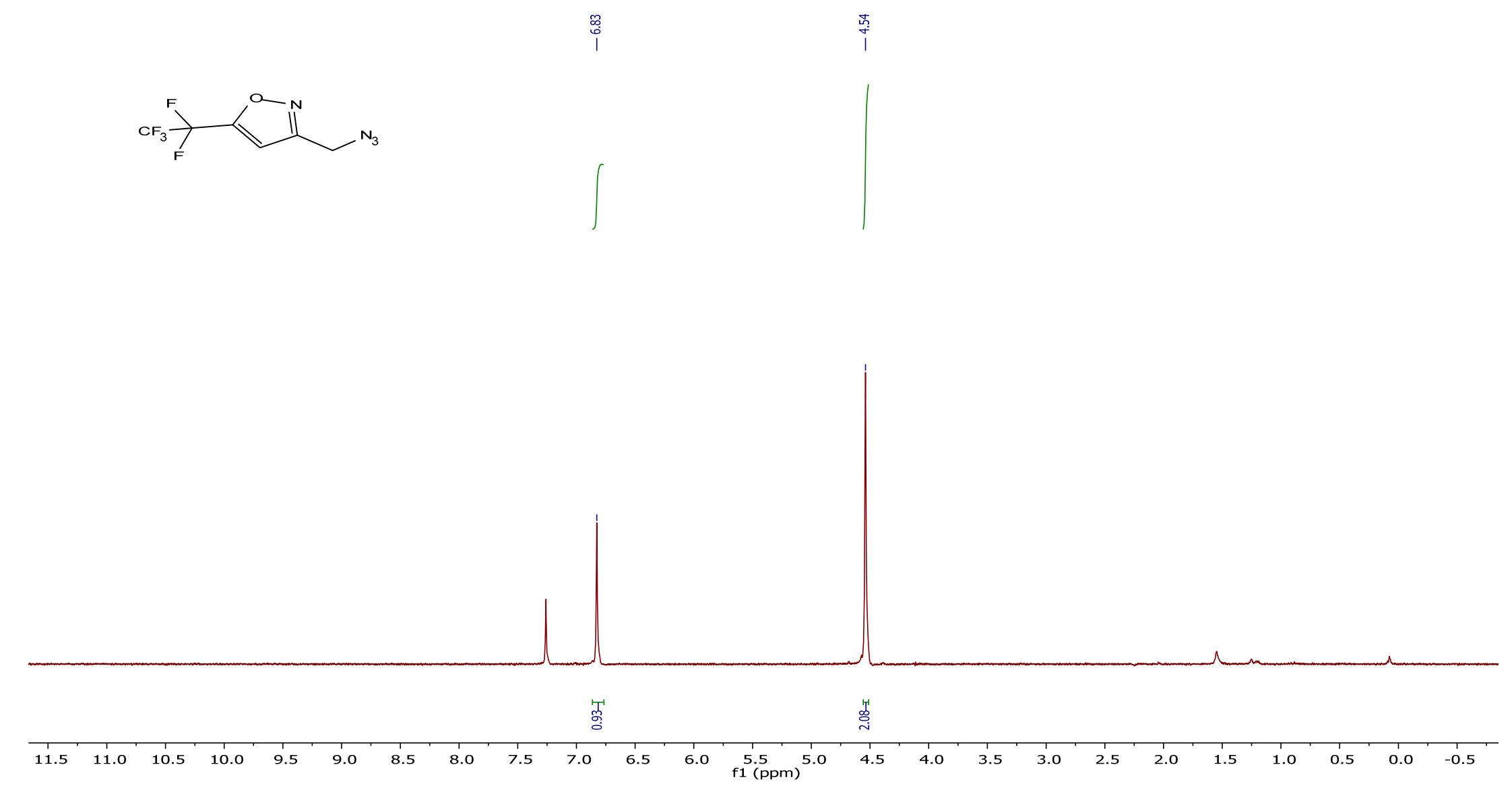


${ }^{13} \mathrm{C}\left\{{ }^{1} \mathrm{H}\right\}$ NMR spectrum of the compound 42d.

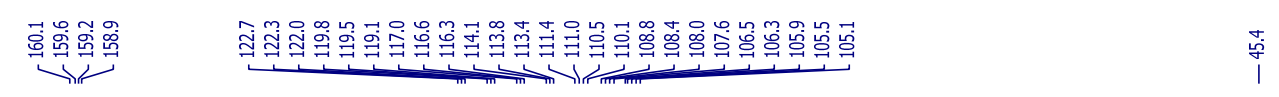

$\overbrace{\mathrm{F}}^{\mathrm{N}} \|_{\mathrm{N}_{3}}^{\mathrm{F}}$

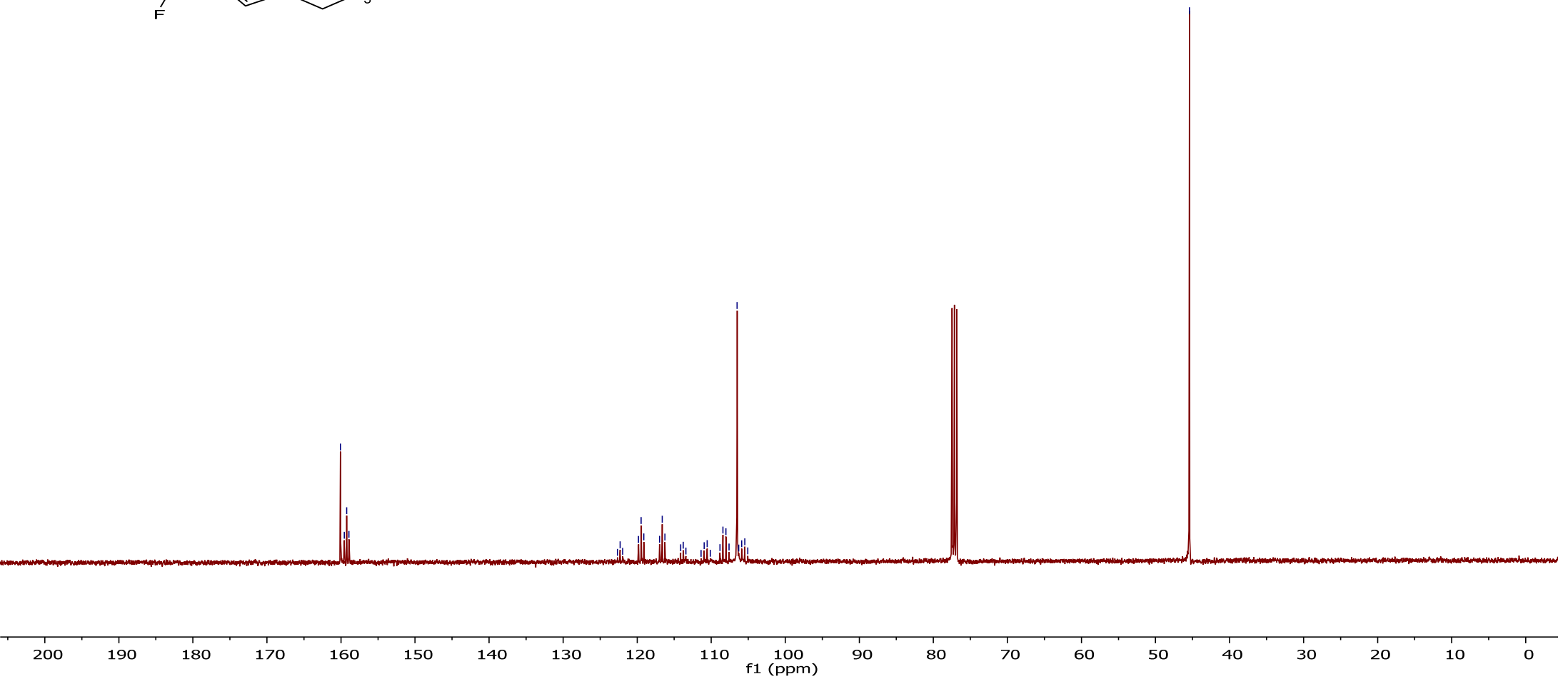


${ }^{19} \mathrm{~F}\left\{{ }^{1} \mathrm{H}\right\}$ NMR spectrum of the compound 42d.
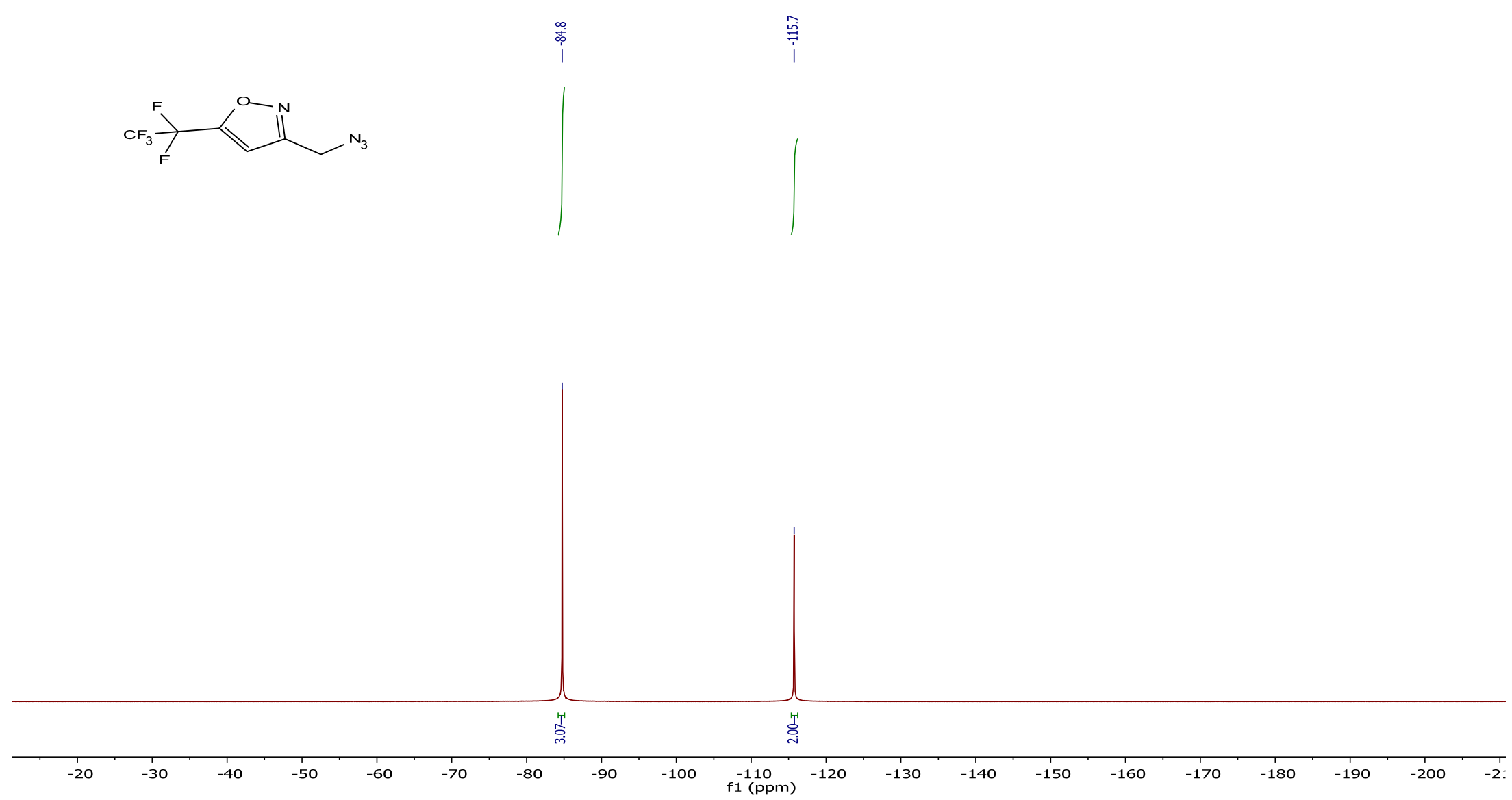
${ }^{1} \mathrm{H}$ NMR spectrum of the compound 38a.

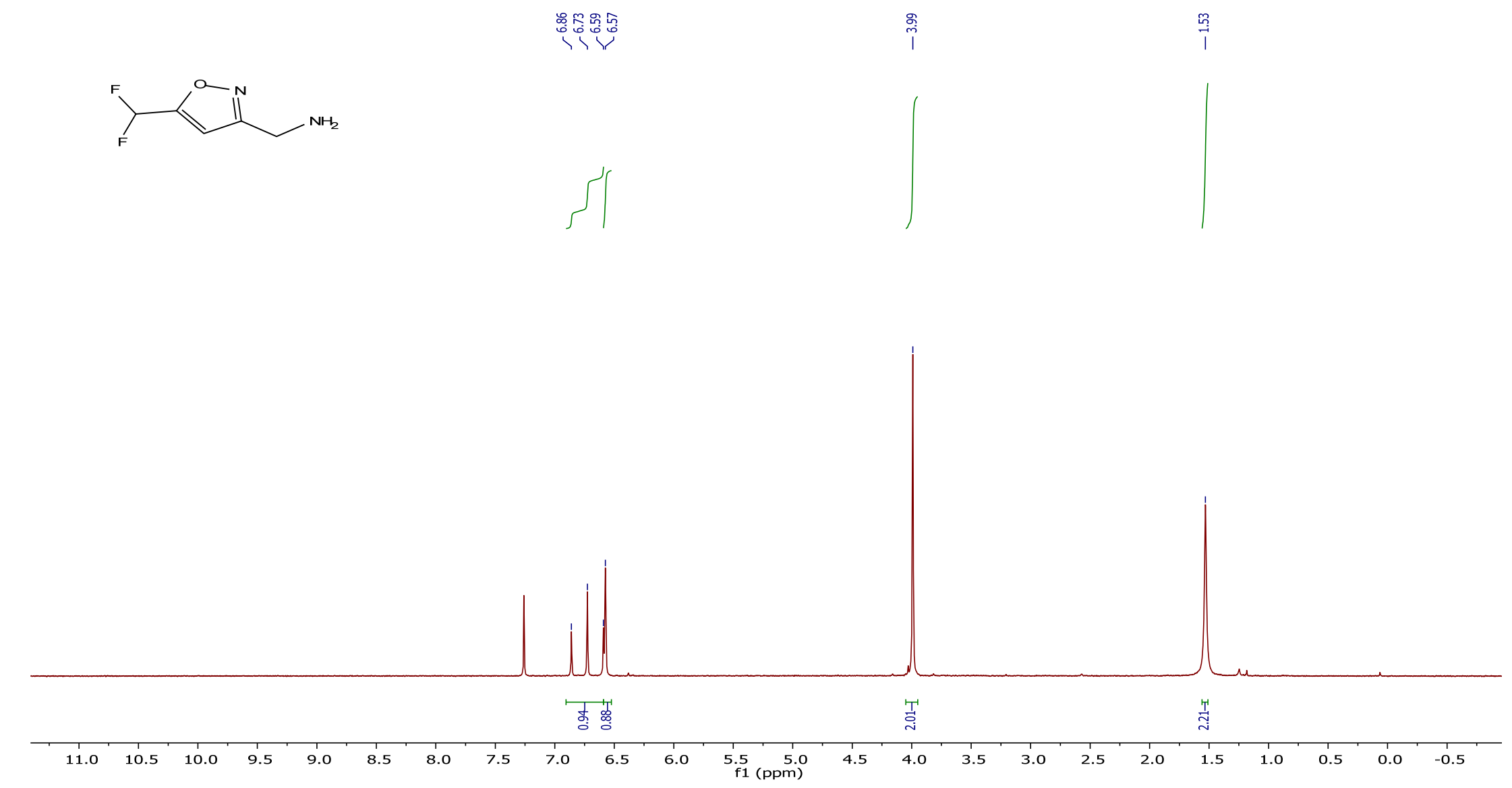


${ }^{13} \mathrm{C}\left\{{ }^{1} \mathrm{H}\right\}$ NMR spectrum of the compound 38a.
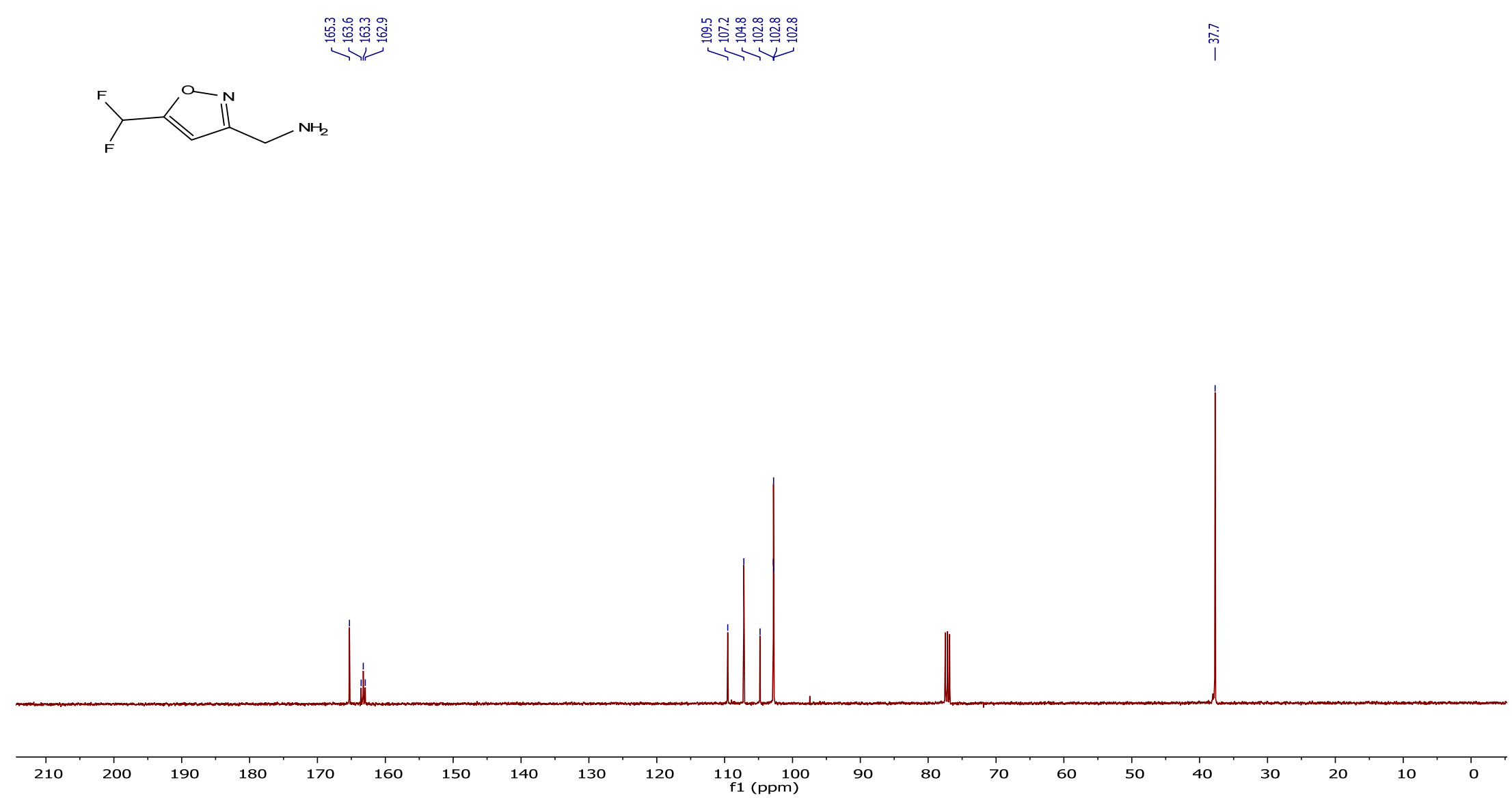
${ }^{19} \mathrm{~F}\left\{{ }^{1} \mathrm{H}\right\}$ NMR spectrum of the compound 38a.

$\stackrel{\stackrel{n}{\infty}}{\underset{i}{i}}$
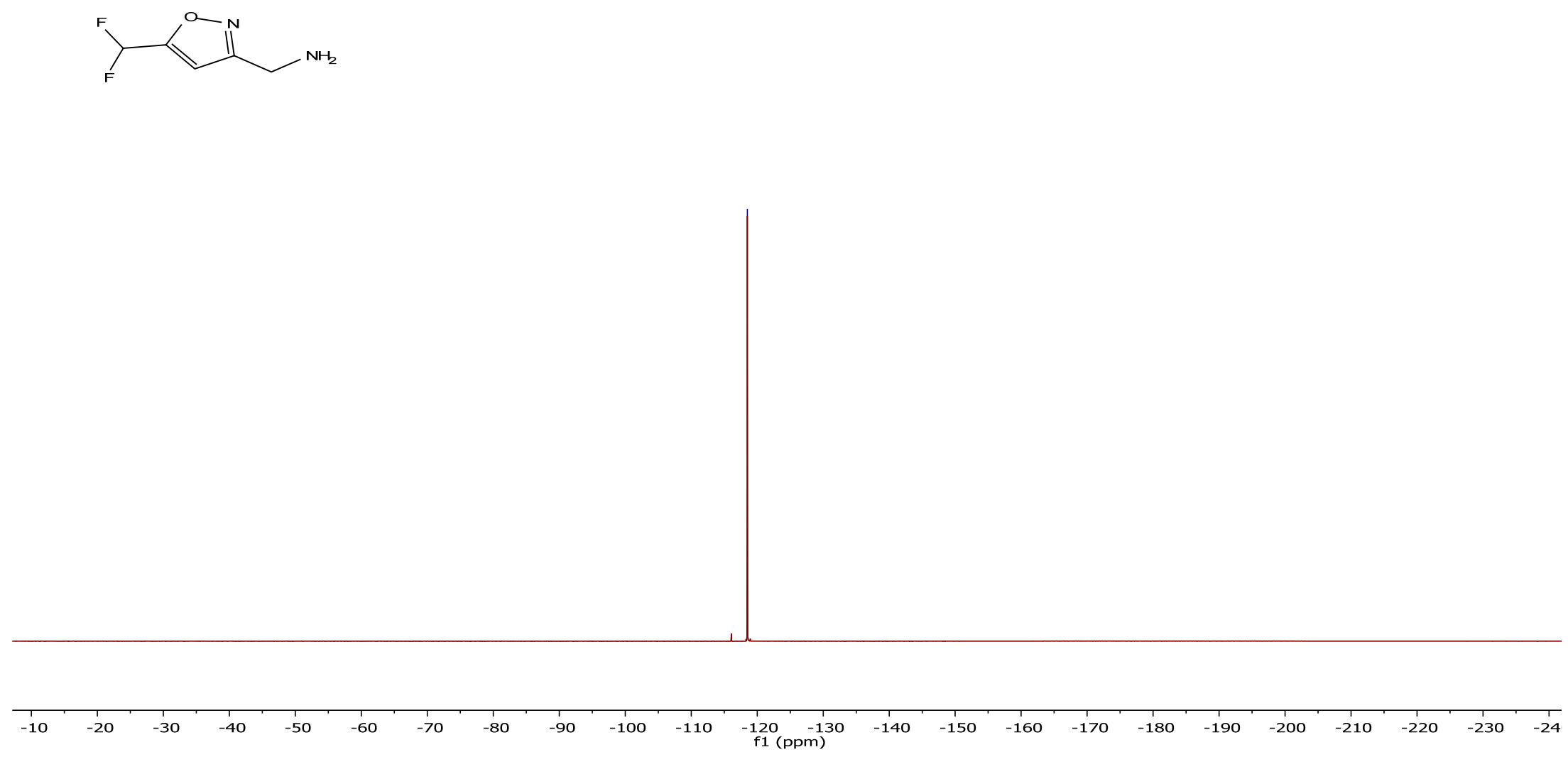
${ }^{1} \mathrm{H}$ NMR spectrum of the compound $\mathbf{3 8 b}$.

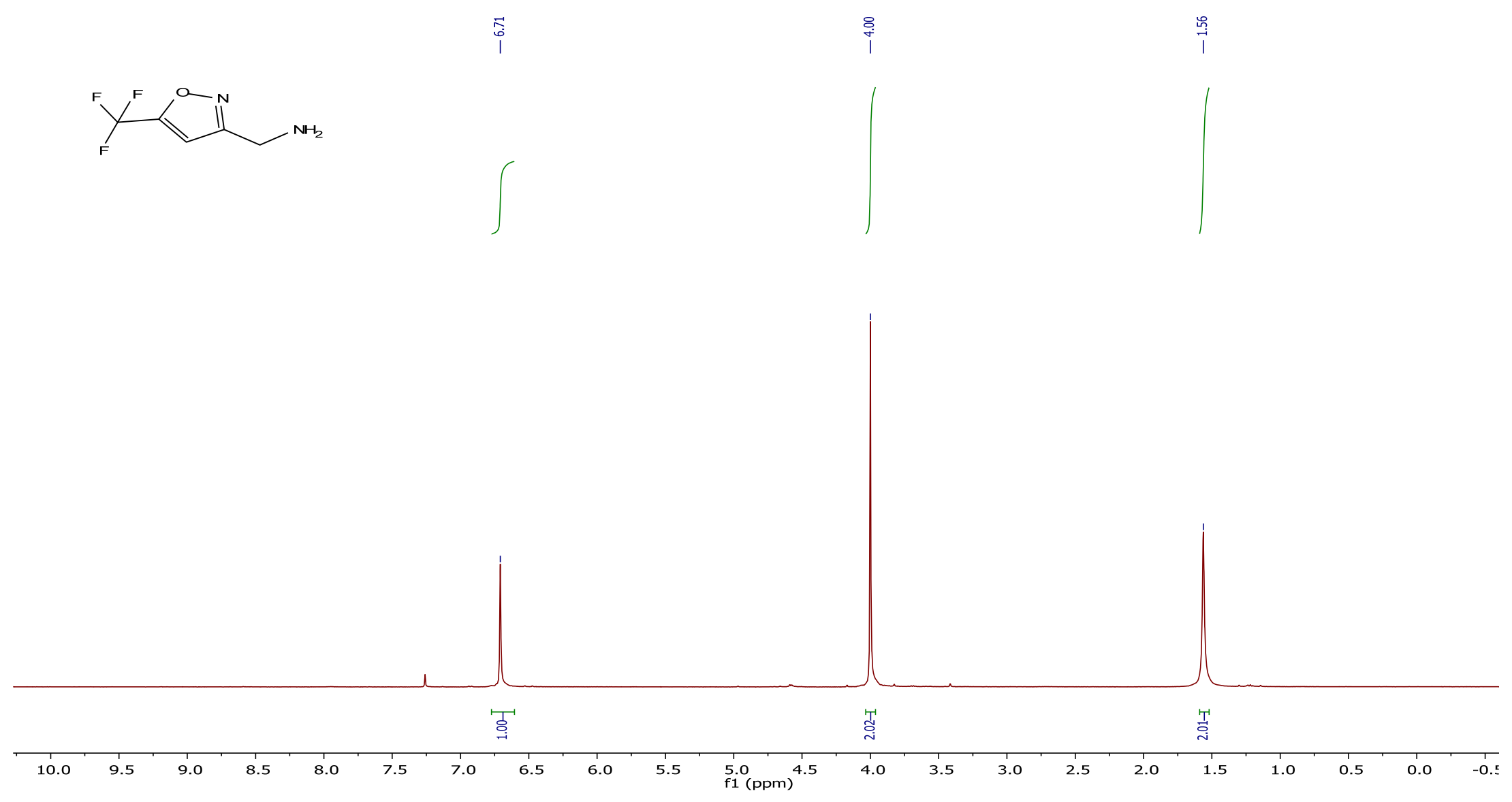


${ }^{13} \mathrm{C}\left\{{ }^{1} \mathrm{H}\right\}$ NMR spectrum of the compound $\mathbf{3 8 b}$.

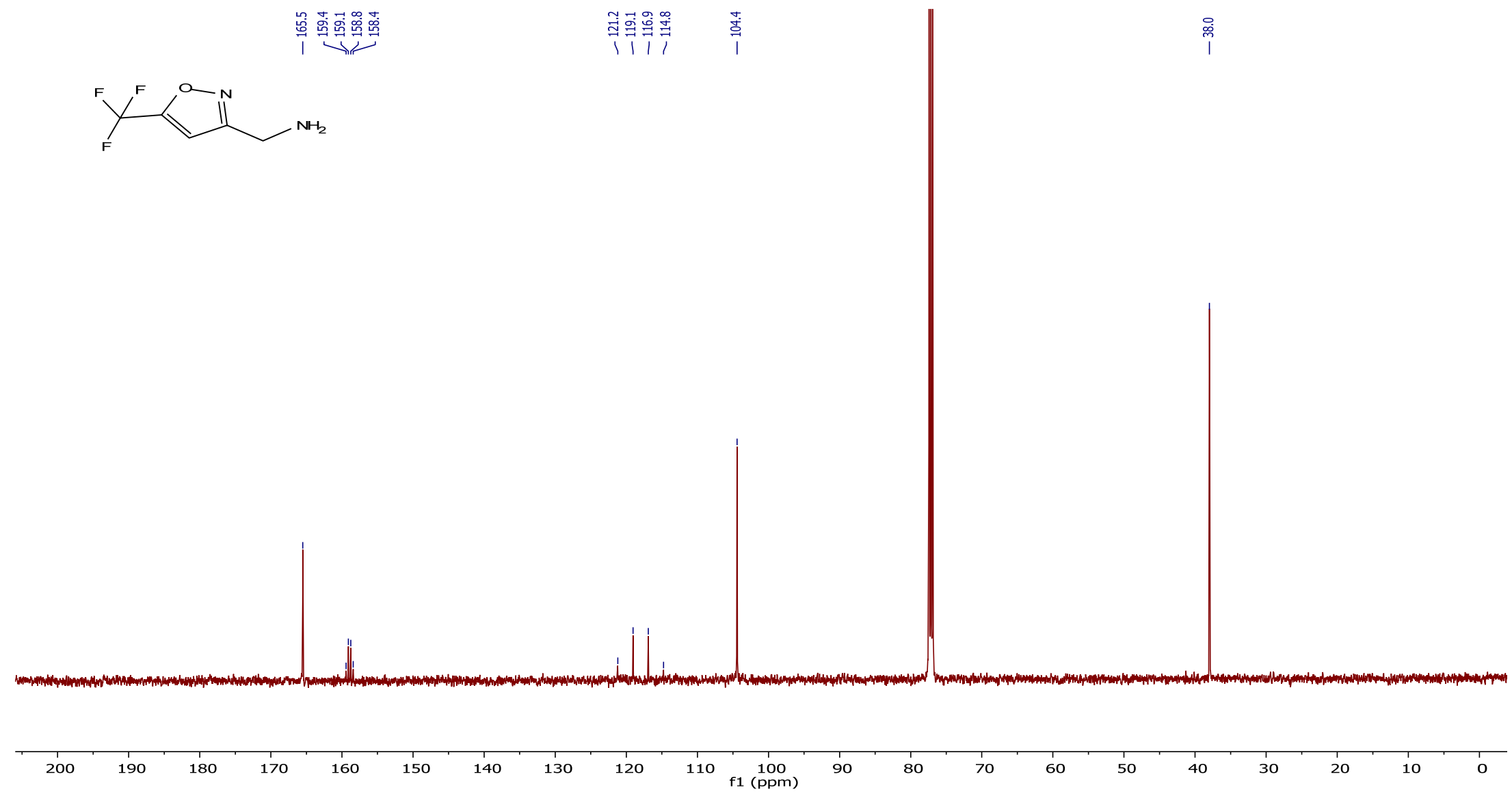


${ }^{19} \mathrm{~F}\left\{{ }^{1} \mathrm{H}\right\}$ NMR spectrum of the compound 38b.

旁

$Y_{F}^{F} \overbrace{}^{N+H}$

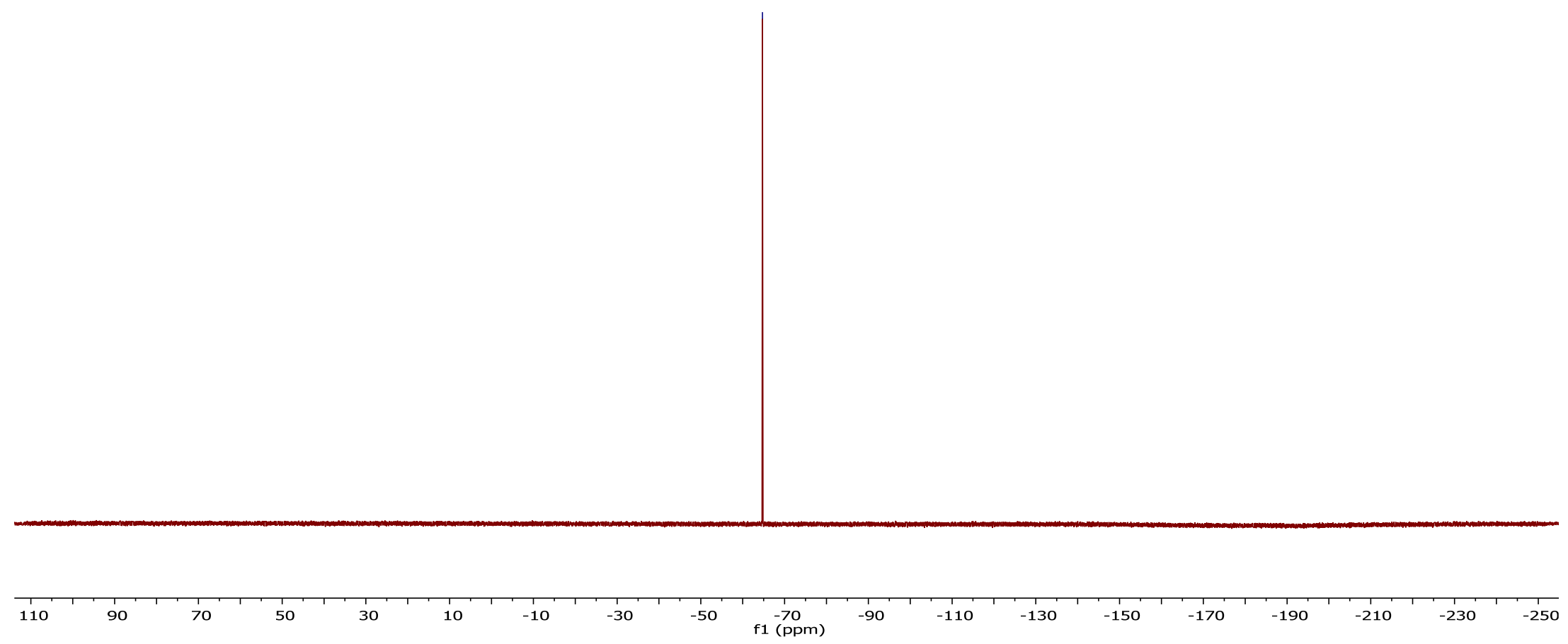


${ }^{1} \mathrm{H}$ NMR spectrum of the compound 38d.

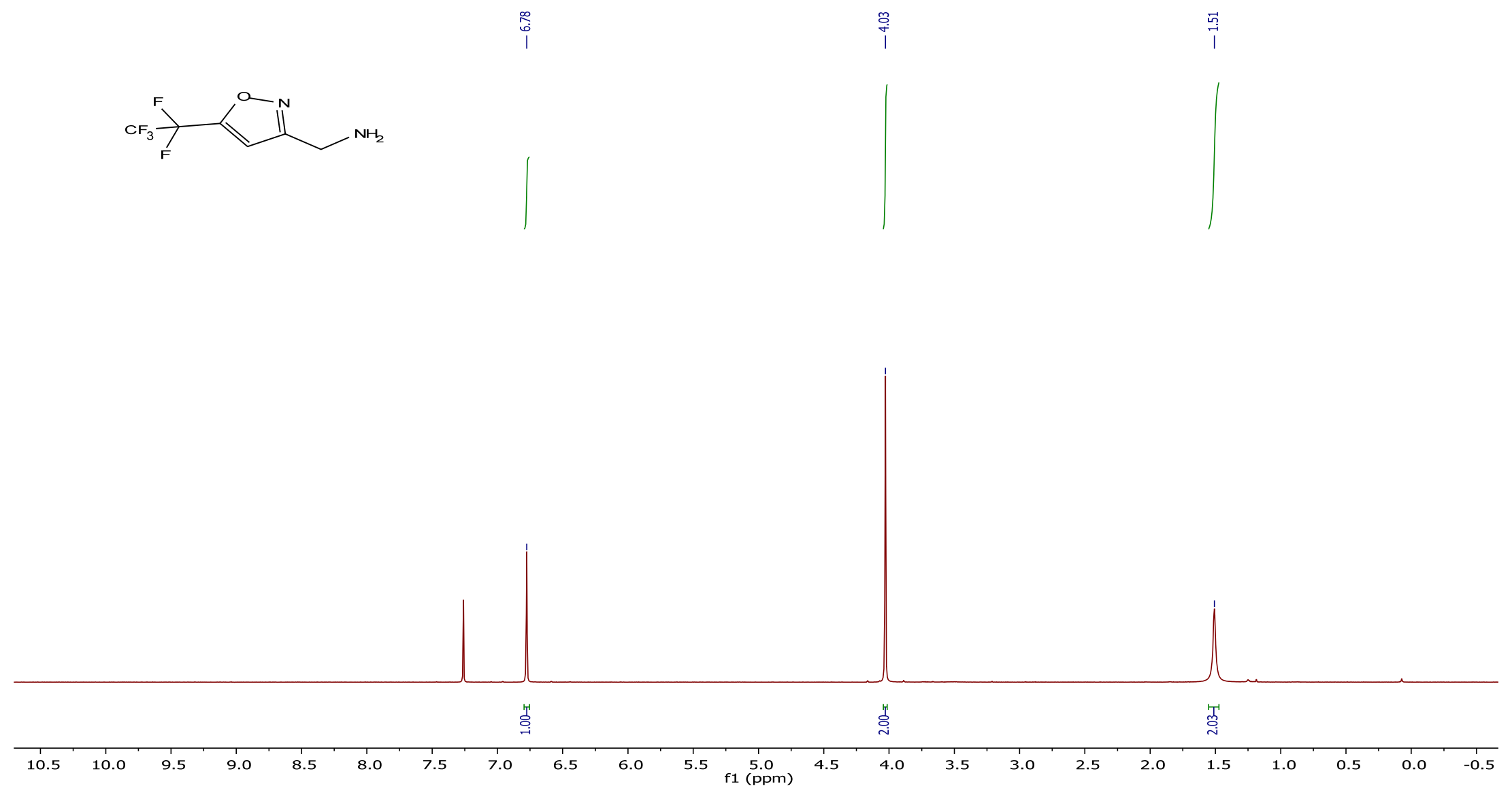


${ }^{13} \mathrm{C}\left\{{ }^{1} \mathrm{H}\right\}$ NMR spectrum of the compound 38d.

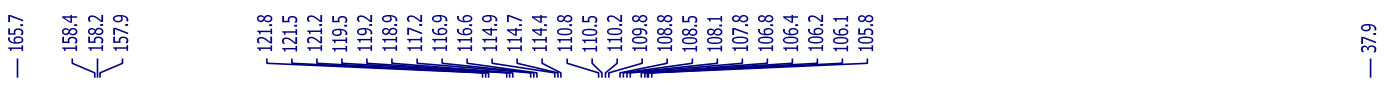

$\overbrace{\mathrm{F}}^{\mathrm{N}} \mathrm{NH}_{2}^{\mathrm{F}}$

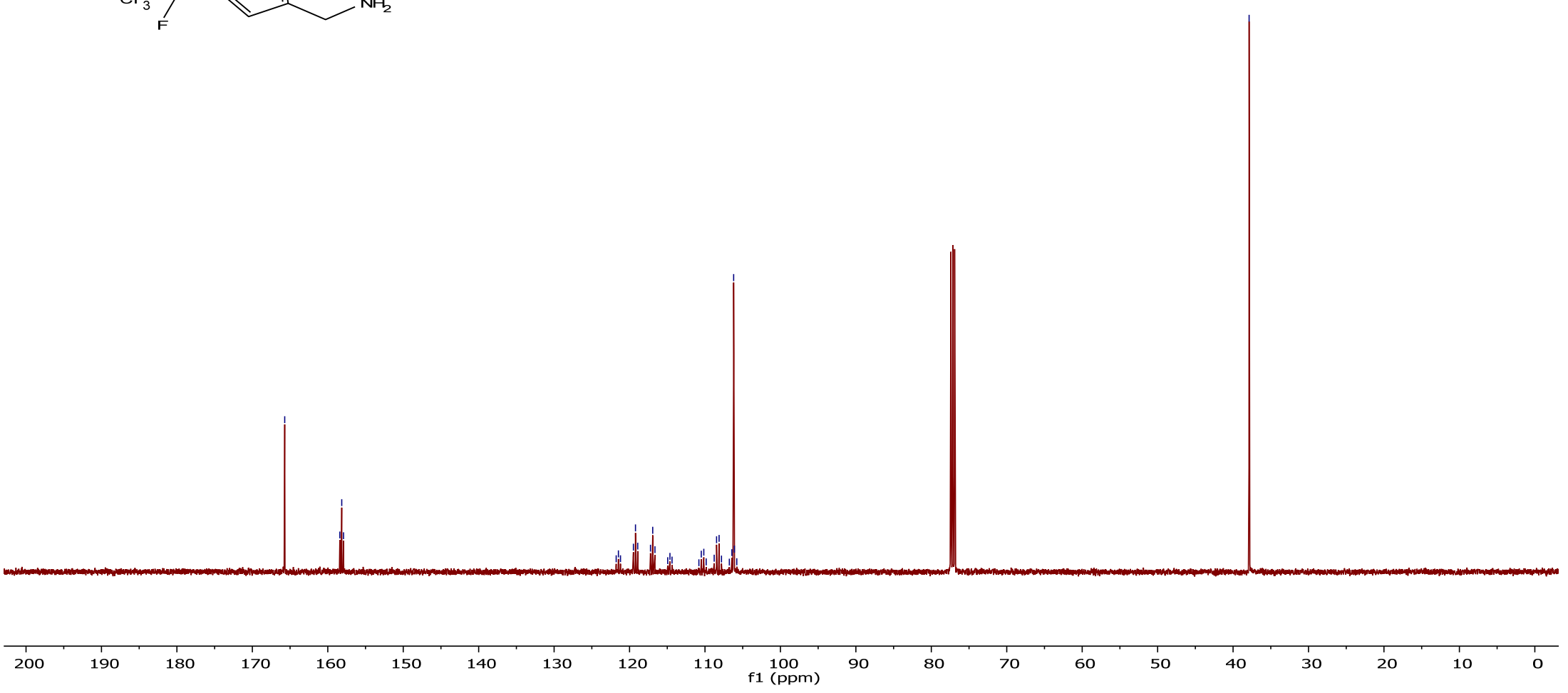


${ }^{19} \mathrm{~F}\left\{{ }^{1} \mathrm{H}\right\}$ NMR spectrum of the compound 38d.
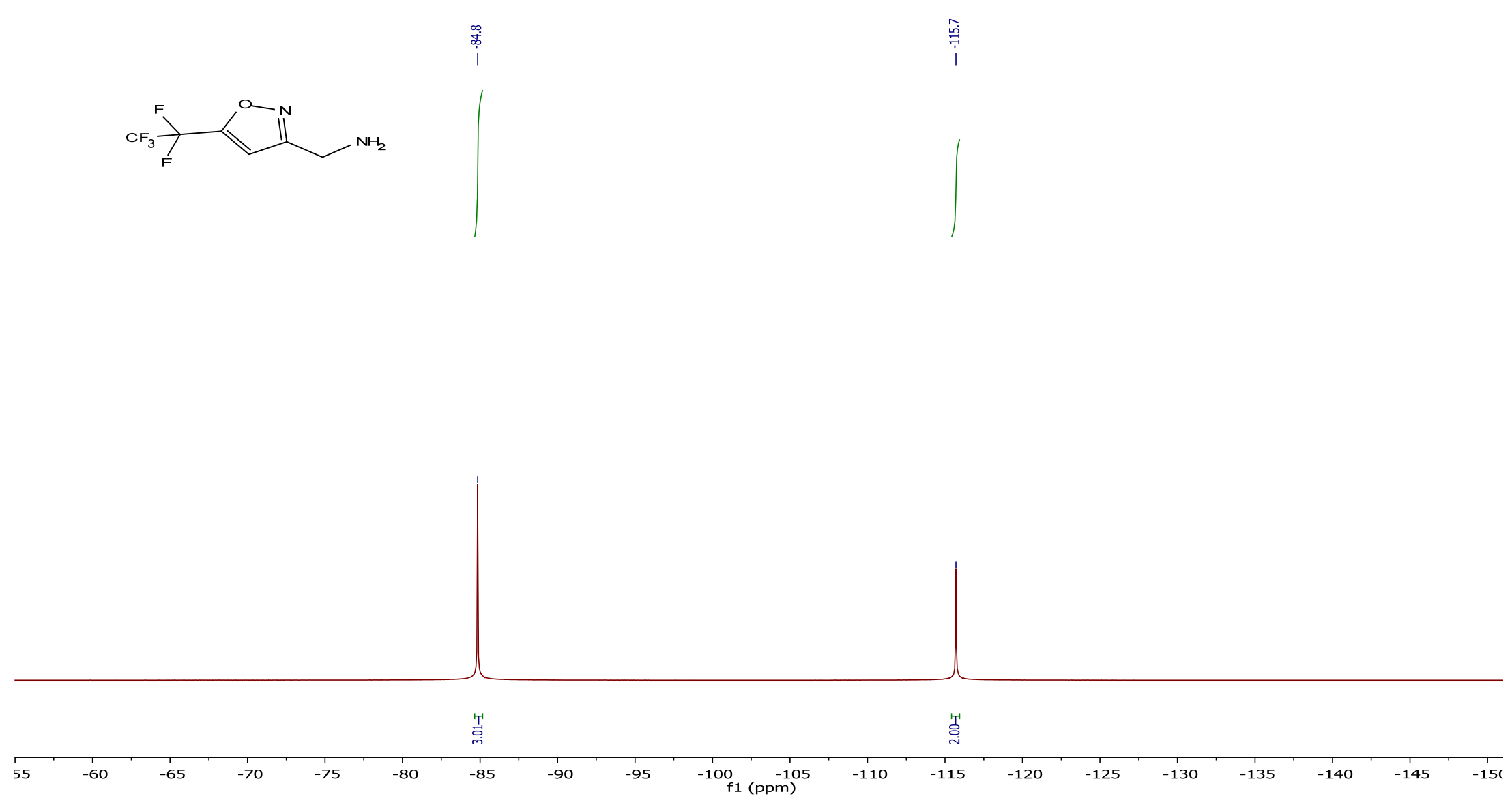
${ }^{1} \mathrm{H}$ NMR spectrum of the compound 39a.
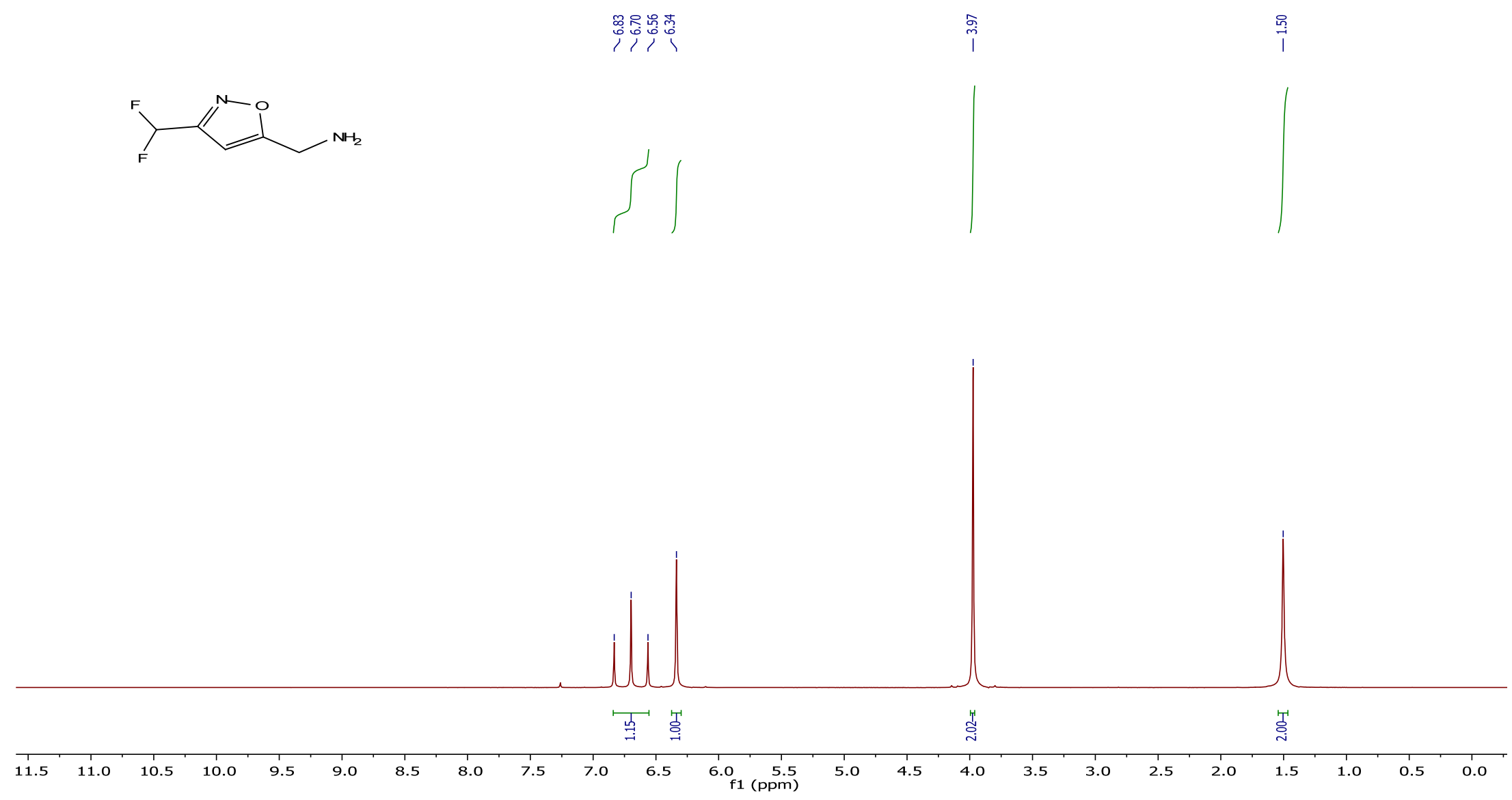
${ }^{13} \mathrm{C}\left\{{ }^{1} \mathrm{H}\right\}$ NMR spectrum of the compound 39a.

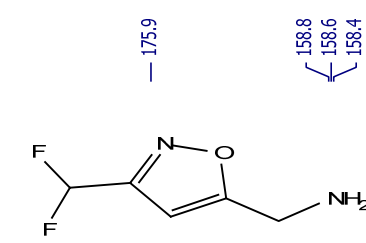

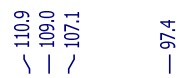

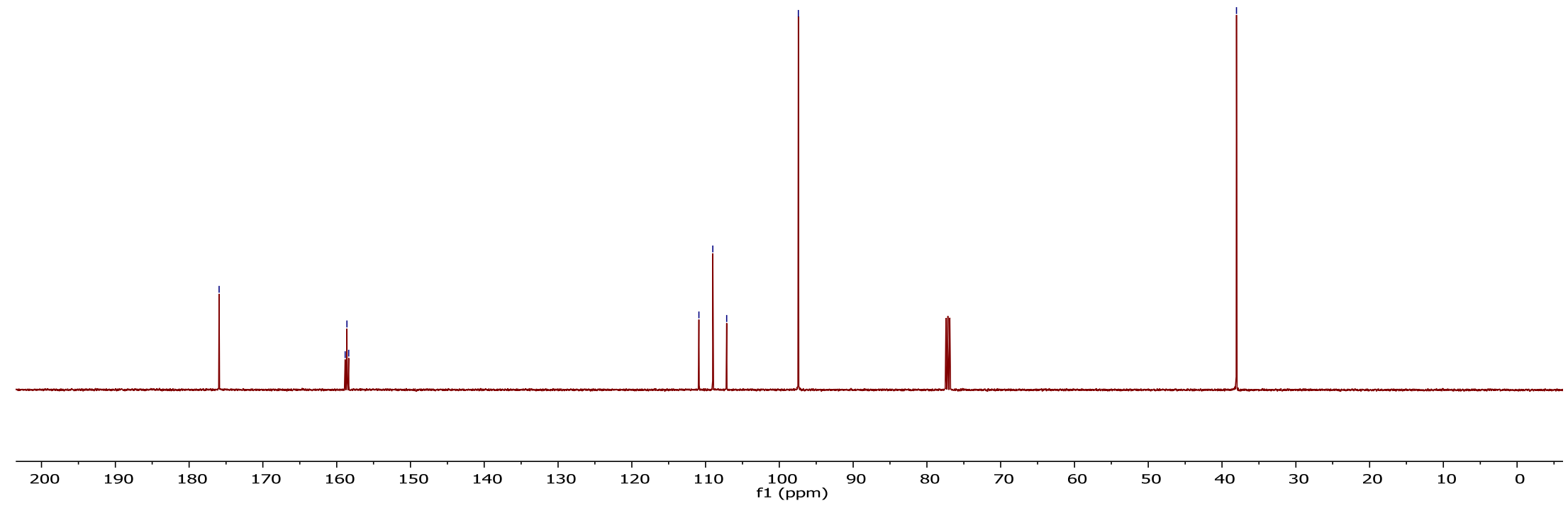


${ }^{19} \mathrm{~F}\left\{{ }^{1} \mathrm{H}\right\}$ NMR spectrum of the compound 39a.

i़
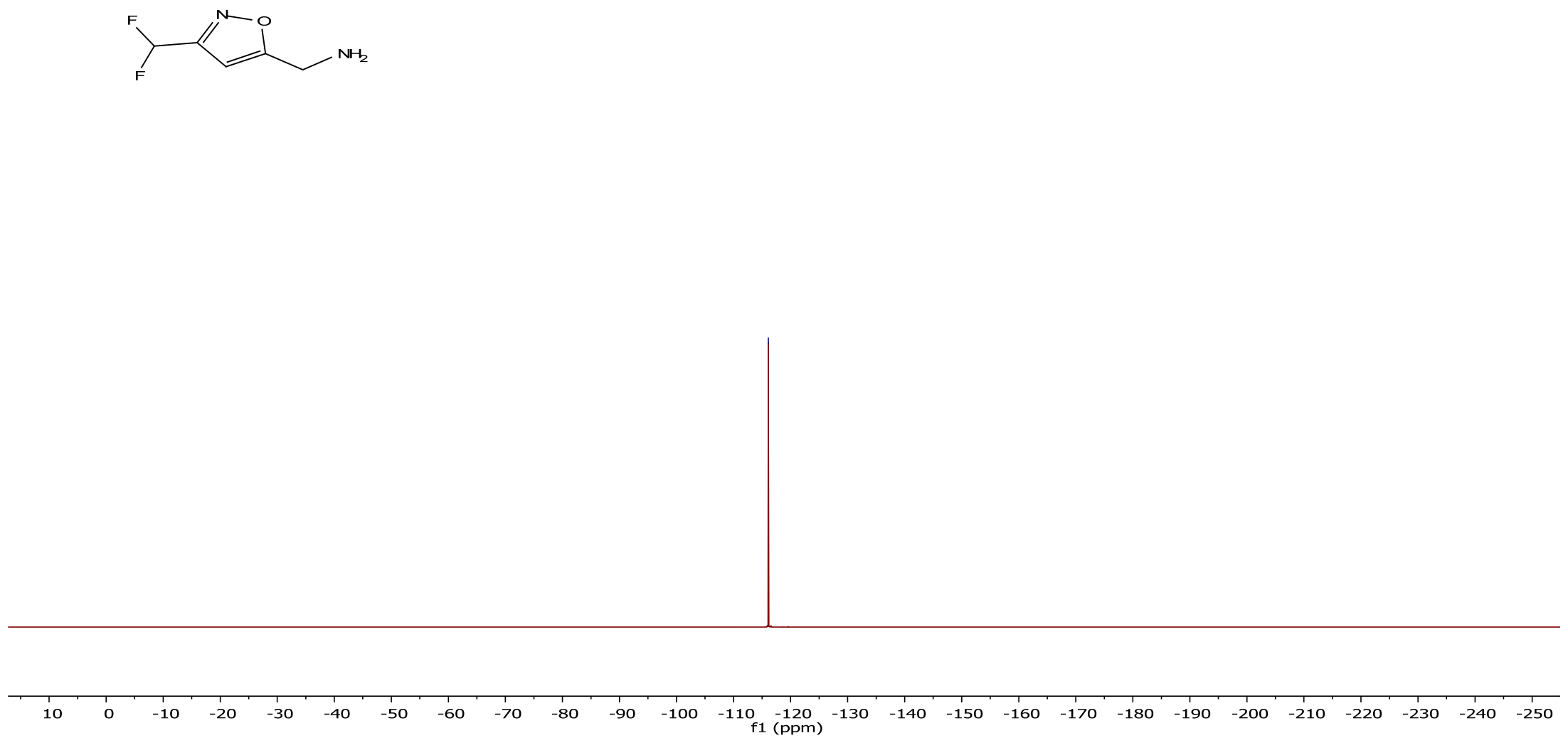
${ }^{1} \mathrm{H}$ NMR spectrum of the compound 44a.
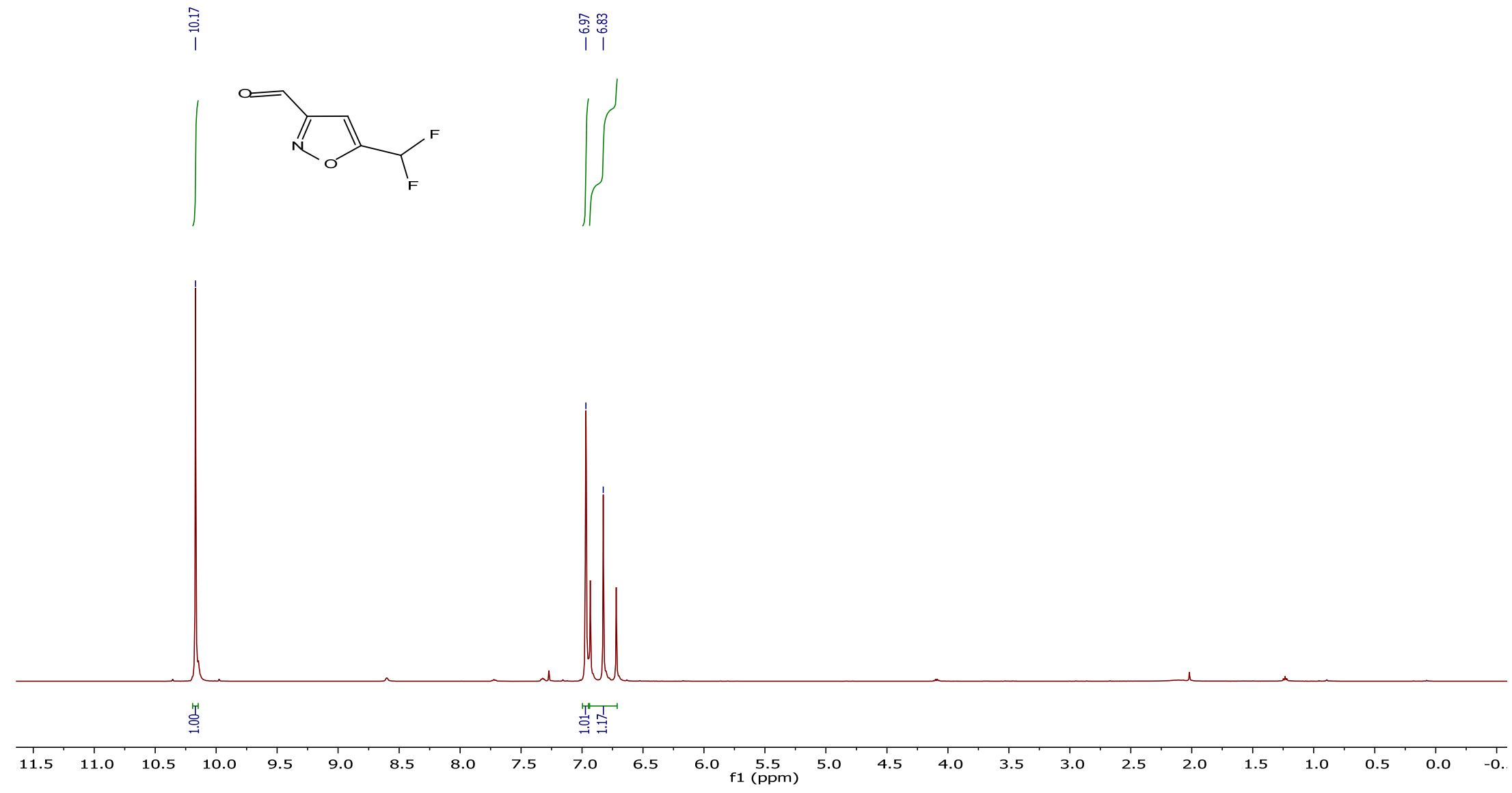
${ }^{13} \mathrm{C}\left\{{ }^{1} \mathrm{H}\right\}$ NMR spectrum of the compound $44 \mathrm{a}$.

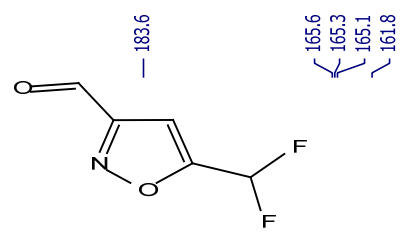

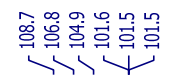

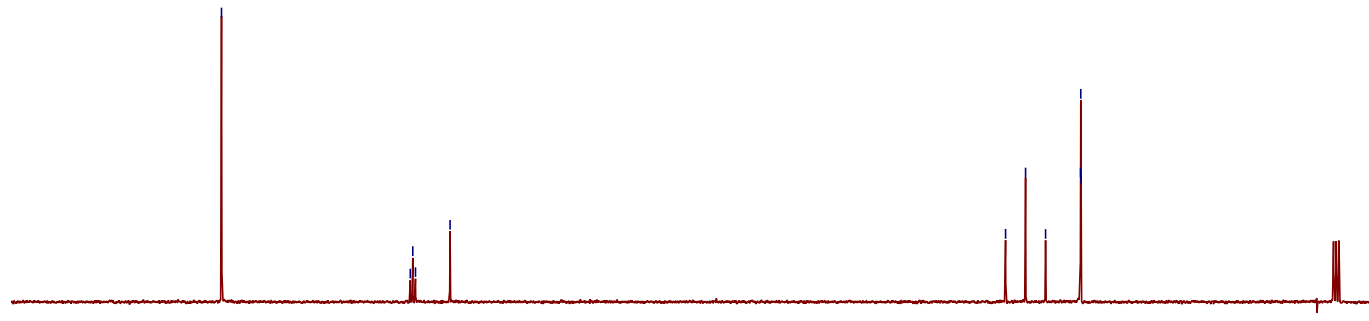

200

190

180

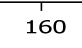


${ }^{19}$ F NMR spectrum of the compound 44a.

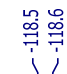
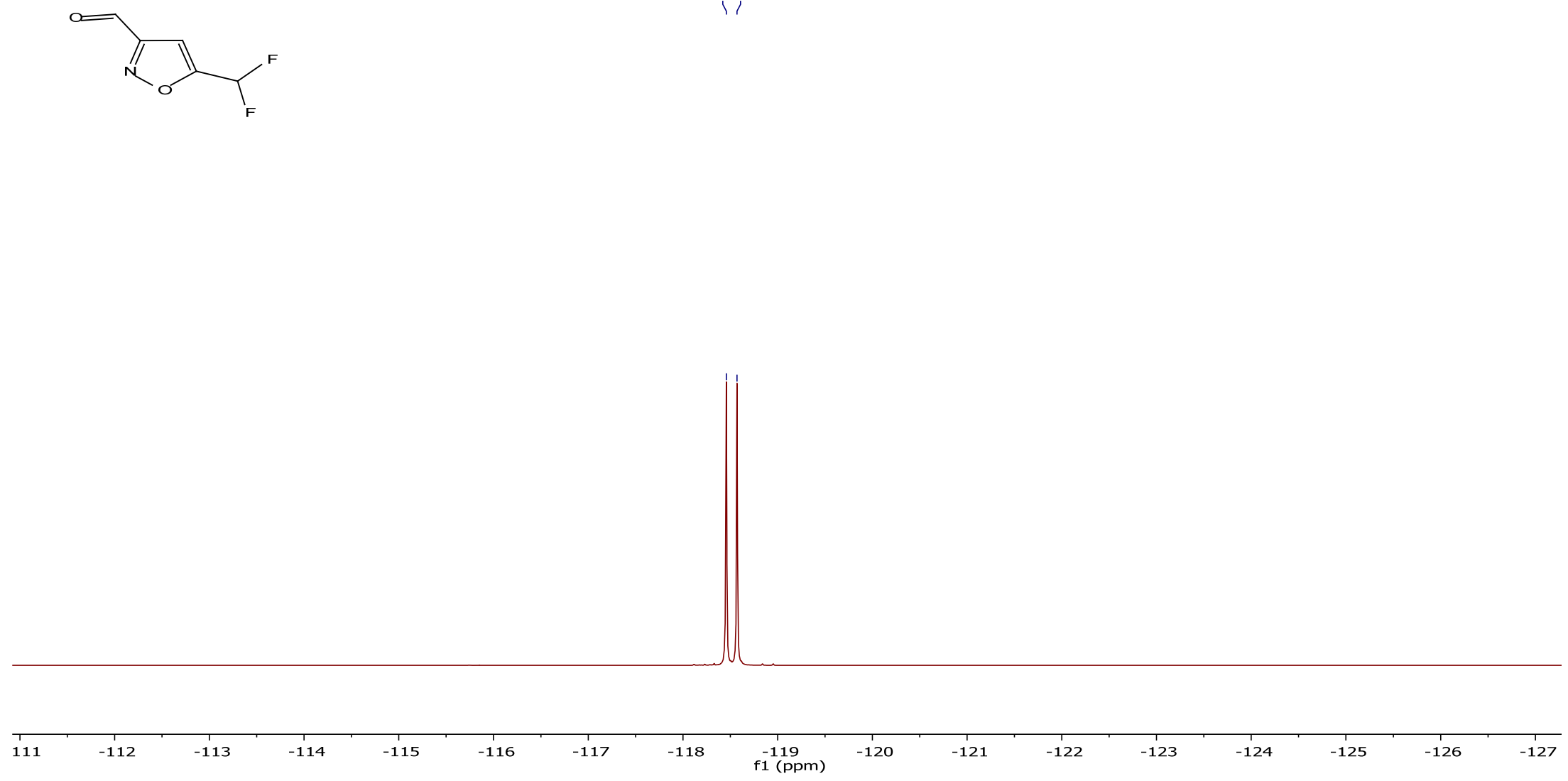
${ }^{1} \mathrm{H}$ NMR spectrum of the compound 45a.

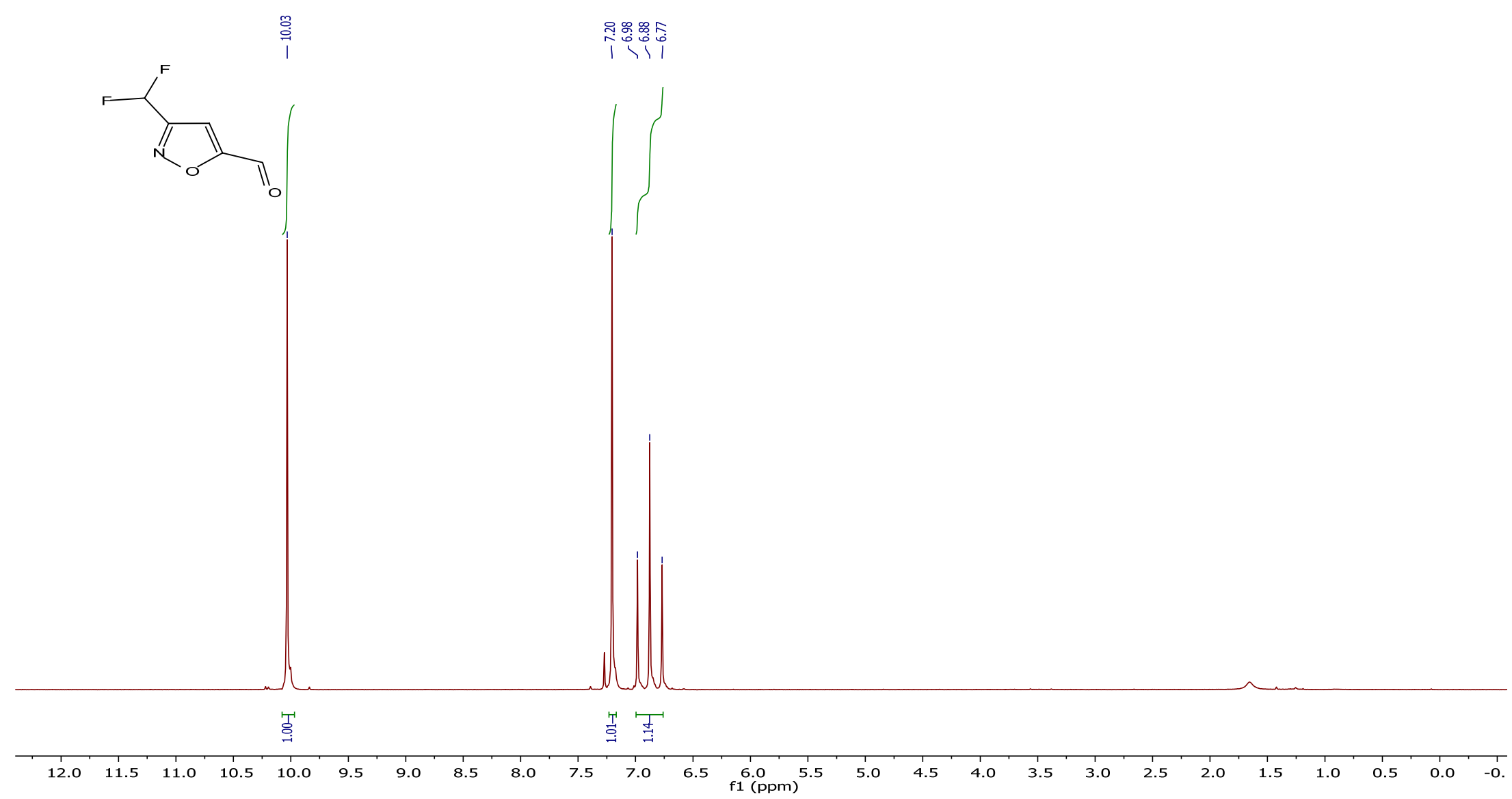


${ }^{13} \mathrm{C}\left\{{ }^{1} \mathrm{H}\right\}$ NMR spectrum of the compound 45a.

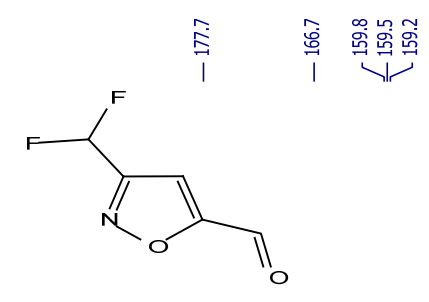

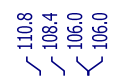

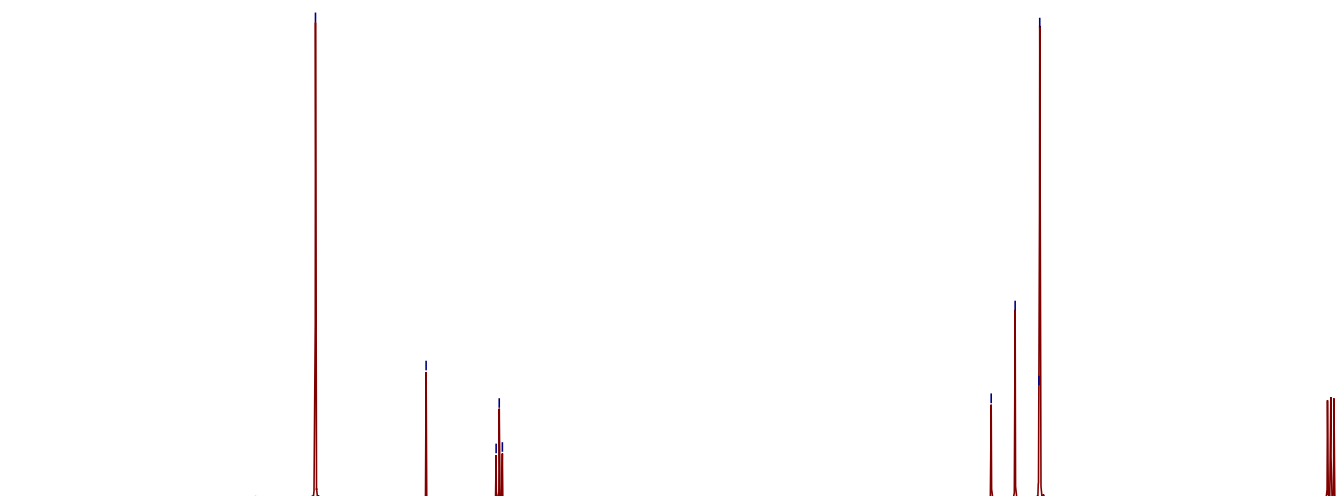

200

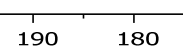

160

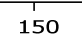

140

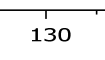

$110 \quad 100$

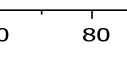

70

60

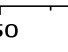

40 
${ }^{19} \mathrm{~F}\left\{{ }^{1} \mathrm{H}\right\}$ NMR spectrum of the compound 45a.

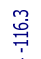
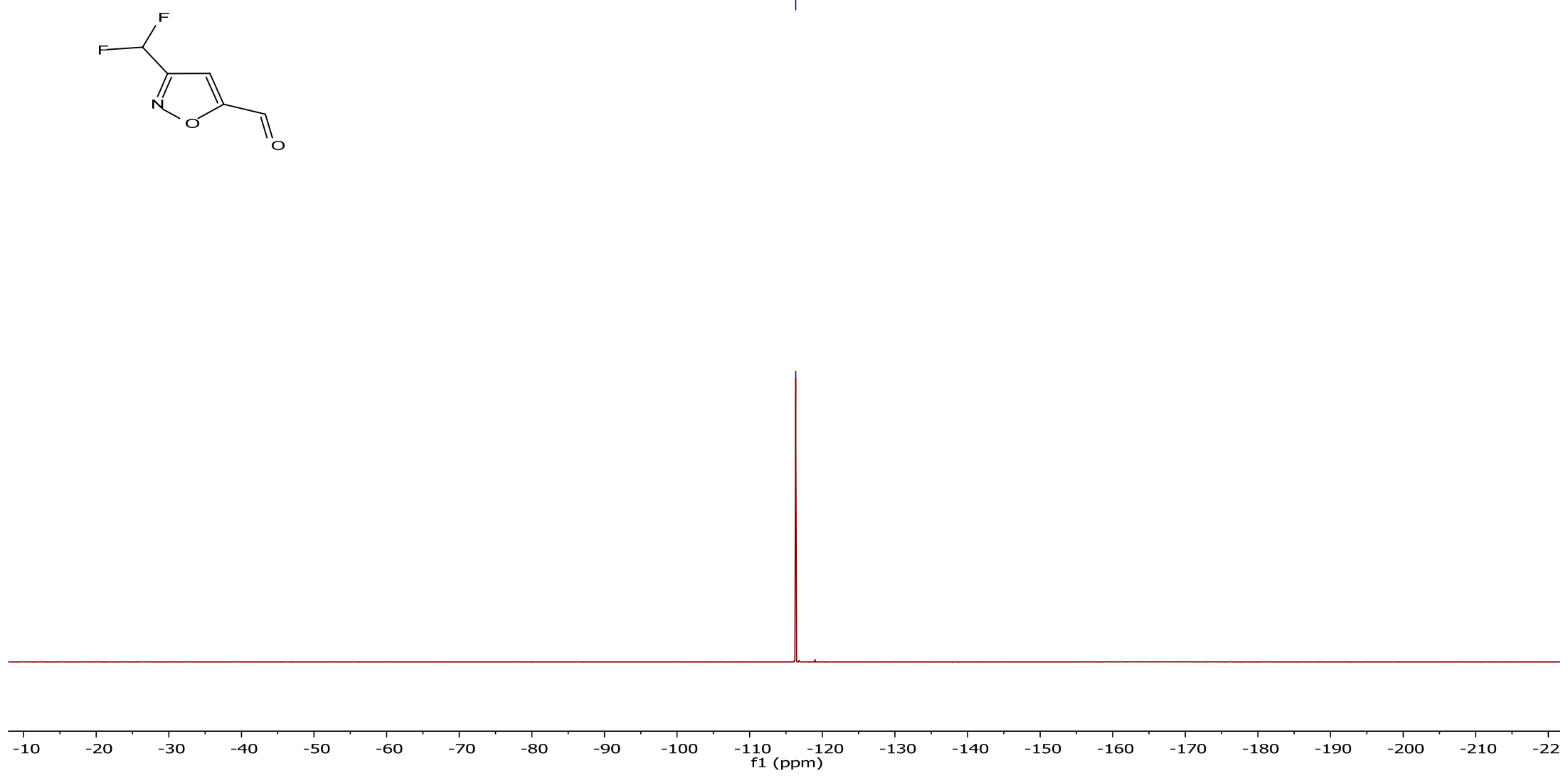
${ }^{1} \mathrm{H}$ NMR spectrum of the compound $\mathbf{4 4 h}$.

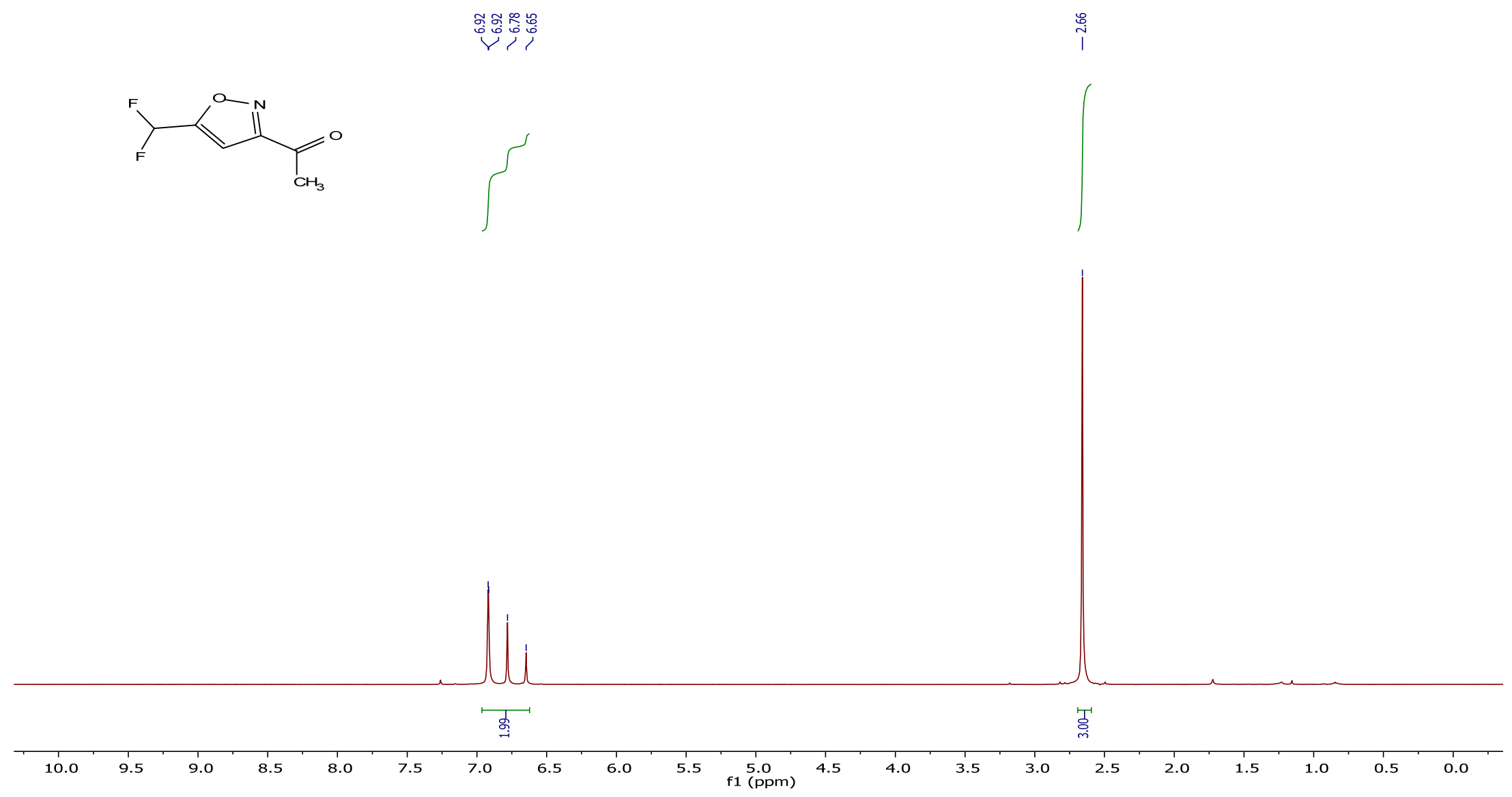


${ }^{13} \mathrm{C}\left\{{ }^{1} \mathrm{H}\right\}$ NMR spectrum of the compound $\mathbf{4 4 h}$.

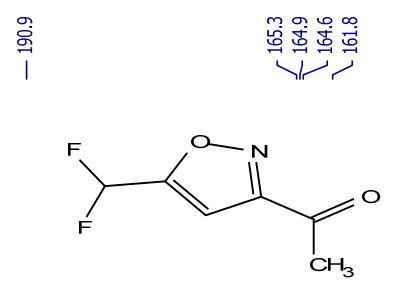

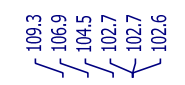

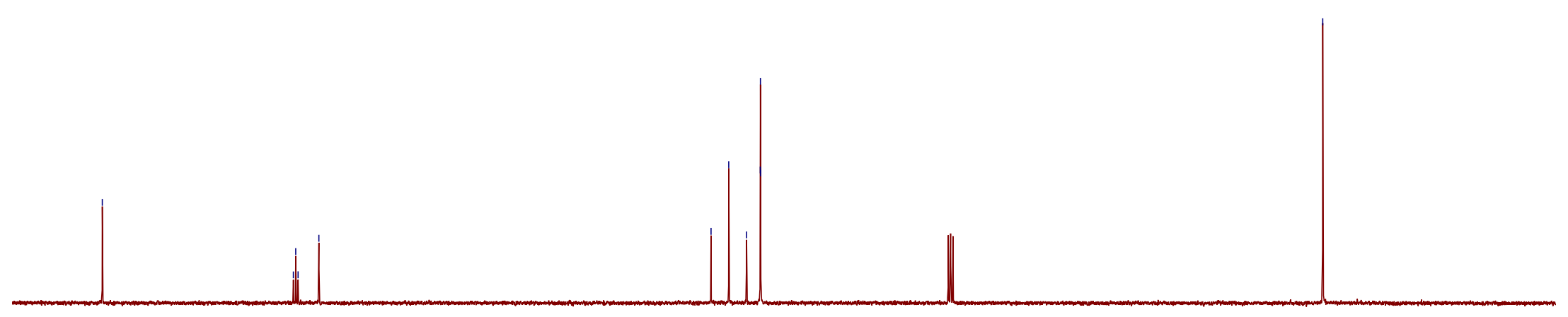

200

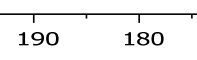

160

140

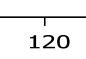

$10 \quad \begin{aligned} & 100 \\ & \mathrm{f} 1(\mathrm{ppm})\end{aligned}$ 
${ }^{19} \mathrm{~F}\left\{{ }^{1} \mathrm{H}\right\}$ NMR spectrum of the compound $\mathbf{4 4 h}$.

$\stackrel{\infty}{\longrightarrow}$<smiles>CC(=O)c1cc(C(F)F)on1</smiles>

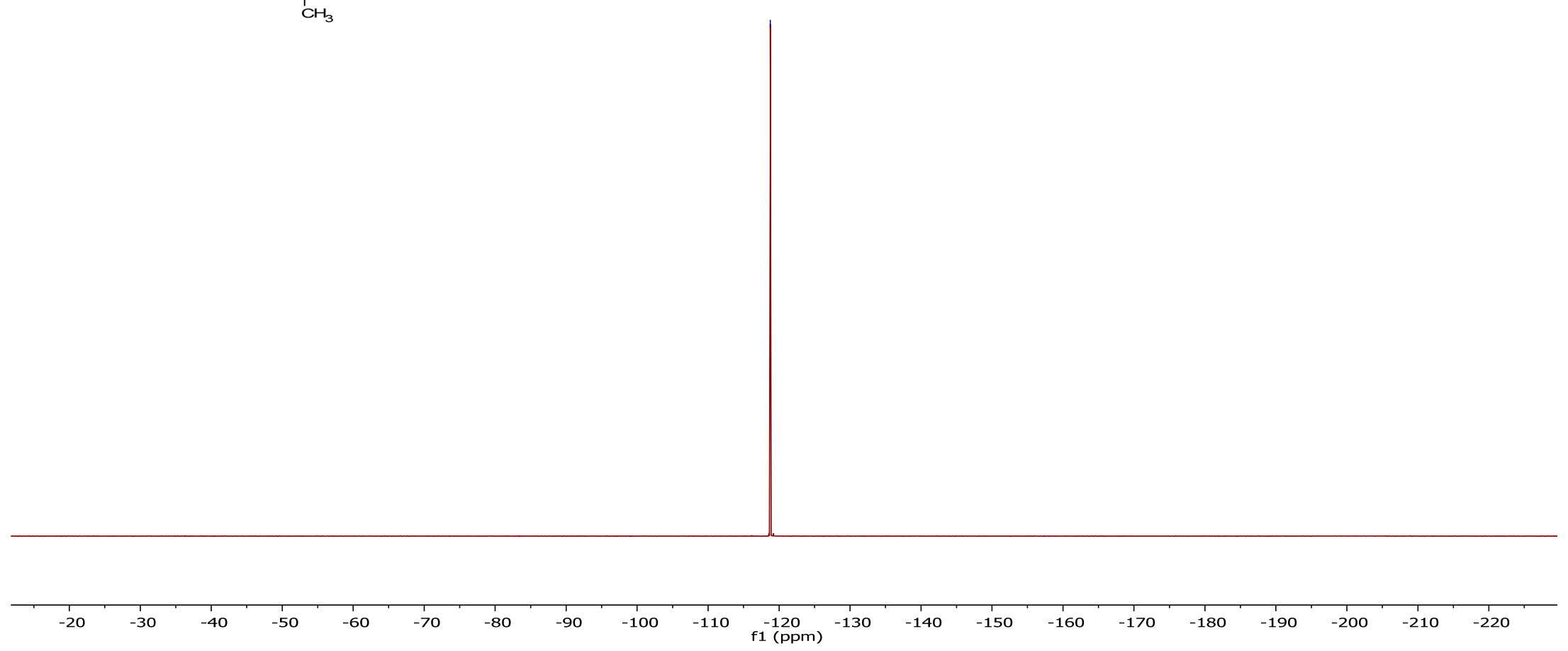


${ }^{1} \mathrm{H}$ NMR spectrum of the compound 46a.

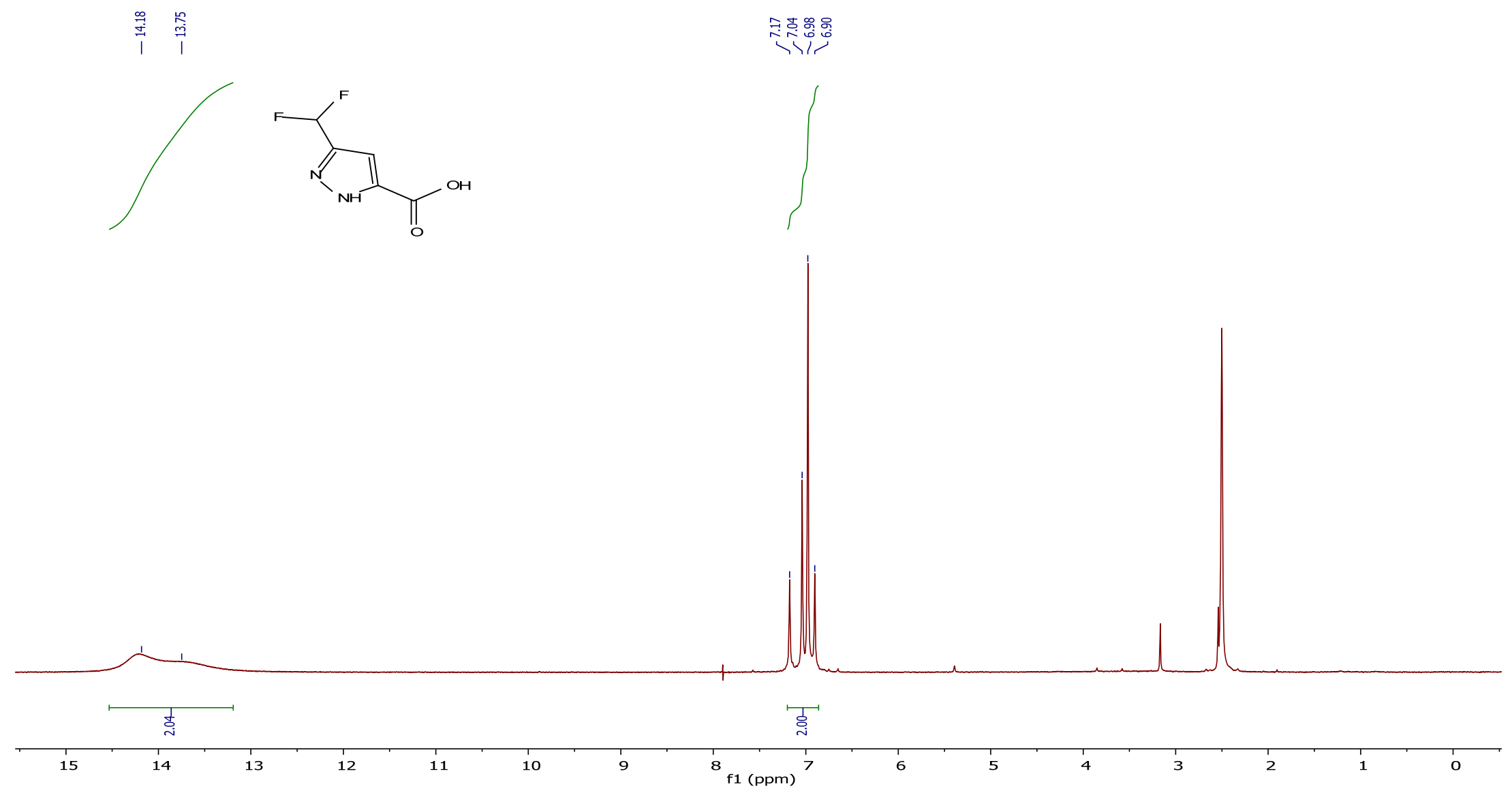




\section{${ }^{13} \mathrm{C}\left\{{ }^{1} \mathrm{H}\right\}$ NMR spectrum of the compound 46a.}

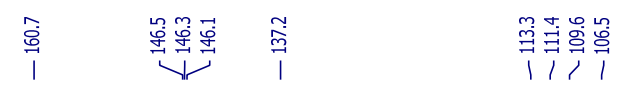<smiles>O=C(O)c1cc(C(F)F)n[nH]1</smiles>

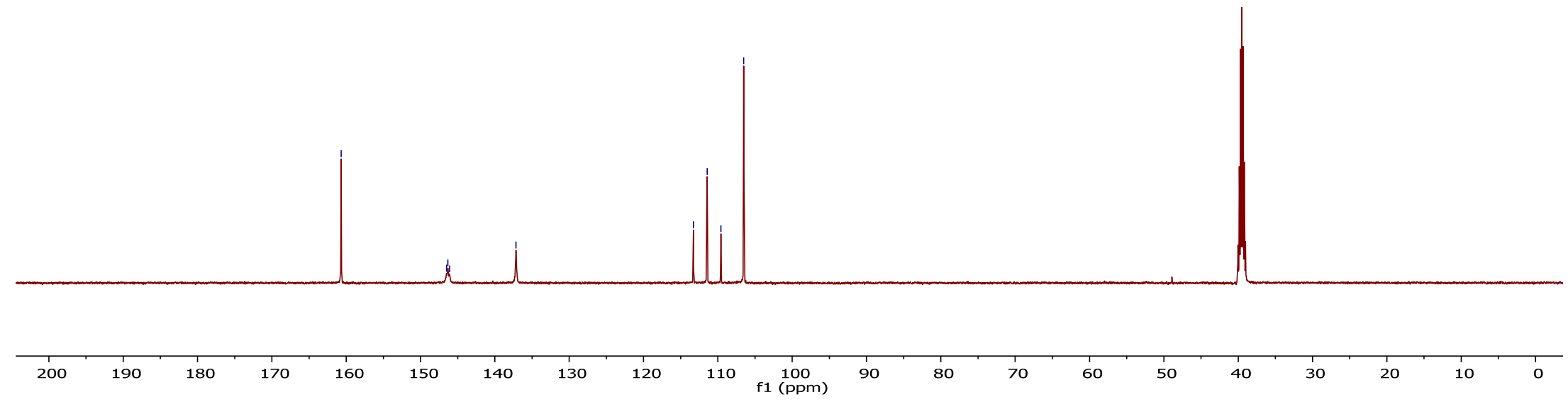


${ }^{19} \mathrm{~F}$ NMR spectrum of the compound $\mathbf{4 6 a}$.

产章
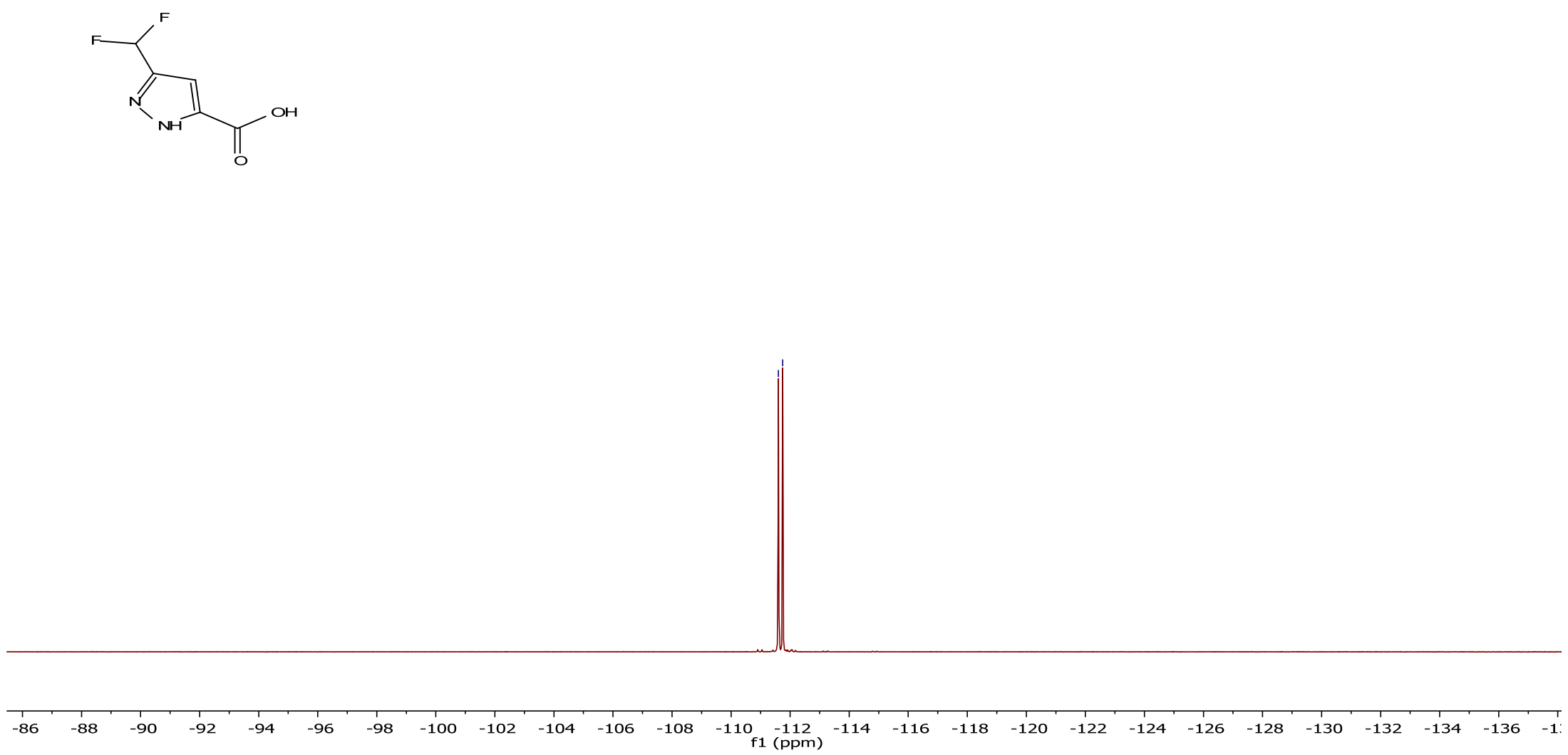

S274 
${ }^{1} \mathrm{H}$ NMR spectrum of the compound $\mathbf{4 7 a}$.

$\stackrel{\widetilde{0}}{\stackrel{0}{?}}$
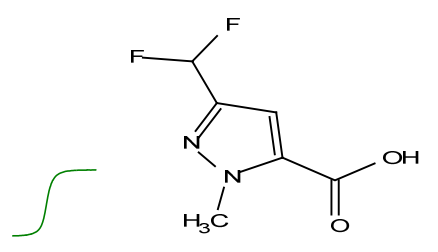

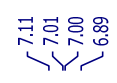

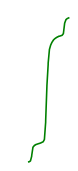

F

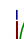

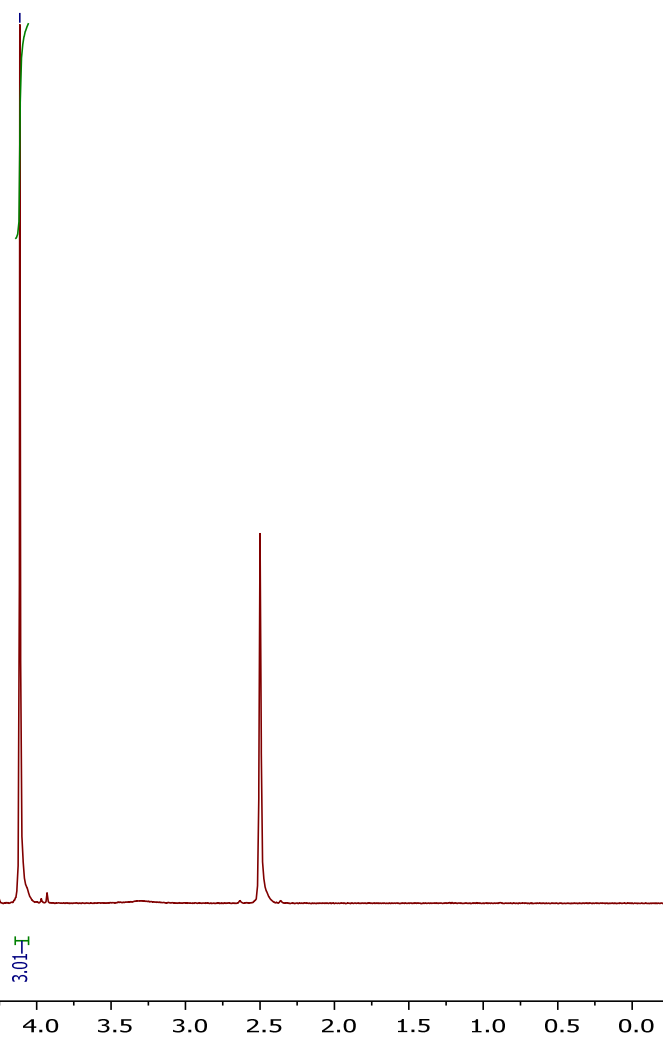

S275 
${ }^{13} \mathrm{C}\left\{{ }^{1} \mathrm{H}\right\}$ NMR spectrum of the compound $47 \mathbf{a}$.

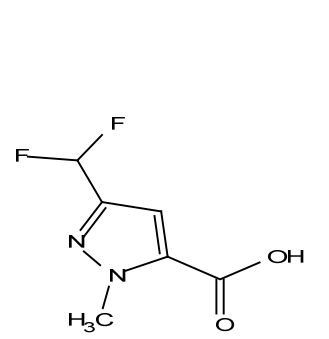

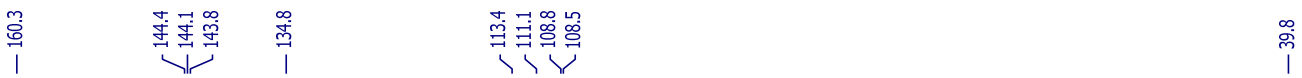

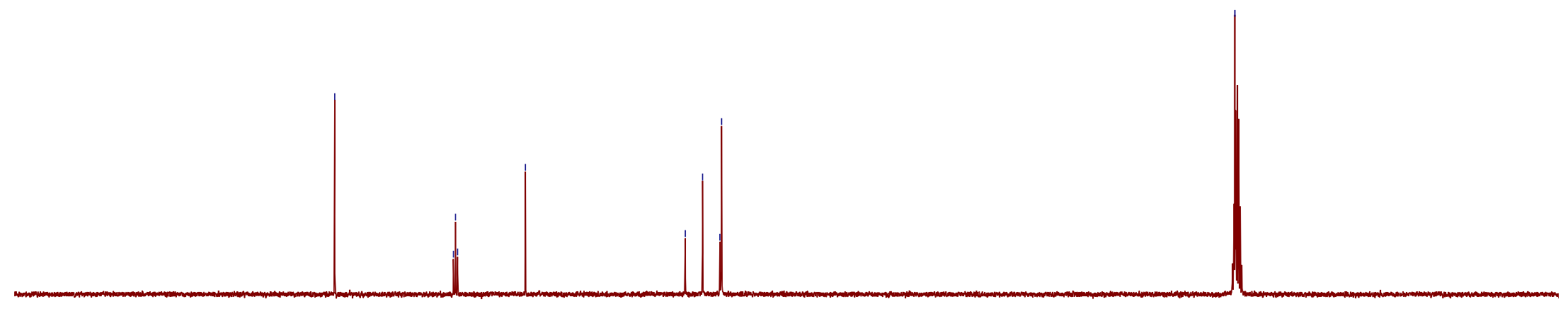

200

190

180
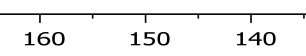

$130 \quad 120$

$110 \quad 100$

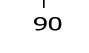

80

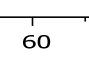

50 
${ }^{19} \mathrm{~F}\left\{{ }^{1} \mathrm{H}\right\}$ NMR spectrum of the compound 47a.

$\vec{\Xi}$
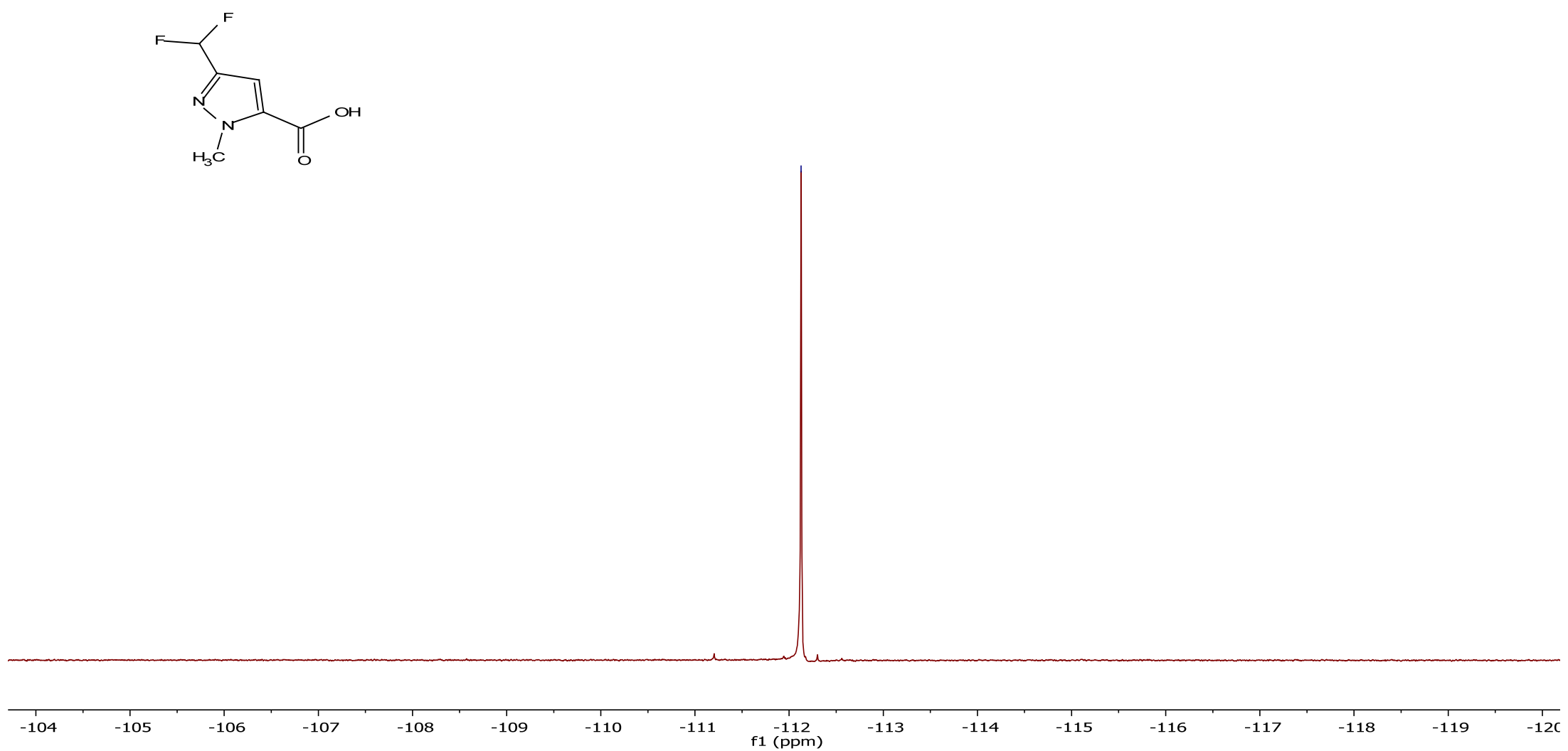
${ }^{1} \mathrm{H}$ NMR spectrum of the compound $4 \mathbf{8}$.

i

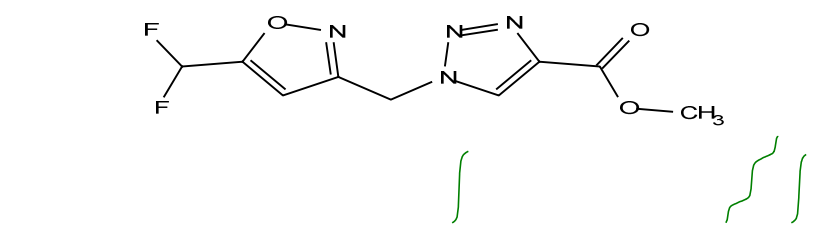

นิ

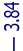
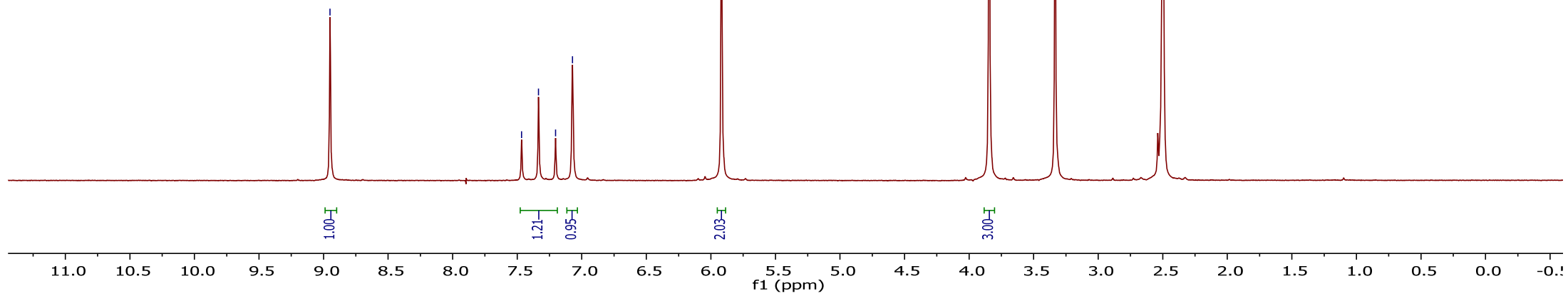

S278 

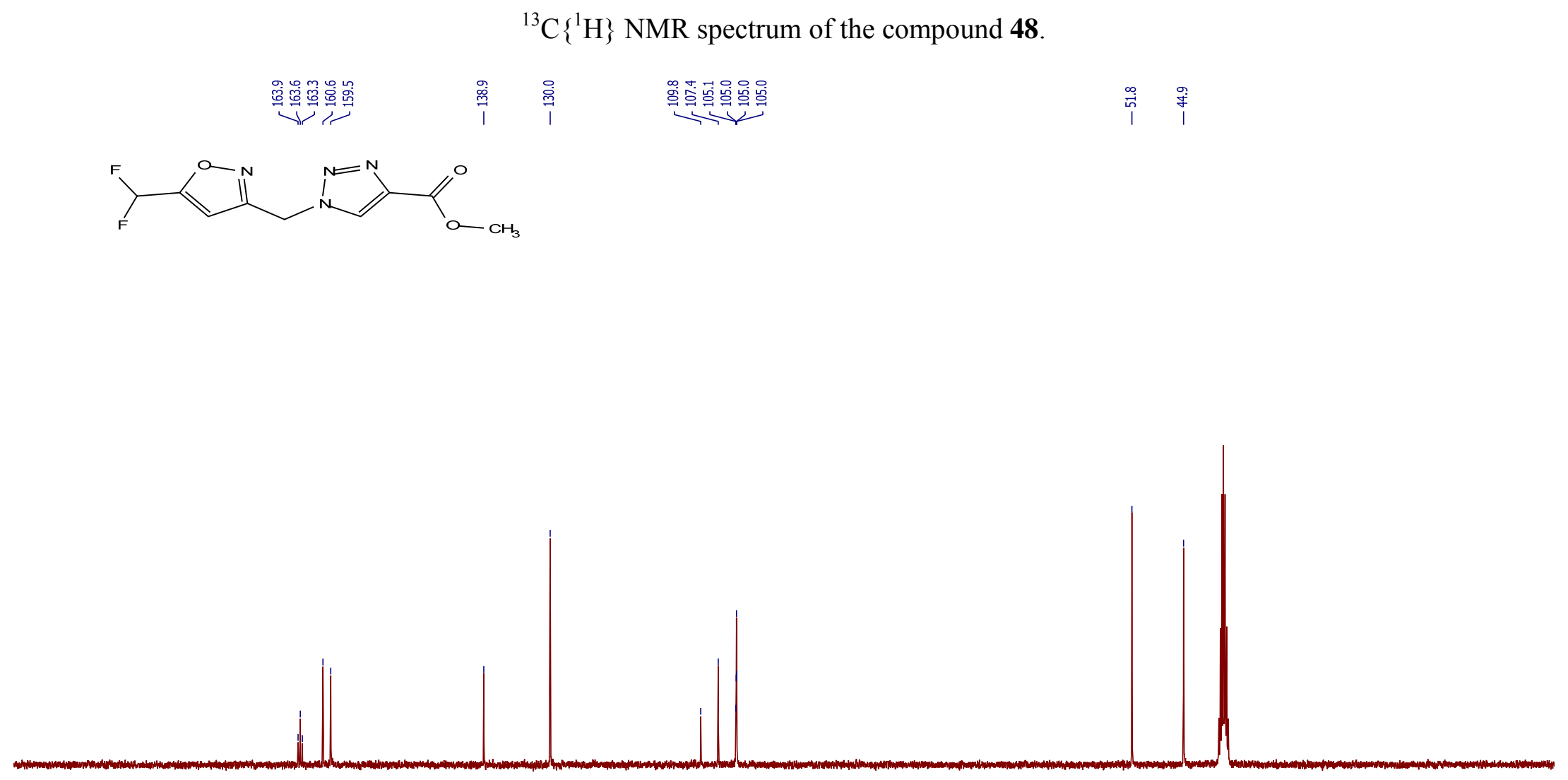

$\frac{1}{200}$
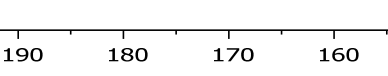
${ }^{19} \mathrm{~F}\left\{{ }^{1} \mathrm{H}\right\}$ NMR spectrum of the compound 48 .

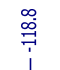
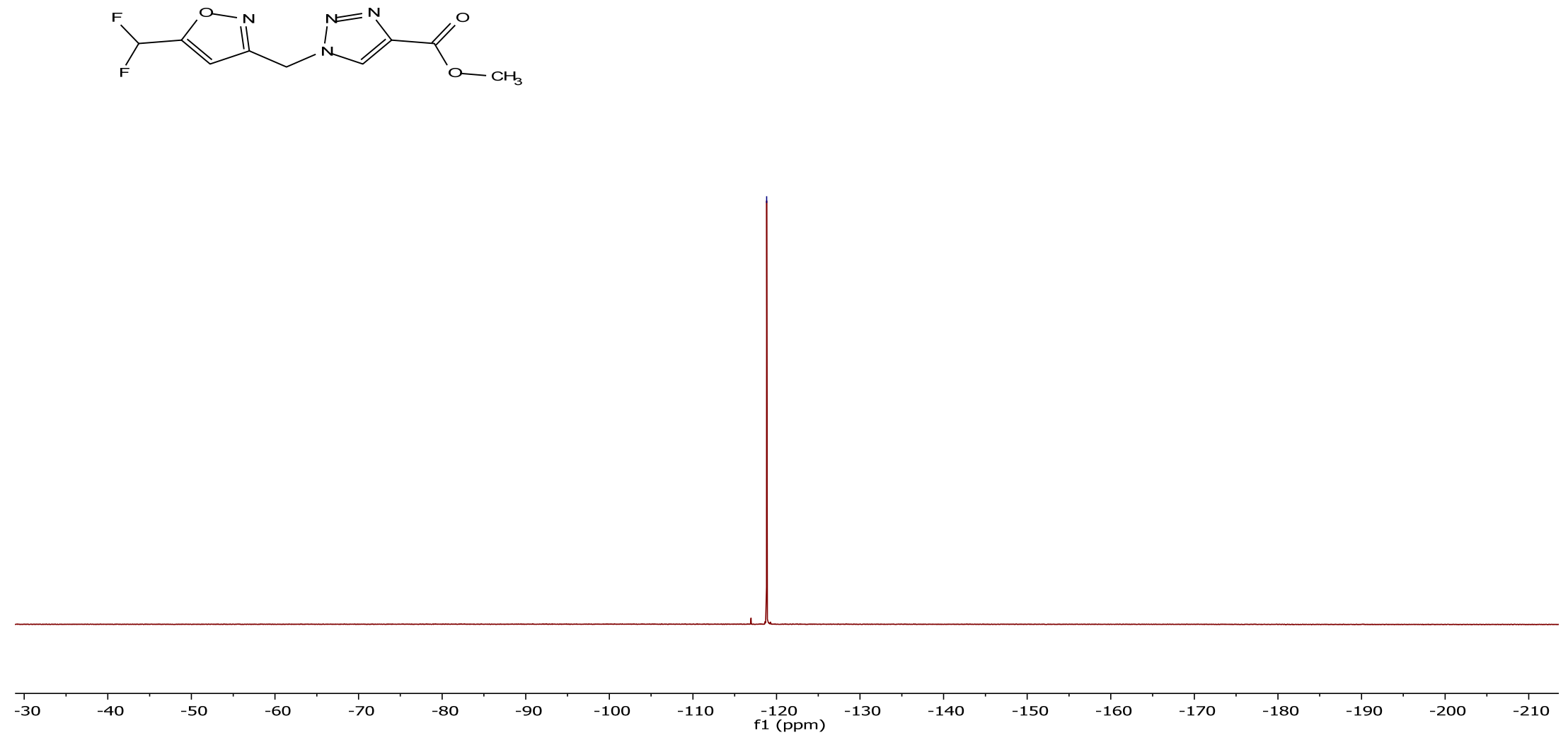
veres
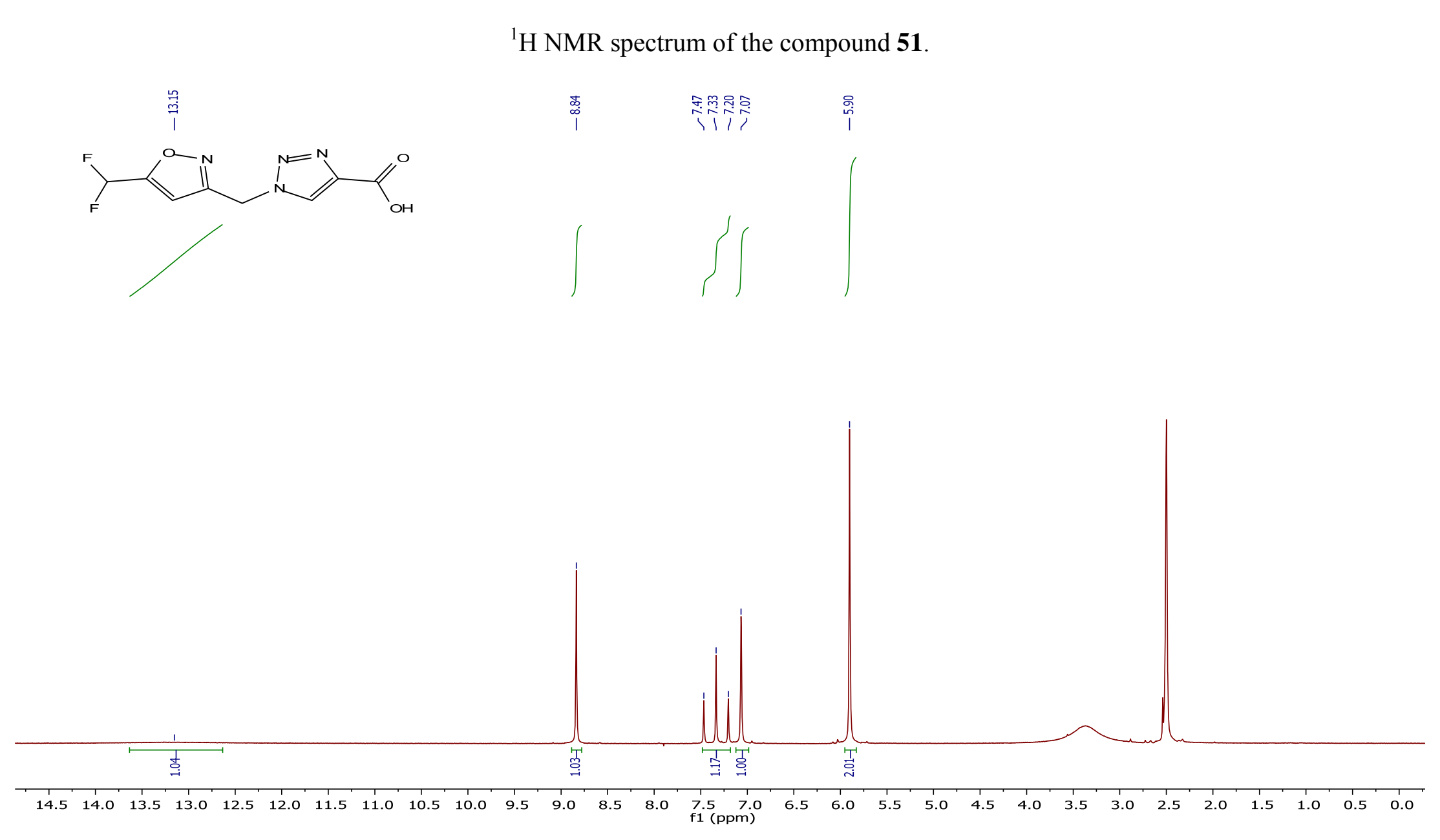

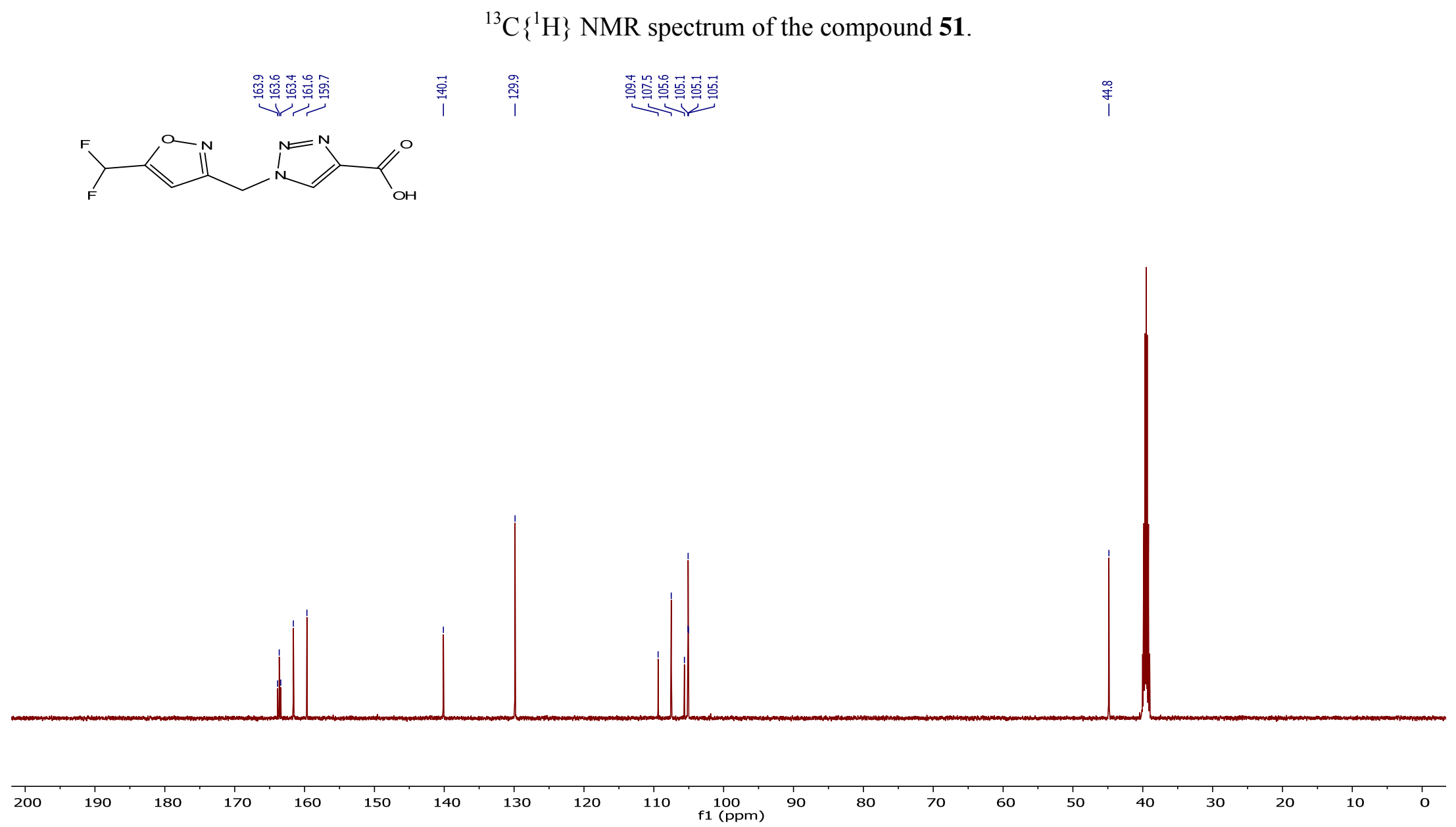
${ }^{19} \mathrm{~F}\left\{{ }^{1} \mathrm{H}\right\}$ NMR spectrum of the compound $\mathbf{5 1}$.

$\stackrel{\Xi}{\stackrel{\varpi}{i}}$
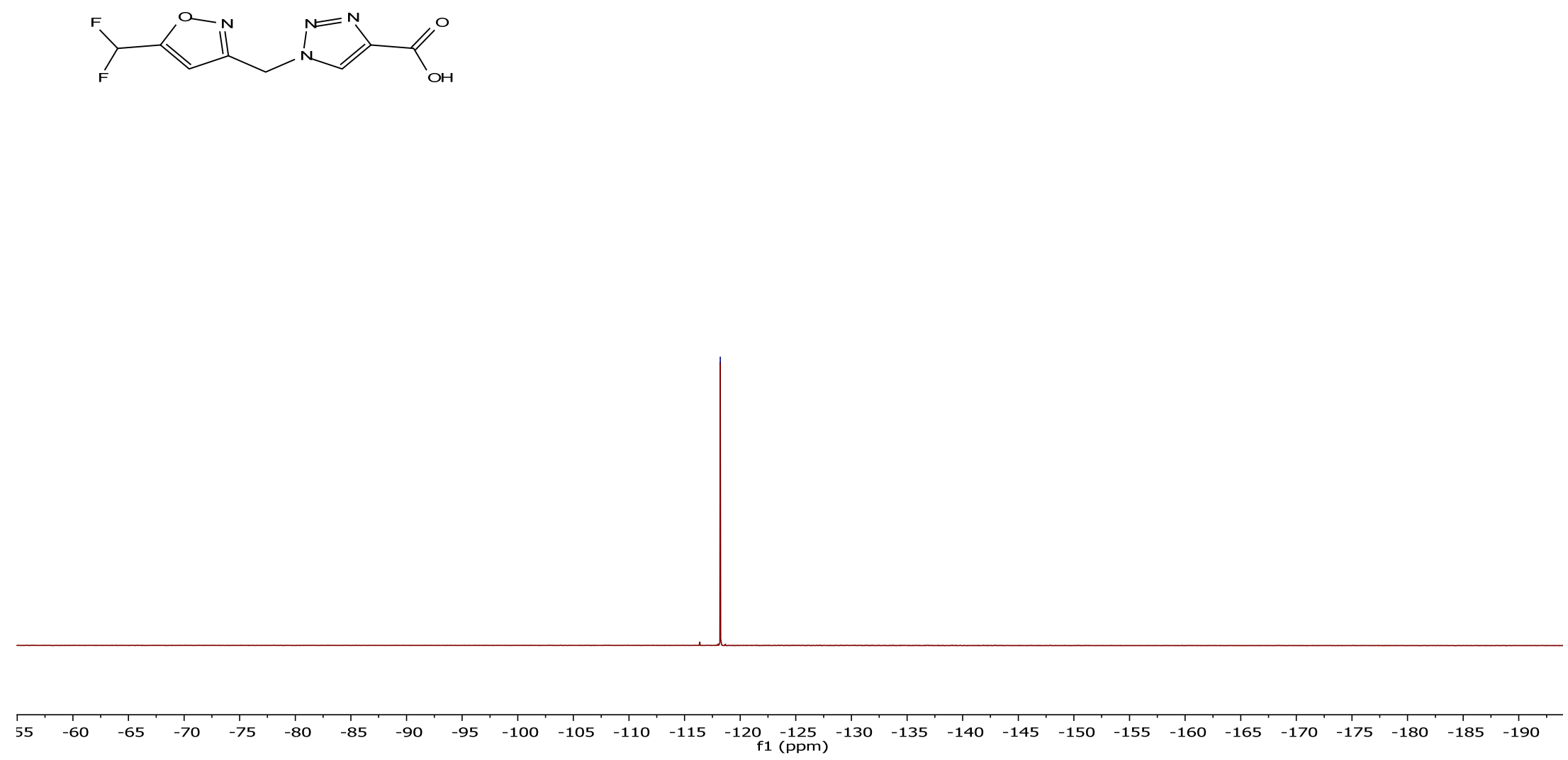
${ }^{1} \mathrm{H}$ NMR spectrum of the compound 49.
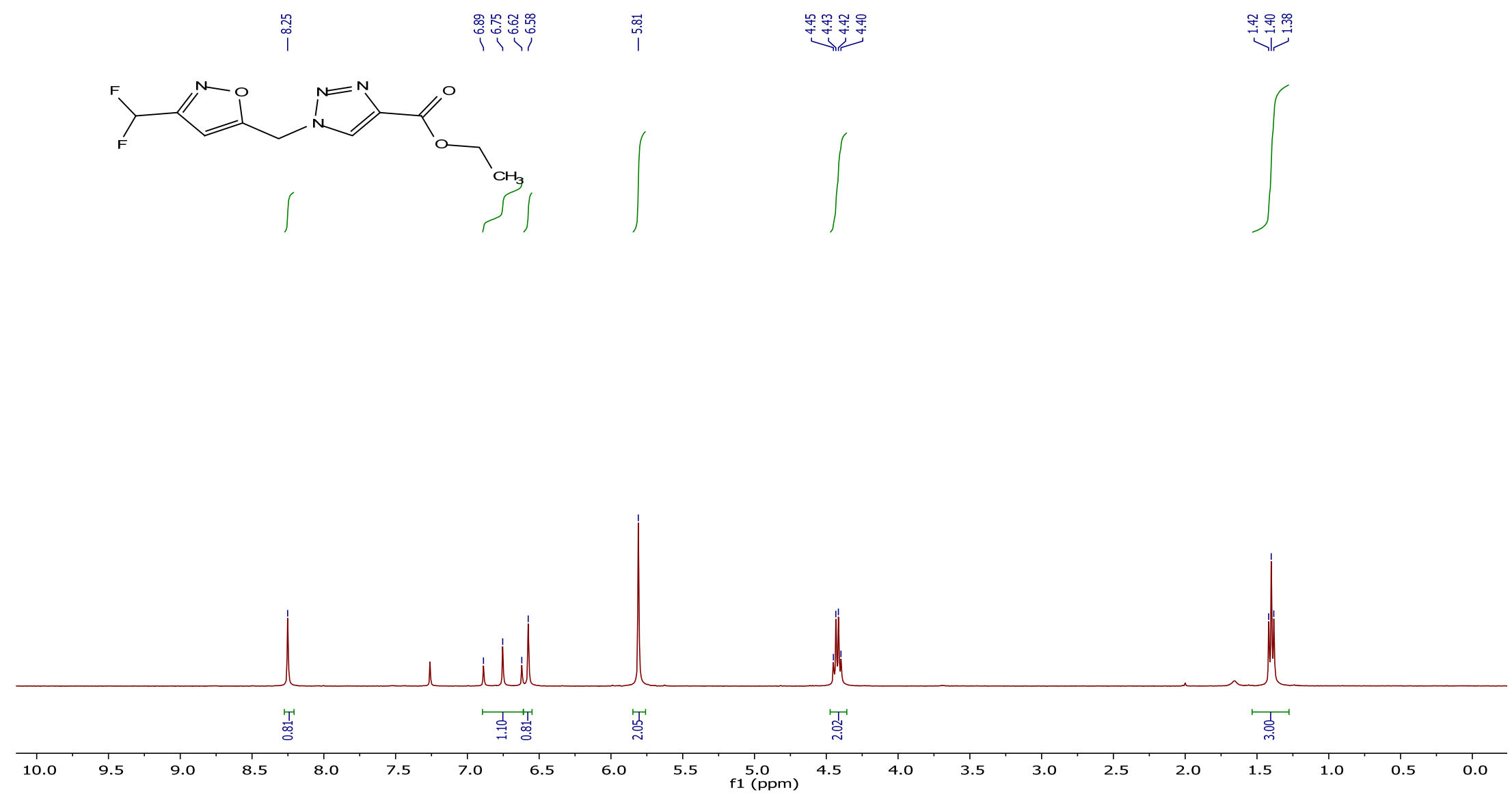


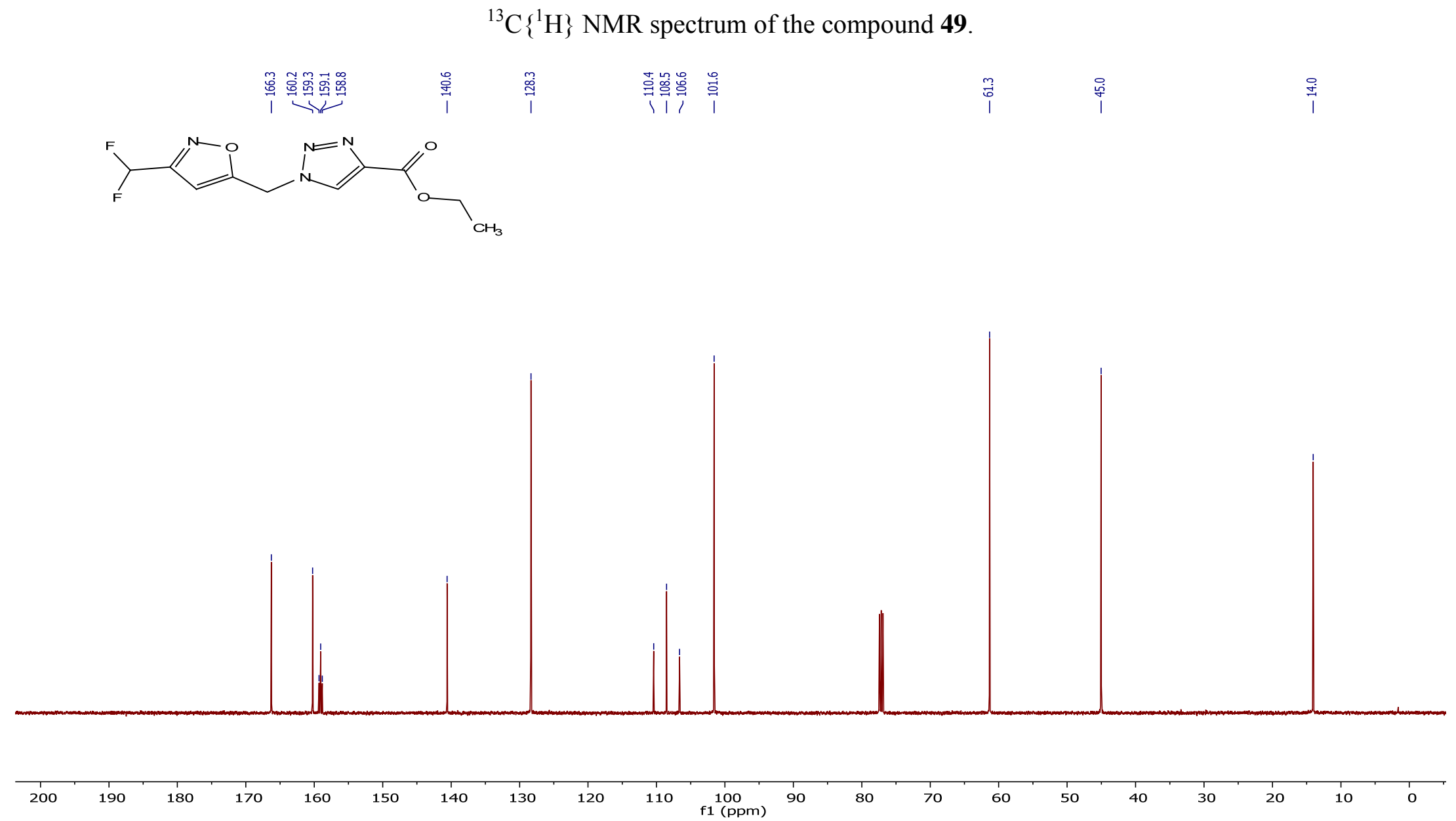


${ }^{19} \mathrm{~F}\left\{{ }^{1} \mathrm{H}\right\}$ NMR spectrum of the compound 49.

冚
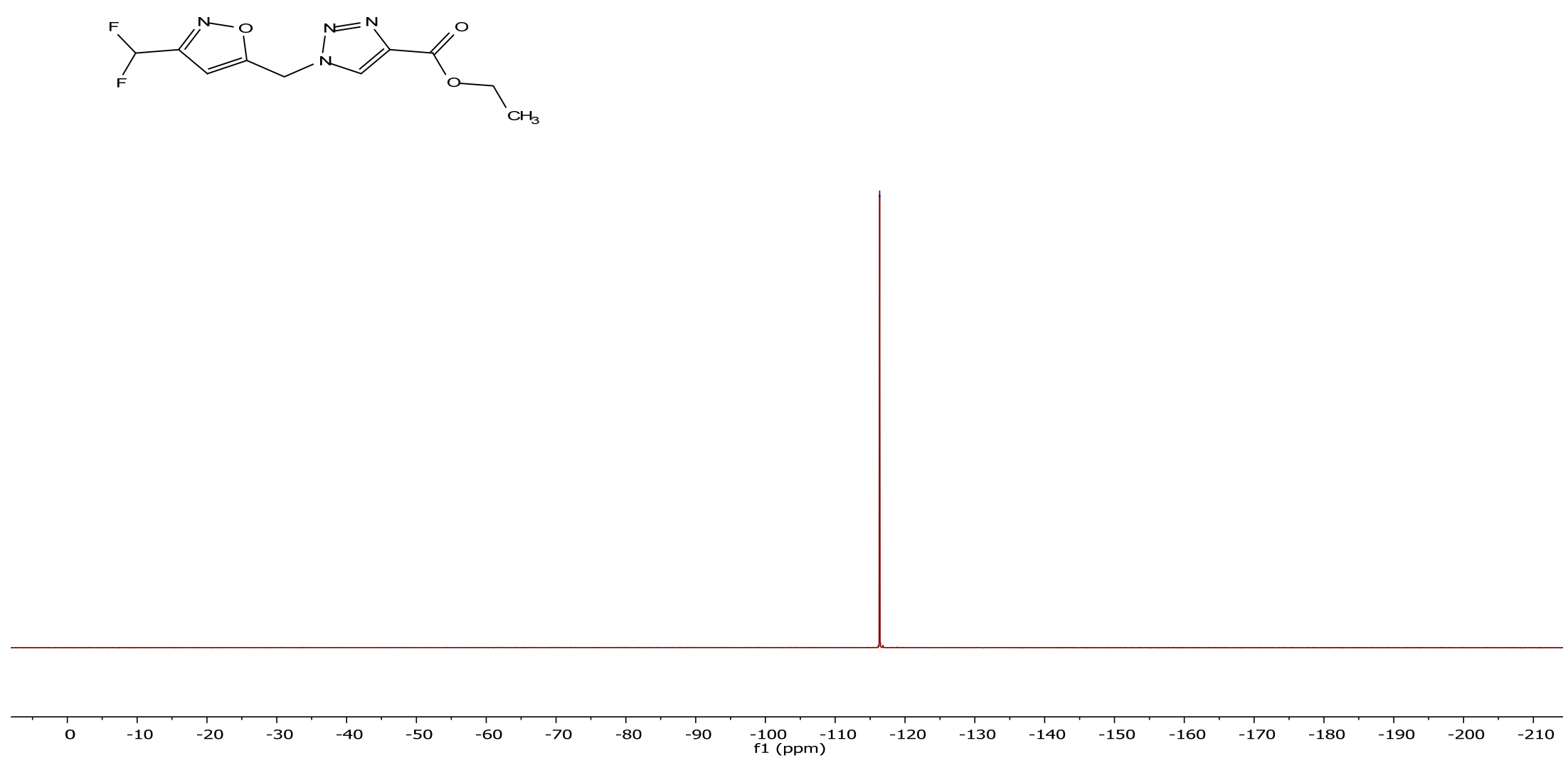
${ }^{1} \mathrm{H}$ NMR spectrum of the compound $\mathbf{5 0}$.

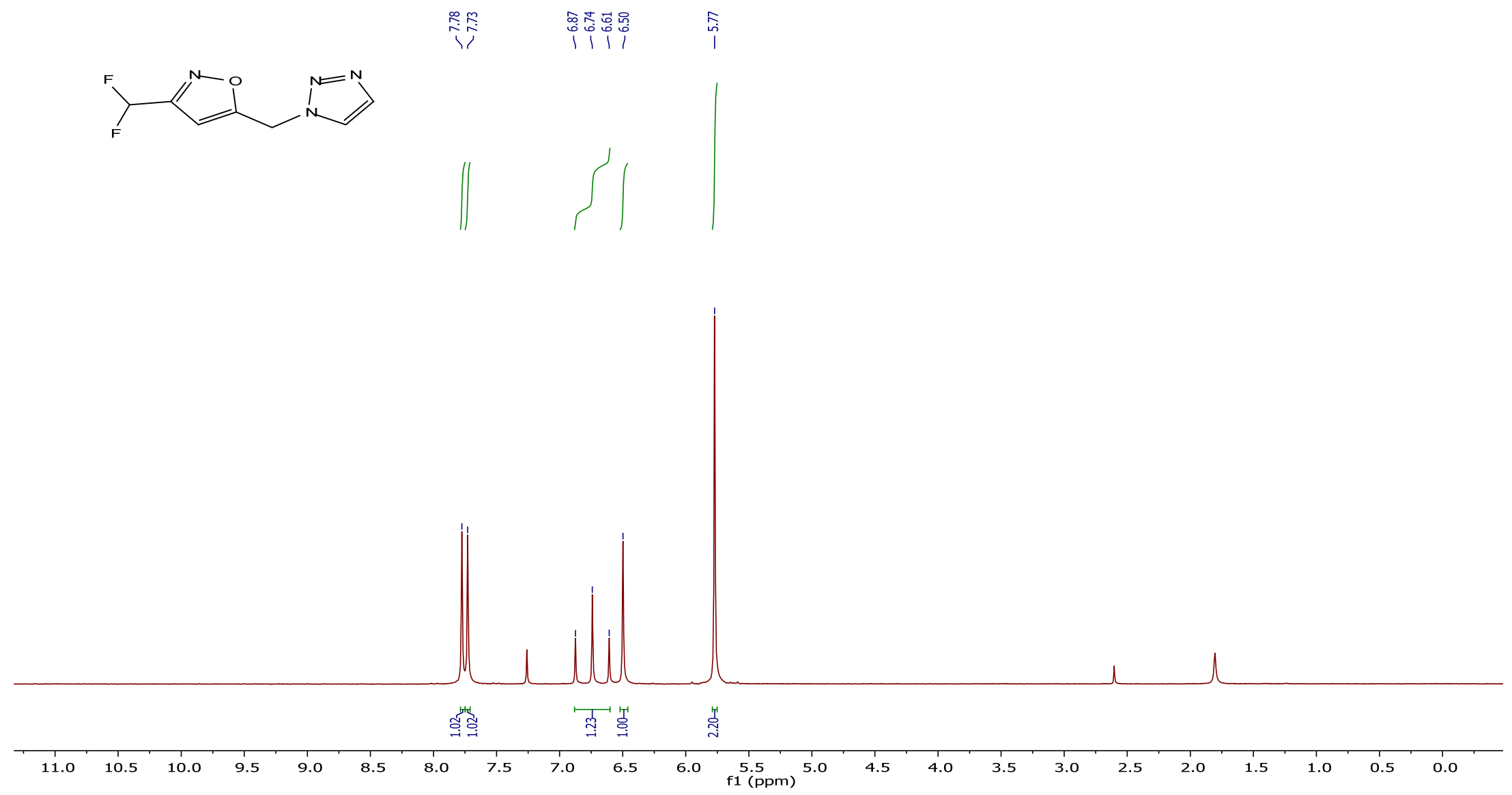




\section{${ }^{13} \mathrm{C}\left\{{ }^{1} \mathrm{H}\right\}$ NMR spectrum of the compound 50.}
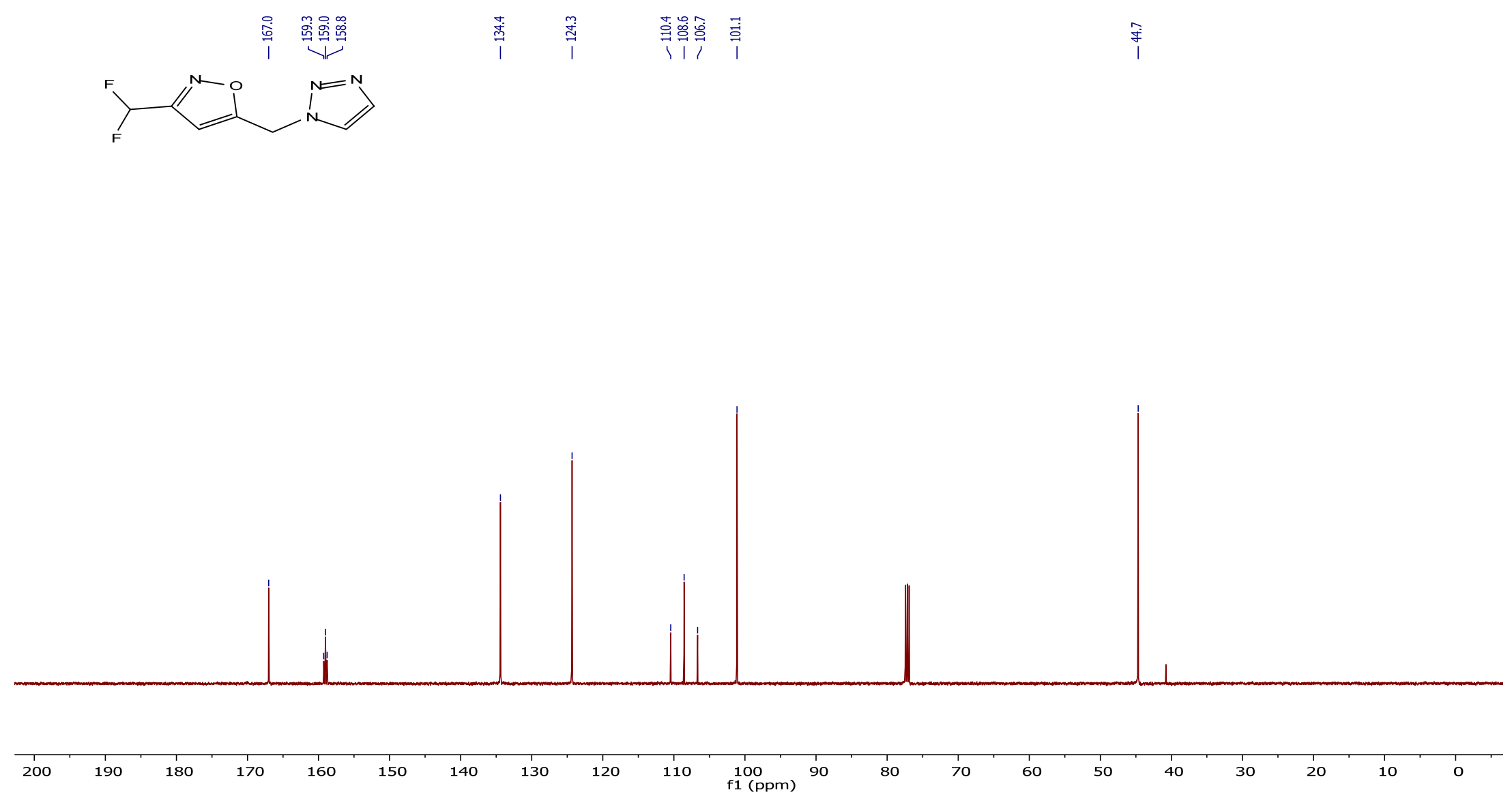
${ }^{19} \mathrm{~F}\left\{{ }^{1} \mathrm{H}\right\}$ NMR spectrum of the compound $\mathbf{5 0 .}$

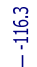
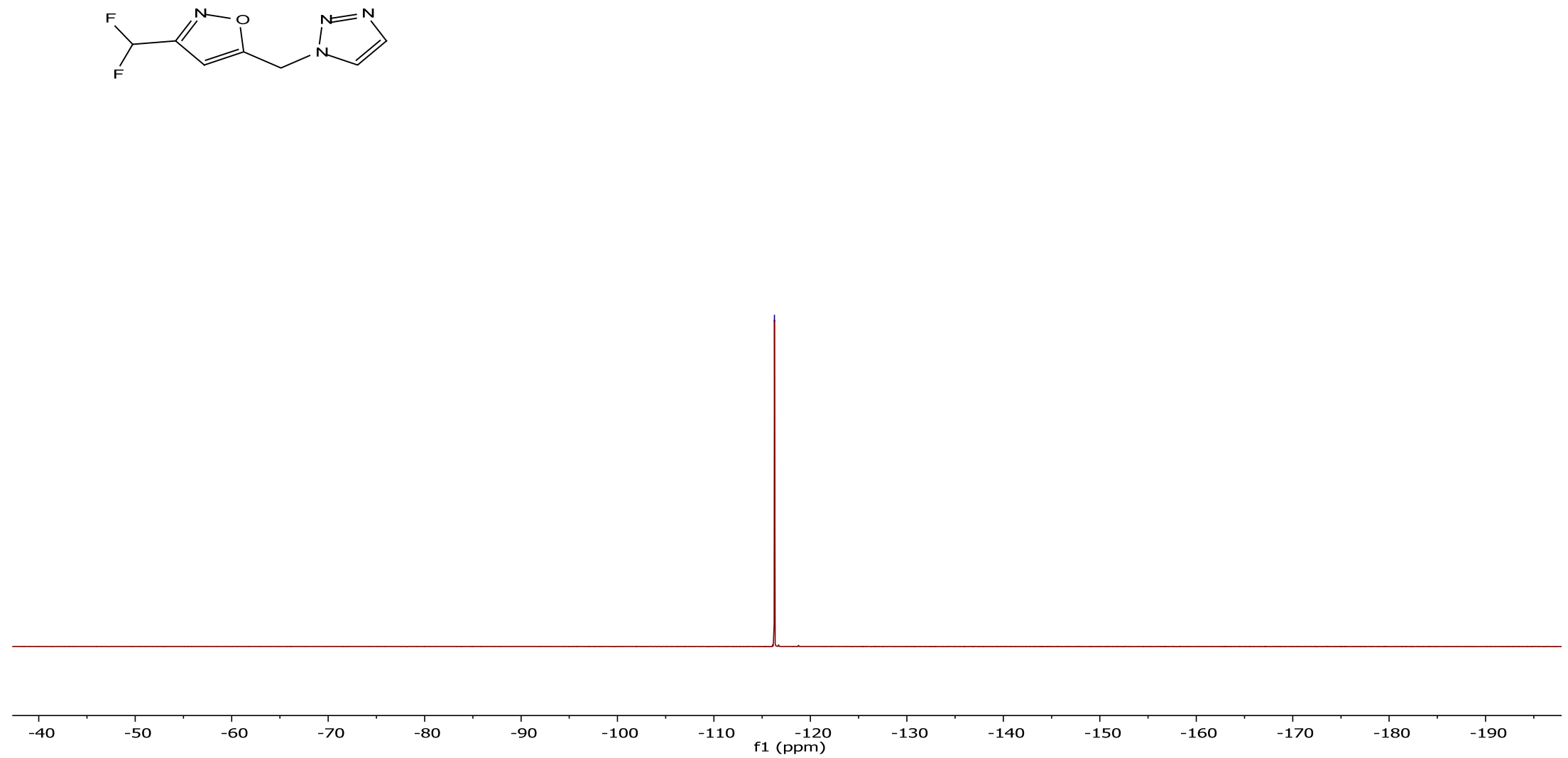
${ }^{1} \mathrm{H}$ NMR spectrum of the compound 52.
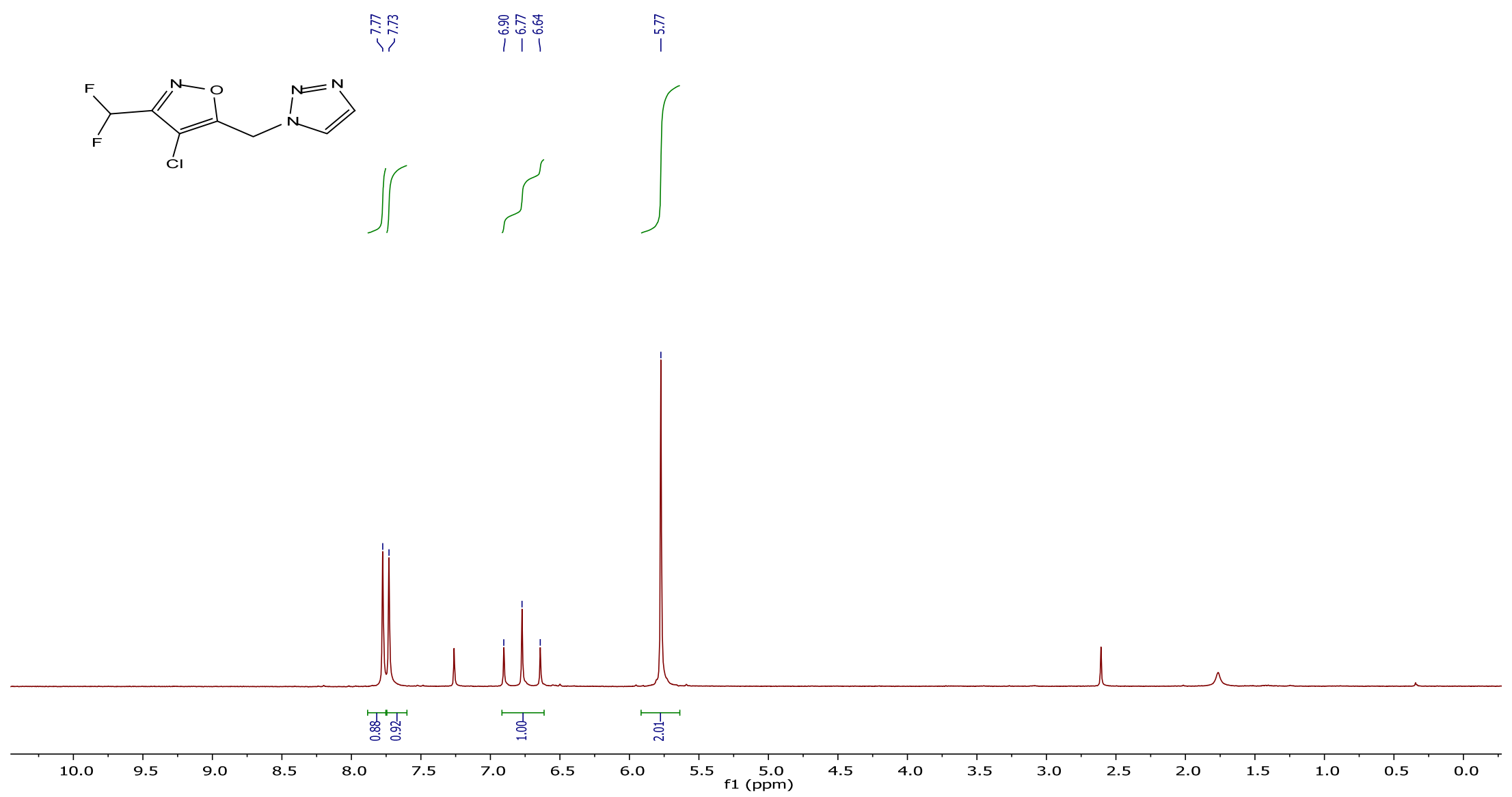


\section{${ }^{13} \mathrm{C}\left\{{ }^{1} \mathrm{H}\right\}$ NMR spectrum of the compound 52.}
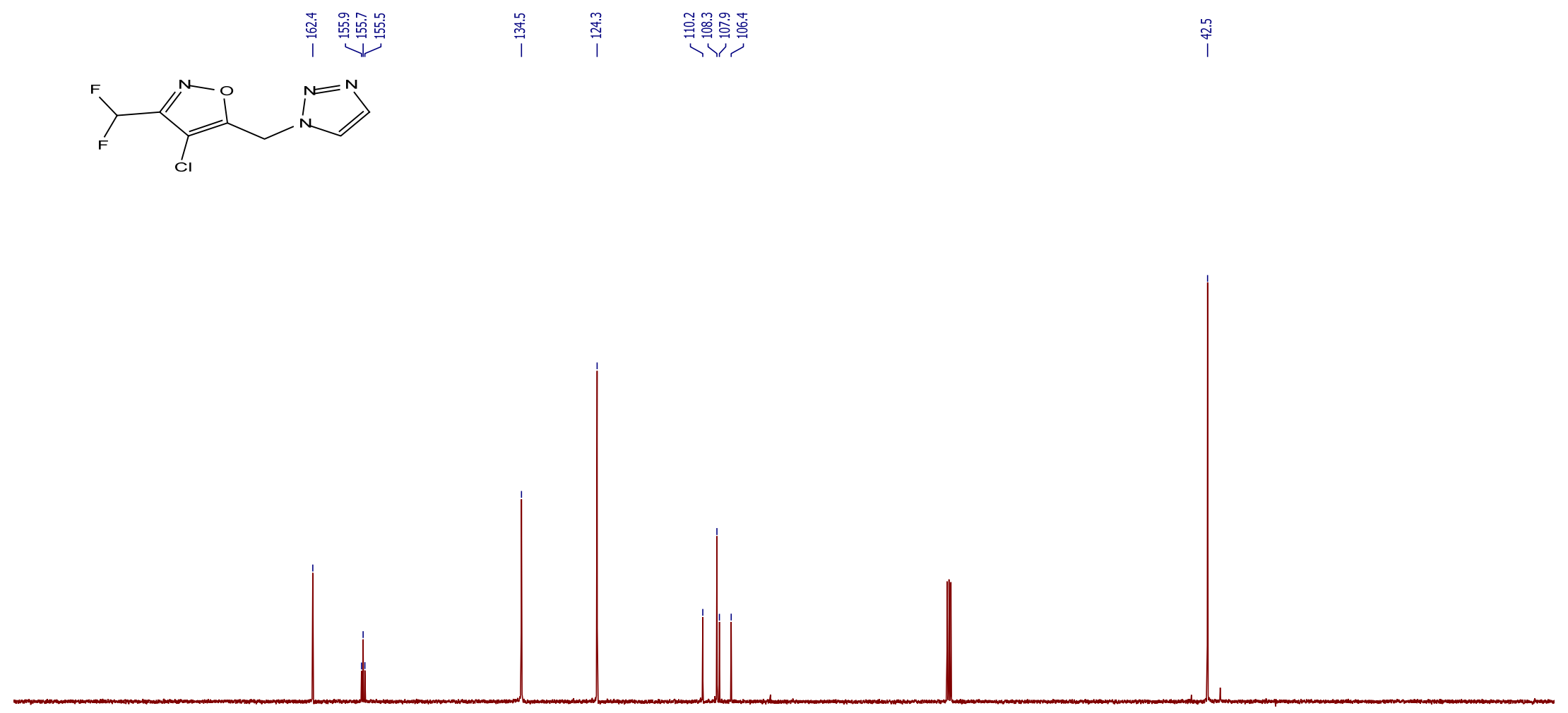
${ }^{19} \mathrm{~F}\left\{{ }^{1} \mathrm{H}\right\}$ NMR spectrum of the compound 52.

$\stackrel{\circ}{\stackrel{9}{i}}$<smiles>FC(F)c1noc(Cn2ccnn2)c1Cl</smiles>

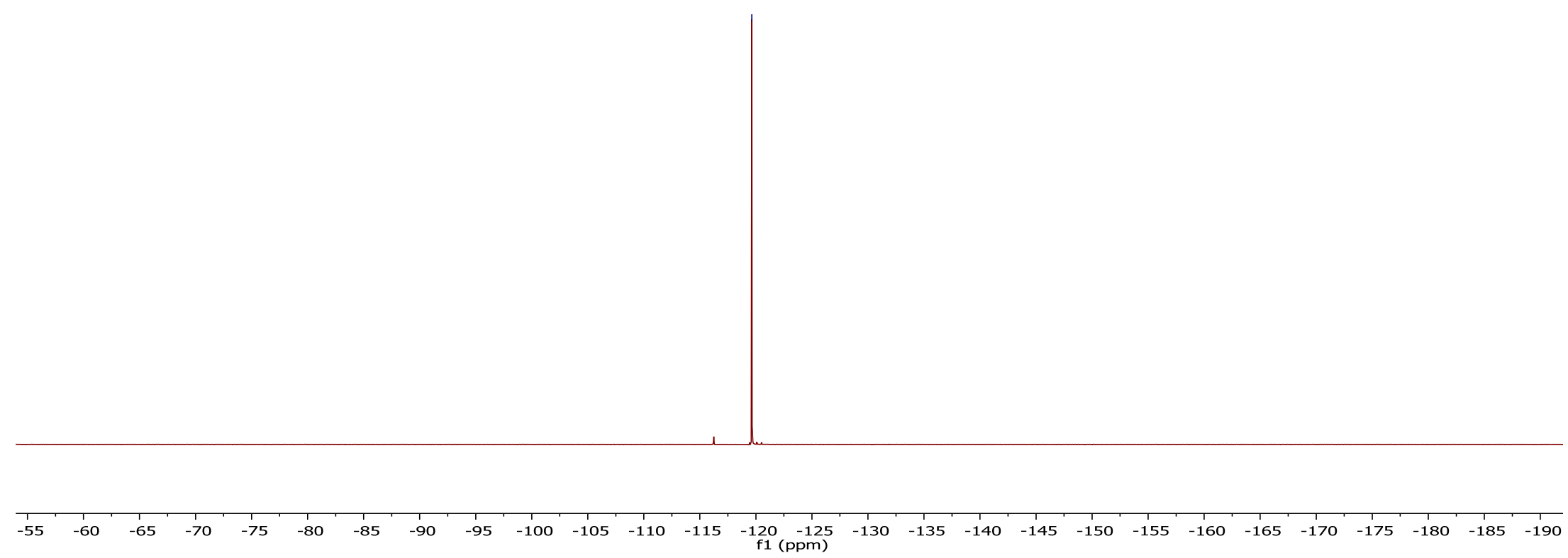




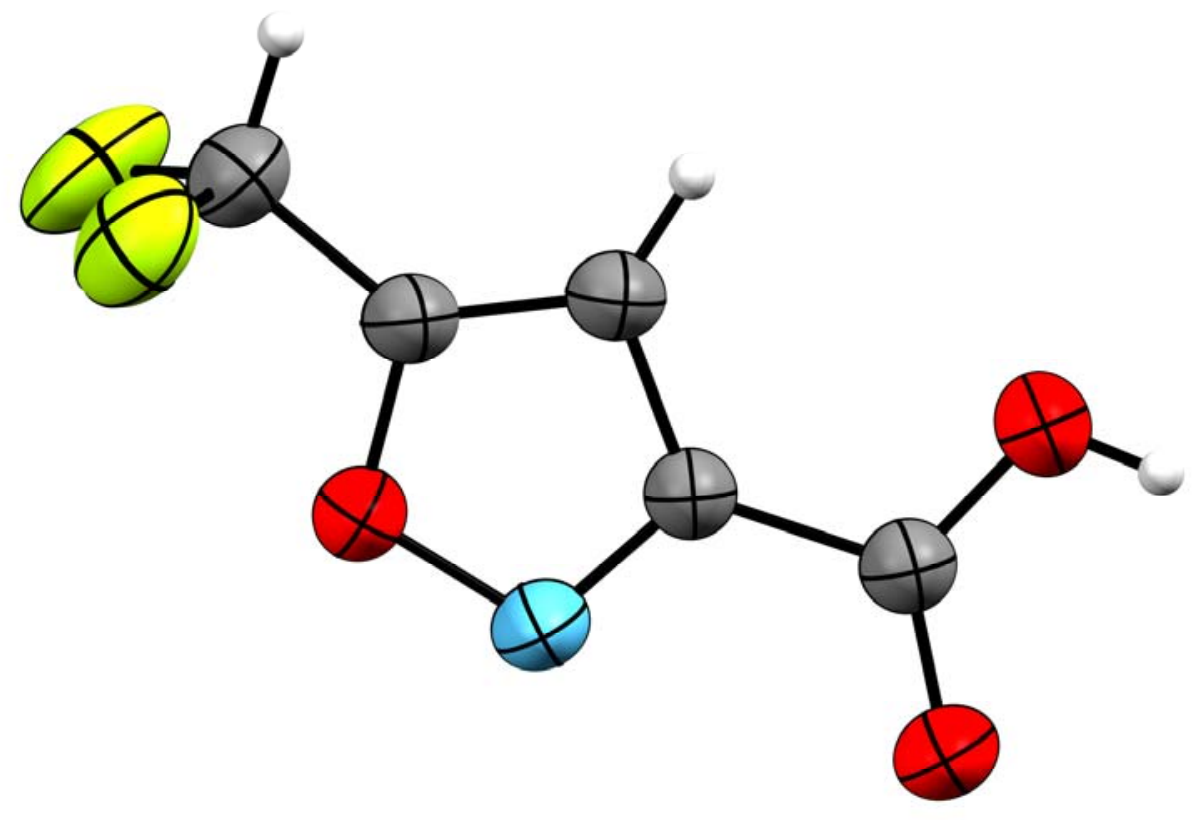

Figure S2. ORTEP diagram for compound 29a. Thermal ellipsoids are shown at the $50 \%$ probability level. 


\section{ORTEP diagram for compound 35g.}

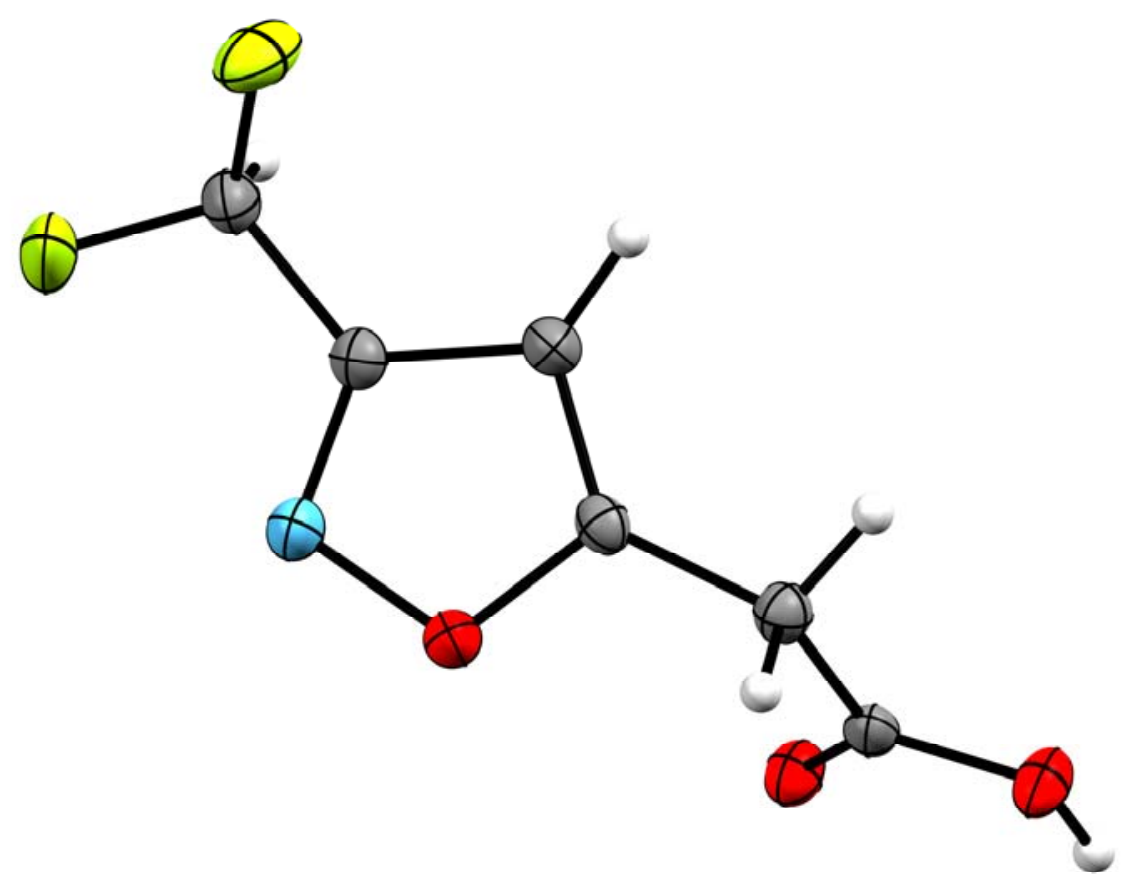

Figure S3. ORTEP diagram for compound 35g. Thermal ellipsoids are shown at the $50 \%$ probability level. 


\section{ORTEP diagram for compound 24h.}

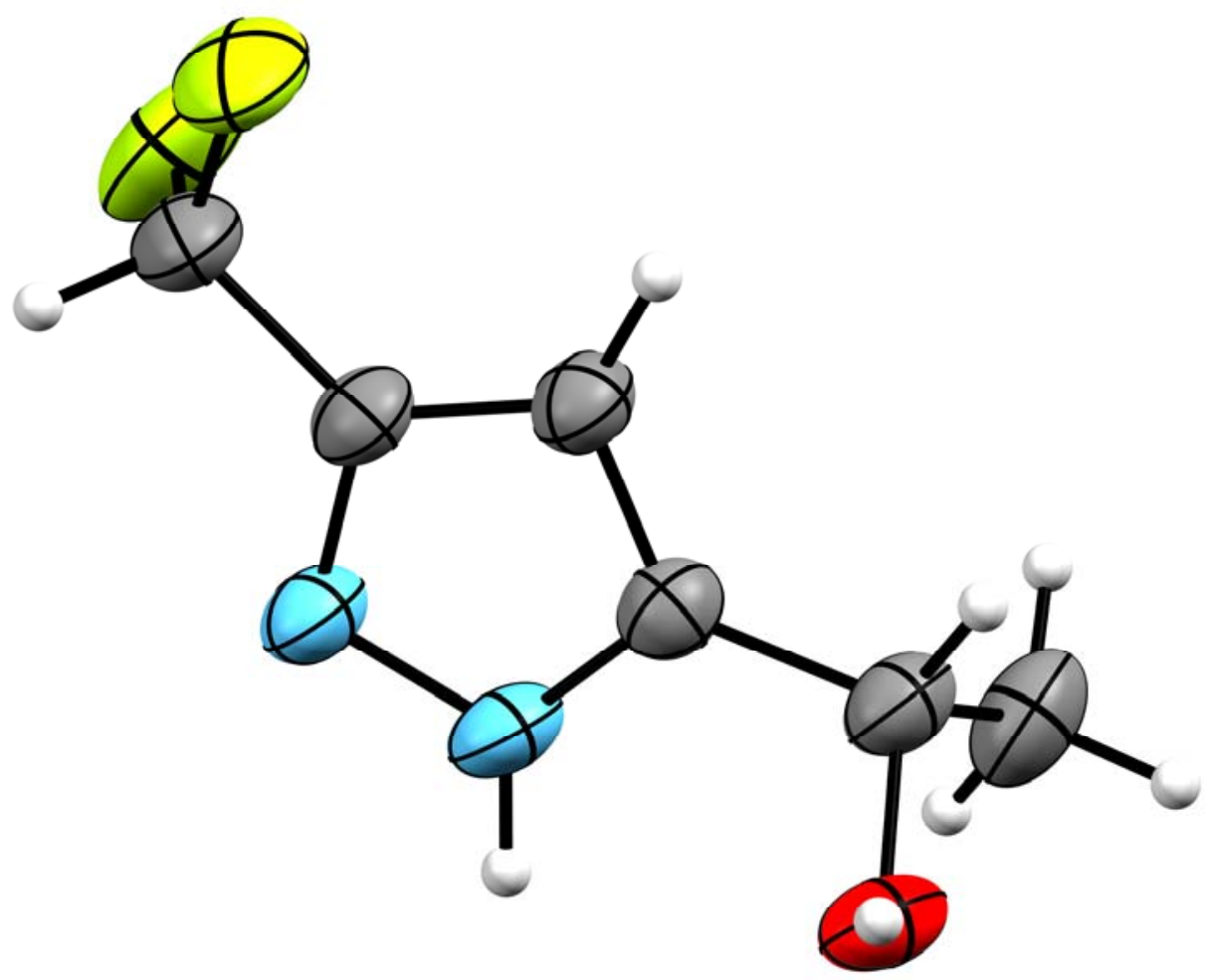

Figure S4. ORTEP diagram for compound 24h. Thermal ellipsoids are shown at the $50 \%$ probability level. 